\title{
A CHARACTERIZATION MODEL WITH SPATIAL AND TEMPORAL RESOLUTION FOR LIFE CYCLE IMPACT ASSESSMENT OF PHOTOCHEMICAL PRECURSORS IN THE UNITED STATES
}

\author{
by \\ Viral Pinakin Shah \\ B. Tech. (Civil Engineering), Indian Institute of Technology Bombay, 2004
}

\author{
Submitted to the Graduate Faculty of \\ Swanson School of Engineering in partial fulfillment \\ of the requirements for the degree of \\ Master of Science
}

University of Pittsburgh 


\section{UNIVERSITY OF PITTSBURGH \\ SWANSON SCHOOL OF ENGINEERING}

This thesis was presented

by

Viral Pinakin Shah

It was defended on

March 27, 2008

and approved by

Dr. Robert J. Ries, Assistant Professor, M.E. Rinker, Sr. School of Building Construction, University of Florida

Dr. Amir Koubaa, Academic Coordinator, Department of Civil and Environmental Engineering

Dr. Melissa M. Bilec, Visiting Research Assistant Professor, Department of Civil and Environmental Engineering

Dr. Mitchell J. Small, Professor, Department of Civil and Environmental Engineering, Carnegie Mellon University

Thesis Advisor: Dr. Robert J. Ries, Assistant Professor, M.E. Rinker, Sr. School of Building Construction, University of Florida 
Copyright (C) by Viral Pinakin Shah

2008 


\title{
A CHARACTERIZATION MODEL WITH SPATIAL AND TEMPORAL RESOLUTION FOR LIFE CYCLE IMPACT ASSESSMENT OF PHOTOCHEMICAL PRECURSORS IN THE UNITED STATES
}

\author{
Viral Pinakin Shah, M.S. \\ University of Pittsburgh, 2008
}

Photochemical pollution is formed due to the chemical reactions of atmospheric $\mathrm{NO}_{\mathrm{x}}$, volatile organic compounds, $\mathrm{CO}$, and $\mathrm{CH}_{4}$ in the presence of sunlight. It is a complex, non-linear process influenced by several parameters which change spatially and temporally. Ozone, which is the most common photochemical, damages human health, ecosystems, and man-made materials. It also contributes to climate change. Traditional life cycle impact assessment methodologies have used aggregated impact factors for a country or even for a continent, neglecting these variations.

This research assesses the geographical and temporal variability in the characterization factors for emissions of $\mathrm{NO}_{\mathrm{x}}$ and VOC over the continental US by developing monthly statelevel factors. A photochemical air quality modeling system (CAMx-MM5-SMOKE) is used to simulate the process of formation, transformation, transport, and removal of photochemical pollutants. Characterization factors are calculated at three levels along the cause-effect chain, namely, fate level, human and ecosystem exposure level, and human effect level.

The results indicate that a spatial variability of one order of magnitude and a temporal variability of two orders of magnitude exist in both the fate level and human exposure and effect level characterization factors for $\mathrm{NO}_{\mathrm{x}}$. The highest temporal variation in the characterization factors for $\mathrm{NO}_{\mathrm{x}}$ is seen in the Northeastern US. The summer time characterization factors for $\mathrm{NO}_{\mathrm{x}}$ are higher than the winter time factors. However, for anthropogenic VOC, the summer time factors are lower than the winter time in almost half of the states. The ecosystem exposure factors for $\mathrm{NO}_{\mathrm{x}}$ and $\mathrm{VOC}$ do not follow a regular pattern and show a spatial variation of about three orders of magnitude. The fate, human exposure, and human effect level factors correlate well as all three are dependent on the atmospheric concentration of ozone. However, they are poorly correlated with the ecosystem exposure factors. Sensitivity analysis of the 
characterization factors for meteorology and emissions inputs shows variation between negative $90 \%$ and positive $180 \%$. This is still lower than the spatial and temporal variations. A life cycle assessment case study is included to illustrate the use of the disaggregated characterization factors. 
TABLE OF CONTENTS

ACKNOWLEDGEMENTS .........................................................................................

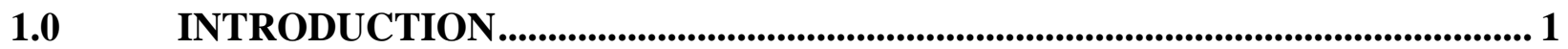

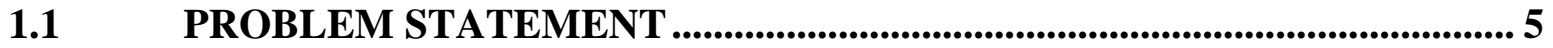

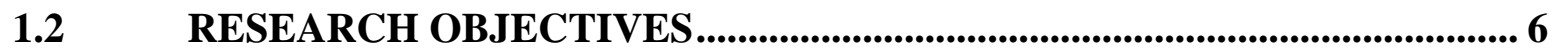

1.3 ORGANIZATION OF THE DOCUMENT .......................................................... 6

2.0 TROPOSPHERIC OZONE: BACKGROUND REVIEW ...................................... 7

2.1 SCIENTIFIC UNDERSTANDING OF PHOTOCHEMICAL SMOG........... 7

2.2 EFFECT OF METEOROLOGY ON OZONE CONCENTRATION........... 10

$2.3 \quad$ SOURCES OF OZONE PRECURSORS ............................................................. 12

$2.4 \quad$ RELATION OF OZONE TO ITS PRECURSORS.......................................... 15

$2.5 \quad$ VARIATIONS IN OZONE CONCENTRATION ........................................ 16

2.6 DETRIMENTAL EFFECTS OF TROPOSPHERIC OZONE ....................... 21

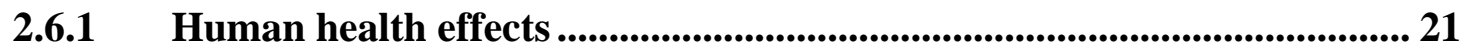

2.6.2 Effects on vegetation and ecosystems ............................................................ 24

2.6.3 Other effects ...................................................................................................... 28

2.7 LEGISLATIVE STANDARDS FOR TROPOSPHERIC OZONE ............... 29

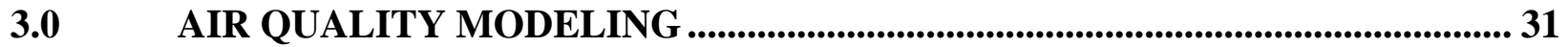

3.1 LAGRANGIAN AND EULERIAN MODELS .................................................. 31 
3.2.1 Chemistry and Transport Model........................................................... 33

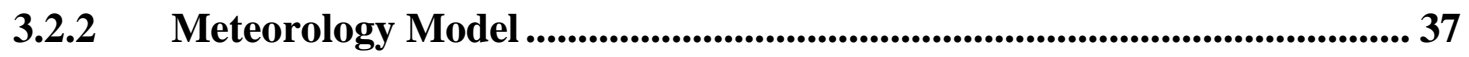

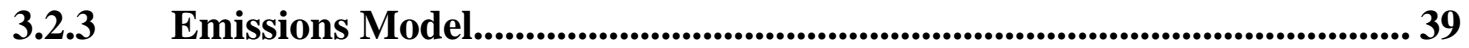

3.2.4 Model evaluation........................................................................................... 40

S.3 SENSITIVITY AND UNCERTAINTY OF PAQMS PREDICTIONS ....... 41

4.0 IMPACT CHARACTERIZATION OF PHOTOCHEMICAL PRECURSORS. 42

4.1 APPROACHES TO CHARACTERIZATION MODELING ....................... 42

4.2 SOPHISTICATION IN CHARACTERIZATION MODELING ............... 45

4.2.1 Depth and breadth of mechanism modeling.......................................... 46

4.2.2 Spatial and temporal resolution of the characterization factors ........... 49

4.3 CHARACTERIZATION OF PHOTO-OXIDANT FORMATION.............. 51

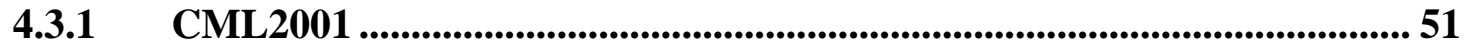

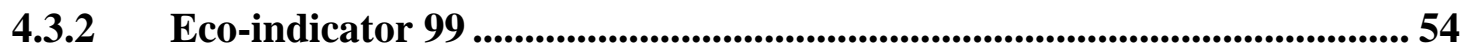

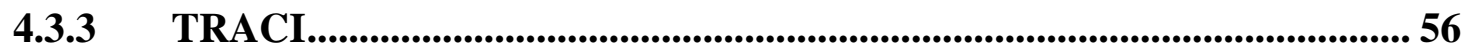

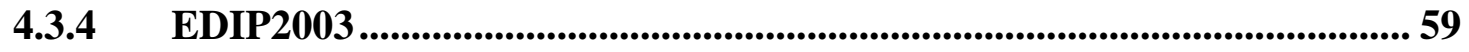

5.0 DESCRIPTION OF THE PROPOSED CHARACTERIZATION MODEL ....... 64

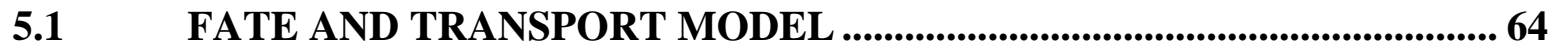

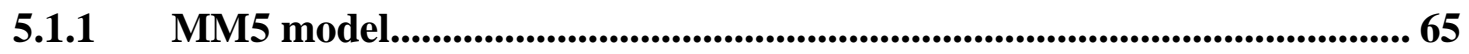

S.1.2 SMOKE system ............................................................................................6 69

5.1.3 CAMx model................................................................................... 74

5.2 CALCULATION OF FATE LEVEL FACTORS .................................. 81

EXPOSURE AND EFFECT MODELS ................................................ 83 
5.4 CALCULATION OF EXPOSURE AND EFFECT LEVEL FACTORS..... 86

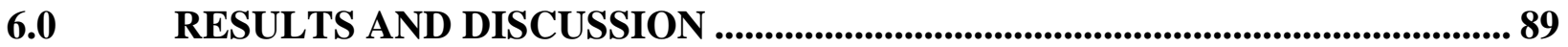

6.1 RESULTS OF THE FATE MODEL …..................................................... 89

6.2 RESULTS OF THE CHARACTERIZATION MODELS................................99

6.2.1 Fate level characterization factors .........................................................99

6.2.2 Exposure level characterization factors....................................................... 110

6.2.3 Effect level characterization factors............................................................. 118

6.3 SENSITIVITY OF THE CHARACTERIZATION FACTORS .................. 125

7.0 ILLUSTRATION OF A DISAGGREGATED LCA.............................................. 136

7.1 DESCRIPTION OF THE STUDY …............................................................. 137

7.2 LIFE CYCLE INVENTORY ............................................................................ 141

7.3 LIFE CYCLE IMPACT ASSESSMENT ....................................................... 146

8.0 CONCLUSION .......................................................................................................... 158

APPENDIX A: MODEL CONFIGURATION FILES ...................................................... 162

APPENDIX B: MODELED EMISSIONS AND CHARACTERIZATION FACTORS .... 168

APPENDIX C: DETAILS OF THE CASE STUDY ......................................................... 194

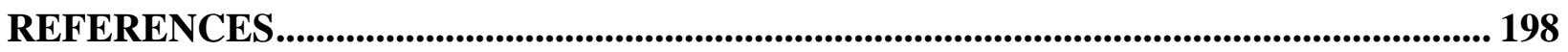




\section{LIST OF TABLES}

Table 4-1 Summary of the photochemical smog characterization models .............................. 63

Table 5-1 Maximum Incremental Reactivity of Carbon Bond IV species ............................... 83

Table 7-1 Regional climate overview for the four locations .............................................. 140

Table 7-2 Building envelope insulation levels at various locations ..................................... 140

Table 7-3 Electricity profiles for the four locations........................................................ 142

Table 7-4 Location of upstream processes for each location............................................... 146

Table A1 Average daily anthropogenic emissions of $\mathrm{NO}_{\mathrm{x}}$ (January-June) ............................ 169

Table A2 Average daily anthropogenic emissions of $\mathrm{NO}_{\mathrm{x}}$ (July-December) .......................... 170

Table A3 Average daily anthropogenic emissions of VOC (January-June) ............................ 171

Table A4 Average daily anthropogenic emissions of VOC (July-December) ......................... 172

Table A5 Average daily biogenic emissions of VOC (January-June) ................................... 173

Table A6 Average daily biogenic emissions of VOC (July-December)................................ 174

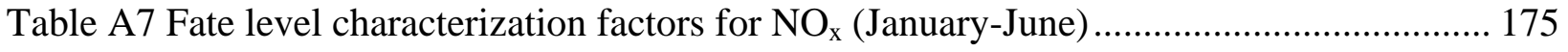

Table A8 Fate level characterization factors for $\mathrm{NO}_{\mathrm{x}}$ (July-December)................................. 176

Table A9 Fate level characterization factors for VOC (January - June) ................................ 177

Table A10 Fate level characterization factors for VOC (July-December) ............................. 178

Table A11 Human exposure level characterization factors for $\mathrm{NO}_{\mathrm{x}}$ (January-June)................. 179

Table A12 Human exposure level characterization factors for $\mathrm{NO}_{\mathrm{x}}$ (July-December) .............. 180

Table A13 Human exposure level characterization factors for VOC (January-June) ................ 181 
Table A14 Human exposure level characterization factors for VOC (July-December).............. 182

Table A15 Ecosystem exposure level characterization factors for NOx (January-June) ............ 183

Table A16 Ecosystem exposure level characterization factors for NOx (July-December) ......... 184

Table A17 Ecosystem exposure level characterization factors for VOC (January-June)............ 185

Table A18 Ecosystem exposure level characterization factors for VOC (July-December) ........ 186

Table A19 Human effect level characterization factors for NOx (January-June) ....................... 187

Table A20 Human effect level characterization factors for NOx (July-December) .................... 188

Table A21 Human effect level characterization factors for VOC (January-June)....................... 189

Table A22 Human effect level characterization factors for VOC (July-December) .................... 190

Table A23 Characterization factors and emissions for the sensitivity scenarios......................... 191

Table A24 Monthly energy consumption by type for the studied cases...................................... 194

Table A25 Emission factors for energy consumption.................................................................... 195

Table A26 LCI and LCIA results for furnace and AC at Pittsburgh ........................................... 195

Table A27 LCI and LCIA results for heat pump at Pittsburgh.................................................... 195

Table A28 LCI and LCIA results for furnace and AC at Minneapolis........................................ 196

Table A29 LCI and LCIA results for furnace and AC at Austin ................................................... 196

Table A30 LCI and LCIA results for furnace and AC at Portland ................................................ 197 


\section{LIST OF FIGURES}

Figure 1-1 Illustration of the four phases of a life cycle assessment ..................................... 2

Figure 1-2 Elements of a life cycle impact assessment ................................................... 3

Figure 1-3 Illustration of an environmental mechanism for the acidification impact category...... 3

Figure 2-1 Schematic representation of ozone chemistry in the atmosphere ........................... 10

Figure 2-2 Source distribution of annual anthropogenic $\mathrm{NO}_{\mathrm{x}}$ and $\mathrm{VOC}$ emissions ................... 14

Figure 2-3 Example of an ozone isopleths diagram......................................................... 15

Figure 2-4 Seasonal average daily maximum 8-hour ozone concentration for May to September

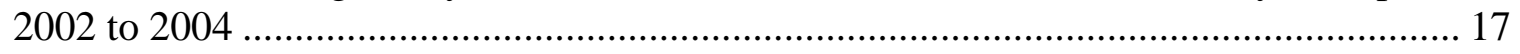

Figure 2-5 Average hourly ozone concentration in US urban areas for April to October 2000 to

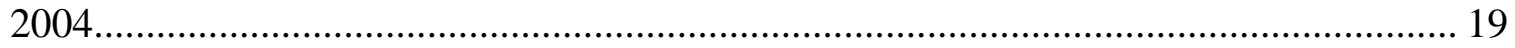

Figure 2-6 Average hourly ozone concentration in US rural areas for April to October 2000 to

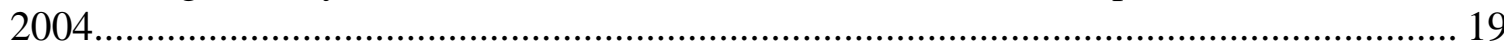

Figure 2-7 Annual trend in the daily 8-hour ozone concentration for May-September 1990 to 2004. 20

Figure 2-8 W126 for the year 2001 calculated using CMAQ model simulation........................ 26

Figure 2-9 Relative yield loss for the 31 NCLAN species at different levels of exposures ......... 28

Figure 3-1 Schematic representation of a Photochemical Air Quality Modeling System and the typical external inputs required............................................................................. 34

Figure 3-2 Schematic representation of relationship between variables and processes of a

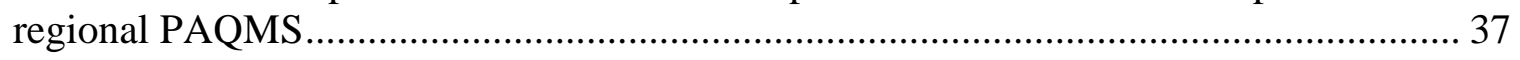

Figure 4-1 Illustration of the approaches for characterization modeling for emission-related impact categories ........................................................................................ 44

Figure 4-2 Illustration of dimensions of sophistication in LCIA ......................................... 46 
Figure 4-3 Illustration of the depth and breadth of mechanism modeling for the acidification impact category in TRACI.............................................................................................. 48

Figure 4-4 Spatial scales of impacts of different impact categories .............................................. 49

Figure 5-1 Schematic diagram of the MM5 modeling system ....................................................... 65

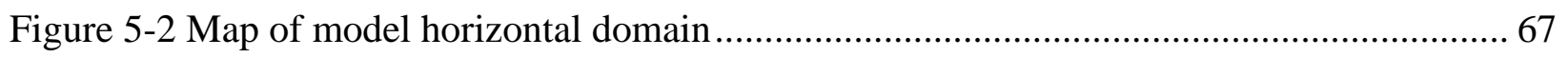

Figure 5-3 Graphical representation of sigma and pressure levels ................................................ 68

Figure 5-4 Processing steps performed by SMOKE .................................................................. 72

Figure 5-5 Illustration of APCA source apportionment scheme ………………………….......... 80

Figure 5-6 Summary of effects of ozone exposure ………………………………………........ 84

Figure 5-7 Summary of the proposed characterization model........................................................ 88

Figure 6-1 Visualization of a sample simulation output.............................................................. 91

Figure 6-2 Visualization of a sample source apportioned ozone concentration ............................ 93

Figure 6-3 Model performance with respect to observed concentrations for the entire domain .. 95

Figure 6-4 Source apportionment of the monthly averaged daily ozone concentration ................ 98

Figure 6-5 Fate level characterization factors for NOx emissions ............................................. 100

Figure 6-6 Fate level characterization factors for VOC emissions ............................................. 101

Figure 6-7 Map of USA showing the states and the four divisions ............................................ 102

Figure 6-8 Daily average anthropogenic emissions of NOx....................................................... 103

Figure 6-9 Daily average MIR weighted anthropogenic emissions of VOC............................... 104

Figure 6-10 Ratio of biogenic and anthropogenic MIR weighted VOC emissions..................... 108

Figure 6-11 Gridded population for the calculation of human characterization factors.............. 111

Figure 6-12 Area covered under forests/crops/grassland ecosystems ......................................... 112

Figure 6-13 Human exposure level factors for NOx ................................................................ 113

Figure 6-14 Human exposure level factors for VOC................................................................... 114

Figure 6-15 Ecosystem exposure level factors for NOx............................................................. 115

Figure 6-16 Ecosystem exposure level factors for VOC ............................................................. 116 
Figure 6-17 Human effect level characterization factors for NOx ....................................... 120

Figure 6-18 Human effect level characterization factors for VOC....................................... 121

Figure 6-19 Normalized maximum and minimum state level characterization factors for NOx 122

Figure 6-20 Normalized maximum and minimum state level characterization factors for VOC123

Figure 6-21 Comparison of anthropogenic emissions for the three scenarios ......................... 127

Figure 6-22 Comparison of ratio of biogenic and anthropogenic VOC for the three scenarios. 128

Figure 6-23 Ozone apportionment to major source categories for the three scenarios .............. 130

Figure 6-24 Comparison of the fate level characterization factors for the three scenarios ........ 132

Figure 6-25 Comparison of human exposure level factors for the three scenarios ................... 133

Figure 6-26 Comparison of ecosystem exposure level factors for the three scenarios.............. 134

Figure 6-27 Comparison of human effect level factors for the three scenario ........................ 135

Figure 7-1 A view of the eQuest model of the building ................................................... 138

Figure 7-2 Results of the HVAC energy use simulation for the building ............................... 143

Figure 7-3 Emission factors for energy derived from fossil fuels ........................................ 144

Figure 7-4 Results of impact assessment for (a) the two systems, and (b) the four locations .... 148

Figure 7-5 Spatial distribution of human exposure impacts for furnace and AC system for Pittsburgh 152 


\section{ACKNOWLEDGEMENTS}

I take this opportunity to thank everyone who has made this thesis possible. Firstly, I thank Dr. Robert J. Ries, for his guidance and support throughout my graduate studies at the University of Pittsburgh. More importantly, I thank him for being a splendid teacher, and inspiring me to work in this area of research. I also thank Dr. Amir Koubaa, Dr. Mitchell Small, and Dr. Melissa Bilec for serving on my thesis committee, and contributing with their insightful feedback. I am thankful to the Department of Civil and Environmental Engineering, and the 3 Rivers Wet Weather Demonstration Project for providing me with financial support during my graduate study. Lastly, I thank Arpita Mitra, my fiancé, for her constant motivation and patience. 


\subsection{INTRODUCTION}

Life Cycle Assessment (LCA) is a tool to model and analyze the environmental effects of specific human activities. The Society of Environmental Toxicological and Chemistry (SETAC) describes LCA as "an objective process to evaluate the environmental burdens associated with a product, process, or activity by identifying and quantifying energy and material usage and environmental releases, to assess the impact of those energy and material uses and releases to the environment, and to evaluate and implement opportunities to effect environmental improvements. The assessment includes the entire life cycle of the product, process, or activity, encompassing extracting and processing raw materials; manufacturing, transportation, and distribution; use/re-use/maintenance; recycling; and final disposal.” [Fava et al 1991]

A LCA is divided into four phases as shown in Figure 1-1. The goal of an LCA identifies the intended application, the motivation, and its intended audience. The scope delineates the product systems or processes to be studied, the system boundaries, the environmental stressors considered, the data requirements, and all assumptions and limitations of the study. The Life Cycle Inventory (LCI) is the process of compilation of the material and energy inputs, and emissions to air, water, and land associated with the life cycle of the system. Life Cycle Impact Assessment (LCIA) assesses the environmental effects of the inputs and outputs of the system. In the Interpretation phase the results of the LCI and LCIA are combined, the goal and scope are reviewed, and the results of the LCA are evaluated to identify opportunities of system improvement to minimize the environmental burden.

ISO 14040 [1997] defines LCIA as "(the phase) aimed at understanding and evaluating the magnitude and significance of the potential environmental impacts of a product system.” The LCIA is further divided into several steps, some of these steps are mandatory, while others are optional. Figure 1-2 illustrates these steps. 


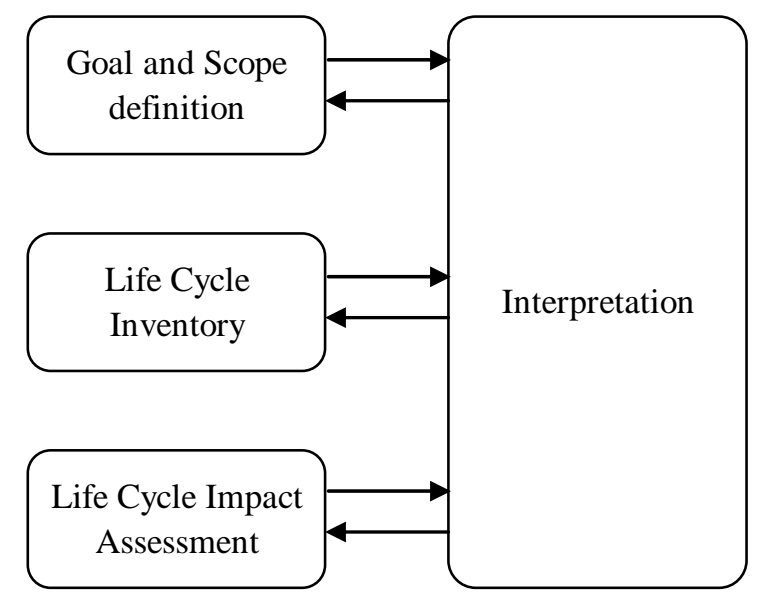

Figure 1-1 Illustration of the four phases of a life cycle assessment [Adapted from International Organization for Standardization (ISO) 14040 1997]

The first step of a LCIA is the selection of impact categories, category indicators, and characterization models. An impact category is a class representing environmental issues of concern to which LCI results may be assigned [ISO 14042 2000]. Climate change, photochemical oxidant formation, and loss of biodiversity are some examples of impact categories. The impact categories of concern for a particular LCA are usually identified at the goal and scope definition stage.

A category indicator is a quantifiable representation of an impact category [ISO 14042 2000]. For example, metric tons (MT) of $\mathrm{CO}_{2}$ equivalents is a suitable category indicator for the climate change impact category. A category indicator is a single index for the representation of all environmental interventions in a particular impact category. Therefore, emissions of methane, halocarbons, etc. are all represented in terms of $\mathrm{CO}_{2}$ equivalents. Each impact category is based on an environmental mechanism. The mechanism is the system of physical, chemical, and biological processes linking the LCI results to the category indicator [ISO 14042 2000]. These environmental mechanisms are also known as impact pathways, a term used in other environmental impact assessment methods. An example of an environmental mechanism for the acidification impact category, following the emission of $\mathrm{SO}_{2}$ is illustrated in Figure 1-3. 


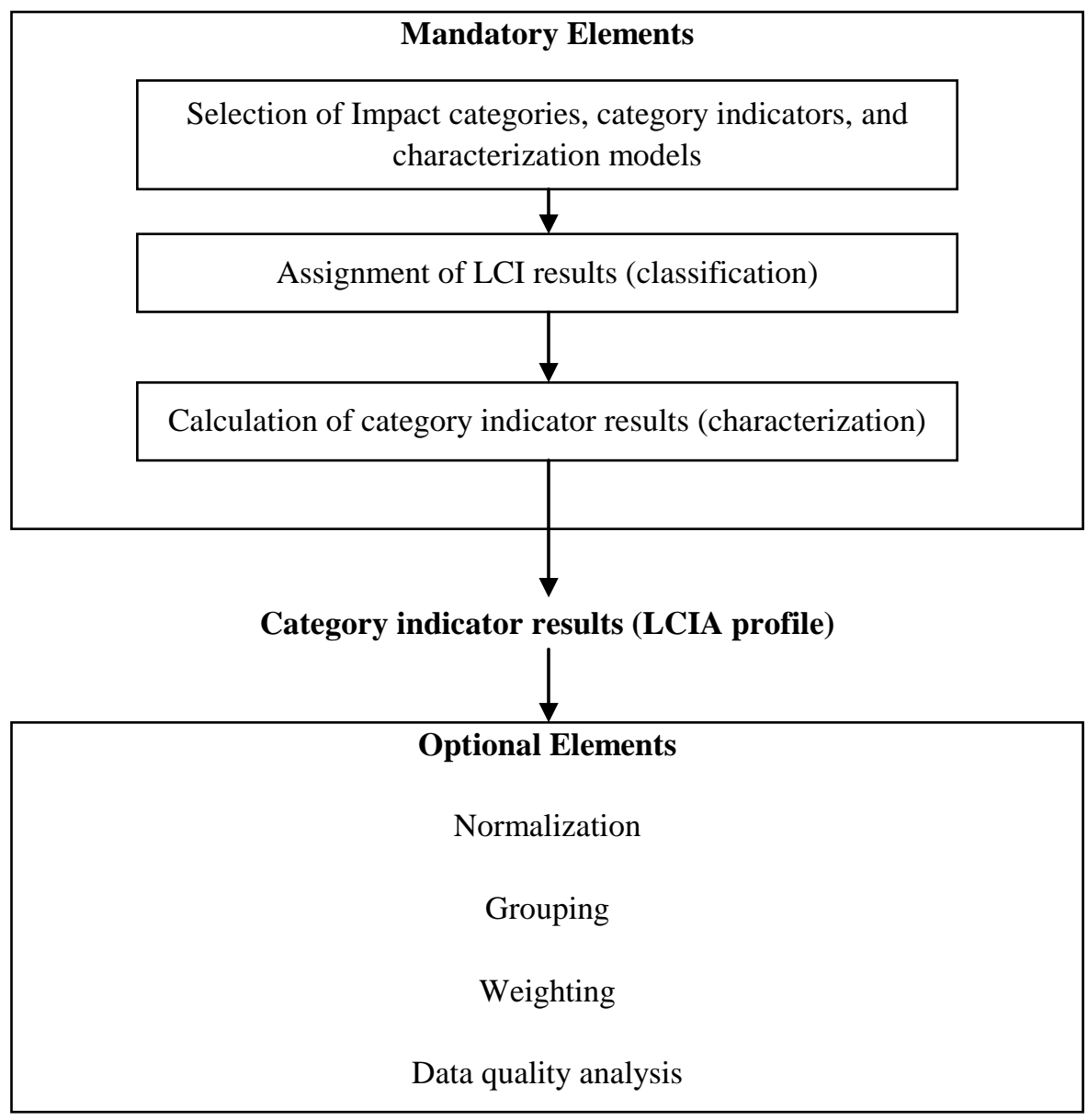

Figure 1-2 Elements of a life cycle impact assessment [Adapted from ISO 14042 2000]

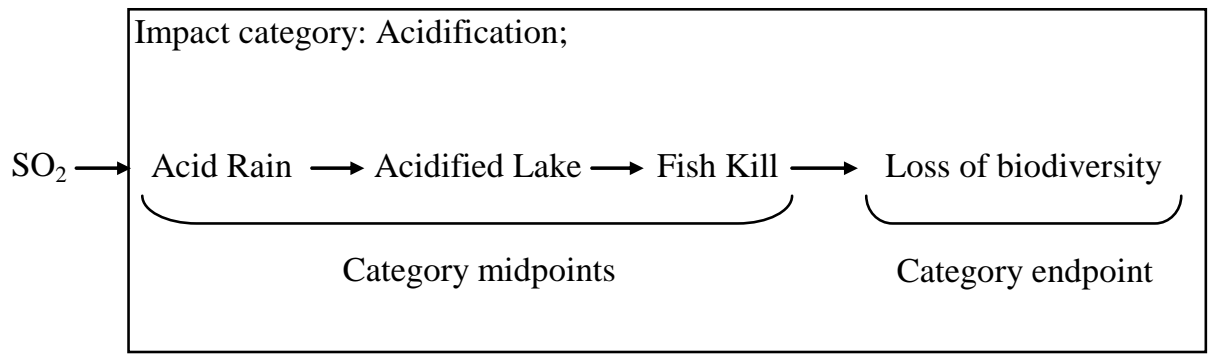

Figure 1-3 Illustration of an environmental mechanism for the acidification impact category [Adapted from Fava et al 1993] 
Category endpoints are environmental impacts that are of direct societal concern [Udo de Haes et al 1999]. Loss of biodiversity, human mortality, damage to man-made materials, and depletion of natural resources are some examples of category endpoints. Category midpoints are between the environmental interventions and the category endpoints [Udo de Haes et al 1999] as illustrated in Figure 1-3. Category endpoints are grouped into classes, known as areas of protection, which have some well recognizable value for society [Udo de Haes et al 1999]. Human health, ecological health, and resource depletion, are the key areas of protection identified by Fava et al [1993]. Udo de Haes et al [1999] have suggested the use of human health, natural resources, natural environment, and man-made environment as the areas of protection.

Characterization models reflect the environmental mechanisms by describing the relationship between the LCI results, the category indicators, and category endpoints [ISO 14042 2000]. Characterization factors are derived from characterization models. For instance, 1 MT of emission of methane can be mathematically converted to $\mathrm{MT}$ of $\mathrm{CO}_{2}$ equivalents using the Intergovernmental Panel on Climate Change (IPCC) model as the characterization model. The characterization factor is the ratio of the quantity of the category indicator generated to the quantity of a particular input or output in the LCI result. In the case of climate change, the characterization factor is the MT of $\mathrm{CO}_{2}$ equivalents per MT of methane emission [ISO 14042 2000].

The second step is the classification of the inventory results into impact categories. This step requires the knowledge of the environmental effects of the output in the LCI data. In some cases, a particular entry can be classified into more than one impact categories. For example, toluene can result in the formation of ozone, and is also a known carcinogen. The third step is characterization, which is the conversion of the LCI results to category indicators and category endpoints using the characterization factors described above.

Several LCIA methods have been developed which have followed these steps, and provide pre-calculated characterization factors for a large number of environmental interventions. These methods have also been incorporated into standard LCA software. Thus, typically, a LCA practitioner can avoid these detailed LCIA steps. 


\subsection{PROBLEM STATEMENT}

Standard LCIA methods provide a single characterization factor for each intervention-impact link. Some of these characterization factors are valid globally (e.g. global warming potential for climate change impact) while others are representative of the conditions in a particular country (e.g. USA) or a small continent (e.g. Europe). Photochemical oxidant formation has always been a basic impact category of LCIA (Fava et al 1993). However, the level of photochemical oxidant formation varies significantly among regions, and between urban and rural areas within a region [United States Environmental Protection Agency (USEPA) 2006a]. It also shows high diurnal and seasonal variations. Traditional LCIA methods have neglected this spatial and temporal variation in photochemical oxidant formation, although the importance of higher spatial resolution has been long recognized [Fava et al 1993]. A coarser spatial and temporal resolution, especially for impacts like photochemical oxidant formation, increases the uncertainty of the LCA results. Recent LCIA methods, like TRACI [Bare et al 2003] and EDIP2003 [Hauschild and Potting 2005], address the issue of spatial variability by incorporating spatially resolved characterization factors. EDIP2003 is a European LCIA method and its results are not very relevant for the United States. TRACI is the only LCIA method that has been developed for the US, but has certain limitations in the models and the impact indicators used for the calculation of the photochemical oxidant formation characterization factors. These methods are described further in Section 4.3.

LCIA methods also differ in the level up to which the intervention-impact link is incorporated. Many LCIA methods are damage oriented methods, that is, they determine the impacts up to the category endpoints. Certain methods, including TRACI, end their impact calculations at the category midpoint level. This is due to the increasing uncertainty in quantitative modeling beyond the midpoint level [Bare et al 2003]. However, the importance of extending the impact assessment to include category endpoints has been also been recognized [Bare et al 2000]. 


\subsection{RESEARCH OBJECTIVES}

The current research focuses on developing a new characterization model for the photochemical oxidant formation impact category. The main objectives of the study are:

a. Assess the spatial and temporal variability in photochemical oxidant formation per unit anthropogenic emission of oxides of nitrogen $\left(\mathrm{NO}_{\mathrm{x}}\right.$ ) and volatile organic compounds (VOC) over the coterminous United States.

b. Develop monthly state-level characterization factors at the category midpoint level for the photochemical oxidant formation impact category.

c. Incorporate human dose-response relationship to extend the impact calculation from the category midpoints to the category endpoints.

d. Illustrate, using a case study, the difference between the results obtained from a traditional (aggregated) LCA and a spatially and temporally resolved LCA.

\subsection{ORGANIZATION OF THE DOCUMENT}

The remaining document is divided into seven additional chapters. Chapter 2.0 is a review of the atmospheric processes involving ozone and its precursors. It gives a brief overview of the adverse effects of exposure to ozone and other photochemical products, and the current legislation to minimize the risk of these effects. Chapter 3.0 discusses advances in the atmospheric dispersion modeling of ozone and its precursors. The modeling system is known as Photochemical Air Quality Modeling System (PAQMS). Chapter 4.0 provides a brief description of the characterization models for photo-oxidant formation employed in current impact assessment methods. Chapter 5.0 details the characterization method and models used in the current study to develop spatially and temporally differentiated characterization factors for US conditions. The results of the modeling, the characterization factors, and their sensitivity are presented in Chapter 6.0. Chapter 7.0 presents an example case study that illustrates the use of spatially and temporally resolved inventory and characterization factors, which reduce uncertainty over traditional LCA methods. 


\subsection{TROPOSPHERIC OZONE: BACKGROUND REVIEW}

Ozone $\left(\mathrm{O}_{3}\right)$ is a highly reactive gas that is primarily found in the stratosphere at the height of 10 to $40 \mathrm{~km}$ above the earth's surface. The stratospheric ozone is formed entirely due to natural processes involving molecular and atomic oxygen which combine in the presence of sun's radiation. The stratospheric ozone absorbs the harmful ultraviolet (UVB) radiation and protects life on earth. However, a small amount of ozone is also formed near the earth's surface in the troposphere. The tropospheric ozone has several adverse effects on human and ecosystem health, and also on some man-made materials. Tropospheric ozone is a secondary air pollutant since it is not directly emitted to the atmosphere in significant quantities. Ozone and other short-lived oxidants, such as peroxyacetyl nitrate (PAN) and hydrogen peroxide, are formed due to the photochemical reactions of oxides of nitrogen $\left(\mathrm{NO}_{\mathrm{x}}\right)$ and volatile organic compounds (VOC). Carbon monoxide (CO) and methane $\left(\mathrm{CH}_{4}\right)$ also participate in photochemical reactions, but to a smaller extent. The mixture of oxidants is referred to as photochemical smog. Photochemical smog is often observed over dense urban areas during summer evenings and has become a persistent environmental issue.

\subsection{SCIENTIFIC UNDERSTANDING OF PHOTOCHEMICAL SMOG}

Tropospheric ozone is primarily formed due to photochemical reactions involving $\mathrm{NO}_{\mathrm{x}}(\mathrm{NO}+$ $\mathrm{NO}_{2}$ ) and VOC, which are known as ozone precursors. In the absence of VOC, $\mathrm{NO}_{\mathrm{x}}$ undergoes a series of photochemical reactions that, both, form and destroy ozone such that the net ozone accumulation is zero. This set of equations is described below.

$$
\left.\mathrm{NO}_{2}+h v \rightarrow \mathrm{NO}+:{ }^{3} \mathrm{P}\right)
$$




$$
\begin{gathered}
: \mathrm{O}\left({ }^{3} \mathrm{P}\right)+\mathrm{O}_{2} \rightarrow \mathrm{O}_{3} \\
\mathrm{NO}+\mathrm{O}_{3} \rightarrow \mathrm{NO}_{2}+\mathrm{O}_{2}
\end{gathered}
$$

In the above reactions, $h v$ represents the ultraviolet radiation. Nitrogen dioxide is dissociated into nitric oxide and atomic oxygen in the presence of sunlight. The atomic oxygen reacts with the oxygen molecule in the air to form ozone. Ozone, in turn, reacts quickly with nitric oxide to form nitrogen dioxide and oxygen. This set of reactions results in no net formation or destruction of any compounds. However, this reaction sequence is disturbed by the presence of VOC, CO or $\mathrm{CH}_{4}$ in the atmosphere.

VOC are oxidized by the hydroxyl radical $(\mathrm{OH})$ present in the atmosphere. The oxidized VOC substitute ozone in equation 3 (above), thus reacting with $\mathrm{NO}$ to form $\mathrm{NO}_{2}$ without consuming $\mathrm{O}_{3}$. The other products of this reaction undergo further reactions, eventually forming more oxidants. This results in the net accumulation of ozone in the atmosphere. The largest source of $\mathrm{OH}$ radicals in the atmosphere is the combination of excited atomic oxygen with water vapor present in the atmosphere. One source of excited atomic oxygen is the photolytic decomposition of $\mathrm{O}_{3}$.

$$
\begin{array}{r}
\mathrm{O}_{3}+h v \rightarrow \mathrm{O}_{2}+\cdot{ }^{\bullet}\left({ }^{1} \mathrm{D}\right) \\
: \mathrm{O}\left({ }^{1} \mathrm{D}\right)+\mathrm{H}_{2} \mathrm{O} \rightarrow 2^{\bullet} \mathrm{OH}
\end{array}
$$

The set of reactions leading to the formation of ozone are illustrated below using methane which is the simplest hydrocarbon.

$$
\mathrm{CH}_{4}+{ }^{\bullet} \mathrm{OH} \rightarrow{ }^{\bullet} \mathrm{CH}_{3}+\mathrm{H}_{2} \mathrm{O}
$$

The methane reaction with hydroxyl radical produces methyl radical which reacts rapidly with oxygen molecule to form methylperoxy radical.

$$
\cdot \mathrm{CH}_{3}+\mathrm{O}_{2} \stackrel{\mathrm{M}}{\longrightarrow} \mathrm{H}_{3} \mathrm{CO}_{2}^{\cdot}
$$


The methylperoxy radical leads to the production of methoxy radical from reaction with NO, inhibiting the consumption of $\mathrm{O}_{3}$. The methoxy radical reacts with oxygen to form formaldehyde and hydroperoxide radical. Formaldehyde further decomposes into carbon monoxide and eventually ozone. The hydroperoxide radical oxidizes more $\mathrm{NO}$ to form $\mathrm{NO}_{2}$.

$$
\begin{gathered}
\mathrm{H}_{3} \mathrm{CO}_{2}^{\bullet}+\mathrm{NO} \rightarrow \mathrm{H}_{3} \mathrm{CO}^{\bullet}+\mathrm{NO}_{2} \\
\mathrm{H}_{3} \mathrm{CO}+\mathrm{O}_{2} \rightarrow \mathrm{HCHO}+\mathrm{HO}_{2}^{\cdot} \\
\mathrm{HO}_{2}^{\bullet}+\mathrm{NO} \rightarrow \mathrm{NO}_{2}+{ }^{\bullet} \mathrm{OH} \\
\mathrm{HCHO}+h v \rightarrow{ }^{\bullet} \mathrm{CHO}+\mathrm{H}^{\bullet} \\
{ }^{\circ} \mathrm{CHO}+\mathrm{O}_{2} \rightarrow \mathrm{CO}+\mathrm{HO}_{2}^{\bullet} \\
\mathrm{CO}+{ }^{\bullet} \mathrm{HO}+\mathrm{O}_{2} \stackrel{\mathrm{M}}{\longrightarrow} \mathrm{HO}_{2}^{\cdot}
\end{gathered}
$$

Other VOC undergo similar oxidation reactions which further oxidize $\mathrm{NO}$ to $\mathrm{NO}_{2}$. Hundreds of VOC are present in the polluted atmosphere and undergo thousands of different chain reactions to produce ozone. Many of these reaction mechanisms, particularly those of aromatic hydrocarbons, are, currently, not fully understood. During night time, when the concentration of hydroxyl and hydroperoxy radicals is low, $\mathrm{NO}_{3}$ can oxidize VOC. In coastal areas and certain other environments chloride and bromide radicals can act as VOC oxidizing agents. In addition to gas phase reactions, other reactions also occur in the aqueous medium within cloud droplets or airborne particles. Atmospheric ozone is destroyed by photochemical decomposition (reaction 4), reaction with NO (reaction 3), reaction with hydroperoxy radical during low concentration of NO (reaction 14), or physical deposition to the surface.

$$
\mathrm{O}_{3}+\mathrm{HO}_{2}^{\cdot} \rightarrow \cdot+\mathrm{HO}+2 \mathrm{O}_{2}
$$

A schematic representation of the chemical and physical transformation of ozone is shown in Figure 2-1. 


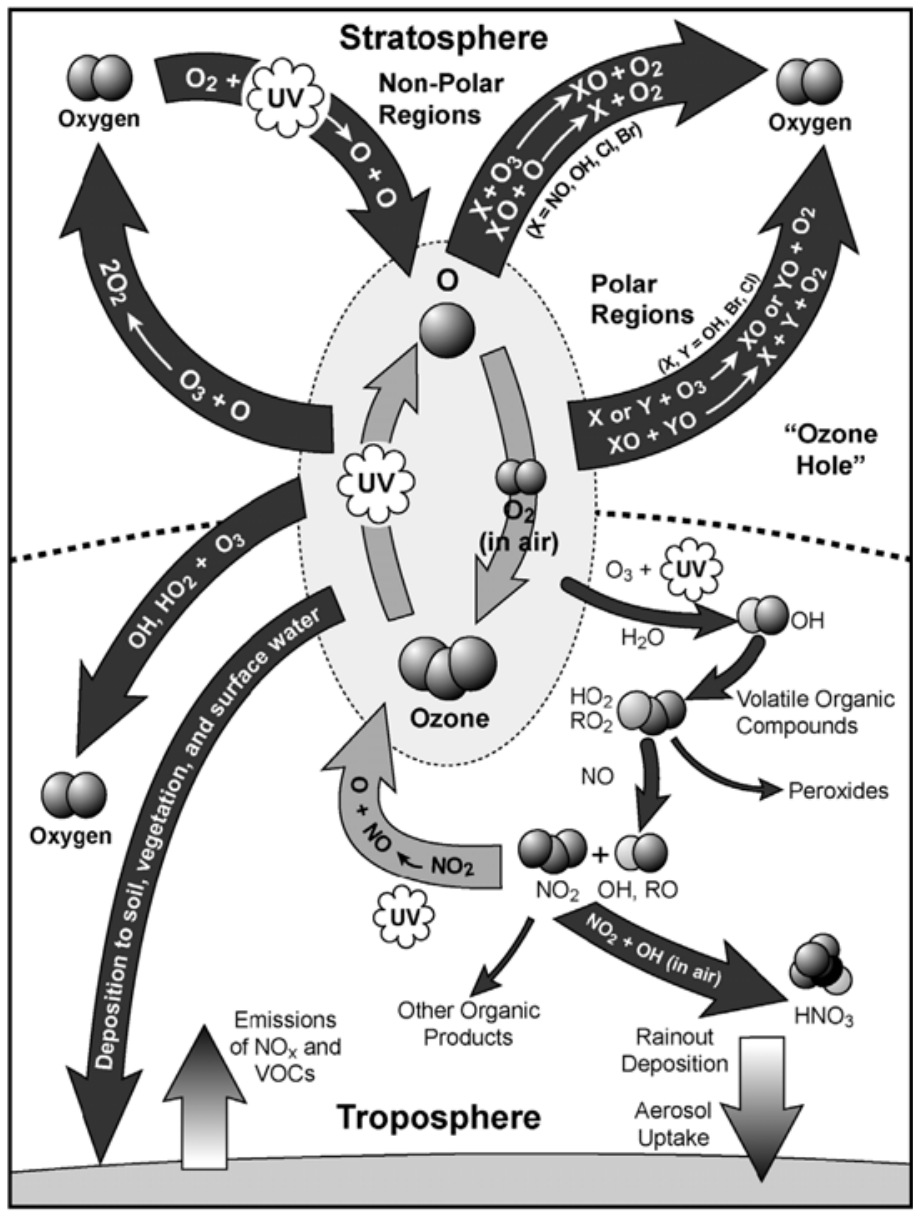

Figure 2-1 Schematic representation of ozone chemistry in the atmosphere [USEPA 2006a]

\subsection{EFFECT OF METEOROLOGY ON OZONE CONCENTRATION}

"Meteorology is the study of the structure, thermodynamics, and dynamics of the atmosphere, particularly the lower parts of the atmosphere in which most of the active weather phenomena and processes take place.” [Arya 1999] Since the atmosphere acts as the stage where all the transformations related to ozone occur, meteorology has the strongest influence on this process. Much of the action takes place in the troposphere because of the relatively stable structure of the stratosphere. The troposphere is further subdivided into the Planetary Boundary Layer (PBL) and the free troposphere. The PBL is the portion of the troposphere which is influenced by the earth's surface. Changes at the earth's surface are manifest in the PBL within a time scale of one hour to 
a day. The PBL is characterized by sharp variations in temperature, wind speeds, and pollutant concentrations, and variable depth due to the air-surface interaction and the effect of the free troposphere above the PBL. The thermodynamical properties of the PBL are determined by the thermal properties of the surface, and the energy transfer mechanisms. The surface roughness and topography affect the dynamic properties of the PBL [Arya 1999]. The depth of the PBL is known as the mixing height as several vertical motions due to turbulence and convection in the PBL disperse the pollutants well in the air within this layer.

Horizontal transport of the pollutant occurs through the process of advection and turbulent diffusion. Advection is the horizontal propagation of the mean wind. Pollutant particles are transported to other locations along the wind direction and in proportion to the wind speed. Long range transport of pollutants occurs mainly through the advective transport by large scale winds in the free troposphere. Turbulent wind flows are highly irregular, rotational, dissipative, and diffusive motions. In common terminology it is referred to as wind gust. These help in mixing the pollutants in several random directions. A high degree of turbulence is mostly observed near the surface of the earth, primarily due to surface roughness. Turbulent motions are generally confined to a smaller scale compared to the advective motions. Pollutant concentrations are linked to the effects of these advective and turbulent motions. They have a cleansing effect in places of high pollutant concentrations but affect the quality of air in the surrounding areas. The scale of ozone and precursor transportation, especially in the free troposphere, is thousands of kilometers. The pollutants transported with these stable winds result in the long range, intercontinental transport of pollutants. Subsidence in high pressure systems, following the cold fronts, brings down these pollutants from the free troposphere into the PBL [USEPA 2006a]. Thus, although the tropospheric ozone formation is essentially an urban phenomenon, it manifests strong effects regionally, and also globally.

Some amount of ozone is transported downward from the stratosphere to the troposphere through a process known as tropopause folding. Tropopause folding is a local drop in the height of the tropopause layer bringing down the stratospheric air. This stratospheric air gets mixed horizontally with in the upper troposphere. This ozone can then be transported vertically to the surface. This phenomenon has the maximum effect in late winter and early spring. However, the contribution of this ozone to surface concentration is usually not significant. It is typically less than $5 \%$ of the zonal average at the surface [Fiore et al 2002]. 
Ultraviolet radiation initiates the photolytic reactions leading to the formation of ozone and also affects some of the intermediate steps. Thus ozone concentrations have a significant direct dependence on solar radiation. Besides, solar radiation has an effect on the thermodynamics of the air mass. Radiation intensity varies diurnally, seasonally, and also with latitude. Cloud cover has a strong effect on the radiation reaching the PBL. The actinic flux is also associated with the concentration, distribution, and refractive index of aerosols. Clouds and aerosol reflect and scatter the incident UV radiation thus impacting the formation or destruction of ozone [USEPA 2006b].

Temperature is another meteorological variable which has a strong relationship with ozone concentration. Typically ozone concentration increases with increasing temperature. One possible explanation for this correlation is the increase in the thermal decomposition of peroxyacetyl nitrate (PAN) which is a reservoir of $\mathrm{NO}_{\mathrm{x}}$. PAN is formed due to the combination of acetylperoxy radicals with $\mathrm{NO}_{2}$. Higher temperatures affect the thermodynamics of the atmosphere and are generally associated with stagnant conditions. They also increase the emission of biogenic (natural) VOC (e.g., isoprene). The major episodes of high ozone concentration occur during periods of slow moving, high pressure systems, observed mainly during summer and early fall in Eastern US. High pressure systems cause subsidence or sinking air. The sinking air reduces the vertical mixing of pollutants in the PBL. High pressure systems are accompanied by warm air, cloudless skies, and light wind. Light winds and less vertical mixing reduce the dispersion of pollution. Higher availability of radiation and temperature in summer boost the photochemical activity and formation of ozone [National Research Council (NRC) 1991].

\subsection{SOURCES OF OZONE PRECURSORS}

As mentioned previously, tropospheric ozone is formed by the photochemical reactions of its precursors, which are oxides of nitrogen ( $\mathrm{NO}+\mathrm{NO}_{2}$, collectively referred to as $\mathrm{NO}_{\mathrm{x}}$ ), volatile organic compounds (VOC), and to a small extent carbon monoxide (CO), and methane $\left(\mathrm{CH}_{4}\right)$. VOC are compounds that are volatile in standard atmospheric conditions because of their high vapor pressures. However, not all VOC contribute to the formation of ozone. For regulatory 
purposes, VOC have been defined as any organic compound that participates in photochemical reactions. Hundreds of compounds are classified as VOC. Methane, which is technically a VOC, has a very low reactivity and long atmospheric lifetimes, and is usually not considered a VOC. CO has a very low reactivity and contributes to ozone formation only in highly polluted urban areas. Its main source of emission is automobile exhaust. The emission sources of $\mathrm{NO}_{\mathrm{x}}$ and VOC can be classified into anthropogenic and biogenic categories. The US EPA compiles a National Emissions Inventory (NEI) for the United States every 3 years. The NEI is a comprehensive database of the source and the amount of anthropogenic emissions of criteria air pollutants as well as hazardous air pollutants in the United States. Most of the data in the NEI is supplied by the states and local agencies, tribes, the industry under the Consolidated Emissions Reporting Rule (CERR).

The NEI summarizes the emission data into 12 sector categories which cover all the known anthropogenic sources of pollution. These categories are: (a) Fertilizer and Livestock, which includes fertilizer application and livestock waste, (b) Electric Generating Units (EGU), (c) Industrial, commercial and residential fuel combustion, (d) Residential wood, which include wood stoves and fireplaces, (e) Waste disposal, which include emissions from landfills and open burning, (f) Fires, which include wildfires, prescribed fires, and agricultural and other burning, (g) Industrial processes, which include emissions from commercial cooking, metal production, chemical manufacturing, petroleum refineries, oil and gas plants, pulp and paper, and cement not due to fuel combustion, (h) On-road, (i) Non-road, (j) Road dust, (k) Solvent use, which include industrial as well as non-industrial, and (l) Miscellaneous processes, like construction, gas stations, agriculture, etc.[USEPA 2002] The contribution of these sectors to the $\mathrm{NO}_{\mathrm{x}}$ and VOC emissions for the year 2002 is detailed in Figure 2-2. The total anthropogenic emissions of $\mathrm{NO}_{\mathrm{x}}$ in the year 2002 were estimated at 19 million MT, and those of VOC were 18.5 million MT.

$\mathrm{NO}_{\mathrm{x}}$ is formed due to the combustion of nitrogen present in the atmosphere and in the fuel during high-temperature processes. Therefore, the major source of $\mathrm{NO}_{\mathrm{x}}$ is fossil fuel combustion. VOC emitted from automobiles are hydrocarbons formed due to incomplete combustion of fuel or from its direct vaporization. Solvent evaporation from industrial and domestic applications is also a major source of VOC. 


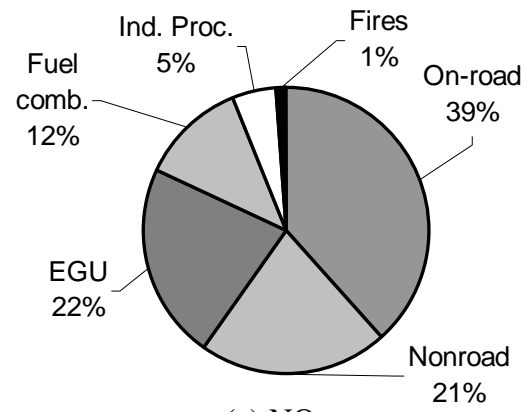

(a) NOx

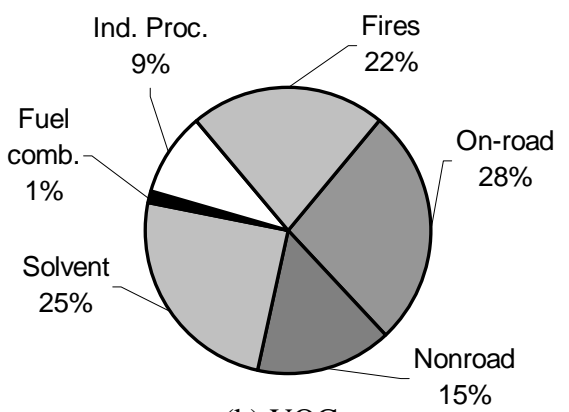

(b) VOC

Figure 2-2 Source distribution of annual anthropogenic $\mathrm{NO}_{\mathrm{x}}$ and VOC emissions

[USEPA 2002]

In addition to anthropogenic emissions, biogenic emissions of $\mathrm{NO}_{\mathrm{x}}$ and $\mathrm{VOC}$ are also significant. Lightning, soils, and oceans are the natural sources of $\mathrm{NO}_{\mathrm{x}}$. Both nitrifying and denitrifying bacteria in the soil produce $\mathrm{NO}_{\mathrm{x}}$. The emission rate depends on fertilization levels and temperature. $\mathrm{NO}_{\mathrm{x}}$ produced from lightning has little impact on surface level ozone. Biogenic sources contribute about 3 million $\mathrm{MT}$ of annual $\mathrm{NO}_{\mathrm{x}}$ half of which is from soils. Plants are made up of thousands of organic compounds. Many of these compounds volatilize from the leaves and branches of the plants and trees. Isoprene is the most abundant of the biogenic VOC emitted. It contribution is about 35\% of the total biogenic VOC. During summer, the rate of emissions is very high due to higher temperatures and more sunlight. Most of the annual VOC is emitted during the summer months. An increase in ambient temperature from $25^{\circ} \mathrm{C}$ to $35^{\circ} \mathrm{C}$ increases the emission rate by 4 times [NRC 1991]. The emission is also higher in the lower latitudes than in the upper latitudes. The annual biogenic emission of VOC is about 4.5 million MT, some of which is non-reactive [USEPA 2006b]. Biogenic emissions are only a small portion of the total ozone precursor emissions. Thus most of the tropospheric ozone formation is due to anthropogenic activity. 


\subsection{RELATION OF OZONE TO ITS PRECURSORS}

Ozone is one of the few pollutants which do not have a one-one relation with its precursors. As described previously, ozone formation and destruction is a complex process, involving several reaction paths and compounds. As a result, ozone concentrations change non-linearly with the concentration of its precursors. The $\mathrm{VOC}-\mathrm{NO}_{\mathrm{x}}-\mathrm{O}_{3}$ relation is expressed graphically in the form of ozone isopleths (surfaces of constant concentrations) plotted in between the $\mathrm{NO}_{\mathrm{x}}$ and VOC concentration axes. An example of an ozone isopleth is shown in Figure 2-3. Instead of concentrations, the emission rates of $\mathrm{NO}_{\mathrm{x}}$ and VOC are plotted on the axes.

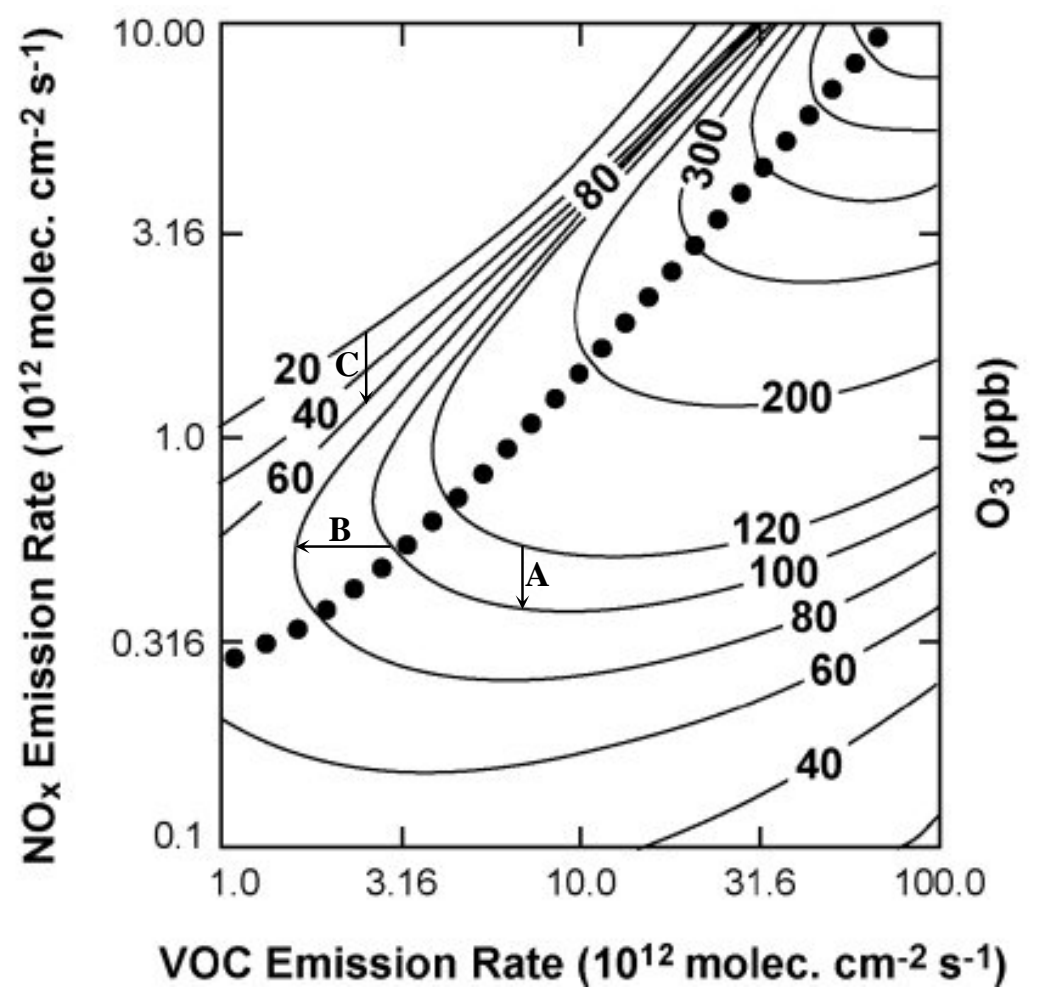

Figure 2-3 Example of an ozone isopleths diagram

[USEPA 2006b]

Ozone isopleths can be divided into two regions by a ridge running from the lower right corner to the upper left corner. The ridge is shown by the dotted line in Figure 2-3. The right side 
of the ridge is characterized by ozone formed under conditions where the availability of $\mathrm{NO}_{\mathrm{x}}$ is the limiting factor. This condition is typical of the suburbs, rural areas and other continental areas. In these regions the net production of ozone decreases with the decrease in $\mathrm{NO}_{\mathrm{x}}$ concentration (shown by change 'A' in Figure 2-3). The relation of ozone concentration with VOC is similar. At low VOC concentrations ozone is directly proportional to the VOC present. A decrease in VOC causes a decrease in ozone (shown by change ' $B$ '). However, at very high $\mathrm{NO}_{\mathrm{x}}$ concentrations, found typically in very dense urban areas, there is a net destruction of ozone by reaction with $\mathrm{NO}$. Also, at high $\mathrm{NO}_{\mathrm{x}}$ concentration, hydroxyl radicals are consumed by $\mathrm{NO}_{2}$ instead of oxidizing VOC to initiate ozone formation. Thus at these high $\mathrm{NO}_{\mathrm{x}}$ levels, a decrease in $\mathrm{NO}_{\mathrm{x}}$ at constant VOC actually results in an increase in ozone concentration (shown as change ' $C$ ' in Figure 2-3). Hence, recently, the upper left region is also referred to as $\mathrm{NO}_{\mathrm{x}}$-saturated instead of VOC-limited. This example isopleth is for a particular mixture of VOC gases. The isopleth is highly sensitive to the composition of the VOC mixture since their reactivity exhibit a large variation. The isopleth is also sensitive to the prevailing meteorological conditions which significantly affect ozone formation. Thus, there exists a complex relationship between the concentration of ozone and the emissions of its precursors, making the development of ozone control strategies a difficult task.

\subsection{VARIATIONS IN OZONE CONCENTRATION}

The USEPA has established the Air Quality System (AQS, formerly known as AIRS) to monitor the concentrations of ozone and various criteria air pollutants throughout the United States. Ozone is routinely monitored at several locations and a detailed hourly data from the year 1970 is publicly available at the AQS website [http://www.epa.gov/ttn/airs/airsaqs/]. Analysis of this ozone concentration data has shown that the concentration of ozone exhibits a large variability among different locations and at different times at a particular location. This is a direct consequence of the fact that the formation and destruction processes of ozone are dependent on several variable factors, as discussed above. A map of USA showing the spatial variability in ozone concentration is presented in Figure 2-4. 


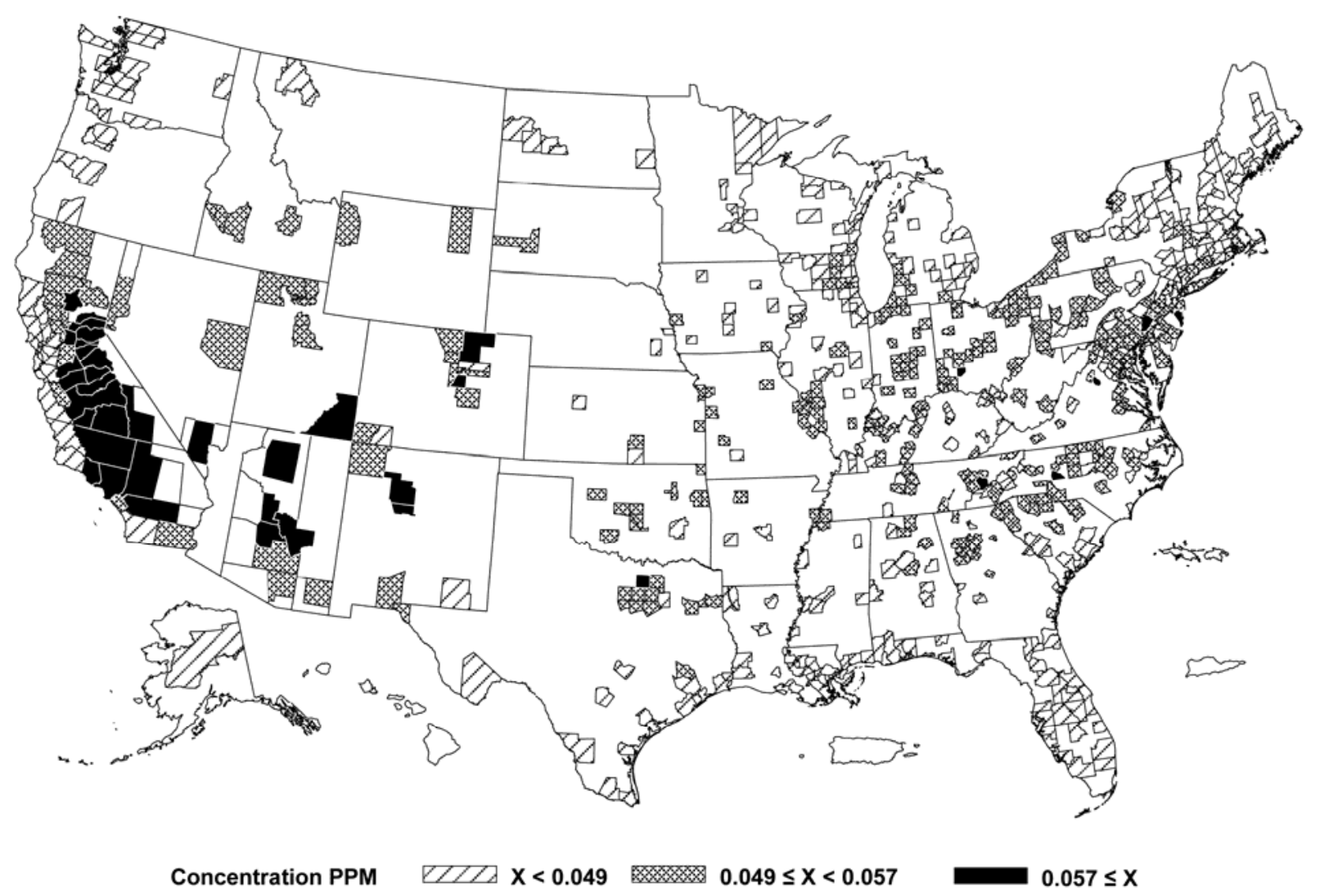

Figure 2-4 Seasonal average daily maximum 8-hour ozone concentration for May to September 2002 to 2004

[USEPA 2006a]

The ozone concentration is actually a volumetric mixing ratio expressed in parts per million (ppm) or parts per billion (ppb). The blank data in Figure 2-4 represents the absence of ozone monitors in these counties. The data shown is for the months of May to September, which is defined as the ozone season in US. This is the period when maximum occurrences of high ozone episodes are observed. High concentrations of ozone are seen in California, some states in the Southwest, and in many places in the East. Variations also exist within each county, with the urban areas having relatively higher concentrations than suburban and rural areas. However, concentrations in rural areas are influenced by nearby urban centers. Besides, many high emission sources like power plants and highways are located in the non-urban areas. Higher ozone concentrations are persistent in rural areas, as lower amounts of ozone are scavenged by 
$\mathrm{NO}$ or $\mathrm{HO}_{2}$. The diurnal patterns in ozone concentration are related to the photochemical activity, the contribution of transported ozone by nocturnal jets, the depth of the boundary layer, and the vertical mixing in the PBL. Figure 2-5 and Figure 2-6 show the diurnal patterns of average 1-h ozone concentration in urban and non-urban areas respectively.

In Figure 2-5 and Figure 2-6 the box represents the interquartile range and the whiskers represent the maxima and minima. It is seen that the maximums in, both, the urban and nonurban areas occur during the mid-afternoons and do not differ significantly in value. High photochemical activity and higher emissions are responsible for these higher concentrations. The average hourly concentration peaks at around $0.06 \mathrm{ppm}$. However, the variation in rural concentration is flatter compared to urban concentration. The high rural concentration during the day persists during the night, whereas the high urban concentration decreases rapidly. This is due to lower titration of ozone with NO in the rural areas.

Most of the ozone maximums occur during the summer months from June to August due to the presence of conditions conducive to ozone formation and accumulation. However, in many relatively remote locations, like national parks, in Western US, high ozone concentrations are observed in the late winter to spring time months of March to June. Even during winter and early spring, photochemical activity does not totally cease, but proceeds at a lower rate. In high altitude regions this photochemical activity is relatively high. Stratospheric ozone exchange is a small portion of the spring time maximum. Spring time maximums in sites at higher latitudes are attributed to the buildup of precursors during the winter months in the arctic regions which result in large ozone formation with the arrival of spring. Long range transport of pollutants from Asia reaches its peak during the spring before it is lifted by the cold front originating in Siberia. Ozone has longer lifetime in spring due to relatively stable conditions which contributes to higher accumulation [USEPA 2006a].

The tropospheric ozone which is not formed due to anthropogenic emissions in North America (USA, Canada, and Mexico) is known as Policy Relevant Background (PRB) ozone, or simply, background ozone. PRB ozone implies the amount of ozone which cannot be reduced by implementing controls and formulating policy within North America. PRB includes ozone which is transported from outside the North American region (e.g. from Asia), ozone which is formed due to biogenic emissions from any part of the world, or ozone transported vertically downwards from the stratosphere [USEPA 2006a]. 


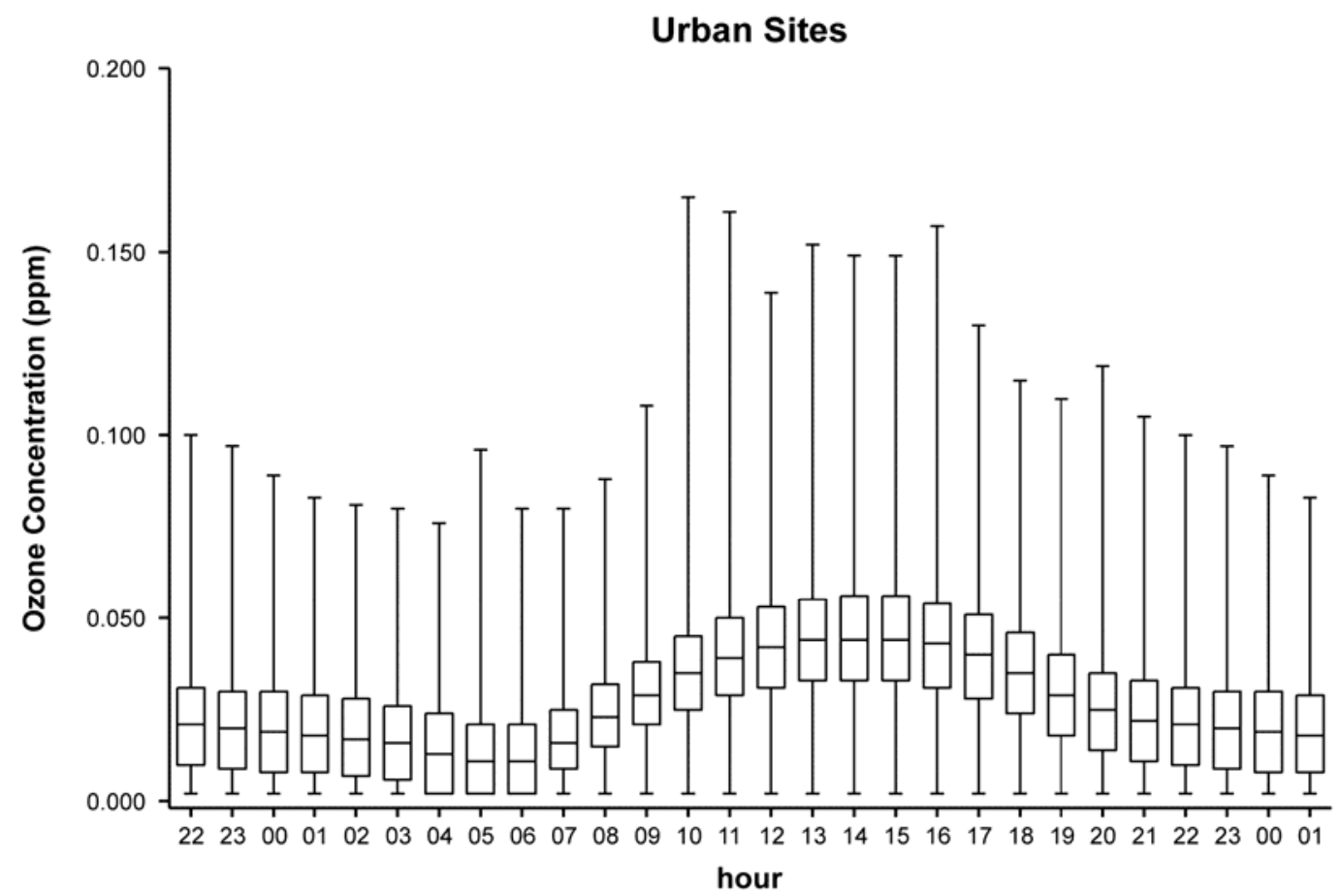

Figure 2-5 Average hourly ozone concentration in US urban areas for April to October 2000 to 2004 [USEPA 2006a]

\section{Rural (CASTNET) Sites}

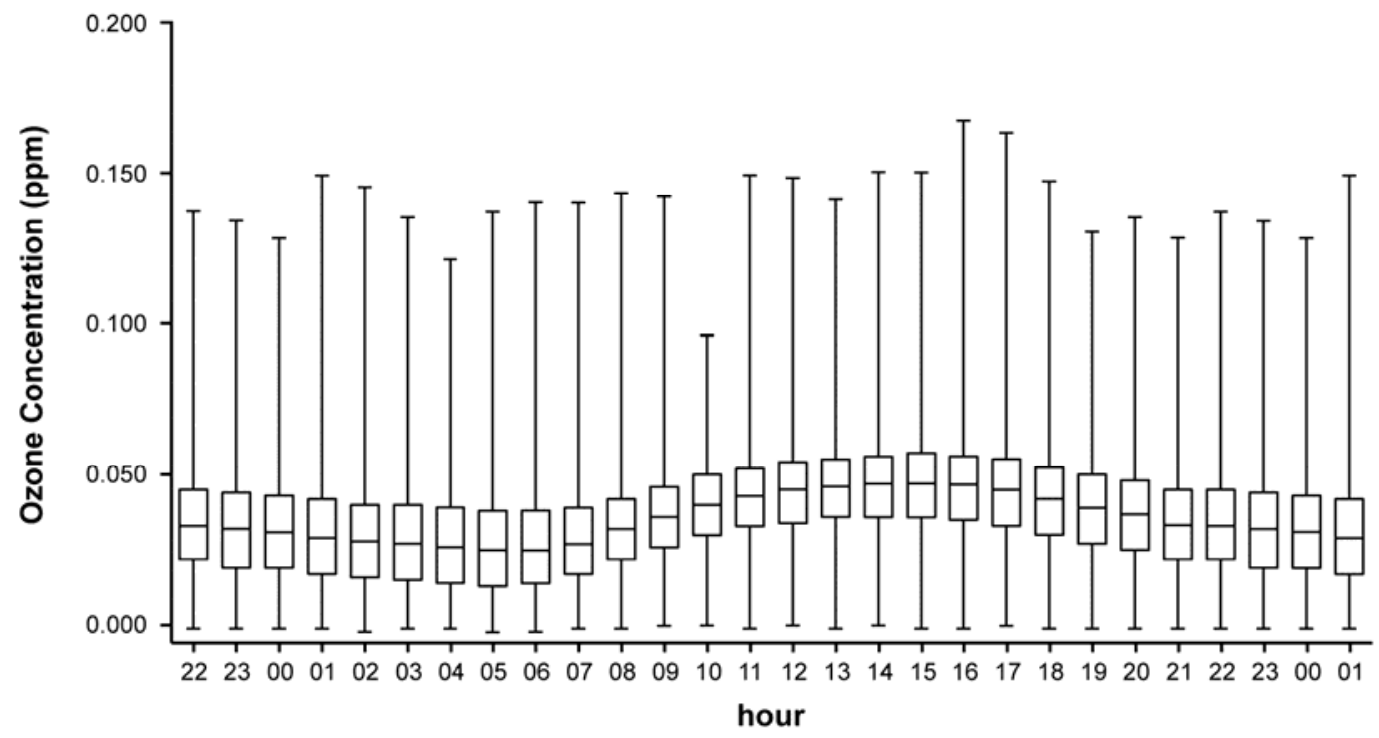

Figure 2-6 Average hourly ozone concentration in US rural areas for April to October 2000 to 2004 [USEPA 2006a] 
Global chemistry and transport models are the only reliable source for estimating the PRB ozone concentrations. These models mathematically simulate the chemical transformations and transport of ozone and its precursors in the atmosphere, globally. Fiore et al [2002] estimated the maximum afternoon summer concentration in the range of 15-35 ppb. PRB concentrations depend on the altitude of the location, the season, and the total ozone concentration. Higher PRB ozone concentration is observed at higher altitudes $(>1.5 \mathrm{~km})$ due to free tropospheric exchange. The PRB concentration is higher in spring than in summer. The PRB ozone concentration is the highest at ozone concentrations of about $50 \mathrm{ppb}$. At higher total ozone concentrations the PRB ozone component decreases and is usually less than 25 ppb [USEPA 2006a].

There have been no significant year to year variations in the average ozone concentrations in the US. Figure 2-7 shows the ozone season mean daily 8-h concentration for the years 1990 to 2004 . The whiskers on the boxes show the $10^{\text {th }}$ and the $90^{\text {th }}$ percentile values. A considerable decrease in the peak concentration is seen after 1995. The difference in the annual ozone concentrations after 1995 is due to the variability in annual climates.

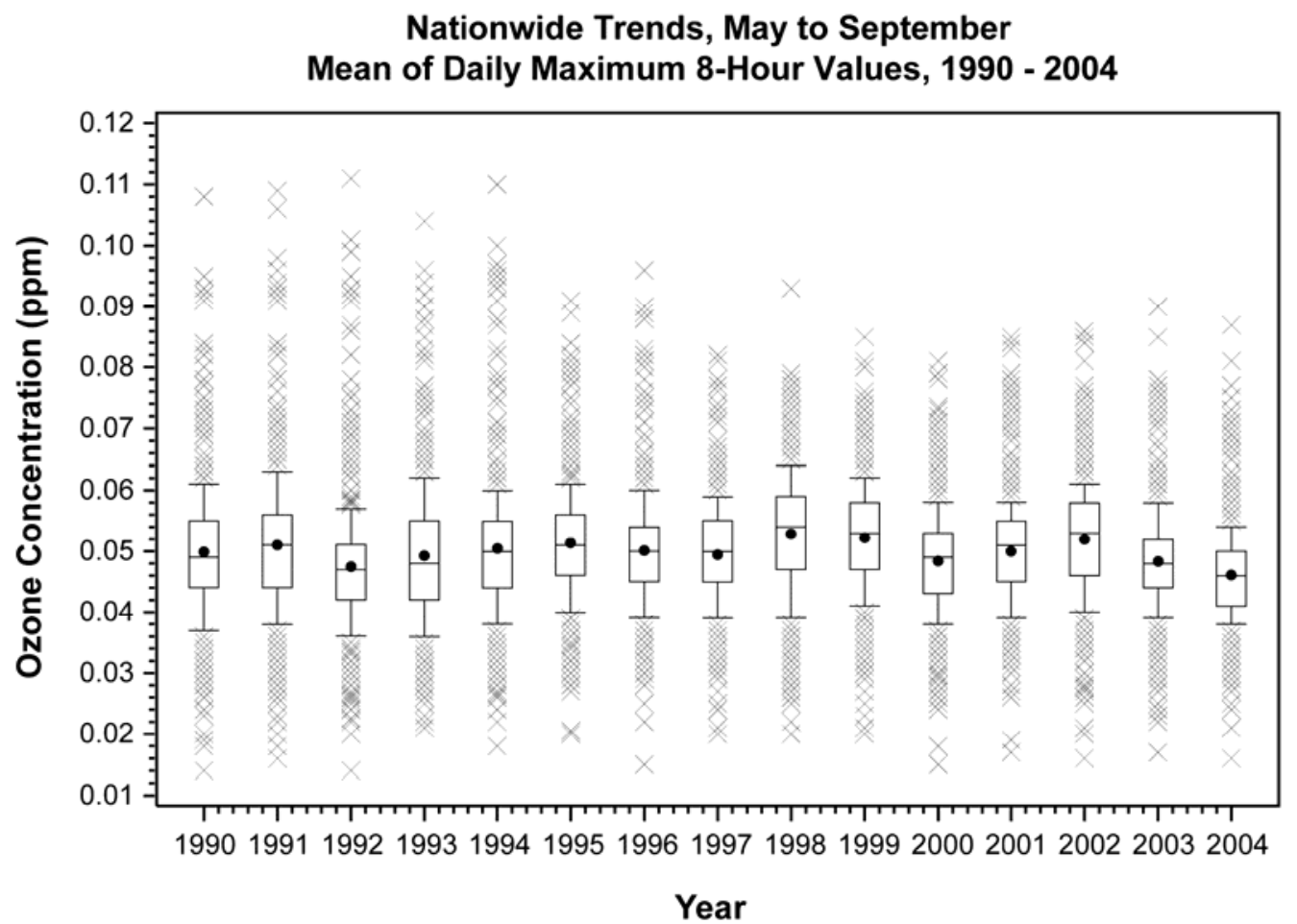

Figure 2-7 Annual trend in the daily 8-hour ozone concentration for May-September 1990 to 2004 [USEPA 2006a] 


\subsection{DETRIMENTAL EFFECTS OF TROPOSPHERIC OZONE}

Exposure to tropospheric ozone is a cause of several respiratory and cardiovascular health effects, and even mortality in humans. Plants and ecosystems are adversely affected due to interference in photosynthesis, and tissue damage on exposure to high ozone concentration. Ozone has an influence on the ground-level UV radiation, thus affecting UV-related health outcomes. Tropospheric ozone is a well-known greenhouse gas and contributes to global warming. It has an indirect forcing effect due to its chemical interrelation with methane, $\mathrm{NO}_{\mathrm{x}}$, non-methane hydrocarbons and CO. Man-made materials like elastomers, fibers, dyes and paints are known to be damaged by exposure to ozone.

\subsubsection{Human health effects}

There is a large body of experimental and epidemiologic evidence supporting the adverse effects of human exposure to tropospheric ozone. These studies have evaluated the short term, repeated, and long term effects of ozone exposure. A brief overview of these effects which has been summarized from the USEPA’s Air Quality Criteria Document [USEPA 2006a] is presented here.

Short term exposure to ozone causes lung function decrements and increased respiratory symptoms in healthy as well as more susceptible individuals. Lung function decrements are measured by the Forced Expiratory Volume in 1 second or $\mathrm{FEV}_{1}$. This is the volume of air that an individual can forcibly exhale in 1 second after maximum inspiration. A human exposure study has indicated a decrease in $\mathrm{FEV}_{1}$ of more than $10 \%$ for $23 \%$ of the test population for a 0.08ppm exposure for 6.6 hours with moderate exercise. The responses of individuals to ozone

exposure vary considerably, and appear to decline with increasing age starting at 18 years. Repeated ozone exposure develops a level of tolerance in the subjects, but this tolerance is lost within one week without exposure. Epidemiologic studies have found a decrease in lung function during ozone episodes particularly among children, asthmatic patients, and outdoor workers.

Clinical studies on healthy humans at $0.08 \mathrm{ppm}$ to $0.12 \mathrm{ppm}$ exposures during moderate to heavy exercise is shown to exhibit cough symptoms, chest pain on deep inspiration, and 
shortness of breath. Epidemiological studies show similar effects of cough, wheeze, formation of phlegm, shortness of breath, and increase in medication use in asthmatic children during ozone episodes. Repeated exposure develops a short term tolerance to these effects. Inflammation of the respiratory tracts has been observed in clinical studies on healthy individuals. Inflammation has been seen even in the absence of pulmonary effects. Toxicological studies on animals show lung inflammation, damage to airway epithelial tissues, and increase in susceptibility to infections. Epidemiological evidence shows similar inflammation in children during 1 hour exposure to ozone concentration of $0.1 \mathrm{ppm}$. Ozone exposure studies show an increase in airway responsiveness in response to bronchial allergens or other stimuli. Epidemiological studies have shown a robust positive correlation between respiratory-related hospital admissions or asthma emergency department visits and ambient ozone concentrations. This increase is supported by the pulmonary and respiratory effects observed in the clinical studies.

Recent evidence suggests that ozone exposure increases the risk of cardiovascular morbidity. One of the plausible mechanisms is the release of ozone-induced platelet activating factor that may lead to clot formation. There is limited, but highly suggestive, clinical and epidemiological evidence supporting the cardiovascular impacts of ozone.

Several epidemiological studies have investigated the relation between short-term mortality and ozone concentration. They have found a positive correlation between the increase in the number of non-accidental deaths and increase in ozone concentration. These studies are either city-specific or multi-city, and some of them adjust for variation due to the presence of particulate matter and changing seasons. The specific mechanism causing mortality is not known for certain, but the plausible pathway can be either due to pulmonary effects of lung damage leading to inflammation and/or decreased lung function, or due to cardiovascular effects.

A recent epidemiological study by Bell et al [2004] used the databases developed as part of the National Morbidity, Mortality, and Air Pollution Study. 95 large communities across the US were studied for the years 1987 to 2000 to investigate the association between short-term ozone exposure and mortality. They used single-lag as well as distributed-lag models to estimate community-specific mortality rates related to ozone exposure. Single-lag models relate the concentration of ozone on a particular day to mortality rates on a subsequent day. For example a 1 day lag model relates the mortality on a particular day to the ozone concentration on the previous day. Distributed-lag models are useful in estimating the effects of an ozone episode, 
which are typically longer than a day to mortality on a single day. A lag of up to 6 days was used to calculate the mortality associated with the daily average ozone concentration for the previous week. Distributed-lag models capture the short-term cumulative effect of ozone exposures which single-lag models cannot. The distributed-lag regression models were used to determine the association of community specific relative mortality rates and ambient ozone concentrations at the first stage. These associations were adjusted for weather, particulate matter, seasonality, and long term trends. The community specific rates showed that for an increase of $10 \mathrm{ppb}$ in the previous week's average ozone concentration the corresponding change in mortality rates was between $-0.73 \%$ and $1.77 \%$. This heterogeneity is due to city-specific factors like trends in pollution levels, use of air-conditioning, percentage of time spent outdoors, and socioeconomic factors.

The community-specific rates were then combined to derive a national average taking into account the spatial differences. The results showed that a $10 \mathrm{ppb}$ increase in the dailyaverage ozone concentration for the previous week increased the daily mortality by $0.52 \%$, nationally. This estimate was slightly lower than other recent US single or multi-city studies or meta-analysis. These studies reported estimates in a range of $0.78 \%$ to $1.37 \%$ [Bell et al 2004]. The results did not show a significantly higher rate for susceptible population groups, like older persons and those having lung and heart diseases. This only means that these groups are not at a higher relative risk, but overall, since the baseline mortality rates in these groups are higher, the number of mortalities is, still, large. However, it is important to note that ozone is only one of the gases in the photochemical air pollution mixture. Other hazardous compounds such as PAN are also present in this mixture. Such epidemiological studies consider ozone as a surrogate measure which represents the entire mixture of photochemical pollutants. The degree to which ozone acts as a surrogate, misclassifying toxicity, varies with locations, and a more suitable, more generic metric for photochemical pollution exposure has not been, yet, determined.

To determine whether the effect was only due to higher concentrations (8h average $>0.08$ ppm), the same analysis was performed for days where the average concentration was less than $0.06 \mathrm{ppm}$. The resulting association was a $0.18 \%$ overall increase in mortality rates for a $10 \mathrm{ppb}$ increase in concentration. A related study by Bell et al [2006] showed the absence of a threshold for the effects of ozone above the background concentration of 15-35 ppb. Therefore, any amount of ozone formed due to anthropogenic precursors poses a risk to public health. It 
suggests that there exists no safe level of ozone concentration below which there are no human health effects. Any regulation on ozone levels is, thus, a tradeoff between costs of implementation, and the effect of ozone on public health.

Studies investigating the long term exposure to ozone have been suggestive, but inconclusive, of impaired lung function development in children. Chronic exposure studies on animals have shown the occurrence of structural changes in the respiratory tracts, and the effect might be cumulative. However, a clear understanding of the long term human effects at ambient ozone levels does not, currently, exist [USEPA 2006a].

\subsubsection{Effects on vegetation and ecosystems}

Crops, plants, tress, and entire ecosystems are affected by exposure to ozone. Most of the research on vegetation effects has been limited to the species level, with few studies at the ecosystem level. These studies have provided clearer understanding of the effects of ozone on the plants at the cellular, physiological, and mechanistic levels. A summary of the effects based on USEPA NAAQS review document [USEPA 2007a] is presented here.

The primary mode of ozone uptake in plants is through the stomatal openings in the leaves. The rate of uptake is dependent on several factors, namely, the ambient concentration, the orientation of the leaf within its surroundings, the effect of other plants in the vicinity, adsorption on leaf cuticle, stems, etc. It is also affected by abiotic factors like humidity, temperature, $\mathrm{CO}_{2}$ concentration, soil fertility, water availability, and the presence of other pollutants. Hence, the ozone flux which reaches the sensitive sites after entering through the stomata varies diurnally, daily, and seasonally. The ozone entering through the stomata undergoes chemical transformation within the leaf air spaces, or within the water lining the cells, to produce toxic chemicals like carbonyls, hydrogen peroxide, and other radicals. These toxics diffuse within the cell, initiating the ozone injury. Ozone hinders photosynthesis by direct impact to chloroplast function, or through blockage of stomatal openings and reduce uptake of $\mathrm{CO}_{2}$.

Injury to a sufficiently large number of cells produces effect at the physiological level. These effects include visible foliar injury and premature senescence, effects on carbohydrate production and allocation, reduced growth and reproduction, and reduced plant vigor. These 
effects are dependent on the cumulative nature and temporal dynamics of the ozone exposure. Long lived plants also exhibit a "carry over” effect where the exposure in one year affects growth in subsequent years. Plants also have the capability of detoxification when the cumulative exposure is relatively low.

Therefore, the exposure of plants and trees is better expressed by a cumulative parameter. SUM06 and W126 are two such indices which have been used to characterize ozone exposure to plants. SUM06 is the 3-month sum of all hourly ozone concentrations greater or equal to 0.06 ppm during the 12 hour period from 8 am to $8 \mathrm{pm}$. The 3 months are consecutive months resulting in the maximum SUM06. These represent the growing season for the crops and trees. W126 is similar to SUM06, except that it is a sigmoidally weighted function of the ozone concentration. The weighting factor can is expressed mathematically as

$$
\mathrm{w}_{\mathrm{c}}=\frac{1}{1+4403 e^{-126 \mathrm{c}}}
$$

where, $\mathrm{c}$ is the hourly concentration of ozone, and $\mathrm{w}_{\mathrm{c}}$ is the weighting factor.

W126 is the preferred index as it does not entirely neglect values below $0.06 \mathrm{ppm}$ which is about the same as the average ozone concentration in US. Besides, no biological threshold to effects exists at $0.06 \mathrm{ppm}$. W126 is also helpful in regulatory application as the weights for concentrations below the PRB levels are very low. Besides, any errors in measurement or estimation of concentration near $0.06 \mathrm{ppm}$ do not have a significant effect on the index, unlike in SUM06. The W126 for the year 2001 developed using regional photochemical model CMAQ is shown in Figure 2-8.

The National Crop Loss Assessment Network (NCLAN) studied the concentrationresponse characteristic for 31 crops. The crop response measured was the loss in biomass due to ozone exposure. These studies were conducted in Open Top Chambers (OTC) with varying parameters like sites, water regimes, and exposure conditions for each species. The concentration-response relations are Weibull functions, where the relative loss is given as

$$
R Y L=1-\exp \left(-\Delta O_{3} / \gamma\right)^{\lambda}
$$




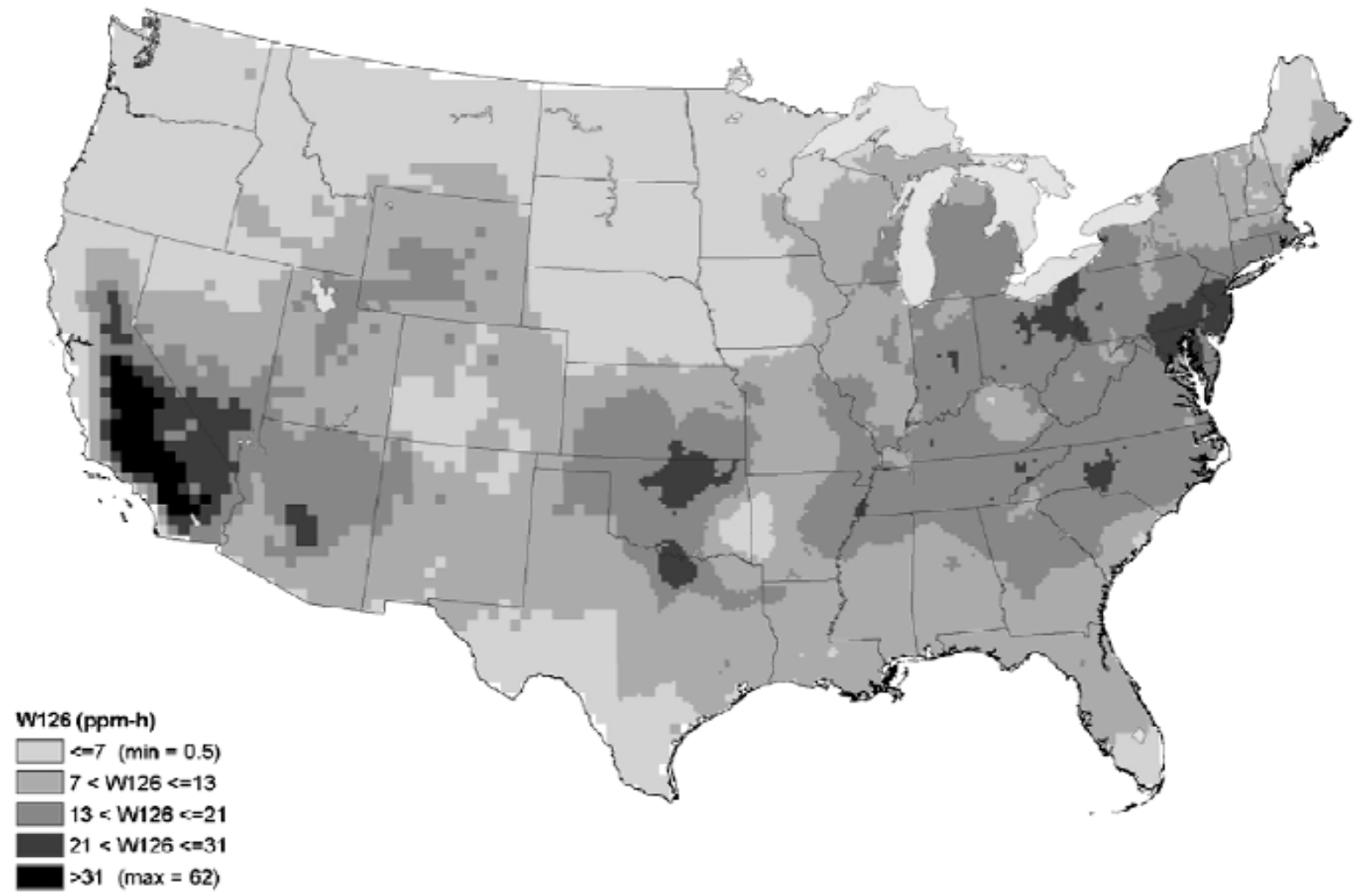

Figure 2-8 W126 for the year 2001 calculated using CMAQ model simulation [USEPA 2007] 
In equation 16, $\lambda$ and $\gamma$ are parameters which are dependent on the crop species and $\Delta \mathrm{O}_{3}$ is the change in ozone concentration index. A similar function is defined for biomass loss in tree seedlings and yield loss in fruits and vegetables with varying $\lambda$ and $\gamma$ values. The range of values for percentage relative yield loss for the 31 NCLAN crop species at various levels of exposure is shown in Figure 2-9. However, it should be noted that these effects are only short term (one growing season) effects of ozone exposure. These C-R functions are relevant for plant species which are harvested and replanted each year. The long term effects on perennial trees are relatively less severe compared to annual crops. Some species show an increasing effect in subsequent years, while in other species, the effects become less significant over time. Evergreen species have been found to be less sensitive to ozone exposure than deciduous species. Tree seedlings show greater sensitivity than mature trees in most of the physiological processes. There is a large variation in the sensitivities of different tree species, and the effect of competition from ozone tolerant species exacerbates the effect on more sensitive species. Due to the presence of such factors, the effects on mature trees and other perennial plant species have not been, yet, quantified adequately.

Our current knowledge of the actual effects of ozone exposure on ecosystems is very limited. No ecosystem-level studies have been performed to determine the pathways in which ozone exposure alters ecosystem functions. A possible effect of ozone exposure at the ecosystem-level is due to scaling up of the effects at the individual species level. This affects ecosystem processes like energy and material flows, competition between species, and net primary productivity. Ozone exposure can make the ecosystem more vulnerable to natural disturbances by affecting the water balance, tolerance to wind, and resistance to diseases and insects. For ecosystems with mixed species, the overall growth rate may be unaffected due to an increase in the competitive growth of ozone tolerant species.

In order to aid regulatory decision making, the effects on vegetation have been converted to an economic index. This is particularly true for agricultural crops whose economic value is well documented. For other plants, trees and ecosystems, this is not possible, as our understanding of the true economic value of their services is unknown. 


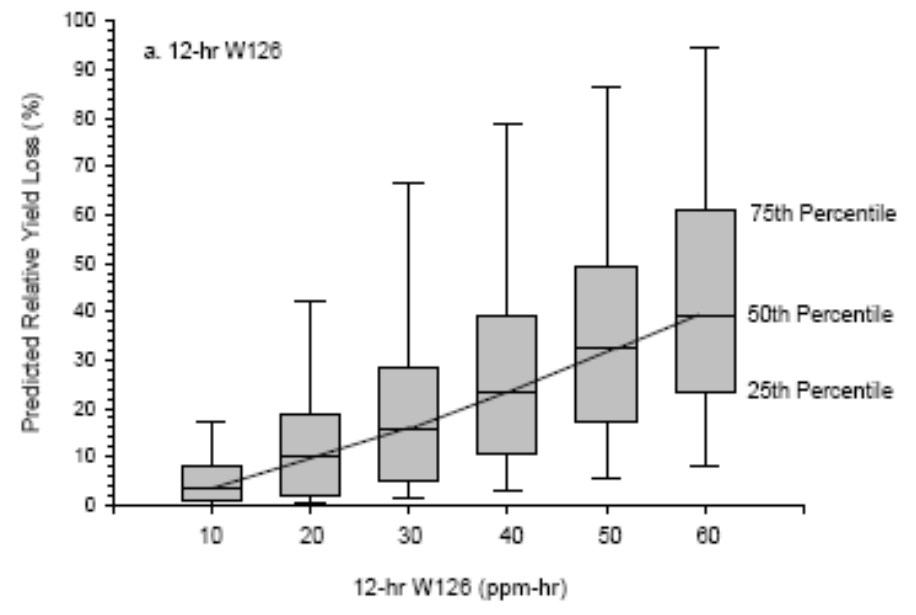

Figure 2-9 Relative yield loss for the 31 NCLAN species at different levels of exposures [USEPA 2007]

\subsubsection{Other effects}

In addition to the direct effects on human and ecosystem health, the presence of higher levels of ozone in the atmosphere has other effects, too. Ozone affects the radiation balance of the atmosphere by absorbing UV-B radiation. Exposure to UV-B radiation is known to cause erythema, skin cancers, ocular effects, and immune system responses. Therefore, an increase in tropospheric ozone concentration could have some public health benefits. Recent studies have reported that UV-B is necessary for the synthesis of Vitamin D in the skin which offsets the adverse effects of UV-B. However, a direct relationship between tropospheric ozone and the UV-B change induced effects has not been determined within reasonable certainty.

Ozone is a greenhouse gas. It traps the infrared emissions reflected from the earth's surface resulting in surface warming. The Intergovernmental Panel on Climate Change [Forster et al 2007] recognizes tropospheric ozone as an important positive radiative forcing component. The increase in the radiative forcing from 1750 to 2005 due to tropospheric ozone is $0.35 \mathrm{~W} / \mathrm{m}^{2}$, as compared to the radiative forcing of $1.66 \mathrm{~W} / \mathrm{m}^{2}$ due to $\mathrm{CO}_{2}$, and 0.48 for $\mathrm{CH}_{4}$. 
Besides the direct positive forcing mechanism of ozone, ozone has an indirect negative effect. Photolysis of ozone produces hydroxyl radical which is important in the removal of methane, hydrofluorocarbons, and other reactive VOC. However, an estimate of this indirect effect is very uncertain due to difficulty in estimating changes in global abundance of hydroxyl radical.

Ozone reacts with many important man-made materials like elastomers, fibers, dyes, and paints decreasing their useful life and aesthetic appearance. The primary reaction involved in this process is the breaking of the carbon-carbon bond in the man made polymer by oxidization due to ozone. This affects the tensile strength of elastomers and fabrics, and causes fading in textile dyes and artist's pigments [USEPA 2006a].

\subsection{LEGISLATIVE STANDARDS FOR TROPOSPHERIC OZONE}

The adverse effects of ozone on human health, vegetation, and other materials have long been identified. Most of the developed countries have implemented legislations to set standards which act as upper limits to the ambient ozone concentrations. These standards are intended to protect the sensitive receptors from exposure to ozone. Most of these standards focus on the human health effects, while some also address vegetation health. These standards were earlier considered to be safe levels below which the effects of exposure are minimal. However, recent scientific research, as discussed previously, has shown that no such threshold to exposure exists, either in human beings or vegetation. Even low levels of exposure have significant effects on the sensitive species. Hence, the current standards represent a balance between the risks of exposure (including public health risks) and the financial burden of implementing those standards.

In the United States, the USEPA has set the National Ambient Air Quality Standard (NAAQS) which regulates many air pollutants including ozone. The first NAAQS set $0.08 \mathrm{ppm}$ as the maximum hourly ozone concentration, which was increased to $0.12 \mathrm{ppm}$ after a few years. If an area violates the NAAQS, it is designated as a non-attainment area. A non-attainment area is given a few years to demonstrate and implement policies and emission controls to meet the NAAQS. The NAAQS was revised in the early 1990's and established $0.08 \mathrm{ppm}$ as the three year average of the fourth highest annual 8 hour average value. Because of rounding, this is, in 
effect, is equal to a value of $0.084 \mathrm{ppm}$. In view of the current scientific understanding of the health effects of exposure below the current standard, USEPA has recently proposed a more stringent standard for human health (primary standard), and a separate standard for vegetation health (secondary standard). The USEPA proposes to set the primary standard within the range of 0.070 to $0.075 \mathrm{ppm}$ for the 8 hour average ozone concentration. In recognition of the difference in human and vegetation effects, the USEPA proposes to establish the secondary standard between 7 and $21 \mathrm{ppm}$-hr of the 3 month W126. USEPA has held public hearings on the proposed standards and is expected to implement the new standards by June 2008 [National Ambient Air Quality Standards for Ozone 2007].

The European Union has established a short term, and a long term target for ozone concentrations. In the short term, starting from 2010, the primary standard target is $120 \mu \mathrm{g} / \mathrm{m}^{3}$, which is equal to $0.06 \mathrm{ppm}$ at standard temperature and pressure. This target can be exceeded on a maximum of 25 days in a given calendar year. The secondary target is an AOT40 index value of $18000 \mu \mathrm{g} / \mathrm{m}^{3}$.h (9 ppm-hr) calculated from 1 hour values during the growing season from May to July. The AOT40 is in principle similar to SUM06. It is the sum of the difference between the hourly ozone concentration greater than $80 \mu \mathrm{g} / \mathrm{m}^{3}$ (40 ppb) and $80 \mu \mathrm{g} / \mathrm{m}^{3}$ of values measured between 8 am and $8 \mathrm{pm}$, daily over the given period. The long term target, for 2020, is zero exceedences in the primary standard, and an AOT40 target value of $6000 \mu \mathrm{g} / \mathrm{m}^{3} . \mathrm{h}$ (3 ppm-hr) [European Union 2002].

Japan has the most stringent standard, worldwide, for ozone at $0.06 \mathrm{ppm}$ of maximum 1 hour concentration. China has multiple standards depending on whether the area class is residential, commercial or industrial. The standards are set at 1 hour values of $0.06,0.08$, and $0.12 \mathrm{ppm}$, respectively. The World Health Organization (WHO) has set its global ozone guideline for the daily maximum 8 hour average concentration at $100 \mu \mathrm{g} / \mathrm{m}^{3}(0.05 \mathrm{ppm})$. At this concentration, the WHO estimates a $1-2 \%$ increase in ozone related mortality over the mortality attributable to the background concentration [WHO 2005]. 


\subsection{AIR QUALITY MODELING}

Photochemical Air Quality Modeling Systems (PAQMS) are being widely used nationally and internationally to model the physical and chemical processes responsible for tropospheric ozone. They are being used extensively in air quality regulatory applications, and in scientific investigations of ozone. Separate models have been developed to simulate processes occurring at the urban, regional, and global scales, respectively. Regional scale air quality models have recently found increased use in science and regulation. The Air Quality Analysis Workgroup of the Ozone Transportation Assessment Group (OTAG) used a PAQMS to identify the long range transport patterns of ozone and its precursors, and the effect on ozone concentration at the nonattainment areas in the North-East corridor. Regional Planning Organizations and several states use the PAQMS to establish the effectiveness of their control plans in meeting the NAAQS. The USEPA used a PAQMS for ozone modeling in support of the Clean Air Interstate Rule (CAIR) and for periodic reviews of NAAQS. Internationally, the North American Research Strategy for Tropospheric Ozone (NARSTO) extensively uses PAQMS in their research efforts. In Europe, the EMEP was established under the Convention on Long Range Transboundary Air Pollution. It uses PAQMS to model photochemical and transport processes for tropospheric ozone, assess the various abatement measures, and provide cost-effective emission controls.

\subsection{LAGRANGIAN AND EULERIAN MODELS}

PAQMS can be divided into Lagrangian and Eulerian models based on their spatial representation. Lagrangian and Eulerian models employ two different frames of reference for the dynamics of atmospheric pollutants. In a Lagrangian model, the observer's reference frame moves along with the wind flow. Thus the observer follows a parcel of air for a long period. In 
the Eulerian model, the reference frame is fixed to the earth or any stationary object. The earth's atmosphere is divided into grids and air parcels are transported from one grid cell to another with the flow of air, and diffusion.

Lagrangian models were used for the earliest modeling systems. A parcel of air containing emissions is followed on a trajectory defined by the mean wind speed and direction at each time interval. Some of the Lagrangian models consider vertical diffusion, too. In this case, the parcel of air is expanded in size as it moves along the trajectory. The pollutant species concentration in the air parcel is calculated at each time step taking into account the photochemical reactions occurring within the parcel. The air parcel is followed for a period of a few days to determine the fate of the pollutant. Eulerian models compute the concentration of each species in a given space at a given time with reference to a fixed co-ordinate system. The co-ordinate system is usually a Cartesian system and the modeling domain is divided into finite number of grids in the $\mathrm{x}, \mathrm{y}$, and $\mathrm{z}$ axes. The processes of transportation and photochemical reaction are simulated in each grid cell at each time step.

Since Lagrangian models calculate the concentration of pollution in a fixed mass of air, they require less computational resources than the Eulerian models, which calculate the concentration in each grid cell of the domain. However, Lagrangian models neglect the effects of vertical wind shear and horizontal diffusivity, and thus are more uncertain when simulated over longer periods of time. Another disadvantage is that they provide information only on the trajectory path. Therefore, to develop a spatial field of pollutant concentrations, or to study the temporal variation at any particular point, several trajectories have to be followed. Because of these limitations of Lagrangian models, and the easy availability of fast computers, Eulerian models have gained popularity and have become the dominant photochemical modeling system [Russell 1997].

The first modern Eulerian model was developed by Reynolds et al [1973] for modeling the formation of ozone in the city of Los Angeles. With the increase in awareness of the importance of long range ozone transport, regional scale $(1000 \mathrm{~km})$ models and global models were developed. Most of the current photochemical models can model processes at multiple scales ranging from a few kilometers to thousands of kilometers. The following discussion of PAQMS will be limited to regional scale Eulerian air quality models. 


\subsection{COMPONENTS OF A PAQMS}

A PAQMS is made up of three core components and several auxiliary supporting programs. Each of the component models a different physical or chemical process. These components and the typical data flow between them is shown in Figure 3-1 and described briefly below. The inputs required by a typical regional model from external databases are also shown in Figure 3-1.

\subsubsection{Chemistry and Transport Model}

The Chemistry and Transport model is the heart of the PAQMS. It simulates the processes of dispersion, emission, chemical reaction, and removal of the pollutants from the troposphere. It is based on the Eulerian continuity equation for gases, which has been modified to reflect key atmospheric processes. It is known as the Atmospheric Diffusion Equation (ADE). The mathematical representation of the ADE is

$$
\frac{\partial c_{i}}{\partial t}+\nabla \cdot\left(\bar{U} c_{i}\right)=\nabla \rho \bar{K} \nabla\left(c_{i} / \rho\right)+R_{i}\left(c_{1}, c_{2} \ldots c_{n}, t, T, . .\right)+S_{i}(\bar{x}, t), \quad i=1,2,3 \ldots . . n
$$

where $c_{i}$ is the concentration of the species $i, \bar{U}$, is the three dimensional wind velocity vector, $\bar{K}$ is the turbulent diffusivity vector for the species, $R_{i}$ is the rate of change in the species' concentration due to chemical reactions, and $S_{i}$ is the source/sink term representing emissions and various removal processes. The equation, essentially, states that the rate of change of the pollutant's average concentration is a function of convective transport, turbulent diffusion, chemical reaction, and emission or deposition of the species. The pollutant diffusion, in this case, is diffusion due to atmospheric turbulence. Molecular diffusion of the species has been found to be small compared to the turbulent diffusion, and the former is thus neglected in the ADE [Russell 1997]. 


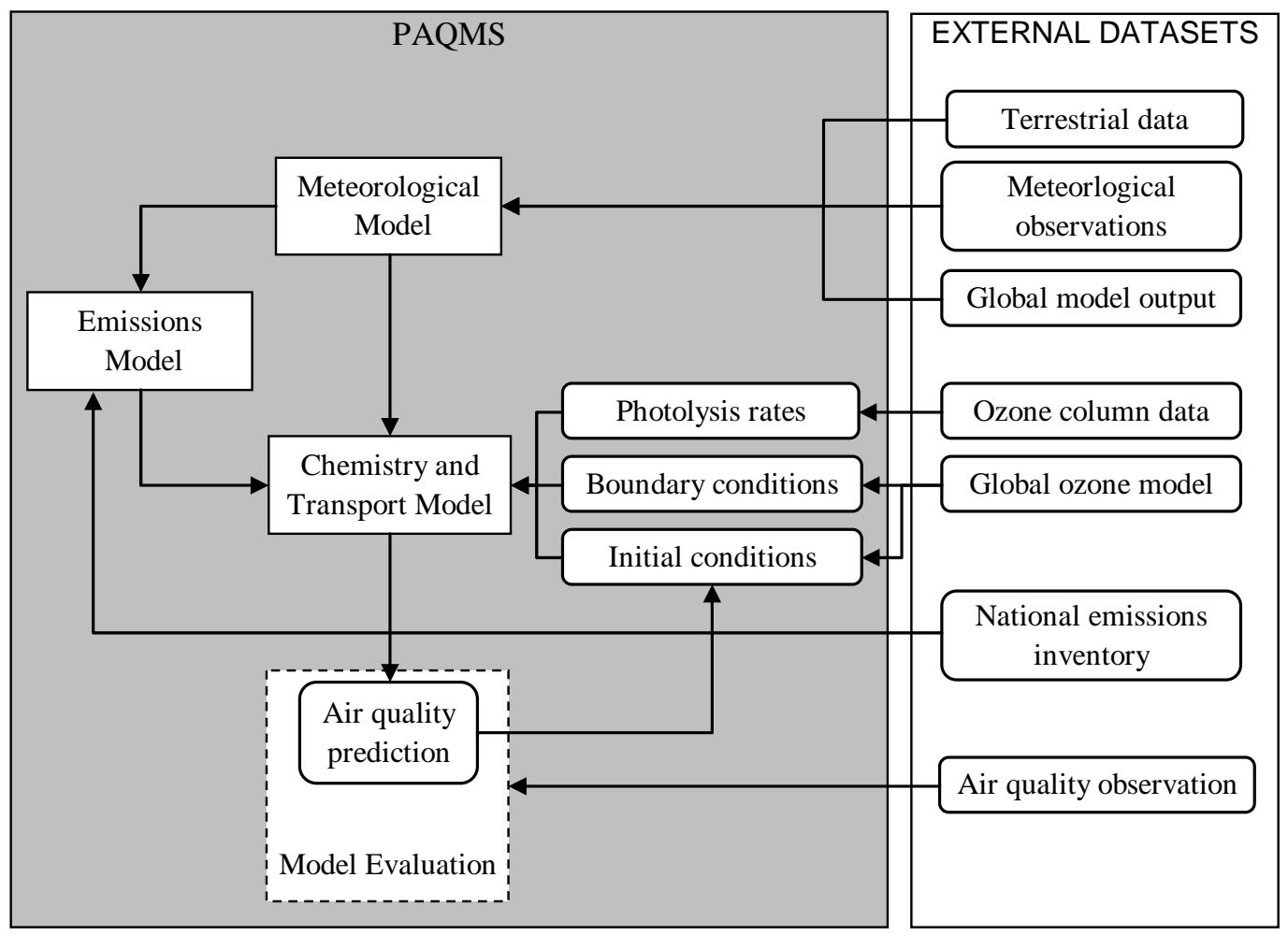

Figure 3-1 Schematic representation of a Photochemical Air Quality Modeling System and the typical external inputs required

Analytical solution to the ADE is known only for a limited set of (mathematically simple) wind, diffusion fields, and chemical reactions. Numerical methods are typically used to solve the ADE. The ADE is solved for each modeled species in each grid cell at each time step of the model run. The physical and chemical processes modeled by the ADE include the following:

a. Pollutant transport: The transport of pollutant species happens through two mechanisms, namely, advective transport and turbulent diffusion. Advective transport takes place in proportion to the time averaged wind vector. Wind flow is predominantly in the horizontal plane. Vertical transport is caused through mechanical turbulence or thermal turbulence. Mechanical turbulence is the formation of eddies when wind flows over rough surfaces. Higher roughness results in greater local turbulence. Surface roughness increases from water bodies, ice/snow, grasslands, croplands, residential areas to dense urban centers. Thermal turbulence is caused due to the heating of the earth's surface 
during the day. The warm air near the surface moves upwards and is replaced by the downward moving cooler air known as convective currents. The closure problem in turbulence is the presence of more unknowns than equations when Reynold's averaging is used in the Navier-Stokes equation. Thus, turbulence problems are solved through parameterizations such as the K-theory, which is used in the ADE. With K-theory, the turbulent transport is parameterized as a constant $(\mathrm{K})$ times the gradient of the average mixing ratios. The value of the constant can be determined using several algorithms from other easily measurable or modeled meteorological parameters [Russell 1997].

b. Pollutant removal / addition: Pollutant deposition on the earth's surface occurs through dry deposition and wet deposition. Dry deposition occurs when the gases are removed at an air-surface interface. Gases, either impact and stick to the surface or react with the surface resulting in their removal. Sedimentation is also a dry deposition process, but its contribution is very small. Dry deposition is usually expressed in terms of deposition velocity which is calculated as the inverse of a sum of resistances. The resistances are aerodynamic resistance between a given height and the laminar sublayer near the surface, resistance due to molecular diffusion in the thin laminar sublayer, and resistance to chemical, biological, and physical interactions at the surface [Russell 1997]. Wet deposition occurs when the gases are scavenged by aerosols, clouds or rain drops. It can occur through the mixing of gases with water, absorption of gas molecules by water droplets, aqueous phase reactions of the pollutants, or diffusion of the gases into precipitating droplets. Deposition depends largely on the solubility of the gas, and on cloud formation and precipitation. Addition of pollutants is due to the biogenic and anthropogenic sources of emissions. The rates of emissions are usually modeled by a separate emission processing program.

c. Chemical transformation: This includes the chemical reactions involving the several chemical compounds present in the atmosphere and also the photodissociation of individual compounds. This results in the destruction of some compounds and the formation of others. Chemical reactions can occur in the gas phase, on the surface of aerosols or droplets, or within the droplets [Russell 1997]. The rates of reactions vary with weather conditions like temperature, solar insolation, pressure, etc. Photochemical processes also result in the elevation of the levels of particulate matter. A few 
photochemical models have been extended to include the formation for aerosols. Particulate matter in the atmosphere has an effect on the photolysis rates of the reactions involved in ozone formation. Also, aerosols with higher absorption capacity result in a decrease in the ozone concentration.

The commonly used multi-scale chemical and transport models in North America are: Community Multiscale Air Quality model (CMAQ), Urban Airshed Model (UAM), Regional Oxidant Model (ROM), and Comprehensive Air quality Model (CAMx).

All the other components and programs of PAQMS, except model evaluation, provide inputs to the chemistry and transport model. The inputs required by chemistry and transport model include: (a) meteorology inputs, (b) emission inputs, (c) topography and land use inputs, (d) atmospheric concentration of pollutants, and (e) a three dimensional grid structure. The inputs are required at each time step of the solution, which is typically one hour. The meteorology, emissions, and concentrations typically vary at each time step. In some air quality models, the vertical grid structure also varies in time.

Meteorology inputs typically provided are: temperature, pressure, wind, cloud/rain/snow water, water vapor, and diffusion coefficients. These are generated by a separate meteorology model. Emissions are the primary source of $\mathrm{NO}_{\mathrm{x}}$ and $\mathrm{VOC}$ which are precursors to the photochemical pollutants. These are either biogenic or anthropogenic in origin. Emissions, in the form required by the chemistry and transport model, are generated by a separate emissions program. For regional models, pollutant concentration on the horizontal and vertical periphery of the domain is another important source of pollutants. These are known as boundary conditions. Boundary conditions are usually provided for the precursors as well as the photochemical products. Initial concentrations of the pollutants are required for the solution of the ADE. These are known as initial conditions. The dependencies between the various inputs and the processes of the PAQMS are depicted in Figure 3-2. 


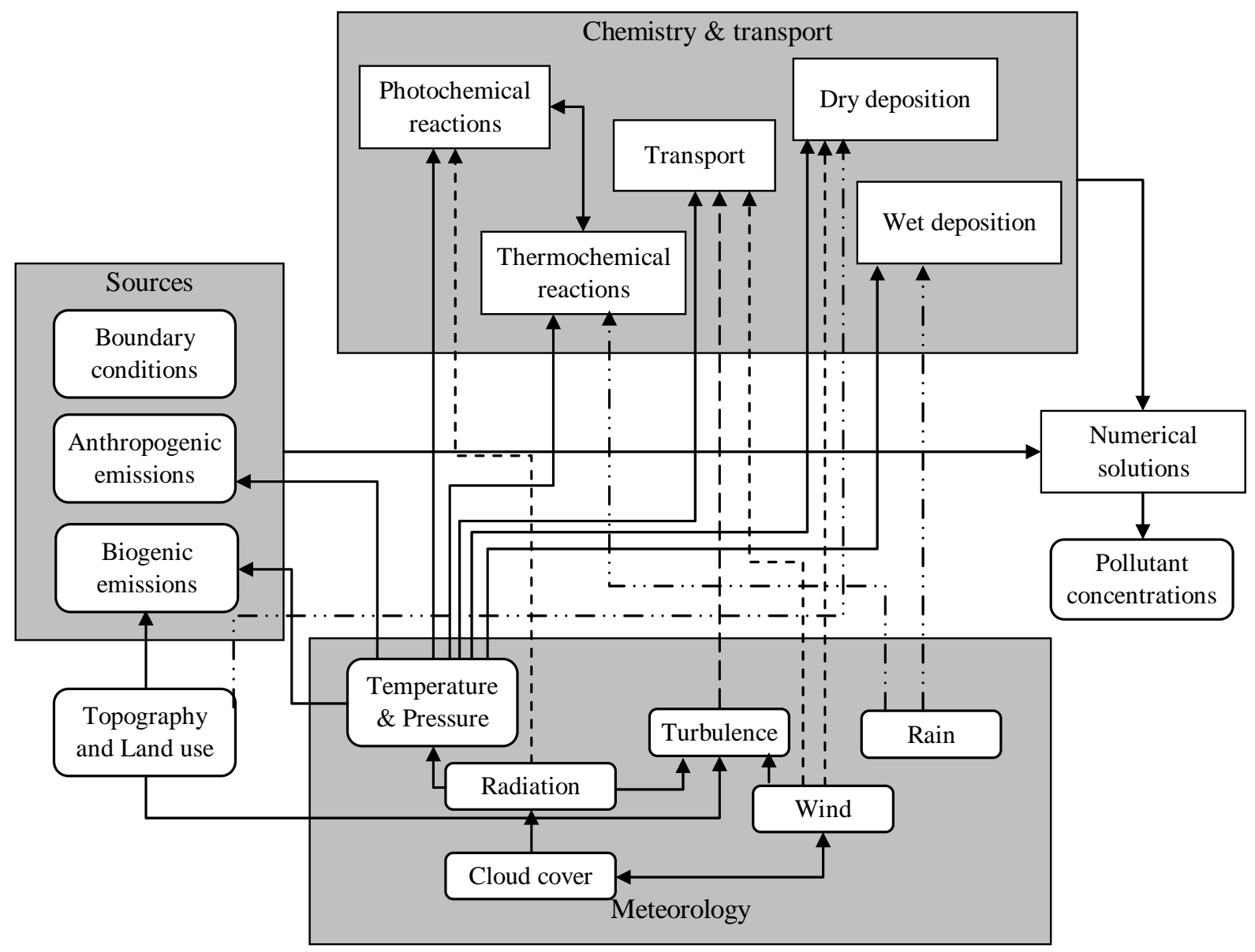

Figure 3-2 Schematic representation of relationship between variables and processes of a regional PAQMS

[Adapted from Russell 1997]

\subsubsection{Meteorology Model}

The meteorology model generates gridded meteorology fields which are essential for the processing of emission rates, chemical transformation, and species transport. The principal meteorology fields produced by a meteorology model are horizontal and vertical wind components, temperature, water vapor mixing ratio, cloud fraction and liquid water content, precipitation, solar actinic flux, sea level pressure, boundary layer depth, turbulence intensity, and surface fluxes for heat, moisture and momentum. The models used in regional PAQMS predict atmospheric motions of wind and turbulence at the mesogamma and mesobeta spatial scale which ranges from 2-200 km, and are known as mesoscale meteorology models. 
Meteorology models can be grouped into three types: (i) diagnostic models that analyze observations at discrete points in the domain, (ii) dynamic models that predict the meteorological fields using numerical integration of the equations of motion, and (iii) four-dimensional data assimilation models which combine the dynamic models with observations at each time step.

Diagnostic models infer the required variables from easily measured variables like temperature, pressure, and humidity. However, they are limited in their spatial and temporal resolution by the location of observation stations and frequency of observations. A high degree of uncertainty is added to the predictions when this sparse data is spatially averaged in three dimensions. Besides, it is difficult to directly measure fields like clouds and vertical velocities required by the PAQMS. Thus diagnostic models are not widely used.

Dynamic models predict the required fields using the basic laws of mechanics and thermodynamics. Dynamic models, in similarity to chemistry and transport models, numerically solve set partial differential equations that are fundamental to atmospheric processes. These equations are mass, momentum, and energy conservation, and thermodynamics of the moving air mass. In addition, the ADE for the three phases of water is also solved at each time step. Initial and boundary conditions are required for the solution of the partial differential equations. The initial and boundary conditions for the model are supplied by a coarser resolution dataset. This dataset is generated by regional or global models which assimilate observational data from various sources and derive the missing data, mathematically. Though, dynamic models are computationally expensive than diagnostic models, they do not require an extensive observation network. The resolution of dynamic models can be as high as $1 \mathrm{~km}$ and is limited only by the availability of computational resources. However, it has been found that the solutions to the model equations are very sensitive to the initial and boundary conditions. Any errors in the initial and boundary conditions accumulate over the duration of the model run and become significant, quickly. This limits the use of these models for long-term weather prediction.

The four-dimensional data assimilation (FDDA) technique is useful in limiting the accumulation of errors when dynamic models are used to simulate historical meteorological conditions. FDDA models use observational data throughout the modeling period to nudge the solution towards the actual. Hence, FDDA models have all the advantages of dynamic models while eliminating their primary disadvantage. FDDA based models are commonly used for producing meteorology fields for PAQMS. The most common meteorology models used in 
North America are: PSU/NCAR Fifth Generation Mesoscale Model (MM5), the Colorado State University's Regional Atmospheric Modeling System (RAMS), and, recently, the Weather Research and Forecasting (WRF) model.

In addition to the strong dependence of air quality on meteorology, there exists a feedback effect of air quality on meteorology. For example, the concentration of tropospheric photochemical pollutants affects the radiation balance of the earth, which, in turn, affects temperature, turbulence, and cloud formation. This feedback effect has been evaluated using coupled models, but it has been shown to be negligible. Besides, it is computationally expensive to recalculate the meteorology fields based on the air quality predictions, particularly when assessing different control strategies [Russell 1997]. Thus, the decoupled or offline approach is used by most of the current air quality models.

\subsubsection{Emissions Model}

Emissions are essential inputs to PAQMS. Emission models process specific data inventories to derive estimates of hourly emissions of $\mathrm{NO}_{\mathrm{x}}, \mathrm{CO}$, and several VOC. These data inventories are a measure of the level of activity which is associated with a particular emission, and are used in the absence of actual emission quantities. For example, total vehicle miles traveled is multiplied with average emissions per mile to calculate vehicular emissions. The emissions model use surrogates, like population or land area, to generate emissions at the model grid resolution from the data inventories which are typically available at state or county level in US. Temporal profiles are applied to derive hourly emission rates from coarsely resolved activity datasets. The emissions are speciated to be compatible with the chemical mechanisms used in the chemistry and transport model. This is particularly applicable for VOC. Typically emissions data inventories report VOC in considerably more detail than required by the chemistry and transport model.

The emissions model is not like the other models of a PAQMS since it is not a mathematical representation of any physical or chemical process. Its function is to apply the necessary factors and weights to generate model ready emissions. Thus, emissions are one of the most uncertain inputs to air quality models. Besides, the performance of the PAQMS is found to be highly sensitive to the emissions input. The uncertainty of the emissions inventory is the most important factor, and also the biggest limitation for the accuracy of the PAQMS to predict 
pollutant concentration [Russell 1997]. The commonly used emissions models in North America are: EPA's Emissions Modeling System-1995 (EMS95), Sparse Matrix Operator Kernel Emissions (SMOKE), and, recently, CONsolidated Community Emissions Processing Tool (CONCEPT).

\subsubsection{Model evaluation}

Model evaluation is necessary to develop confidence in the results obtained from the model simulation. Evaluation is different from validation or verification. Validation and verification imply an absolute correctness of the model. It is not possible to ascertain the absolute correctness

of models of natural systems. Evaluation is the assessment of adequacy of the model science by comparison with empirical data. Evaluation is the process of confirmation of the physical and chemical representations constituting the model [Russell and Dennis 2000]. Thorough model evaluations are essential during the development and testing of the models. During this phase, each module (e.g. chemistry, deposition, etc.) is independently evaluated against physical measurements. For model applications, evaluations determine the acceptability of the performance of the modeling system for the particular domain and period. The typical procedure is to statistically compare the predicted ozone concentrations to those observed in the field. This is known as operational evaluation. A second type of procedure is known as diagnostic evaluation which assesses the response of ozone concentration to changes in inputs, especially precursor emissions. Diagnostic evaluation can be performed by comparing observed and predicted ratios of $\mathrm{NO}_{\mathrm{x}}, \mathrm{VOC}$ and $\mathrm{CO}$, or ratios of secondary products like reactive nitrogen and peroxides [USEPA 2007b].

There are difficulties associated with the evaluation of a regional PAQMS. Pollutant concentrations have a high spatial variability. A PAQMS produces concentrations that are averaged over the volume of the grid cell which is several kilometers in each horizontal dimension. This can produce significant differences in the observation and the predicted concentration in the grid cell containing the observation location. Secondly, it is very difficult and expensive to generate an observation dataset at the spatial and temporal scales for the large domains that regional models cover. 


\subsection{SENSITIVITY AND UNCERTAINTY OF PAQMS PREDICTIONS}

Uncertainty in photochemical models arises from three main sources: (i) the inherent randomness of certain natural processes, like turbulence, (ii) uncertainty in model formulation and structure, and (iii) uncertainty of model inputs. Sensitivity analysis of the model indicates the relative importance of the sources of uncertainties. Sensitivity analyses have been performed for individual modules of the chemistry and transport model as well as for the predictions of a 3-D model.

Stand alone sensitivity analysis of the rate parameters and yields of chemical mechanisms have shown that relative uncertainties in the prediction of ozone concentration varies with the VOC/ $/ \mathrm{NO}_{\mathrm{x}}$ ratio in the range of 25 to $50 \%$ (one sigma) for urban conditions, and 15 to $20 \%$ for

rural conditions. The rate parameters for $\mathrm{NO}_{2}$ photolysis and the reaction of $\mathrm{NO}_{2}$ with $\mathrm{OH}$ to form $\mathrm{HNO}_{3}$ have the maximum contribution to ozone concentration uncertainty. A few studies have evaluated the relative performance of chemical kinetics and advection solvers and have found small uncertainties. The uncertainty associated with the selection of coarser horizontal (20-30 km) and vertical (6-8) grid resolution is believed to be within acceptable limits [Russell and Dennis 2000].

Hanna et al [2001] performed a Monte Carlo simulation to assess the sensitivity and uncertainty associated with 128 model inputs. These inputs include initial conditions, boundary conditions, emissions, wind speed and direction, temperature, relative humidity, cloud cover, and chemical rate parameters for the modeled equations. 100 simulations of the OTAG model run for the July 12-14, 1995 episode were performed. Little information was available on the uncertainty of all the inputs required by the chemistry and transport model. The uncertainty estimates were elicited from experts in the respective fields. The uncertainty in most of the model inputs varied from $20 \%$ to a factor of 2 . Most of the variables were represented as lognormal distributions. The resulting maximum hourly ozone concentration predictions had a 95\% confidence interval of 1.6 times the median. It was seen that the uncertainty in photolysis of $\mathrm{NO}_{2}$ had the maximum contribution to the uncertainty. Uncertainties in wind speed and direction, cloud cover, relative humidity, and biogenic VOC also had significant contributions. 


\subsection{IMPACT CHARACTERIZATION OF PHOTOCHEMICAL PRECURSORS}

Following the classification of the Life Cycle Inventory (LCI) into impact categories, characterization is the process of converting the LCI results into the respective category indicators. ISO 14042 [2000] requires the characterization model to be based on a "distinct identifiable environmental mechanism and/or reproducible empirical observation.” Characterization factors, generated from the characterization model, quantitatively relate the LCI to the impact category indicator. Characterization factors are also referred to as equivalency factors [Fava et al 1993]. The category indicator is the measure of impact for its impact category. Therefore, mathematically, the life cycle impact for a particular impact category is represented as:

$$
\mathrm{I}=\left[\begin{array}{llll}
\mathrm{CF}_{1} & \mathrm{CF}_{2} & \cdots & \mathrm{CF}
\end{array}\right] \times\left[\begin{array}{c}
\mathrm{E}_{1} \\
\mathrm{E}_{2} \\
\vdots \\
\mathrm{E}_{\mathrm{n}}
\end{array}\right]
$$

where $E_{1}, E_{2}, \ldots, E_{n}$ are the environmental interventions classified into the impact category I, having $\mathrm{CF}_{1}, \mathrm{CF}_{2}, \ldots, \mathrm{CF}_{\mathrm{n}}$ as their respective characterization factors.

\subsection{APPROACHES TO CHARACTERIZATION MODELING}

Hofstetter [1998] identifies two approaches that can be used for characterization modeling. The top-down approach, also known as descriptive approach, starts from the observed damages in the areas of protection and relates it to their cause. For example, morbidity and mortality are 
damages to the human health area of protection. Medical science provides us with the diagnosis of the effect which led to the disease or death. The next step is to relate the effect to a specific cause using epidemiological studies. The causal link factors are derived from exposure assessment. Empirical relationships between exposure and pollutant emissions extend the link from the damage to emissions. It can also be possible to identify the source of emissions. The last step is to determine the portion of the emissions which is attributed to the LCI.

The bottom up approach is a prescriptive procedure, in contrast to the descriptive topdown approach. The modeling begins at the quantity of emissions of a pollutant in the LCI. Multimedia fate models are used to predict the exposure to the damage subject. A dose-response relationship, previously established using toxicological studies, links the exposure to the effect on the subject. For example, some particular effect will have a certain probability of causing disease and death in humans. In the damage assessment step, the extent of the damage on the subjects due to morbidity and mortality is judged [Hofstetter 1998]. Both these approaches are illustrated in Figure 4-1.

Fate models are either single compartment models or multimedia models. They model the relationship between the emissions of a pollutant to its environmental (air, water, or soil) concentration. They model their transport, dispersion, and physical and chemical transformation based on the properties of the pollutant and external characteristics, such as meteorology. The PAQMS described in Chapter 3.0 is a fate model for photochemical precursors.

Exposure is the contact between an organism and a pollutant at its outer boundary. Exposure depends on the characteristics of the subject and the concentration and persistence of the pollutant in the exposure media. For example, in humans, behavioral, societal, and physiological characteristics strongly influence exposure. An exposure model's goal is to estimate the exposure as a function of these factors.

The damage assessment step is highly influenced by value judgments. These judgments create a common index for the damages, by assigning a relevance factor to distinct effects. For instance, one day of coughing can be considered half as harmful as one day of asthma attack, and, thus, the relevance factor of the former would be half that of the latter [Hofstetter 1998]. This step has been not been favored by many as it introduces an element of subjectivity in the impact assessment. 


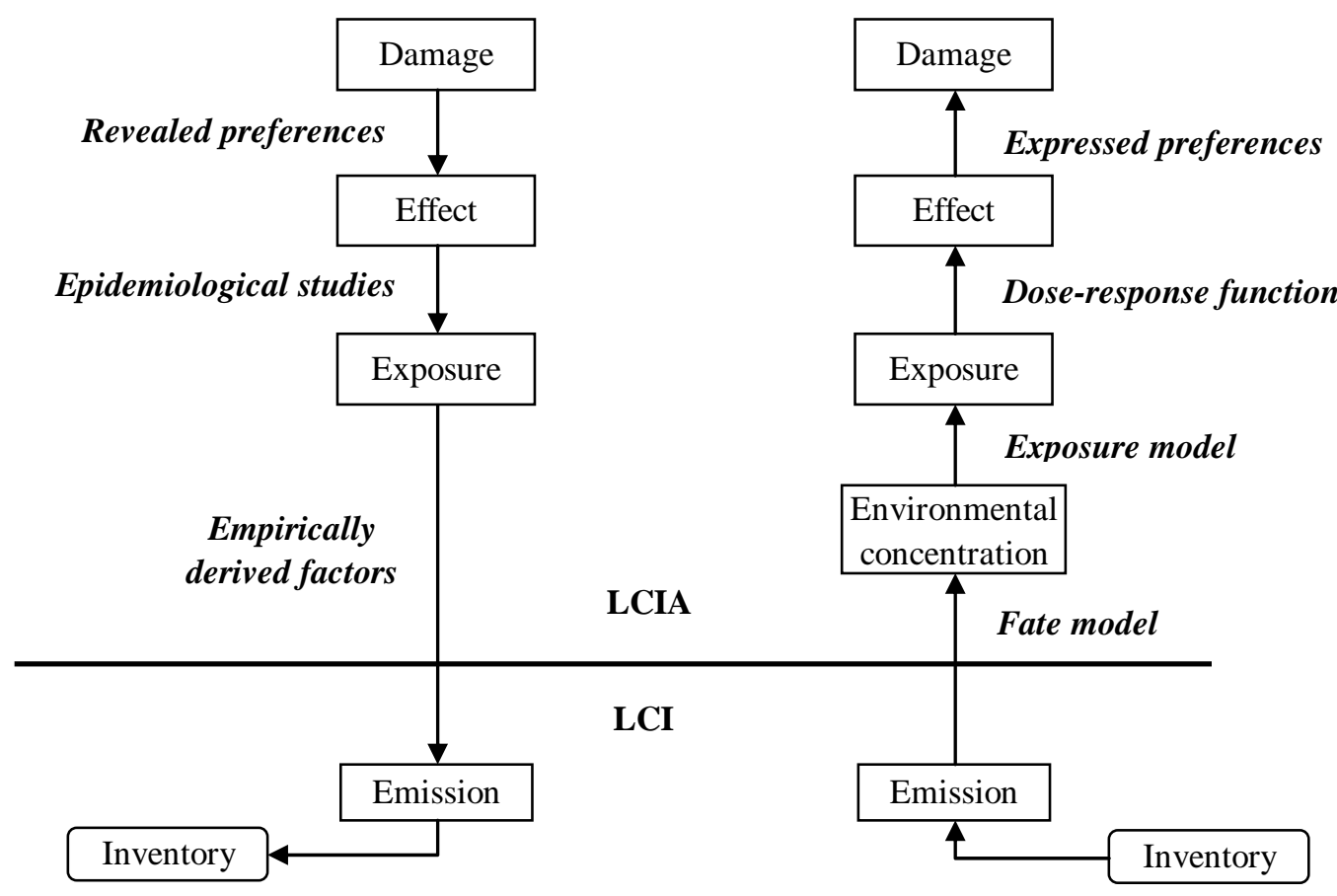

TOP-DOWN PROCEDURE

BOTTOM-UP PROCEDURE

Figure 4-1 Illustration of the approaches for characterization modeling for emission-related impact categories

[Adapted from Hofstetter 1998] 
Hofstetter [1998] states that it is necessary to combine the bottom up and top down approaches two procedures to use all available information to increase the comprehensiveness of the characterization model, and to use the descriptive models as a reliability check for the prescriptive models. A combined approach is also useful in filling any data gaps that may exist in the links.

A generic characterization factor calculated based on the above procedure is expressed mathematically as

$$
\mathrm{CF}(\mathrm{s}, \mathrm{i}, \mathrm{t})=\sum_{\mathrm{j}} \frac{\operatorname{effect}(\mathrm{s}, \mathrm{j}, \mathrm{t})}{\operatorname{emissions}(\mathrm{s}, \mathrm{i})}=\sum_{\mathrm{j}}\left(\frac{\operatorname{fate}(\mathrm{s}, \mathrm{j}, \mathrm{t})}{\operatorname{emissions}(\mathrm{s}, \mathrm{i})}\right) \cdot\left(\frac{\operatorname{exposure}(\mathrm{s}, \mathrm{j}, \mathrm{t})}{\operatorname{fate}(\mathrm{s}, \mathrm{j}, \mathrm{t})}\right) \cdot\left(\frac{\operatorname{effect}(\mathrm{s}, \mathrm{j}, \mathrm{t})}{\operatorname{exposure}(\mathrm{s}, \mathrm{j}, \mathrm{t})}\right)
$$

where, $s$ denotes the pollutant, $i$ is the location of emission, $j$ is the related location of exposure of the receptor, and $t$ is the time period during which the effect is taken into account [Pennington et al 2004].

\subsection{SOPHISTICATION IN CHARACTERIZATION MODELING}

LCIA sophistication corresponds to the level of detail used in the characterization models [United Nations Environment Program 2003]. Different life cycle impact assessment methods use characterization models of different levels of sophistication. Thus different LCIA methods produce different results for the same LCI. This has a direct effect on the reliability of the results of an LCA in decision making. LCA has also faced criticism because of the multiple methodologies of LCIA [Seppala 2003]. Therefore, higher LCIA sophistication provides more scientific credibility to decisions based on LCA [UNEP 2003]. Poor quality of inventory data, the depth of scientific knowledge, availability of appropriate software, and budget constraints are some of the practical considerations that has led to simplification of LCIA methodology. Simplifications include: (i) reduction in spatial and temporal discrimination (or ignoring these

dimensions altogether), (ii) ignoring fate, exposure and effect, (iii) assuming linear doseresponse curves, and/or (iv) ignoring background concentrations of pollutants [UNEP 2003]. 
Sophistication in LCIA can be divided into two broad dimensions: (i) the depth and breadth of environmental mechanism modeling, and (ii) the spatial and temporal resolution of the characterization factors [Potting and Hauschild 2005]. Sophistication in LCIA increases, and uncertainty in interpretation of results decreases, with characterization modeling further down along these directions. This is illustrated in Figure 4-2. The two dimensions of sophistication are discussed below.

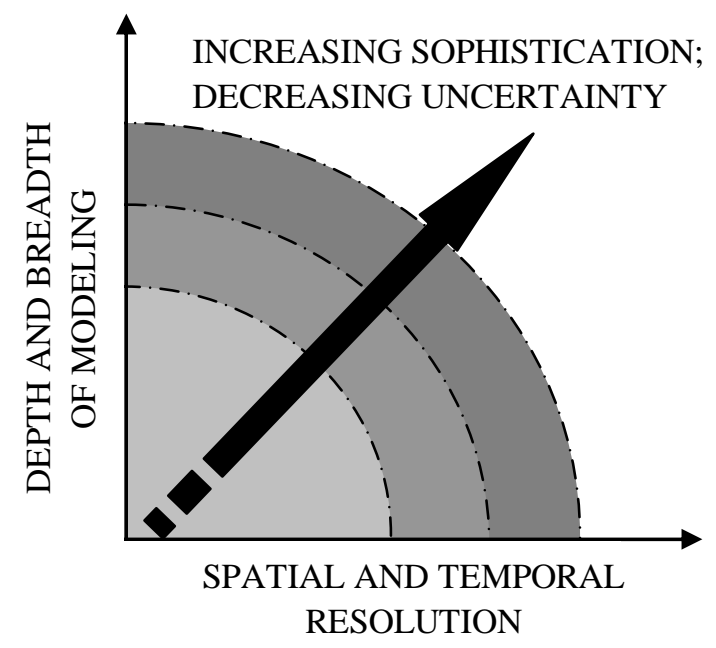

Figure 4-2 Illustration of dimensions of sophistication in LCIA

\subsubsection{Depth and breadth of mechanism modeling}

Depth of mechanism refers to the point along the environmental mechanism where the impact is calculated. Impacts are calculated at either the category midpoints or the category endpoint in the environmental mechanism. Breadth of the mechanism modeling is number of possible impact mechanisms and midpoints and endpoints considered for a particular impact category. For instance, the depth and breadth of the model for the acidification impact category considered in the Tool for the Reduction and Assessment of Chemical and other environmental Impacts (TRACI) is illustrated in Figure 4-3. The depth of the mechanism, as shown, starts at the emission of the pollutants $\left(\mathrm{SO}_{2}\right.$ and $\left.\mathrm{NO}_{\mathrm{x}}\right)$ to the atmosphere and ends at the effects of 
acidification on the ecosystem, which are the category endpoints. Although this impact pathway is illustrated in TRACI, it does not calculate impacts at the endpoint. The impacts are calculated at the midpoint, which is the deposition of the sulfate and nitrate anions for the acidification impact category.

In Figure 4-3, the breadth of the impact category is represented by the three impact pathways that follow the deposition of the anions. A fourth impact pathway that could also be considered is the effect of acid rain on metals, monuments, and historical artifacts [Norris et al 2003] within the man-made materials area of protection. The breadth of the modeling is dependent on the areas of protection that are considered as important in the impact assessment method, and is also limited by the understanding and quantifiability of the impact pathways. A highly sophisticated LCIA would incorporate all known impact pathways in all important areas of protection, so as to provide a comprehensive assessment of the impacts.

The depth in the impact pathway at which the impacts are calculated has been extensively discussed in two earlier workshops [UNEP 2003]. The key difference between calculating impacts at the midpoint and at the endpoint is in the determination of the environmental and societal relevance of the impacts. The indicator at the endpoint represents damage to a well recognized societal value. Thus it is more comprehensible to the decision maker, who may not be a scientist. Endpoint impact calculation also provides a structured approach in determining the relative relevance of different impact categories. However, the argument against using endpoint impact calculation is the limited availability of reliable data and robust models for all pathways beyond the midpoint. This leads to unsubstantiated assumptions and value choices, particularly at the damage assessment step. These values may not be shared by all users. Missing links and data, also, increase the uncertainty of the results. It gives a false sense of accuracy and improvement over the midpoint results [UNEP 2003]. Therefore, the depth and breadth of the characterization model are interrelated. Typically, as the modeling extends beyond the midpoint, several impact pathways can be considered to reach to the endpoint. If the breadth of the model does not include all these pathways, due to unavailability of data and models or due to other value choices, the validity of increasing the depth is questionable. The conclusion of the workshops [UNEP 2003] was that both the midpoint and endpoint approaches have their advantages and disadvantages. The best approach is to use a consistent framework to calculate both the midpoint and the endpoint impacts, and communicate both the results to the decision makers. 


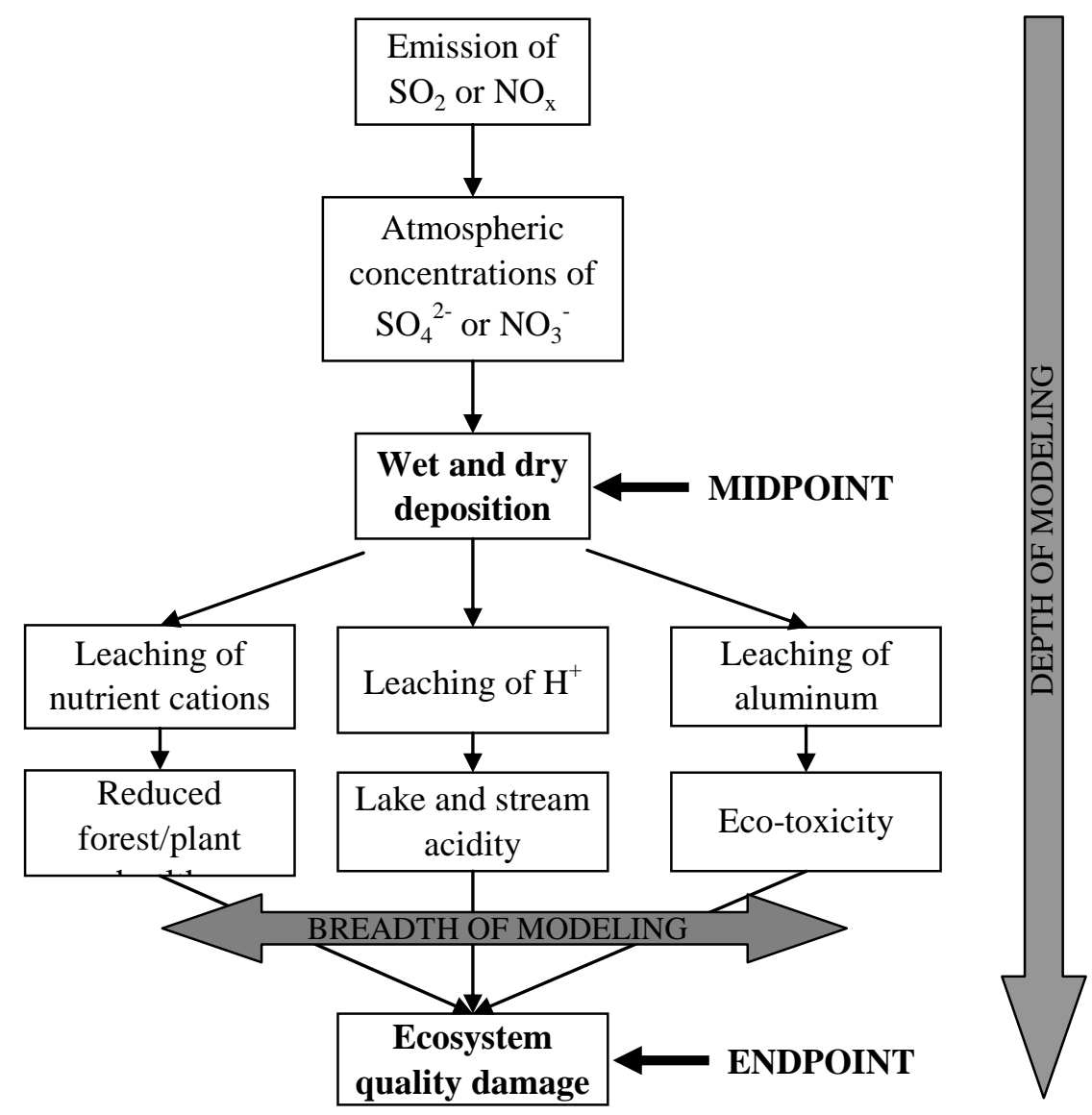

Figure 4-3 Illustration of the depth and breadth of mechanism modeling for the acidification impact category in TRACI

[Bare et al 2003] 


\subsubsection{Spatial and temporal resolution of the characterization factors}

The emissions during the life cycle of a product or a service occur at various locations and at different points in time. The location and time of emissions strongly affect the fate of the pollutants and also their impacts on the receptors. Even though spatial differentiation has been considered important since the early days of LCIA development, it has been neglected in most of the LCIA methods. Neglecting these variations can affect the validity of the decisions based on the LCA results [Potting and Hauschild 2006]. Some impact categories, like climate change and stratospheric ozone depletion, have a global effect, while others, like photochemical oxidant formation and acidification, have a regional effect. Spatial differentiation is relevant for such regional impact categories. Figure 4-4 illustrates the spatial scale of impacts for the common impact categories.

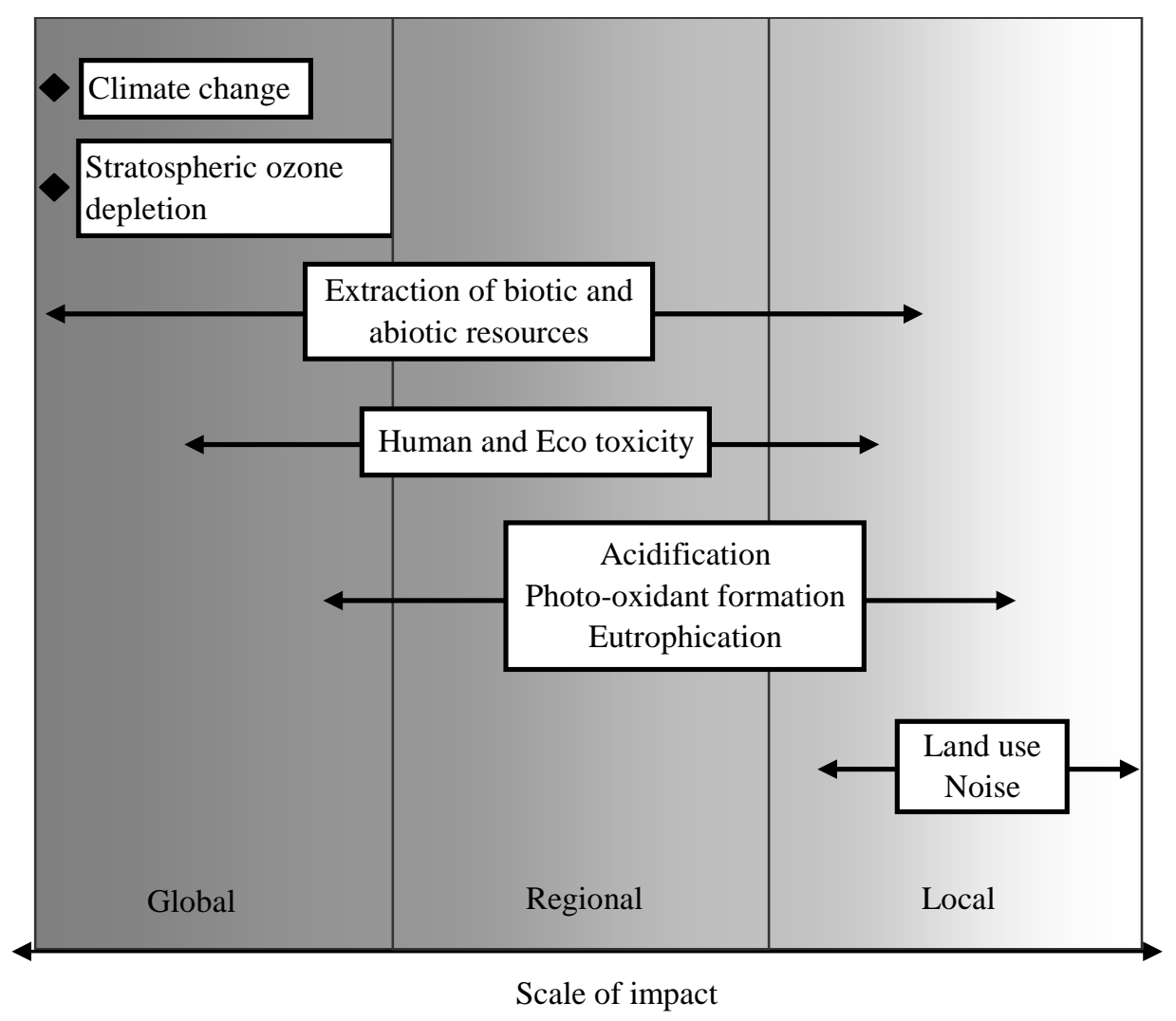

Figure 4-4 Spatial scales of impacts of different impact categories 
Potting and Hauschild [2006] identified three levels of sophistication in spatial resolution of characterization factors. A site-generic characterization factor does not consider any spatial differentiation in sources or the receiving environment. A site-dependent factor spatially differentiates the sources and their subsequent receiving environments. Source categories are defined at a spatial scale of countries or states within countries (50-500 km). Receiving environments are defined at a higher resolution (typically less than $150 \mathrm{~km}$ ). Thus, site dependent characterization factors include variation within and between the receiving environments with respect to each source category. They are also informed by background concentrations of pollutants in the receiving environments, and any thresholds in the concentration-effect relationship. A site-specific characterization factor is very detailed spatially. Only a few sources are considered, and the effects are modeled on receiving environments that are very close to the sources. This level of sophistication does not, usually, add significantly to the accuracy of the results. Besides, the level of spatial resolution typically available in the LCI does not support site specific assessment. The site-dependent level is, thus, the relevant level of sophistication for LCIA [Potting and Hauschild 2006]. It has been argued that spatial differentiation in LCA adds an additional burden on the LCI data collection without adding significantly to the decision-making process. Bellekom [2006] demonstrated that it is feasible and relatively easy to determine the LCI at the site-dependent level. Potting and Hauschild [2005] have demonstrated that the differences in characterization factors between emission sites are larger than the differences in characterization factors between pollutants in the acidification and photochemical oxidant formation impact categories.

Traditional LCIA methods integrate the emissions over the entire life cycle of the product or process. Therefore, all emissions are treated as a pulse rather than a flux distributed over a period of time. The debate on temporal differentiation within LCIA has focused on two separate topics. The first topic looks at the time horizon of impacts and the treatment of present and future emissions. For example, global warming potentials for the climate change impact category can be calculated for 20,50,100, or 500 year periods. Choosing any one period cuts off all future impacts that may potentially occur. The other method proposed is that the future impacts may be discounted as they are less certain and can possibly be avoided [Udo de Haes et al 1999, Hellweg et al 2003]. The second topic of debate is the importance of the calendar time of the emissions. Potting and Hauschild [2005] have demonstrated the variation in the characterization factors for 
different years. This variation is due to the difference in the level of economic activity, and the level of emission control measures which affect the environmental concentration of the pollutants and the background exposure at the receptor sites.

However, the seasonal variation in the impacts has been neglected in LCIA. Important environmental phenomena, like photochemical oxidant formation, acidification, and eutrophication, show large seasonal variability due to the processes responsible for fate and transport of the pollutants, and also due to the seasonal changes in the characteristics of the receiving environment. The level of seasonal variability in photochemical oxidant formation has already been discussed in Chapter 2.0.

The aim of this study is to increase the spatial and temporal resolution of the characterization factors for photochemical oxidant formation, and extend the impact calculation to the category endpoints.

\subsection{CHARACTERIZATION OF PHOTO-OXIDANT FORMATION}

The photochemical oxidant formation impact category is addressed by all the widely accepted LCIA methods. However, there is no standard characterization model used by all these methods. The characterization models of most of these LCIA methods are identical to either the CML2001 method [Guinée et al 2002] or the Eco-indicator99 method [Goedkoop and Spriensma 2001]. The models used by the TRACI method [Bare et al 2003] and EDIP2003 method [Potting and Hauschild 2005] are considerably different from the other two methods. These four common methods for impact characterization of photochemical oxidants are discussed in the following text.

\subsubsection{CML2001}

Guinée et al [2002] recommends the use of the Photochemical Ozone Creation Potential (POCP) metric as the characterization factor for $\mathrm{NO}_{\mathrm{x}}$ and VOC emissions. POCP is defined as the increase in the amount of ozone formed due to a unit increase in the emission of a particular 
precursor. The POCP values for $\mathrm{NO}_{\mathrm{x}}$ and 95 VOC under Western European conditions have been calculated by Derwent [1996]. The POCP value is expressed relative to the POCP value of ethylene which is fixed at 100.

A Lagrangian photochemical model was used to simulate the emissions, chemical reactions, transport, and deposition of ozone, PAN, and hydrogen peroxide and their precursors. The air parcel which is followed continuously has a lateral dimension of $10 \mathrm{~km} \mathrm{x} 10 \mathrm{~km}$ and a height extending up to the top of the boundary layer. The emission rates were obtained from several European emission inventories. The VOC emissions are speciated into 95 individual compounds and methane based on nine source categories. The chemical mechanism comprises of 48 chemical reactions of inorganic compounds, 85 photochemical reactions, and 771 oxidation reactions involving hydroxyl ions and ozone. The initial concentrations of some of the species were set to realistic tropospheric baseline levels.

The simulation consisted of a base case scenario and several alternate scenarios in which the emission of one precursor was increased marginally. In the base case, the emission rates represented the worst case scenario in North-Western Europe. The meteorology condition used in the simulation is a highly idealized anticyclonic situation leading to a westerly wind flow. This westerly trajectory path is usually associated with ozone episodes over the Netherlands and United Kingdom. The air parcels took five days to traverse continental Europe and reach the British Isles. The highest ozone concentration calculated was $0.118 \mathrm{ppm}$ at the border of Wales and England. Most of the trajectories at the end of the simulation had ozone concentrations higher than 0.075 ppm [Derwent et al 1996]. These concentrations show that the emissions and meteorology scenarios considered represent conditions prevalent during high ozone episodes.

In each alternate case, the emission rate of the one precursor was marginally increased uniformly across the entire domain, and the simulation was repeated. The base case trajectory in which the maximum ozone concentration was calculated was monitored for changes in the ozone concentration with respect to the base case. The POCP value of a precursor is calculated as the ratio of the change in the average ozone concentration for the 5-day trajectory due to the addition of the precursor, to the change due to addition of ethylene, multiplied by hundred. Mathematically, the POCP is expressed as, 


$$
\operatorname{POCP}_{\mathrm{i}}=\frac{\frac{\left.\mathrm{O}_{3} \text { (due to addition of VOC(i) }\right)-\mathrm{O}_{3} \text { (basecase) }}{\text { additional emission of VOC(i) }}}{\frac{\mathrm{O}_{3} \text { (due to addition of ethylene) }-\mathrm{O}_{3} \text { (basecase) }}{\text { additional emission of ethylene }}}
$$

Except benzaldehyde, the POCP values of all the VOC studied are positive, that is, they contribute to ozone formation. The POCP value of nitric oxide (NO) is found to be negative, which is due to the high $\mathrm{NO}_{\mathrm{x}}$ conditions employed in the base case simulation. The photooxidant formation impact is thus calculated as,

$$
\text { Photo }- \text { oxidant formation impact }=\sum_{\mathrm{i}} \mathrm{POCP}_{\mathrm{i}} \times \mathrm{m}_{\mathrm{i}}
$$

where $m_{i}$ is the mass of the precursor emitted during the life cycle of the product and $P O C P_{i}$ is the Photochemical Ozone Creation Potential of the emitted substance [Guniée et al 2002].

POCP values for 101 VOC have been calculated under highly polluted North American urban conditions [Derwent et al 2001]. The air parcel is followed for a period of one day, unlike the 5-day period for the European model. Thus the North American model does not represent precursor emissions, and ozone formation and destruction at a regional scale, but at the localized urban scale. Due to the difference in the trajectory period, the slow reacting VOC have lower POCP values in the North American model. The POCP values were calculated from the difference in the peak ozone formation instead of the average ozone concentration in the European model.

In the EDIP1997 method, Hauschild and Wenzel [1998] have used the POCP values as characterization factors. However, they recommend the use of two different sets of POCP values, one for high- $\mathrm{NO}_{\mathrm{x}}$ conditions and the other for low- $\mathrm{NO}_{\mathrm{x}}$ conditions. High- $\mathrm{NO}_{\mathrm{x}}$ condition POCP values have been obtained from Derwent and Jenkin [1991], and the low- $\mathrm{NO}_{\mathrm{x}}$ condition values from Andersson-Sköld et al [1992]. A $\mathrm{NO}_{\mathrm{x}}$ concentration of $0.01 \mathrm{ppm}$ is chosen as the margin between low and high concentration conditions. The low- $\mathrm{NO}_{\mathrm{x}}$ characterization factors are applicable for emissions in Scandinavian countries, and less polluted rural areas in the rest of Europe. 
Since the POCP is a measure of the amount of the additional ozone formed, it describes the impact at the category midpoint. The CML2001 method does not consider any spatial or temporal differentiation. The EDIP1997 method spatially differentiates between emissions in low- $\mathrm{NO}_{\mathrm{x}}$ and high- $\mathrm{NO}_{\mathrm{x}}$ regions.

\subsubsection{Eco-indicator 99}

The photochemical oxidant formation impact in the Eco-indicator 99 method [Goedkoop and Spriensma 2001] is based on the method developed by Hofstetter [1998]. The Eco-indicator 99 method describes the impact at the category endpoints. The endpoints considered for the effect of photo-oxidant formation are: asthmatic attacks, minor restricted activity day (MRAD), respiratory hospital admissions, symptom days, emergency room visits for asthma, and acute mortality. The category indicator for human health impacts is Disability Adjusted Life Years (DALYs). DALYs express the years of life lost due to premature mortality and the years lived with disability of specified severity and duration. One DALY is one lost year of healthy life [Murray and Lopez 1996]. The characterization factor is the product of the fate factor and the effect factor. The impact for a compound is thus expressed as,

$$
\text { Impact }_{\mathrm{i}}=\sum_{\mathrm{i}}\left[\mathrm{F}_{\mathrm{i}} \cdot \mathrm{E}_{\mathrm{im}} \cdot\left(Y L L_{m}+Y L D_{m}\right)\right] \times \mathrm{m}_{\mathrm{i}}
$$

where, $i$ is the pollutant causing photo-oxidant formation, $F$ is the fate factor, $E$ is the effect factor, $m$ is the endpoint, $Y L L$ is Years Life Lost, YLD is Years Lived Disabled, $m$ is the mass of the pollutant emitted [Hofstetter 1998].

The fate factor is the measure of a pollutant's contribution to tropospheric ozone formation. The fate factors are based on the results of the simulations of the EMEP MSC-W ozone model [http://www.emep.int] reported by Simpson [1993]. The EMEP is a Lagrangian model which calculates ozone concentrations every six hours over entire Europe at a 150x150 $\mathrm{km}^{2}$ grid. The air parcels were followed along 96-h trajectories. The meteorology conditions were obtained from a numerical weather prediction model. The model chemistry describes 45 species through 100 chemical reactions. Initial and top boundary conditions were obtained from observations or extrapolated from the model calculations. The anthropogenic emissions of $\mathrm{SO}_{\mathrm{x}}$, 
$\mathrm{NO}_{\mathrm{x}}$, and VOC were from officially submitted data or estimated by the modeling team. Biogenic emissions of isoprene from forests are calculated at each time step using the model's surface temperature fields.

Base case simulations were performed for the summers (April to September) of 1985 and 1989. Three additional emission control scenarios were simulated for the 1989 summer. The scenarios considered were: $50 \%$ reductions in $\mathrm{NO}_{\mathrm{x}}$ emissions, $50 \%$ reductions in anthropogenic VOC emissions, and 50\% $\mathrm{NO}_{\mathrm{x}}$ and anthropogenic VOC reduction. The first and the second scenarios resulted in a 7\% (0.00392 ppm) and 6\% (0.00336 ppm) decrease in the domain wide average ozone concentration, respectively, from the $0.056 \mathrm{ppm}$ baseline concentration. The fate factor for $\mathrm{NO}_{\mathrm{x}}$ and VOC is the reduction in the mass of ozone formed at the surface (1m layer) per unit decrease in precursor emission. The POCP values are used to derive the fate factors for individual VOC based on an average POCP value for the entire VOC emission in Europe. Mathematically, the fate factors for $\mathrm{NO}_{\mathrm{x}}$ and VOC are expressed by Hostetter [1998] as,

$$
\begin{gathered}
\mathrm{F}_{\mathrm{NOx} / \mathrm{VOC}}=\frac{\text { decrease in ozone mass at the surface }}{\text { reduction in emission of precursor }} \\
\mathrm{F}_{\mathrm{i}}=\frac{\mathrm{POCP}_{\mathrm{i}}}{\mathrm{POCP}_{\text {avg }}} \times \mathrm{F}_{\mathrm{VOC}}
\end{gathered}
$$

where $F_{i}$ is the fate factor for a VOC denoted by $i$ and POCP $_{\text {avg }}$ is the average POCP for the entire VOC emission inventory.

The effect factors are calculated from several epidemiological studies which describe the relationship between the various endpoints and an increase in ambient ozone concentration. For example, a study found that an increase of $1 \mu \mathrm{g} / \mathrm{m}^{3}$ was associated with an increase of 4.29 asthma attack cases per 1000 persons per year. This relation is known as a ConcentrationResponse (C-R) function. Similar C-R functions for mortality, MRAD, Respiratory hospital admissions, symptom days, and ERV for asthma were used by Hofstetter [1998]. The C-R function for mortality is expressed as the percent increase in short term non-accidental mortality rate per unit increase in ozone concentration. Thus, the baseline non-accidental mortality rates are required to calculate the increase due to ozone. 
The duration of the disability and the disability weight (severity of the disability) for each respiratory disability are required to calculate the YLD for morbidity endpoints. For example, for ERV for asthma, the disability duration is 3 days and the disability weight is considered as 0.1 [Hofstetter 1998]. YLL is the average life expectancy at the time of death. YLL and YLD values also depend upon whether the discounting of the future and age-weighting is considered [Hofstetter 1998].

Hofstetter [1998] notes that the impact factors for photo-oxidant formation do not differ for the three cultural perspectives as the endpoints with limited evidence were negligible in the total impact. The three most significant endpoints for ozone impacts, in order of their contributions, were: acute mortality, asthma attacks, and symptom days [Hofstetter 1998].

The Eco-indicator 99 method does not employ any spatial or temporal differentiation. The characterization factors are calculated for average European conditions and may not represent the impact of photo-oxidant formation in different countries or at the global level. The characterization factors are calculated at several endpoints of ozone exposures. The DALYs index characterizes the human health area of protection at a fundamental level which can be easily comprehended by both scientists and policy makers.

\subsubsection{TRACI}

The Tool for Reduction and Assessment of Chemical and other environmental Impacts (TRACI) impact assessment method has been specifically developed for North American conditions by the USEPA. TRACI characterizes impacts at the midpoints to avoid the uncertainties associated with effect modeling. The characterization factors for most of the local and regional impacts have been calculated at the state level [Bare et al 2003]. Norris [2003] describes the detailed methodology used for the calculation of the characterization factors for photochemical oxidants.

The amount of ozone formed due to emission of the precursors is the quantity which describes the impact. The category indicator for the impact is expressed in terms of grams of $\mathrm{NO}_{\mathrm{x}}$ equivalents. This is based on the observation by Kasibhatla et al [1998] that a near linear relationship exists between the increase in the regional accumulated ozone concentration and the unit increase in the emission of $\mathrm{NO}_{\mathrm{x}}$. This observation was based on the measured ozone concentration across a large domain during four summer ozone episodes characterized by 
stagnant meteorological conditions. The effect of transport of ozone and $\mathrm{NO}_{\mathrm{x}}$ from outside the domain has not been considered due to the difficulties in quantifying it.

The state level characterization factors are calculated using state level $\mathrm{NO}_{\mathrm{x}}$ sourcereceptor matrices. They describe the relation between the emissions of $\mathrm{NO}_{\mathrm{x}}$ in a particular state to the changes in ambient $\mathrm{NO}_{\mathrm{x}}$ concentration in each receiving region across North America. The change in concentration in each of the receiving state is scaled based on the population in the state to derive the aggregated impact. Thus, the impact is expressed as,

$$
\begin{aligned}
& \text { Photo - oxidant impact }=\sum_{\mathrm{i}} \mathrm{CF}_{\mathrm{i}} \times \mathrm{m}_{\text {NOx eq,i }} \\
& \qquad \mathrm{CF}_{\mathrm{i}}=\sum_{\mathrm{j}} \mathrm{SR}_{\mathrm{ji}} \times \mathrm{s}_{\mathrm{j}}
\end{aligned}
$$

where, $C F_{i}$ is the state-level characterization factor, $m_{N O x}$ eq,i, is the mass of precursors emitted from each state $i$ in mass of $\mathrm{NO}_{\mathrm{x}}$ equivalents, $S R_{j i}$ is the source-receptor coefficient for the receptor state $j$, and $s_{j}$ is a scaling factor based on the population of state $j$.

The source-receptor matrix is calculated from multiple simulations of the Advanced Statistical Trajectory Regional Air Pollution (ASTRAP) model [Shannon 1981] which was developed to support the National Acid Precipitation Assessment Program (NAPAP). ASTRAP is a highly parameterized model that simulates the emission, horizontal transport, vertical diffusion, chemical transformation of $\mathrm{NO}_{\mathrm{x}}$ to nitrate, and the wet and dry deposition of the pollutants. ASTRAP consists of three subprograms. The first subprogram is a one-dimensional (vertical) numerical module treating vertical diffusion, chemical transformation, and dry deposition. The second program calculates the horizontal dispersion and wet deposition of the pollutants at a 6-h interval in a 5-day trajectory. The third subprogram integrates the emission inventory with the vertical and horizontal dispersion statistics to calculate the ambient concentration, and deposited mass of the pollutants. Since the ASTRAP model was developed for simulating acid precipitation, it does not model the photochemical reactions underlying the formation of photo-oxidants.

The $\mathrm{NO}_{\mathrm{x}}$-equivalency factor for VOC is calculated based on empirical and modeling studies. It is the ratio of the change in regional ozone concentration due to a unit change in $\mathrm{NO}_{\mathrm{x}}$ concentration to the change in ozone concentration due to a unit change in VOC concentration. It 
is a measure of the relative efficiency of ozone formation. This ratio has been considered to be 2 in TRACI. It is assumed that the transport and deposition characteristics for $\mathrm{NO}_{\mathrm{x}}$ and VOC are identical and can be described by the source-receptor matrix generated for $\mathrm{NO}_{\mathrm{x}}$ using ASTRAP. The Maximum Incremental Reactivity (MIR) scale of Carter [1998] is used to differentiate between the relative influences of VOC species on ozone formation. Thus, the $\mathrm{NO}_{\mathrm{x}}$ equivalents for VOC is calculated as,

$$
\mathrm{m}_{\mathrm{NOx} \text { eq, }}=2 \times \frac{\mathrm{MIR}_{\mathrm{c}}}{\mathrm{MIR}_{\mathrm{vOC}}} \times \mathrm{m}_{\mathrm{c}}
$$

where, 2 is the ratio of the efficiency of ozone formation, $M I R_{c}$ is the Maximum Incremental Reactivity of VOC $c, M I R_{V O C}$ is the reactivity of a base mixture of VOC, and $m_{c}$ is the mass of the VOC emitted.

The methodology for calculating the MIR values is described in Carter [1994]. The Empirical Kinetic Modeling Approach (EKMA) developed by USEPA is used in the calculation of MIR. The EKMA is a single cell model that simulates the formation of ozone from its precursors for one day ozone episodes. The single cell model represents dynamic injection of pollutants, time-varying changes in inversion height with entrainment of pollutants aloft, and time-varying chemical transformation based on sunlight, temperature and humidity [Carter 1994]. The inputs for the base case scenario are derived from the conditions during specific ozone episodes in 39 US cities. The MIR scenario is derived by adjusting the $\mathrm{NO}_{\mathrm{x}}$ concentration in the base case scenario such that a small addition of the base VOC mixture will have the maximum ozone formation. The incremental reactivity is the increase in the mass of ozone formed for a unit additional mass of each VOC species to the modified $\mathrm{NO}_{\mathrm{x}}$ scenario. Hence, MIR represents the potential of ozone formation in high $\mathrm{NO}_{\mathrm{x}}$ concentrations that are typically seen in highly polluted urban conditions.

The MIR values calculated by Carter [1994] are based on the SAPRC90 chemical mechanism. The updated MIR values that are used in TRACI were calculated by Carter [1998] using an updated SAPRC97 chemical mechanism. The most recent values were calculated using the SAPRC07 mechanism [Carter 2007]. Derwent et al [2001] have reported a high degree of correlation between the POCP values for North America and the MIR values. This is an expected 
outcome considering that the POCP values were also calculated for a single day ozone episode using the same initial conditions as those used by Carter [1994]. In a recent reactivity assessment by the North American Research Strategy for Tropospheric Ozone (NARSTO) a three dimensional Eulerian model was used to calculate the relative reactivities for VOC. The relative reactivities calculated for the modeled regional ozone episode were found to be close to the MIR scale developed using the EKMA model [Carter et al 2003]. However, ozone episodes are typically characterized by high accumulation of the precursors similar to the initial conditions used in the EKMA model.

The characterization factors calculated in TRACI are spatially differentiated at the state level. Aggregated factors were developed for the US census regions, East and West US, and for the entire country based on the probability distribution of the emissions across the country. An important limitation of the TRACI method is the use of two disconnected models (ASTRAP and MIR) to represent the transport and chemical transformation of the precursors, respectively. Secondly, the relative efficiency of ozone formation of $\mathrm{NO}_{\mathrm{x}}$ to that of VOC is based on a limited number of studies and, its spatial variability is also not considered. MIR values are based on highly polluted urban conditions, and do not represent the average conditions prevalent throughout the country. An integrated approach, which is now available with the development of the PAQMS, can increase the sophistication of the ozone fate modeling by addressing all these limitations.

\subsubsection{EDIP2003}

The EDIP2003 impact assessment method was developed using a consensus process by the Danish Environmental Protection Agency. It is an updated version of the EDIP97 method. The characterization factors for all non-global impact categories in EDIP2003 are resolved spatially at the country level for the entire Europe. For the photochemical oxidant formation, acidification, and terrestrial eutrophication impact categories, multiple sets of temporally differentiated characterization factors have been calculated [Hauschild and Potting 2005]. EDIP2003 recognizes that the contribution of a single source to exposure of the receptors is marginal in the multiple source - multiple receptors perspective of environmental problems. Thus the temporal variability of a single source cancels out against the background exposures of the receptors. Thus 
the time dimension of the inventory has been argued to be less important. However, the calendar time in which the event occurs has been recognized as important. Economic activity varies with calendar time thus affecting the emissions and the total environmental load. Therefore, the characterization factors for photochemical oxidant formation have been calculated for 1990, 1995, and 2010 based on the past emission inventories and the emission reduction plans for the future years [Potting and Hauschild 2005].

The Regional Air Pollution Information and Simulation (RAINS) model has been used to generate the photochemical oxidant formation characterization factors for each country. The RAINS model relates the emission of precursors in any country to their impact on the receptor areas. In other words, it gives the source-receptor relationship between the emission of precursors and the concentration of ozone in the boundary layer at the receptor site. It is a reduced-form model derived from multiple simulations of the Lagrangian EMEP model using statistical methods [Potting and Hauschild 2005]. The mathematical expression of the model is as follows:

$$
\left[O_{3}\right]_{j}=k_{j}+\sum_{k=1}^{M}\left(a_{i j} v_{i}+b_{i j} n_{i}+c_{i j} n_{i}^{2}\right)+\alpha_{j} \overline{e n}_{j}^{2}+\overline{e n}_{j} \sum_{k=1}^{M} d_{i j} v_{i}
$$

where,

$\left.O_{3}\right]_{j}$ is the six month (April to September) average afternoon ozone concentration experienced at receptor $j$

$n_{i} \quad$ is the annual anthropogenic $\mathrm{NO}_{\mathrm{x}}$ emission from country $i$

$v_{i} \quad$ is the annual anthropogenic VOC emission from country $i$

$\overline{e n}_{j} \quad$ is the "effective" emission of $\mathrm{NO}_{\mathrm{x}}$ experienced at receptor $j$ over the period. It is the sum of the emissions, which have been adjusted for tropospheric exchange, along the trajectory reaching $j$

$k_{j} \quad$ represents the effects of background concentration of ozone and precursors, and natural VOC emissions

$M \quad$ is the number of countries whose emissions have the maximum contribution to ozone formation at the receptor. $M$ is set to 11

As noted previously, The EMEP model is a single layer model which calculates the concentration of ozone every six hours at the nodes of a $150 \mathrm{~km}$ x $150 \mathrm{~km}$ grid covering entire Europe. Air parcels are followed for a period of 96 hours. The meteorological conditions are 
obtained from the Norwegian Numerical Weather Prediction model. The model has been run for the emission scenarios of 1990, 1995, and 2010. Biogenic emissions of $\mathrm{NO}_{\mathrm{x}}$ and VOC are calculated separately during the model runs. A detailed description of the derivation of the simplified model has been described in Heyes et al [1996, 1997]. 242 emission scenario cases for the EMEP model were used to derive this simplified relationship. Current emission scenario and $70 \%$ reduction in anthropogenic $\mathrm{NO}_{\mathrm{x}}$ and VOC emissions represented the two base cases. Six cases with various emission reductions within the $70 \%$ range were considered for each of the 40 emitter countries. The coefficients of the simplified model were calculated using linear regression. To reduce the effect of inter-annual meteorological variability, these coefficients were calculated for five years (1989, 90, 92-94).

The exposure of both, humans and vegetation, to tropospheric ozone has been considered in the EDIP2003 method. The AOT40 index has been used to calculate the vegetation exposure. AOT40 is the cumulative exceedance of the hourly daytime ozone concentration above the 0.040 ppm (40 ppb) threshold level for the growing season. The growing season for crops is three months and for forest trees is six months. The critical levels of AOT40 have been set by the UNECE Convention on Long-range Transboundary Air Pollution at 3 ppm-hours for crops and 10 ppm-hours for trees. Since the critical level for crop exposure is stricter than that for trees, a uniform critical level equal to that of the former has been used in RAINS. The exceedance of the AOT40 index over the 3ppm-hour level is the measure of the impact on vegetation. Since no thresholds for chronic exposure of humans to ozone have been identified, an index equivalent to AOT40, AOT60, has been used in RAINS. The critical level is set at zero ppm-hours and the impact is measured in terms of the exceedance above this level.

The EDIP2003 method follows an 'only above threshold' approach to impact assessment rather than a 'less is better' approach [Potting and Hauschild 2005]. In this approach, only the entities (humans, vegetation, etc.) that experience exposures higher than the critical levels are valued. Besides, these entities are not differentiated based on the severity of the exceedance. Due to the discontinuous nature of the threshold exceedance impact function, entities that are exposed only slightly less than the critical level and are at the maximum risk are completely neglected. A 'less is better' approach would value all entities equally irrespective of their background exposures. Thus reducing the exposure for an entity exposed high above the threshold would not have any additional preference over reducing the exposure for an entity exposed to very low 
levels. Currently, there is no general consensus in the LCIA community on which one of the approaches is better.

The functional form of the AOT40 or AOT60 index is similar to the expression for average ozone concentration. The characterization factors for human and vegetation impacts for each emitter country are defined as the marginal increase in exposure due to the emission of a unit mass of the precursor. They are obtained from partial differentiation of these AOT60 and AOT40 expressions. Therefore they are expressed as

$$
\begin{gathered}
\mathrm{HE}=\sum_{\mathrm{j}=1}^{\mathrm{N}}\left(\mathrm{AOT}_{\mathrm{j}} \times \mathrm{P}_{\mathrm{j}}\right) \text {, and } \\
\mathrm{VE}=\sum_{\mathrm{j}=1}^{\mathrm{N}}\left[\left(\mathrm{AOT}_{4} 0_{\mathrm{j}}-\mathrm{C}\right) \times u\left(\mathrm{AOT}_{\mathrm{j}}-\mathrm{C}\right) \times \mathrm{LA}_{\mathrm{j}}\right] \\
\mathrm{CF}_{\mathrm{NO}, \mathrm{HE}}(\mathrm{i})=\frac{\partial \mathrm{HE}}{\partial \mathrm{n}_{\mathrm{i}}} \text { and } \mathrm{CF}_{\mathrm{VOC}, \mathrm{HE}}(\mathrm{i})=\frac{\partial \mathrm{HE}}{\partial \mathrm{v}_{\mathrm{i}}} \\
\mathrm{CF}_{\mathrm{NO}, \mathrm{VE}}(\mathrm{i})=\frac{\partial \mathrm{VE}}{\partial \mathrm{n}_{\mathrm{i}}} \text { and } \mathrm{CF}_{\mathrm{VOC}, \mathrm{VE}}(\mathrm{i})=\frac{\partial \mathrm{VE}}{\partial \mathrm{v}_{\mathrm{i}}}
\end{gathered}
$$

where,

$$
\begin{array}{ll}
\mathrm{HE} & \text { is the cumulative human exposure } \\
\mathrm{VE} & \text { is the cumulative vegetation exposure } \\
\mathrm{N} & \text { is the number of receptor sites considered in the model } \\
\mathrm{P}_{\mathrm{j}} & \text { is the population at the receptor site } \mathrm{j} \\
\mathrm{C} & \text { is the critical level (3 ppm-hours) } \\
u & \text { is the unit step function } \\
\mathrm{LA}_{\mathrm{j}} & \text { is the land area at the receptor site } \\
\mathrm{n}_{\mathrm{i}} & \text { is the annual anthropogenic NOx emission from country i } \\
\mathrm{v}_{\mathrm{i}} & \text { is the annual anthropogenic VOC emission from country } \mathrm{i}
\end{array}
$$

The spatially differentiated human and vegetation impact characterization factors for $\mathrm{NO}_{\mathrm{x}}$ and VOC showed a variability of 2 and 4 orders of magnitude, respectively. This reinforces the need for spatial resolution in the characterization of regional impact categories. The observation from the modeling study was that the contribution to ozone formation of $\mathrm{NO}_{\mathrm{x}}$ is roughly twice that of VOC. This further validates the assumption made in TRACI. However, the average 
characterization factor for $\mathrm{NO}_{\mathrm{x}}$ is three times that of VOC, thus showing the uncertainty introduced by not considering exposure effects in the characterization factors. The characterization factors for VOC do not differentiate between individual species. For this, the relative POCP approach that is used in the Eco-indicator 99 method is recommended in EDIP2003, too.

Table 4-1 summarizes the four characterization models described above and the proposed characterization model which is described in detail in Chapter 5.0.

Table 4-1 Summary of the photochemical smog characterization models

\begin{tabular}{|c|c|c|c|c|c|}
\hline Characteristic & CML2001 & EI99 & TRACI & EDIP2003 & Proposed model \\
\hline $\begin{array}{l}\text { Impact } \\
\text { characterization } \\
\text { level(s) }\end{array}$ & Fate level & $\begin{array}{l}\text { Human } \\
\text { Effect level }\end{array}$ & Fate level & $\begin{array}{l}\text { (a) Human } \\
\text { exposure level } \\
\text { (b) Ecosystem } \\
\text { exposure level }\end{array}$ & $\begin{array}{l}\text { (a) Fate level } \\
\text { (b) Human } \\
\text { exposure level } \\
\text { (c) Ecosystem } \\
\text { exposure level } \\
\text { (d) Human effect } \\
\text { level }\end{array}$ \\
\hline $\begin{array}{l}\text { Category } \\
\text { Indicator(s) }\end{array}$ & $\mathrm{ppb} \mathrm{O}_{3}$ & DALY & $\begin{array}{l}\text { g of } \mathrm{NO}_{\mathrm{x}} \\
\text { equivalent }\end{array}$ & $\begin{array}{l}\text { (a) persons.ppm.hr } \\
\text { (b) } \mathrm{m}^{2} \cdot \mathrm{ppm} \cdot \mathrm{hr}\end{array}$ & $\begin{array}{l}\text { (a) } \mathrm{ppb}_{3} \\
\text { (b) ppm.persons } \\
\text { (c) } \mathrm{g} \mathrm{O}_{3} \\
\text { deposited } \\
\text { (d) persons } \\
\text { (mortality) }\end{array}$ \\
\hline $\begin{array}{l}\text { Spatial } \\
\text { applicability }\end{array}$ & Europe & Europe & US & Europe & US \\
\hline $\begin{array}{l}\text { Spatial } \\
\text { differentiation }\end{array}$ & None & $\begin{array}{l}\text { Low } \mathrm{NO}_{\mathrm{x}} \text { and } \\
\text { high } \mathrm{NO}_{\mathrm{x}} \\
\text { regions }\end{array}$ & State level & Country level & State level \\
\hline $\begin{array}{l}\text { Temporal } \\
\text { differentiation }\end{array}$ & None & None & None & Annual & Monthly \\
\hline $\begin{array}{l}\text { Underlying } \\
\text { model(s) }\end{array}$ & $\begin{array}{l}\text { Lagrangian } \\
\text { EMEP }\end{array}$ & $\begin{array}{l}\text { Lagrangian } \\
\text { EMEP, } \\
\text { epidemiology } \\
\text { studies }\end{array}$ & $\begin{array}{l}\text { EKMA and } \\
\text { ASTRAP }\end{array}$ & RAINS & $\begin{array}{l}\text { CAMx, } \\
\text { epidemiology } \\
\text { studies }\end{array}$ \\
\hline
\end{tabular}




\subsection{DESCRIPTION OF THE PROPOSED CHARACTERIZATION MODEL}

The goal of this research is to identify and apply a new characterization model that addresses the limitations in current impact assessment methods for photo-oxidant formation that have been identified in Chapter 4.0. The methodology proposed by Hofstetter [1998] of combining the top down and bottom up approaches will be followed for the development of the characterization model. The advantages of using a combined approach are identified by Hofstetter [1998] and have been mentioned in Section 4-1. A PAQMS will be used to determine the fate and transport of the precursors and photo-oxidants. The exposure of humans and vegetation will be determined using methods similar to the RAINS model. The effect model will be based on epidemiological studies for human health impacts. The following section describes each of these in further detail.

\subsection{FATE AND TRANSPORT MODEL}

The combination of MM5 v3 [http://mmm.ucar.edu/mm5/] (meteorology model), SMOKE v3.2 [http://www.smoke-model.org] (emissions model), and CAMx v4.2 [http://www.camx.com/] (chemistry and transport model) has been selected for this application. These models have been evaluated and used, individually and in combination, in several previous studies [USEPA 2005a, Tonnesen et al 2005, Lee et al 2007]. A brief description of each of these models is presented below. 


\subsubsection{MM5 model}

The MM5 model has been developed by National Center for Atmospheric Research (NCAR) in association with The Pennsylvania State University. The model had been undergoing continuous development since the early 70's until 2001. Currently, it has been “frozen” and is not being further developed. The Weather Research and Forecasting (WRF) [http://www.wrf-model.org] model is the latest to be developed by NCAR. However, MM5 continues to be routinely used for air quality applications. It is a limited-area, terrain following sigma-coordinate, nonhydrostatic model. It is based on the prognostic equations that predict the three dimensional wind vector, temperature, water vapor mixing ratio, and pressure. The choice of MM5 is based on its prognostic nature, its suitability for the scale of the application, its past performance for photochemical modeling, thorough documentation, and the public availability of its Fortran90 source code.

The core model is supported by several programs which together form the MM5 modeling system. A graphical representation of the modeling system and the associated data flows are shown in Figure 5-1.

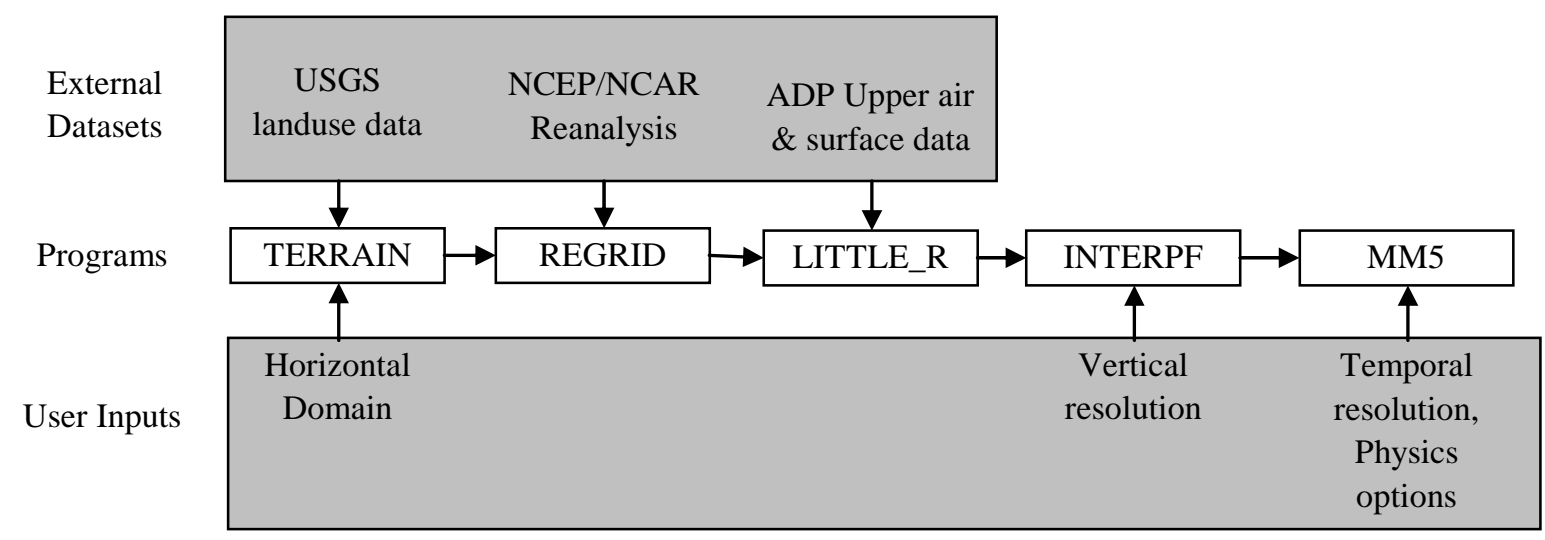

Figure 5-1 Schematic diagram of the MM5 modeling system 
TERRAIN is the first program to be executed in the MM5 system. The functions of this program are (i) to define the mesoscale domain in either the Lambert conformal, polar stereographic, or Mercator map projections, (ii) to generate topographic and land use data for the selected model domain from the external datasets, and (iii) to calculate the map scale factors and Coriolis parameter. It requires the user to define a map projection, domain parameters like horizontal extent and grid dimension, and select the appropriate resolution of topographic dataset and categories of land use datasets.

The horizontal domain selected for the current application encompasses the continental United States and parts of Canada and Mexico. The map projection is the Lambert conformal centered at $40^{\circ} \mathrm{N}$ and $97^{\circ} \mathrm{W}$ with true latitudes at $33^{\circ} \mathrm{N}$ and $45^{\circ} \mathrm{N}$. The horizontal domain extends $5940 \mathrm{~km}$ in the east-west direction and $4500 \mathrm{~km}$ in the north-south direction forming a 165x125 horizontal grid with grid size of $36 \mathrm{~km}$. The choice of grid size has a significant effect on the computing requirements of the model. A previous study by Cohen [2004] compares the model results for $4 \mathrm{~km}, 12 \mathrm{~km}$, and $36 \mathrm{~km}$ grid sizes. The results show only a slight variation between the three grid resolutions. EPA [2007b] recommends $36 \mathrm{~km}$ as the maximum grid size for regional scale modeling. A map showing the extents of the domain is presented in Figure 5-2.

REGRID follows in execution after TERRAIN. Its purpose is to read archived gridded meteorological forecasts on pressure levels generated by another coarser, larger scale model and interpolate them to the map projection and grid defined in the previous step, for the time period and at the time interval input by the user. The time period selected for each of the present simulations is 7 or 8 days and the output time step is six hours. The year 2001 is selected as the base year for the simulation as the most recent emissions data is currently available for 2001. The task of REGRID is shared by two separate programs. The program PREGRID reads in the archived data and REGRIDDER performs the interpolation. Several datasets can be used as the input for REGRID. These include National Center for Environment Protection's Global Data Assimilation System (NCEP GDAS), NCEP/NCAR Reanalysis, and NCEP Eta. The NCEP/NCAR Reanalysis dataset is used for the current model. It is a global dataset with horizontal resolution of $209 \mathrm{~km}, 28$ vertical levels, and 6hour interval. It is available to NCAR users at the UCAR website [http://www.ucar.edu/]. 


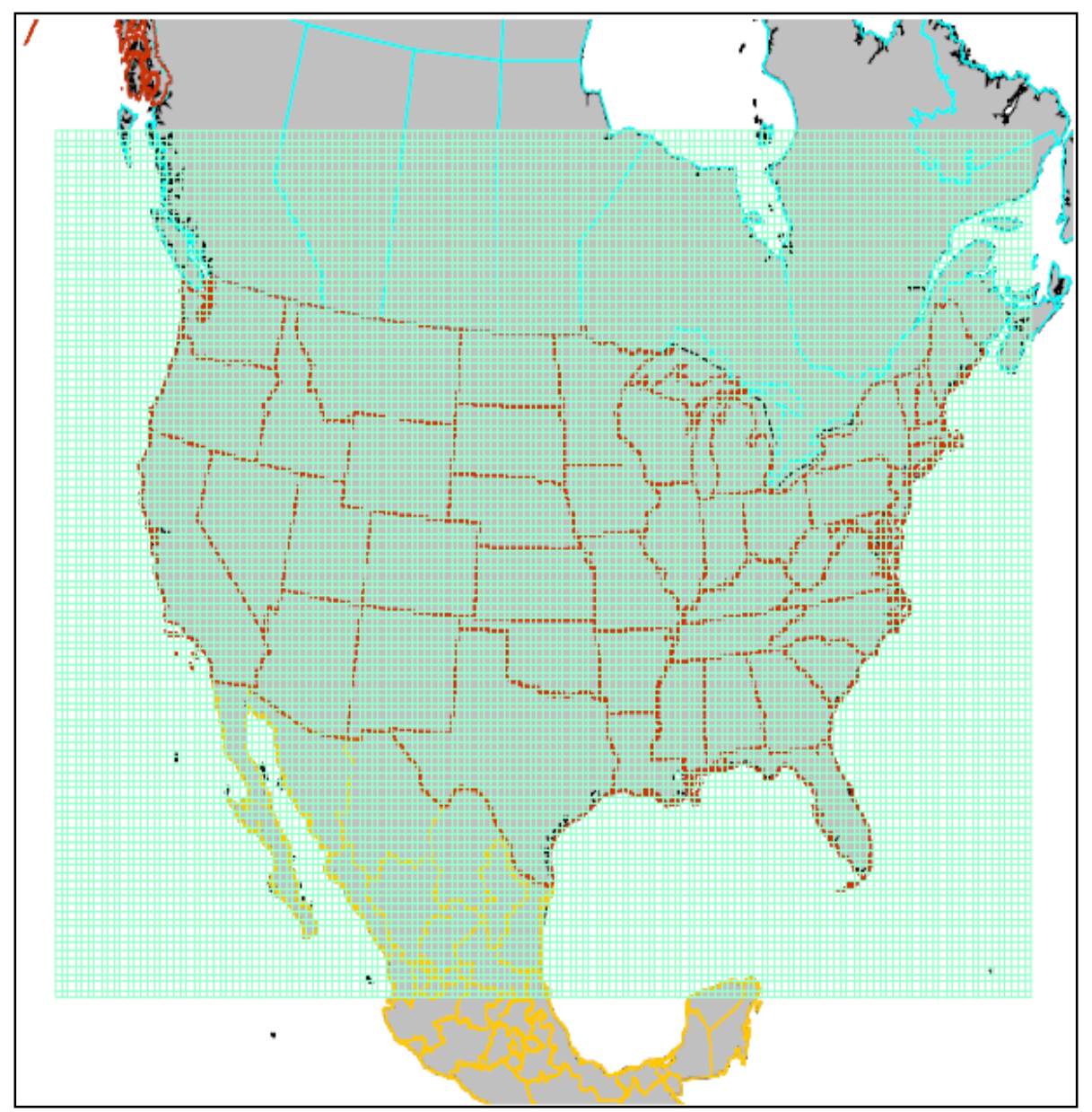

Figure 5-2 Map of model horizontal domain

LITTLE_R performs the objective analysis wherein the first guess fields generated by REGRID are refined by incorporating information from observations. It is an optional step but it is important for estimating actual conditions. The observation datasets used are the NCEP ADP Global Upper Air Observation Subsets, which include radiosonde, pibals, and aircraft data [http://dss.ucar.edu/datasets/ds353.4/], and NCEP ADP Global Surface Observations [http://dss.ucar.edu/datasets/ds464.0/]. Both these datasets are available at the NCAR website for users with NCAR accounts. The output from LITTLE_R will be used as the initial and lateral boundary conditions, as well as, during FDDA. Hence, it is required to run both, REGRID and LITTLE_R, for the entire modeling period.

INTERPF performs the vertical interpolation of the pressure level data to the user selected sigma-levels. A graphical representation of the difference between pressure levels and sigma levels is shown in Figure 5-3. The modeling domain has been resolved into twenty eight vertical 
layers with the top layer at a pressure of $100 \mathrm{mb}$. The layers near the surface are finer to adequately resolve the processes within the planetary boundary layer. Out of twenty eight, about eighteen layers lie in the planetary boundary layer. EPA [2007b] does not give a minimum requirement for the number of vertical layers. It recommends the use of the currently accepted standards for vertical resolution which is about 30 layers.
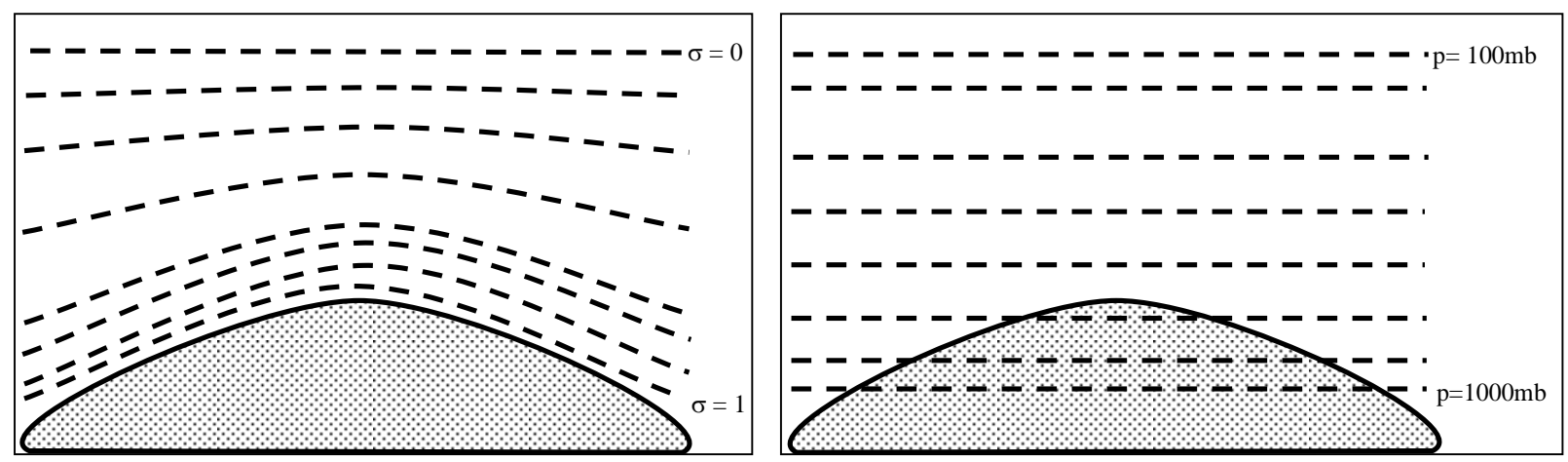

Figure 5-3 Graphical representation of sigma and pressure levels

The output from INTERPF is used as input by MM5, which is the numerical weather prediction program. MM5 computes the solutions to the equations of pressure, temperature, wind, and moisture using spatial and temporal finite differencing techniques. In addition to the numerical solution to these equations at the spatial and temporal resolution, parameterization of certain physical processes is required. These physical processes are sub-grid, too brief, complex, or poorly understood. Parameterization accounts for the cumulative effect of these processes without actually modeling them. In MM5, the parameterized processes are: (i) cumulus convection, which represent vertical mixing and rainfall due to convective clouds, (ii) planetary boundary layer processes and diffusion, which represent the vertical mixing in the PBL due to turbulence, (iii) microphysics of moisture and cloud formation, (iv) radiation, and (v) interaction with land surface. There are several alternate schemes for the representation of these processes, each having a set of assumptions and range of applicability. A detailed discussion of these parameterizations is beyond the scope of this document. Further details of these schemes are available at the MM5 website [http://www.mmm.ucar.edu/mm5/]. There is an interaction among 
the processes, and the choice of parameterization schemes needs to be checked for compatibility. A sensitivity study was performed for the Western Regional Air Partnership (WRAP) [KemballCook et al 2004] comparing the different sets of parameterization schemes. The model domain was the continental US. The comparison did not show a significant difference in MM5 performance for different parameterization schemes. Another study [Mao et al 2006] has shown that notwithstanding the difference in meteorological variables, the performance of the overall photochemical model is comparable. For the current application, the schemes used are: KainFritsch for cumulus parameterization, Pleim-Xiu for PBL processes and land soil model, Reisner2 for moisture microphysics, and CCM2 radiation scheme. Most of these options are identical to those selected in an evaluation study of MM5 simulation results for the year 2001 [McNally 2003].

Four Dimensional Data Assimilation (FDDA) is performed using the method of analysis nudging, where a nudging term is added to the prognostic equations for wind, temperature, and water vapor. These terms nudge the solution towards the analysis data processed by LITTLE_R. Nudging is performed for above-surface temperature, wind, and humidity at one hour steps, which is the model output interval.

\subsubsection{SMOKE system}

SMOKE was created by MCNC Environmental Modeling Center (now Baron Advanced Meteorological Systems), and later modified and improved by USEPA. It is currently maintained by the Carolina Environment Program at the University of North Carolina, Chapel Hill. As the name indicates, SMOKE employs the sparse-matrix approach to spatially and temporally resolve, and speciate the emission inventory as required by the chemistry and transport program. The traditional method of emission processing is based on a network of pipes and filters. In other words, the data files contain all the attributes acquired from previous processes. At every processing stage a part of the data is filtered and combined with additional data, and rewritten to the file. This increases the memory and storage requirements of the program. The sparse-matrix approach executes all the processes in parallel. It generates matrices for the gridding, speciation, and temporal allocation of the emissions inventory which are merged in the final step. 
The emissions data is obtained from the SMOKE formatted emissions datasets generated for air quality modeling in support for the Clean Air Interstate Rule (CAIR) [USEPA 2005b]. These datasets are publicly available at the USEPA website [ftp://ftp.epa.gov/EmisInventory/2001nmp/]. They are based on the 1999 National Emissions Inventory (1999 NEI) [http://www.epa.gov/ttn/chief/net/1999inventory.html] which is collected and maintained by the USEPA under the Consolidated Emissions Reporting Rule (CERR) [http://www.epa.gov/ttn/chief/cerr/index.html]. The emissions information is collected from numerous state and local air agencies, tribes, and from industry. To efficiently compile this data, USEPA has developed a detailed Source Classification Code (SCC) system covering all types of emission sources. These act as unique identification numbers for each source category, and make it easier to define the speciation, temporal, and spatial properties of each source.

Broadly, the sources of emissions can be divided into four categories. These are:

a. Point sources: Point sources of emissions are individual facilities which have large annual emissions (greater than 100 tons per year of $\mathrm{NO}_{\mathrm{x}}$ or VOC) or have lesser emissions (greater than 25 tons per year of VOC) but are located in a non-attainment zone. The facilities include Electric Generating Units (EGU) and non-EGUs like chemical manufacturers, iron and steel plants, petroleum refineries, solvent coating units, etc. The individual facilities are required to submit their annual estimated emissions along with ancillary parameters like, stack longitude and latitude, stack diameter, height, exit gas temperature, velocity, SCC, etc. Emissions from point sources can be easily monitored and regulated. Point sources contribute close to $30 \%$ of the total anthropogenic $\mathrm{NO}_{\mathrm{x}}$ (mainly from EGUs) and about $15 \%$ of anthropogenic VOC.

b. Stationary area sources: Emission sources which are distributed over a large spatial area and are not mobile are called stationary area sources or nonpoint sources. These include residential emissions, architectural coatings, agriculture emissions, smaller industrial emissions, etc. The amount of emissions from nonpoint sources cannot be measured directly due to their distributed nature. The emissions estimates for these sources are derived from the spatially allocated activity level and documented emission factors like those in AP42 [http://www.epa.gov/ttn/chief/ap42]. The spatial allocation to a particular county may be based on the actual level of activity in the county or on a surrogate to proportionally distribute the total emissions among the counties. For example, to calculate the county-level 
emission figures for residential heating using natural gas, the annual state-level natural gas consumption is divided to the respective counties based on the detailed housing information of the US Census Bureau and multiplied by the corresponding AP42 emissions factor. Prescribed and wildfire sources are either considered as stationary area sources or point sources. Area sources account for about $10 \%$ of anthropogenic $\mathrm{NO}_{\mathrm{x}}$ and more than $50 \%$ of anthropogenic VOC (mainly from solvent use and fires).

c. Mobile sources: Mobile sources can be divided further into onroad sources and nonroad sources. Nonroad sources include vehicles and other moving equipment that do not travel on roadways. These include construction vehicles, locomotives, lawn mowers, marine vehicles, etc. Similar to area sources, the emissions from these sources cannot be measured directly. The National Mobile Inventory Model (NMIM) [http://www.epa.gov/otaq/nmim.htm] developed by USEPA is used to calculate county-level mobile emissions. The NMIM employs the NONROAD model [http://www.epa.gov/otaq/nonrdmdl.htm] for nonroad sources and MOBILE6 [http://www.epa.gov/otaq/mobile.htm] for onroad sources. The core of these models is the National County Database which contains county-level activity data like Vehicle Miles Traveled (VMT) which is divided into 28 vehicle classes and 12 roadways types, population estimates for various nonroad equipment, and ancillary data such as fuel properties and monthly average temperature and humidity. The emission factor for nonroad and onroad equipment is a function of the vehicle type, age activity, fuel's chemical composition, and the weather conditions for the particular time period. NMIM aggregates the mobile source emissions data on a monthly basis. Mobile sources contribute close to $60 \%$ of anthropogenic $\mathrm{NO}_{\mathrm{x}}$ (40\% onroad) and about 35\% of anthropogenic VOC (23\% onroad).

d. Biogenic Sources: Vegetation is the single largest source of VOC emissions in the United States. The total amount of VOC emissions from vegetation is greater than all anthropogenic sources combined. Biogenic $\mathrm{NO}_{\mathrm{x}}$ is primarily released from soils and is about $5 \%$ of anthropogenic emission. The amount of emission is strongly dependent on the temperature and solar radiation. The USEPA developed Biogenic Emissions Inventory System (BEIS) [http://www.epa.gov/AMD/biogen.html] to estimate biogenic emissions of $\mathrm{NO}_{\mathrm{x}}$ and VOC. In addition to the meteorology parameters, it requires land use/land cover datasets, species composition, and leaf mass density at the desired spatial resolution. 
The sequence of steps followed by SMOKE and the supporting data required for generating model ready emissions is represented in Figure 5-4. SMOKE is run using shell scripts which set all the shell environment variables required by the programs. The procedure followed by SMOKE is the same for all anthropogenic emission sources. The steps followed in the processing of biogenic emissions are a little different.

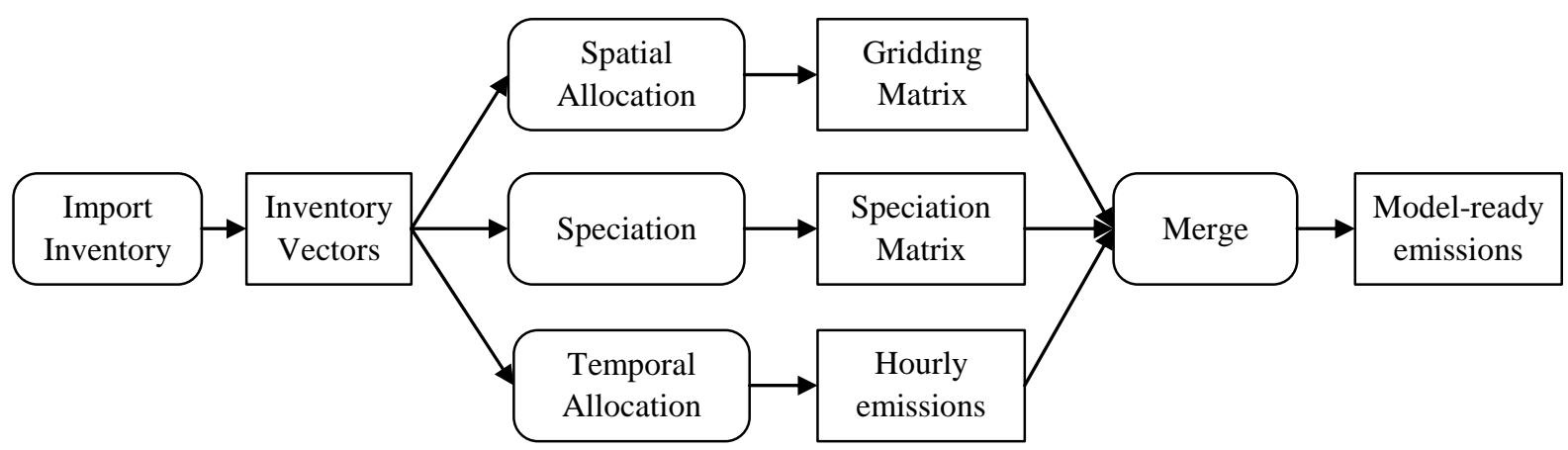

(a) Processing steps for anthropogenic stationary and mobile area source emissions

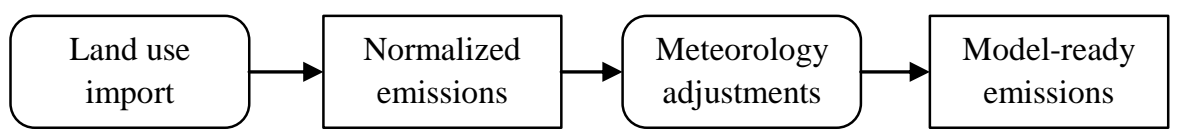

(b) Processing steps for biogenic emissions

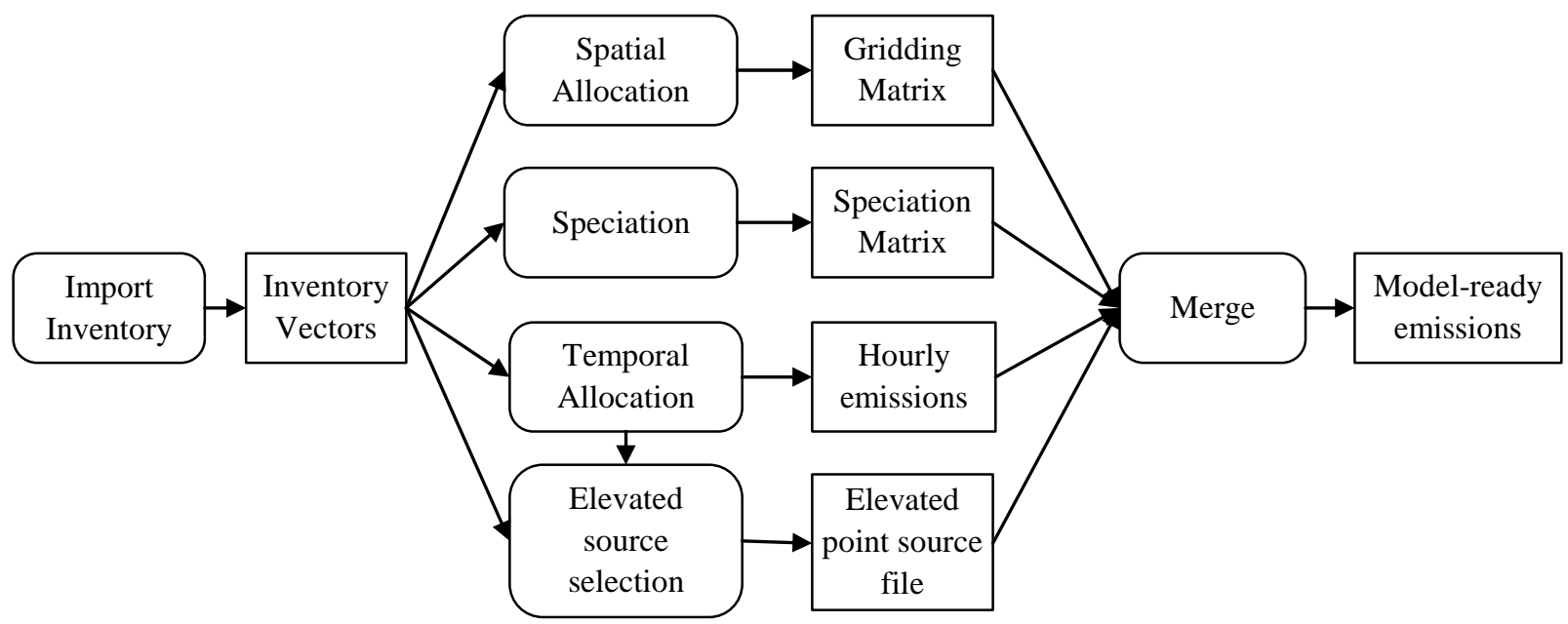

(c) Processing steps for point source emissions

Figure 5-4 Processing steps performed by SMOKE

[http://www.smoke-model.org/version2.4/html/] 
The following is a brief description of each of these steps.

a. Inventory Import: This is performed by the SMKINVEN program for anthropogenic emission classes and by RAWBIO (BEIS2) or NORMBEIS3 (BEIS3) for biogenic sources. The SMOKE formatted NEI dataset is used as input for anthropogenic emissions. NORMBEIS3 imports normalized emissions from the Biogenic Emissions Land cover Database (BELD3), which contains vegetation data at $1 \mathrm{~km}$ resolution for North America. SMKINVEN activities include checking the raw inventory data for format and duplicity of records, fill in missing values for point source stack parameters, record sorting, and conversion of units.

b. Spatial processing: Spatial processing is the distribution of county-level/state-level emissions to a user specified grid. The program GRDMAT generates a gridding matrix containing allocation factors for the corresponding grid cells for each emission record in the SMKINVEN output file. The area and mobile sources are distributed based on the user specified surrogate for each SCC. Since the records for point sources contain their geographic location, they are entirely allocated to the intersecting grid cell. The inventory for biogenic sources is already gridded into a finer resolution. The aggregation of the biogenic emission inventory to the required resolution takes place during the inventory import step.

c. Chemical speciation processing: The inventory reports emissions for pollutants aggregated as $\mathrm{NO}_{\mathrm{x}}$ and VOC. The chemistry and transport models use a small set of compounds, known as model species, to represent the atmospheric chemical reactions. (This is discussed further in Section 5.1.3). SPCMAT uses a speciation profile to convert emission of $\mathrm{NO}_{\mathrm{x}}$ and VOC to emissions of the model species. The speciation profile file contains separate pollutant to species conversion factors for a variety of source categories besides a set of average factors. The choice of speciation profile is dependent on the chemical mechanism used in the chemistry and transport Model. The output from SPCMAT is a mole-based speciation matrix for each record of the inventory.

d. Temporal processing: The purpose of temporal processing is to create hourly emission estimates from the annual or monthly files. The hourly distribution of emissions is based on a set of monthly, weekly, and diurnal factors representing activity patterns for each source category. Temporal processing is performed by the TEMPORAL program for anthropogenic sources and TMPBEIS3 for biogenic sources. Since biogenic emissions are dependent on weather conditions, hourly gridded meteorology files are required for their temporal 
processing. For this purpose, the Meteorology Chemistry Interface Processor (MCIP) [http://www.cmascenter.org/] is used to convert the MM5 output to a format readable by TMPBEIS3.

e. Merging: The final step is to merge all the matrices with the emissions inventory for selected classes to generate model ready, gridded, speciated hourly emissions. The current study requires the generation of separate files for anthropogenic and biogenic sources. Merging is performed by SMKMERGE. The format of the output file and the units of emissions depend on the chemistry and transport model. CAMx requires emissions to be expressed in moles/hr for gases arranged in a specific format in a fortran binary file. In addition, CAMx requires the physical parameters like stack height and diameter, gas exit temperature and velocity, etc for elevated point sources. CAMx calculates the plume rise for these sources based on meteorology conditions and allocates the emissions to the appropriate vertical layer. This data is provided to CAMx in an ASCII formatted elevated point source file.

\subsubsection{CAMx model}

CAMx is a Eulerian chemistry and transport model developed, maintained, and updated by Environ Corporation and is a leading model used in PAQMS. It is available publicly at the CAMx website [http://www.camx.com]. It is a "one atmosphere" model since it integrates the processes for gaseous as well as particulate pollutants. It is a multi-scale model and can be used for urban as well as continental domains. In addition to the basic chemistry and transport simulations, CAMx includes useful analysis features like Ozone Source Apportionment Technology (OSAT), Decoupled Direct Method (DDM) for sensitivity analysis, and Process Analysis (PA). The OSAT and DDM features are of particular importance to the current research, and are the principal reason for selecting CAMx among other photochemical models. CAMx has been used by USEPA for air quality modeling in support of NAAQS and CAIR, and by several state and regional agencies.

The Eulerian continuity equation (Equation 17) is numerically solved at each time step at each grid cell. The equation is split into components representing each physical process (emission, advection, diffusion, chemistry, and removal) that calculate the contribution of that process to the change in concentration of a model species. The time step is internally determined 
by CAMx such that the Courants-Friedrichs-Levy number (the ratio of wind speed to grid spacing) is less than 1 . Time steps typically vary in the range of 5-15 minutes for cell size of 10$50 \mathrm{~km}$. At each time step for a particular grid cell, CAMx follows the processing sequence of injection of emissions, horizontal advection, vertical advection, vertical diffusion, horizontal diffusion, wet scavenging, and finally chemistry. Dry deposition is not treated as a separate process. The dry deposition velocity is used as the lower limit for vertical diffusion.

As discussed earlier, emissions are treated as either distributed surface emissions from area, mobile or biogenic sources or as elevated point sources from large industrial plants like EGUs, refineries, steel plants, etc. Surface emissions are injected into the lowest vertical layer as they are considered to lack the momentum required to reach upper layers. Plume rise from elevated point sources above the stack top is calculated by CAMx based on the stack diameter, exhaust gas temperature and velocity, and meteorological conditions. The emissions are injected into the grid cell at a height, above the stack host cell, equal to the plume rise.

Pollutant transport through advection in CAMx is mass conservative as well as mass consistent. Mass conservation is the ability to account for all emissions and removal of mass of each species during time integration. To maintain mass conservation CAMx uses density, instead of volumetric ratios, as the measure for the amount of each species. The model is mass consistent if the quantity of pollutant transported is equivalent to the atmospheric momentum field. CAMx uses several numerical techniques to minimize mass inconsistency.

Wet deposition is an important removal process for gaseous pollutants with high solubility. It can occur through any of different processes which include mixing of gas and condensed water, absorption of gas molecules, aqueous phase reactions, precipitation of droplets, and diffusion into falling precipitation. CAMx models the process of wet deposition through a scavenging model, which relates the rate of change in pollutant concentration to the initial concentration by a scavenging coefficient calculated based on meteorological conditions. Dry deposition is modeled as a first-order mechanism where the flux of the pollutant deposited is the product of a dry deposition velocity and the pollutant concentration in the lowest layer. The dry deposition velocity depends on the reactivity, solubility, diffusivity, local meteorological conditions, and season-specific surface characteristics.

The photochemical reactions taking place in the atmosphere involve a large number of chemical species which undergo a larger number of reactions. It is computationally expensive to 
model all these species and reactions. Besides, our level of knowledge of many processes that would have to be represented is not fully developed [Carter et al 1999]. Hence, chemical mechanisms are used as a means to condense the several reactions into a few representative reactions involving a fraction of the reactants and products. This is done by "lumping" of the chemical compounds into representative species, known as model species. The rationale behind the lumping technique is that organic compounds can be divided into groups that have similar chemical properties, or follow similar reaction mechanisms in the formation of ozone. Even though the non-ozone products of the reactions differ significantly, these are secondary for the purpose of ozone modeling. Typically, inorganic species $\left(\mathrm{NO}_{2}, \mathrm{CO}\right.$, etc.) are represented explicitly.

Two commonly used lumping schemes are: lumped structure and lumped molecule. In the lumped structure approach, the organic species are grouped together according to bond type (carbon single bond, carbonyl bond, etc.). The Carbon Bond 4 (CB4) mechanism [Grey et al 1989] is an example of a lumped structure mechanism. In the lumped molecule approach, the species having similar reactivity towards the formation of ozone are grouped together. SAPRC99 [Carter 2000] is based on the lumped molecular approach. Both the CB4 and SAPRC99 mechanisms are available in CAMx. The SAPRC mechanism is scientifically advanced than the older CB4 mechanism. However, the computational resources required for SAPRC are higher than CB4. Besides, there is only a slight difference in the results from the two mechanisms [Morris et al 2004]. A modified version of CB4 mechanism adopted by the OTAG group will be used for the present study [Yarwood et al 2005a]. It is represented in CAMx as Mechanism 3.

The modified CB4 mechanism involves 96 chemical reactions and 14 inorganic and 24 organic species, most of which are short-lived intermediate radicals [Environ 2006]. In the CB4 mechanism, inorganic species are treated explicitly. In organic species, formaldehyde, ethane, and isoprene are treated explicitly. All other VOC are lumped into three carbon bond surrogates and two molecular surrogates. The carbon bond surrogates are PAR (single bond), OLE (double bond), and ALD2 (higher aldehydes). The molecular surrogates are TOL (monoalkylbenzne), and XYL (dialkylbenzene). Some of the modeled reactions denote photolytic decomposition of the model species, the rates of which are dependent on parameters like cloud cover, total ozone column, solar zenith angle, altitude, atmospheric haze, and surface albedo. The rates of the other reactions are dependent on meteorological variables like temperature and pressure. 
In addition to the emissions file, CAMx requires meteorological inputs for eight variables: precipitation (for wet/dry deposition), cloud depth (for photolysis rates), height and pressure for each vertical layer (for vertical grid definition, reaction rates, and plume rise), X/Y wind vector (for advection), temperature (for transport, plume rise, dry and wet deposition, and reaction rates), water vapor (for chemistry, and dry/wet deposition), and vertical diffusivity (for pollutant transport). In addition the land use/topography data, used for calculating the surface roughness for dry deposition, is also obtained from the MM5 output. A program named MM5CAMX available at the CAMx website [http://www.camx.com/down] is used to convert the MM5 output to CAMx compatible format. In the current study, the vertical diffusivity coefficients are calculated in MM5CAMX using the O'Brien scheme. This scheme uses a simplified cubic profile of the vertical diffusion coefficients within the PBL. The horizontal diffusion coefficients are calculated in CAMx at each time step. They are calculated using the deformation approach, thus are proportional to the straining and shearing of the wind field in each grid cell.

The albedo/haze/ozone values for each grid cell, required by CAMx to calculate photolysis rates, are obtained from the Total Ozone Mapping Spectrometer [http://toms.gsfc.nasa.gov] observations maintained at the Goddard Space Flight Centre website. The dataset contains the total (stratospheric and tropospheric) ozone column depth for a global grid. The data is extracted using the AHOMAP program available at the CAMx website [http://www.camx.com/down]. Another program TUV is used to generate photolysis rates table for the selected mechanism's chemical reactions. The rate values tabulated correspond to the range of pertinent physical conditions present over the entire modeling domain. The daily albedo/haze/ozone file and the land use file are the required inputs.

For every run, CAMx requires a set of initial conditions over the entire domain. Besides, since it is a regional model, it requires boundary conditions for the four lateral boundaries and the top boundary for the simulation period. For subsequent runs that are continuous in time, the end concentration file output by CAMx can be used as initial conditions. From the users perspective, the initial and boundary conditions are the most difficult to prepare. Different approaches have been used by researchers for generation of these inputs. A 'clean condition' is where the initial and boundary concentration of all species in the entire domain is zero. This method has been used in the CAIR model runs [USEPA 2005a]. Starting from clean conditions, 
the model is run for a few simulation days as an initial spin-up period. This will give sufficient time for the model to reach near-real conditions in the PBL. However, the effect of the higher concentration, especially of ozone, in the free troposphere may not be fully accounted for [Environ et al 2003]. A clean lateral boundary condition assumes no intercontinental transport of pollutants over the Pacific and Atlantic oceans. Fiore et al [2002] have demonstrated the significance of transport of Asian and European emissions to North America. A method recently developed [Byun et al 2005], which is similar to the MM5 method of generating boundary conditions, is to extract the relevant data from the simulation of the global ozone model, GEOSCHEM [http://www-as.harvard.edu/chemistry/trop/geos]. In the absence of gridded output files, the visualization of the results of the 2001 GEOS-CHEM model run [http://www.atmos.washington.edu/ jaegle/geoso3_start.html] is used to estimate the initial, four lateral and top boundary conditions. In the current study, the boundary conditions are held constant for each simulation month.

The horizontal grid configuration is continued from the MM5 model except that the domain has been reduced from $165 \times 125$ cells to $148 \times 112$ cells to eliminate boundary effects. CAMx does not require the user to input the vertical grid parameters. The vertical grid structure is obtained from the MM5CAMX output. It is not necessary to maintain the vertical grid structure defined in MM5. MM5CAMX offers the flexibility to merge adjacent MM5 layers and use a coarser resolution in CAMx. For the current study, CAMx is configured to use 14 vertical grids. Since MM5's vertical grids follow sigma levels, the vertical grid structure of CAMx can vary at each grid cell and time-step. The model is simulated for each day of the simulation year, which is chosen as 2001. In addition to the diagnostic and log files, CAMx outputs the average concentration of the model species at each model time interval (usually one hour), the wet/dry deposition of the species, and an instantaneous concentration at the end of the simulation period (one day) in three separate files. Optionally, output from the selected probing tool is also written.

CAMx provides the capability of four very useful probing tools related to gas phase chemistry. These tools are: Ozone Source Apportionment Technology (OSAT), Direct Decoupled Method (DDM) for sensitivity analysis, Process Analysis, and Reactive Tracers. The OSAT is a method to trace the geographical source, time, and type of emission associated with the ozone concentration at each grid cell. OSAT will be used in the current study to identify the ozone impact with the state in which the responsible emission occurred. Further details of this 
method are given below. The DDM method is useful to test the sensitivity of the ozone concentration to any particular emission source. In other words, it calculates the change in ozone concentration at a particular location to a very small change in the quantity of emissions. The Process Analysis method calculates the contribution of each physical and chemical process to ozone formation. The Reactive Tracer method is used to track the changes that a particular model species undergoes during its life. A limitation of CAMx is that two or more methods cannot be used simultaneously in a single simulation.

The OSAT and DDM methods are most suitable for the purpose of the current study. There is an essential difference between these two methods. OSAT analyzes the contribution of each emission source to the ozone already formed at a location. It looks only at the current scenario that is modeled. On the other hand, DDM resolves what would be the effect of a small change in the emission scenario. Both these methods measure source apportionments, but, theoretically, OSAT considers the entire ozone whereas DDM considers only the last microgram of ozone formed. Besides, there is a significant amount of additional storage and computing time required for a DDM application than a similar OSAT application. It has been reported that OSAT, too, can predict the change in ozone concentration to a small change in emission quite well [Yarwood et al 2003].

OSAT involves the use of additional model species which act as tracers to the pollutants tracked. They are spectators to the normal photochemical reactions of CAMx and, therefore, are known as passive tracers. They mirror the emission, transport, diffusion, removal and chemical processes that the tracked pollutants undergo. The emissions from a particular geographical area of interest and/or emission category can be grouped together into source groupings and each group can be tracked separately. For every unit of $\mathrm{NO}_{\mathrm{x}} / \mathrm{VOC}$ emission from a source grouping an equal amount of $\mathrm{NO}_{\mathrm{x}} / \mathrm{VOC}$ tracer associated with that source is emitted at the same location. For the VOC tracers the emissions of different VOC are added on the basis of the number of carbon atoms. Two additional ozone tracer species associated with the source groupings are modeled. They are: O3N which represents ozone formed under $\mathrm{NO}_{\mathrm{x}}$ limited conditions, and O3V representing ozone formed under $\mathrm{NO}_{\mathrm{x}}$ saturated conditions. When the CAMx chemical reactions result in the production of ozone at any time step/grid cell, the amount of ozone formed is distributed to the O3N or O3V species for a source group based on the proportion of the $\mathrm{NO}_{\mathrm{x}}$ or VOC tracer of that source group present in the cell. For VOC, the allocation is in proportion to 
the quantity of VOC weighted by their Maximum Incremental Reactivity (MIR). In case of destruction or removal of ozone, the reduction in all the ozone tracers is in proportion to their quantities present in the cell. The transport of the tracer species is based on similar weighting principles. As a result of this technique, it is possible to apportion the ozone formation at any grid cell/ time step to its source group. The source groups are usually geographical with further subdivision into emission categories (point / area / mobile / biogenic). OSAT is a very important tool in decision making for policy related to emission control as it indicates, although not precisely, how the benefits could be maximized.

CAMx incorporates Antropogenic Precursor Culpability Assessment (APCA) which is a variation of OSAT. In OSAT, the ozone formed is allocated to the participating source group based only on the conditions it was formed. It does not consider the characteristics of the source groups themselves. APCA favors the allocation of respnsibility to anthropogenic sources over biogenic sourcces. For instance, a common situation is when ozone is formed under $\mathrm{NO}_{\mathrm{x}}$ saturated conditions in the presence of anthropogenic $\mathrm{NO}_{\mathrm{x}}$ and biogenic VOC precursors. OSAT will allocate the ozone formed to the source grouping associated with the biogenic emissions, whereaes APCA would associate it to the anthroogenic $\mathrm{NO}_{\mathrm{x}}$ source group. The rationale behind this is that since biogenic VOC emissions cannot be controlled, the ozone should be allocated to the controllable anthropogenic emissions. Figure 5-5 illustrates the allocation scheme followed in APCA.

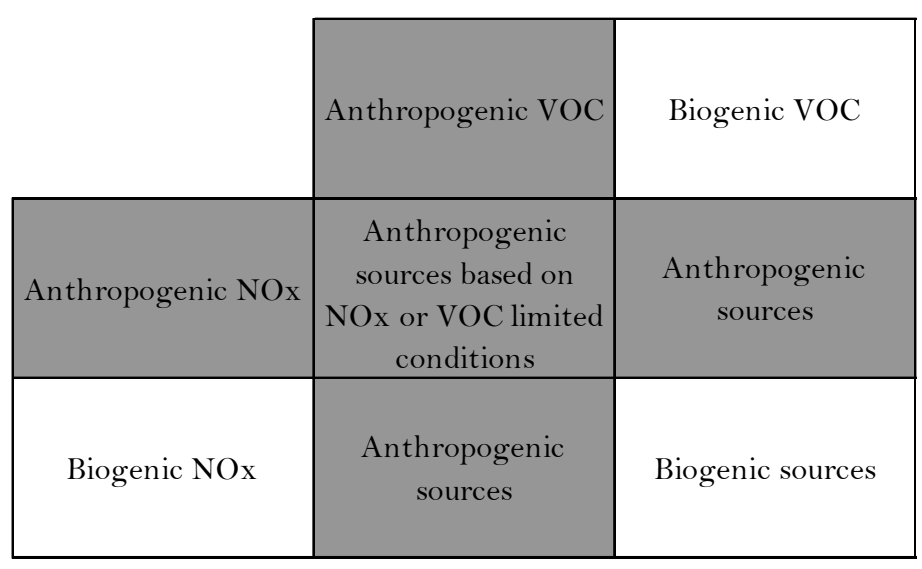

Figure 5-5 Illustration of APCA source apportionment scheme 
The APCA technique will be used for the current study. The entire modeling domain is divided into 52 source regions, the 48 coterminus states, Washington DC, Canada, Mexico, and the inland water bodies and oceans. They are further divided into anthropogenic and biogenic categories. The total tracer species modeled is thus 424, which includes 106 species (52 species for each of the 2 categories and 1 each for initial and boundary conditions) each for $\mathrm{NO}_{\mathrm{x}}$ tracer, VOC tracer, O3N and O3V. The geographical division is input using a source map file which contains the source group for each grid cell. While assigning grid cells that are intersected by the boundaries of two or more states, the cell is assigned to the state which has the maximum population in that cell. The APCA outputs two files, one containing the hourly concentration of the O3N and O3V species, and the other containing the end of siulation concentration for all the 424 species.

\subsection{CALCULATION OF FATE LEVEL FACTORS}

Fate and transport of pollutants is the first link in the cause-effect chain. This has been determined using the PAQMS described above. This has also, traditionally, been the mid-point impact for photochemical ozone formation in LCIA. Due to the limitations of using either the midpoint or the endpoint factors, the impacts will be characterized at three levels.

The characterization factors are calculated at the fate (midpoint) level, the exposure level, and at the effect (end point) level. The method of calculation of the fate level characterization factor is illustrated below.

The fate level characterization factor is defined as amount of ozone formed per unit emission of its precursor $\left(\mathrm{NO}_{\mathrm{X}} / \mathrm{VOC}\right)$. The quantity of ozone is not expressed as the absolute mass but as the average surface level concentration. For a state $L$, the fate level characterization factor for $\mathrm{NO}_{\mathrm{x}}$ for month $M$ is calculated as

$$
\text { CFFate }_{L, N O x, M}=\frac{1 / D \sum_{d=1}^{D_{M}} 1 / n_{k} \sum_{k=1}^{n_{k}}\left[O_{3} N\right]_{a v g, k, L, d} \times 1000}{1 / D \sum_{d=1}^{D_{M}} E_{N O x, L, d}}
$$


where, $\left[\mathrm{O}_{3} N\right]_{a v g, k, L, d}$ is the average surface level anthropogenic sourced ozone tracer (in ppm) in grid cell $k$ for day $d, E_{N O x, L, d}$ (in MT) is the anthropogenic $\mathrm{NO}_{\mathrm{x}}$ emission from state $L$ on day d. $n_{k}$ represents the number of cells which contribute to $95 \%$ of the surface ozone mass present in the domain on day $d$ that is attributed to state $L$. The quantity of anthropogenic $\mathrm{NO}_{\mathrm{x}}$ emitted from state $L$ is the sum of the hourly $\mathrm{NO}$ and $\mathrm{NO}_{2}$ emissions at each grid cell lying in the state boundary.

$$
E_{N O x, L}=\left\{\sum_{t=100 G M T}^{2400 G M T} \sum_{i \in L}\left\{N O_{i, t}+N O_{2 i, t}\right\}\right\}
$$

The total daily emissions and daily average ozone are calculated based on Greenwich Meriden Time (GMT). The average ozone concentration attributed to state $L$ is

$$
\left[O_{3} N\right]_{a v g, k, L}=\frac{1}{24}\left\{\sum_{t=100 G M T}^{2400 G M T}\left[O_{3} N\right]_{k, L, t}\right\}
$$

The same method is followed for VOC, except that the $O 3 N$ species is replaced by $O 3 \mathrm{~V}$, and the total emissions of VOC is the reactivity weighted sum of the modeled VOC species (species of the CB4 mechanism). The reactivity weighting is used to provide a common base to the VOC mixtures emitted from each state. The MIR of the VOC species, expressed in mass of ozone formed per unit mass of the species emitted, is used as the reactivity index. Therefore,

$$
E_{V O C, L}=\sum_{t=100 G M T}^{2400 G M T} \sum_{i \in L} \sum_{j=1}^{8}\left\{V O C_{j, i, t} \times M I R_{j}\right\}
$$

The MIR corresponds to the quantity of ozone formed when the conditions are most sensitive to VOC emissions. MIR values for several individual VOC have been calculated by Carter [2007]. The MIR values for the CB4 species is presented in Table 5-1. 
Table 5-1 Maximum Incremental Reactivity of Carbon Bond IV species

[Environ 2006]

\begin{tabular}{lcccc}
\hline $\begin{array}{l}\text { CB4 } \\
\text { Species }\end{array}$ & $\begin{array}{c}\text { No. of Carbon } \\
\text { atoms }\end{array}$ & $\begin{array}{c}\text { MIR } \\
\left(\mathrm{mol} \mathrm{O}_{3} / \text { mol species) }\right.\end{array}$ & $\begin{array}{c}\text { Molecular } \\
\mathrm{Wt}^{(\mathrm{g} / \mathrm{mol})^{\dagger}}\end{array}$ & $\begin{array}{c}\text { MIR } \\
\text { (g O }_{3} / \mathrm{g} \text { species) }\end{array}$ \\
\hline ALD2 & 2 & 3.007 & 32 & 4.51 \\
ETH & 2 & 2.328 & 28 & 3.99 \\
FORM & 1 & 5.771 & 30 & 9.23 \\
ISOP & 5 & 4.375 & 68 & 3.08 \\
OLE & 2 & 4.831 & 32 & 7.25 \\
PAR & 1 & 0.346 & 16 & 1.04 \\
TOL & 7 & 0.514 & 112 & 0.22 \\
XYL & 8 & 2.383 & 128 & 0.89 \\
\hline
\end{tabular}

$\dagger$ The molecular weight is calculated as 16 times the number of carbon atoms for species not represented explicitly. This corresponds to the average carbon/oxygen/hydrogen proportion in a VOC [Yarwood et al 2005b].

\subsection{EXPOSURE AND EFFECT MODELS}

The adverse effects of exposure to ozone were discussed in Chapter 2.0. These are summarized in Figure 5-6. The effects of ozone can be categorized into three areas of protection: human health, ecosystem quality, and manmade materials. Human health effects include premature mortality, and morbidity due to effects on the respiratory and cardiovascular systems. Effects on ecosystem include selective mortality of susceptible species leading to loss of biodiversity, and inhibition of key ecosystem functions like carbon sequestration. Ozone has adverse effects on biotic (crops) as well as abiotic (materials) manmade environment. Long term elevated concentration of ozone can contribute to climate change [USEPA 2007]. 


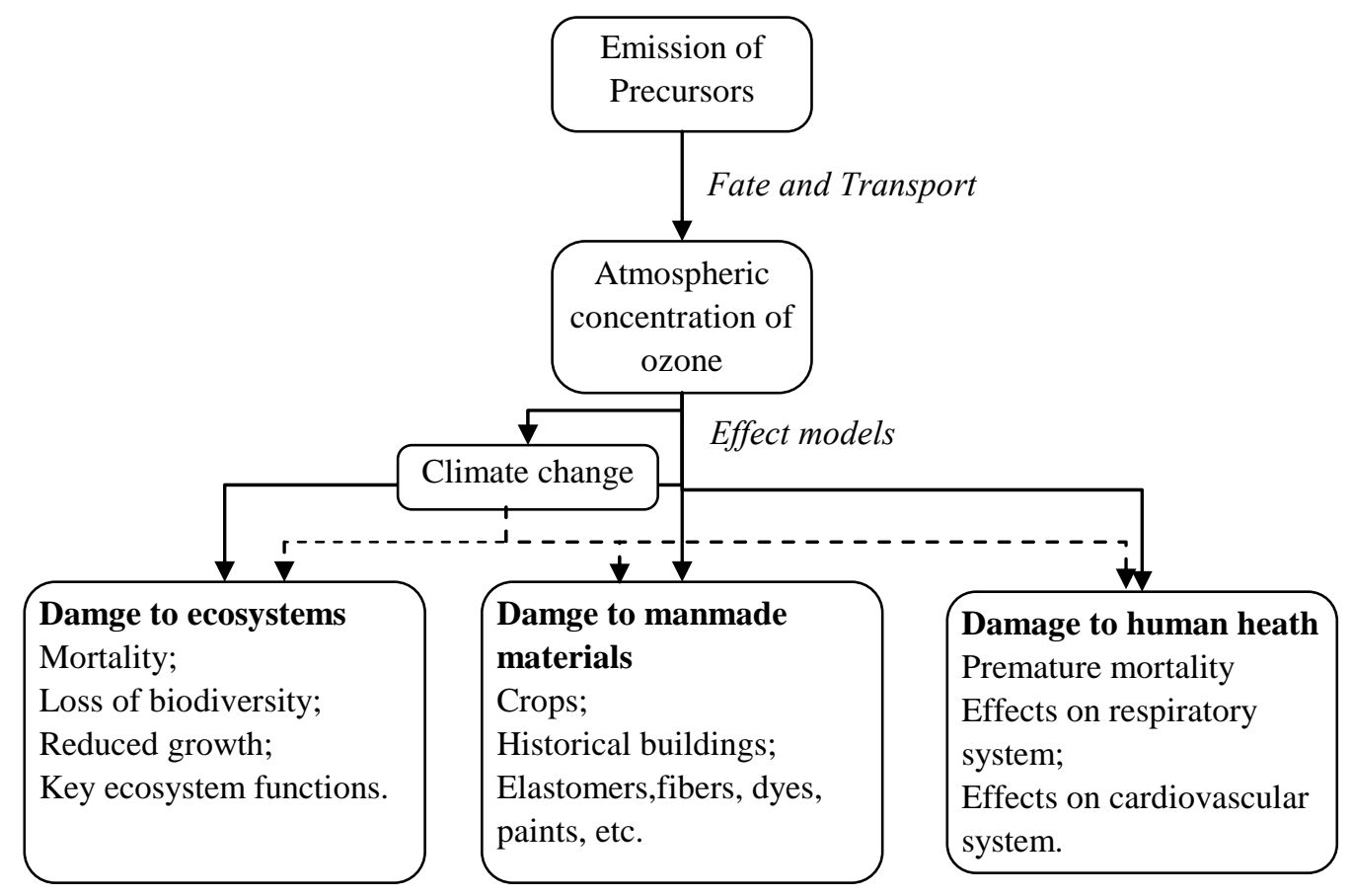

Figure 5-6 Summary of effects of ozone exposure

The result obtained from the epidemiology study conducted by Bell et al [2004] is used as the effect model for human health. The concentration-response function derived in the study showed that a $10 \mathrm{ppb}$ increase in the daily-average ozone concentration for the previous week increased the daily mortality by $0.52 \%$, nationally. The human health impact is calculated as the increase in mortality associated with additional ozone concentration above the policy relevant background concentration. The policy relevant background concentration is the uncontrollable (for a particular country) fraction of the total ozone. It includes ozone formed due to precursor emissions from vegetation, or the long range transport of precursors and ozone. The ozone associated with emissions from each state represents the 'controllable' ozone formed from anthropogenic sources in that state. Hence it is equal to the increase in state-attributed ozone concentration above the policy relevant background level.

The C-R functions for morbidity have been developed for a few locations, and may not represent the average effects across the country. The effects on respiratory and cardiovascular systems can be determined using exposure-response functions developed using toxicological 
studies. However, the determination of this relationship requires detailed modeling based on varying parameters such as demographic distributions, residential variables (presence of air conditioners, etc.), physiological variables, human activity patters, meteorological conditions, micro-environment concentrations, and the dose-effect relationships [USEPA 2007a]. These parameters are location and time specific and cannot be generalized. A reduced-form parametric model describing the concentration-effect relationship has not been developed. A detailed end effect modeling exercise, for locations representing the conditions of the entire North American modeling domain, is a time-consuming process, requires data that is currently publicly unavailable, and beyond the scope of this study.

As noted in Chapter 2.0, the knowledge of effects of ozone on ecosystem quality is very limited, and mathematical models have been developed only for tree seedling growth. Thus, the effect-level factors for ecosystem quality based on currently available data will address only a very small fraction of the effects. In the manmade materials area of protection, mathematical C-R relationships have been developed only for select crops. In view of these limitations, the effect level factors for ecosystem quality and manmade materials will not be calculated.

The exposure level characterization factor lies in between the effect level and the fate level factors in the cause-effect chain. It refines the fate level (mid-point) factors by incorporating the population (for human health impacts) and area of agricultural, forest, and range land (for ecosystem quality impacts) that is potentially exposed to the ozone concentration. They do not contain the assumptions, uncertainties, and limitations of end point modeling, but are better estimates for the damage than the fate level factors. 


\subsection{CALCULATION OF EXPOSURE AND EFFECT LEVEL FACTORS}

The exposure level characterization factor for human health impact due to $\mathrm{NO}_{\mathrm{x}}$ is defined as

$$
C F_{L, N O x, h u m, \exp }=\frac{\sum_{k \in D}\left[O_{3} N\right]_{a v g, k, L} \times p_{k}}{E_{N O x, L}}
$$

where, $p_{k}$ is the population of the grid cell $k$ as per the 2001 US Census. It is derived using the population surrogate dataset used in SMOKE. The exposure level characterization factor for ecosystem health is based on the predicted flux of ozone received by the ecosystem. The flux of ozone is calculated as the total mass of ozone deposited in each grid cell, in each month, through, both, dry and wet deposition processes. Since CAMx does not output a source apportioned deposition file, the contribution of each source to the total deposited mass is determined from the source distribution of the surface-level ozone concentration in each grid cell and at each time step. Mathematically, this relation for $\mathrm{NO}_{\mathrm{x}}$ is

$$
C F_{L, N O x, e c o, \exp }=\frac{\sum_{k \in D V}\left[O_{3} N d\right]_{t o t a l, k, L} \times A_{k}}{E_{N O x, L}}
$$

where, $D V$ is the part of the domain which is under vegetation cover, $A_{k}$ is the area of each grid cell in sq. km., and $\left[\mathrm{O}_{3} \mathrm{Nd}\right]_{\text {total, }, \mathrm{L}, \mathrm{i}}$ is the mass of ozone deposited in grid cell $k$ in each month attributed to state $L$. It is calculated as

$$
\left[O_{3} N d\right]_{t o t a l, k, L}=\sum_{d=1}^{D_{M}} \sum_{t=100 G M T}^{2400 G M T}\left(\frac{\left[O_{3} N\right]_{k, L, t}}{\left[O_{3}\right]_{k, t o t a l, t}}\right) \times\left(O_{3} D D_{k, \text { total }, t}+O_{3} W D_{k, \text { total }, t}\right)
$$


where $O 3 D D$ and $O 3 W D$ are the mass (in grams) of the total ozone deposited in grid cell $k$ at time $t$. The areas covered by vegetation have been derived from the USGS land-use / land-cover datasets used in the CAMx model. The exposure level characterization factors for VOC have similar expressions.

The human health effect level characterization factor for $\mathrm{NO}_{\mathrm{x}}$ is based on the C-R function calculated by Bell et al [2004] and is given as

$$
C F_{L, N O x, \text { hum }, \text { eff }}=\frac{\sum_{k \in D}\left[O_{3} N\right]_{\text {avg }, k, L} \times 1000 \frac{p p b}{p p m} \times \frac{m_{k}}{365} \times 0.00052}{E_{N O x, L}}
$$

where $D$ is the entire horizontal modeling domain, $m_{k}$ is the annual mortality in grid cell $k$ excluding non-resident deaths and deaths resulting from injuries or other external causes. $m_{k}$ is derived from the county-level annual mortality by distributing it to the corresponding grid cells in proportion to the population distribution. The county level annual mortality data is obtained from Centers for Disease Control and Prevention's Wide-ranging ONline Data for Epidemiological Research (CDC-WONDER) [http://wonder.cdc.gov]. It is the average mortality for the period 1999-2005 corresponding to ICD 10 codes corresponding to non-accidental mortality. The effect-level characterization factor for VOC is calculated similarly.

The C-R function of $0.052 \%$ increase per ppb increase in average ozone concentration represents the average coefficient for the range of data analyzed by Bell et al [2004]. This coefficient varies with the ambient ozone concentration. Therefore, the increase in concentration from $30 \mathrm{ppb}$ to $40 \mathrm{ppb}$ would have a lower coefficient compared to the coefficient for an increase from $70 \mathrm{ppb}$ to $80 \mathrm{ppb}$. The average coefficient aggregates this non-linear function.

A summary of the proposed characterization model described in this chapter is illustrated in Figure 5-7. 


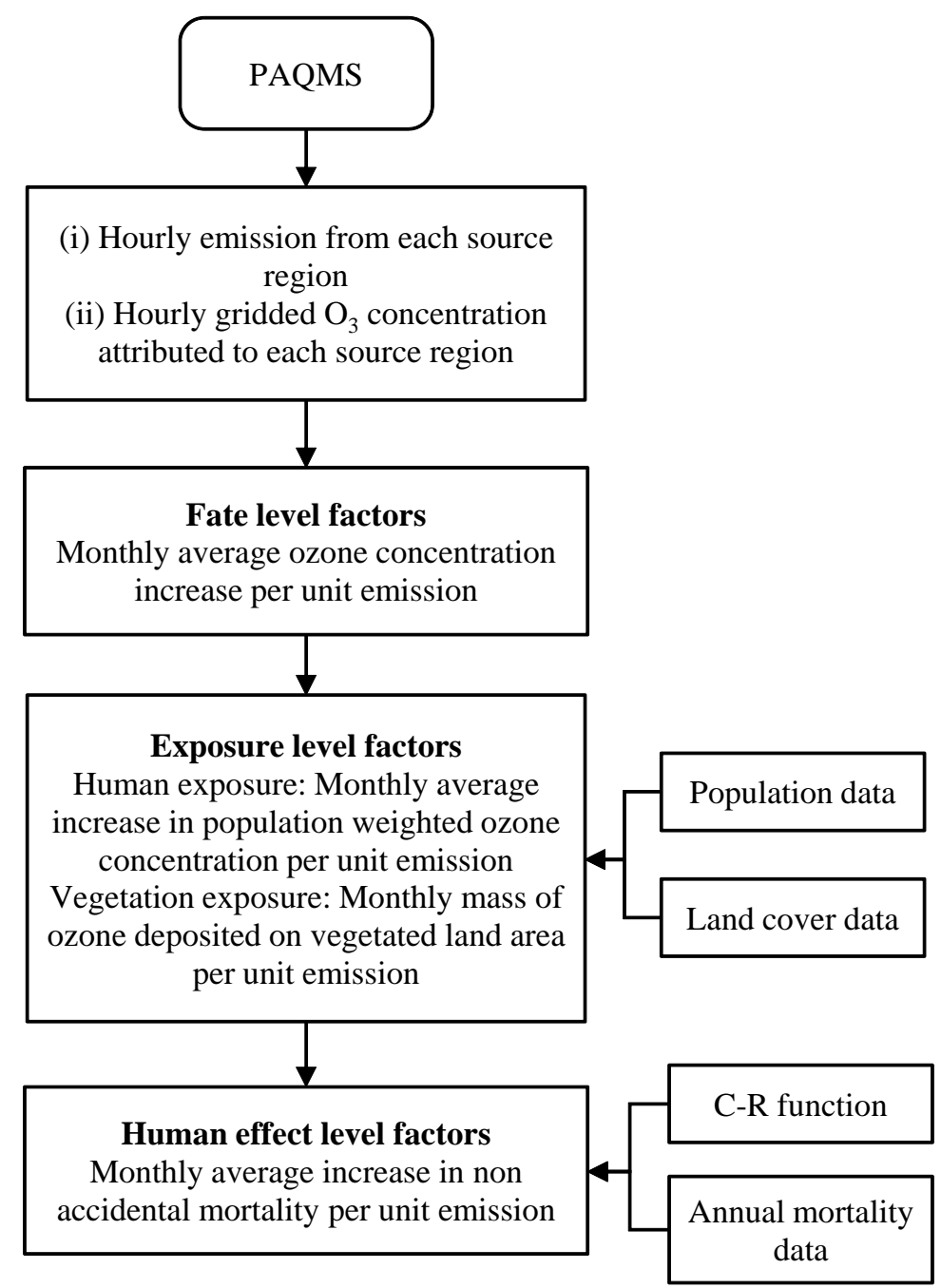

Figure 5-7 Summary of the proposed characterization model 


\subsection{RESULTS AND DISCUSSION}

The proposed characterization model for the impacts on human and ecosystem health has been described in Chapter 5.0. The fate and transport of photochemical products and their precursors is simulated for the year 2001. Three levels of characterization factors are calculated at the fate, exposure, and effect levels. The results of the simulation are presented in this section.

\subsection{RESULTS OF THE FATE MODEL}

The fate and transport model predicts the atmospheric ozone concentration in the atmosphere for the entire domain, and also its source distribution. Each state in the coterminous USA including Washington DC has been identified as a distinct source in the model. This source attribution of ozone formation has been used to calculate the state specific characterization factors.

Among the output files, four files are used for the calculation of characterization factors. These include the following: (a) concentration file for selected modeled chemical species (photochemical products and their precursors), (b) dry and wet deposited mass of ozone, (c) concentration file for the non-reactive tracer species of $\mathrm{NO}_{\mathrm{x}}$ and $\mathrm{VOC}$ that are emitted from each source region, and (d) a diagnostic file that gives, besides other information, the modeled anthropogenic emissions from each state. Visualizations of a very small set of sample outputs are shown in Figure 6-1 and Figure 6-2.

Figure 6-1 shows the predicted ozone concentration across the domain on 4 June, 2001, at 0000, 6000, 1200, and 1800 hours GMT. The GMT is used for visualization as well for calculating the characterization factors to avoid the difficulties due to the presence of four time zones in the modeled domain. As is usual during the summer, the highest ozone concentrations are seen on the East Coast and in some parts of California. The predicted ozone concentration 
lies in the range of over $0.070 \mathrm{ppm}$ in some areas in late evening to about $0.020 \mathrm{ppm}$ in a few areas at early morning. Figure 6-2 is a sample output from the source apportionment module. It shows the predicted surface concentration of ozone which is attributed to anthropogenic $\mathrm{NO}_{\mathrm{x}}$ and VOC emissions from Pennsylvania. It shows snapshots of predictions at 1800 GMT on 4, 5, 6, and 7 May, 2001. It can be seen that there is non-uniformity in the pattern of distribution of the photo-oxidants due to changing meteorological conditions. Most of the ozone formed due to emissions in Pennsylvania is concentrated over the surrounding states. However, the effects of emissions in Pennsylvania can be seen as far as North Carolina on one day and over Canada on the next day. The prevalent wind direction in US is from the west towards the East, therefore, some of the ozone formed around Pennsylvania is transported to the Atlantic Ocean

An important aspect of fate and transport modeling is the comparison of the predicted concentration field and the observed field concentrations. However, a detailed model evaluation is beyond the scope of this study. A comparison between the predicted and observed concentration is an indicator of the performance of the modeling system, which includes the meteorology and emission models in addition to the chemistry and transport model. As noted in Chapter 3.0, differences in predicted and observed concentration can arise due to uncertainty in the inputs as well as in the model formulation. Figure 6-3 shows the performance of the model for the entire simulation period. Figure 6-3 (a) is a comparison between the domain averages of the predicted and observed maximum 8-hour concentrations at each grid cell for each day. The observed concentrations are obtained from the Air Quality System (AQS) yearly raw data maintained by the USEPA [http://www.epa.gov/ttn/airs/airsaqs]. Therefore, the domain includes only those sites that are monitored under the AQS. The predicted concentration is the concentration in the grid cell in which the field monitor is located. The predicted concentration is not expected to be exactly equal to the observed concentrations, because of the coarse grid resolution, both, horizontally and vertically, in addition to the uncertainty in model inputs and parameters. 


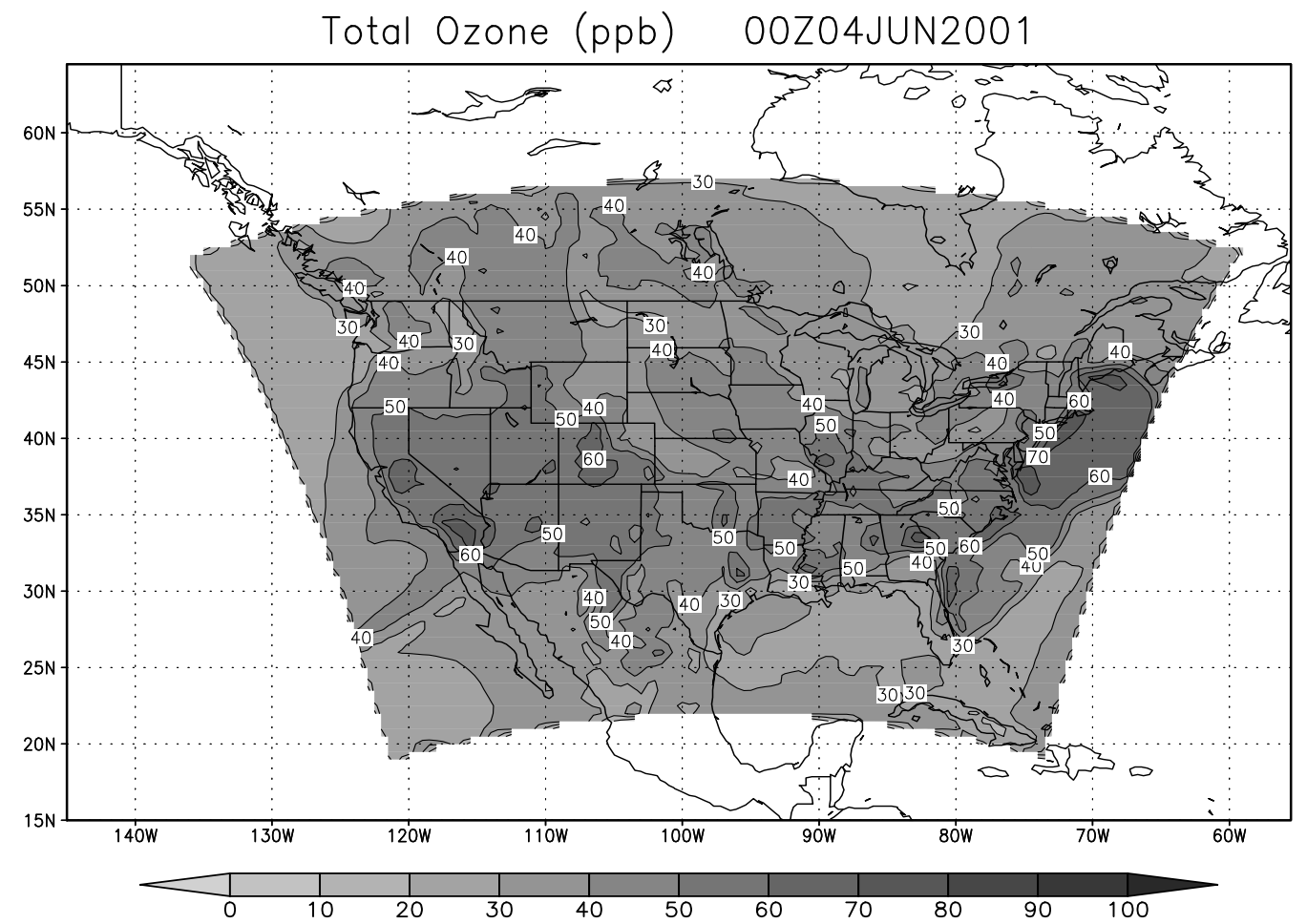

(a) Predicted ozone concentration (in ppb) at 0000 GMT on June 04,2001

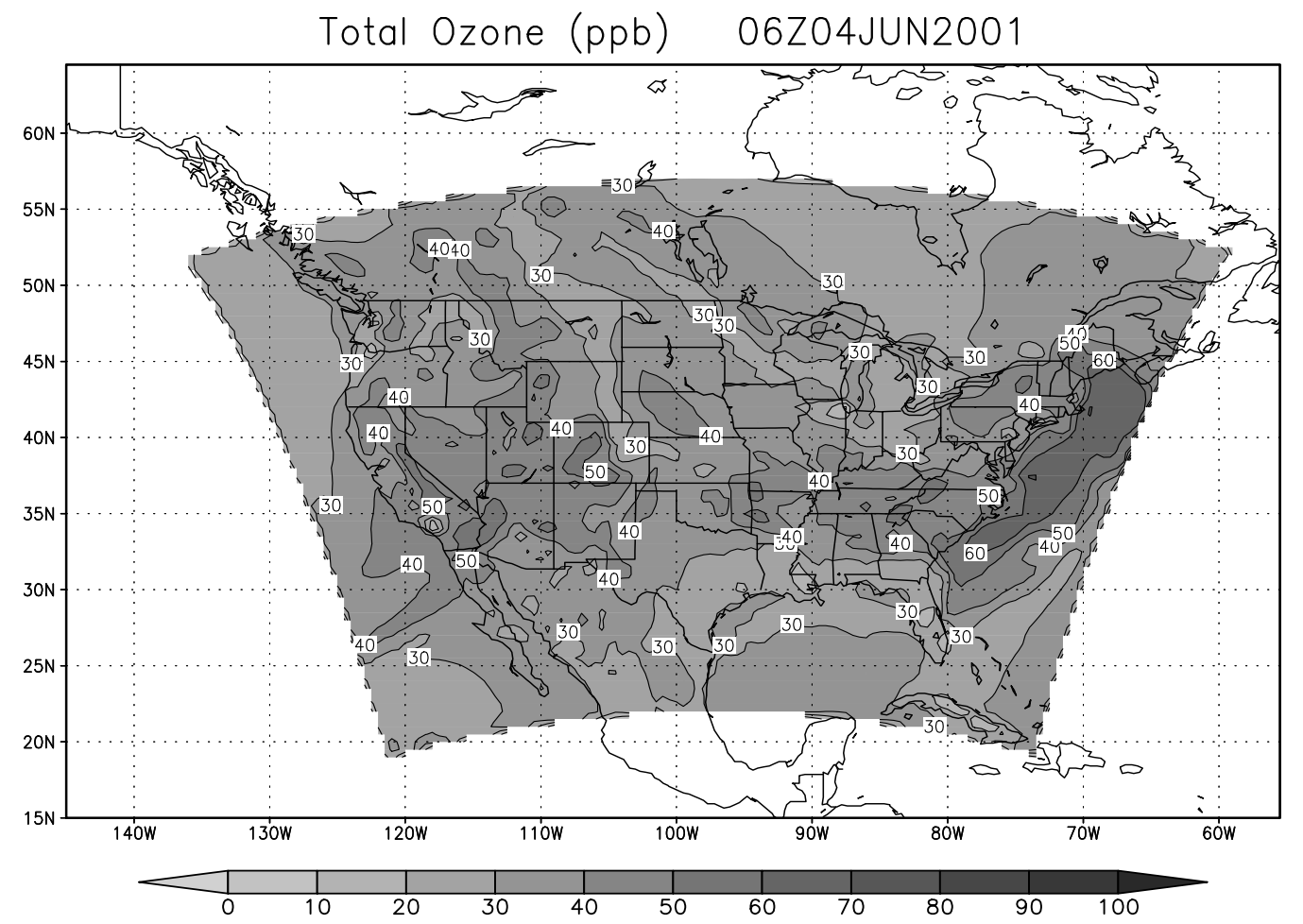

(b) Predicted ozone concentration (in ppb) at 0600 GMT on June 04,2001

Figure 6-1 Visualization of a sample simulation output 


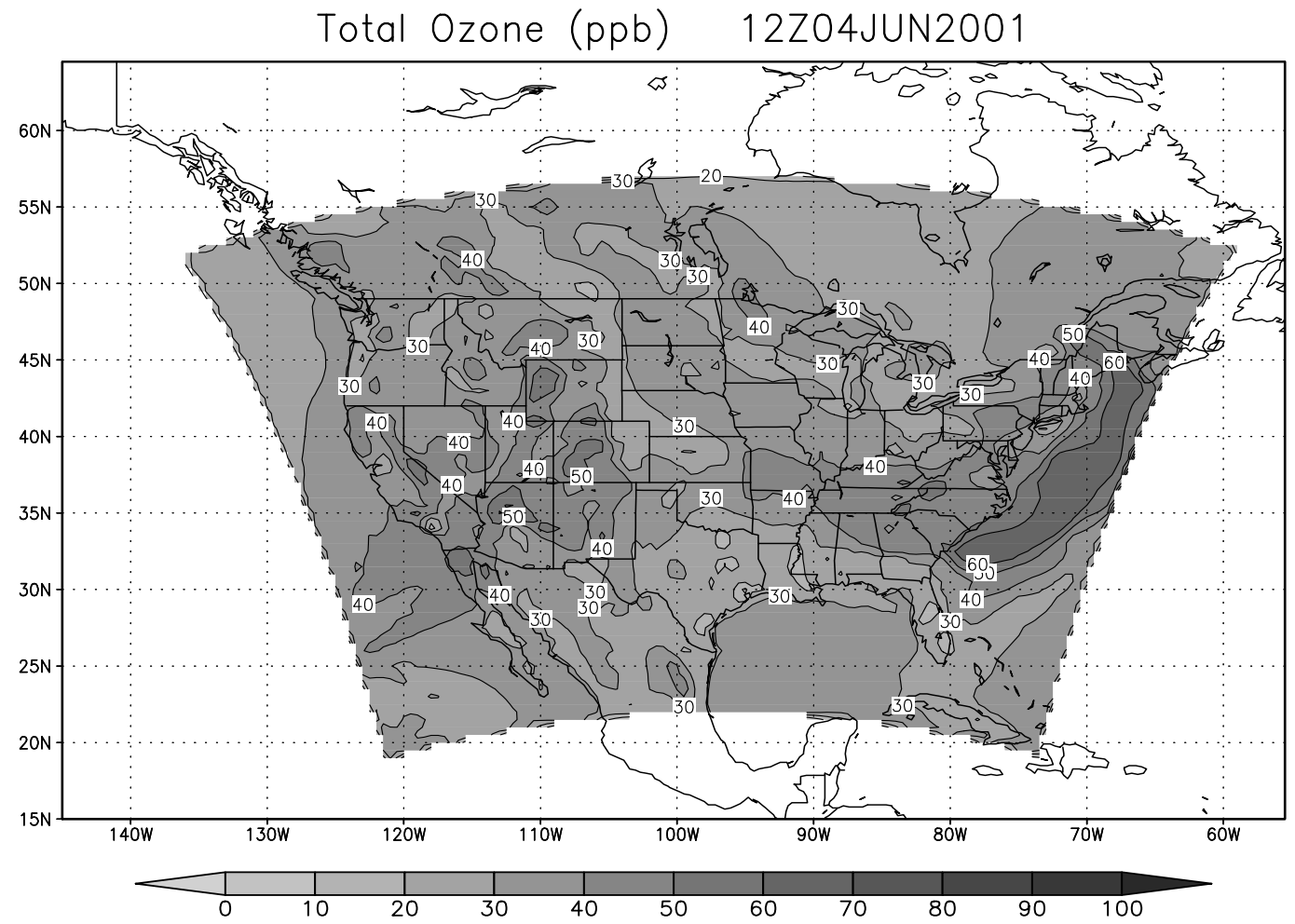

(c) Predicted ozone concentration (in ppb) at 1200 GMT on June 04,2001

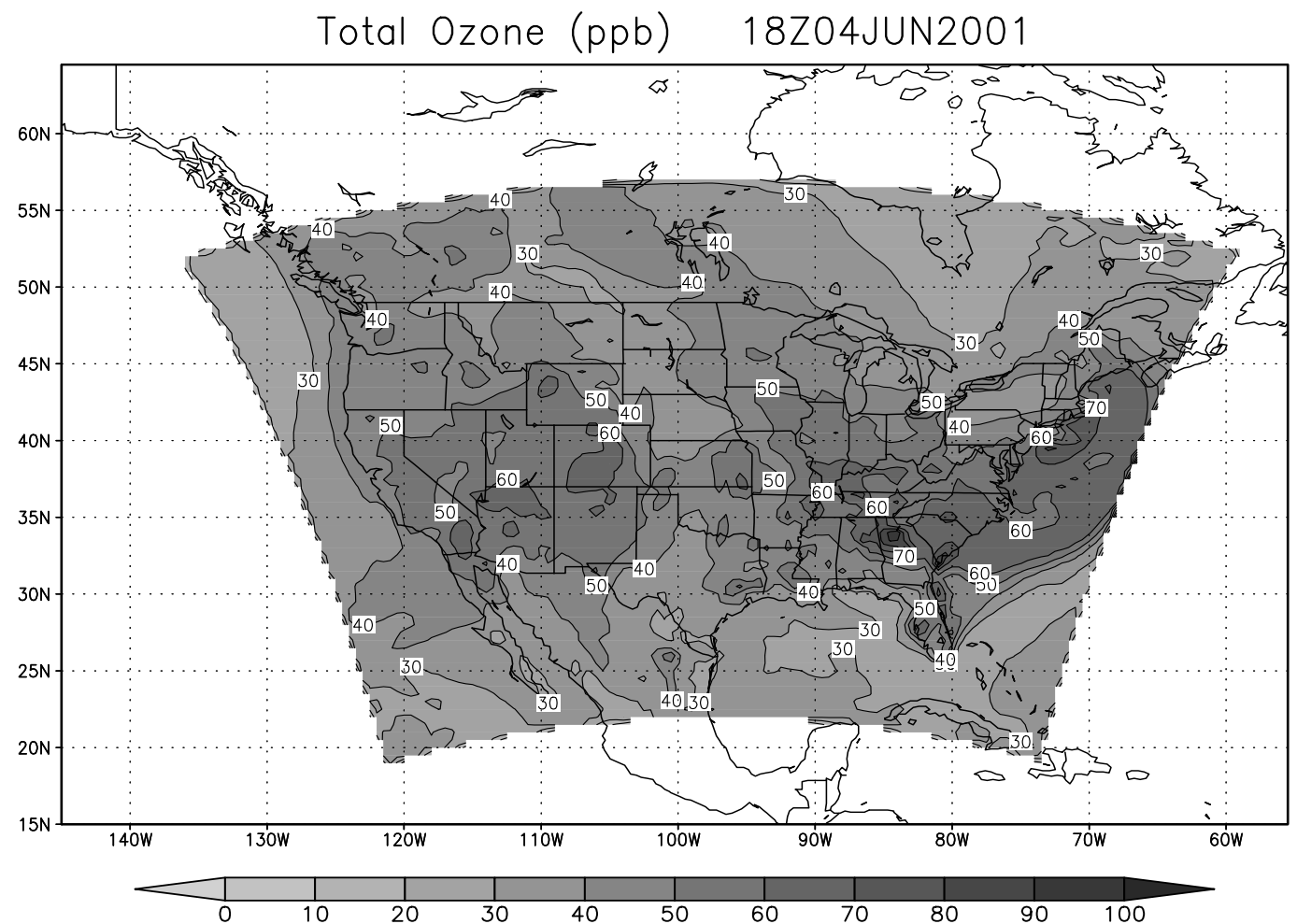

(d) Predicted ozone concentration (in ppb) at 1800 GMT on June 04,2001

Figure 6-1 continued 


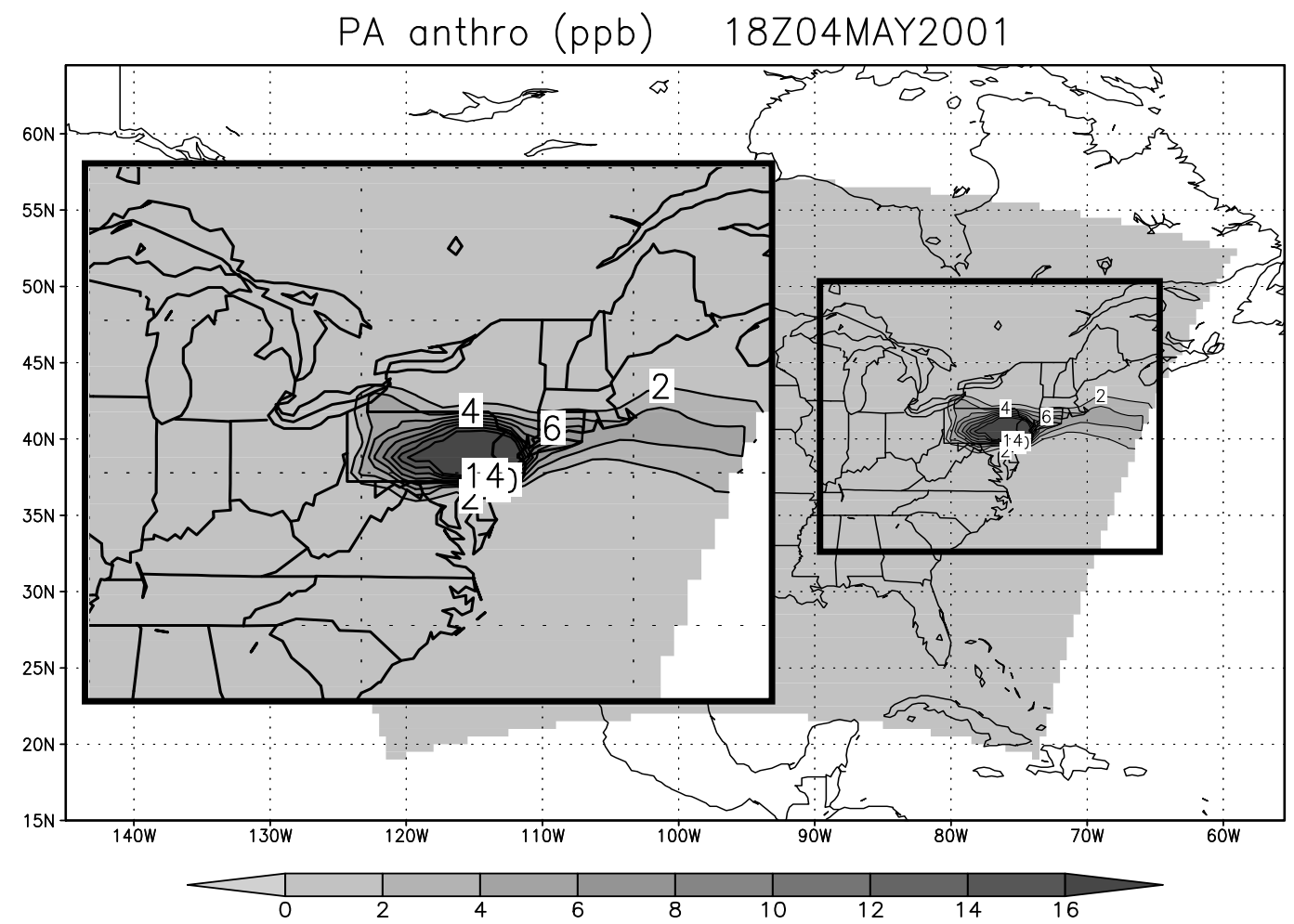

(a) Predicted ozone (in ppb) due to PA anthropogenic emissions at 1800 GMT on May 04,2001

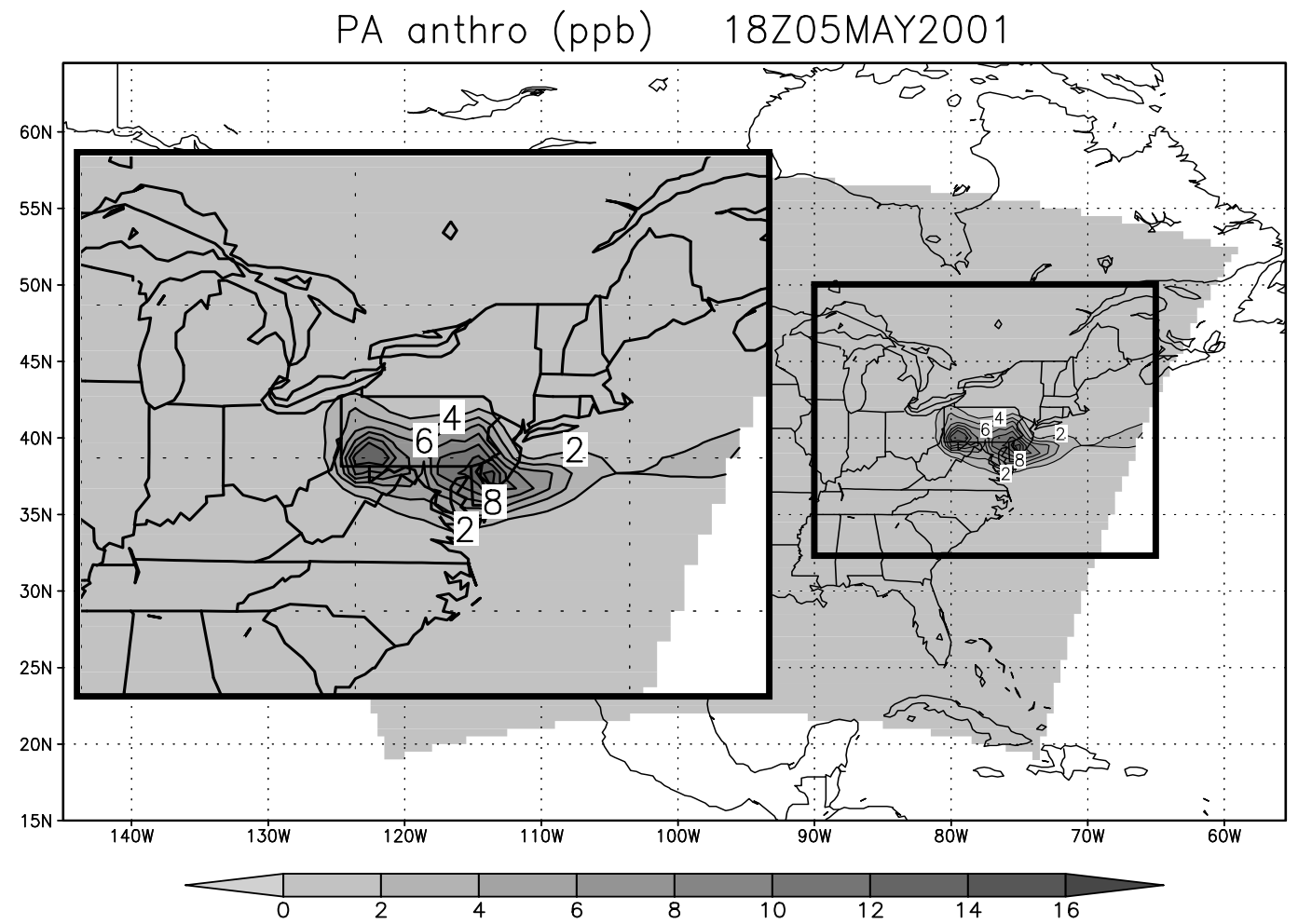

(b) Predicted ozone (in ppb) due to PA anthropogenic emissions at 1800 GMT on May 05,2001

Figure 6-2 Visualization of a sample source apportioned ozone concentration 


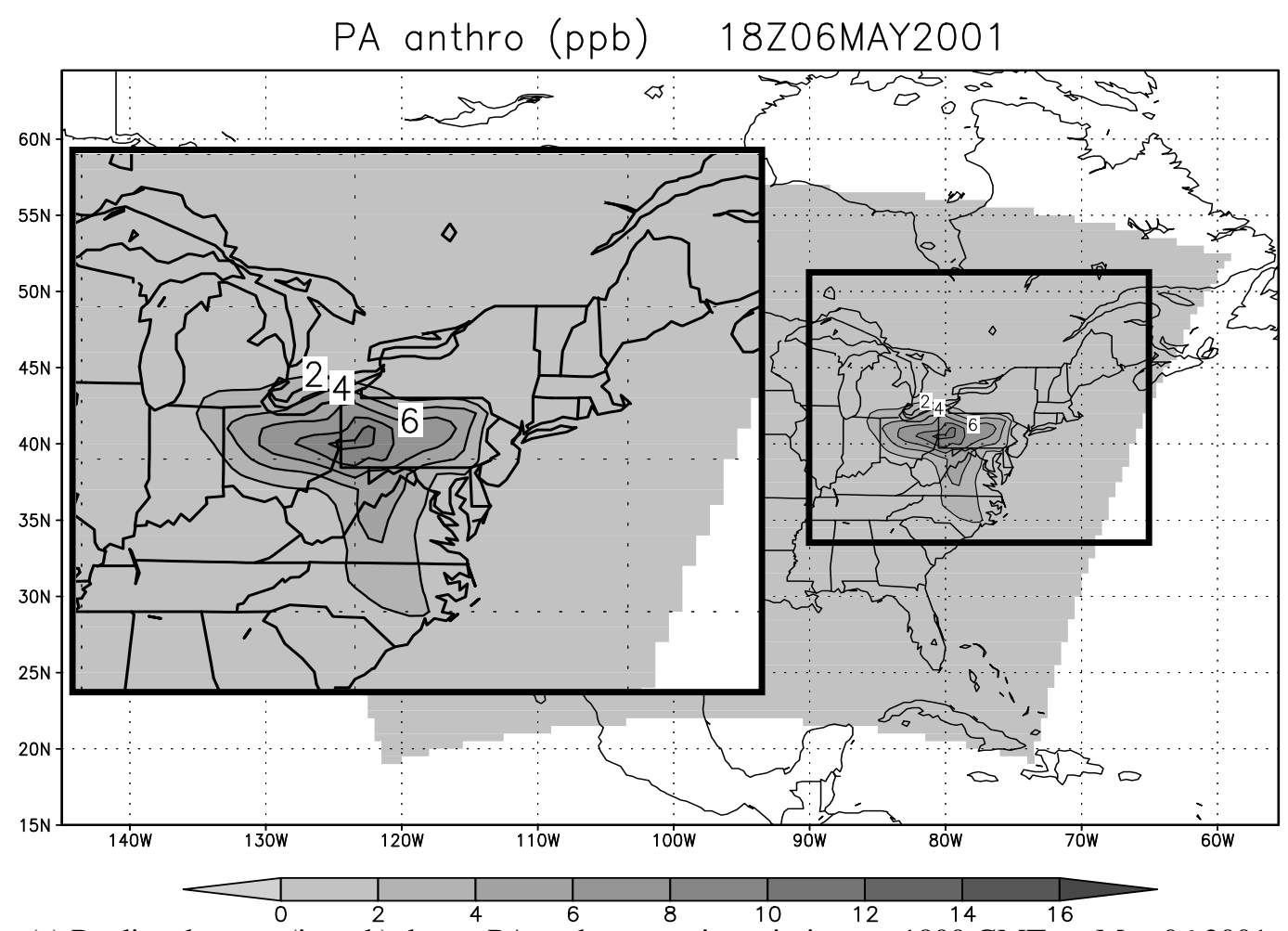

(c) Predicted ozone (in ppb) due to PA anthropogenic emissions at 1800 GMT on May 06,2001

PA anthro (ppb) 18Z07MAY2001

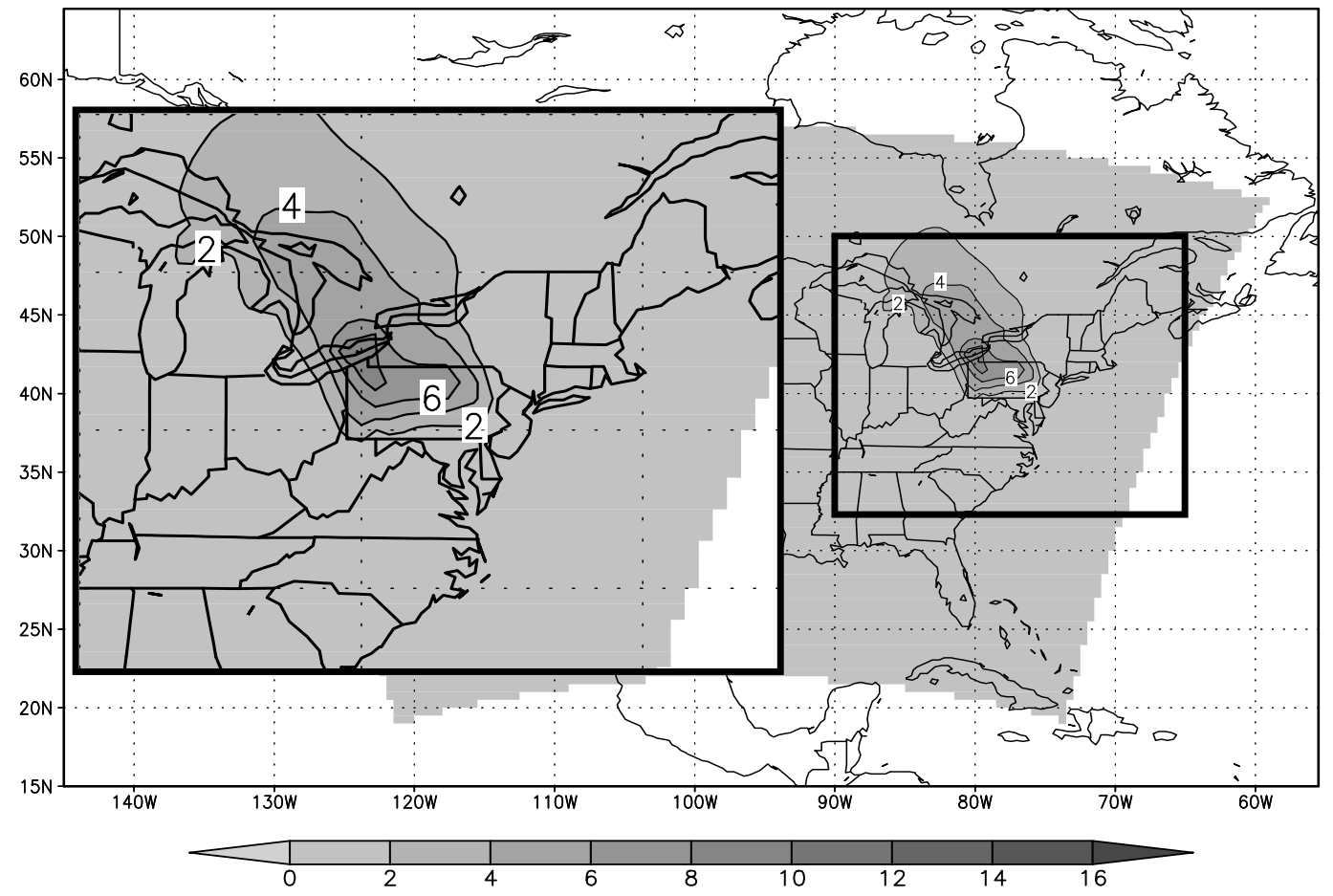

(d) Predicted ozone (in ppb) due to PA anthropogenic emissions at 1800 GMT on May 07,2001

Figure 6-2 continued 


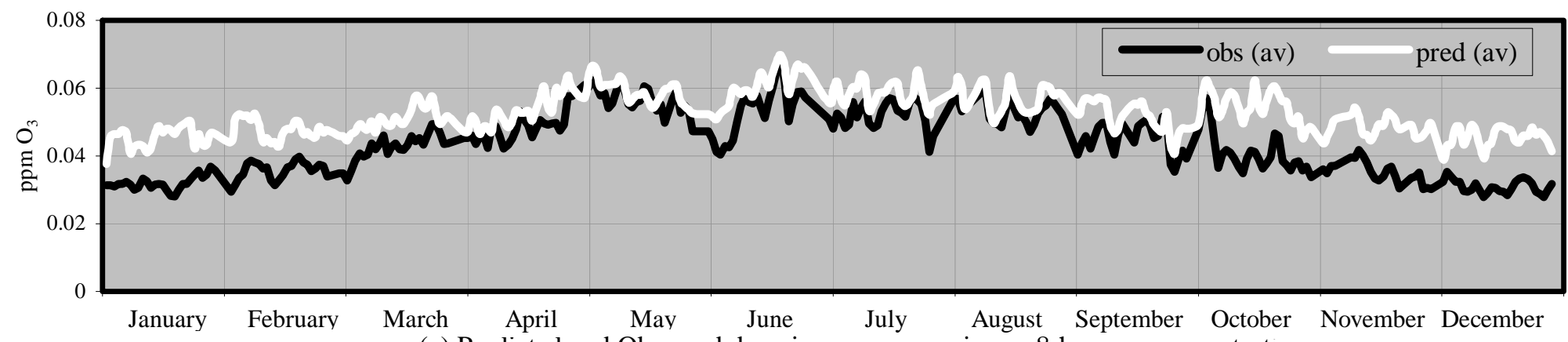

(a) Predicted and Observed domain average maximum 8-h ozone concentratıon

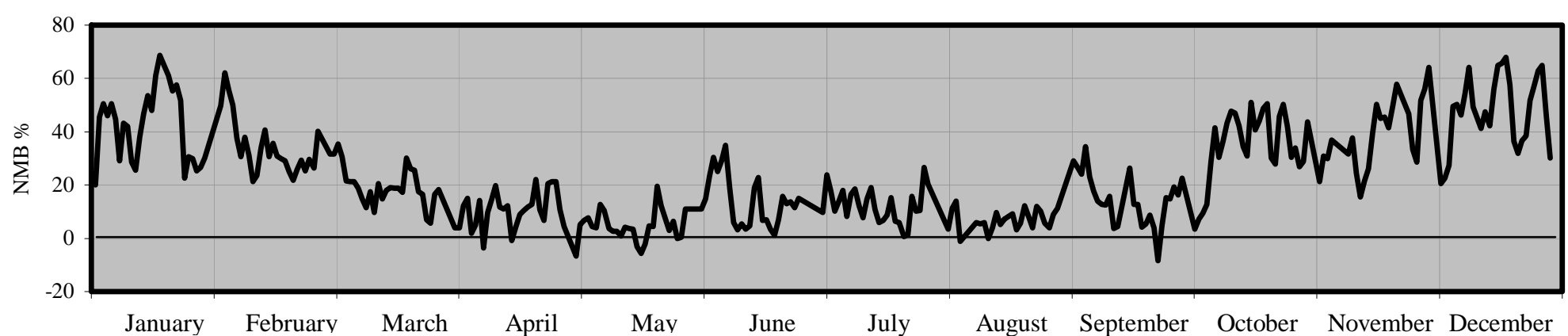

(b) Normalized mean bias (NMB) for the entire simulation perıod

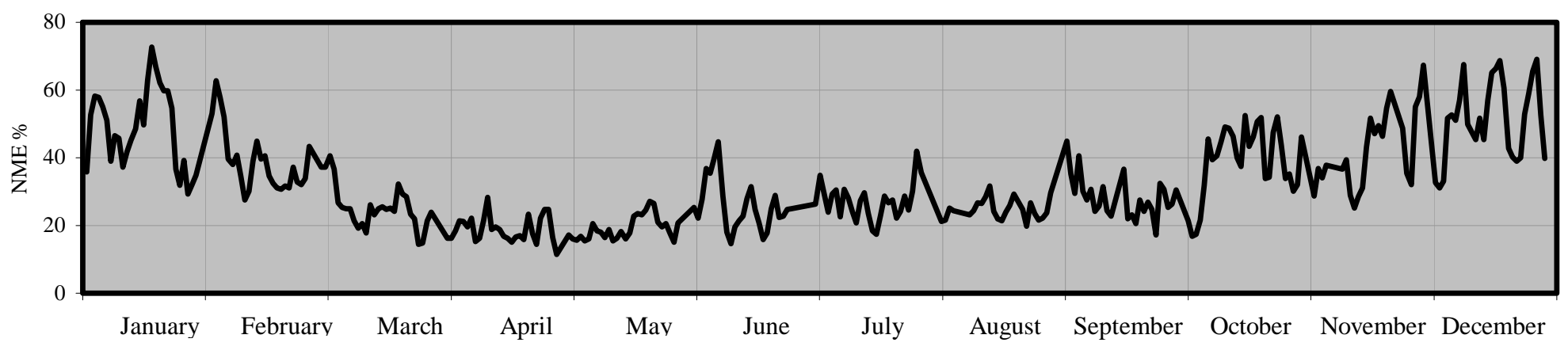

(c) Normalized mean error (NME) for the entire simulation period

Figure 6-3 Model performance with respect to observed concentrations for the entire domain 
Figure 6-3 (b) and (c) show the Normalized Mean Bias (NMB) and the Normalized Mean Error (NME) for the simulation period. These are two indices commonly used for model performance evaluation [USEPA 2007b]. Mathematically, they are defined as

$$
\mathrm{NMB}=\frac{\sum_{i=1}^{N}\left(P_{i}-O_{i}\right)}{\sum_{i=1}^{N} O_{i}} \text { and } \mathrm{NME}=\frac{\sum_{i=1}^{N}\left|P_{i}-O_{i}\right|}{\sum_{i=1}^{N} O_{i}}
$$

where $P_{i}$ and $O_{i}$ are the predicted and observed daily maximum 8-hour concentrations. They represent average deviation or error as a percentage of the average ozone across the domain.

From Figure 6-3, it is clear that the predicted concentrations follow the same trend as the observed concentration, although they are over-predicting for almost the entire simulation period. The model performs very well in the period of March to September. The NME and NMB, during this period lie below $40 \%$ and $20 \%$, respectively. During the winter months, the performance decreases when the NME and NMB lie in the range of 20 to $75 \%$. It is seen that the predicted concentrations are closely matched with the observed when the domain average 8-h maximum ozone concentration is above $0.04 \mathrm{ppm}$. One explanation for the relatively poor performance of the model in the winter months could be that these models, particularly the chemistry and transport models, are used extensively to model short ozone episodes which occur during the summer months (March to September). Hence, most of the calibration and parameter estimation for the model has been performed for simulations involving such meteorological conditions. Therefore, the model might be over-predicting the concentrations during the winter months. Uncertainties in the meteorology, emission models, and choice of initial and boundary conditions have also have a significant effect on the uncertainty of the predicted concentrations.

Notwithstanding the model performance, the predicted concentration field will be used to calculate the impact factors. Using a modified concentration obtained by reducing the predicted concentration by a factor equal to the normalized error was considered. However, this was discarded due to the limited spatial coverage of the AQS domain, which is seen in Figure 2-4.

Figure 6-4 shows the contribution of the major groups of sources in the monthly average 24 hour ozone concentration. These major groups are: (i) anthropogenic ozone which is the ozone attributed to all anthropogenic emissions from the model domain, (ii) biogenic ozone 
which is attributed to the biogenic emissions in the domain, and (iii) domain boundary ozone which is the component transported from the lateral and the top boundaries or formed due to precursors entering from the boundary. It can be seen that the contribution of the ozone attributed to boundary conditions is substantial. In the summer months, from May to September, the boundary condition ozone is at least $60 \%$ of total ozone concentration across the entire domain. In the winter months, this contribution increases to more than $90 \%$. The anthropogenic component is the highest during the summer months, where it reaches around $25 \%$, but decreases sharply in the winter. The biogenic component contributes relatively less during the entire year. Another very small component of the total ozone is the ozone attributed to initial conditions. This has not been shown in Figure 6-4.

Fiore et al [2003] have calculated the afternoon background concentration over US for the year 2001 using the GEOS-CHEM global model. They simulated one base scenario and two additional scenarios, one with all anthropogenic emissions set to zero, and the other with only North American anthropogenic emissions set to zero. The contribution of each source was calculated from the reduction in ozone from the base case. It was found that the background, that is, all ozone which is not formed due to North American anthropogenic emissions generally lies in the range of 15-35 ppb. It is highest during the winter and decreases during the spring. It further decrease during the summer months. The results obtained from the current simulation follow a similar pattern. However, the ozone formed due to boundary conditions in the current simulation has a slightly higher relative contribution than the contribution of background concentration seen by Fiore et al [2003]. An important difference between the background concentrations and boundary conditions is that boundary conditions, typically, include ozone which can be attributed to anthropogenic or biogenic emissions of the domain. The boundary conditions for the current simulation are time-invariant concentrations representing the monthly average conditions at the boundary. A part of the boundary ozone and precursors could have actually been formed due to the emissions from certain US states, which are then transported back into the model domain. The source apportionment procedure cannot capture this since the boundary conditions are a model input and are defined outside the simulation. The model treats these as just another anthropogenic source of precursors and, in this case, it also includes transported ozone. Thus, the ozone attributed to boundary conditions should be actually higher than the background concentration. 


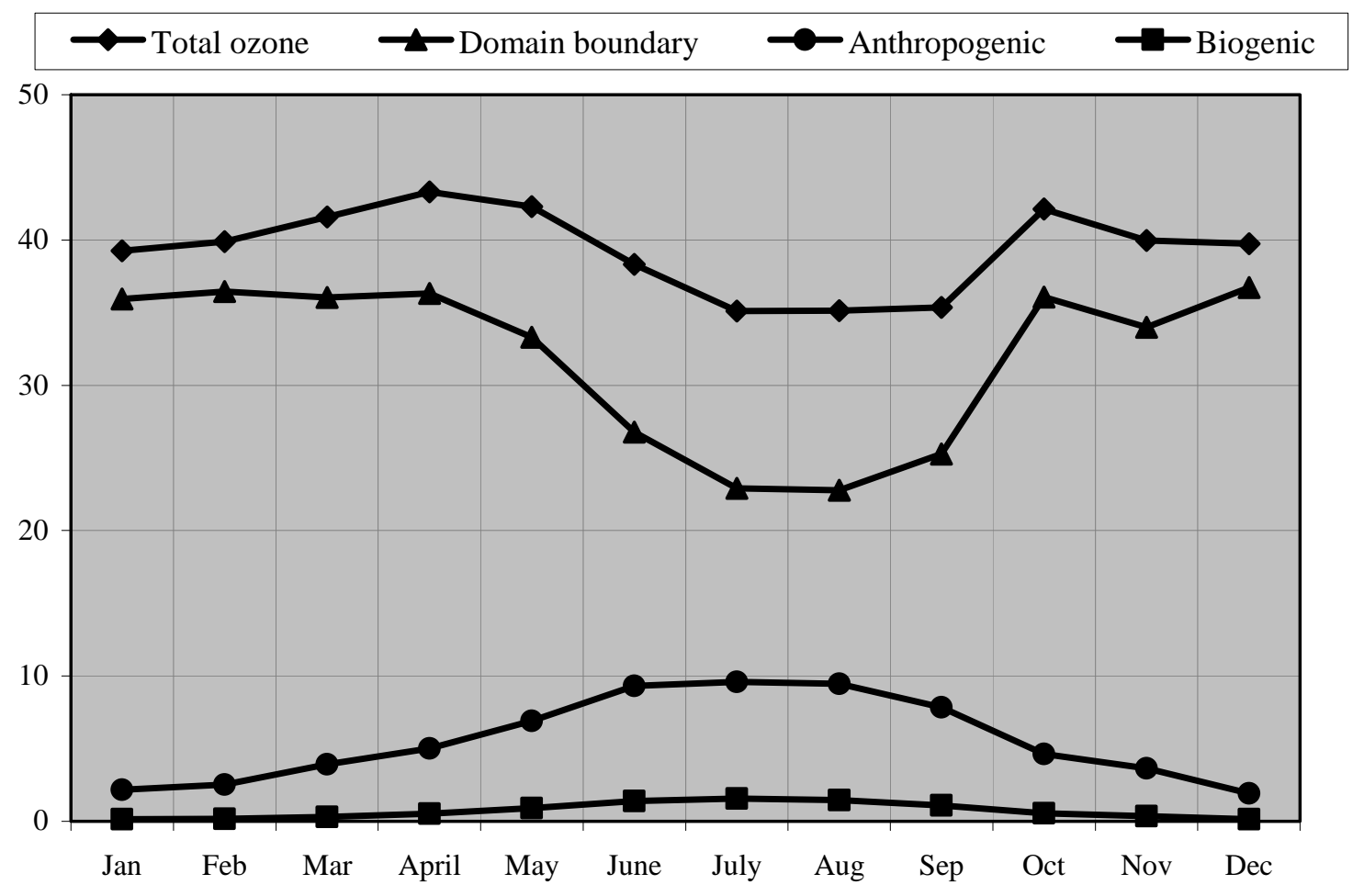

Figure 6-4 Source apportionment of the monthly averaged daily ozone concentration 


\subsection{RESULTS OF THE CHARACTERIZATION MODELS}

Three levels of characterization factors are calculated for each state in the continental US for every month of the year. The results of the characterization model are discussed below.

\subsubsection{Fate level characterization factors}

The fate level characterization factor represents the average increase in surface ozone concentration in the areas affected by $\mathrm{NO}_{\mathrm{x}}$ and VOC emissions in a state. The fate level characterization factors are presented in Figure 6-5 and Figure 6-6. The units of the fate level factors for $\mathrm{NO}_{\mathrm{x}}$ and VOC are in ppb per MT of emissions, except that, for VOC, the emissions are weighted with the dimensionless MIR values. From top to bottom, the US states are divided into four sections that are similar to the four US census regions. The divisions are shown in Figure 6-7. The characterization factors on the Y-axis are plotted on a logarithmic scale. For each state, the monthly factors are shown with vertical bars starting with January on the left. The horizontal line represents the annual average characterization factor for the US. 

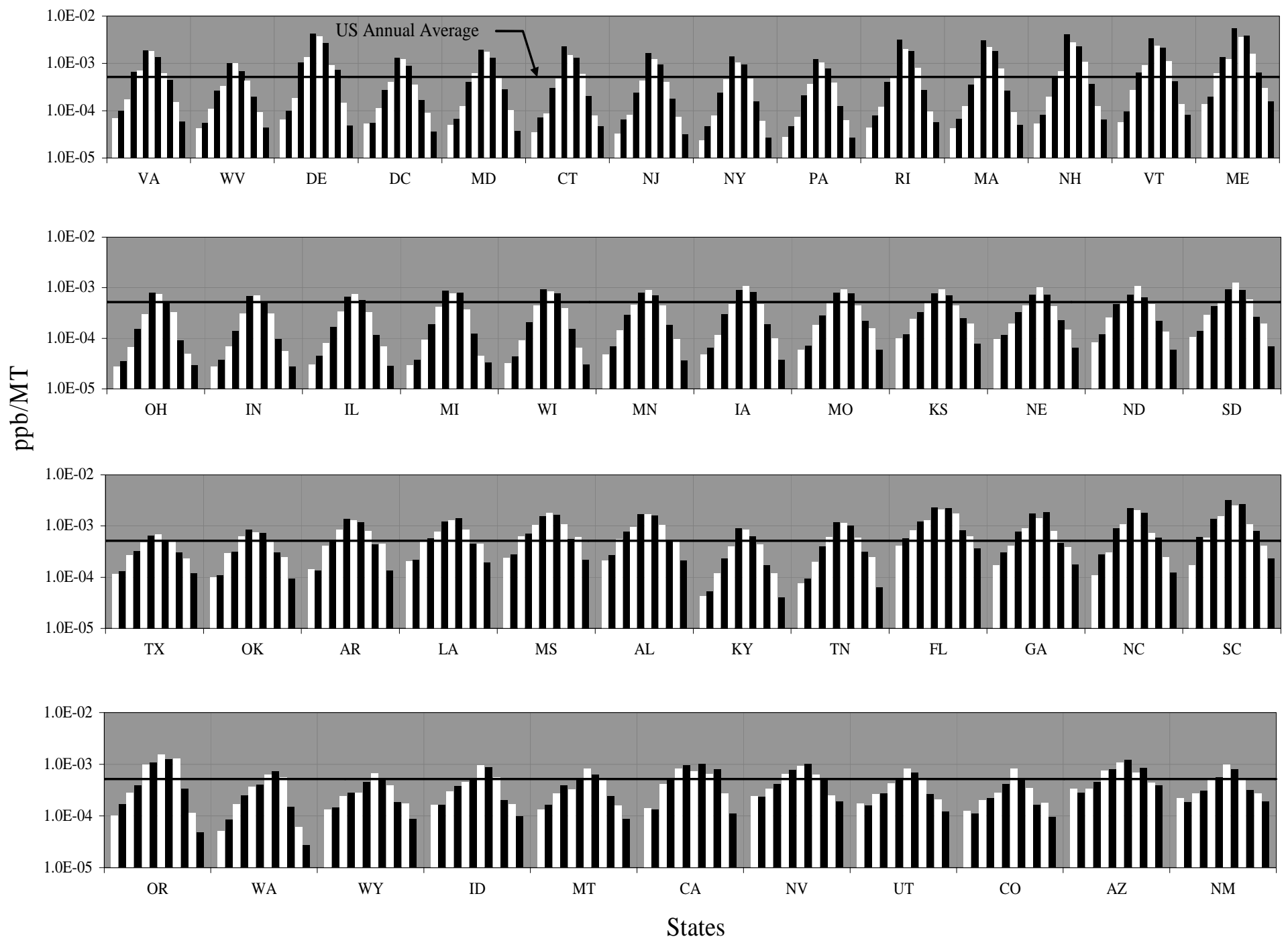

Figure 6-5 Fate level characterization factors for NOx emissions 
$\square$ January $\square$ February $\square$ March $\square$ April $\square$ May $\square$ June July $\square$ August September $\square$ October $\square$ November $\square$ December
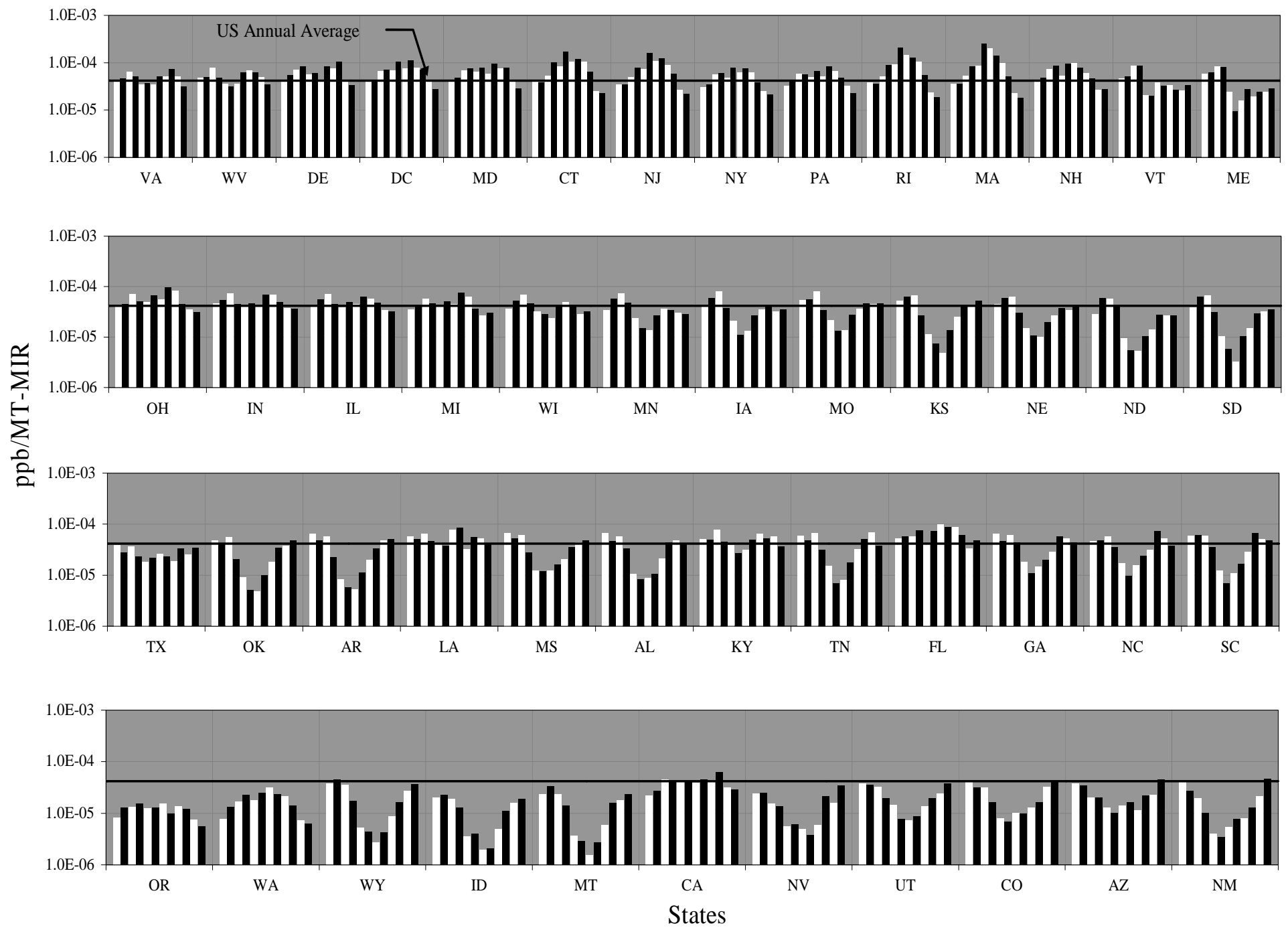

Figure 6-6 Fate level characterization factors for VOC emissions 


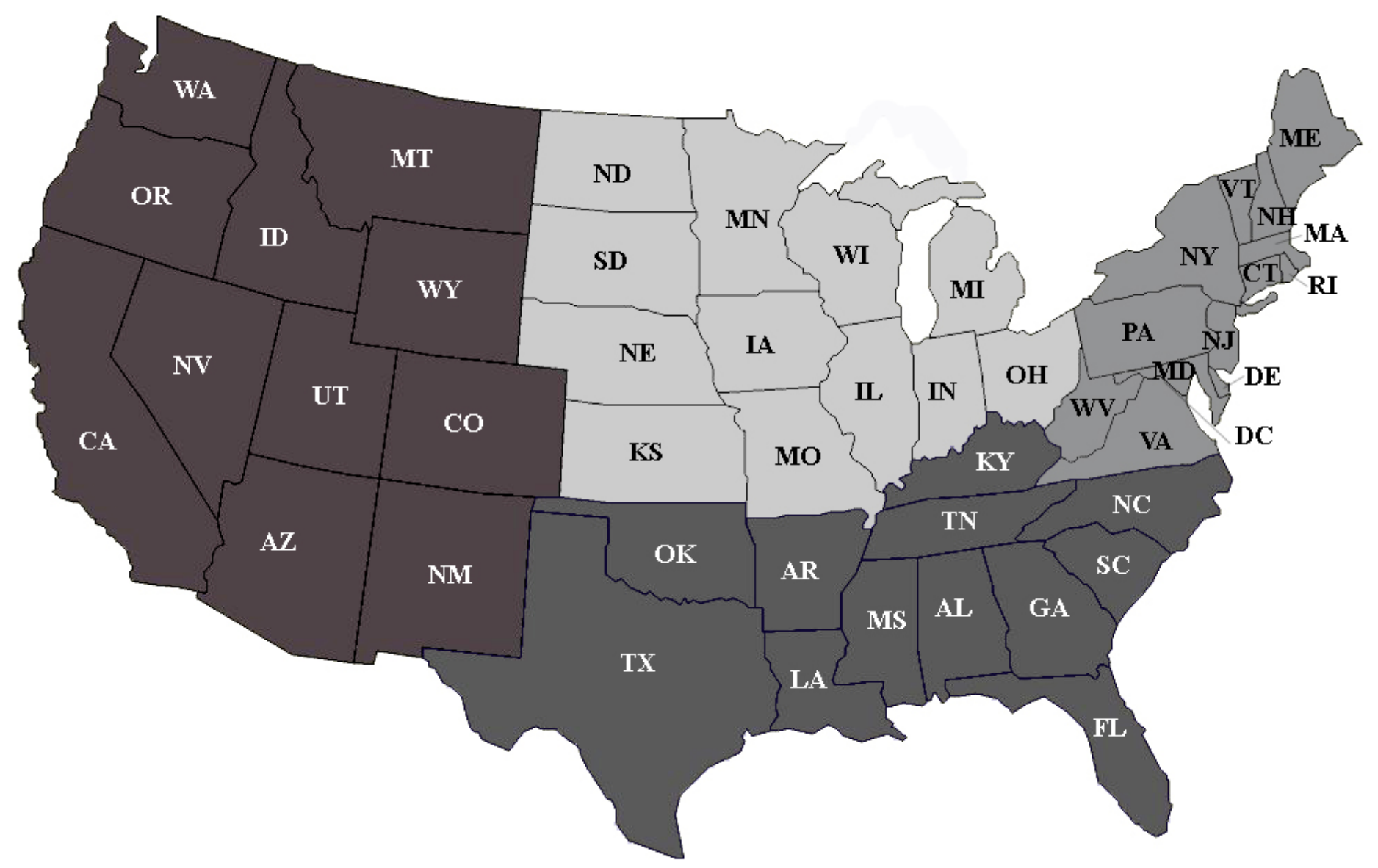

Figure 6-7 Map of USA showing the states and the four divisions 


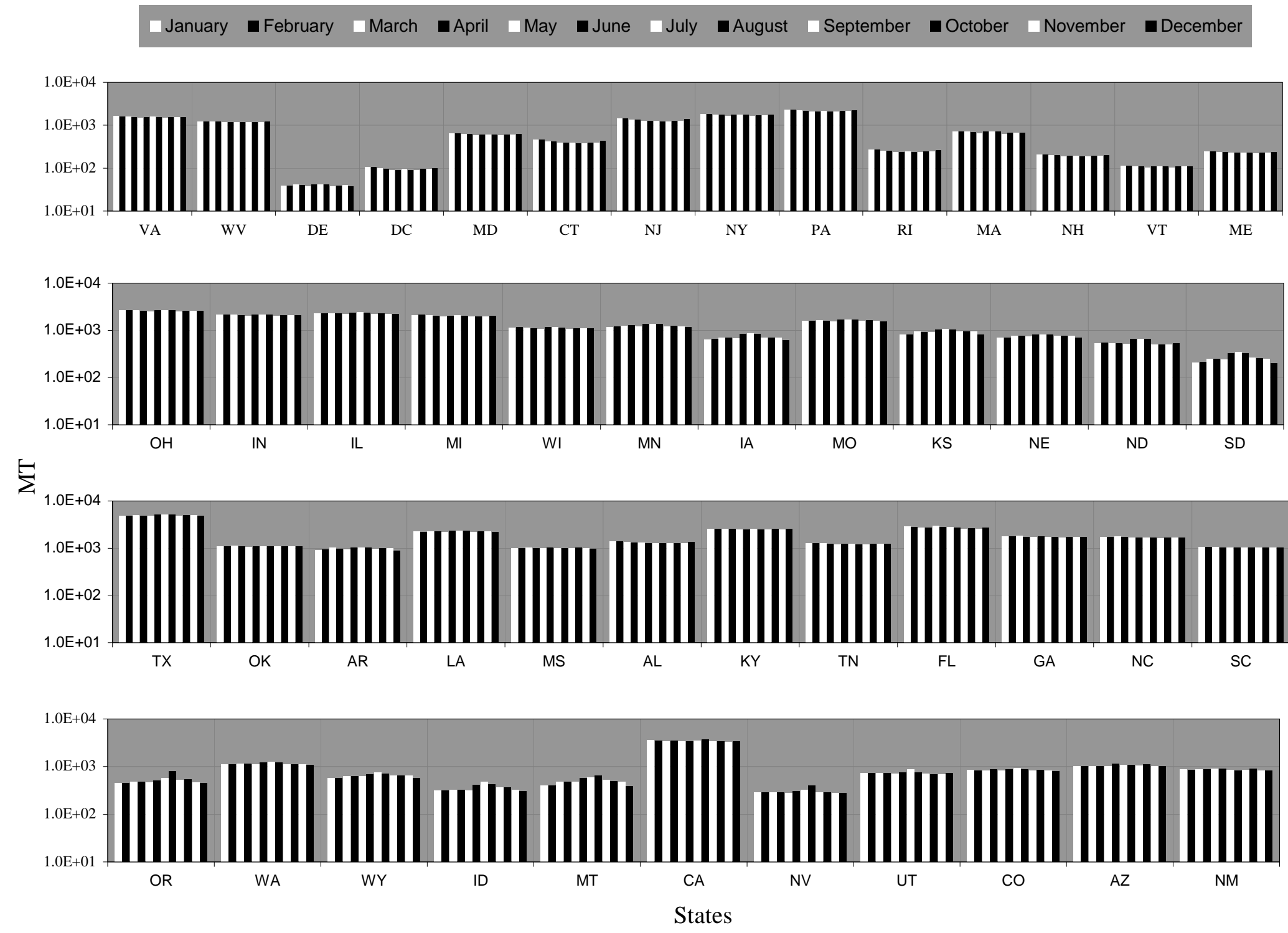

Figure 6-8 Daily average anthropogenic emissions of NOx 


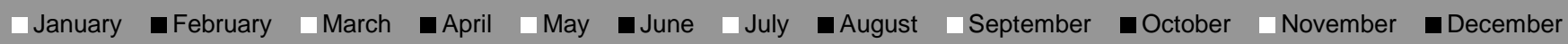
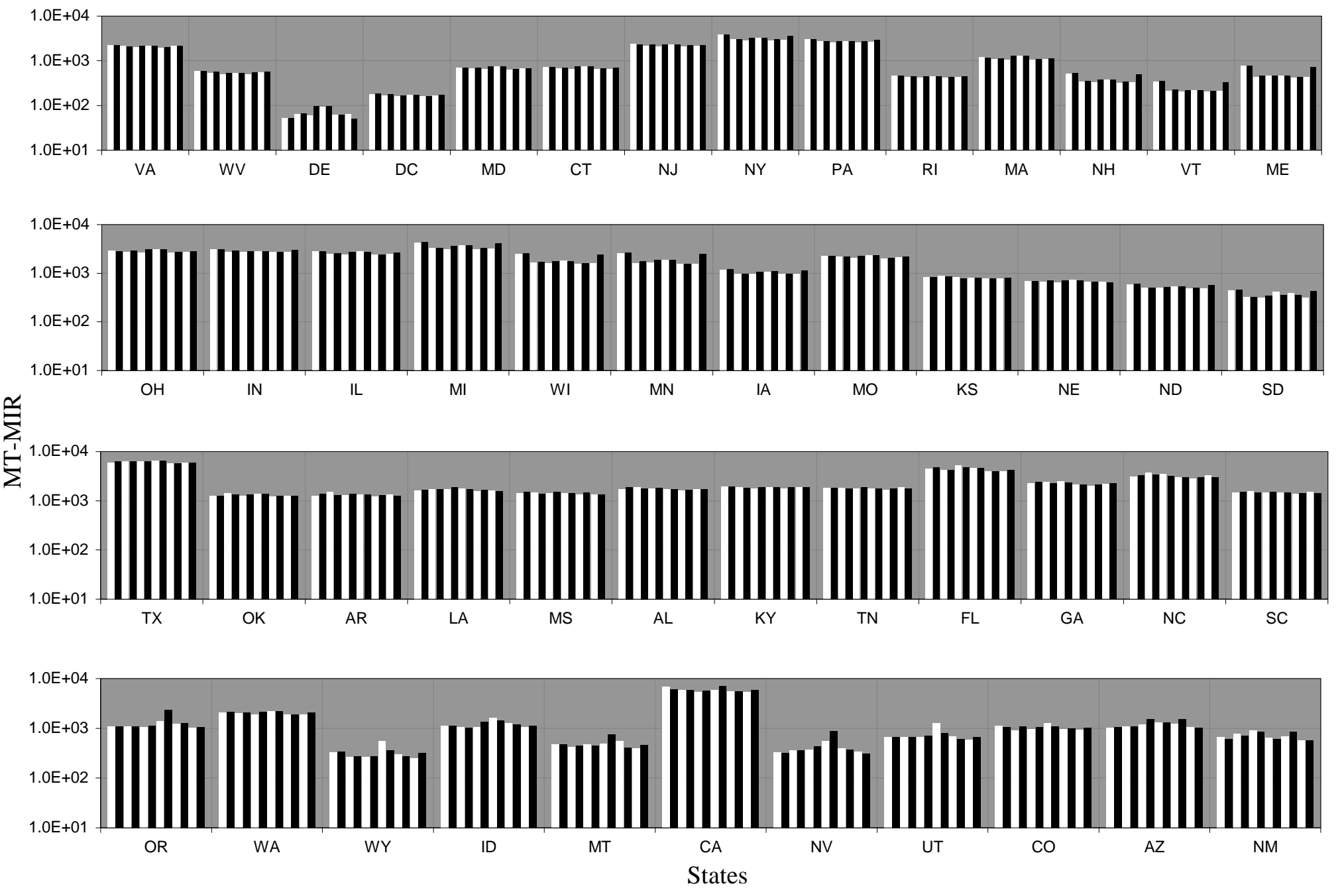

Figure 6-9 Daily average MIR weighted anthropogenic emissions of VOC 
The daily average emissions of $\mathrm{NO}_{\mathrm{x}}$ and VOC for each state for each month are shown in Figure 6-8 and Figure 6-9. These are not the state level emissions outputted by SMOKE, but the emissions considered in the CAMx OSAT model for each source region. There is a negligible difference in these two sets since the grid size limits the resolution of the state edges. The emissions of VOC are weighted with the average MIR values for the VOC species to reduce the effect of variability in reactivity.

As can be seen in Figure 6-8, there is very little variability in the anthropogenic $\mathrm{NO}_{\mathrm{x}}$ emissions from any state throughout the year. As expected, the variability across the states is very high. For instance, the anthropogenic $\mathrm{NO}_{\mathrm{x}}$ emissions in Texas are more than 100 times the emissions from smaller states like Delaware. The average emissions are lesser in the sparsely populated, although large, Western states, like Wyoming, Idaho, etc. than in the smaller Eastern states like New Jersey, Massachusetts, etc. The pattern for anthropogenic VOC emissions is similar to that of $\mathrm{NO}_{\mathrm{x}}$. However, the intra-annual variability of VOC emissions is little higher than that of $\mathrm{NO}_{\mathrm{x}}$. A few spikes in the emissions in the summer months are seen in the western states. The $\mathrm{NO}_{\mathrm{x}}$ and MIR weighted VOC emissions are of the same order of magnitude. However, the average MIR for the VOC mixture is in the range of 1.5 to 2.

The fate level characterization factors for $\mathrm{NO}_{\mathrm{x}}$, shown in Figure 6-5, represent the increase in monthly average daily ozone concentration in $\mathrm{ppb}$ in the affected region per MT increase in monthly average daily emissions. For example, the entire daily $\mathrm{NO}_{\mathrm{x}}$ emission from Pennsylvania in the month of May of about 2000 MT results in an increase of about $0.4 \mathrm{ppb}$ in the daily ozone concentration in the affected areas above the background concentration for the whole month. This is a small number, but it is important to consider that it is the 24 hour average concentration and is averaged over the almost the entire affected area which include places that are only marginally affected. Besides, the contribution of anthropogenic emissions to total ozone over the US is small compared to the background concentration. Conversely, a $10 \%$ increase in emission in Pennsylvania on any day in May will increase the 24 hour average concentration by $0.04 \mathrm{ppb}$ on, probably, the same day or on the following day.

The fate level characterization factors for $\mathrm{NO}_{\mathrm{x}}$ exhibit a very high variability across, both, the months and the states. However, the intra-annual variability is much larger than the spatial variability. Across US, the characterization factors are the highest in the summer months of June to August and decrease substantially during the winter months. For the Northern states the winter 
time factors are about two orders of magnitude lesser than the summer time factors, and for the Southern states these are about one order of magnitude lower. This is due to the higher temperatures and radiation in the Southern latitudes during the winter months. The highest factors, overall, are observed in the smaller North-Eastern and Southern states of Delaware, Maine, Rhode Island, the Carolinas, Mississippi, and Florida despite the Southern states having considerably higher temperatures than the Northern states. This reinforces that ozone concentration is not only a function of several meteorological variables in addition to temperature, but is also non-linearly dependent upon the regional emission rates. In the summer, solar radiation does not vary significantly with latitude, whereas this variation is much higher in the winter. That is another reason, besides temperature, why the intra-annual variation in the Northern states is much higher than in the Southern states.

The variability in the fate level characterization factors for VOC, shown in Figure 6-5, is high, too. However, on an average the intra-annual variability in VOC factors is smaller compared to the variability for $\mathrm{NO}_{\mathrm{x}}$. The intra-annual variability for VOC lies within one order of magnitude. An interesting observation is that the peaks are found in the non-summer months (December to March) in more than half of the states. This is less pronounced in the NorthEastern states. This effect is as a result of the strong dependence of biogenic VOC emissions on temperature and solar radiation. During the winter months, the relative contribution of biogenic VOC to the total VOC emissions decreases significantly, especially in the Southern states, thus increasing the contribution of anthropogenic VOC in ozone formation. Since most of the ozone in the region is formed under $\mathrm{NO}_{\mathrm{x}}$-limited conditions, the net decrease in the emissions of VOC does not have an effect on the total ozone formation. Figure 6-10 shows the ratio of MIR weighted biogenic and anthropogenic VOC emissions. By comparing it with Figure 6-6, it is seen that the states having a high contribution of biogenic VOC are the ones where the characterization factors for anthropogenic VOC peak in the winters. For instance, this effect is clearly discernible in states like Montana, Wyoming, and South Dakota, whereas it is less in states like New York, New Jersey, Ohio, Illinois, etc.

The US annual average characterization factors for $\mathrm{NO}_{\mathrm{x}}$ and VOC are 5.2E-04 ppb/MT and 4.2E-05 ppb/MT-MIR, respectively. The US annual average is the emission weighted mean of the characterization factors. It is calculated as 


$$
\text { US annual average } \mathrm{CF}=\frac{\sum_{s=1}^{49} \sum_{t=1}^{12} \mathrm{CF}_{s t} \cdot \mathrm{E}_{s t}}{\sum_{s=1}^{49} \sum_{t=1}^{12} \mathrm{E}_{s t}}
$$

where, $i$ and $m$ represent the state and month, respectively, and $C F$ and $E$ are the characterization factors and state emissions, respectively. Upon considering an average MIR value of 1.75, the factor for VOC increases to 7.4E-05 ppb/MT of VOC. This implies that $1 \mathrm{MT}$ of anthropogenic $\mathrm{NO}_{\mathrm{x}}$ is, on an average, 7 times more effective then $1 \mathrm{MT}$ of anthropogenic VOC in increasing surface level ozone concentration. It is important to note that the ozone attributed to anthropogenic $\mathrm{NO}_{\mathrm{x}}$ also includes ozone formed due to biogenic VOC under VOC limited conditions, which would have normally been attributed to VOC. As can be seen from Figure 6-10, the biogenic VOC emissions can be as high as 20 times the anthropogenic emissions. Thus, the overall ratio of the characterization factors of $\mathrm{NO}_{\mathrm{x}}$ and VOC is slightly higher than the value of 2 assumed in TRACI and obtained in EDIP2003. The MIR value of $\mathrm{NO}_{\mathrm{x}}$, recently calculated by Carter [http://pah.cert.ucr.edu/ carter/SAPRC/] is about 25, which is about 14 times the MIR for the average VOC mix. Thus, $\mathrm{NO}_{\mathrm{x}}$ is about 14 times more potent in ozone formation compared to an average VOC mix. However, since the MIR values are calculated at conditions of extreme sensitivity for each of the precursors, they represent the extreme values of the reactivity range. These conditions are not typical of the domain average. As observed, the domain average relative potential of ozone formation of anthropogenic NOx is about 7 times that of anthropogenic VOC. 


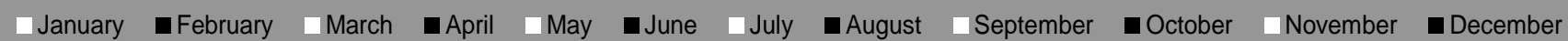

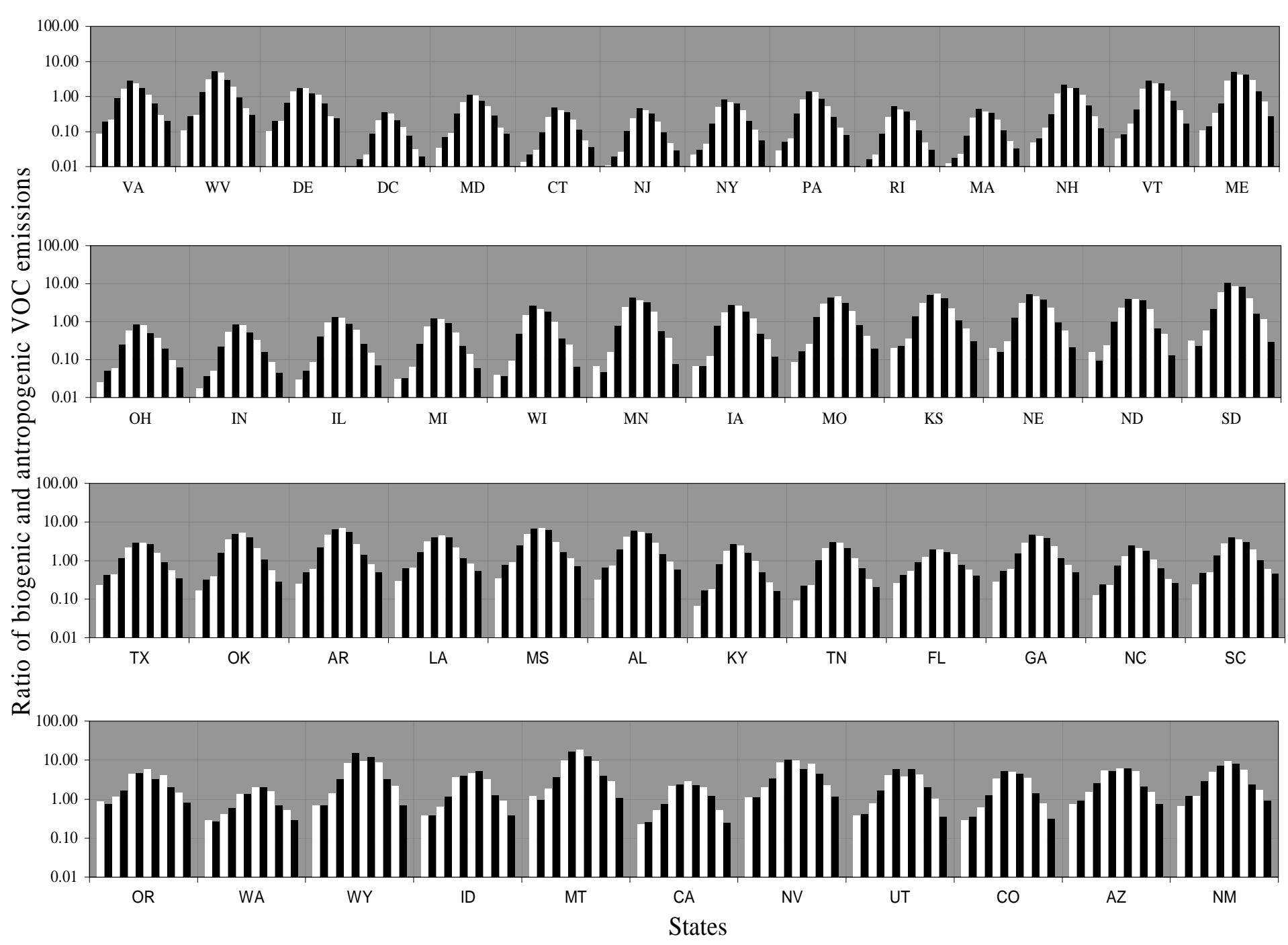

Figure 6-10 Ratio of biogenic and anthropogenic MIR weighted VOC emissions 
To measure the variability in the characterization factors, two coefficients of variations (COV) are calculated. The first, $C O V_{S}$, represents the spatial variability, the second, $C O V_{T}$, represents the variability in time. These are calculated using the following expressions.

$$
\begin{gathered}
\operatorname{COV}_{\mathrm{S}}=\underset{\mathrm{X}}{=} \sqrt{\frac{1}{49} \sum_{\mathrm{s}=1}^{49}\left(\overline{\mathrm{X}}_{\mathrm{s}}-\overline{\mathrm{X}}\right)^{2}} \quad \text { where } \quad \overline{\mathrm{X}}_{\mathrm{s}}=\frac{\sum_{\mathrm{t}=1}^{12} \mathrm{CF}_{\mathrm{st}} \cdot \mathrm{E}_{\mathrm{st}}}{\sum_{\mathrm{t}=1}^{12} \mathrm{E}_{\mathrm{st}}} \\
\operatorname{COV}_{\mathrm{T}}=\underset{\mathrm{x}}{=} \sqrt{\frac{1}{12} \sum_{\mathrm{t}=1}^{12}\left(\overline{\mathrm{X}}_{\mathrm{t}}-\overline{\mathrm{X}}\right)^{2}} \quad \text { where } \quad \overline{\mathrm{X}}_{\mathrm{t}}=\frac{\sum_{\mathrm{s}=1}^{49} \mathrm{CF}_{\mathrm{st}} \cdot \mathrm{E}_{\mathrm{st}}}{\sum_{\mathrm{s}=1}^{49} \mathrm{E}_{\mathrm{st}}} \\
=\sum_{\mathrm{X}=1}^{49} \sum_{t=1}^{12} \mathrm{CF}_{s t} \cdot \mathrm{E}_{s t} \\
\sum_{s=1}^{49} \sum_{t=1}^{12} \mathrm{E}_{s t}
\end{gathered}
$$

The $\mathrm{COV}_{\mathrm{S}}$ and $\mathrm{COV}_{\mathrm{T}}$ for $\mathrm{NO}_{\mathrm{x}}$ fate level characterization factors is $59 \%$ and $74 \%$ respectively, while those for VOC factors are $45 \%$ and $15 \%$, respectively. This indicates that, overall, the spatial as well as temporal variation in the $\mathrm{NO}_{\mathrm{x}}$ factors is high, whereas for VOC factors, the temporal variation, overall, is not very significant. However, this is an overall trend, and the variations for individual states can be different.

As seen in Figure 6-2, a significant portion of the ozone formed on the Eastern coast is transported towards the Atlantic, and out of the model domain. A small fraction of it also transported across the Atlantic Ocean and reaches Europe. The portion of the ozone formed over the Atlantic Ocean is not captured in the calculation of the characterization factors. However, this is not considered to be very important since it does not have any considerable effect on terrestrial ecosystems or humans. 


\subsubsection{Exposure level characterization factors}

The exposure-level characterization factors incorporate the potentially affected human population or ecosystem area due to the ambient ozone concentrations or ozone deposition. This is a step further from the fate level factors which represent the average surface concentration of ozone and are not informed of the underlying human or natural environment. The exposure level characterization model does not actually calculate the exposure of individual receptors, but is only a crude representation. They are based on the assumption that the actual exposure is directly proportional to the ambient concentration or deposition flux, and it is not significantly affected by other factors. This assumption, of course, is far from real, but the scope of the present study limits further sophistication of exposure modeling.

Exposure level factors are developed separately for human and ecosystem exposure for $\mathrm{NO}_{\mathrm{x}}$ and VOC emissions. The population in each grid cell and the area under vegetation that is considered for the calculation of the characterization factors are shown in Figure 6-11 and Figure 6-12, respectively. The characterization factors for human exposure are shown in Figure 6-13 and Figure 6-14; whereas the factors for ecosystem exposure are shown in Figure 6-15 and Figure 6-16. Human exposure level factors are expressed in the units of ppm-persons/MT for $\mathrm{NO}_{\mathrm{x}}$ and ppm-persons/MT-MIR for VOC. The ecosystem exposure factors are expressed as the monthly deposited mass per unit emissions, that is, g/month-MT for $\mathrm{NO}_{\mathrm{x}}$ and $\mathrm{g}$ /month-MT-MIR for VOC. In similarity with the fate level factors, these are plotted on a logarithmic scale indicating their high variability.

The US annual average human exposure level factors for $\mathrm{NO}_{\mathrm{x}}$ and VOC are $41.6 \mathrm{ppm}-$ persons/MT and $4.9 \mathrm{ppm}$-persons/MT. Since the concentration, in units of ppm, is the 24 hour average, it is equivalent to an exposure of 1 ppm-day. The exposure level factors are cumulative. In other words, $1 \mathrm{MT}$ of $\mathrm{NO}_{\mathrm{x}}$ emission increase on a day results in a $41.6 \mathrm{ppm}$-days-persons population exposure increase, and $1 \mathrm{MT}$ of $\mathrm{NO}_{\mathrm{x}}$ emission increase for every day of the week will increase the population exposure by 7 times the daily exposure. 


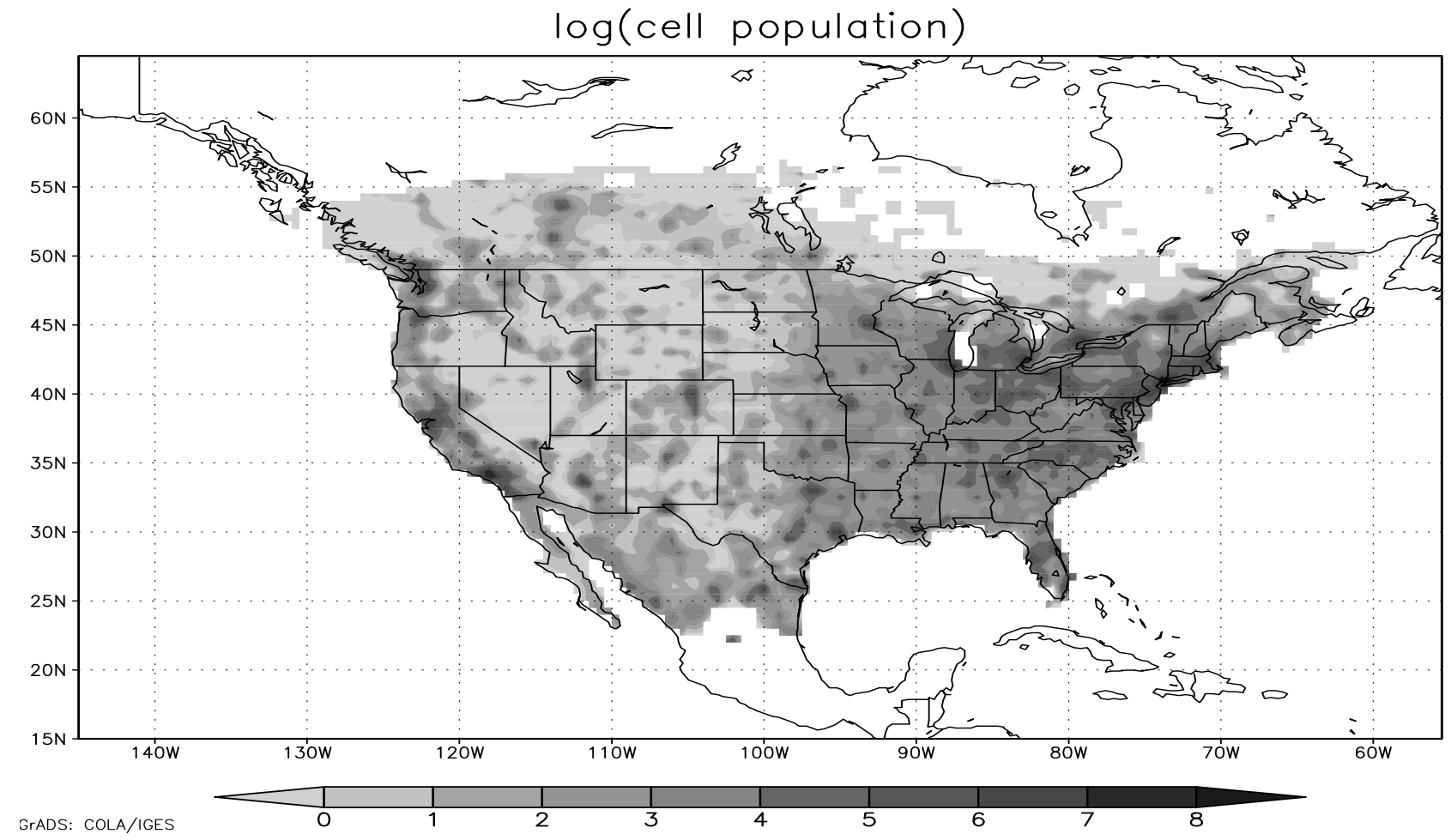

Figure 6-11 Gridded population for the calculation of human characterization factors 


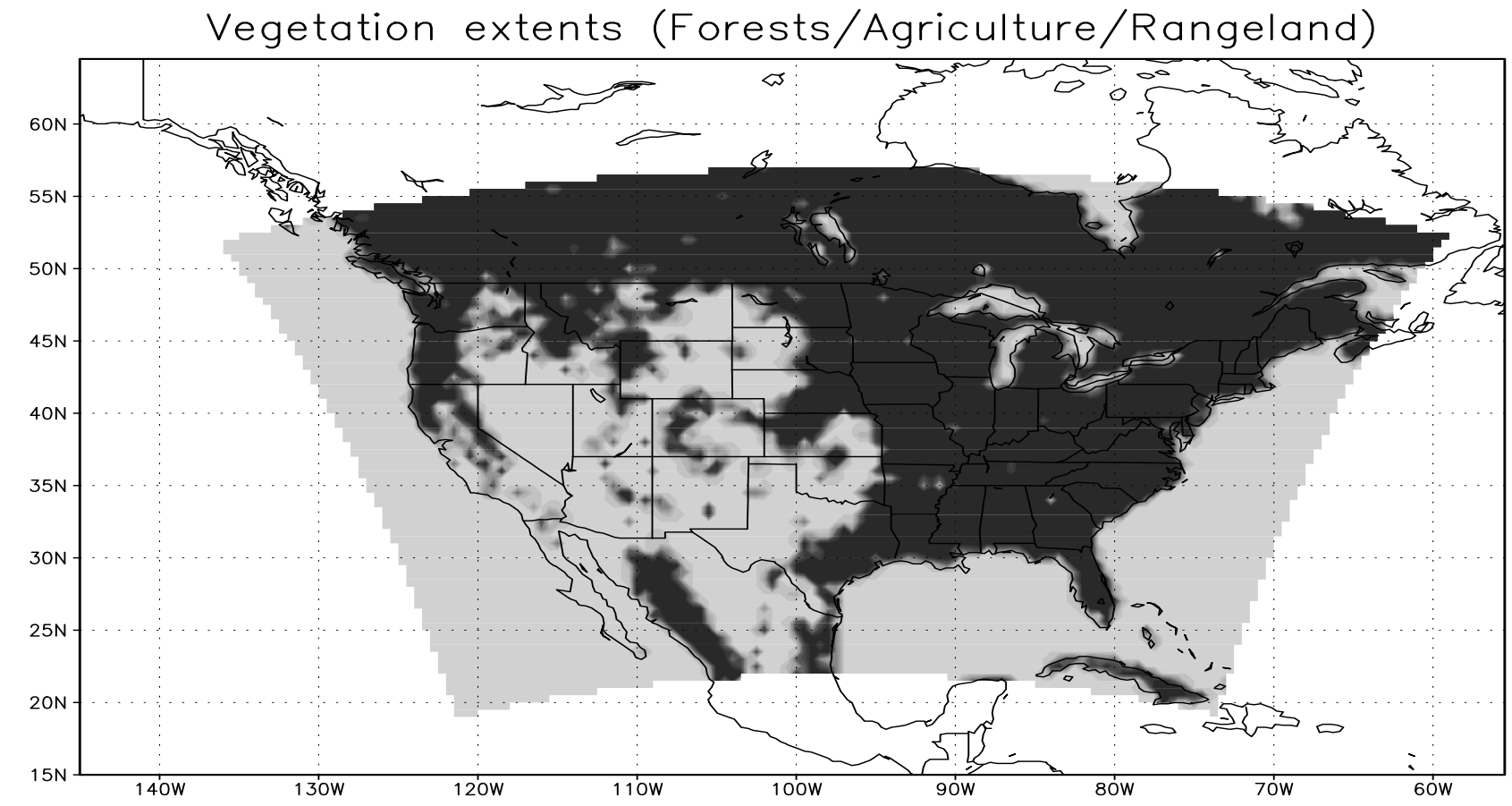

GrADS: COLA/IGES

Figure 6-12 Area covered under forests/crops/grassland ecosystems 


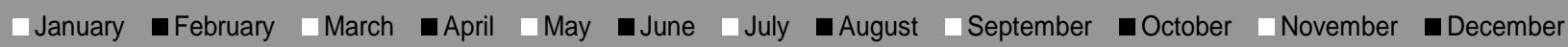
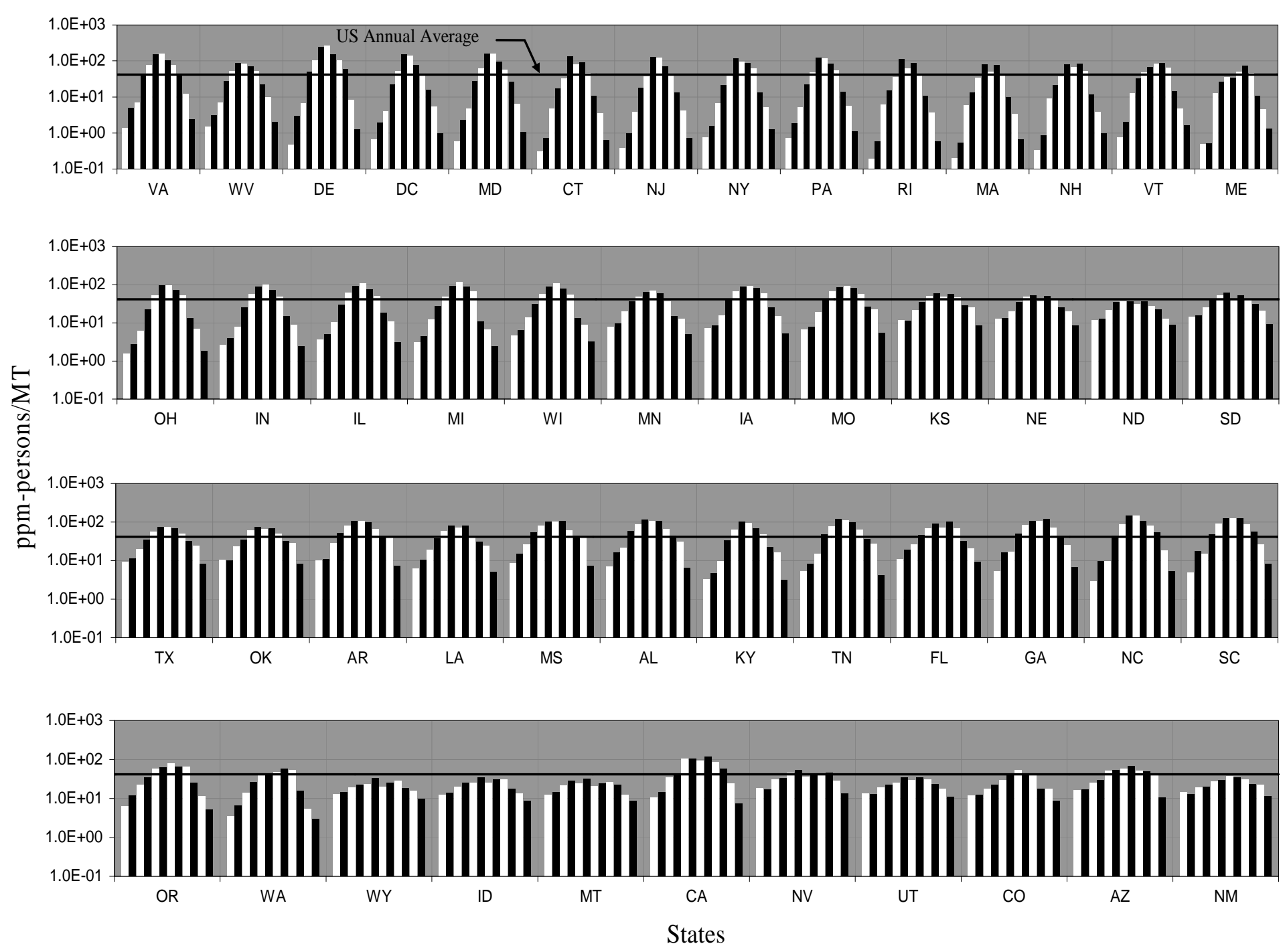

Figure 6-13 Human exposure level factors for NOx 


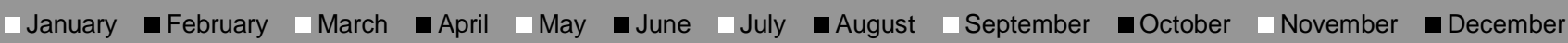
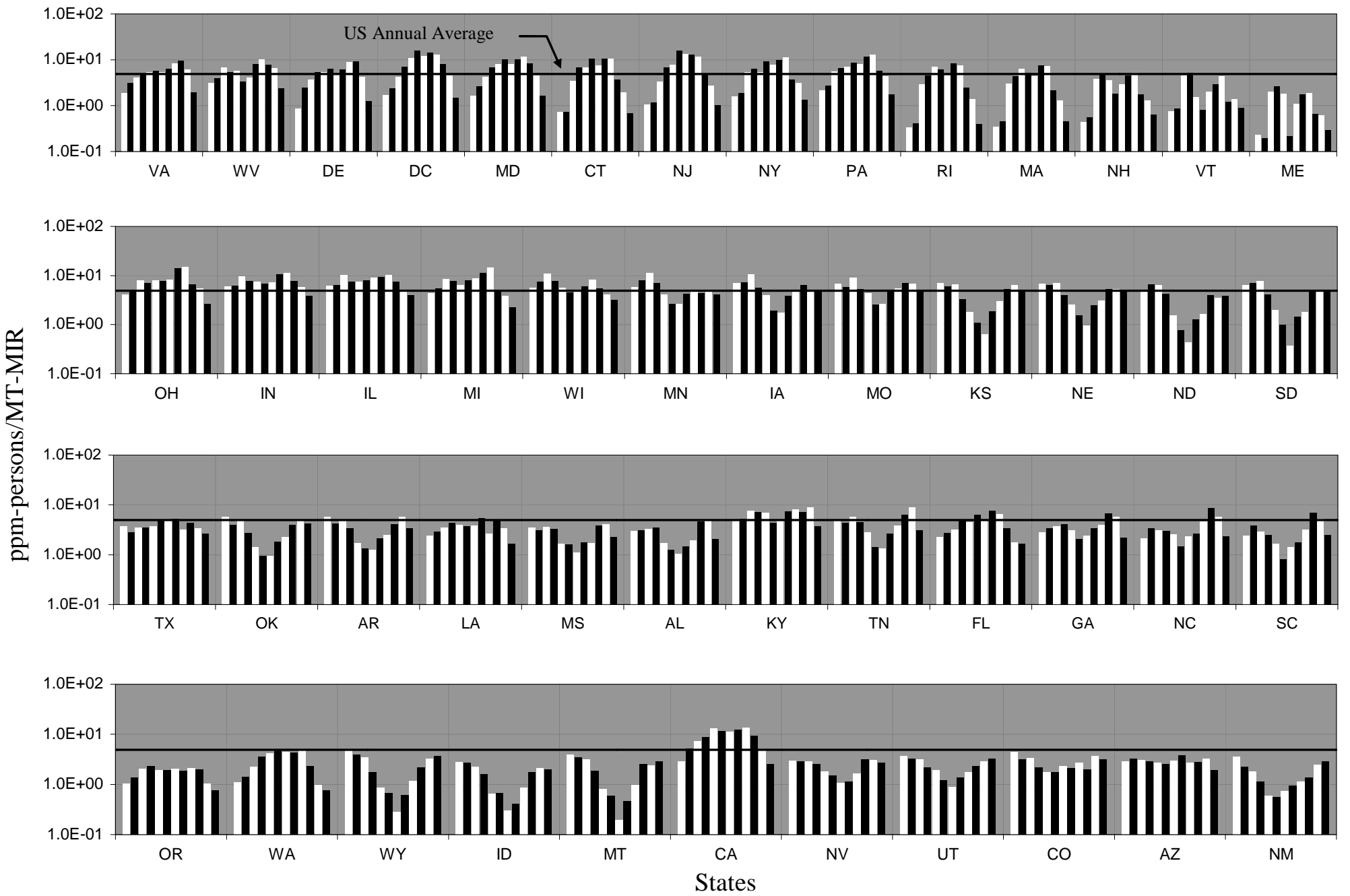

Figure 6-14 Human exposure level factors for VOC 


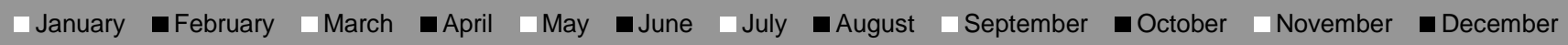
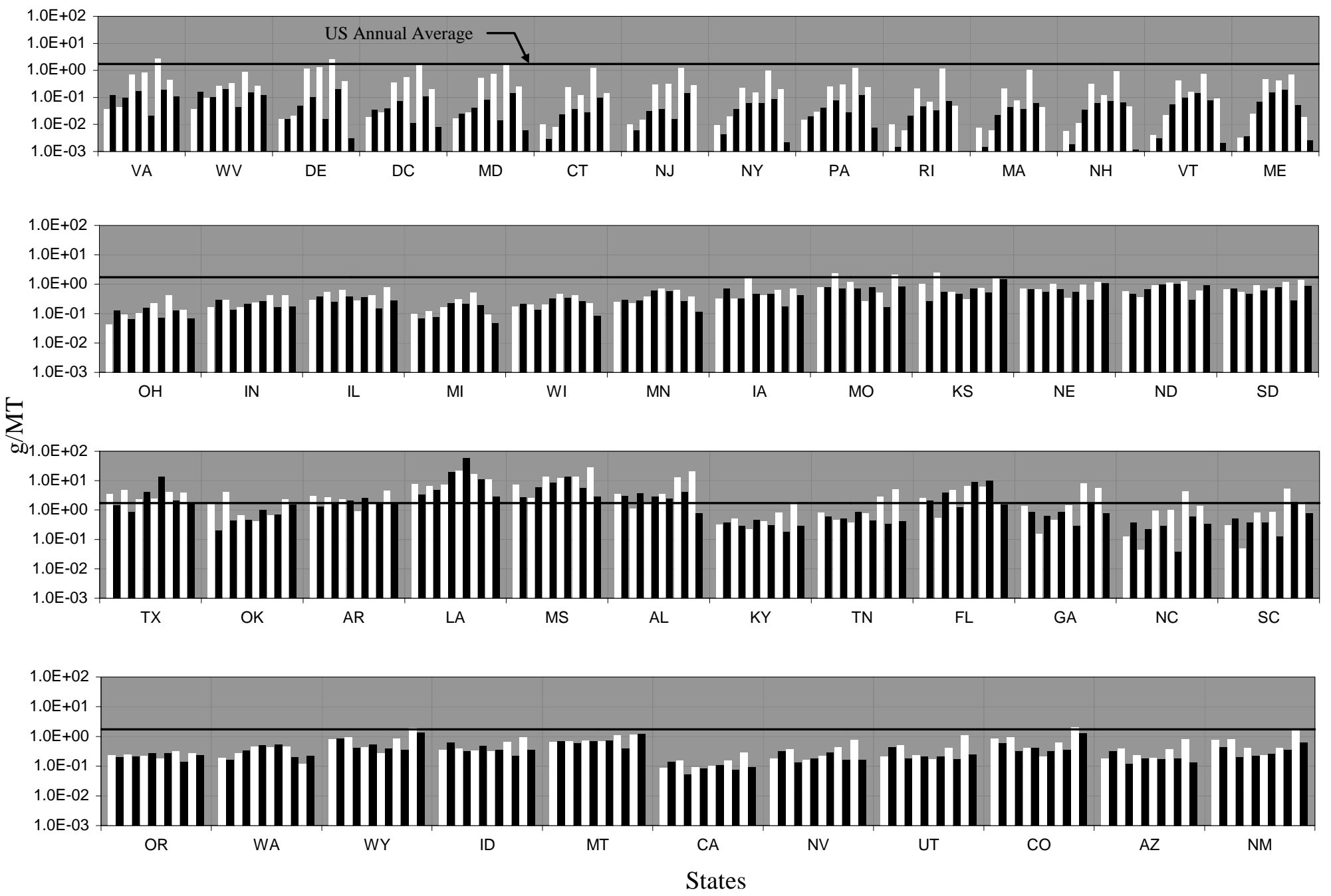

Figure 6-15 Ecosystem exposure level factors for NOx 


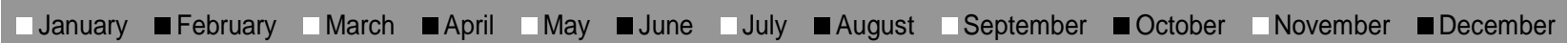
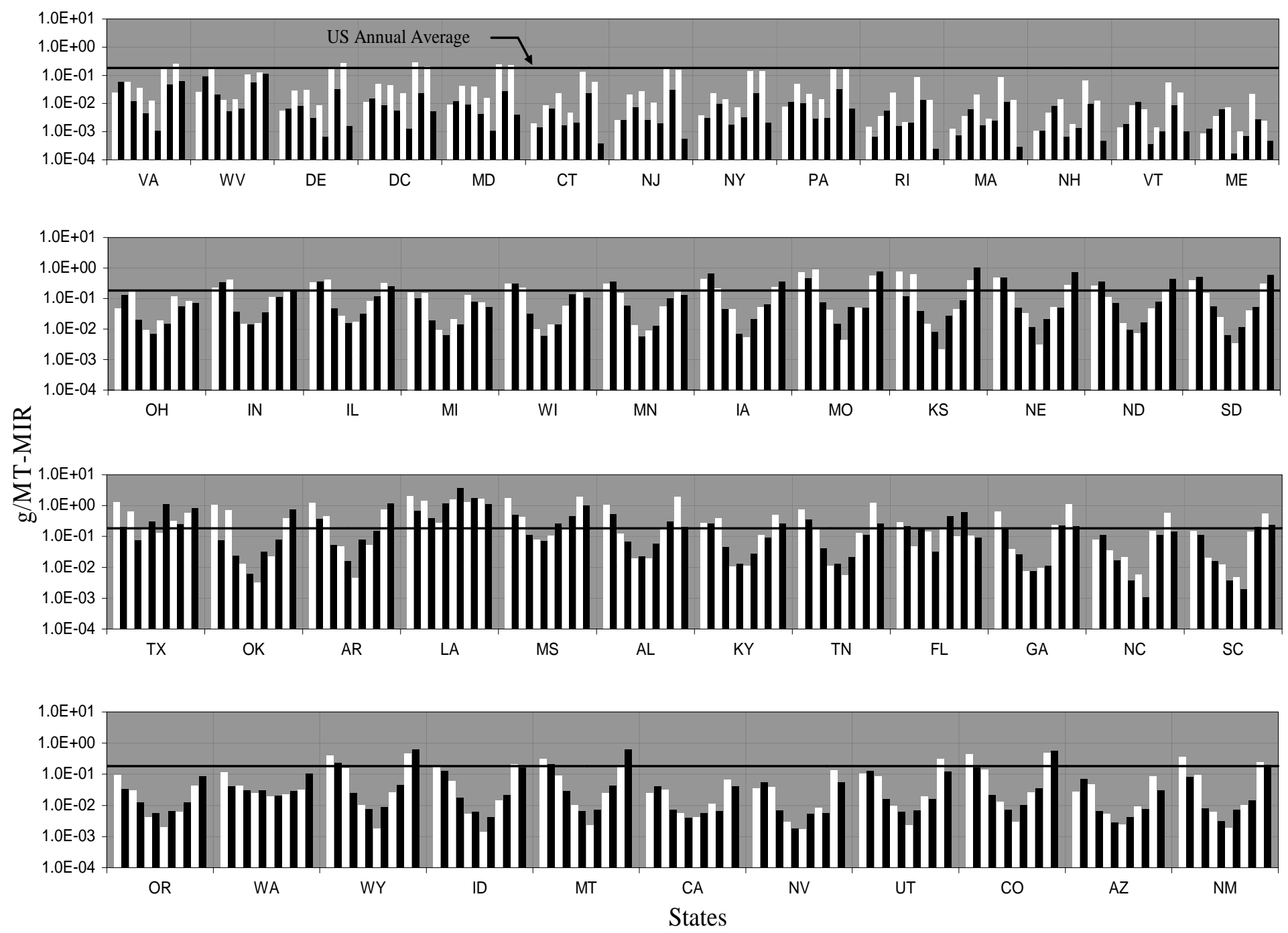

Figure 6-16 Ecosystem exposure level factors for VOC 
The human exposure level characterization factor is based on the assumption of linearity and the non-threshold nature of the exposure-response relation. They represent the assumption that overall impact for one person exposed to a concentration of $0.060 \mathrm{ppm}$ for six days is the same as the impact for three persons exposed to 0.120 ppm for one day. In the absence of any known thresholds or significant non-linear effects in the concentration ranges of concern, this assumption is reasonable.

The human exposure level factors follow a pattern of variability similar to the fate level factors. The characterization factors in the North-Eastern states have a very high (almost three orders of magnitude) temporal variability which decreases in the southern and western states. As expected the sparsely populated states, like Montana, Idaho, Wyoming, etc. have lower than average characterization factors. Even in the North-East the human exposure factors for states like Maine and New Hampshire are relatively closer to average compared to the fate level factors. The $\mathrm{COV}_{\mathrm{S}}$ and $\mathrm{COV}_{\mathrm{T}}$ of the human exposure-level factors for $\mathrm{NO}_{\mathrm{x}}$ are $31 \%$ and $76 \%$ respectively, indicating a lesser variability than the fate level factors for $\mathrm{NO}_{\mathrm{x}}$.

The human exposure level factors for VOC do not show much change in the variability pattern from the fate level factors, too. The highest characterization factors are seen in the NorthEastern states, whereas, all states, except California, in the West have lower than average characterization factors. The lower characterization factors in the summers are seen here, too, in almost half of the states. The $\mathrm{COV}_{\mathrm{S}}$ and $\mathrm{COV}_{\mathrm{T}}$ for the human level exposure factors for VOC are $42 \%$ and $25 \%$, respectively.

The ecosystem exposure factors represent the average mass of ozone deposited in a day on agricultural, forest, or rangeland ecosystems through dry and wet deposition processes. As discussed in Chapter 5.0, wet deposition is influenced, in addition to the physical properties of ozone, by rate of precipitation and presence of clouds within the grid cells. Dry deposition, on the other hand, is dependent on turbulent diffusion in the boundary layer, which is dependent on several other meteorological factors, and the surface characteristics on which the deposition takes place. Dry deposition is the dominant removal mechanism for ozone, since ozone is sparingly soluble in water at standard atmospheric conditions. Even though the deposition of ozone is expressed as a linear function of the ambient ozone concentration, the multiplication factor (dry deposition velocity) is largely influenced by local meteorology and surface conditions. Thus the ecosystem exposure characterization factors do not follow a pattern similar 
to the concentration dependent characterization factors. For both $\mathrm{NO}_{\mathrm{x}}$ and $\mathrm{VOC}$, the characterization factors are the highest for the Southern states of Louisiana, Mississippi, Alabama, Texas, etc., and are the lowest in the North-Eastern states like New York, Maine, Washington DC, etc. The maximum variation between the states is more than two orders of magnitude. The $\mathrm{COV}_{\mathrm{S}}$ for the $\mathrm{NO}_{\mathrm{x}}$ and VOC characterization factors are $139 \%$ and $115 \%$. There is a high inter-annual variation, especially in the North-Eastern states, and the variation does not follow a clear pattern. For instance, in the North-East there is a fluctuation of about one order of magnitude in the months from May to August. This shows the high sensitivity of deposition on short term meteorological conditions. Due to the influence of biogenic emissions on source attribution, many states have lower characterization factors for VOC in the summer months. The highest factor for VOC is for the state of Louisiana in the month of September. The $\mathrm{COV}_{\mathrm{T}}$ for NOx and VOC are $60 \%$ and $70 \%$, respectively.

The US annual average ecosystem exposure level factors for $\mathrm{NO}_{\mathrm{x}}$ and VOC are 1.71 g/MT and $0.19 \mathrm{~g} / \mathrm{MT}-\mathrm{MIR}$, respectively. Similar to the human exposure factors, the ecosystem exposure is also cumulative. For example, an emission of 1 MT of a VOC mixture of MIR 1 for all days of a week will result in the total ecosystem exposure to $1.4 \mathrm{~g}$ of ozone.

\subsubsection{Effect level characterization factors}

Effect level characterization factors are calculated only for human mortality. Our knowledge on the effects on ecosystems is too limited to enable us to quantify the effects. As noted in Chapter 5.0, the concentration-response relationship obtained by Bell et al [2004] is used to calculate the

effect level factors. These are shown for $\mathrm{NO}_{\mathrm{x}}$ and VOC in Figure 6-17 and Figure 6-18, respectively.

The effect level characterization factors represent mortality increase per unit emission of precursors and are given in the units of persons/MT for $\mathrm{NO}_{\mathrm{x}}$ and persons/MT-MIR for VOC. They follow a variability pattern very similar to the exposure level factors. This is as expected, as the variability in the rate of non-accidental mortality across the US, especially at the model resolution, does not vary considerably. The US annual average factors for $\mathrm{NO}_{\mathrm{x}}$ and VOC are 4.03E-04, and 4.93E-05, respectively. In other words, a 100 MT daily increase in anthropogenic $\mathrm{NO}_{\mathrm{x}}$ emission throughout the year will result in about 14 additional annual mortalities. The 
$\mathrm{COV}_{\mathrm{T}}$ and $\mathrm{COV}_{\mathrm{S}}$ for the $\mathrm{NO}_{\mathrm{x}}$ characterization factors are $42 \%$ and $79 \%$, while those for VOC factors are $46 \%$ and $26 \%$. These coefficients of variation are very similar to those for the human exposure and fate level characterization factors.

A summary of the variation of all the characterization factors is presented in Figure 6-19 and Figure 6-20. On the vertical axis are the maximum and minimum characterization factors for each state normalized to the US annual average. The vertical axis is plotted on a logarithmic scale. The vertical I-shaped bars indicate the normalized maximum and minimum values of the characterization factors. 

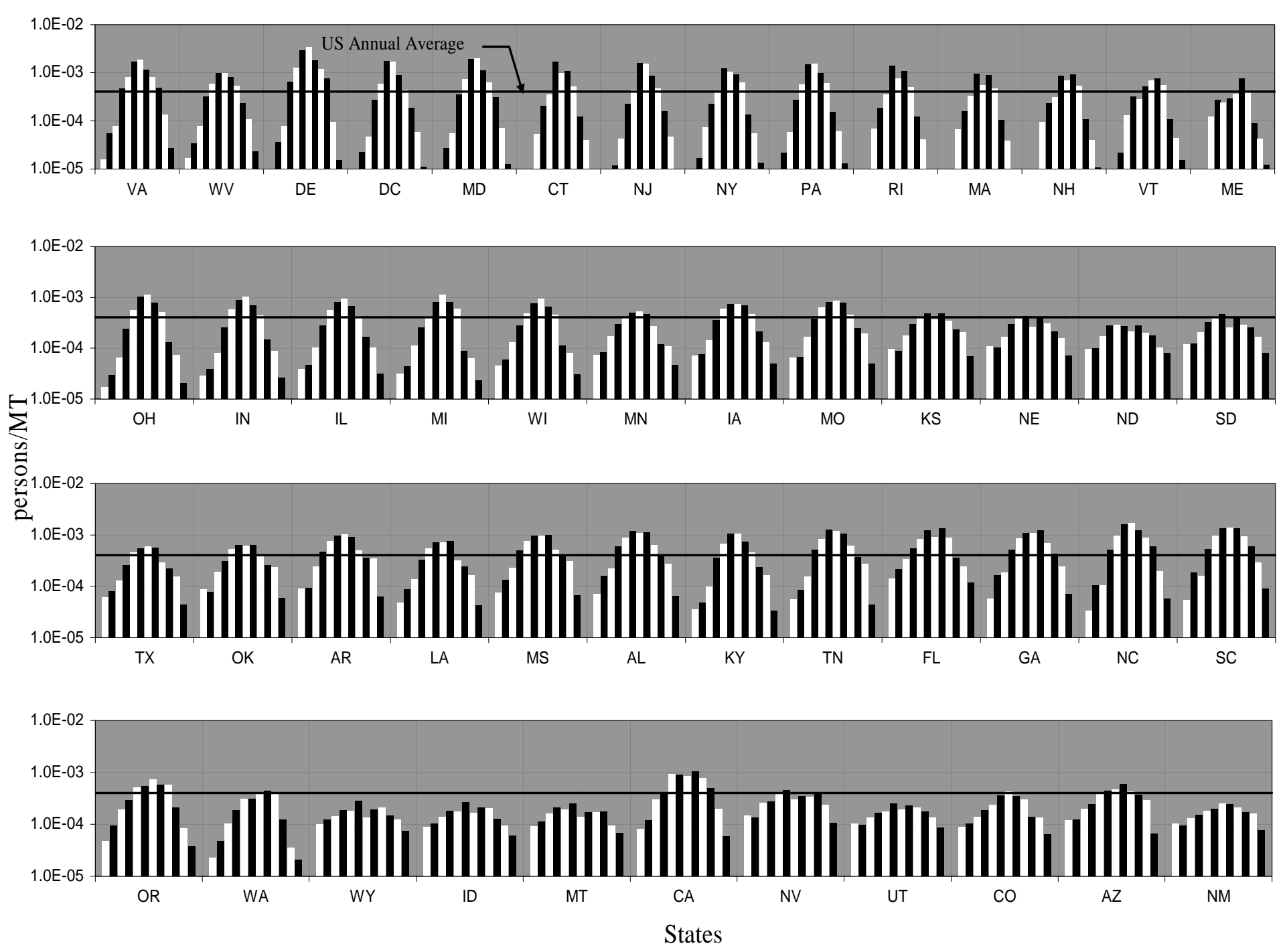

Figure 6-17 Human effect level characterization factors for NOx 

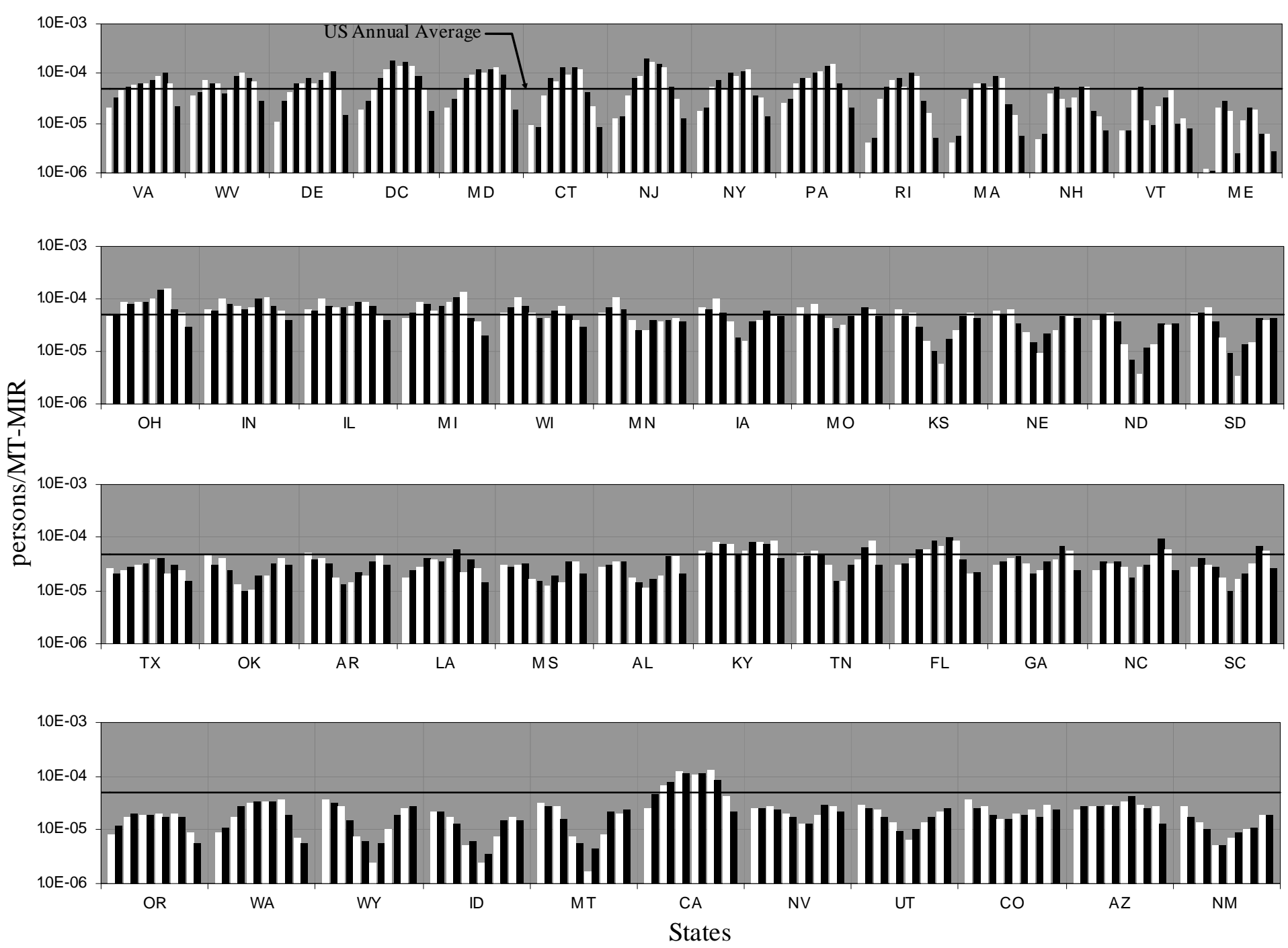

Figure 6-18 Human effect level characterization factors for VOC 
CF-Fate Hum-Exp $\quad$ Hum-Eff Eco-Exp
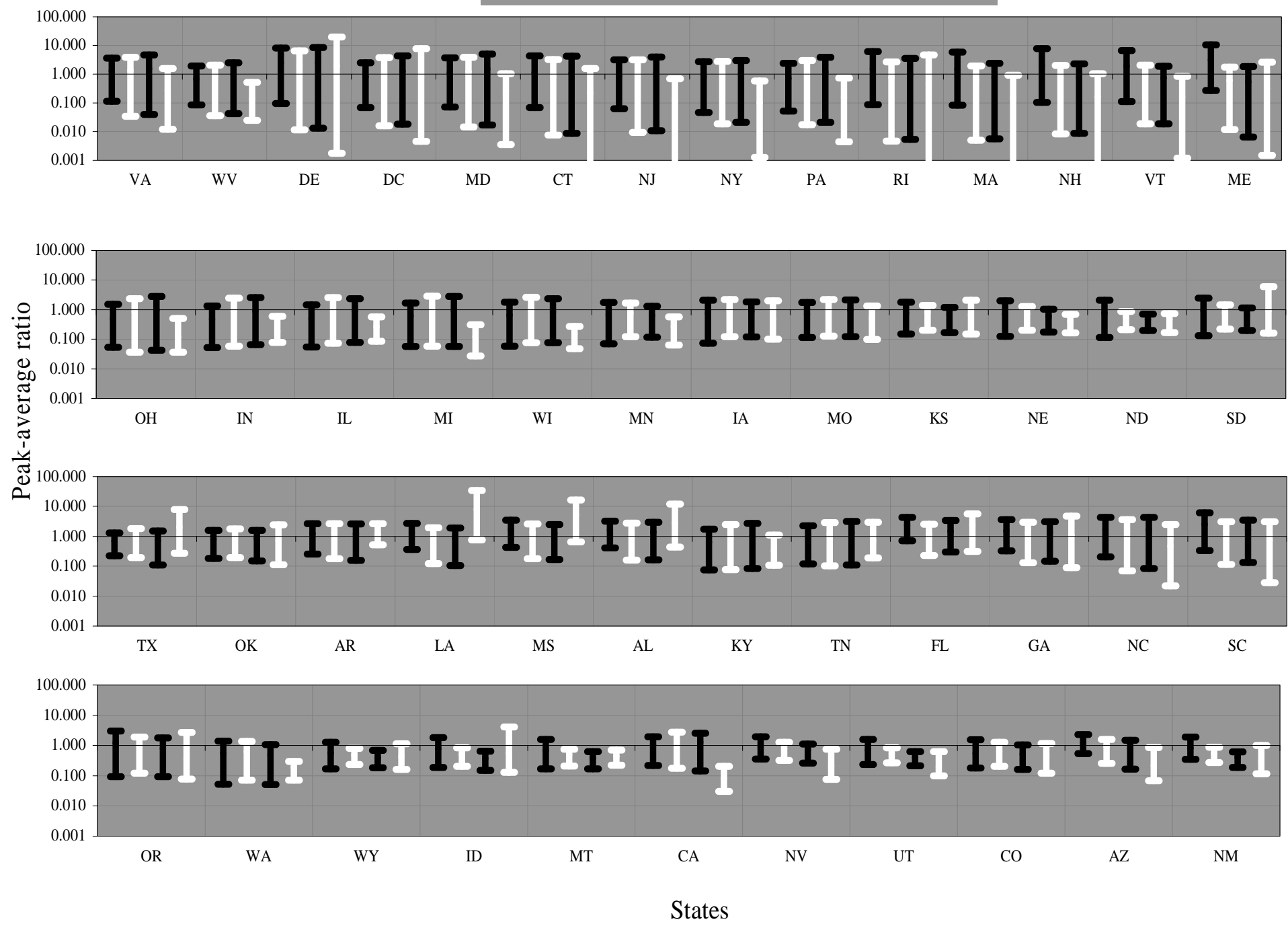

Figure 6-19 Normalized maximum and minimum state level characterization factors for NOx 

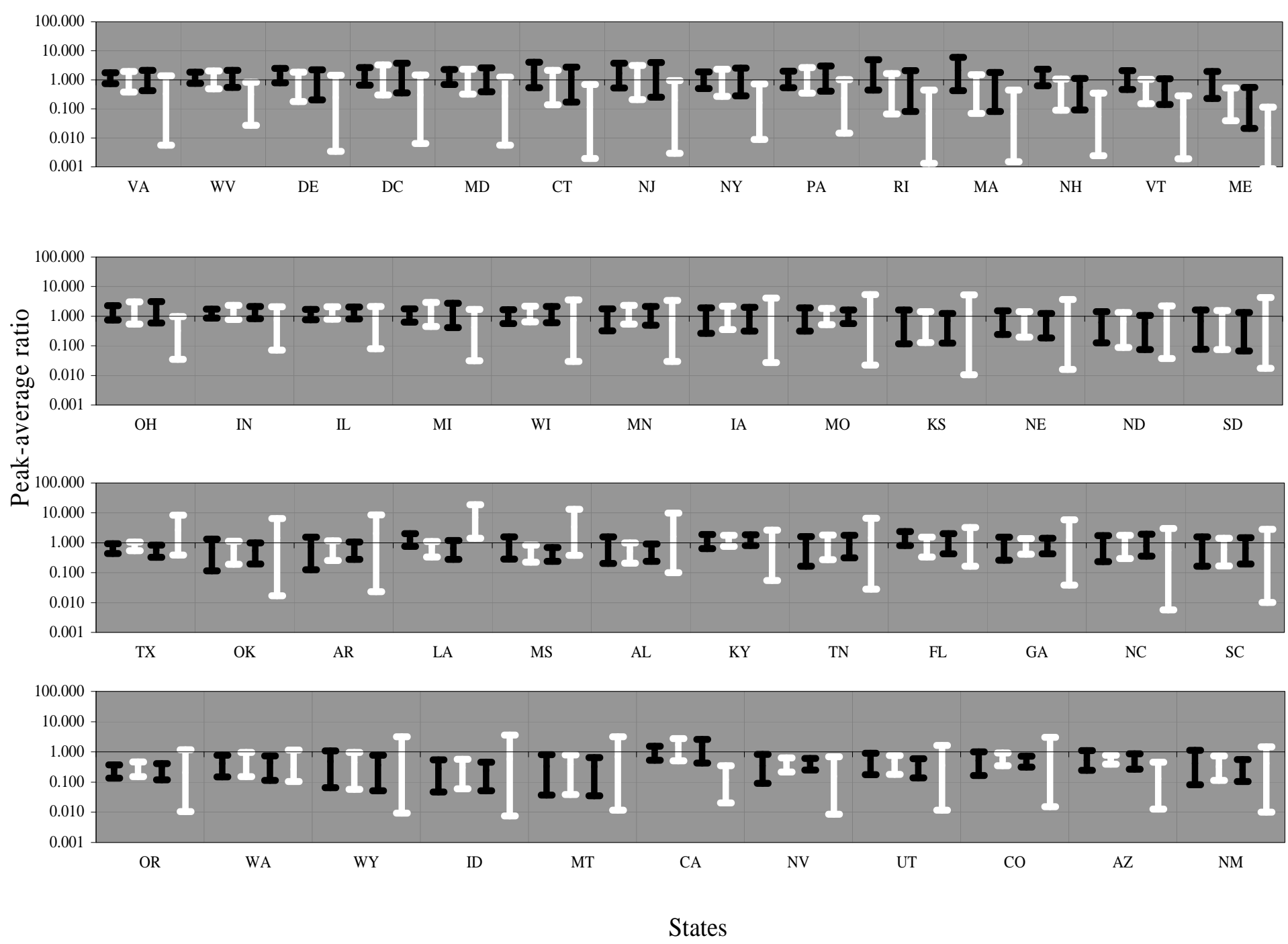

Figure 6-20 Normalized maximum and minimum state level characterization factors for VOC 
It is seen in Figure 6-19 and Figure 6-20 that the fate level, human exposure, and the human effect level characterization factors have very similar variability. The human exposure and human effect level factors are further closely matched. In the states near the Eastern coast, the normalized human exposure and effect level factors are lower than the normalized fate level factors in the winter months. This is mainly due to the continental high pressure forcing winds carrying the ozone and precursors towards the east and over the ocean. This is seen in the $\mathrm{NO}_{\mathrm{x}}$ characterization factors for Delaware, where the fate level factors have a temporal variation of two orders of magnitude while the temporal variation of human exposure and effect level factors is about three orders of magnitude. A similar increase in variation is also seen in the VOC characterization factors for the Eastern states.

As expected, most of the sparsely populated Western states, like Nevada, Utah, New Mexico, Wyoming, etc. have smaller peaks in their human exposure and effect level factors than the fate level factors. Conversely, most of the densely populated states, like California, Ohio, Indiana, Illinois, etc. have higher peaks in the human exposure and effect level factors. However, the moderately populated states in the North-East, like Maine, Vermont, Massachusetts, etc. are influenced by pollutant transport towards the sparsely populated north or towards the ocean.

The VOC fate, human exposure and effect level, that is, the concentration-dependent characterization factors are affected significantly by the relative amount of biogenic emissions. States, like Idaho, Montana, Wyoming, etc, which have the highest relative amount of biogenic emissions have the lowest characterization factors. Conversely, states like Massachusetts, Rhode Island, have the highest concentration-dependent characterization factors. The temporal variability in the concentration- dependent characterization factors for $\mathrm{NO}_{\mathrm{x}}$ reflects the variability in meteorology affecting the formation and retention of atmospheric ozone; whereas, the temporal variability in factors for VOC is driven by the meteorological processes and land use types that affect the relative contribution of biogenic emissions.

For the ecosystem exposure factors, the trend indicates that the characterization factors decrease in the northern states. The highest characterization factors are found in the southern states, like Louisiana, and the lowest in states like Maine, Vermont, North Hampshire, etc. This is the result of lower vegetation resistances to deposition at higher temperatures and solar radiation. However for southern states like Arizona and Nevada, the characterization factors are relatively lower. This is due to the prevalence of semi-arid and desert ecosystems in these states. 
It is assumed that desert ecosystems do not suffer any significant damage due to ozone exposure and hence are not considered in the calculation of the exposure factors. In general, considerable analysis is required to determine the specific factors affecting the variability in deposition patterns. The scope of this research limits further analysis of this aspect.

\subsection{SENSITIVITY OF THE CHARACTERIZATION FACTORS}

Meteorology and emissions form an integral part of the PAQMS. The processes of ozone formation, transport, and destruction are highly influenced by these two inputs to the chemistry and transport model. However, they do not remain constant every year. Large variations in annual climate are common. The annual change in anthropogenic emissions is not as high as annual climate changes. However, biogenic emissions are greatly influenced by meteorology conditions, and can have higher variation. Besides, the current accuracy in predicting meteorology variations for future years is relatively low. On the other hand, ample data exists to model the growth in emissions and the effects of regulatory control strategies on future year inventories. Sensitivity analysis is performed to get a rough estimate of the magnitude of variation in the characterization factors due to the variation in meteorology and emissions. Two scenarios, referred to as Scenario 1 and Scenario 2, are modeled. The scenario used to develop the characterization factors described in the previous sections is referred to as the Base case.

Scenario 1 is modeled to test the sensitivity of the characterization factors primarily to meteorology conditions. A new set of characterization factors are calculated for the month of July using 2006 meteorology instead of the 2001 meteorology used in the base case. Nationally, 2006 is the second warmest year on record (record period: 1895-2007) and also the second warmest July. Temperatures in all states were either much-above or above normal [http://www.ncdc.noaa.gov/oa/climate/research/2006/jul/national.html]. The year 2001 is currently (2008), the seventh warmest year on record. Overall, the July of 2001 is the nineteenth warmest on record. However, the spatial variation was large. Most of the Eastern states experienced below normal to much-below normal temperatures. Most of the western and southern states experienced above normal or much-above normal temperatures, while in some of the states the temperatures were near normal. The difference in precipitation for the two periods 
was not large. The precipitation in July 2006 was below normal, while in July 2001 it was near normal [http://www.ncdc.noaa.gov/oa/climate/research/2001/jul/us_national.html]. The temperature and precipitation cannot describe the meteorological conditions completely, but they act as key indicators. Most meteorological processes are driven by temperature variations, and wet deposition with precipitation is a large sink for $\mathrm{NO}_{\mathrm{x}}$. Besides the meteorology input to the chemistry and transport model, the albedo/haze/ozone input file has also been regenerated from observed conditions in July 2006. The emission inputs for this scenario were the same as those used for 2001. The biogenic emissions, too, were not adjusted for the changing meteorological conditions. Other inputs, like initial and boundary conditions, and land use characteristics, remained unchanged.

Scenario 2 was developed to determine the effect of variation in emissions. The new set of characterization factors were based on the year 2020 emission inventory estimated by USEPA for the ozone NAAQS proposal. Future year inventories are based on modeling the growth in emissions due to increase in economic activity, changes in the mix of production within and between sectors, replacement of capital equipment, changes in population, land use, etc. They also include modeling of controls mandated under the Clean Air Act or other regulations, and other voluntary controls and initiatives. A detailed description of the growth and control models used for generating this emission inventory is provided in the USEPA Regulatory Impact Analysis for the NAAQS proposals [USEPA 2005c]. Scenario 2 is obtained by changing the emissions inputs of Scenario 1. Thus the meteorology conditions of July 2006 are used in the simulation. Biogenic emissions are also recalculated based on the July 2006 meteorology. The emissions of $\mathrm{NO}_{\mathrm{x}}$ and VOC used in Scenario 2 are compared with those used in the base case and Scenario 1 in Figure 6-21. The upper two bar charts in Figure 6-21 are the daily $\mathrm{NO}_{\mathrm{x}}$ emissions in each state, and the lower two bar charts are the daily, MIR weighted VOC emissions. The ratio of biogenic and anthropogenic emissions for Scenario 2 and that for the base case and Scenario 1 are compared in Figure 6-22. 

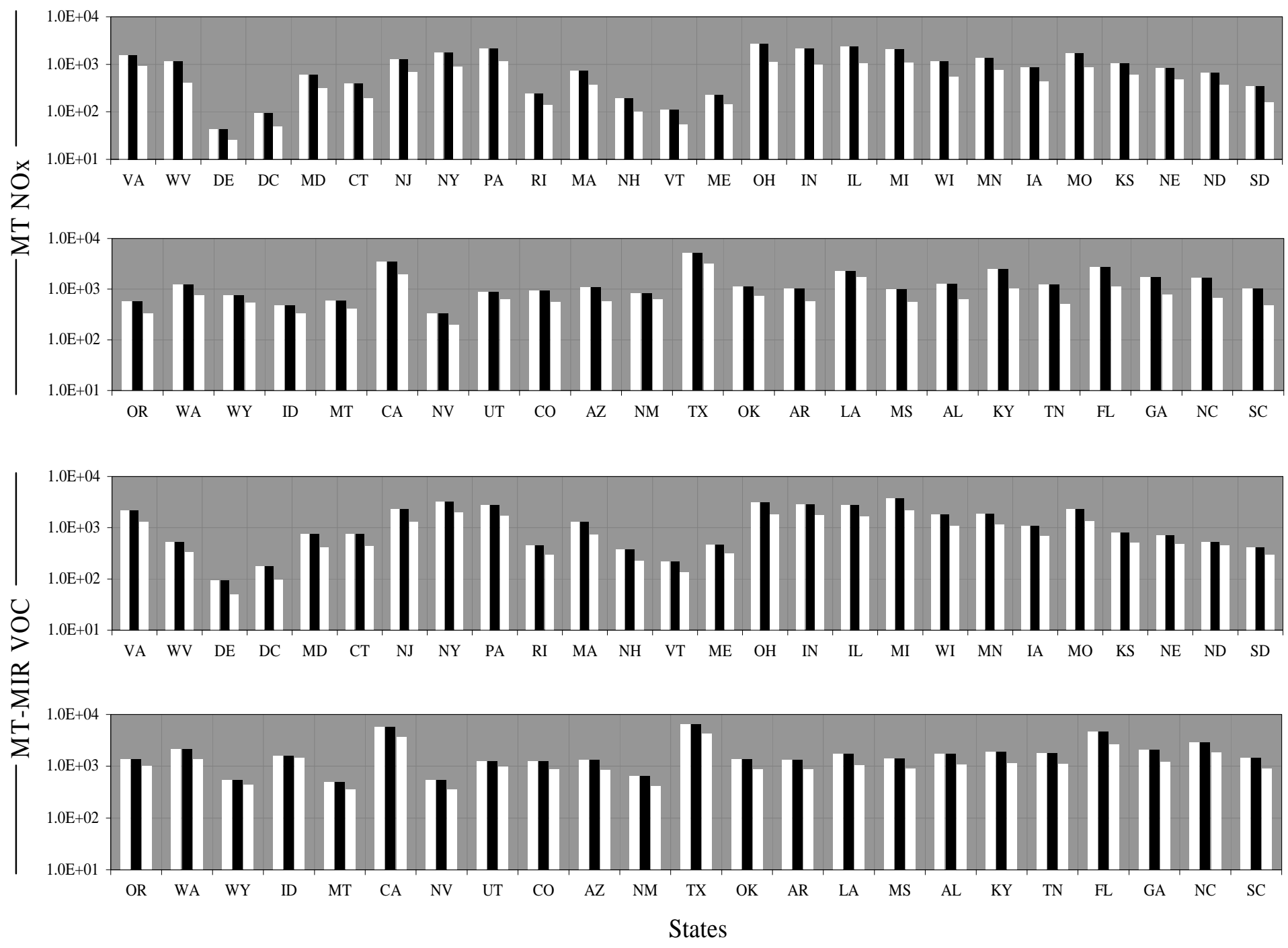

Figure 6-21 Comparison of anthropogenic emissions for the three scenarios 


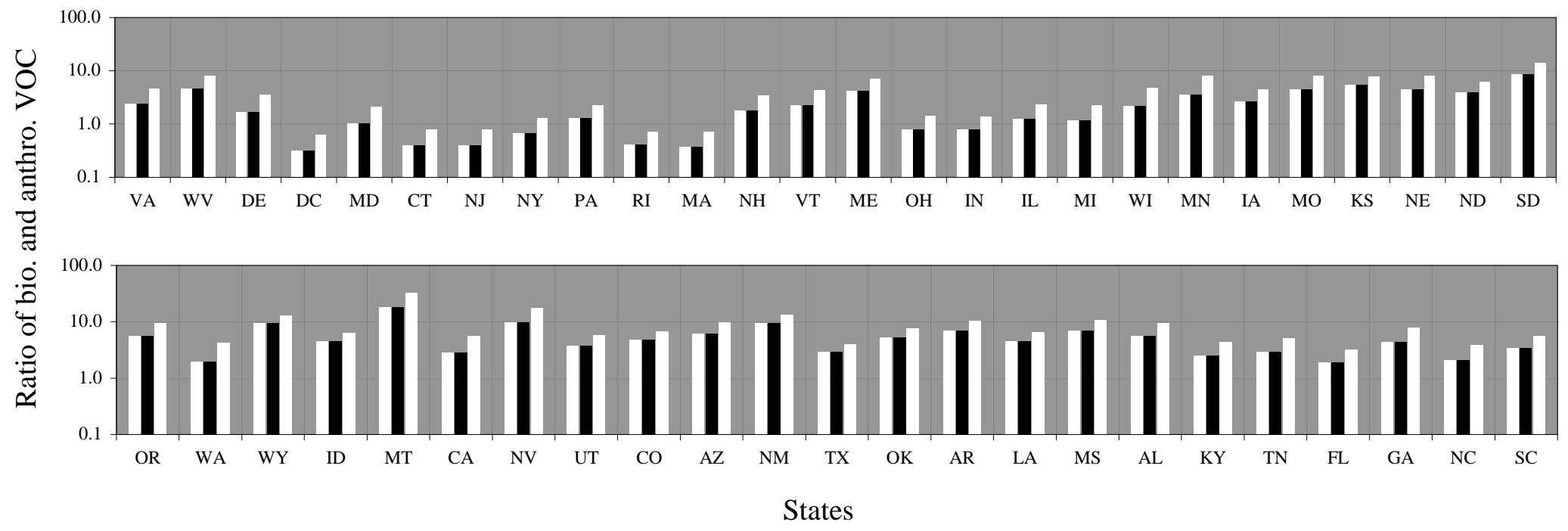

Figure 6-22 Comparison of ratio of biogenic and anthropogenic VOC for the three scenarios 
Despite the growth in economic activity and population, it is expected that the emissions will reduce in 2020. The reduction in emissions is due to increase in efficiencies, replacement of capital equipment, and, above all, due to regulatory requirements. This decrease in emissions from 2001 to 2020 is seen in Figure 6-21. The decrease in $\mathrm{NO}_{\mathrm{x}}$ emission lies in the range of 25 to $65 \%$ with an average decrease of $45 \%$. The decrease in the MIR weighted VOC emissions lies between 10 and 47\%, with an average decrease of 35\%.

It is seen in Figure 6-22 that the ratio of the biogenic and anthropogenic emissions for Scenario 2 increases from the base case. This increase lies in the range of 35 to 126\%, with an average of $75 \%$. A part of this increase in the ratio is due to decrease in the anthropogenic VOC. The other part of this increase is because of the actual increase in the biogenic VOC emissions due to the unusually high temperatures experienced throughout the country in July 2006. The change in the biogenic VOC emissions lies in the range of -11 to $38 \%$, with an overall average increase of $11 \%$. Due to this increase in biogenic emissions, despite the decrease in the anthropogenic VOC emissions, the total VOC emissions for many states increased in Scenario 2. The decrease in the biogenic VOC emissions in July 2006 was seen in the Southern states of Florida, Texas, New Mexico, Louisiana, Mississippi, and Oklahoma.

Figure 6-23 shows the trend in the domain wide total ozone average and the contribution of the major sources. From the base case to Scenario 1 there is a very slight (less than 1\%) increase in the average total ozone. The increase in the ozone attributed to the boundary, anthropogenic, and biogenic sources increases in proportion to their contributions. From Scenario 1 to Scenario 2, the emissions of $\mathrm{NO}_{\mathrm{x}}$ throughout the domain decrease substantially, whereas the domain-wide VOC emissions decrease less than 5\%. The total ozone formed in the domain shows a strong decline in Scenario 2. This is support of the observation that most of the ozone formed in the US is formed under $\mathrm{NO}_{\mathrm{x}}$ saturated conditions. Thus a sharp decline in total ozone is seen as a result of the decrease in $\mathrm{NO}_{\mathrm{x}}$ emissions. There is a small increase in the ozone formed from only biogenic sources because of the higher biogenic emissions of both $\mathrm{NO}_{\mathrm{x}}$ and VOC. 


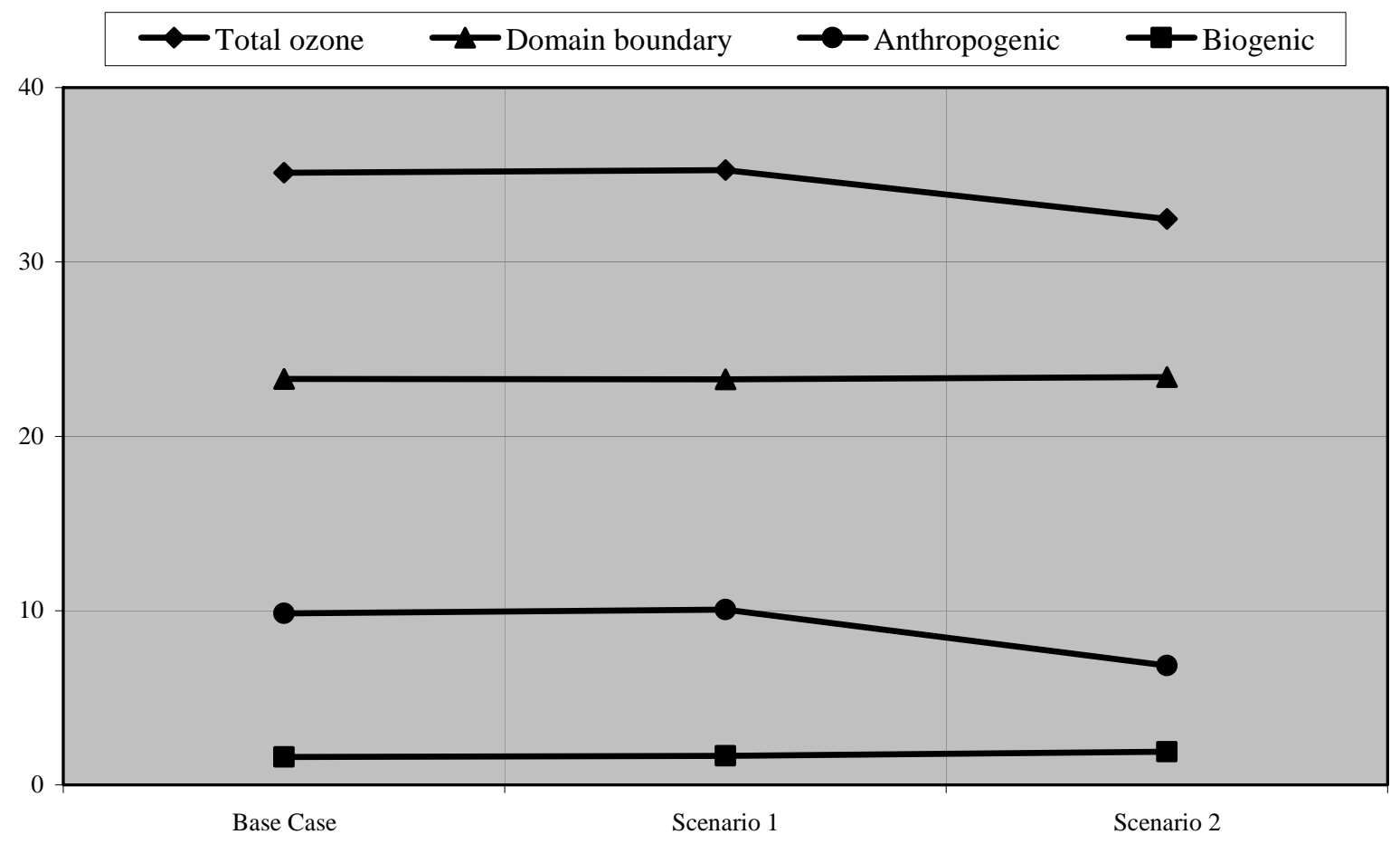

Figure 6-23 Ozone apportionment to major source categories for the three scenarios

The fate level factors for the 49 states, for the base case and the two sensitivity scenarios are presented in Figure 6-24. The upper two charts are the factors for $\mathrm{NO}_{\mathrm{x}}$ and the lower two are the factors for VOC. As seen in Figure 6-24, the characterization factors for $\mathrm{NO}_{\mathrm{x}}$ follow an overall increase from the base case to Scenario 1. This is in line with expectation as the temperatures in most parts of the country in 2006 were much higher than in 2001. However, some states like North and South Dakota, Nebraska, Louisiana, and Florida showed a decrease in the factors. The change in the characterization factors was in the range of $-21 \%$ and $72 \%$. The increase in the US average $\mathrm{NO}_{\mathrm{x}}$ fate level characterization factor for July, from 2001 to 2006, was $15.8 \%$. A domain wide increase in the $\mathrm{NO}_{\mathrm{x}}$ characterization factors is seen from comparison of Scenario 1 and Scenario 2, except for Montana, which showed a 4\% decrease. The maximum increase in the characterization factors was 65\%, and the increase in the US average $\mathrm{NO}_{\mathrm{x}}$ characterization factor was 32\% with respect to Scenario 1. Thus, despite the decrease in the total ozone formed, the ozone formed per unit $\mathrm{NO}_{\mathrm{x}}$ emission increases. This stems from the nonlinear relationship between ozone and $\mathrm{NO}_{\mathrm{x}}$. 
In case of the VOC characterization factors, there is no clear pattern; some states show a decrease while others show an increase. The change in the characterization factors is in the range of $-44 \%$ to $75 \%$. Thus, the US average factor increases, marginally, by $2 \%$. The Scenario 2 VOC characterization factors for all the states show a decrease compared to Scenario 1 . This decrease is in the range of $20 \%$ to $75 \%$. This is due to the high increase in the amount of biogenic emissions relative to the anthropogenic VOC emissions as seen in Figure 6-22. Thus, the decrease in the ozone concentration attributed to anthropogenic VOC is more relative to the decrease in emissions.

Figure 6-25 and Figure 6-27 show the comparison of the human exposure and human effect level characterization factors for $\mathrm{NO}_{\mathrm{x}}$ and VOC. As expected, the pattern of change in these characterization factors is similar to that observed in the fate level factors. For the human exposure and effect level factors, the change in the US averages for $\mathrm{NO}_{\mathrm{x}}$ and VOC from base case to Scenario 1 lie in the range of $10 \%$ to $18 \%$. The change in the US average from Scenario 1 to Scenario 2 for $\mathrm{NO}_{\mathrm{x}}$ is about $68 \%$ and for VOC is a decrease of about $10 \%$. The change in the ecosystem exposure factors, seen in Figure 6-26, is a decrease from base case to Scenario 1, except for Florida, which shows a very sharp increase, both for $\mathrm{NO}_{\mathrm{x}}$ and VOC. In similarity to the fate level factors for $\mathrm{NO}_{\mathrm{x}}$, there is an increase in the characterization factors due to decrease in emissions. When the anthropogenic VOC emissions are decreased (Scenario 1 to Scenario 2), increases as well as decreases are seen in the characterization factors; increases mostly in the Northern states.

However, in both the scenarios it is seen that the spatial variation in the characterization factors is more than one order of magnitude higher than the variation due to change in meteorological conditions or emissions. Thus, despite the uncertainty in the state-level characterization factors due to the model inputs, a higher uncertainty is introduced in LCIA by neglecting the spatial variability of ozone formation.

The very high sensitivity of the characterization factors to two of the model inputs indicates the uncertainty inherent in the current factors. Several simulations involving different scenarios are required to quantify this uncertainty and arrive at average characterization factors. It is also indicative of the difficulties in impact characterization of such regional environmental problems within the highly aggregating LCA framework. 


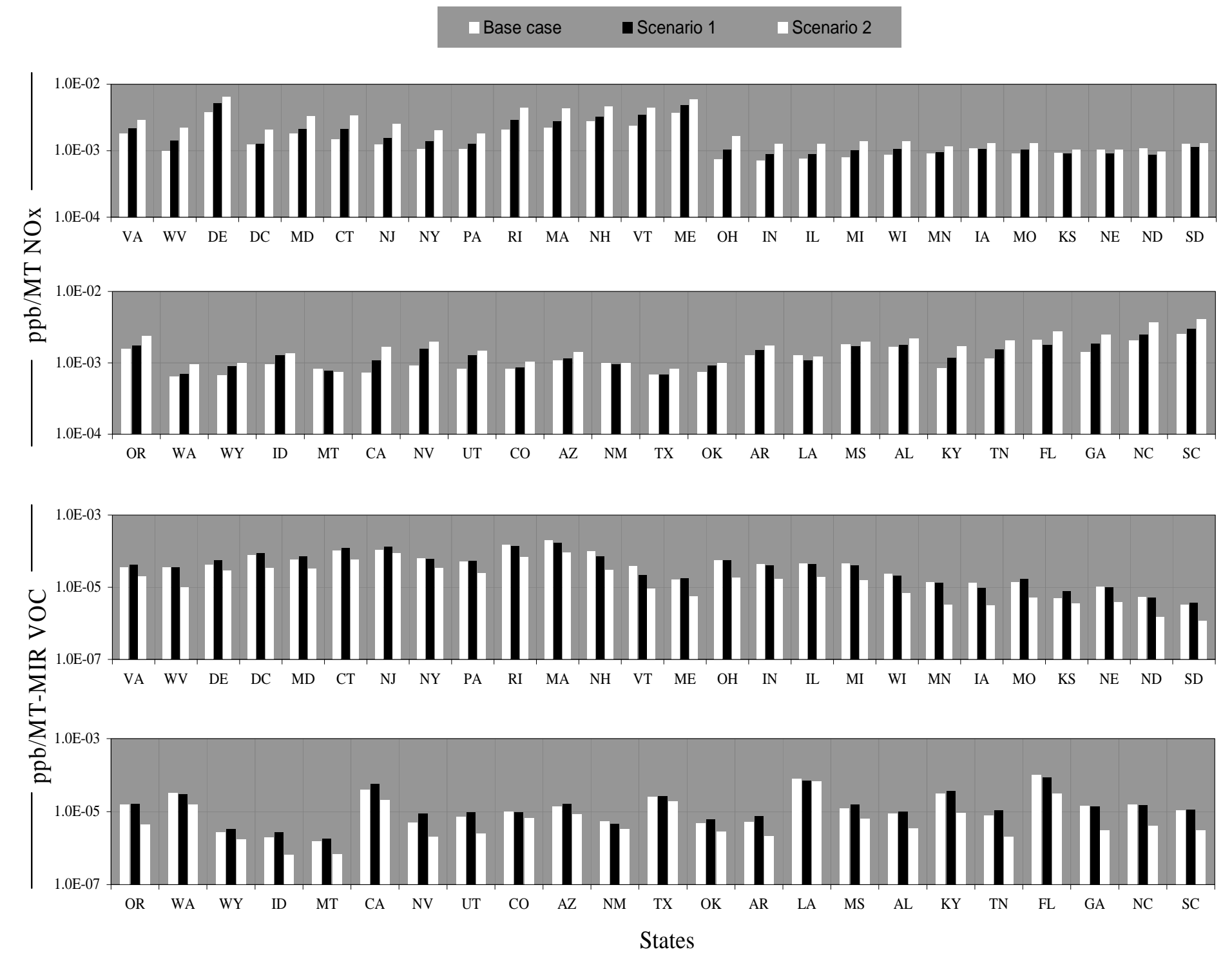

Figure 6-24 Comparison of the fate level characterization factors for the three scenarios 

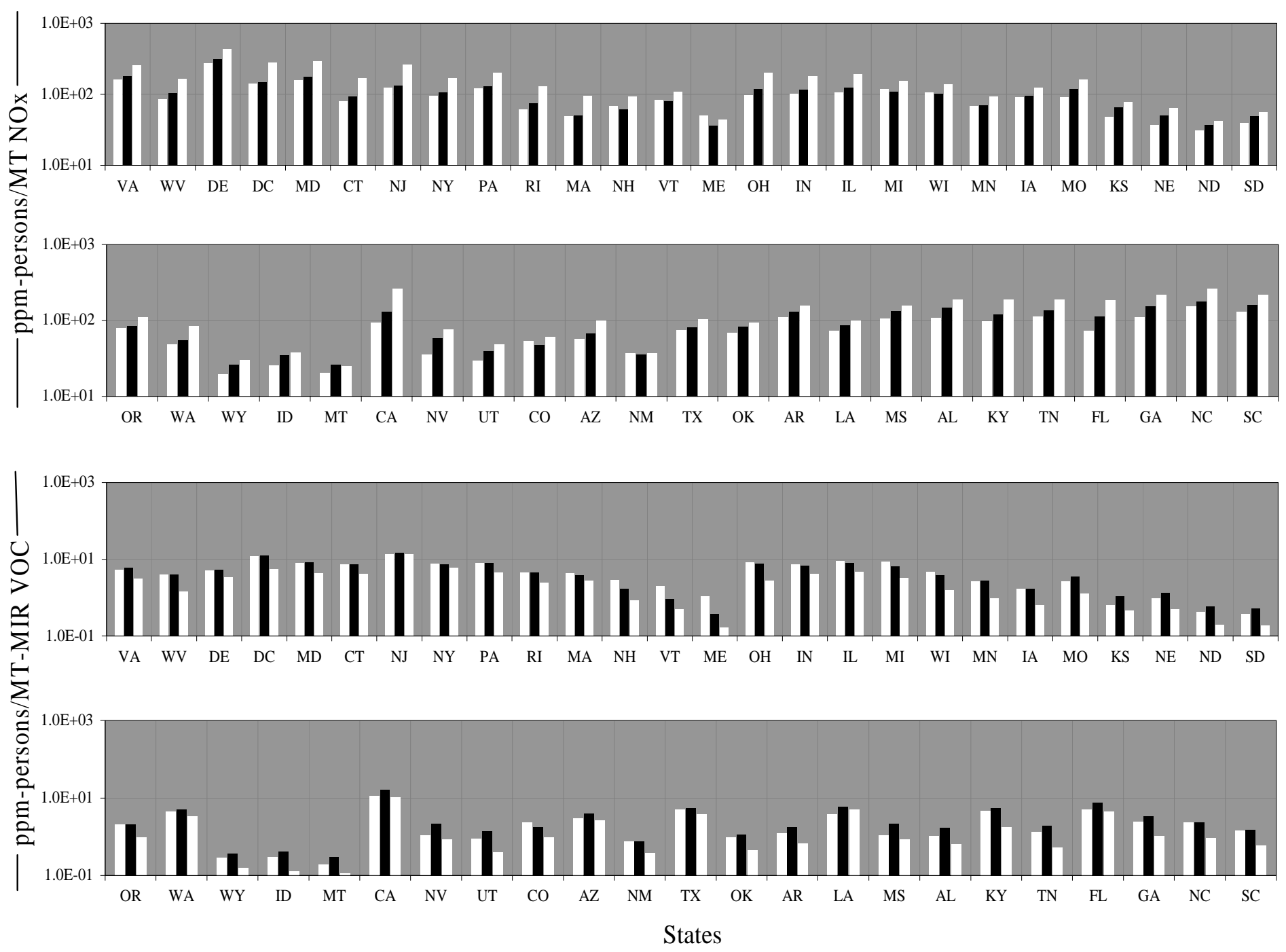

Figure 6-25 Comparison of human exposure level factors for the three scenarios 

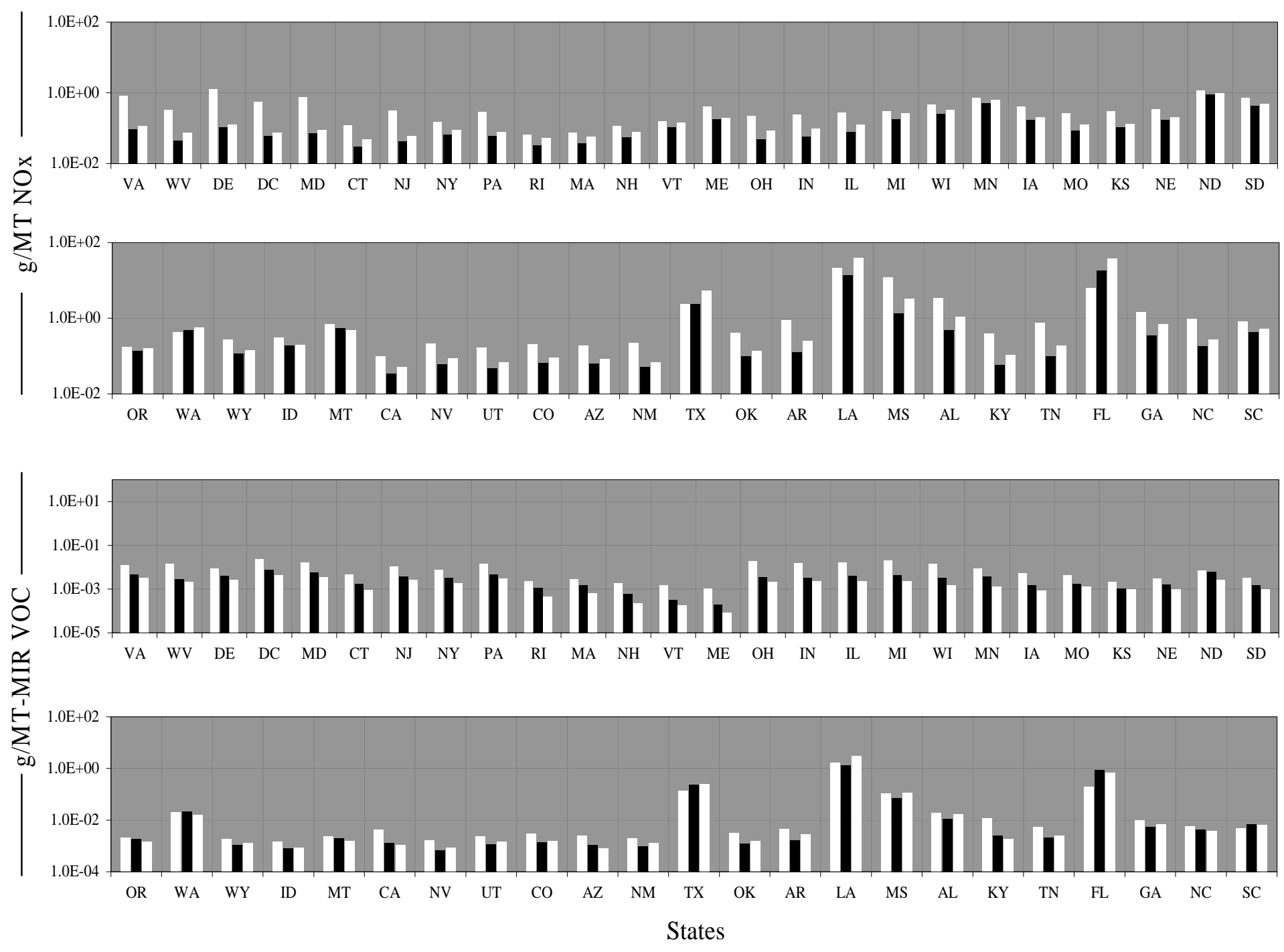

Figure 6-26 Comparison of ecosystem exposure level factors for the three scenarios 

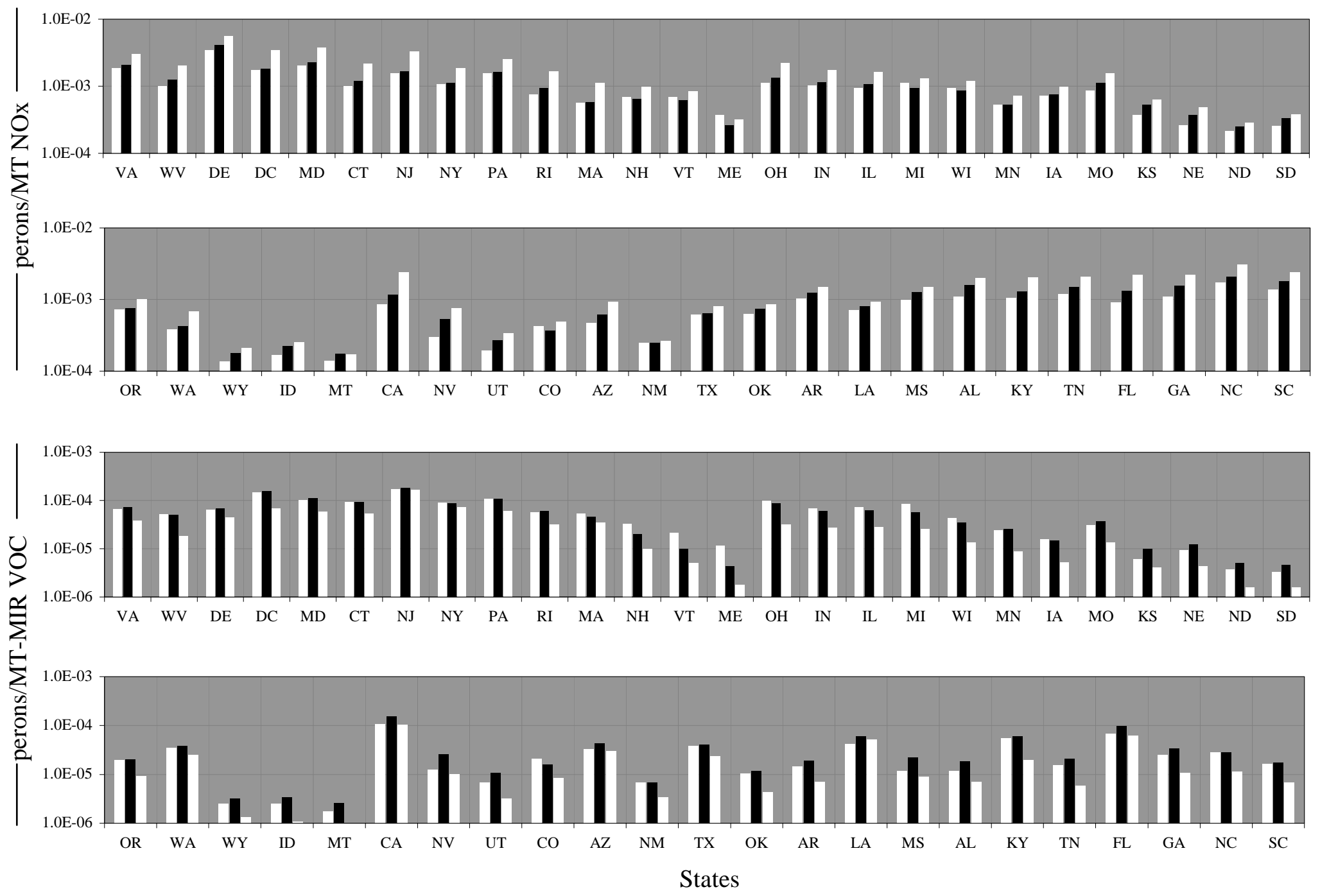

Figure 6-27 Comparison of human effect level factors for the three scenario 


\subsection{ILLUSTRATION OF A DISAGGREGATED LCA}

This section presents a case study illustrating the use of the characterization factors that were presented in Chapter 6.0. A spatially and temporally resolved LCA requires a suitably resolved LCI that is used along with the resolved impact characterization factors. Many times, it is relatively easy to obtain an inventory which is spatially resolved, at least, at the state level. However, the temporal resolution of LCI is usually very limited. Most of the standard inventory databases lack spatial and temporal details. An LCA practitioner or researcher has to make a greater effort to incorporate these details. However, there are some LCA application areas where the spatial and temporal details are easily available. The illustrative case study is an example of such an application. The purpose is not to develop a spatially and temporally resolved LCI, but to present a comparison between a coarsely and finely resolved LCA.

The case study is based on an earlier study by Shah et al [2008]. It is divided into two parts. The first is a comparison of energy use of two residential heating and cooling systems in Pittsburgh, PA. The first system uses a central natural gas powered furnace for heating, and a direct exchange split system for cooling. This system represents the majority of the heating and cooling systems in US households. The second system uses an electric air-air heat pump for heating as well as cooling. The heat pump is similar to an air conditioner, but can reverse the refrigeration cycle to heat the house. The heat pump system is growing in popularity, mainly because of its higher efficiency, and lower cost per unit of heat supplied compared to the furnace. The aim of this part of the study is to determine the effects of the varying temporal profile of the emissions.

The second part of the case study will focus on the change in impacts due to the change in the location of the building. For this part, the building will be, hypothetically, located at Pittsburgh, PA; Minneapolis, MN; Austin, TX; and Portland, OR. These locations lie in different climate zones and, therefore, have different heating and cooling requirements. Another 
difference among these locations is the electricity fuel mix, that is, the proportion of coal, natural gas, nuclear, renewable energy, etc. used for electricity generation. These differences are described further in this chapter.

\subsection{DESCRIPTION OF THE STUDY}

The study uses eQuest v3.60 [http://doe2.com/equest/] to simulate the thermal performance of the building. eQuest is based on the widely used DOE-2 software, but has a user friendly interface, making it easier to use. It also includes a 'project wizard' which guides a novice user at each modeling step. The building is defined as a set of several elements, such as floors, walls, windows, roof, etc, which form the building envelope. In addition, it also uses the data on the number of users, building use schedule, thermostat settings, lighting, room occupancy, HVAC and other mechanical and electrical systems, etc. It relies on hourly weather data for a particular year and location to calculate the external thermal loading. The external thermal loading also depends on the properties of the building envelope, building orientation, location, time-zone, etc. The internal load for the building is due to the occupants, lighting, and other equipment in the building. The heat required to be added or removed to maintain a fixed temperature is the sum of the external and the internal loads.

This addition or removal of heat is performed by the HVAC systems defined in the model. The central heating and cooling systems are the primary systems, whereas the air or water distribution systems are known as secondary systems. eQuest offers multiple options for the design of these systems. The energy required by these systems is dependent upon the total loading, and the efficiency of the systems.

The building selected for this study is a single family residence measuring $179 \mathrm{~m}^{2}$ (1938 sq. ft.) in livable area. It has an L-shaped plan with the two legs having equal lengths. A view of the modeled building is presented in Figure 7-1. The two longer faces are oriented towards the West and the South. It has two stories above ground and an unconditioned basement below. The roof has a pitch of $30^{\circ}$ with one foot of overhangs on all sides. It has a crawl space between the second floor and the roof. The walls and the roof are constructed as per standard wood construction. The wall siding and roofing have colors with medium radiation absorption. 


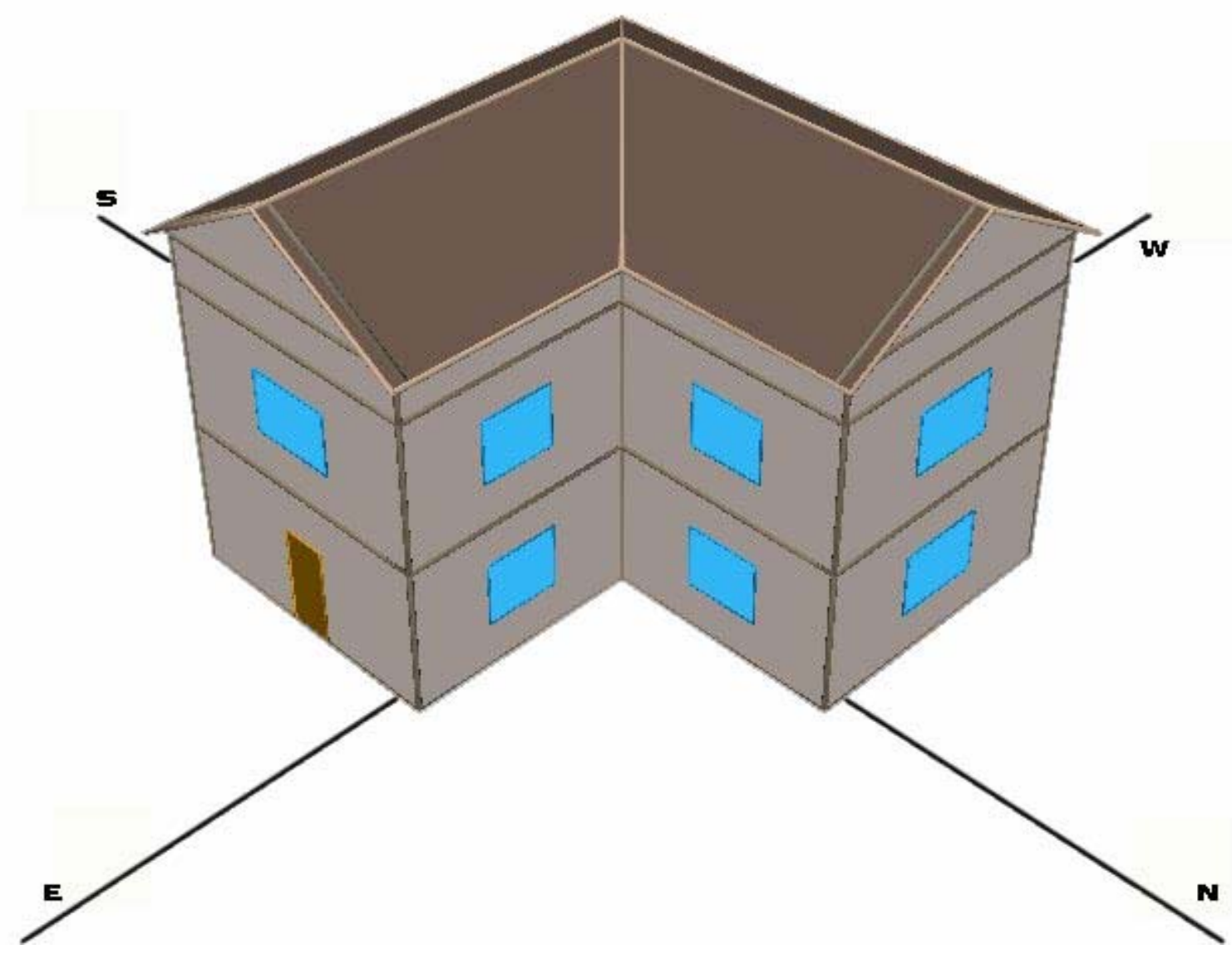

Figure 7-1 A view of the eQuest model of the building 
The insulation in the walls, floor, and ceilings is dependent upon the standard practice in each of the locations. The standard is usually the minimum recommendations of the International Energy Conservation Code (IECC) [International Code Council 2003] which are based on the building's climate zone. The heating and cooling requirements at these locations, which are a function of the climate zone, are summarized in Table 7-1. The IECC recommendations for the insulation levels have been used in this study. The heat resistivities (R-values) for the insulations at the different locations are shown in Table 7-2. In addition to the insulation, the recommended thermal properties of the windows also vary with the climate. The thermal properties of glass are defined by its heat conductivity (U-factor) and the Solar Heat Gain Coefficient (SHGC). The Ufactor and SHGC of glass are also presented in Table 7-2. The windows are assumed to be equally distributed on the four sides, with $14 \%$ of the net face area covered by windows. One opaque door is located, each, on the East and the West face.

The building is assumed to be occupied partially during the day between 7 am and $5 \mathrm{pm}$. The assumed maximum occupancy of the house is 4 persons. Additional internal loads are mainly due to internal lighting, and miscellaneous equipment. The thermostat is set at $78^{\circ}$ and $70^{\circ}$ for cooling and heating, respectively, during the occupied hours. During unoccupied hours, the temperatures are set at $85^{\circ}$, and $60^{\circ}$, respectively.

The central heating and cooling equipment studied at the four locations is the natural gas furnace, and the DX condenser. The burner assembly of the furnace uses an electrical spark to ignite a mixture of natural gas and air. The air, warmed from the combustion of the natural gas, rises up to the heat exchanger where the heat is transferred to the air flowing through the duct network in the house. The Annual Fuel Utilization Efficiency (AFUE) of the furnace chosen for the study is $94 \%$. AFUE is the average ratio of the heat generated to the fuel energy consumed during an entire year. It includes all heat losses due to seasonal variations and occupant temperature control.

The DX condenser is the commonly used split air-conditioning system. The DX coils that are located outside the house cool the refrigerant. The refrigerant travels between the condenser and heat exchanger that is located within the building, thus cooling the air inside. The Seasonal Energy Efficiency Ratio (SEER) of the unit is assumed to be 18. The SEER is the total cooling output averaged over the cooling season per unit electric input. 
Heat pumps, generally, have a lower efficiency compared to air-conditioners because of their reversibility. The key difference between a furnace and a heat pump is that the furnace generates heat by converting chemical energy from fossil fuels; whereas, the heat pump uses electricity to transfer the heat from outside to the inside. This greatly increases the heating capacity of the heat pump per unit of energy consumed. Thus, for a HSPF of 8.2, the heat pump can supply $2.4 \mathrm{KWh}$ of thermal energy per KWh of electrical energy. This efficiency is a function of the outside temperature. It decreases as the temperature decreases. At temperatures below $20^{\circ} \mathrm{F}$, the outside heat is very low for the heat pump to be economical, and thus it uses the electrical resistance coils to generate heat.

Table 7-1 Regional climate overview for the four locations

[Shah et al 2008]

\begin{tabular}{lcc}
\hline Location & $\begin{array}{c}\text { Heating degree days } \\
\left(\text { Base } 65^{\circ} \mathrm{F}\right)\end{array}$ & $\begin{array}{c}\text { Cooling degree days } \\
\left(\text { Base } 65^{\circ} \mathrm{F}\right)\end{array}$ \\
\hline Pittsburgh, PA & 5829 & 726 \\
Minneapolis, MN & 7876 & 699 \\
Austin, TX & 1648 & 2974 \\
Portland, OR & 4400 & 390 \\
\hline
\end{tabular}

Table 7-2 Building envelope insulation levels at various locations

[Shah et al 2008]

\begin{tabular}{|c|c|c|c|c|}
\hline Building Component & Pittsburgh, PA & Minneapolis, MN & Austin, TX & Portland, OR \\
\hline $\begin{array}{l}\text { Glazing U-factor, } \\
\mathrm{W} / \mathrm{K} \cdot \mathrm{m}^{2}\left(\mathrm{Btu} / \mathrm{h} \cdot{ }^{\circ} \mathrm{F} \cdot \mathrm{ft}^{2}\right)\end{array}$ & $2.3(0.40)$ & $2.0(0.35)$ & $3.7(0.65)$ & $2.6(0.45)$ \\
\hline $\begin{array}{l}\text { Glazing Solar Heat Gain } \\
\text { Coefficient }\end{array}$ & 0.55 & 0.55 & 0.40 & 0.55 \\
\hline $\begin{array}{l}\text { Floor Insulation, } \\
\mathrm{K}^{\prime} \mathrm{m}^{2} / \mathrm{W}\left(\mathrm{h}^{\circ} \mathrm{F} \mathrm{Ft}^{2} / \mathrm{Btu}\right)\end{array}$ & $5.3(30)$ & $5.3(30)$ & 3.4 (19) & $3.4(19)$ \\
\hline 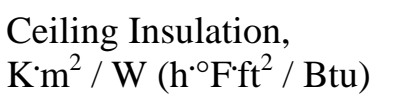 & $6.7(38)$ & $8.7(49)$ & $5.3(30)$ & $5.3(30)$ \\
\hline 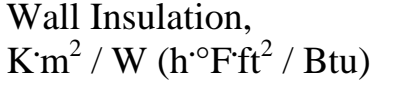 & $3.2(18)$ & $3.7(21)$ & $2.3(13)$ & $2.3(13)$ \\
\hline
\end{tabular}




\subsection{LIFE CYCLE INVENTORY}

For simplicity, this study limits the inventory only to the annual energy use for the heating and cooling of the building. It does not consider the entire life cycle of the systems, which should include the upstream processes of manufacturing and transportation of the systems, the energy usage and maintenance throughout the life of the systems, and their final recycling, or disposal. Thus, the inventory studied is not truly a life cycle inventory.

The simulation of the building with the furnace and AC is performed for the four locations. For Pittsburgh, the building is simulated with the heat pump, too. All simulations are performed using the weather conditions for 2001. The results of the simulation are presented in Figure 7-2. The vertical axis represents the energy consumption, in KWh, for each month. Based on the end use, the energy demand is divided into heating and cooling. The heating energy is obtained from either direct combustion of natural gas in residential furnaces or in the form of electricity from an electricity generating unit for the heat pump. The heating energy is, thus, further divided into thermal energy and electrical energy.

From Figure 7-2, it is seen that the annual heating demand is considerably higher for all the four locations compared with the cooling demand. As expected, for Minneapolis, which is the northernmost of the four locations, it is 17 times the cooling demand; whereas for Austin, the southernmost location, it is about 2 times. The period having no heating requirement varies from 3 months (in Portland and Minnesota) to 7 months (in Austin). For the heat pump in Pittsburgh, the annual heating energy requirement is $27 \%$ lower than the annual heating energy requirement of a furnace. This is because of the high efficiency of the heat pump in transferring heat.

In addition to the difference in the weather related energy demands at the four locations, the four locations also differ in their electricity fuel mix. Table 7-3 presents the average proportion of electricity obtained from each fuel. These proportions vary inter as well as intra annually. However, the percentages presented in Table 7-3 are the annual averages for the year 2004. These electricity profiles are not specific to the individual cities, but are state-wide averages. 
Table 7-3 Electricity profiles for the four locations

[Shah et al 2008]

\begin{tabular}{lcccc}
\hline Fuel source & Pennsylvania & Minnesota & Texas & Oregon \\
\hline Coal (\%) & 55 & 65 & 38 & 7 \\
Natural gas (\%) & 5 & 3 & 48 & 25 \\
Nuclear (\%) & 35 & 25 & 10 & 0 \\
Petroleum and other gases (\%) & 2 & 2 & 2 & 1 \\
Hydropower \& other renewables (\%) & 3 & 5 & 2 & 67 \\
\hline
\end{tabular}

The difference in the fuel mix is important, as it affects the emissions of $\mathrm{NO}_{\mathrm{x}}$ and VOC per unit electricity generated. $\mathrm{NO}_{\mathrm{x}}$ and VOC are generated during processing and combustion of fossil fuels. Thus, states which depend heavily on fossil fuels for their electricity have higher unit emissions. For example, Texas obtains $88 \%$ of its electricity from coal, natural gas and other fossil fuels. On the other extreme, 67\% of Oregon's electricity is obtained from hydropower and the remaining from fossil fuels. It is assumed in this study that $\mathrm{NO}_{\mathrm{x}}$ and VOC are emitted only during the process of deriving electricity from fossil fuels. Thus, this study does not consider the fossil fuel combusted, solvents manufactured, or other sources of emissions during the construction of the electricity generation infrastructure or during its operation. Similarly, it does not consider the emissions during the construction of the infrastructure for other fuels, too. Thus nuclear energy, hydropower, and renewable sources are assumed to have zero emissions.

The emission factors for $\mathrm{NO}_{\mathrm{x}}$ and VOC are presented in Figure 7-3. The emission for VOC is weighted with the MIR for the emitted VOC mixture from each process. The VOC speciation profiles are obtained from the speciation profile data used in SMOKE. The emission factors have been calculated from the US LCI database maintained by National Renewable Energy Laboratory [http://www.nrel.gov/lci]. Figure 7-3 (a) shows the emission factors per KWh of thermal energy derived from natural gas in a residential furnace. The emission factors per KWh of electricity derived from natural gas are given in Figure 7-3 (b). 

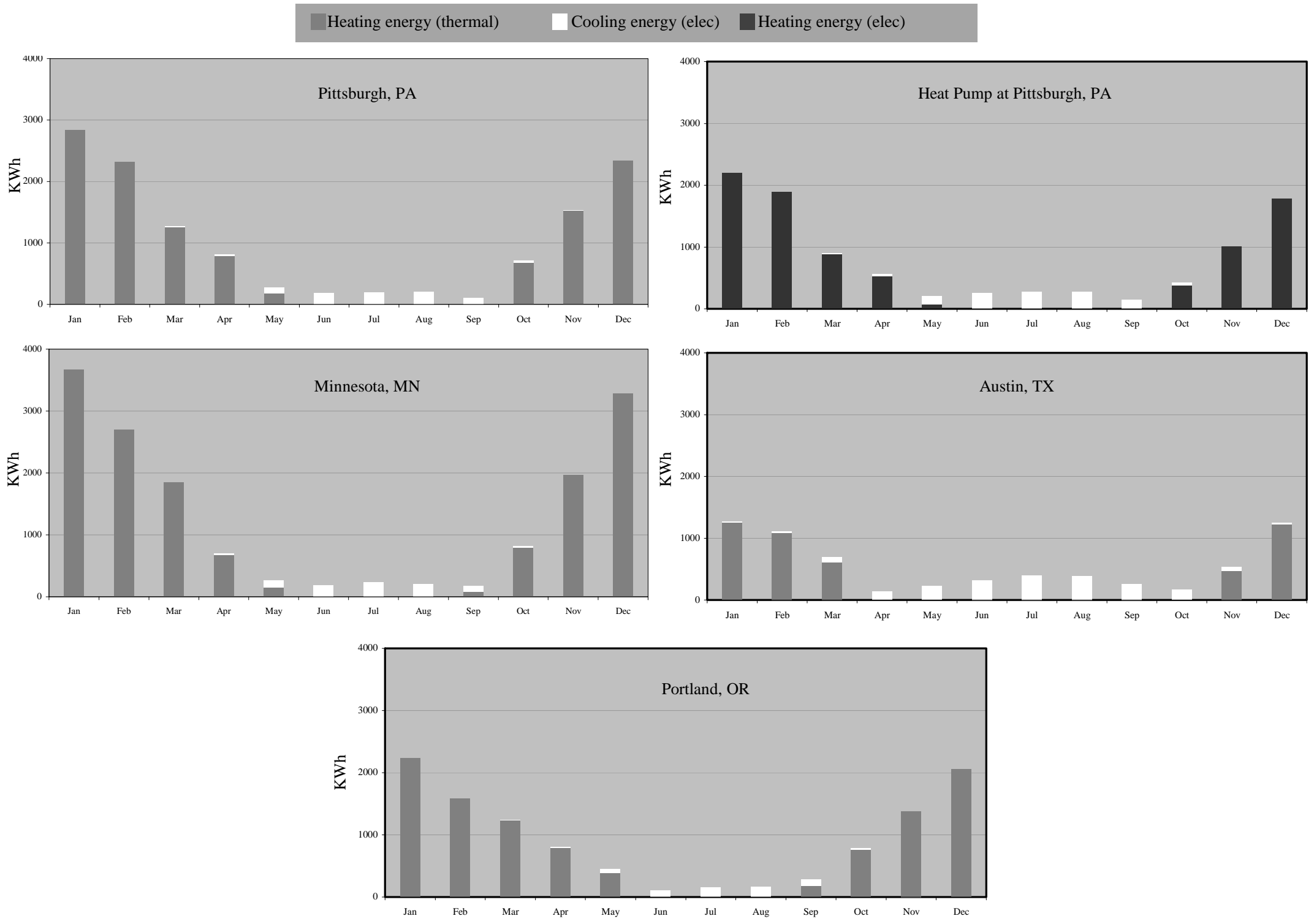

Figure 7-2 Results of the HVAC energy use simulation for the building 


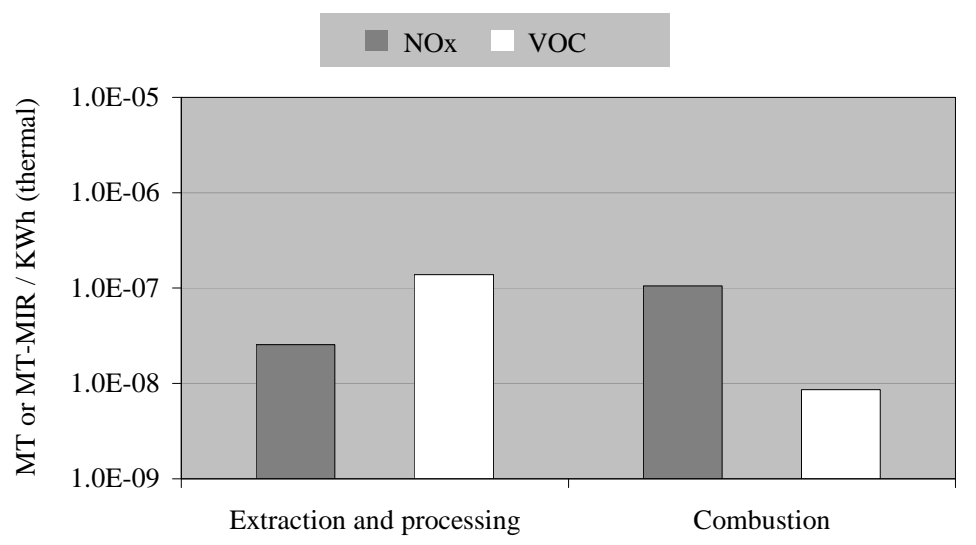

(a) Natural gas in residential furnace

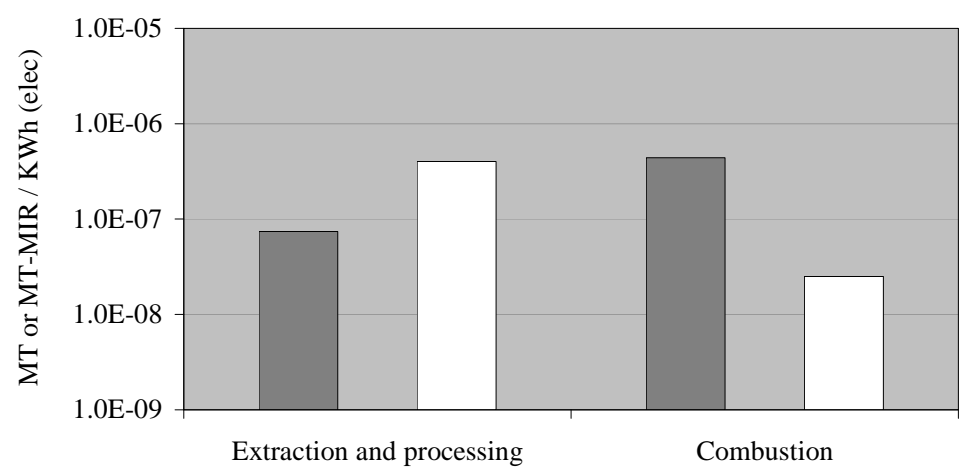

(b) Natural gas in electricity boilers

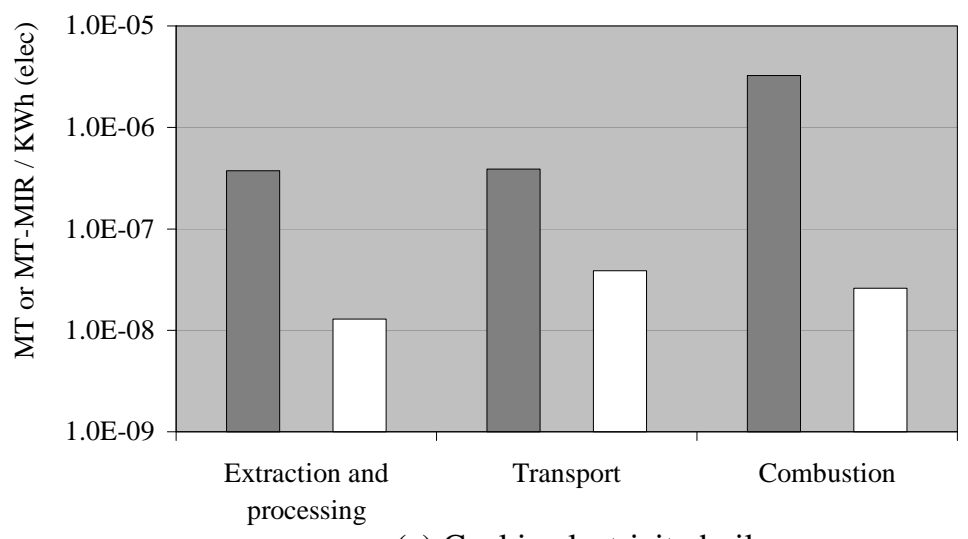

(c) Coal in electricity boilers

Figure 7-3 Emission factors for energy derived from fossil fuels 
Extraction and processing, and combustion are the two processes included for calculation of emission factors for natural gas usage. Emissions during distribution of gas are assumed to be negligible. The emission factors for a unit of electricity from natural gas are about 33\% higher than the emission factors for thermal energy. This represents the efficiency of conversion of thermal energy to electricity at the generation units. The VOC emitted during the extraction and processing of natural gas is about 15 times higher than the emission from combustion. On the other hand, the emissions of $\mathrm{NO}_{\mathrm{x}}$ during combustion are 4 times higher than during extraction and processing.

The emission factors for coal are shown in Figure 7-3 (c). The processes of extraction and processing, transportation of coal from the processing plant to the electricity plant, and its combustion are considered. The transportation emissions shown are for Minneapolis and Portland. For Pittsburgh and Austin these are about 10 times lower, due to their proximity to coal deposits. In case of coal, the $\mathrm{NO}_{\mathrm{x}}$ emissions from combustion are higher than the combined emission from the upstream processes. VOC emissions are almost evenly distributed among the three processes for larger transportation distances. It is seen that the overall $\mathrm{NO}_{\mathrm{x}}$ emission factors for coal generated electricity are 7.3 times the emission factors for natural gas generated electricity; whereas the VOC emission factors for coal are 0.4 times those for natural gas.

The combustion process and the upstream processes do not occur at the same locations. For example, natural gas extraction and processing for the fuel received in Pennsylvania happens in the Gulf of Mexico. The states where the upstream processes are located for coal and natural gas are given in Table 7-4. The two largest sources of natural gas for the US are Alaska and Gulf of Mexico (Texas, Louisiana, and Alabama). Coal, on the other hand, is locally available in Pennsylvania, and Texas. Wyoming is the major source of coal for the other two cities. Coal is assumed to be transported in diesel powered locomotives to the electricity generating units. For Minneapolis and Portland, the trains pass through South Dakota and Idaho, respectively, on their routes. Thus, coal transportation emissions are distributed in three states based on the distance covered within each state.

A LCI which is disaggregated spatially at the state level and temporally at the month level is formed by combining the monthly energy usage, the electricity profiles, emission factors, and the locations of the upstream processes. 
Table 7-4 Location of upstream processes for each location

\begin{tabular}{lcccc}
\hline Fuel: Upstream process & Pittsburgh & Minneapolis & Austin & Portland \\
\hline $\begin{array}{l}\text { Natural gas: Extraction and } \\
\text { processing }\end{array}$ & LA & AS & TX & AS \\
Coal: Extraction and processing & PA & WY & TX & WY \\
Coal: Transportation & PA (100 mi) & $\begin{array}{c}\text { WY (250 mi) } \\
\text { SD (400 mi) } \\
\text { MN (350 mi) }\end{array}$ & TX (100 mi) & ID (330 mi) \\
& & & OR (330 mi) \\
\hline
\end{tabular}

\subsection{LIFE CYCLE IMPACT ASSESSMENT}

The characterization factors developed in Chapter 6.0 will be used to calculate the impact of the disaggregated LCI. Impacts will be calculated using the human and ecosystem exposure level factors for the respective states and months of the emission processes. However, Alaska, the source of natural gas for Minnesota and Oregon, is located outside the model domain. The characterization factors for Alaska have not been calculated. It is assumed that due to the extremely cold climate the ozone formation in Alaska is negligible compared to other states.

The results of the disaggregated impact assessment will be compared to results obtained using aggregated characterization factors. The MIR values for the compounds will be used as the characterization factors. Jane Bare [2008] has indicated that the forthcoming revision for TRACI would continue to use MIR values for characterizing photochemical smog impacts. Since the emissions of VOC are already MIR weighted, the MIR for $\mathrm{NO}_{\mathrm{x}}$ is, additionally, required. The MIR values for $\mathrm{NO}$ and $\mathrm{NO}_{2}$ have been recently calculated by Carter [http://pah.cert.ucr.edu/ carter/SAPRC/]. These have been calculated by adjusting the VOC concentrations such that a unit emission of $\mathrm{NO}_{\mathrm{x}}$ produces the maximum ozone. The US average values for $\mathrm{NO}$ and $\mathrm{NO}_{2}$ are 25.4 and $17.2 \mathrm{~g} \mathrm{O}_{3} / \mathrm{g}$ compound, respectively. Considering a 9:1 ratio for the emissions of $\mathrm{NO}$ and $\mathrm{NO}_{2}$, the emission weighted $\mathrm{NO}_{\mathrm{x}}$ MIR value is 24.6. 
The results of impact characterization, using both the aggregated and disaggregated characterization methods, are presented in Figure 7-4. Figure 7-4 (a) presents the comparison of the impacts for furnace and AC, and heat pump for Pittsburgh. Figure 7-4 (b) presents the impacts for the four locations. The impacts are cumulative for the entire year and are shown separately for the summer (April to September) and winter (October to March) months. The impacts are normalized to the worst case in each category.

It is seen in Figure 7-4 (a) that for the aggregated characterization method the impact of the furnace and AC system is only $16 \%$ of the impact of the heat pump. More than $80 \%$ of the impact of the heat pump is during the winter months; whereas it is about half for the furnace and AC. This is because, during the winter, the heat pump has higher electricity consumption which has a high $\mathrm{NO}_{\mathrm{x}}$ emission factor. Besides, the MIR value for $\mathrm{NO}_{\mathrm{x}}$ is about 9 times the MIR value for the average VOC mixture. Even though the energy consumption of furnace is 14 times that of the AC, the impacts for the summer and winter are almost equal.

The results of the disaggregated impact assessment still show a clear preference of furnace and AC over the heat pump. However, the difference in their relative performance has decreased substantially. The impacts of the furnace and AC system are $60 \%$ and $70 \%$ of the impacts of the heat pump in the human and ecosystem exposure categories, respectively. For human exposure, the summer time impacts are in the range of $80-90 \%$ of the annual impacts for both the systems, despite the higher energy consumption in winter. This is due to the coal dominated electricity profile of Pennsylvania, and the considerably higher human exposure characterization factors for $\mathrm{NO}_{\mathrm{x}}$ in the summer months. Thus, the greater heating efficiency of the heat pump and the resulting savings in energy consumption has no environmental benefit for ozone impacts. The lower efficiency of the heat pump in transferring the heat from inside to the outside compared to the AC is the important criteria. This effect is also seen in the aggregated impacts, but the use of temporally resolved factors for human exposure places greater weight on emissions during summer when most of the ozone episodes occur. 


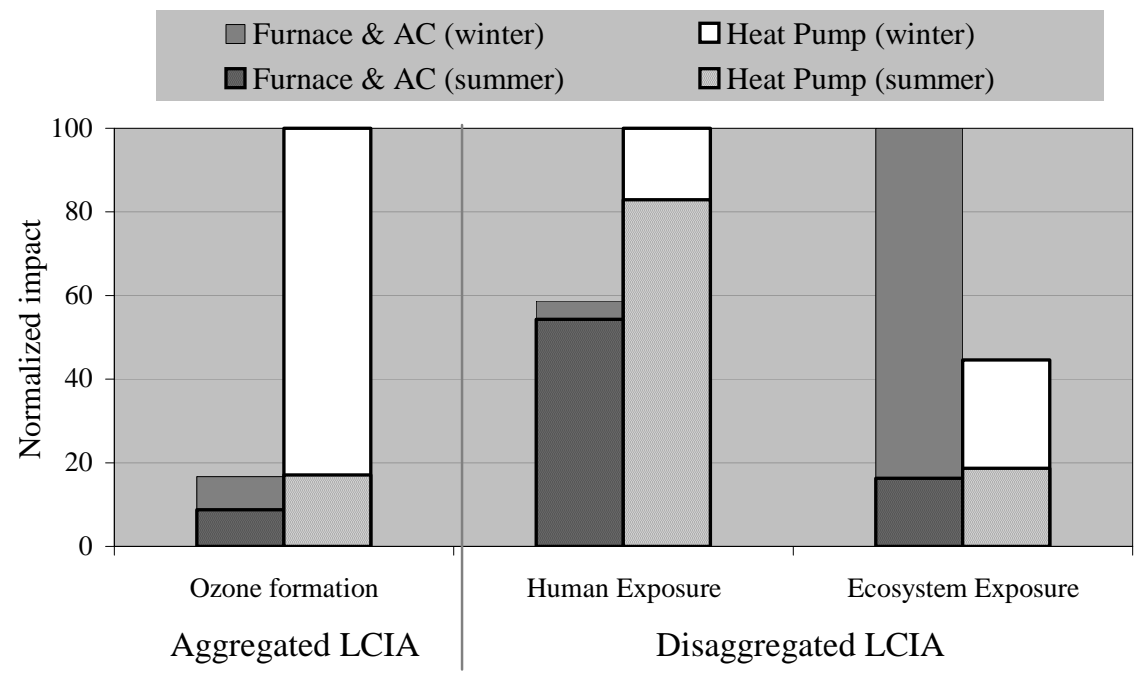

(a)

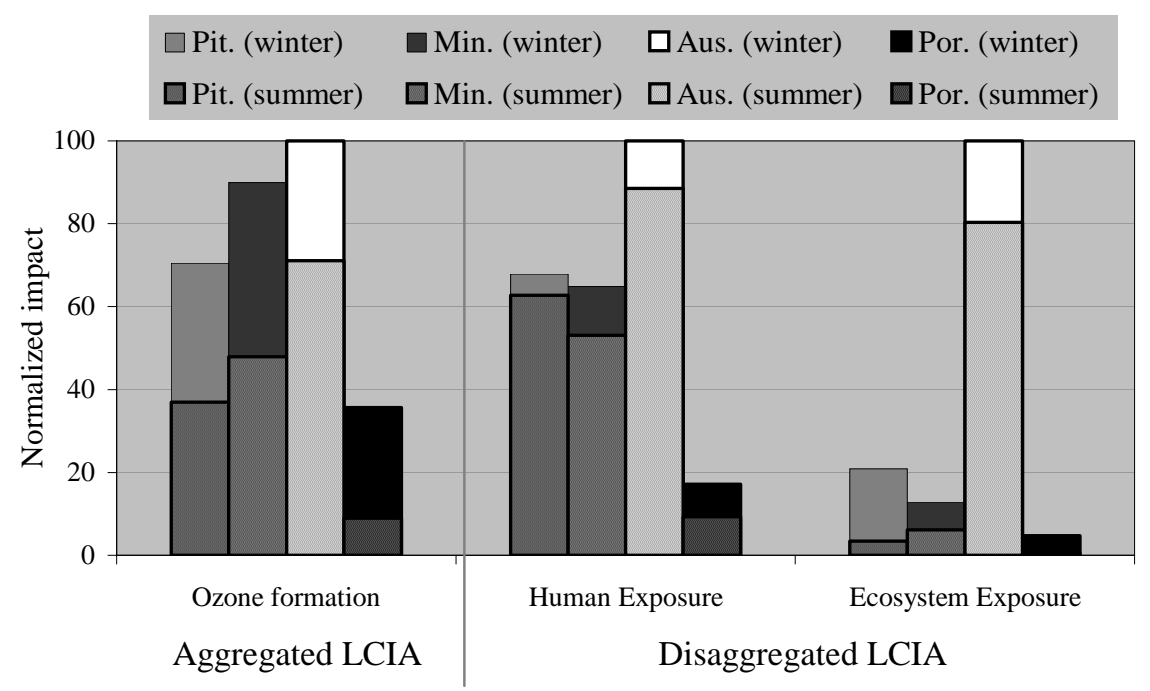

(b)

Figure 7-4 Results of impact assessment for (a) the two systems, and (b) the four locations 
For ecosystem exposure, more than $80 \%$ of the impacts of the furnace and AC are experienced in the winter. This is because Louisiana, which has the highest ecosystem exposure characterization factors, is the source of natural gas for Pennsylvania. The consumption of energy derived from natural gas for the furnace is about 25 times that of the heat pump. If Pennsylvania would also have obtained its natural gas from Alaska, the furnace's impact on ecosystem would have been lower than the ecosystem impact for the heat pump. Despite the 8 times higher electricity consumption of the heat pump in the winter, the relative impacts on ecosystem are almost equal. This is, again, due to higher ozone formation and deposition potential during the summer months.

The results of the aggregated impact assessment for the four locations show that the highest impact is for Austin, followed closely by Minneapolis. Impacts for Pittsburgh and Portland are $70 \%$ and $35 \%$ of those of Austin, respectively. The summer time impacts range from $25 \%$ to $70 \%$. For Austin, the impacts are the highest due to the high electricity consumption by the AC. The AC electricity consumption in Texas is almost equal to the sum of the AC consumption for the other three locations. However, the smaller percentage of coal in the electricity profile of Texas partly compensates for this higher electricity usage. The summer impacts in Portland are lower than the winter impacts because of the low proportion of fossil fuels in its electricity profile. The winter period impacts are directly proportional to the energy consumption, and are highest for Minneapolis. The true winter period for Austin is, actually, shorter than October to March. Thus the winter time impacts (October to March) include some cooling impacts, too. Similarly, for the other three places, some heating was required in April and May. It is important to note that these heating and cooling requirements are only for the year 2001, and may vary inter-annually.

A disaggregated impact calculation also shows highest impacts for Austin in, both, the human and ecosystem exposure categories. (Figure 7-4 b) As seen in Figure 7-4 (b), the impacts during summer are magnified more than the winter impacts. However, the impacts for Pittsburgh are higher than the impacts for Minneapolis in both categories. This is mainly because the impacts are dominated by coal combustion in the boilers, and because of the higher summer time $\mathrm{NO}_{\mathrm{x}}$ and VOC characterization factors for Pennsylvania compared to Minnesota. Besides, the characterization factors for emissions during coal processing and extraction are higher for coal 
combusted in Pittsburgh. The greater transportation of coal to Minnesota and Oregon does not change the overall ranking.

In the ecosystem exposure impact category, the impacts are dominated by winter period impacts, except for Austin, where the winter energy consumption and, thus, impacts are relatively low. For Minneapolis and Portland, the unit impacts of natural gas (sourced in Alaska) are lower, than for Pittsburgh and Austin. Besides, there is less inter-annual variation in the ecosystem exposure factors. Thus the summer and winter impacts in Minneapolis are almost equal. For Portland, the lower fossil fuel consumption combined with lower emission and characterization factors is the reason for lower impacts.

The monthly spatial distribution of the human exposure impacts for the natural gas furnace and AC system in Pittsburgh are presented in Figure 7-5. 86\% of the annual human exposure impact is in the months of May to September. During these months, coal is the primary source of energy. Natural gas contributes only 5\% to Pennsylvania's electricity mix. Thus, almost all emissions due to extraction and processing, and combustion processes occur in Pennsylvania. As a result, the majority of the cumulative exposure, during these months, occurs to the populations of Pennsylvania, New York, and New Jersey. Canada and the surrounding US states of Ohio, West Virginia, Virginia, Maryland, Connecticut, Massachusetts, and North Carolina are also significantly affected. The Western states remain mostly unaffected.

However, the spatial distribution of the impact changes in the winter months. The 'centre of impact' gradually moves southwards as winter approaches. There are two reasons behind this change. Firstly, during the winter months, natural gas becomes the primary source of energy for thermal comfort. Natural gas that reaches Pennsylvania is extracted and processed in the Gulf of Mexico. Hence, a significant quantity of emissions occurs near or within the state of Louisiana. Besides, during the winter, the Southern states have a relatively higher potential for ozone formation than the Northern states. The characterization factors for VOC emissions are also higher during the winter months compared to the summer months. Thus, Texas and Louisiana experience majority of the exposure due to residential heating in Pennsylvania during winter. The impacts experienced in Pennsylvania are also significant. Almost all of the US Eastern states are affected due to natural gas combustion in Pennsylvania in the winter months. Considerable impacts are experienced in Mexico and Canada. Smaller impacts are felt as far as California, too. 
In the above discussion, it is seen that using state and month specific characterization factors can change the order of performance ranking. It also affects the differences between the relative performances in each location. The relative differences also considerably vary between the human and ecosystem impact categories. Besides, there are important differences in the spatial distribution of the impacts in the summer and winter months. The spatial distribution of impact is useful in differential weighting of the impacts based on location when the impacts due to processes in one state affect the population in other states or other countries. For example, human health effects experienced in Canada or Mexico due to emissions in Pennsylvania can have different weighting than the impacts experienced within the US. These emphasize the importance of using a spatially and temporally resolved characterization method, and also the importance of moving from fate level towards effect level impact characterization. 


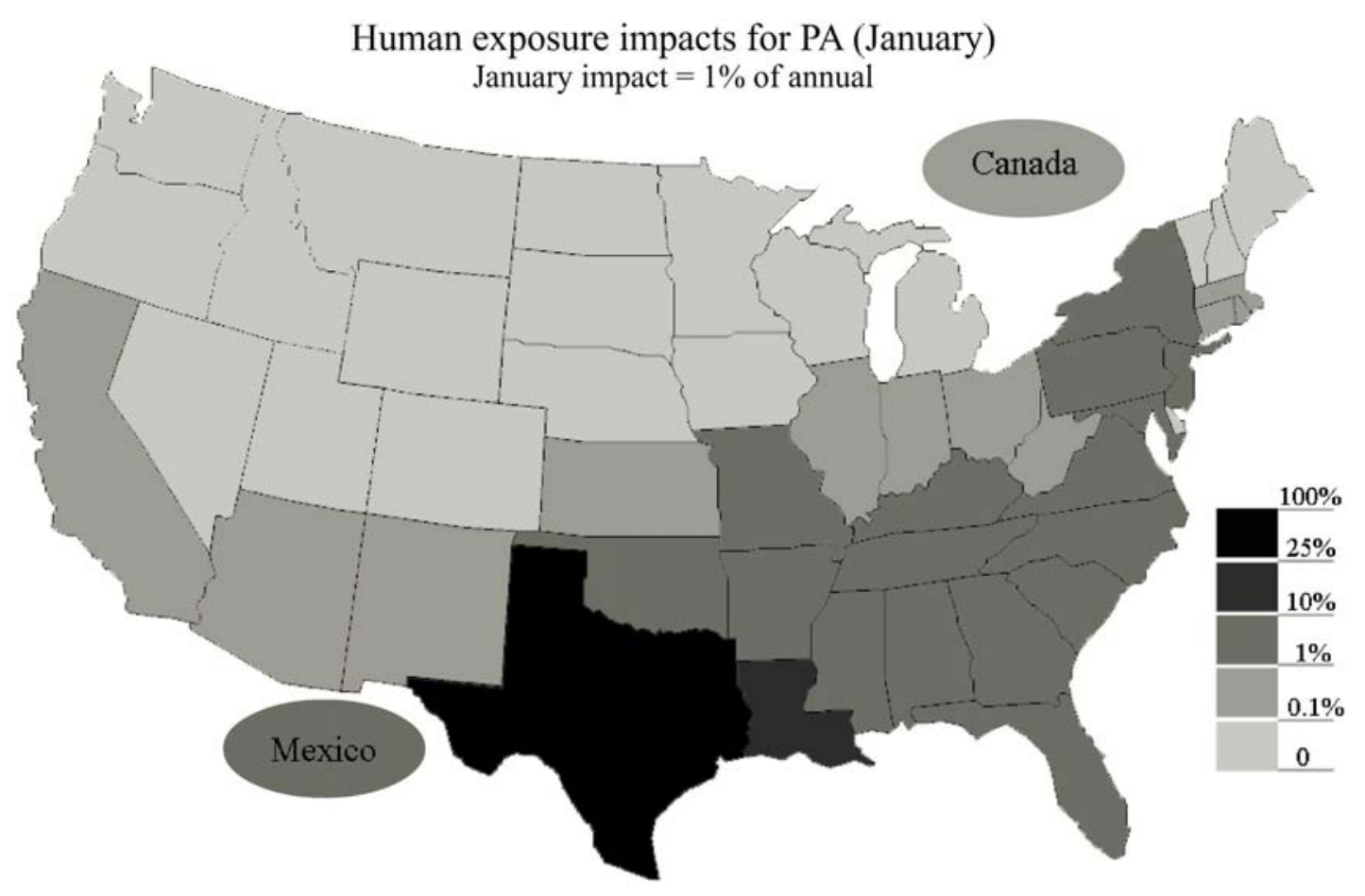

(a) January

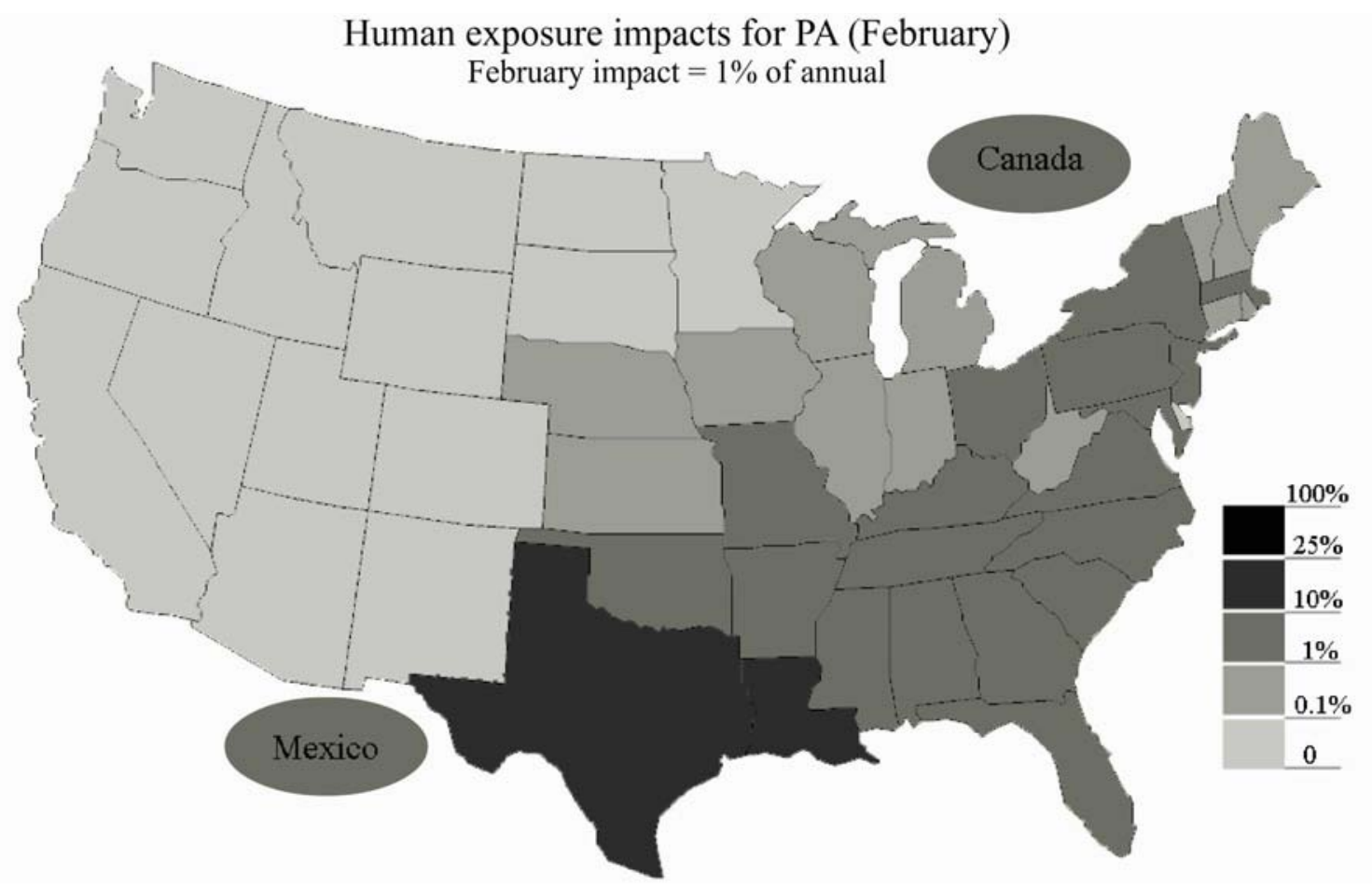

(b) February

Figure 7-5 Spatial distribution of human exposure impacts for furnace and AC system for Pittsburgh 


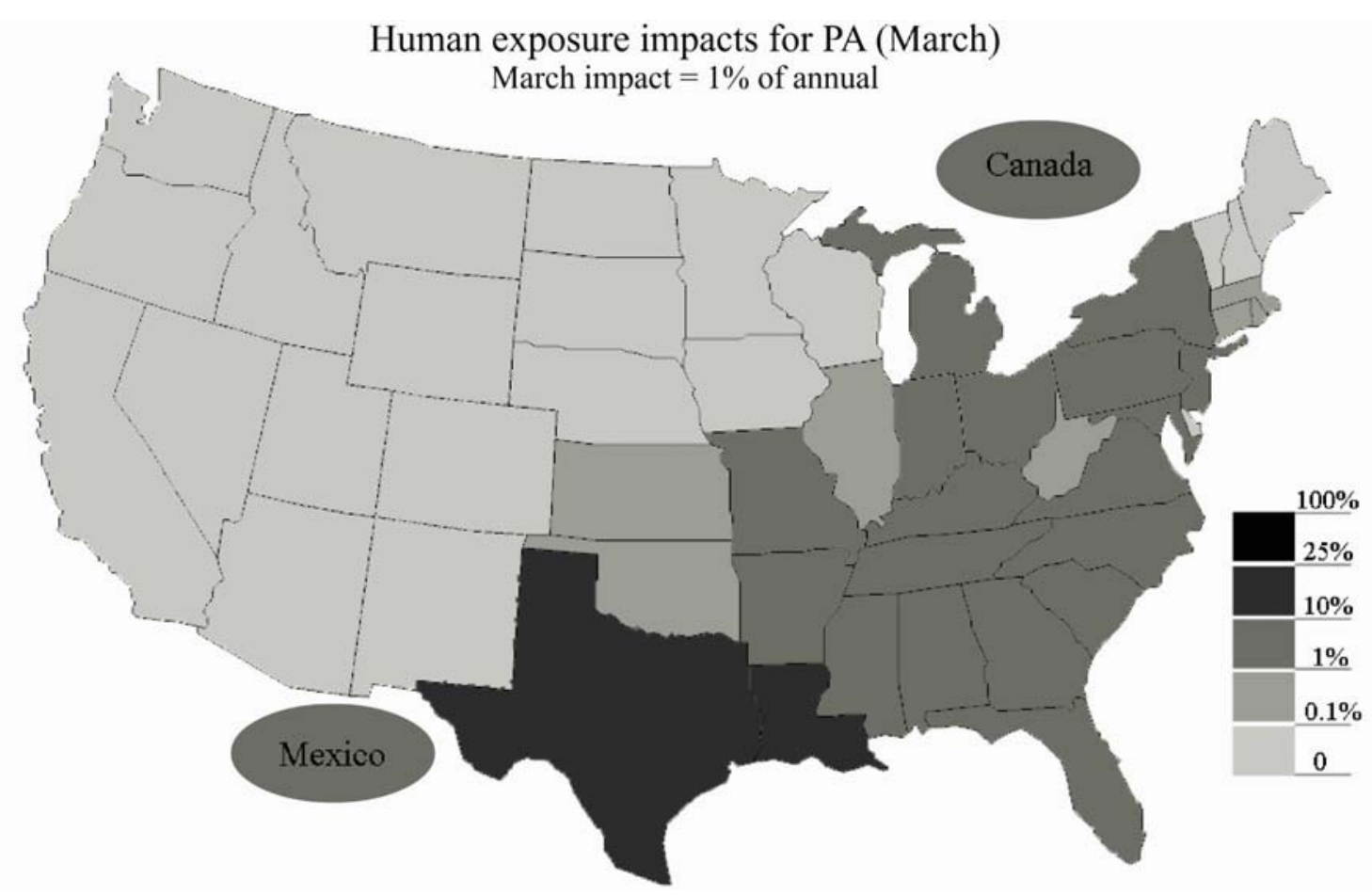

(c) March

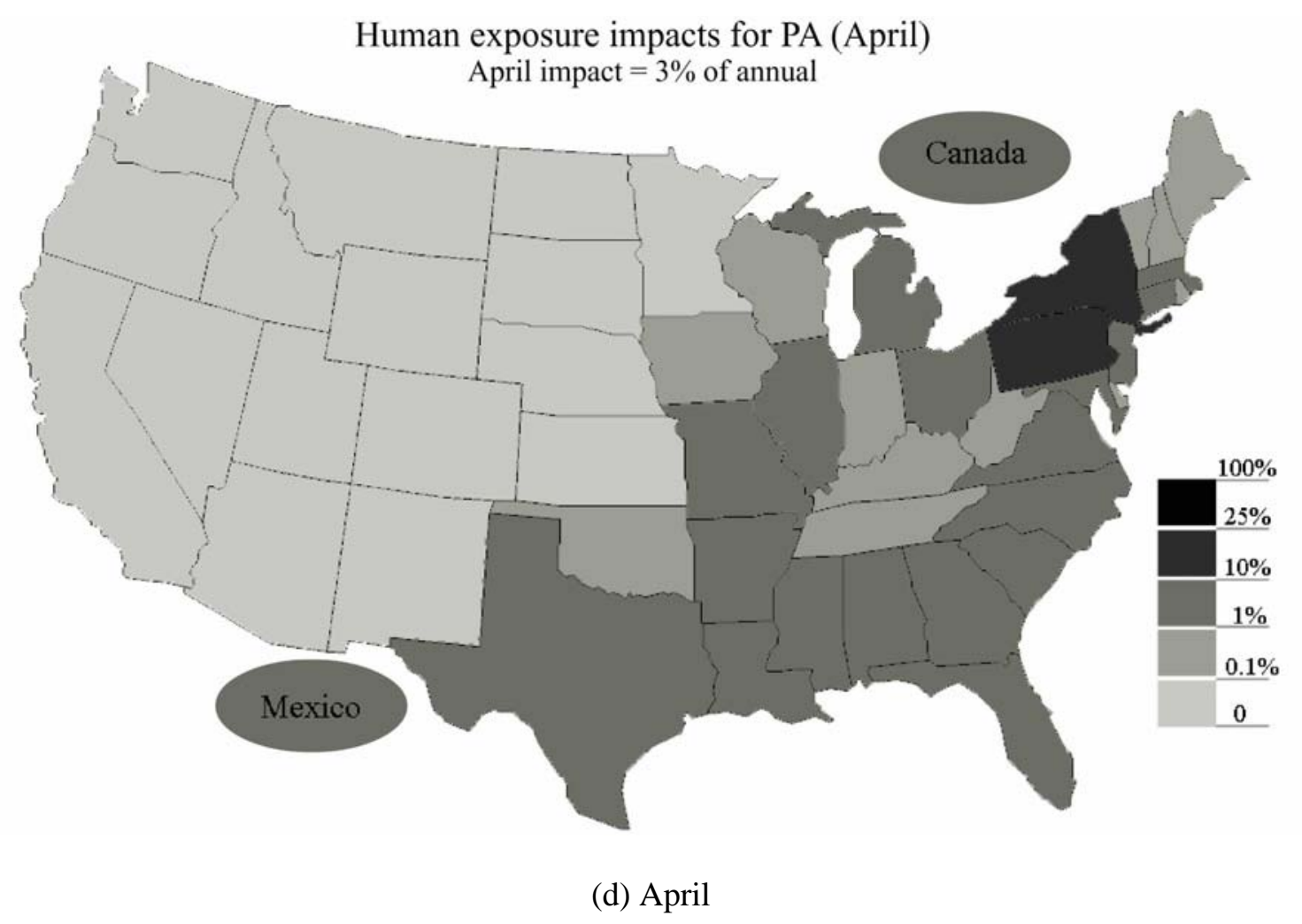

Figure 7-5 continued 


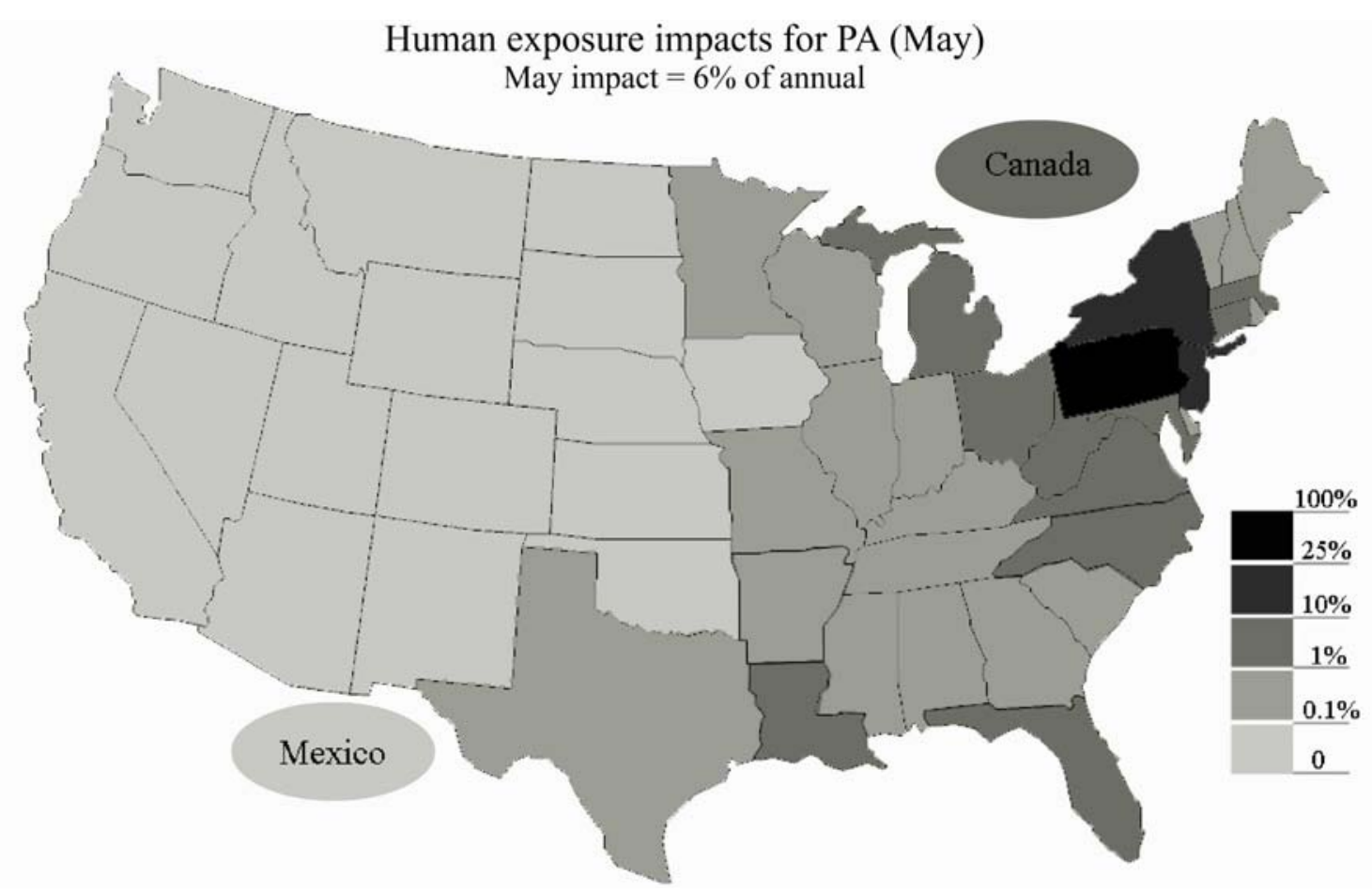

(e) May

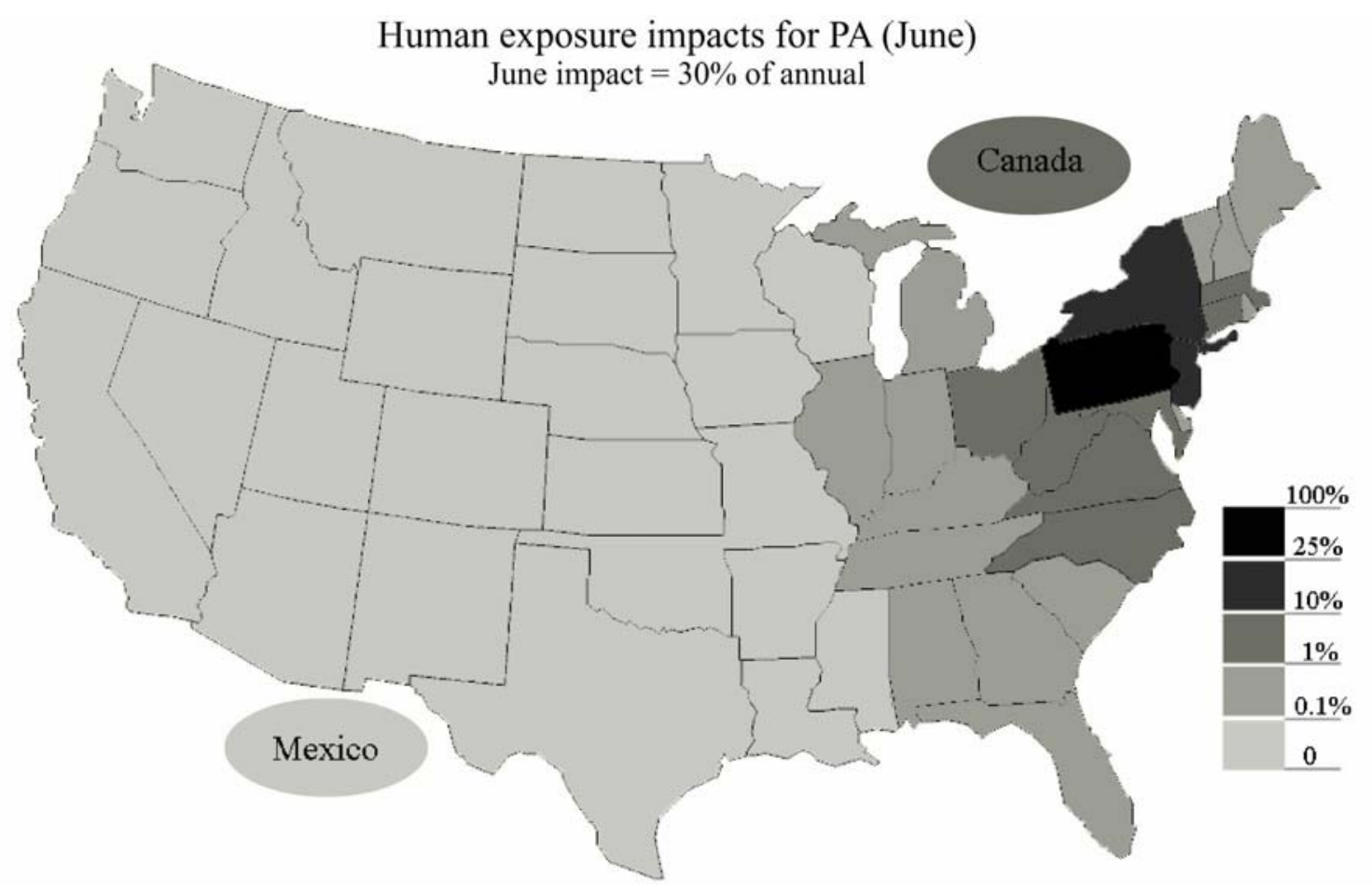

(f) June

Figure 7-5 continued 
Human exposure impacts for PA (July)

July impact $=31 \%$ of annual

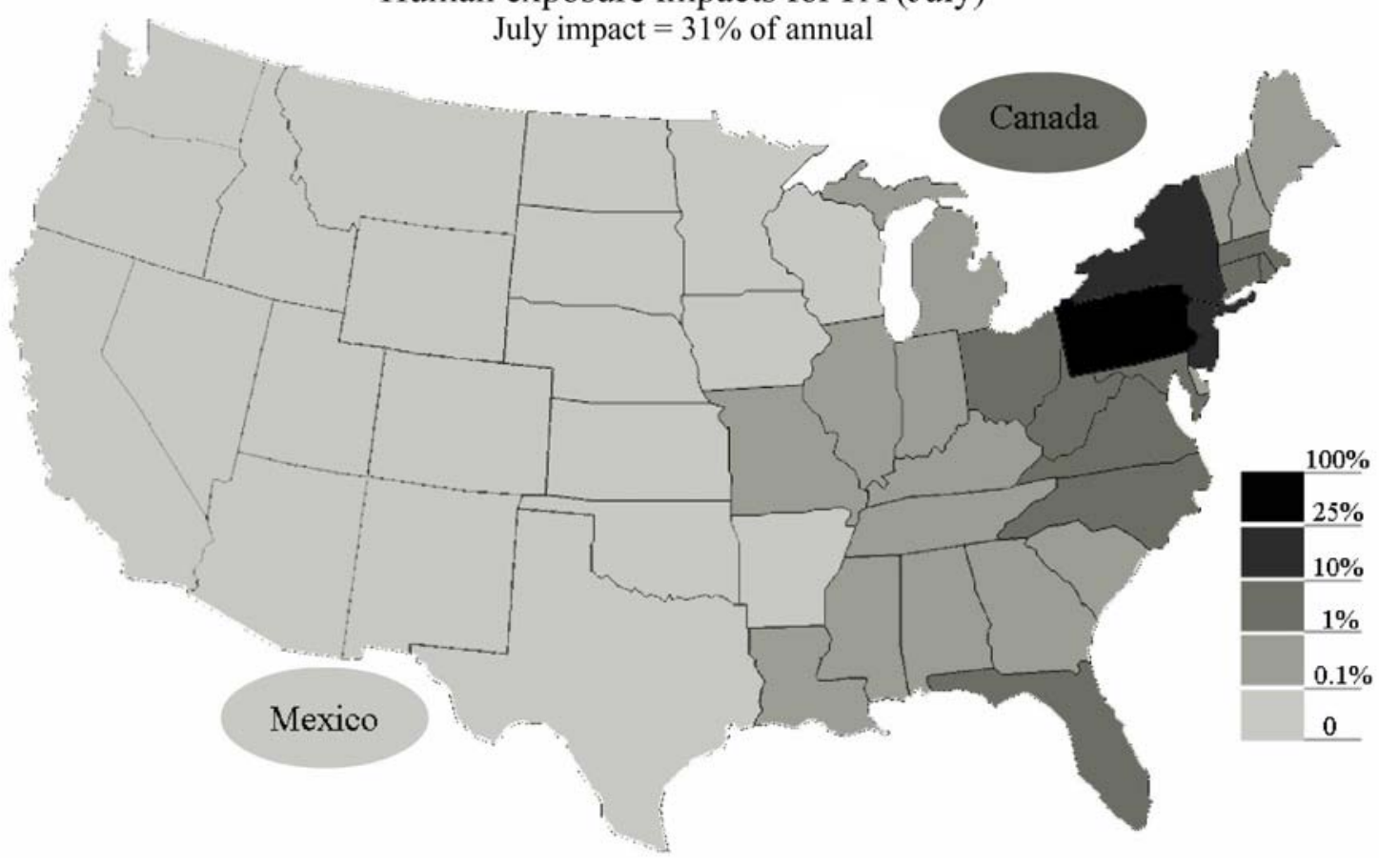

(g) July

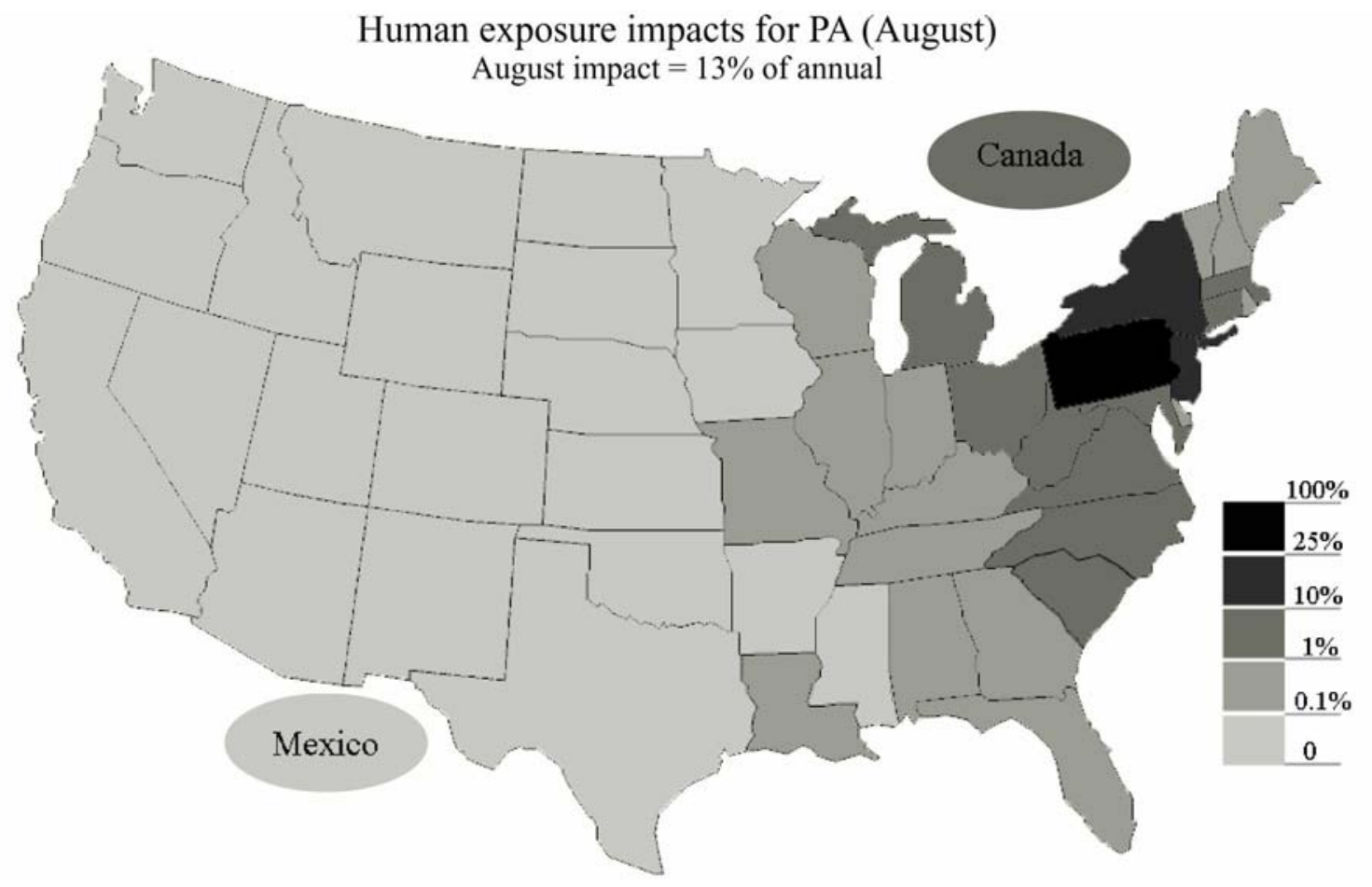

(h) August

Figure 7-5 continued 


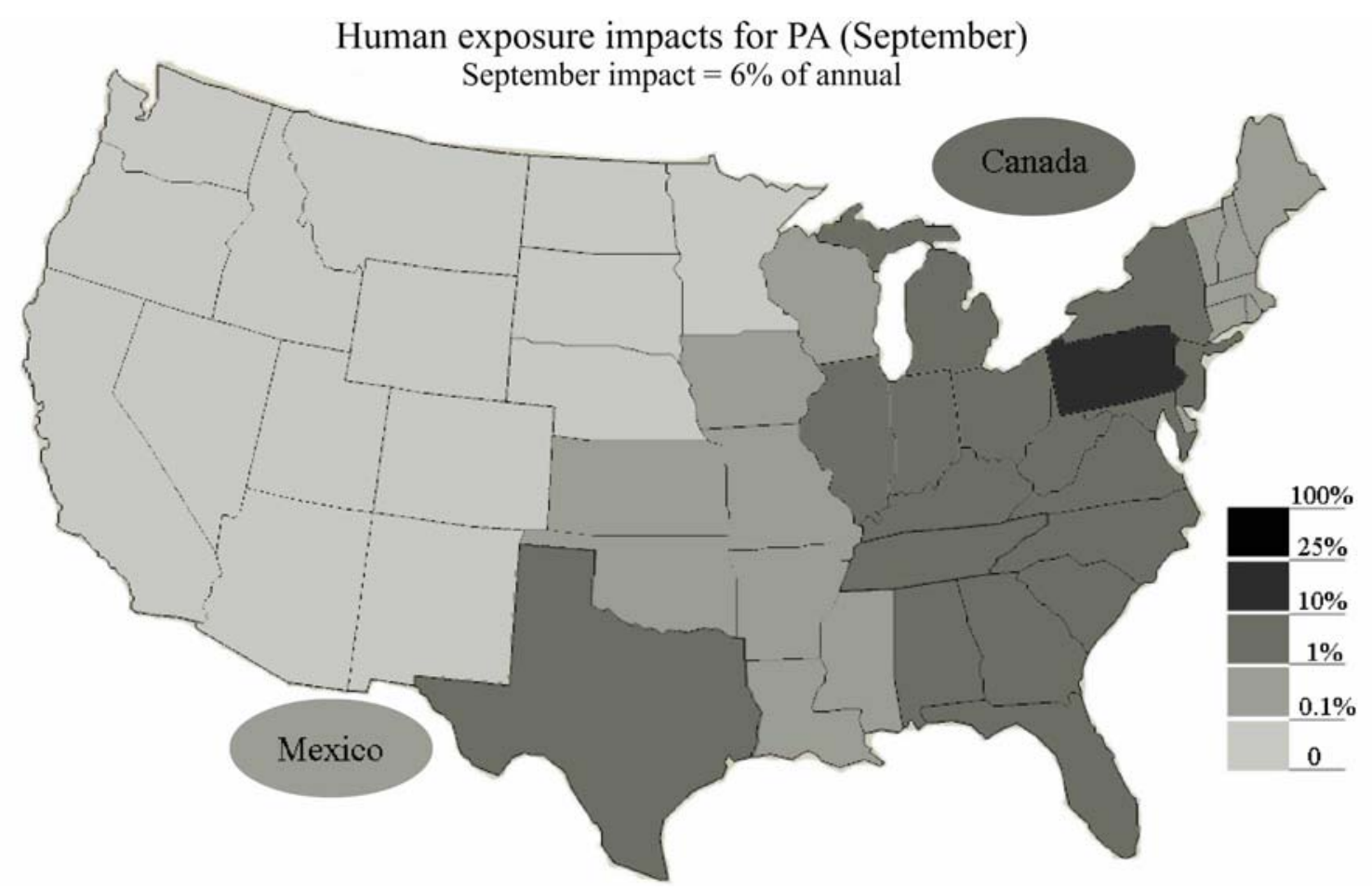

(i) September

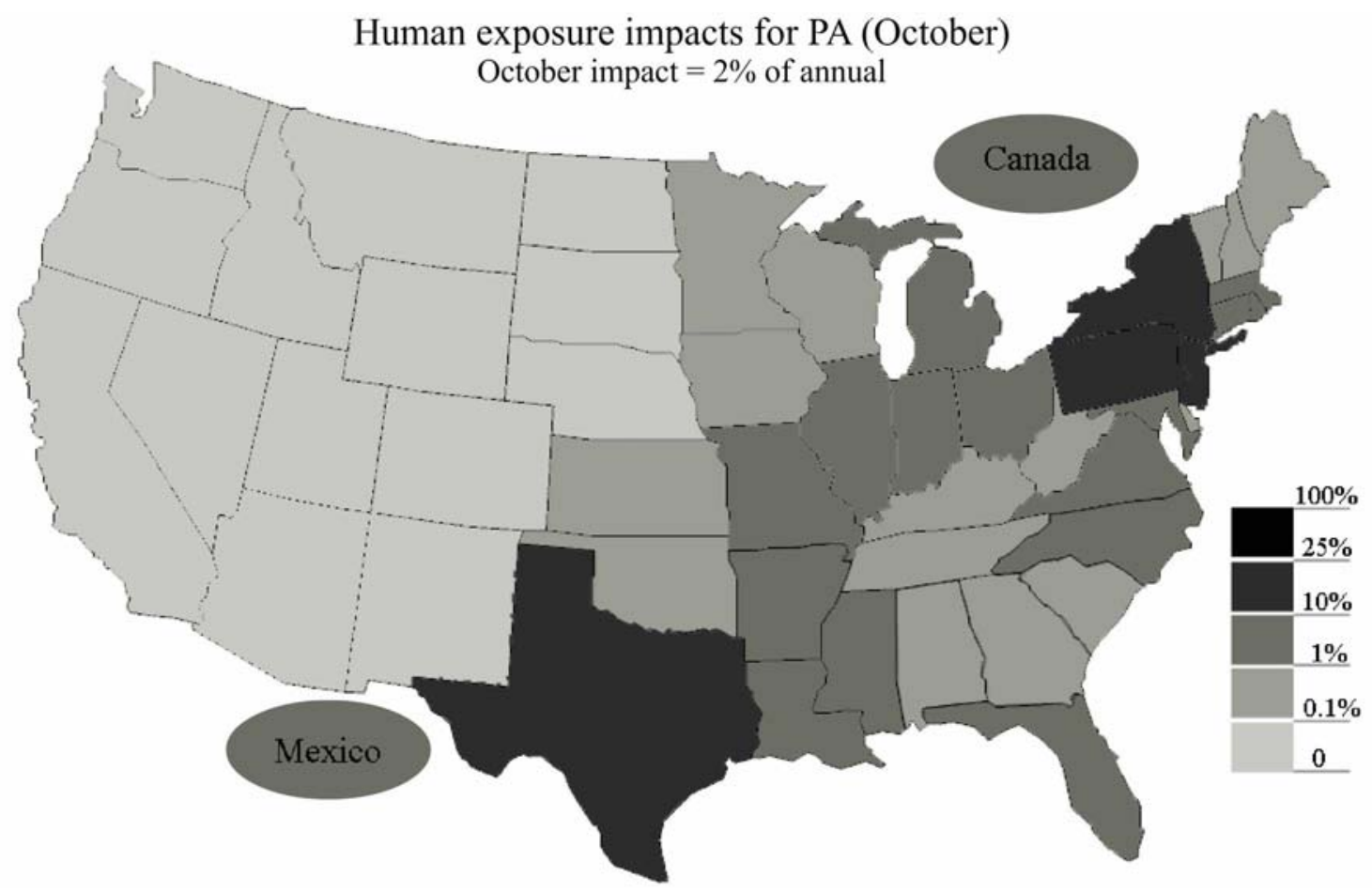

(j) October

Figure 7-5 continued 


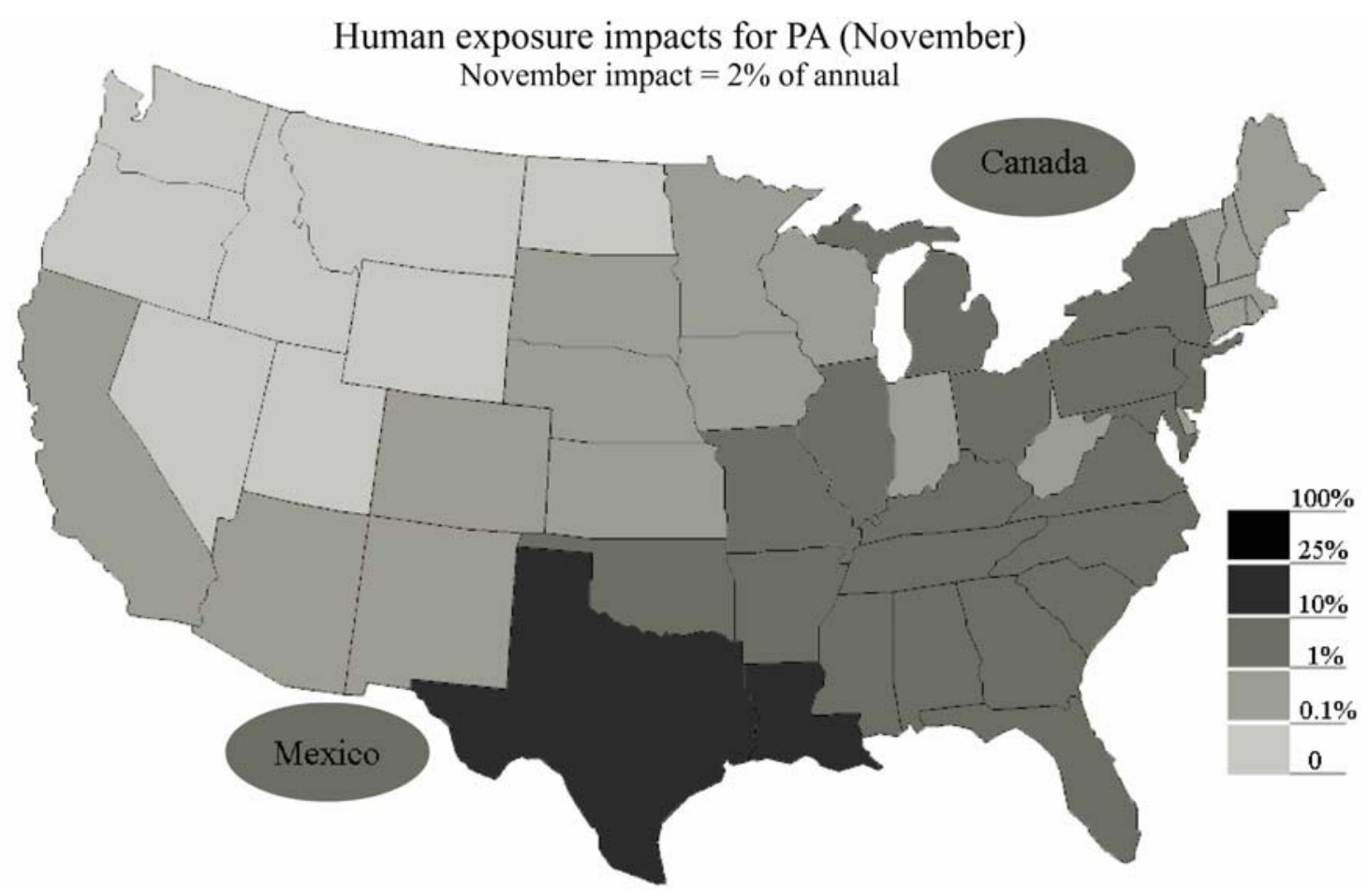

(k) November

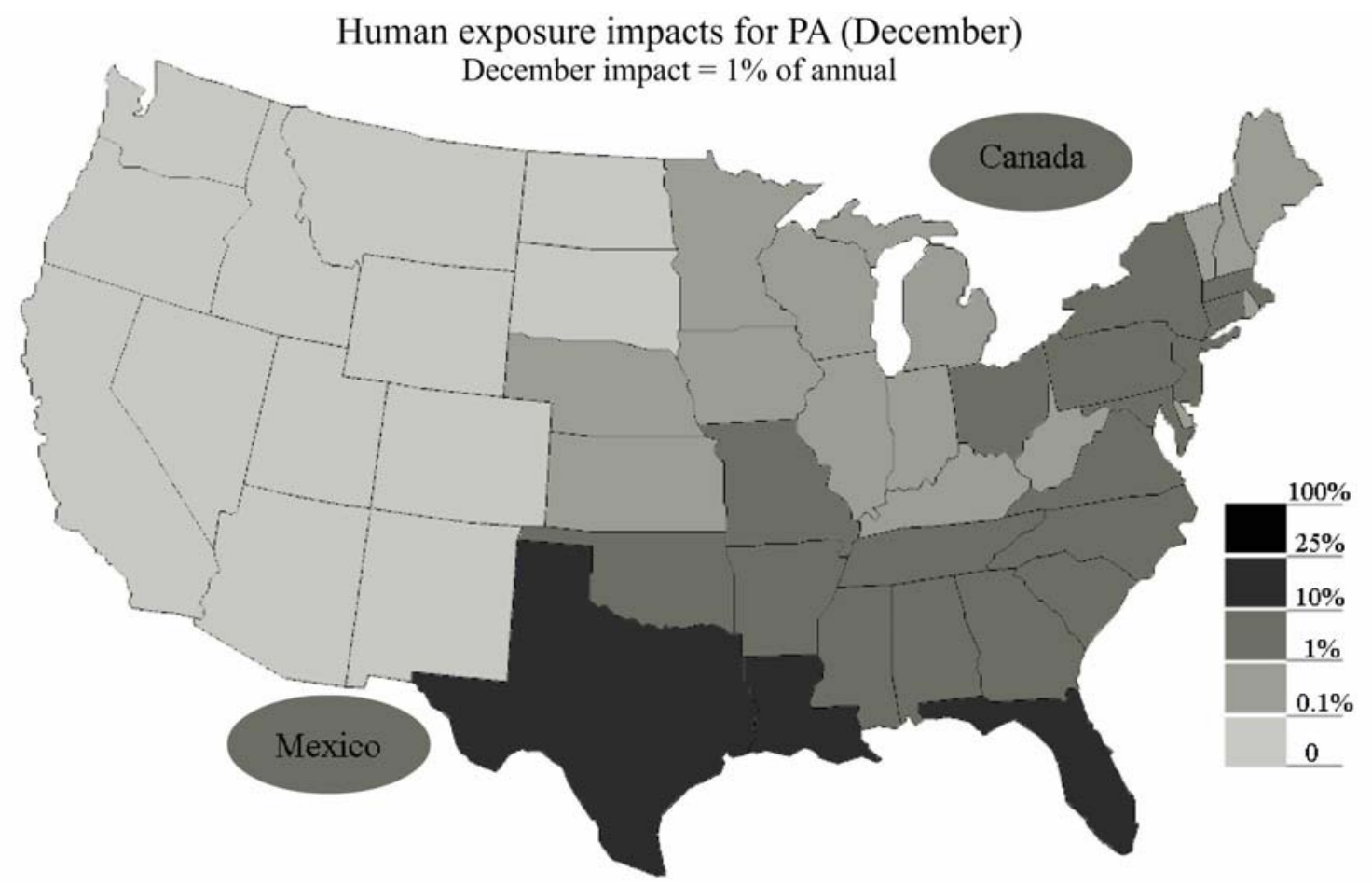

(l) December

Figure 7-5 continued 


\subsection{CONCLUSION}

Year long simulation of the MM5-SMOKE-CAMx photochemical air quality modeling system was performed to determine the variability in the life cycle impact characterization factors for photochemical oxidant formation due to the location and time of emission. The study considered $\mathrm{NO}_{\mathrm{x}}$ and VOC as the important photochemical precursors, and ozone as the indicator oxidant species. Characterization factors for anthropogenic $\mathrm{NO}_{\mathrm{x}}$ and VOC were developed for each of the 48 continental states and Washington DC and for each month of the year. The factors were developed at the fate, human and vegetation exposure, and human effect levels.

A very high spatial and temporal variability was seen in the characterization factors. For any location, the difference between the maximum and minimum fate level and human exposure and effect level characterization factors for $\mathrm{NO}_{\mathrm{x}}$ was between one and two orders of magnitude. The highest variability in these ozone concentration-based characterization factors was seen in the North-Eastern US and the lowest in the upper Mid-West and interior West. For any given month the maximum difference in the characterization factors across the spatial domain was close to one order of magnitude. The concentration-based characterization factors for $\mathrm{NO}_{\mathrm{x}}$ were the highest in the months of June to September.

The concentration-based VOC characterization factors showed a relatively lesser variation overall, although the variation was close to two orders of magnitude for a few states. The intra-annual variation for most of the states was within one order of magnitude. During the summer months, the spatial variation in concentration-based VOC characterization factors was about two orders of magnitude. The summer time characterization factors for VOC were lower than the winter time factors in almost half of the states. This was due to the high proportion (up to 20 times the anthropogenic emissions) of biogenic VOC emissions in the summer months which resulted in lower attribution of ozone formation to anthropogenic sources. On an average, the concentration-based characterization factors for VOC were 6 to 10 times lower than the 
characterization factors for $\mathrm{NO}_{\mathrm{x}}$. Considering an average VOC MIR of 1.75, the concentration based VOC characterization factors per MT are between 3.7 and 5.7 times the characterization factors for $\mathrm{NO}_{\mathrm{x}}$.

The ecosystem exposure characterization factors are based on the amount of ozone deposited to vegetated surfaces. These showed a very high spatial and temporal variation, too. The variation was the highest in the North East US. It was more than three orders of magnitude for some states. No clear temporal pattern emerged for the $\mathrm{NO}_{\mathrm{x}}$ characterization factors, because of the dependence of deposition processes on many meteorological factors. For VOC, a pattern similar to the concentration-based factors was seen. The highest factors for $\mathrm{NO}_{\mathrm{x}}$ and $\mathrm{VOC}$ were for the states of Louisiana, Texas, Mississippi, Alabama, and Florida.

Overall, no significant change in variability is seen in moving from fate level factors to human exposure or human effect level factors. However, the ecosystem exposure level factors have a variability of one order of magnitude higher, both temporally and spatially, compared to the other factors.

A sensitivity analysis of the characterization factors to the meteorology and emission inputs was performed. The variation in the concentration based $\mathrm{NO}_{\mathrm{x}}$ and $\mathrm{VOC}$ factors due to change in meteorological conditions was in the range of $-65 \%$ and $100 \%$; whereas the change in the ecosystem exposure factors is between $-90 \%$ and $180 \%$. The decrease in $\mathrm{NO}_{\mathrm{x}}$ and VOC emissions showed an overall increase in the $\mathrm{NO}_{\mathrm{x}}$ characterization factors (up to $100 \%$ increase), but an overall decrease (up to 73\%) in the VOC characterization factors. Although variation due to the sensitivity of the factors to meteorology and emissions inputs is high, it is still smaller than the spatial and temporal variations.

A case study of the annual heating and cooling energy usage of a building using two systems, and at four different locations was presented. The case study illustrated the use of the characterization factors developed in the study. The results of the disaggregated impact assessment were compared with those obtained using conventional methods. Significant differences in the results were observed, particularly due to the higher weighting of the emissions during summer when the conditions are favorable to ozone formation. Significant differences in the spatial distribution of the impacts between the summer and winter months are also observed.

Spatial and temporal differentiation takes into account the differences in fate and transport of the pollutant, and the exposure and effect on the sensitive human or ecosystem 
population. Adequate resolution for seasonal and regional processes, like photochemical ozone formation, is important to reduce the uncertainty in impact assessment and improve the decision making power.

Future research in this area can address some of the limitations of this study as well as look at other related ideas. A few such ideas are as follows:

a. A relatively high difference between the predicted and observed ozone concentration was seen in the results of the winter months. An investigation of this difference would improve the reliability of the characterization factors.

b. In view of the limitations on the resolution of inventory data, the study used the state political boundaries to group the model grid cells into source areas. Smaller source regions divided based on land areas, populations, or other surrogates can be used to resolve the spatial aggregation in the characterization factors of larger states.

c. The fate and transport model was simulated using a particular set of inputs (meteorology, emissions, IC, BC, etc.), model parameters (reaction mechanism, source apportionment technique, etc.), and the models (CAMx, MM5, SMOKE) themselves. Each of these influences the characterization factors that are calculated from the predicted ozone concentrations. The limited sensitivity analysis of the factors performed in this study was indicative of the variation expected. However, a thorough sensitivity analysis is required to actually define the confidence intervals for the characterization factors.

d. The characterization model failed to include human impact end points, besides short-term mortality, due to the limited availability of concentration-response functions applicable for the entire domain. Our understanding of the effects of ozone on ecosystems is currently limited, but developing rapidly. As newer studies dealing with human and ecosystem endpoints, and also with the effects of ozone on climate change and man made materials, are published, their results can be coupled with the existing predictions of the fate and transport model to increase the breadth and depth of the impact characterization.

e. To evaluate the actual ozone exposure to human population, the predictions of the fate and transport model can be used as an input to an exposure assessment model that predicts the concentration of ozone in the inhaled air. Exposure modeling requires extensive data on the demographics, activity patterns, microenvironment conditions, etc. The predictions of the 
exposure model can be used with the dose-response relationships to calculate the effect on humans.

f. Spatial and temporal differentiation can be extended to all regional and seasonal impact categories. Spatial differentiation at the mid point level of the impact categories of acidification, aquatic eutrophication, and exposure to particulate matter has been incorporated in TRACI. A study of the temporal variation of the factors for these categories can be a step forward. Spatial differentiation, too, can be extended to human and ecosystem toxicity and terrestrial euthrophication.

g. Spatial and temporal differentiation of the characterization factors has a limited benefit if the inventory is aggregated. An emphasis on incorporating some form of spatial and temporal information within standard LCI databases, and using adequately resolved characterization factors will greatly increase the reliability of a standard LCA. 


\section{APPENDIX A}

\section{MODEL CONFIGURATION FILES}

A copy of the files showing the user configuration for the MM5 and CAMx model simulations are presented in this section.

\section{A.1 MM5 CONFIGURATION}

Below is a copy of the mmlif file, located by default in \$MM5DIR/Run directory. It contains the configurations used during compilation and at runtime.

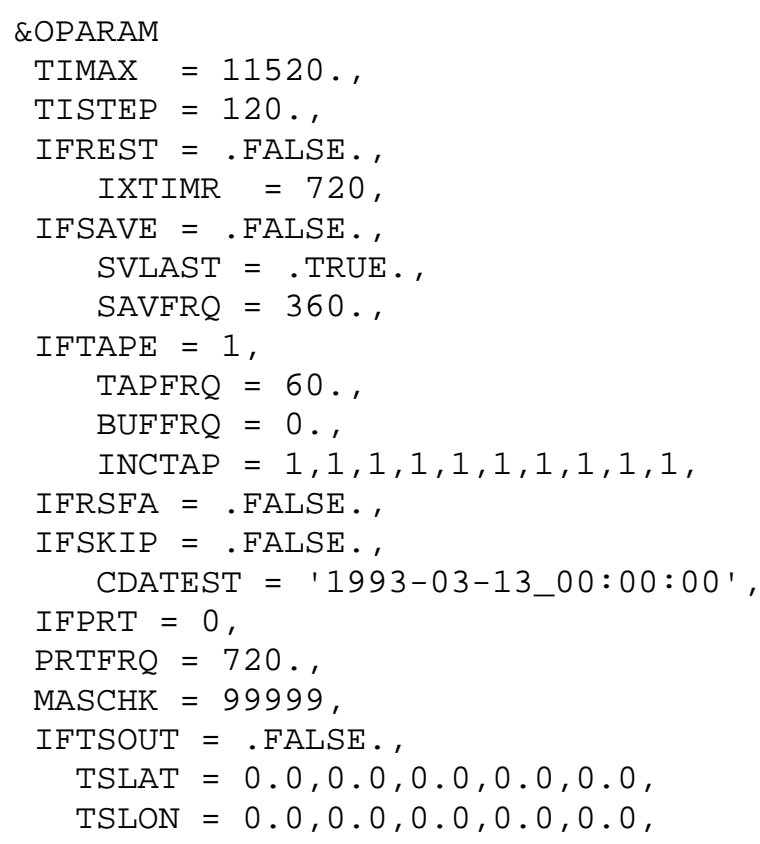




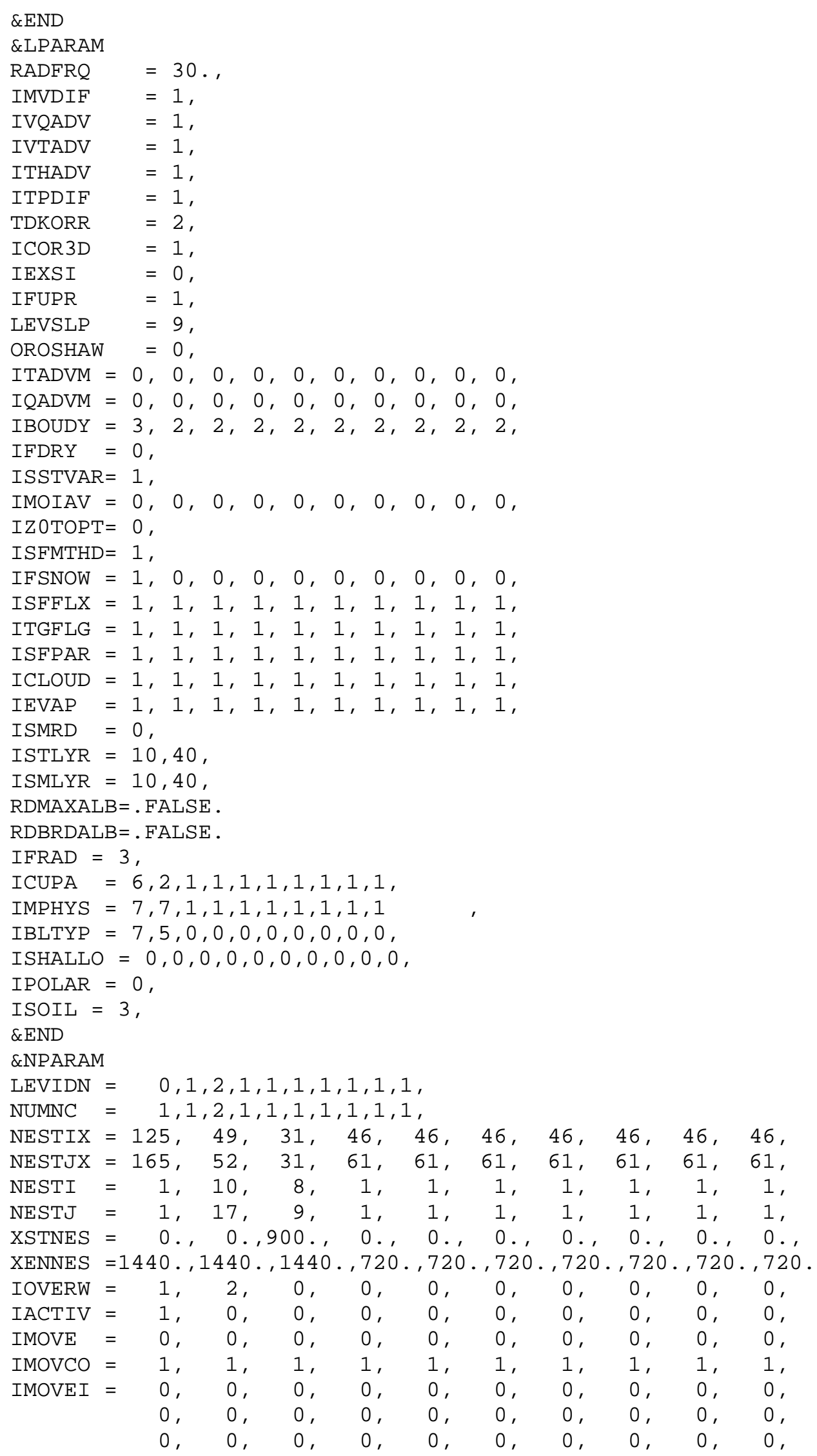




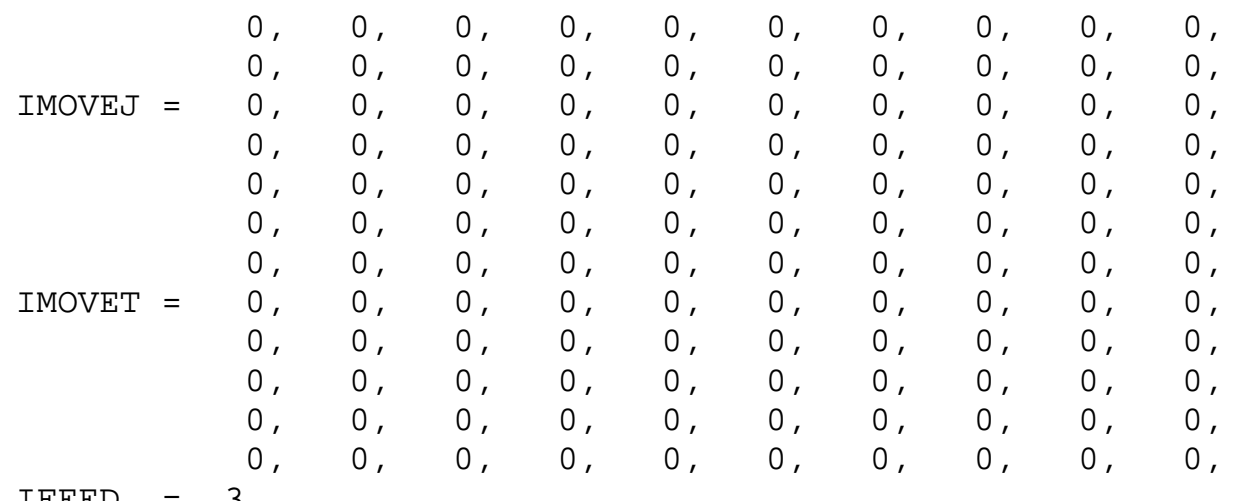

$$
\begin{aligned}
& \text { \&END } \\
& \text { \&PPARAM } \\
& \text { ZZLND }=0.1 \\
& \text { ZZWTR }=0.0001, \\
& \text { ALBLND }=0.15 \text {, } \\
& \text { THINLD }=0.04, \\
& \text { XMAVA }=0.3, \\
& \text { CONF }=1.0, \\
& \text { \&END } \\
& \text { \&FPARAM } \\
& \text { FDASTA }=\odot ., 0 ., 0 ., 0 ., 0 ., 0 ., 0 ., 0 ., 0 ., 0 . \\
& \text { FDAEND }=1440 ., 0 ., 0 ., 0 ., 0 ., 0 ., 0 ., 0 ., 0 ., 0 ., \\
& \text { I4D }=1, \odot, \odot, \odot, \odot, \odot, \odot, \odot, \odot, \odot \text {, } \\
& \odot, \odot, \odot, \odot, \odot, \odot, \odot, \odot, \odot, \odot \text {, } \\
& \text { DIFTIM }=360 ., 720 ., 0 ., 0 ., 0 ., 0 ., 0 ., 0 ., 0 ., 0 ., \\
& \text { 360., 180., }, ., 0 ., 0 ., 0 ., 0 ., 0 ., 0 ., 0 ., \\
& \text { IWIND }=1,1,0,0,0,0,0,0,0,0 \text {, } \\
& 1,1,0,0,0,0,0,0,0,0 \text {, } \\
& \mathrm{GV}=2.5 \mathrm{E}-4,1.0 \mathrm{E}-4,0 ., 0 ., 0 ., 0 ., 0 ., 0 ., 0 ., 0 ., \\
& \text { 2.5E-4, 1. } 0 \mathrm{E}-4,0 ., 0 ., 0 ., 0 ., 0 ., 0 ., 0 ., 0 ., \\
& \text { ITEMP }=1,1,0,0, \odot, \odot, \odot, \odot, \odot, \odot \text {, } \\
& 1,1,0,0,0,0,0,0,0,0 \text {, } \\
& \mathrm{GT}=2.5 \mathrm{E}-4,1.0 \mathrm{E}-4,0 ., 0 ., 0 ., 0 ., 0 ., 0 ., 0 ., 0 ., \\
& \text { 2.5E-4, 1. } 0 \mathrm{E}-4,0 ., 0 ., 0 ., 0 ., 0 ., 0 ., 0 ., 0 ., \\
& \text { IMOIS }=1,1,0,0,0,0,0,0,0,0 \text {, } \\
& 1,1,0,0,0,0,0,0,0,0 \text {, } \\
& \mathrm{GQ}=1 . \mathrm{E}-5,1 . \mathrm{E}-5, \Theta_{.}, \Theta_{.}, \Theta_{.}, \Theta_{.}, 0 ., 0 ., 0 ., 0 ., \\
& \text { 1.E-5, 1.E-5, } 0 ., 0 ., 0 ., 0 ., 0 ., 0 ., 0 ., 0 ., \\
& \text { IROT }=\odot, \odot, \odot, \odot, \odot, \odot, \odot, \odot, \odot, \odot \text {, } \\
& \mathrm{GR}=5 . \mathrm{E} 6,5 . \mathrm{E} 6,0 ., 0 ., 0 ., 0 ., 0 ., 0 ., 0 ., 0 ., \\
& \text { INONBL }=0,1,1,1,1,1,1,1,1,1 \text {, } \\
& 0,1,1,1,1,1,1,1,1,1 \text {, } \\
& 1,1,1,1,1,1,1,1,1,1 \text {, } \\
& 1,1,1,1,1,1,1,1,1,1 \text {, } \\
& \text { RINBLW=250 . , } \\
& \text { NPFG }=50 \text {, } \\
& \text { I4DI }=\odot, \Theta, \odot, \Theta, \odot, \odot, \odot, \odot, \odot, \odot \text {, } \\
& \text { ISWIND }=1,0, \odot, 0, \odot, \odot, \odot, \odot, \odot, \odot \text {, } \\
& \text { GIV }=4 . E-4,4 . E-4,0 ., 0 ., 0 ., 0 ., 0 ., 0 ., 0 ., 0 ., \\
& \text { ISTEMP }=1,0,0, \odot, \odot, \odot, \odot, \odot, 0,0 \text {, } \\
& \text { GIT }=4 . \mathrm{E}-4,4 . \mathrm{E}-4,0 ., 0 ., 0 ., 0 ., 0 ., 0 ., 0 ., 0 ., \\
& \text { ISMOIS }=1, \odot, \odot, \odot, \odot, \odot, \odot, \odot, \odot, \odot \text {, } \\
& \text { GIQ }=4 . \mathrm{E}-4,4 . \mathrm{E}-4,0 ., 0 ., 0 ., 0 ., 0 ., 0 ., 0 ., 0 ., \\
& \text { RINXY=240 . , }
\end{aligned}
$$


RINSIG $=0.001$,

TWIND0=40. $\odot$,

NPFI $=20$,

$I O N F=2$,

IDYNIN $=0$,

DTRAMP $=60$. ,

\&END

\section{A.2 CAMX CONFIGURATION}

Below is a copy of the CAMx.in file, which is the user input for the CAMx program.

\&CAMx_Control

Run_Message $=$ 'CAMx 4.20 - Mech 3(CB4) - US36 148x112 20060730'

[--- Model clock control ---

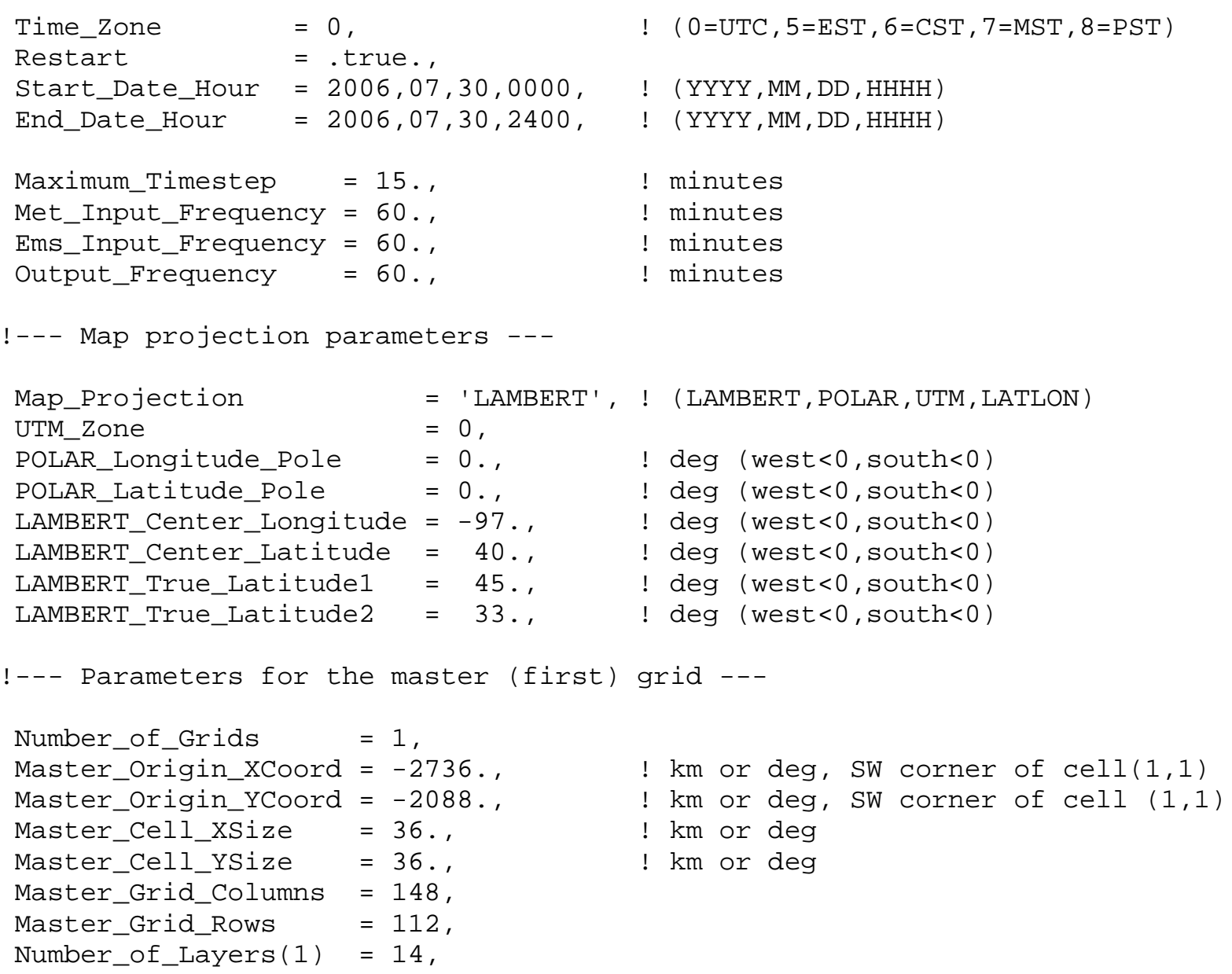


!--- Model options ---

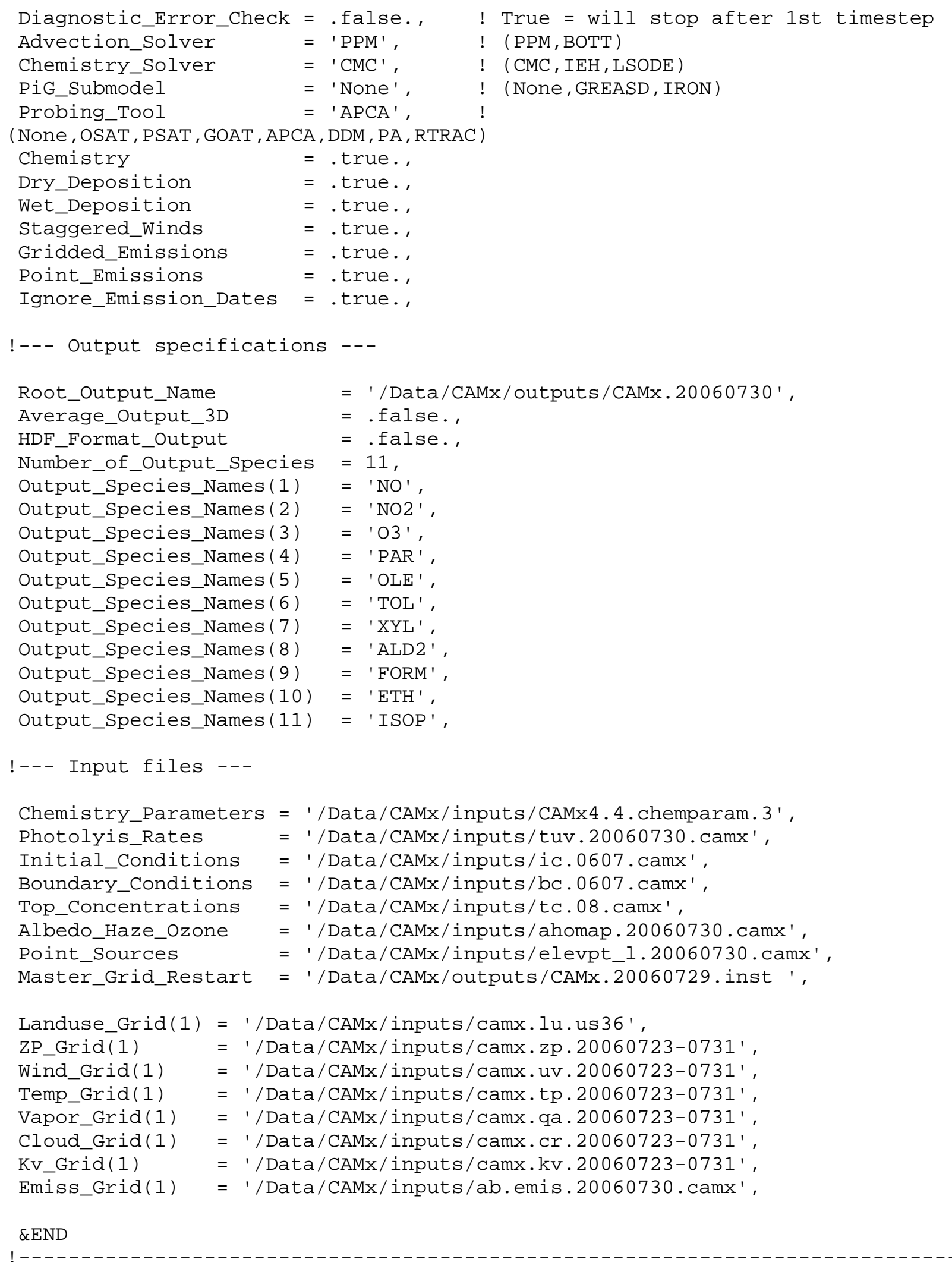

\&SA_Control 
SA_File_Root

SA_Master_SfC_Output

SA_Nested_Sfc_Output

SA_Summary_Output

SA_Stratify_Boundary

SA_Number_of_Source_Regions

SA_Number_of_Source_Groups

Use_Leftover_Group

Number_of_Timing_Releases
= '/Data/CAMx/outputs/CAMx.20060730',

$=$.true.,

$=$.false.,

$=$.true.,

$=$.false.,

$=52$,

$=2$,

$=$.true.,

$=\Theta$,

SA_Receptor_Definitions = '/Data/CAMx/inputs/receptor_def',

SA_Source_Area_Map(1)= '/Data/CAMX/inputs/source_map',

SA_Source_Area_Map $(2)=$ ' ',

SA_Master_Restart = '/Data/CAMX/outputs/CAMx.20060729.sa.inst ',

SA_Nested_Restart = ' ',

SA_Points_Group(1) = ' ',

SA_Emiss_Group_Grid $(1,1)=$ 'Data/CAMx/inputs/b.emis.20060730.camx',

SA_Emiss_Group_Grid $(1,2)=$ ' ',

\&END 


\section{APPENDIX B}

\section{MODELED EMISSIONS AND CHARACTERIZATION FACTORS}

The monthly state-level emissions and the characterization factors calculated in this study are

presented in this section. The emissions and characterization factors for the two sensitivity scenarios are also tabulated.

(Continued on next page) 
Table A1 Average daily anthropogenic emissions of $\mathrm{NO}_{\mathrm{x}}$ (January-June)

\begin{tabular}{|c|c|c|c|c|c|c|c|}
\hline \multicolumn{2}{|l|}{ State } & January & February & March & April & May & June \\
\hline Oregon & OR & $4.5 \mathrm{E}+02$ & $4.6 \mathrm{E}+02$ & $4.8 \mathrm{E}+02$ & $4.8 \mathrm{E}+02$ & $4.7 \mathrm{E}+02$ & $5.1 \mathrm{E}+02$ \\
\hline Washington & WA & $1.1 \mathrm{E}+03$ & $1.1 \mathrm{E}+03$ & $1.1 \mathrm{E}+03$ & $1.2 \mathrm{E}+03$ & $1.1 \mathrm{E}+03$ & $1.2 \mathrm{E}+03$ \\
\hline Wyoming & $\overline{W Y}$ & $5.8 \mathrm{E}+02$ & $5.8 \mathrm{E}+02$ & $6.2 \mathrm{E}+02$ & $6.3 \mathrm{E}+02$ & $6.2 \mathrm{E}+02$ & $6.9 \mathrm{E}+02$ \\
\hline Idaho & ID & $3.1 \mathrm{E}+02$ & $3.1 \mathrm{E}+02$ & $3.3 \mathrm{E}+02$ & $3.2 \mathrm{E}+02$ & $3.2 \mathrm{E}+02$ & $4.1 \mathrm{E}+02$ \\
\hline Montana & MT & $4.0 \mathrm{E}+02$ & $4.0 \mathrm{E}+02$ & $4.7 \mathrm{E}+02$ & $4.8 \mathrm{E}+02$ & $4.9 \mathrm{E}+02$ & $5.8 \mathrm{E}+02$ \\
\hline California & CA & $3.6 \mathrm{E}+03$ & $3.5 \mathrm{E}+03$ & $3.4 \mathrm{E}+03$ & $3.5 \mathrm{E}+03$ & $3.4 \mathrm{E}+03$ & $3.4 \mathrm{E}+03$ \\
\hline Nevada & $\mathrm{NV}$ & $2.8 \mathrm{E}+02$ & $2.8 \mathrm{E}+02$ & $2.8 \mathrm{E}+02$ & $2.9 \mathrm{E}+02$ & $2.8 \mathrm{E}+02$ & $3.0 \mathrm{E}+02$ \\
\hline Utah & UT & $7.4 \mathrm{E}+02$ & $7.4 \mathrm{E}+02$ & $7.2 \mathrm{E}+02$ & $7.2 \mathrm{E}+02$ & $7.1 \mathrm{E}+02$ & $7.5 \mathrm{E}+02$ \\
\hline Colorado & $\mathrm{CO}$ & $8.4 \mathrm{E}+02$ & $8.2 \mathrm{E}+02$ & $8.3 \mathrm{E}+02$ & $8.6 \mathrm{E}+02$ & $8.2 \mathrm{E}+02$ & $8.8 \mathrm{E}+02$ \\
\hline Arizona & $\overline{\mathrm{AZ}}$ & $1.0 \mathrm{E}+03$ & $1.0 \mathrm{E}+03$ & $1.0 \mathrm{E}+03$ & $1.0 \mathrm{E}+03$ & $1.0 \mathrm{E}+03$ & $1.1 \mathrm{E}+03$ \\
\hline New Mexico & NM & $8.6 \mathrm{E}+02$ & $8.4 \mathrm{E}+02$ & $8.8 \mathrm{E}+02$ & $8.7 \mathrm{E}+02$ & $9.0 \mathrm{E}+02$ & $8.9 \mathrm{E}+02$ \\
\hline Texas & $\mathrm{TX}$ & $4.9 \mathrm{E}+03$ & $4.8 \mathrm{E}+03$ & $4.9 \mathrm{E}+03$ & $4.9 \mathrm{E}+03$ & $4.8 \mathrm{E}+03$ & $5.1 \mathrm{E}+03$ \\
\hline Oklahoma & $\mathrm{OK}$ & $1.1 \mathrm{E}+03$ & $1.1 \mathrm{E}+03$ & $1.1 \mathrm{E}+03$ & $1.1 \mathrm{E}+03$ & $1.1 \mathrm{E}+03$ & $1.1 \mathrm{E}+03$ \\
\hline Arkansas & $\overline{\mathrm{AR}}$ & $9.1 \mathrm{E}+02$ & $9.3 \mathrm{E}+02$ & $1.0 \mathrm{E}+03$ & $9.5 \mathrm{E}+02$ & $9.4 \mathrm{E}+02$ & $1.0 \mathrm{E}+03$ \\
\hline Louisiana & $\overline{\mathrm{LA}}$ & $2.2 \mathrm{E}+03$ & $2.2 \mathrm{E}+03$ & $2.3 \mathrm{E}+03$ & $2.2 \mathrm{E}+03$ & $2.2 \mathrm{E}+03$ & $2.3 \mathrm{E}+03$ \\
\hline Mississippi & MS & $1.0 \mathrm{E}+03$ & $1.0 \mathrm{E}+03$ & $1.0 \mathrm{E}+03$ & $9.9 \mathrm{E}+02$ & $9.9 \mathrm{E}+02$ & $1.0 \mathrm{E}+03$ \\
\hline Alabama & $\mathrm{AL}$ & $1.4 \mathrm{E}+03$ & $1.4 \mathrm{E}+03$ & $1.4 \mathrm{E}+03$ & $1.3 \mathrm{E}+03$ & $1.3 \mathrm{E}+03$ & $1.3 \mathrm{E}+03$ \\
\hline Kentucky & KY & $2.6 \mathrm{E}+03$ & $2.5 \mathrm{E}+03$ & $2.5 \mathrm{E}+03$ & $2.5 \mathrm{E}+03$ & $2.5 \mathrm{E}+03$ & $2.5 \mathrm{E}+03$ \\
\hline Tennessee & TN & $1.3 \mathrm{E}+03$ & $1.3 \mathrm{E}+03$ & $1.3 \mathrm{E}+03$ & $1.2 \mathrm{E}+03$ & $1.2 \mathrm{E}+03$ & $1.2 \mathrm{E}+03$ \\
\hline Florida & FL & $2.9 \mathrm{E}+03$ & $2.8 \mathrm{E}+03$ & $2.8 \mathrm{E}+03$ & $2.8 \mathrm{E}+03$ & $3.0 \mathrm{E}+03$ & $2.8 \mathrm{E}+03$ \\
\hline Georgia & GA & $1.8 \mathrm{E}+03$ & $1.8 \mathrm{E}+03$ & $1.8 \mathrm{E}+03$ & $1.7 \mathrm{E}+03$ & $1.7 \mathrm{E}+03$ & $1.8 \mathrm{E}+03$ \\
\hline North Carolina & $\mathrm{NC}$ & $1.7 \mathrm{E}+03$ & $1.7 \mathrm{E}+03$ & $1.8 \mathrm{E}+03$ & $1.7 \mathrm{E}+03$ & $1.7 \mathrm{E}+03$ & $1.7 \mathrm{E}+03$ \\
\hline South Carolina & SC & $1.1 \mathrm{E}+03$ & $1.1 \mathrm{E}+03$ & $1.1 \mathrm{E}+03$ & $1.0 \mathrm{E}+03$ & $1.0 \mathrm{E}+03$ & $1.0 \mathrm{E}+03$ \\
\hline Virginia & $\mathrm{VA}$ & $1.6 \mathrm{E}+03$ & $1.6 \mathrm{E}+03$ & $1.6 \mathrm{E}+03$ & $1.5 \mathrm{E}+03$ & $1.5 \mathrm{E}+03$ & $1.5 \mathrm{E}+03$ \\
\hline West Virginia & WV & $1.2 \mathrm{E}+03$ & $1.2 \mathrm{E}+03$ & $1.2 \mathrm{E}+03$ & $1.2 \mathrm{E}+03$ & $1.2 \mathrm{E}+03$ & $1.2 \mathrm{E}+03$ \\
\hline Delaware & $\mathrm{DE}$ & $3.9 \mathrm{E}+01$ & $3.9 \mathrm{E}+01$ & $4.1 \mathrm{E}+01$ & $4.0 \mathrm{E}+01$ & $3.8 \mathrm{E}+01$ & $4.2 \mathrm{E}+01$ \\
\hline District of Colum & $\overline{\mathrm{DC}}$ & $1.1 \mathrm{E}+02$ & $1.0 \mathrm{E}+02$ & $1.0 \mathrm{E}+02$ & $9.7 \mathrm{E}+01$ & $9.0 \mathrm{E}+01$ & $9.1 \mathrm{E}+01$ \\
\hline Maryland & $\mathrm{MD}$ & $6.4 \mathrm{E}+02$ & $6.4 \mathrm{E}+02$ & $6.2 \mathrm{E}+02$ & $6.1 \mathrm{E}+02$ & $5.9 \mathrm{E}+02$ & $6.0 \mathrm{E}+02$ \\
\hline Connecticut & CT & $4.5 \mathrm{E}+02$ & $4.5 \mathrm{E}+02$ & $4.2 \mathrm{E}+02$ & $4.1 \mathrm{E}+02$ & $3.8 \mathrm{E}+02$ & $3.8 \mathrm{E}+02$ \\
\hline New Jersey & $\mathrm{NJ}$ & $1.4 \mathrm{E}+03$ & $1.4 \mathrm{E}+03$ & $1.3 \mathrm{E}+03$ & $1.3 \mathrm{E}+03$ & $1.2 \mathrm{E}+03$ & $1.2 \mathrm{E}+03$ \\
\hline New York & NY & $1.8 \mathrm{E}+03$ & $1.8 \mathrm{E}+03$ & $1.7 \mathrm{E}+03$ & $1.7 \mathrm{E}+03$ & $1.6 \mathrm{E}+03$ & $1.7 \mathrm{E}+03$ \\
\hline Pennsylvania & $\mathrm{PA}$ & $2.3 \mathrm{E}+03$ & $2.3 \mathrm{E}+03$ & $2.2 \mathrm{E}+03$ & $2.2 \mathrm{E}+03$ & $2.1 \mathrm{E}+03$ & $2.1 \mathrm{E}+03$ \\
\hline Rhode Island & RI & $2.7 \mathrm{E}+02$ & $2.7 \mathrm{E}+02$ & $2.6 \mathrm{E}+02$ & $2.5 \mathrm{E}+02$ & $2.4 \mathrm{E}+02$ & $2.4 \mathrm{E}+02$ \\
\hline Massachusetts & MA & $7.0 \mathrm{E}+02$ & $7.0 \mathrm{E}+02$ & $6.9 \mathrm{E}+02$ & $6.9 \mathrm{E}+02$ & $6.5 \mathrm{E}+02$ & $7.1 \mathrm{E}+02$ \\
\hline New Hampshire & $\mathrm{NH}$ & $2.1 \mathrm{E}+02$ & $2.1 \mathrm{E}+02$ & $2.0 \mathrm{E}+02$ & $2.0 \mathrm{E}+02$ & $1.8 \mathrm{E}+02$ & $1.9 \mathrm{E}+02$ \\
\hline Vermont & $\mathrm{VT}$ & $1.1 \mathrm{E}+02$ & $1.1 \mathrm{E}+02$ & $1.1 \mathrm{E}+02$ & $1.1 \mathrm{E}+02$ & $1.0 \mathrm{E}+02$ & $1.1 \mathrm{E}+02$ \\
\hline Maine & $\mathrm{ME}$ & $2.4 \mathrm{E}+02$ & $2.4 \mathrm{E}+02$ & $2.3 \mathrm{E}+02$ & $2.3 \mathrm{E}+02$ & $2.3 \mathrm{E}+02$ & $2.3 \mathrm{E}+02$ \\
\hline Ohio & $\mathrm{OH}$ & $2.7 \mathrm{E}+03$ & $2.7 \mathrm{E}+03$ & $2.6 \mathrm{E}+03$ & $2.6 \mathrm{E}+03$ & $2.5 \mathrm{E}+03$ & $2.6 \mathrm{E}+03$ \\
\hline Indiana & IN & $2.1 \mathrm{E}+03$ & $2.1 \mathrm{E}+03$ & $2.1 \mathrm{E}+03$ & $2.1 \mathrm{E}+03$ & $2.0 \mathrm{E}+03$ & $2.2 \mathrm{E}+03$ \\
\hline Illinois & $\mathrm{IL}$ & $2.3 \mathrm{E}+03$ & $2.3 \mathrm{E}+03$ & $2.3 \mathrm{E}+03$ & $2.2 \mathrm{E}+03$ & $2.2 \mathrm{E}+03$ & $2.4 \mathrm{E}+03$ \\
\hline Michigan & MI & $2.1 \mathrm{E}+03$ & $2.1 \mathrm{E}+03$ & $2.0 \mathrm{E}+03$ & $2.0 \mathrm{E}+03$ & $1.9 \mathrm{E}+03$ & $2.0 \mathrm{E}+03$ \\
\hline Wisconsin & WI & $1.1 \mathrm{E}+03$ & $1.1 \mathrm{E}+03$ & $1.1 \mathrm{E}+03$ & $1.1 \mathrm{E}+03$ & $1.1 \mathrm{E}+03$ & $1.1 \mathrm{E}+03$ \\
\hline Minnesota & MN & $1.2 \mathrm{E}+03$ & $1.2 \mathrm{E}+03$ & $1.2 \mathrm{E}+03$ & $1.3 \mathrm{E}+03$ & $1.2 \mathrm{E}+03$ & $1.3 \mathrm{E}+03$ \\
\hline Iowa & IA & $6.4 \mathrm{E}+02$ & $6.5 \mathrm{E}+02$ & $7.0 \mathrm{E}+02$ & $6.9 \mathrm{E}+02$ & $6.7 \mathrm{E}+02$ & $8.4 \mathrm{E}+02$ \\
\hline Missouri & MO & $1.6 \mathrm{E}+03$ & $1.6 \mathrm{E}+03$ & $1.6 \mathrm{E}+03$ & $1.6 \mathrm{E}+03$ & $1.5 \mathrm{E}+03$ & $1.7 \mathrm{E}+03$ \\
\hline Kansas & $\mathrm{KS}$ & $8.2 \mathrm{E}+02$ & $8.2 \mathrm{E}+02$ & $9.4 \mathrm{E}+02$ & $9.2 \mathrm{E}+02$ & $9.0 \mathrm{E}+02$ & $1.0 \mathrm{E}+03$ \\
\hline Nebraska & $\mathrm{NE}$ & $6.9 \mathrm{E}+02$ & $7.0 \mathrm{E}+02$ & $7.6 \mathrm{E}+02$ & $7.6 \mathrm{E}+02$ & $7.4 \mathrm{E}+02$ & $8.2 \mathrm{E}+02$ \\
\hline North Dakota & ND & $5.3 \mathrm{E}+02$ & $5.4 \mathrm{E}+02$ & $5.2 \mathrm{E}+02$ & $5.3 \mathrm{E}+02$ & $5.1 \mathrm{E}+02$ & $6.4 \mathrm{E}+02$ \\
\hline South Dakota & SD & $2.1 \mathrm{E}+02$ & $2.1 \mathrm{E}+02$ & $2.5 \mathrm{E}+02$ & $2.5 E+02$ & $2.4 \mathrm{E}+02$ & $3.2 \mathrm{E}+02$ \\
\hline
\end{tabular}


Table A2 Average daily anthropogenic emissions of $\mathrm{NO}_{\mathrm{x}}$ (July-December)

\begin{tabular}{|c|c|c|c|c|c|c|c|}
\hline \multicolumn{2}{|l|}{ State } & July & August & September & October & November & December \\
\hline Oregon & OR & $5.7 \mathrm{E}+02$ & $7.9 \mathrm{E}+02$ & $5.3 \mathrm{E}+02$ & $5.4 \mathrm{E}+02$ & $4.7 \mathrm{E}+02$ & $4.4 \mathrm{E}+02$ \\
\hline Washington & WA & $1.2 \mathrm{E}+03$ & $1.2 \mathrm{E}+03$ & $1.1 \mathrm{E}+03$ & $1.1 \mathrm{E}+03$ & $1.1 \mathrm{E}+03$ & $1.1 \mathrm{E}+03$ \\
\hline Wyoming & WY & $7.5 \mathrm{E}+02$ & $7.1 \mathrm{E}+02$ & $6.5 \mathrm{E}+02$ & $6.5 \mathrm{E}+02$ & $6.4 \mathrm{E}+02$ & $5.8 \mathrm{E}+02$ \\
\hline Idaho & ID & $4.8 \mathrm{E}+02$ & $4.2 \mathrm{E}+02$ & $3.7 \mathrm{E}+02$ & $3.7 \mathrm{E}+02$ & $3.2 \mathrm{E}+02$ & $3.1 \mathrm{E}+02$ \\
\hline Montana & MT & $5.9 \mathrm{E}+02$ & $6.5 \mathrm{E}+02$ & $5.2 \mathrm{E}+02$ & $4.9 \mathrm{E}+02$ & $4.9 \mathrm{E}+02$ & $3.9 \mathrm{E}+02$ \\
\hline California & CA & $3.5 \mathrm{E}+03$ & $3.7 \mathrm{E}+03$ & $3.4 \mathrm{E}+03$ & $3.4 \mathrm{E}+03$ & $3.3 \mathrm{E}+03$ & $3.4 \mathrm{E}+03$ \\
\hline Nevada & NV & $3.3 \mathrm{E}+02$ & $4.0 \mathrm{E}+02$ & $2.8 \mathrm{E}+02$ & $2.9 \mathrm{E}+02$ & $2.8 \mathrm{E}+02$ & $2.8 \mathrm{E}+02$ \\
\hline Utah & $\begin{array}{l}\text { UT } \\
\end{array}$ & $8.8 \mathrm{E}+02$ & $7.6 \mathrm{E}+02$ & $7.0 \mathrm{E}+02$ & $6.9 \mathrm{E}+02$ & $6.9 \mathrm{E}+02$ & $7.4 \mathrm{E}+02$ \\
\hline Colorado & $\mathrm{CO}$ & $9.3 \mathrm{E}+02$ & $8.8 \mathrm{E}+02$ & $8.2 \mathrm{E}+02$ & $8.4 \mathrm{E}+02$ & $8.3 \mathrm{E}+02$ & $8.1 \mathrm{E}+02$ \\
\hline Arizona & $\overline{\mathrm{AZ}}$ & $1.1 \mathrm{E}+03$ & $1.1 \mathrm{E}+03$ & $1.0 \mathrm{E}+03$ & $1.1 \mathrm{E}+03$ & $1.0 \mathrm{E}+03$ & $1.0 \mathrm{E}+03$ \\
\hline New Mexico & NM & $8.4 \mathrm{E}+02$ & $8.3 \mathrm{E}+02$ & $8.4 \mathrm{E}+02$ & $8.9 \mathrm{E}+02$ & $8.2 \mathrm{E}+02$ & $8.3 \mathrm{E}+02$ \\
\hline Texas & TX & $5.2 \mathrm{E}+03$ & $5.2 \mathrm{E}+03$ & $4.9 \mathrm{E}+03$ & $4.9 \mathrm{E}+03$ & $4.9 \mathrm{E}+03$ & $4.8 \mathrm{E}+03$ \\
\hline Oklahoma & $\overline{\mathrm{OK}}$ & $1.1 \mathrm{E}+03$ & $1.1 \mathrm{E}+03$ & $1.1 \mathrm{E}+03$ & $1.1 \mathrm{E}+03$ & $1.1 \mathrm{E}+03$ & $1.1 \mathrm{E}+03$ \\
\hline Arkansas & $\overline{\mathrm{AR}}$ & $1.0 \mathrm{E}+03$ & $1.0 \mathrm{E}+03$ & $9.6 \mathrm{E}+02$ & $9.8 \mathrm{E}+02$ & $9.8 \mathrm{E}+02$ & $8.9 \mathrm{E}+02$ \\
\hline \begin{tabular}{|l} 
Louisiana \\
\end{tabular} & $\overline{L A}$ & $2.3 \mathrm{E}+03$ & $2.3 \mathrm{E}+03$ & $2.2 \mathrm{E}+03$ & $2.3 \mathrm{E}+03$ & $2.2 \mathrm{E}+03$ & $2.2 \mathrm{E}+03$ \\
\hline Mississippi & MS & $1.0 \mathrm{E}+03$ & $1.0 \mathrm{E}+03$ & $9.8 \mathrm{E}+02$ & $1.0 \mathrm{E}+03$ & $9.9 \mathrm{E}+02$ & $9.6 \mathrm{E}+02$ \\
\hline Alabama & $\overline{\mathrm{AL}}$ & $1.3 \mathrm{E}+03$ & $1.3 \mathrm{E}+03$ & $1.2 \mathrm{E}+03$ & $1.3 \mathrm{E}+03$ & $1.3 \mathrm{E}+03$ & $1.4 \mathrm{E}+03$ \\
\hline Kentucky & KY & $2.5 \mathrm{E}+03$ & $2.5 \mathrm{E}+03$ & $2.5 E+03$ & $2.5 \mathrm{E}+03$ & $2.5 E+03$ & $2.5 \mathrm{E}+03$ \\
\hline Tennessee & TN & $1.2 \mathrm{E}+03$ & $1.2 \mathrm{E}+03$ & $1.2 \mathrm{E}+03$ & $1.2 \mathrm{E}+03$ & $1.2 \mathrm{E}+03$ & $1.2 \mathrm{E}+03$ \\
\hline Florida & FL & $2.8 \mathrm{E}+03$ & $2.7 \mathrm{E}+03$ & $2.6 \mathrm{E}+03$ & $2.6 \mathrm{E}+03$ & $2.6 \mathrm{E}+03$ & $2.7 \mathrm{E}+03$ \\
\hline Georgia & $\overline{\mathrm{GA}}$ & $1.7 \mathrm{E}+03$ & $1.7 \mathrm{E}+03$ & $1.7 \mathrm{E}+03$ & $1.7 \mathrm{E}+03$ & $1.7 \mathrm{E}+03$ & $1.7 \mathrm{E}+03$ \\
\hline North Carolina & $\mathrm{NC}$ & $1.7 \mathrm{E}+03$ & $1.7 \mathrm{E}+03$ & $1.6 \mathrm{E}+03$ & $1.7 \mathrm{E}+03$ & $1.7 \mathrm{E}+03$ & $1.7 \mathrm{E}+03$ \\
\hline South Carolina & $\mathrm{SC}$ & $1.0 \mathrm{E}+03$ & $1.0 \mathrm{E}+03$ & $1.0 \mathrm{E}+03$ & $1.0 \mathrm{E}+03$ & $1.0 \mathrm{E}+03$ & $1.0 \mathrm{E}+03$ \\
\hline Virginia & VA & $1.6 \mathrm{E}+03$ & $1.5 \mathrm{E}+03$ & $1.5 \mathrm{E}+03$ & $1.5 \mathrm{E}+03$ & $1.5 \mathrm{E}+03$ & $1.5 \mathrm{E}+03$ \\
\hline West Virginia & WV & $1.2 \mathrm{E}+03$ & $1.2 \mathrm{E}+03$ & $1.2 \mathrm{E}+03$ & $1.2 \mathrm{E}+03$ & $1.2 \mathrm{E}+03$ & $1.2 \mathrm{E}+03$ \\
\hline Delaware & $\mathrm{DE}$ & $4.2 \mathrm{E}+01$ & $4.1 \mathrm{E}+01$ & $3.8 \mathrm{E}+01$ & $3.9 \mathrm{E}+01$ & $4.0 \mathrm{E}+01$ & $3.8 \mathrm{E}+01$ \\
\hline District of Colum & DC & $9.4 \mathrm{E}+01$ & $8.9 \mathrm{E}+01$ & $9.0 \mathrm{E}+01$ & $9.4 \mathrm{E}+01$ & $9.4 \mathrm{E}+01$ & $1.0 \mathrm{E}+02$ \\
\hline Maryland & MD & $6.1 \mathrm{E}+02$ & $5.9 \mathrm{E}+02$ & $5.8 \mathrm{E}+02$ & $6.0 \mathrm{E}+02$ & $6.0 \mathrm{E}+02$ & $6.2 \mathrm{E}+02$ \\
\hline Connecticut & CT & $3.9 \mathrm{E}+02$ & $3.8 \mathrm{E}+02$ & $3.7 \mathrm{E}+02$ & $3.9 \mathrm{E}+02$ & $3.9 \mathrm{E}+02$ & $4.3 \mathrm{E}+02$ \\
\hline New Jersey & NJ & $1.3 \mathrm{E}+03$ & $1.2 \mathrm{E}+03$ & $1.2 \mathrm{E}+03$ & $1.3 \mathrm{E}+03$ & $1.2 \mathrm{E}+03$ & $1.4 \mathrm{E}+03$ \\
\hline New York & NY & $1.8 \mathrm{E}+03$ & $1.7 \mathrm{E}+03$ & $1.6 \mathrm{E}+03$ & $1.7 \mathrm{E}+03$ & $1.7 \mathrm{E}+03$ & $1.7 \mathrm{E}+03$ \\
\hline Pennsylvania & $\overline{\mathrm{PA}}$ & $2.1 \mathrm{E}+03$ & $2.1 \mathrm{E}+03$ & $2.1 \mathrm{E}+03$ & $2.1 \mathrm{E}+03$ & $2.1 \mathrm{E}+03$ & $2.2 \mathrm{E}+03$ \\
\hline Rhode Island & RI & $2.4 \mathrm{E}+02$ & $2.3 E+02$ & $2.3 \mathrm{E}+02$ & $2.4 \mathrm{E}+02$ & $2.4 \mathrm{E}+02$ & $2.6 \mathrm{E}+02$ \\
\hline Massachusetts & MA & $7.2 \mathrm{E}+02$ & $7.0 \mathrm{E}+02$ & $6.3 \mathrm{E}+02$ & $6.5 \mathrm{E}+02$ & $6.6 \mathrm{E}+02$ & $6.7 \mathrm{E}+02$ \\
\hline New Hampshire & $\mathrm{NH}$ & $1.9 \mathrm{E}+02$ & $1.9 \mathrm{E}+02$ & $1.8 \mathrm{E}+02$ & $1.9 \mathrm{E}+02$ & $1.9 \mathrm{E}+02$ & $2.0 \mathrm{E}+02$ \\
\hline Vermont & VT & $1.1 \mathrm{E}+02$ & $1.1 \mathrm{E}+02$ & $1.0 \mathrm{E}+02$ & $1.1 \mathrm{E}+02$ & $1.1 \mathrm{E}+02$ & $1.1 \mathrm{E}+02$ \\
\hline Maine & $\mathrm{ME}$ & $2.3 \mathrm{E}+02$ & $2.2 \mathrm{E}+02$ & $2.2 \mathrm{E}+02$ & $2.2 \mathrm{E}+02$ & $2.3 \mathrm{E}+02$ & $2.4 \mathrm{E}+02$ \\
\hline Ohio & $\mathrm{OH}$ & $2.7 \mathrm{E}+03$ & $2.6 \mathrm{E}+03$ & $2.5 \mathrm{E}+03$ & $2.6 \mathrm{E}+03$ & $2.6 \mathrm{E}+03$ & $2.6 \mathrm{E}+03$ \\
\hline Indiana & IN & $2.2 \mathrm{E}+03$ & $2.1 \mathrm{E}+03$ & $2.0 \mathrm{E}+03$ & $2.1 \mathrm{E}+03$ & $2.1 \mathrm{E}+03$ & $2.1 \mathrm{E}+03$ \\
\hline Illinois & $\overline{\mathrm{IL}}$ & $2.4 \mathrm{E}+03$ & $2.3 \mathrm{E}+03$ & $2.2 \mathrm{E}+03$ & $2.2 \mathrm{E}+03$ & $2.2 \mathrm{E}+03$ & $2.2 \mathrm{E}+03$ \\
\hline Michigan & MI & $2.1 \mathrm{E}+03$ & $2.0 \mathrm{E}+03$ & $1.9 \mathrm{E}+03$ & $2.0 \mathrm{E}+03$ & $2.0 \mathrm{E}+03$ & $2.0 \mathrm{E}+03$ \\
\hline Wisconsin & WI & $1.1 \mathrm{E}+03$ & $1.1 \mathrm{E}+03$ & $1.1 \mathrm{E}+03$ & $1.1 \mathrm{E}+03$ & $1.1 \mathrm{E}+03$ & $1.1 \mathrm{E}+03$ \\
\hline Minnesota & MN & $1.4 \mathrm{E}+03$ & $1.3 \mathrm{E}+03$ & $1.2 \mathrm{E}+03$ & $1.2 \mathrm{E}+03$ & $1.2 \mathrm{E}+03$ & $1.2 \mathrm{E}+03$ \\
\hline Iowa & IA & $8.5 \mathrm{E}+02$ & $8.3 \mathrm{E}+02$ & $6.8 \mathrm{E}+02$ & $7.0 \mathrm{E}+02$ & $6.9 \mathrm{E}+02$ & $6.2 \mathrm{E}+02$ \\
\hline Missouri & MO & $1.7 \mathrm{E}+03$ & $1.7 \mathrm{E}+03$ & $1.6 \mathrm{E}+03$ & $1.6 \mathrm{E}+03$ & $1.6 \mathrm{E}+03$ & $1.5 \mathrm{E}+03$ \\
\hline Kansas & KS & $1.0 \mathrm{E}+03$ & $1.0 \mathrm{E}+03$ & $9.3 \mathrm{E}+02$ & $9.4 \mathrm{E}+02$ & $9.3 \mathrm{E}+02$ & $8.0 \mathrm{E}+02$ \\
\hline Nebraska & $\mathrm{NE}$ & $8.2 \mathrm{E}+02$ & $8.1 \mathrm{E}+02$ & $7.5 \mathrm{E}+02$ & $7.6 \mathrm{E}+02$ & $7.5 \mathrm{E}+02$ & $6.8 \mathrm{E}+02$ \\
\hline North Dakota & ND & $6.5 \mathrm{E}+02$ & $6.4 \mathrm{E}+02$ & $5.0 \mathrm{E}+02$ & $5.0 \mathrm{E}+02$ & $5.0 \mathrm{E}+02$ & $5.3 \mathrm{E}+02$ \\
\hline South Dakota & SD & $3.4 \mathrm{E}+02$ & $3.2 \mathrm{E}+02$ & $2.6 \mathrm{E}+02$ & $2.6 \mathrm{E}+02$ & $2.4 \mathrm{E}+02$ & $2.0 \mathrm{E}+02$ \\
\hline
\end{tabular}


Table A3 Average daily anthropogenic emissions of VOC (January-June)

\begin{tabular}{|c|c|c|c|c|c|c|c|}
\hline \multicolumn{2}{|l|}{ State } & January & February & March & April & May & June \\
\hline Oregon & OR & $1.1 \mathrm{E}+03$ & $1.1 \mathrm{E}+03$ & $1.1 \mathrm{E}+03$ & $1.1 \mathrm{E}+03$ & $1.1 \mathrm{E}+03$ & $1.1 \mathrm{E}+03$ \\
\hline Washington & WA & $2.1 \mathrm{E}+03$ & $2.1 \mathrm{E}+03$ & $2.0 \mathrm{E}+03$ & $2.0 \mathrm{E}+03$ & $1.9 \mathrm{E}+03$ & $2.1 \mathrm{E}+03$ \\
\hline Wyoming & WY & $3.3 \mathrm{E}+02$ & $3.4 \mathrm{E}+02$ & $2.6 \mathrm{E}+02$ & $2.7 \mathrm{E}+02$ & $2.7 \mathrm{E}+02$ & $2.7 \mathrm{E}+02$ \\
\hline Idaho & ID & $1.1 \mathrm{E}+03$ & $1.1 \mathrm{E}+03$ & $1.1 \mathrm{E}+03$ & $1.0 \mathrm{E}+03$ & $1.0 \mathrm{E}+03$ & $1.3 \mathrm{E}+03$ \\
\hline Montana & MT & $4.8 \mathrm{E}+02$ & $4.8 \mathrm{E}+02$ & $4.3 \mathrm{E}+02$ & $4.4 \mathrm{E}+02$ & $4.7 \mathrm{E}+02$ & $4.4 \mathrm{E}+02$ \\
\hline California & CA & $6.8 \mathrm{E}+03$ & $6.0 \mathrm{E}+03$ & $5.9 \mathrm{E}+03$ & $5.7 \mathrm{E}+03$ & $5.4 \mathrm{E}+03$ & $5.6 \mathrm{E}+03$ \\
\hline Nevada & $\mathrm{NV}$ & $3.2 \mathrm{E}+02$ & $3.2 \mathrm{E}+02$ & $3.6 \mathrm{E}+02$ & $3.6 \mathrm{E}+02$ & $3.7 \mathrm{E}+02$ & $4.4 \mathrm{E}+02$ \\
\hline Utah & UT & $6.7 \mathrm{E}+02$ & $6.7 \mathrm{E}+02$ & $6.7 \mathrm{E}+02$ & $6.7 \mathrm{E}+02$ & $6.5 \mathrm{E}+02$ & $7.1 \mathrm{E}+02$ \\
\hline Colorado & $\mathrm{CO}$ & $1.1 \mathrm{E}+03$ & $1.1 \mathrm{E}+03$ & $9.1 \mathrm{E}+02$ & $1.1 \mathrm{E}+03$ & $9.7 \mathrm{E}+02$ & $1.1 \mathrm{E}+03$ \\
\hline Arizona & $\overline{\mathrm{AZ}}$ & $1.0 \mathrm{E}+03$ & $1.0 \mathrm{E}+03$ & $1.1 \mathrm{E}+03$ & $1.1 \mathrm{E}+03$ & $1.2 \mathrm{E}+03$ & $1.5 \mathrm{E}+03$ \\
\hline New Mexico & NM & $6.7 \mathrm{E}+02$ & $6.0 \mathrm{E}+02$ & $7.8 \mathrm{E}+02$ & $7.1 \mathrm{E}+02$ & $9.0 \mathrm{E}+02$ & $8.6 \mathrm{E}+02$ \\
\hline Texas & $\mathrm{TX}$ & $6.0 \mathrm{E}+03$ & $6.2 \mathrm{E}+03$ & $6.2 \mathrm{E}+03$ & $6.2 \mathrm{E}+03$ & $6.3 \mathrm{E}+03$ & $6.3 \mathrm{E}+03$ \\
\hline Oklahoma & $\mathrm{OK}$ & $1.3 \mathrm{E}+03$ & $1.3 \mathrm{E}+03$ & $1.4 \mathrm{E}+03$ & $1.3 \mathrm{E}+03$ & $1.2 \mathrm{E}+03$ & $1.3 \mathrm{E}+03$ \\
\hline Arkansas & AR & $1.2 \mathrm{E}+03$ & $1.4 \mathrm{E}+03$ & $1.5 \mathrm{E}+03$ & $1.3 \mathrm{E}+03$ & $1.3 \mathrm{E}+03$ & $1.3 \mathrm{E}+03$ \\
\hline Louisiana & LA & $1.6 \mathrm{E}+03$ & $1.6 \mathrm{E}+03$ & $1.7 \mathrm{E}+03$ & $1.7 \mathrm{E}+03$ & $1.7 \mathrm{E}+03$ & $1.9 \mathrm{E}+03$ \\
\hline Mississippi & MS & $1.4 \mathrm{E}+03$ & $1.5 \mathrm{E}+03$ & $1.4 \mathrm{E}+03$ & $1.4 \mathrm{E}+03$ & $1.4 \mathrm{E}+03$ & $1.5 \mathrm{E}+03$ \\
\hline Alabama & $\mathrm{AL}$ & $1.7 \mathrm{E}+03$ & $1.9 \mathrm{E}+03$ & $1.9 \mathrm{E}+03$ & $1.8 \mathrm{E}+03$ & $1.8 \mathrm{E}+03$ & $1.8 \mathrm{E}+03$ \\
\hline Kentucky & KY & $1.9 \mathrm{E}+03$ & $1.9 \mathrm{E}+03$ & $1.8 \mathrm{E}+03$ & $1.8 \mathrm{E}+03$ & $1.8 \mathrm{E}+03$ & $1.9 \mathrm{E}+03$ \\
\hline Tennessee & TN & $1.8 \mathrm{E}+03$ & $1.8 \mathrm{E}+03$ & $1.8 \mathrm{E}+03$ & $1.8 \mathrm{E}+03$ & $1.7 \mathrm{E}+03$ & $1.8 \mathrm{E}+03$ \\
\hline Florida & FL & $4.5 \mathrm{E}+03$ & $4.7 \mathrm{E}+03$ & $4.3 \mathrm{E}+03$ & $4.2 \mathrm{E}+03$ & $5.3 \mathrm{E}+03$ & $4.8 \mathrm{E}+03$ \\
\hline Georgia & GA & $2.3 \mathrm{E}+03$ & $2.4 \mathrm{E}+03$ & $2.4 \mathrm{E}+03$ & $2.3 \mathrm{E}+03$ & $2.5 \mathrm{E}+03$ & $2.3 \mathrm{E}+03$ \\
\hline North Carolina & $\mathrm{NC}$ & $3.1 \mathrm{E}+03$ & $3.2 \mathrm{E}+03$ & $3.8 \mathrm{E}+03$ & $3.4 \mathrm{E}+03$ & $3.5 \mathrm{E}+03$ & $3.2 \mathrm{E}+03$ \\
\hline South Carolina & SC & $1.5 \mathrm{E}+03$ & $1.5 \mathrm{E}+03$ & $1.6 \mathrm{E}+03$ & $1.5 \mathrm{E}+03$ & $1.5 \mathrm{E}+03$ & $1.5 \mathrm{E}+03$ \\
\hline Virginia & $\mathrm{VA}$ & $2.2 \mathrm{E}+03$ & $2.2 \mathrm{E}+03$ & $2.2 \mathrm{E}+03$ & $2.1 \mathrm{E}+03$ & $2.0 \mathrm{E}+03$ & $2.2 \mathrm{E}+03$ \\
\hline West Virginia & WV & $5.9 \mathrm{E}+02$ & $5.9 \mathrm{E}+02$ & $5.4 \mathrm{E}+02$ & $5.7 \mathrm{E}+02$ & $5.0 \mathrm{E}+02$ & $5.3 \mathrm{E}+02$ \\
\hline Delaware & $\mathrm{DE}$ & $5.2 \mathrm{E}+01$ & $5.2 \mathrm{E}+01$ & $6.4 \mathrm{E}+01$ & $6.6 \mathrm{E}+01$ & $6.1 \mathrm{E}+01$ & $9.5 \mathrm{E}+01$ \\
\hline District of Colum & $\overline{\mathrm{DC}}$ & $1.8 \mathrm{E}+02$ & $1.8 \mathrm{E}+02$ & $1.7 \mathrm{E}+02$ & $1.8 \mathrm{E}+02$ & $1.6 \mathrm{E}+02$ & $1.7 \mathrm{E}+02$ \\
\hline Maryland & $\mathrm{MD}$ & $7.0 \mathrm{E}+02$ & $7.0 \mathrm{E}+02$ & $6.7 \mathrm{E}+02$ & $7.0 \mathrm{E}+02$ & $6.4 \mathrm{E}+02$ & $7.6 \mathrm{E}+02$ \\
\hline Connecticut & CT & $7.4 \mathrm{E}+02$ & $7.2 \mathrm{E}+02$ & $6.7 \mathrm{E}+02$ & $7.0 \mathrm{E}+02$ & $6.5 \mathrm{E}+02$ & $7.4 \mathrm{E}+02$ \\
\hline New Jersey & NJ & $2.3 \mathrm{E}+03$ & $2.3 \mathrm{E}+03$ & $2.2 \mathrm{E}+03$ & $2.3 \mathrm{E}+03$ & $2.1 \mathrm{E}+03$ & $2.3 \mathrm{E}+03$ \\
\hline New York & NY & $3.8 \mathrm{E}+03$ & $3.8 \mathrm{E}+03$ & $3.0 \mathrm{E}+03$ & $3.1 \mathrm{E}+03$ & $2.9 \mathrm{E}+03$ & $3.2 \mathrm{E}+03$ \\
\hline Pennsylvania & $\mathrm{PA}$ & $3.0 \mathrm{E}+03$ & $3.0 \mathrm{E}+03$ & $2.7 \mathrm{E}+03$ & $2.8 \mathrm{E}+03$ & $2.6 \mathrm{E}+03$ & $2.8 \mathrm{E}+03$ \\
\hline Rhode Island & RI & $4.7 \mathrm{E}+02$ & $4.7 \mathrm{E}+02$ & $4.4 \mathrm{E}+02$ & $4.5 \mathrm{E}+02$ & $4.2 \mathrm{E}+02$ & $4.5 \mathrm{E}+02$ \\
\hline Massachusetts & MA & $1.2 \mathrm{E}+03$ & $1.2 \mathrm{E}+03$ & $1.1 \mathrm{E}+03$ & $1.1 \mathrm{E}+03$ & $1.1 \mathrm{E}+03$ & $1.3 \mathrm{E}+03$ \\
\hline New Hampshire & $\mathrm{NH}$ & $5.3 \mathrm{E}+02$ & $5.3 \mathrm{E}+02$ & $3.4 \mathrm{E}+02$ & $3.5 \mathrm{E}+02$ & $3.3 \mathrm{E}+02$ & $3.8 \mathrm{E}+02$ \\
\hline Vermont & $\mathrm{VT}$ & $3.5 \mathrm{E}+02$ & $3.6 \mathrm{E}+02$ & $2.1 \mathrm{E}+02$ & $2.2 \mathrm{E}+02$ & $2.0 \mathrm{E}+02$ & $2.2 \mathrm{E}+02$ \\
\hline Maine & $\mathrm{ME}$ & $7.7 \mathrm{E}+02$ & $7.9 \mathrm{E}+02$ & $4.4 \mathrm{E}+02$ & $4.6 \mathrm{E}+02$ & $4.6 \mathrm{E}+02$ & $4.6 \mathrm{E}+02$ \\
\hline Ohio & $\mathrm{OH}$ & $2.9 \mathrm{E}+03$ & $2.9 \mathrm{E}+03$ & $2.7 \mathrm{E}+03$ & $2.9 \mathrm{E}+03$ & $2.7 \mathrm{E}+03$ & $3.1 \mathrm{E}+03$ \\
\hline Indiana & IN & $3.1 \mathrm{E}+03$ & $3.1 \mathrm{E}+03$ & $2.8 \mathrm{E}+03$ & $2.9 \mathrm{E}+03$ & $2.7 \mathrm{E}+03$ & $2.8 \mathrm{E}+03$ \\
\hline Illinois & $\mathrm{IL}$ & $2.8 \mathrm{E}+03$ & $2.8 \mathrm{E}+03$ & $2.5 \mathrm{E}+03$ & $2.6 \mathrm{E}+03$ & $2.4 \mathrm{E}+03$ & $2.7 \mathrm{E}+03$ \\
\hline Michigan & MI & $4.2 \mathrm{E}+03$ & $4.3 \mathrm{E}+03$ & $3.2 \mathrm{E}+03$ & $3.3 \mathrm{E}+03$ & $3.1 \mathrm{E}+03$ & $3.7 \mathrm{E}+03$ \\
\hline Wisconsin & WI & $2.5 \mathrm{E}+03$ & $2.6 \mathrm{E}+03$ & $1.7 \mathrm{E}+03$ & $1.7 \mathrm{E}+03$ & $1.6 \mathrm{E}+03$ & $1.8 \mathrm{E}+03$ \\
\hline Minnesota & MN & $2.5 \mathrm{E}+03$ & $2.7 \mathrm{E}+03$ & $1.6 \mathrm{E}+03$ & $1.8 \mathrm{E}+03$ & $1.6 \mathrm{E}+03$ & $1.9 \mathrm{E}+03$ \\
\hline Iowa & IA & $1.2 \mathrm{E}+03$ & $1.2 \mathrm{E}+03$ & $9.7 \mathrm{E}+02$ & $9.8 \mathrm{E}+02$ & $9.7 \mathrm{E}+02$ & $1.1 \mathrm{E}+03$ \\
\hline Missouri & MO & $2.2 \mathrm{E}+03$ & $2.2 \mathrm{E}+03$ & $2.2 \mathrm{E}+03$ & $2.1 \mathrm{E}+03$ & $2.1 \mathrm{E}+03$ & $2.3 \mathrm{E}+03$ \\
\hline Kansas & $\mathrm{KS}$ & $8.3 \mathrm{E}+02$ & $8.2 \mathrm{E}+02$ & $8.8 \mathrm{E}+02$ & $8.4 \mathrm{E}+02$ & $8.2 \mathrm{E}+02$ & $7.8 \mathrm{E}+02$ \\
\hline Nebraska & $\mathrm{NE}$ & $6.8 \mathrm{E}+02$ & $6.9 \mathrm{E}+02$ & $6.7 \mathrm{E}+02$ & $7.0 \mathrm{E}+02$ & $6.5 \mathrm{E}+02$ & $7.0 \mathrm{E}+02$ \\
\hline North Dakota & ND & $5.9 \mathrm{E}+02$ & $6.1 \mathrm{E}+02$ & $5.0 \mathrm{E}+02$ & $5.0 \mathrm{E}+02$ & $5.0 \mathrm{E}+02$ & $5.3 \mathrm{E}+02$ \\
\hline South Dakota & SD & $4.4 \mathrm{E}+02$ & $4.5 \mathrm{E}+02$ & $3.2 \mathrm{E}+02$ & $3.2 \mathrm{E}+02$ & $3.2 \mathrm{E}+02$ & $3.4 \mathrm{E}+02$ \\
\hline
\end{tabular}


Table A4 Average daily anthropogenic emissions of VOC (July-December)

\begin{tabular}{|c|c|c|c|c|c|c|c|}
\hline State & & July & August & September & October & November & December \\
\hline Oregon & OR & $1.4 \mathrm{E}+03$ & $2.3 \mathrm{E}+03$ & $1.2 \mathrm{E}+03$ & $1.3 \mathrm{E}+03$ & $1.0 \mathrm{E}+03$ & $1.0 \mathrm{E}+03$ \\
\hline Washington & WA & $2 E+03$ & $2.2 \mathrm{E}+03$ & $1.9 \mathrm{E}+03$ & $1.9 \mathrm{E}+03$ & $9 \mathrm{E}+03$ & $2.0 \mathrm{E}+03$ \\
\hline Wyoming & $\overline{W Y}$ & $4 \mathrm{E}+02$ & $.6 \mathrm{E}+02$ & $.0 \mathrm{E}+02$ & $.7 \mathrm{E}+02$ & $5 \mathrm{E}+02$ & $2 \mathrm{E}+02$ \\
\hline Idaho & ID & $6 \mathrm{E}+03$ & $.4 \mathrm{E}+03$ & $2 \mathrm{E}+03$ & $1.2 \mathrm{E}+03$ & $\overline{0 E+03}$ & $1.1 \mathrm{E}+03$ \\
\hline Montana & MT & $\overline{0 E+02}$ & $7.5 \mathrm{E}+02$ & $5.5 \mathrm{E}+02$ & $4.1 \mathrm{E}+02$ & $4.0 \mathrm{E}+02$ & $4.6 \mathrm{E}+02$ \\
\hline California & CA & $5.8 \mathrm{E}+03$ & $7.0 \mathrm{E}+03$ & $5.5 \mathrm{E}+03$ & $5.5 \mathrm{E}+03$ & $5.4 \mathrm{E}+03$ & $5.8 \mathrm{E}+03$ \\
\hline Nevada & NV & $5.4 \mathrm{E}+02$ & $8.8 \mathrm{E}+02$ & $4.0 \mathrm{E}+02$ & $3.7 \mathrm{E}+02$ & $3.4 \mathrm{E}+02$ & $3.0 \mathrm{E}+02$ \\
\hline Utah & $\overline{\mathrm{UT}}$ & $.3 \mathrm{E}+03$ & $8.0 \mathrm{E}+02$ & $6.8 \mathrm{E}+02$ & $6.0 \mathrm{E}+02$ & $5.9 \mathrm{E}+02$ & $6.5 \mathrm{E}+02$ \\
\hline Colorado & $\overline{\mathrm{CO}}$ & $3 \mathrm{E}+03$ & $1.1 \mathrm{E}+03$ & $9.7 \mathrm{E}+02$ & $9.8 \mathrm{E}+02$ & $9.2 \mathrm{E}+02$ & $1.0 \mathrm{E}+03$ \\
\hline Arizona & $\overline{\mathrm{AZ}}$ & $3 \mathrm{E}+03$ & $1.3 \mathrm{E}+03$ & $1.2 \mathrm{E}+03$ & $1.5 \mathrm{E}+03$ & $1.0 \mathrm{E}+03$ & $1.0 \mathrm{E}+03$ \\
\hline New Mexico & NM & $5 \mathrm{E}+02$ & $6.1 \mathrm{E}+02$ & $6.8 \mathrm{E}+02$ & $8.5 \mathrm{E}+02$ & $5.6 \mathrm{E}+02$ & $5.6 \mathrm{E}+02$ \\
\hline Texas & TX & $5 \mathrm{E}+03$ & $6.5 \mathrm{E}+03$ & $5.8 \mathrm{E}+03$ & $5.9 \mathrm{E}+03$ & $5.9 \mathrm{E}+03$ & $6.0 \mathrm{E}+03$ \\
\hline Oklahoma & OK & $4 \mathrm{E}+03$ & $1.4 \mathrm{E}-$ & $1.2 \mathrm{E}+03$ & $1.2 \mathrm{E}+03$ & +03 & $.2 \mathrm{E}+03$ \\
\hline Arkansas & $\overline{\mathrm{AR}}$ & $3 \mathrm{E}+03$ & $3 \mathrm{E}-$ & $2 \mathrm{E}+03$ & $1.3 \mathrm{E}+03$ & +03 & $.2 \mathrm{E}+03$ \\
\hline Louisiana & $\overline{\mathrm{LA}}$ & $7 \mathrm{E}+03$ & $7 \mathrm{E}$ & $6 \mathrm{E}-$ & $1.6 \mathrm{E}+03$ & $1.6 \mathrm{E}$ & $1.6 \mathrm{E}+03$ \\
\hline Mississippi & $\overline{\mathrm{MS}}$ & $4 \mathrm{E}+03$ & $4 \mathrm{E}^{+}$ & $\overline{3 \mathrm{E}+}$ & $1.4 \mathrm{E}+03$ & $1.3 \mathrm{E}+03$ & $1.3 \mathrm{E}+03$ \\
\hline Alabama & $\mathrm{AL}$ & $.7 E+03$ & $1.7 \mathrm{E}^{+}$ & $1.6 \mathrm{E}+03$ & $1.7 \mathrm{E}+03$ & $1.7 \mathrm{E}+03$ & $1.7 \mathrm{E}+03$ \\
\hline Kentucky & KY & $1.9 \mathrm{E}+03$ & $1.9 \mathrm{E}^{+}$ & $1.8 \mathrm{E}^{+}$ & $1.9 \mathrm{E}+03$ & $1.9 \mathrm{E}+03$ & $1.9 \mathrm{E}+03$ \\
\hline Tennessee & TN & $1.8 \mathrm{E}+03$ & $1.8 \mathrm{E}+03$ & $1.7 \mathrm{E}^{+}$ & $1.8 \mathrm{E}+03$ & $1.9 \mathrm{E}+03$ & $1.8 \mathrm{E}+03$ \\
\hline Florida & $\overline{\mathrm{FL}}$ & $4.7 \mathrm{E}+03$ & $4.7 \mathrm{E}+03$ & $4.0 \mathrm{E}+$ & $4.0 \mathrm{E}+03$ & $3.9 \mathrm{E}+03$ & $4.3 E+03$ \\
\hline Georgia & $\overline{\mathrm{GA}}$ & $2.1 \mathrm{E}+03$ & $2.1 \mathrm{E}+$ & $2.0 \mathrm{E}-$ & $2.1 \mathrm{E}+03$ & $2.1 \mathrm{E}$ & $2.3 \mathrm{E}+03$ \\
\hline North Carolina & $\mathrm{NC}$ & $2.9 \mathrm{E}+03$ & $2.9 \mathrm{E}+$ & $2.8 \mathrm{E}-$ & $3.0 \mathrm{E}+03$ & $3.2 \mathrm{E}+03$ & $3.0 \mathrm{E}+03$ \\
\hline South Carolina & $\mathrm{SC}$ & $4 \mathrm{E}+03$ & $\overline{5 \mathrm{E}+}$ & $1.4 \mathrm{E}+$ & $1.4 \mathrm{E}+03$ & $1.5 \mathrm{E}$ & $1.4 \mathrm{E}+03$ \\
\hline Virginia & VA & $2 \mathrm{E}+03$ & 33 & 3 & $2.0 \mathrm{E}+03$ & 03 & $2.2 \mathrm{E}+03$ \\
\hline West Virginia & WV & $3 \mathrm{E}+02$ & & 22 & $5.6 \mathrm{E}+02$ & 02 & $5.7 \mathrm{E}+02$ \\
\hline Delaware & $\mathrm{DE}$ & $9.4 \mathrm{E}+01$ & 1 & 1 & $6.3 \mathrm{E}+01$ & 01 & $5.1 \mathrm{E}+01$ \\
\hline$\overline{\text { District of } \mathrm{Cr}}$ & $\overline{\mathrm{DC}}$ & $7 \mathrm{E}+02$ & & 2 & $1.6 \mathrm{E}+02$ & 02 & $1.8 \mathrm{E}+02$ \\
\hline Maryland & $\overline{M D}$ & $7.6 \mathrm{E}+02$ & & $6.4 \mathrm{E}$ & $6.5 \mathrm{E}+02$ & $\overline{02}$ & $6.8 \mathrm{E}+02$ \\
\hline Connecticut & CT & $7.5 \mathrm{E}+02$ & $7.5 \mathrm{E}-$ & $6.5 \mathrm{E}+02$ & $6.9 \mathrm{E}+02$ & $6.6 \mathrm{E}+02$ & $6.9 \mathrm{E}+02$ \\
\hline New Jersey & $\mathrm{NJ}$ & $2.3 \mathrm{E}+03$ & $2.3 \mathrm{E}-$ & $2.1 \mathrm{E}+$ & $2.2 \mathrm{E}+03$ & $2.2 \mathrm{E}^{+}$ & $2.3 \mathrm{E}+03$ \\
\hline New York & NY & $3.2 \mathrm{E}+03$ & $3.2 \mathrm{E}^{+}$ & $2.8 \mathrm{E}^{+}$ & $3.0 \mathrm{E}+03$ & $2.9 \mathrm{E}+03$ & $3.7 \mathrm{E}+03$ \\
\hline Pennsylvania & $\overline{\mathrm{PA}}$ & $2.8 \mathrm{E}+03$ & $2.8 \mathrm{E}+03$ & $2.6 \mathrm{E}+03$ & $2.8 \mathrm{E}+03$ & $2.7 \mathrm{E}+03$ & $2.9 \mathrm{E}+03$ \\
\hline Rhode Island & RI & $4.5 \mathrm{E}+02$ & $4.5 \mathrm{E}+02$ & $4.2 \mathrm{E}+02$ & $4.4 \mathrm{E}+02$ & $4.3 \mathrm{E}+02$ & $4.5 \mathrm{E}+02$ \\
\hline Massachusetts & MA & $1.3 \mathrm{E}+03$ & & $1.0 \mathrm{E}+03$ & $1.1 \mathrm{E}+03$ & $1.1 \mathrm{E}+03$ & $1.1 \mathrm{E}+03$ \\
\hline New Hampsl & $\mathrm{NH}$ & $3.8 \mathrm{E}+02$ & $3.8 \mathrm{E}+02$ & $3.2 \mathrm{E}+\mathrm{C}$ & $3.4 \mathrm{E}+02$ & $3.4 \mathrm{E}+02$ & $5.0 \mathrm{E}+02$ \\
\hline Vermont & VT & $2.2 \mathrm{E}+02$ & 2 & $2.0 \mathrm{E}+$ & $2.1 \mathrm{E}+02$ & $2.1 \mathrm{E}+02$ & $3.3 \mathrm{E}+02$ \\
\hline Maine & ME & $6 \mathrm{E}+1$ & & 2 & $4.4 \mathrm{E}+02$ & & $7.4 \mathrm{E}+02$ \\
\hline Ohio & $\mathrm{OH}$ & $E+03$ & 3 & $2.7 \mathrm{E}$ & $2.7 \mathrm{E}+03$ & $2.8 \mathrm{E}$ & $2.8 \mathrm{E}+03$ \\
\hline Indiana & IN & $\mathrm{E}+03$ & 3 & 775 & $2.7 \mathrm{E}+03$ & $.8 \mathrm{E}$ & $3.0 \mathrm{E}+03$ \\
\hline & $\mathrm{IL}$ & $3+03$ & & & $2.4 \mathrm{E}+03$ & 03 & $2.6 \mathrm{E}+03$ \\
\hline Michigan & MI & $3.7 \mathrm{E}+03$ & $3.7 \mathrm{E}+$ & $3.1 \mathrm{E}+03$ & $3.3 \mathrm{E}+03$ & $3.2 \mathrm{E}+03$ & $4.1 \mathrm{E}+03$ \\
\hline Wisconsin & WI & $1.8 \mathrm{E}+03$ & $1.8 \mathrm{E}+03$ & $1.6 \mathrm{E}+03$ & $1.6 \mathrm{E}+03$ & $1.6 \mathrm{E}+03$ & $2.4 \mathrm{E}+03$ \\
\hline Minnesota & MN & $1.9 \mathrm{E}+03$ & $1.9 \mathrm{E}+$ & $1.5 \mathrm{E}+03$ & $1.5 \mathrm{E}+03$ & $1.6 \mathrm{E}+03$ & $2.4 \mathrm{E}+03$ \\
\hline Iowa & IA & $1.1 \mathrm{E}+03$ & $1.1 \mathrm{E}+03$ & $9.7 \mathrm{E}+02$ & $9.7 \mathrm{E}+02$ & $9.8 \mathrm{E}+02$ & $1.1 \mathrm{E}+03$ \\
\hline Missouri & $\mathrm{MO}$ & $2.3 \mathrm{E}+03$ & $2.3 \mathrm{E}+03$ & $2.0 \mathrm{E}+03$ & $2.1 \mathrm{E}+03$ & $2.1 \mathrm{E}+03$ & $2.2 \mathrm{E}+03$ \\
\hline Kansas & KS & $8.1 \mathrm{E}+02$ & $7.9 \mathrm{E}+$ & $7.7 \mathrm{E}-$ & $7.7 \mathrm{E}+02$ & $7.7 \mathrm{E}+02$ & $8.0 \mathrm{E}+02$ \\
\hline Nebraska & $\mathrm{NE}$ & $7.2 \mathrm{E}+02$ & $7.1 \mathrm{E}+$ & $6.5 \mathrm{E}+02$ & $6.6 \mathrm{E}+02$ & $6.6 \mathrm{E}+02$ & $6.5 \mathrm{E}+02$ \\
\hline North Dak & ND & $5.3 \mathrm{E}+02$ & & $4.9 \mathrm{E}+02$ & $5.0 \mathrm{E}+02$ & $4.9 \mathrm{E}+02$ & $5.8 \mathrm{E}+02$ \\
\hline South Dakota & SD & $4.2 \mathrm{E}+02$ & $3.5 \mathrm{E}+02$ & $4.0 \mathrm{E}+02$ & $3.6 \mathrm{E}+02$ & $3.1 \mathrm{E}+02$ & $4.2 \mathrm{E}+02$ \\
\hline
\end{tabular}


Table A5 Average daily biogenic emissions of VOC (January-June)

\begin{tabular}{|c|c|c|c|c|c|c|c|}
\hline State & & January & February & March & April & May & June \\
\hline Oregon & OR & $9.2 \mathrm{E}+02$ & $8.2 \mathrm{E}+02$ & $1.3 \mathrm{E}+03$ & $1.8 \mathrm{E}+03$ & $4.7 \mathrm{E}+03$ & $5.1 \mathrm{E}+03$ \\
\hline Washington & WA & $6.0 \mathrm{E}+02$ & $5.5 \mathrm{E}+02$ & $8.3 \mathrm{E}+02$ & $1.2 \mathrm{E}+03$ & $2.6 \mathrm{E}+03$ & $2.9 \mathrm{E}+03$ \\
\hline Wyoming & WY & $2.3 \mathrm{E}+02$ & $2.3 \mathrm{E}+02$ & $3.7 \mathrm{E}+02$ & $8.8 \mathrm{E}+02$ & $2.2 \mathrm{E}+03$ & $4.1 \mathrm{E}+03$ \\
\hline Idaho & ID & $4.3 \mathrm{E}+02$ & $4.3 \mathrm{E}+02$ & $6.6 \mathrm{E}+02$ & $1.2 \mathrm{E}+03$ & $3.7 \mathrm{E}+03$ & $5.2 \mathrm{E}+03$ \\
\hline Montana & MT & $5.6 \mathrm{E}+02$ & $4.5 \mathrm{E}+02$ & $7.9 \mathrm{E}+02$ & $1.6 \mathrm{E}+03$ & $4.6 \mathrm{E}+03$ & $7.2 \mathrm{E}+03$ \\
\hline California & CA & $1.5 \mathrm{E}+03$ & $1.6 \mathrm{E}+03$ & $3.0 \mathrm{E}+03$ & $4.2 \mathrm{E}+03$ & $1.2 \mathrm{E}+04$ & $1.3 \mathrm{E}+04$ \\
\hline Nevada & NV & $3.7 \mathrm{E}+02$ & $3.5 \mathrm{E}+02$ & $6.9 \mathrm{E}+02$ & $1.2 \mathrm{E}+03$ & $3.2 \mathrm{E}+03$ & $4.4 \mathrm{E}+03$ \\
\hline Utah & UT & $2.5 \mathrm{E}+02$ & $2.8 \mathrm{E}+02$ & $5.1 \mathrm{E}+02$ & $1.1 \mathrm{E}+03$ & $2.7 \mathrm{E}+03$ & $4.1 \mathrm{E}+03$ \\
\hline Colorado & $\mathrm{CO}$ & $3.1 \mathrm{E}+02$ & $3.8 \mathrm{E}+02$ & $5.5 \mathrm{E}+02$ & $1.4 \mathrm{E}+03$ & $3.2 \mathrm{E}+03$ & $5.5 \mathrm{E}+03$ \\
\hline Arizona & AZ & $7.4 \mathrm{E}+02$ & $9.3 \mathrm{E}+02$ & $1.6 \mathrm{E}+03$ & $2.7 \mathrm{E}+03$ & $6.3 \mathrm{E}+03$ & $8.1 \mathrm{E}+03$ \\
\hline New Mexico & NM & $4.5 \mathrm{E}+02$ & $7.1 \mathrm{E}+02$ & $9.3 \mathrm{E}+02$ & $2.0 \mathrm{E}+03$ & $4.5 \mathrm{E}+03$ & $6.1 \mathrm{E}+03$ \\
\hline Texas & $\mathrm{TX}$ & $1.4 \mathrm{E}+03$ & $2.7 \mathrm{E}+03$ & $2.8 \mathrm{E}+03$ & $7.2 \mathrm{E}+03$ & $1.4 \mathrm{E}+04$ & $1.8 \mathrm{E}+04$ \\
\hline Oklahoma & OK & $2.2 \mathrm{E}+02$ & $4.1 \mathrm{E}+02$ & $5.5 \mathrm{E}+02$ & $2.1 \mathrm{E}+03$ & $4.4 \mathrm{E}+03$ & $6.5 \mathrm{E}+03$ \\
\hline Arkansas & $\mathrm{AR}$ & $3.1 \mathrm{E}+02$ & $7.0 \mathrm{E}+02$ & $9.1 \mathrm{E}+02$ & $2.9 \mathrm{E}+03$ & $6.0 \mathrm{E}+03$ & $8.8 \mathrm{E}+03$ \\
\hline Louisiana & LA & $4.7 \mathrm{E}+02$ & $1.0 \mathrm{E}+03$ & $1.1 \mathrm{E}+03$ & $2.8 \mathrm{E}+03$ & $5.5 \mathrm{E}+03$ & $7.6 \mathrm{E}+03$ \\
\hline Mississippi & MS & $4.9 \mathrm{E}+02$ & $1.2 \mathrm{E}+03$ & $1.3 \mathrm{E}+03$ & $3.5 \mathrm{E}+03$ & $7.1 \mathrm{E}+03$ & $1.0 \mathrm{E}+04$ \\
\hline Alabama & $\mathrm{AL}$ & $5.6 \mathrm{E}+02$ & $1.3 \mathrm{E}+03$ & $1.4 \mathrm{E}+03$ & $3.5 \mathrm{E}+03$ & $7.3 \mathrm{E}+03$ & $1.1 \mathrm{E}+04$ \\
\hline Kentucky & $\mathrm{KY}$ & $1.3 \mathrm{E}+02$ & $3.2 \mathrm{E}+02$ & $3.3 \mathrm{E}+02$ & $1.5 \mathrm{E}+03$ & $3.2 \mathrm{E}+03$ & $4.9 \mathrm{E}+03$ \\
\hline Tennessee & TN & $1.7 \mathrm{E}+02$ & $4.2 \mathrm{E}+02$ & $4.3 \mathrm{E}+02$ & $1.8 \mathrm{E}+03$ & $3.7 \mathrm{E}+03$ & $5.7 \mathrm{E}+03$ \\
\hline Florida & FL & $1.2 \mathrm{E}+03$ & $2.0 \mathrm{E}+03$ & $2.4 \mathrm{E}+03$ & $3.9 \mathrm{E}+03$ & $6.6 \mathrm{E}+03$ & $9.4 \mathrm{E}+03$ \\
\hline Georgia & GA & $6.5 \mathrm{E}+02$ & $1.3 \mathrm{E}+03$ & $1.5 \mathrm{E}+03$ & $3.5 \mathrm{E}+03$ & $7.2 \mathrm{E}+03$ & $1.1 \mathrm{E}+04$ \\
\hline North Carolina & $\mathrm{NC}$ & $4.0 \mathrm{E}+02$ & $8.0 \mathrm{E}+02$ & $8.6 \mathrm{E}+02$ & $2.6 \mathrm{E}+03$ & $4.6 \mathrm{E}+03$ & $7.9 \mathrm{E}+03$ \\
\hline South Carolina & SC & $3.6 \mathrm{E}+02$ & $7.3 \mathrm{E}+02$ & $7.7 \mathrm{E}+02$ & $2.0 \mathrm{E}+03$ & $4.0 \mathrm{E}+03$ & $6.2 \mathrm{E}+03$ \\
\hline Virginia & VA & $1.9 \mathrm{E}+02$ & $4.2 \mathrm{E}+02$ & $4.7 \mathrm{E}+02$ & $1.9 \mathrm{E}+03$ & $3.3 \mathrm{E}+03$ & $6.2 \mathrm{E}+03$ \\
\hline West Virginia & WV & $6.4 \mathrm{E}+01$ & $1.6 \mathrm{E}+02$ & $1.6 \mathrm{E}+02$ & $7.7 \mathrm{E}+02$ & $1.6 \mathrm{E}+03$ & $2.7 \mathrm{E}+03$ \\
\hline Delaware & $\mathrm{DE}$ & $5.4 \mathrm{E}+00$ & $1.0 \mathrm{E}+01$ & $1.3 \mathrm{E}+01$ & $4.4 \mathrm{E}+01$ & $8.3 \mathrm{E}+01$ & $1.6 \mathrm{E}+02$ \\
\hline District of Colum & DC & $1.4 \mathrm{E}+00$ & $3.0 \mathrm{E}+00$ & $3.6 \mathrm{E}+00$ & $1.5 \mathrm{E}+01$ & $3.2 \mathrm{E}+01$ & $6.0 \mathrm{E}+01$ \\
\hline Maryland & MD & $2.4 \mathrm{E}+01$ & $4.9 \mathrm{E}+01$ & $5.9 \mathrm{E}+01$ & $2.2 \mathrm{E}+02$ & $4.4 \mathrm{E}+02$ & $8.4 \mathrm{E}+02$ \\
\hline Connecticut & $\mathrm{CT}$ & $9.9 \mathrm{E}+00$ & $1.5 \mathrm{E}+01$ & $2.0 \mathrm{E}+01$ & $6.7 \mathrm{E}+01$ & $1.7 \mathrm{E}+02$ & $3.6 \mathrm{E}+02$ \\
\hline New Jersey & NJ & $2.5 \mathrm{E}+01$ & $4.3 \mathrm{E}+01$ & $5.7 \mathrm{E}+01$ & $2.3 \mathrm{E}+02$ & $5.1 \mathrm{E}+02$ & $1.0 \mathrm{E}+03$ \\
\hline New York & $\mathrm{NY}$ & $8.5 \mathrm{E}+01$ & $1.1 \mathrm{E}+02$ & $1.3 \mathrm{E}+02$ & $5.1 \mathrm{E}+02$ & $1.4 \mathrm{E}+03$ & $2.6 \mathrm{E}+03$ \\
\hline Pennsylvania & PA & $8.5 \mathrm{E}+01$ & $1.5 \mathrm{E}+02$ & $1.7 \mathrm{E}+02$ & $8.7 \mathrm{E}+02$ & $2.1 \mathrm{E}+03$ & $3.9 \mathrm{E}+03$ \\
\hline Rhode Island & RI & $4.9 \mathrm{E}+00$ & $7.6 \mathrm{E}+00$ & $9.8 \mathrm{E}+00$ & $3.9 \mathrm{E}+01$ & $1.1 \mathrm{E}+02$ & $2.3 \mathrm{E}+02$ \\
\hline Massachusetts & MA & $1.5 \mathrm{E}+01$ & $2.1 \mathrm{E}+01$ & $2.5 \mathrm{E}+01$ & $8.6 \mathrm{E}+01$ & $2.7 \mathrm{E}+02$ & $5.6 \mathrm{E}+02$ \\
\hline New Hampshire & $\mathrm{NH}$ & $2.5 \mathrm{E}+01$ & $3.4 \mathrm{E}+01$ & $4.3 \mathrm{E}+01$ & $1.1 \mathrm{E}+02$ & $4.0 \mathrm{E}+02$ & $8.1 \mathrm{E}+02$ \\
\hline Vermont & VT & $2.2 \mathrm{E}+01$ & $3.0 \mathrm{E}+01$ & $3.6 \mathrm{E}+01$ & $9.4 \mathrm{E}+01$ & $3.4 \mathrm{E}+02$ & $6.0 \mathrm{E}+02$ \\
\hline Maine & $\mathrm{ME}$ & $8.1 \mathrm{E}+01$ & $1.1 \mathrm{E}+02$ & $1.5 \mathrm{E}+02$ & $2.8 \mathrm{E}+02$ & $1.3 \mathrm{E}+03$ & $2.3 \mathrm{E}+03$ \\
\hline Ohio & $\mathrm{OH}$ & $7.3 \mathrm{E}+01$ & $1.5 \mathrm{E}+02$ & $1.6 \mathrm{E}+02$ & $7.1 \mathrm{E}+02$ & $1.6 \mathrm{E}+03$ & $2.6 \mathrm{E}+03$ \\
\hline Indiana & IN & $5.5 \mathrm{E}+01$ & $1.1 \mathrm{E}+02$ & $1.4 \mathrm{E}+02$ & $6.3 \mathrm{E}+02$ & $1.4 \mathrm{E}+03$ & $2.3 \mathrm{E}+03$ \\
\hline Illinois & IL & $8.3 \mathrm{E}+01$ & $1.4 \mathrm{E}+02$ & $2.1 \mathrm{E}+02$ & $1.0 \mathrm{E}+03$ & $2.2 \mathrm{E}+03$ & $3.6 \mathrm{E}+03$ \\
\hline Michigan & MI & $1.3 \mathrm{E}+02$ & $1.4 \mathrm{E}+02$ & $2.0 \mathrm{E}+02$ & $8.4 \mathrm{E}+02$ & $2.3 \mathrm{E}+03$ & $4.4 \mathrm{E}+03$ \\
\hline Wisconsin & WI & $9.9 \mathrm{E}+01$ & $9.3 \mathrm{E}+01$ & $1.5 \mathrm{E}+02$ & $8.1 \mathrm{E}+02$ & $2.3 \mathrm{E}+03$ & $4.6 \mathrm{E}+03$ \\
\hline Minnesota & $\mathrm{MN}$ & $1.7 \mathrm{E}+02$ & $1.2 \mathrm{E}+02$ & $2.4 \mathrm{E}+02$ & $1.4 \mathrm{E}+03$ & $3.9 \mathrm{E}+03$ & $7.9 \mathrm{E}+03$ \\
\hline Iowa & IA & $7.6 \mathrm{E}+01$ & $8.0 \mathrm{E}+01$ & $1.2 \mathrm{E}+02$ & $7.5 \mathrm{E}+02$ & $1.7 \mathrm{E}+03$ & $2.9 \mathrm{E}+03$ \\
\hline Missouri & $\mathrm{MO}$ & $1.9 \mathrm{E}+02$ & $3.7 \mathrm{E}+02$ & $5.6 \mathrm{E}+02$ & $2.8 \mathrm{E}+03$ & $6.1 \mathrm{E}+03$ & $9.8 \mathrm{E}+03$ \\
\hline Kansas & KS & $1.7 \mathrm{E}+02$ & $1.8 \mathrm{E}+02$ & $3.0 \mathrm{E}+02$ & $1.1 \mathrm{E}+03$ & $2.5 \mathrm{E}+03$ & $3.9 \mathrm{E}+03$ \\
\hline Nebraska & NE & $1.4 \mathrm{E}+02$ & $1.1 \mathrm{E}+02$ & $2.0 \mathrm{E}+02$ & $8.8 \mathrm{E}+02$ & $2.0 \mathrm{E}+03$ & $3.6 \mathrm{E}+03$ \\
\hline North Dakota & $\mathrm{ND}$ & $9.2 \mathrm{E}+01$ & $5.6 \mathrm{E}+01$ & $1.2 \mathrm{E}+02$ & $4.8 \mathrm{E}+02$ & $1.2 \mathrm{E}+03$ & $2.1 \mathrm{E}+03$ \\
\hline South Dakota & SD & $1.4 \mathrm{E}+02$ & $1.0 \mathrm{E}+02$ & $1.8 \mathrm{E}+02$ & $6.9 \mathrm{E}+02$ & $1.8 \mathrm{E}+03$ & $3.5 \mathrm{E}+03$ \\
\hline
\end{tabular}


Table A6 Average daily biogenic emissions of VOC (July-December)

\begin{tabular}{|c|c|c|c|c|c|c|c|}
\hline State & & July & August & September & October & November & December \\
\hline Oregon & OR & $7.9 \mathrm{E}+03$ & $7.5 \mathrm{E}+03$ & $5.1 \mathrm{E}+03$ & $2.5 \mathrm{E}+03$ & $1.5 \mathrm{E}+03$ & $8.2 \mathrm{E}+02$ \\
\hline Washington & $\overline{\text { WA }}$ & $4.3 \mathrm{E}+03$ & $4.6 \mathrm{E}+03$ & $3.0 \mathrm{E}+03$ & $1.3 \mathrm{E}+03$ & $9.7 \mathrm{E}+02$ & $5.8 \mathrm{E}+02$ \\
\hline Wyoming & WY & $5.2 \mathrm{E}+03$ & $4.4 \mathrm{E}+03$ & $2.6 \mathrm{E}+03$ & $8.8 \mathrm{E}+02$ & $5.3 E+02$ & $2.2 \mathrm{E}+02$ \\
\hline Idaho & ID & $7.4 \mathrm{E}+03$ & $7.4 \mathrm{E}+03$ & $4.1 \mathrm{E}+03$ & $1.5 \mathrm{E}+03$ & $9.2 \mathrm{E}+02$ & $4.2 \mathrm{E}+02$ \\
\hline Montana & MT & $9.1 \mathrm{E}+03$ & $9.3 E+03$ & $5.0 \mathrm{E}+03$ & $1.6 \mathrm{E}+03$ & $1.2 \mathrm{E}+03$ & $4.9 \mathrm{E}+02$ \\
\hline California & CA & $1.7 \mathrm{E}+04$ & $1.6 \mathrm{E}+04$ & $1.1 \mathrm{E}+04$ & $6.6 \mathrm{E}+03$ & $2.8 \mathrm{E}+03$ & $1.4 \mathrm{E}+03$ \\
\hline Nevada & $\mathrm{NV}$ & $5.3 \mathrm{E}+03$ & $5.1 \mathrm{E}+03$ & $3.2 \mathrm{E}+03$ & $1.6 \mathrm{E}+03$ & $7.6 \mathrm{E}+02$ & $3.5 \mathrm{E}+02$ \\
\hline Utah & UT & $4.8 \mathrm{E}+03$ & $4.5 \mathrm{E}+03$ & $2.9 \mathrm{E}+03$ & $1.2 \mathrm{E}+03$ & $5.9 \mathrm{E}+02$ & $2.3 \mathrm{E}+02$ \\
\hline Colorado & $\mathrm{CO}$ & $6.2 \mathrm{E}+03$ & $4.8 \mathrm{E}+03$ & $3.4 \mathrm{E}+03$ & $1.4 \mathrm{E}+03$ & $7.0 \mathrm{E}+02$ & $3.2 \mathrm{E}+02$ \\
\hline Arizona & $\mathrm{AZ}$ & $8.1 \mathrm{E}+03$ & $7.9 \mathrm{E}+03$ & $6.2 \mathrm{E}+03$ & $3.1 \mathrm{E}+03$ & $1.6 \mathrm{E}+03$ & $7.4 \mathrm{E}+02$ \\
\hline New Mexico & $\mathrm{NM}$ & $6.2 \mathrm{E}+03$ & $4.9 \mathrm{E}+03$ & $3.8 \mathrm{E}+03$ & $2.0 \mathrm{E}+03$ & $9.4 \mathrm{E}+02$ & $5.1 \mathrm{E}+02$ \\
\hline Texas & TX & $1.9 \mathrm{E}+04$ & $1.7 \mathrm{E}+04$ & $9.3 \mathrm{E}+03$ & $5.4 \mathrm{E}+03$ & $3.3 E+03$ & $2.1 \mathrm{E}+03$ \\
\hline Oklahoma & $\overline{\mathrm{OK}}$ & $7.4 \mathrm{E}+03$ & $5.4 \mathrm{E}+03$ & $2.6 \mathrm{E}+03$ & $1.3 \mathrm{E}+03$ & $7.1 \mathrm{E}+02$ & $3.5 \mathrm{E}+02$ \\
\hline Arkansas & AR & $9.5 \mathrm{E}+03$ & $7.4 \mathrm{E}+03$ & $3.3 \mathrm{E}+03$ & $1.8 \mathrm{E}+03$ & $1.1 \mathrm{E}+03$ & $6.0 \mathrm{E}+02$ \\
\hline Louisiana & LA & $7.9 \mathrm{E}+03$ & $7.0 \mathrm{E}+03$ & $3.4 \mathrm{E}+03$ & $1.9 \mathrm{E}+03$ & $1.4 \mathrm{E}+03$ & $8.3 E+02$ \\
\hline Mississippi & MS & $9.8 \mathrm{E}+03$ & $8.6 \mathrm{E}+03$ & $4.0 \mathrm{E}+03$ & $2.4 \mathrm{E}+03$ & $1.6 \mathrm{E}+03$ & $9.4 \mathrm{E}+02$ \\
\hline Alabama & $\mathrm{AL}$ & $9.7 \mathrm{E}+03$ & $8.7 E+03$ & $4.6 \mathrm{E}+03$ & $2.5 \mathrm{E}+03$ & $1.6 \mathrm{E}+03$ & $1.0 \mathrm{E}+03$ \\
\hline Kentucky & $\mathrm{KY}$ & $4.7 \mathrm{E}+03$ & $3.0 \mathrm{E}+03$ & $1.8 \mathrm{E}+03$ & $9.7 \mathrm{E}+02$ & $5.1 \mathrm{E}+02$ & $3.0 \mathrm{E}+02$ \\
\hline Tennessee & TN & $5.2 \mathrm{E}+03$ & $3.8 \mathrm{E}+03$ & $2.0 \mathrm{E}+03$ & $1.1 \mathrm{E}+03$ & $6.2 \mathrm{E}+02$ & $3.6 \mathrm{E}+02$ \\
\hline Florida & $\overline{\text { FL }}$ & $9.0 \mathrm{E}+03$ & $7.9 \mathrm{E}+03$ & $5.8 E+03$ & $3.1 \mathrm{E}+03$ & $2.3 \mathrm{E}+03$ & $1.8 \mathrm{E}+03$ \\
\hline Georgia & GA & $9.2 \mathrm{E}+03$ & $7.9 \mathrm{E}+03$ & $4.7 \mathrm{E}+03$ & $2.5 \mathrm{E}+03$ & $1.6 \mathrm{E}+03$ & $1.1 \mathrm{E}+03$ \\
\hline North Carolina & $\mathrm{NC}$ & $6.1 \mathrm{E}+03$ & $5.3 \mathrm{E}+03$ & $3.0 \mathrm{E}+03$ & $1.9 \mathrm{E}+03$ & $1.1 \mathrm{E}+03$ & $7.8 \mathrm{E}+02$ \\
\hline South Carolina & SC & $5.0 \mathrm{E}+03$ & $4.4 \mathrm{E}+03$ & $2.6 \mathrm{E}+03$ & $1.5 \mathrm{E}+03$ & $9.1 \mathrm{E}+02$ & $6.4 \mathrm{E}+02$ \\
\hline Virginia & VA & $5.1 \mathrm{E}+03$ & $3.8 \mathrm{E}+03$ & $2.2 \mathrm{E}+03$ & $1.3 \mathrm{E}+03$ & $6.4 \mathrm{E}+02$ & $4.3 E+02$ \\
\hline West Virginia & WV & $2.5 \mathrm{E}+03$ & $1.6 \mathrm{E}+03$ & $9.6 \mathrm{E}+02$ & $5.2 \mathrm{E}+02$ & $2.5 \mathrm{E}+02$ & $1.7 \mathrm{E}+02$ \\
\hline Delaware & $\overline{\mathrm{DE}}$ & $1.6 \mathrm{E}+02$ & $1.1 \mathrm{E}+02$ & $6.8 \mathrm{E}+01$ & $3.8 \mathrm{E}+01$ & $1.8 \mathrm{E}+01$ & $1.2 \mathrm{E}+01$ \\
\hline District of Colun & DC & $5.4 \mathrm{E}+01$ & $3.6 \mathrm{E}+01$ & $2.2 \mathrm{E}+01$ & $1.2 \mathrm{E}+01$ & $5.3 \mathrm{E}+00$ & $3.4 \mathrm{E}+00$ \\
\hline Maryland & $\mathrm{MD}$ & $7.9 \mathrm{E}+02$ & $5.7 E+02$ & $3.3 E+02$ & $1.9 \mathrm{E}+02$ & $8.6 \mathrm{E}+01$ & $5.8 \mathrm{E}+01$ \\
\hline Connecticut & $\overline{\mathrm{CT}}$ & $3.0 \mathrm{E}+02$ & $2.7 \mathrm{E}+02$ & $1.4 \mathrm{E}+02$ & $7.7 \mathrm{E}+01$ & $3.6 \mathrm{E}+01$ & $2.4 \mathrm{E}+01$ \\
\hline New Jersey & NJ & $9.3 \mathrm{E}+02$ & $7.2 \mathrm{E}+02$ & $4.0 \mathrm{E}+02$ & $2.1 \mathrm{E}+02$ & $9.8 \mathrm{E}+01$ & $6.4 \mathrm{E}+01$ \\
\hline New York & $\mathrm{NY}$ & $2.2 \mathrm{E}+03$ & $2.0 \mathrm{E}+03$ & $1.1 \mathrm{E}+03$ & $5.9 \mathrm{E}+02$ & $3.3 \mathrm{E}+02$ & $2.1 \mathrm{E}+02$ \\
\hline Pennsylvania & PA & $3.7 \mathrm{E}+03$ & $2.4 \mathrm{E}+03$ & $1.4 \mathrm{E}+03$ & $7.2 \mathrm{E}+02$ & $3.5 \mathrm{E}+02$ & $2.3 \mathrm{E}+02$ \\
\hline Rhode Island & RI & $1.9 \mathrm{E}+02$ & $1.7 \mathrm{E}+02$ & $8.9 \mathrm{E}+01$ & $4.7 \mathrm{E}+01$ & $2.1 \mathrm{E}+01$ & $1.3 \mathrm{E}+01$ \\
\hline Massachusetts & $\overline{\mathrm{MA}}$ & $4.7 \mathrm{E}+02$ & $4.2 \mathrm{E}+02$ & $2.3 \mathrm{E}+02$ & $1.2 \mathrm{E}+02$ & $5.7 \mathrm{E}+01$ & $3.7 \mathrm{E}+01$ \\
\hline New Hampshire & $\mathrm{NH}$ & $6.7 \mathrm{E}+02$ & $6.5 \mathrm{E}+02$ & $3.6 \mathrm{E}+02$ & $1.9 \mathrm{E}+02$ & $9.3 \mathrm{E}+01$ & $6.0 \mathrm{E}+01$ \\
\hline Vermont & VT & $5.0 \mathrm{E}+02$ & $5.1 \mathrm{E}+02$ & $2.9 \mathrm{E}+02$ & $1.5 \mathrm{E}+02$ & $8.5 \mathrm{E}+01$ & $5.4 \mathrm{E}+01$ \\
\hline Maine & $\mathrm{ME}$ & $1.9 \mathrm{E}+03$ & $2.0 \mathrm{E}+03$ & $1.2 \mathrm{E}+03$ & $6.1 \mathrm{E}+02$ & $3.1 \mathrm{E}+02$ & $2.0 \mathrm{E}+02$ \\
\hline Ohio & $\overline{\mathrm{OH}}$ & $2.5 \mathrm{E}+03$ & $1.5 \mathrm{E}+03$ & $9.9 \mathrm{E}+02$ & $5.1 \mathrm{E}+02$ & $2.7 \mathrm{E}+02$ & $1.7 \mathrm{E}+02$ \\
\hline Indiana & IN & $2.3 \mathrm{E}+03$ & $1.5 \mathrm{E}+03$ & $8.8 \mathrm{E}+02$ & $4.2 \mathrm{E}+02$ & $2.3 \mathrm{E}+02$ & $1.3 \mathrm{E}+02$ \\
\hline Illinois & IL & $3.5 \mathrm{E}+03$ & $2.4 \mathrm{E}+03$ & $1.4 \mathrm{E}+03$ & $6.3 \mathrm{E}+02$ & $3.7 \mathrm{E}+02$ & $1.8 \mathrm{E}+02$ \\
\hline Michigan & MI & $4.3 \mathrm{E}+03$ & $3.4 \mathrm{E}+03$ & $1.6 \mathrm{E}+03$ & $7.2 \mathrm{E}+02$ & $4.5 \mathrm{E}+02$ & $2.4 \mathrm{E}+02$ \\
\hline Wisconsin & WI & $3.9 \mathrm{E}+03$ & $3.2 \mathrm{E}+03$ & $1.5 \mathrm{E}+03$ & $5.7 \mathrm{E}+02$ & $4.0 \mathrm{E}+02$ & $1.5 \mathrm{E}+02$ \\
\hline Minnesota & $\mathrm{MN}$ & $6.8 \mathrm{E}+03$ & $6.1 \mathrm{E}+03$ & $2.7 \mathrm{E}+03$ & $8.7 \mathrm{E}+02$ & $5.7 \mathrm{E}+02$ & $1.8 \mathrm{E}+02$ \\
\hline Iowa & IA & $2.8 \mathrm{E}+03$ & $2.0 \mathrm{E}+03$ & $1.2 \mathrm{E}+03$ & $4.7 \mathrm{E}+02$ & $3.4 \mathrm{E}+02$ & $1.3 \mathrm{E}+02$ \\
\hline Missouri & $\mathrm{MO}$ & $1.1 \mathrm{E}+04$ & $6.9 \mathrm{E}+03$ & $3.8 \mathrm{E}+03$ & $1.6 \mathrm{E}+03$ & $8.7 \mathrm{E}+02$ & $4.1 \mathrm{E}+02$ \\
\hline Kansas & $\mathrm{KS}$ & $4.4 \mathrm{E}+03$ & $3.2 \mathrm{E}+03$ & $1.7 \mathrm{E}+03$ & $8.2 \mathrm{E}+02$ & $5.0 \mathrm{E}+02$ & $2.4 \mathrm{E}+02$ \\
\hline Nebraska & $\mathrm{NE}$ & $3.3 \mathrm{E}+03$ & $2.6 \mathrm{E}+03$ & $1.5 \mathrm{E}+03$ & $6.3 \mathrm{E}+02$ & $3.8 \mathrm{E}+02$ & $1.4 \mathrm{E}+02$ \\
\hline North Dakota & ND & $2.1 \mathrm{E}+03$ & $2.0 \mathrm{E}+03$ & $1.0 \mathrm{E}+03$ & $3.2 \mathrm{E}+02$ & $2.3 \mathrm{E}+02$ & $7.5 \mathrm{E}+01$ \\
\hline South Dakota & SD & $3.6 \mathrm{E}+03$ & $3.0 \mathrm{E}+03$ & $1.6 \mathrm{E}+03$ & $5.6 \mathrm{E}+02$ & $3.6 \mathrm{E}+02$ & $1.2 \mathrm{E}+02$ \\
\hline
\end{tabular}


Table A7 Fate level characterization factors for $\mathrm{NO}_{\mathrm{x}}$ (January-June)

\begin{tabular}{|c|c|c|c|c|c|c|c|}
\hline State & & January & February & March & April & May & June \\
\hline Oregon & OR & $1.0 \mathrm{E}-04$ & 7.1E-05 & $1.2 \mathrm{E}-04$ & $3.9 \mathrm{E}-04$ & $4.3 \mathrm{E}-04$ & $1.1 \mathrm{E}-03$ \\
\hline Washington & WA & $5.1 \mathrm{E}-05$ & $4.5 \mathrm{E}-05$ & $9.7 \mathrm{E}-05$ & $2.5 \mathrm{E}-04$ & $2.1 \mathrm{E}-04$ & 4.1E-04 \\
\hline Wyoming & WY & $1.3 \mathrm{E}-04$ & $2.5 \mathrm{E}-04$ & $5.8 \mathrm{E}-04$ & $2.9 \mathrm{E}-04$ & $6.6 \mathrm{E}-04$ & $4.5 \mathrm{E}-04$ \\
\hline Idaho & ID & $1.6 \mathrm{E}-04$ & $4.6 \mathrm{E}-05$ & $9.3 \mathrm{E}-05$ & $3.8 \mathrm{E}-04$ & $1.4 \mathrm{E}-04$ & $5.4 \mathrm{E}-04$ \\
\hline Montana & MT & $1.3 \mathrm{E}-04$ & $1.4 \mathrm{E}-04$ & $3.0 \mathrm{E}-04$ & $3.8 \mathrm{E}-04$ & $3.4 \mathrm{E}-04$ & $\overline{5.0 \mathrm{E}-04}$ \\
\hline California & $\mathrm{CA}$ & $1.4 \mathrm{E}-04$ & $7.6 \mathrm{E}-05$ & $2.4 \mathrm{E}-04$ & $5.0 \mathrm{E}-04$ & $5.2 \mathrm{E}-04$ & $9.7 \mathrm{E}-04$ \\
\hline Nevada & NV & $2.4 \mathrm{E}-04$ & $2.1 \mathrm{E}-04$ & $2.7 \mathrm{E}-04$ & $4.1 \mathrm{E}-04$ & $5.0 \mathrm{E}-04$ & 7.6E-04 \\
\hline Utah & UT & $1.7 \mathrm{E}-04$ & $1.8 \mathrm{E}-04$ & $2.8 \mathrm{E}-04$ & $2.7 \mathrm{E}-04$ & $4.6 \mathrm{E}-04$ & $5.0 \mathrm{E}-04$ \\
\hline Colorado & $\mathrm{CO}$ & $1.3 \mathrm{E}-04$ & $8.5 \mathrm{E}-05$ & $1.8 \mathrm{E}-04$ & $2.2 \mathrm{E}-04$ & $2.4 \mathrm{E}-04$ & $4.1 \mathrm{E}-04$ \\
\hline Arizona & $\mathrm{AZ}$ & $3.4 \mathrm{E}-04$ & $2.8 \mathrm{E}-04$ & $3.1 \mathrm{E}-04$ & $4.5 \mathrm{E}-04$ & $6.5 \mathrm{E}-04$ & 8.1E-04 \\
\hline New Mexico & NM & $2.2 \mathrm{E}-04$ & $2.5 \mathrm{E}-04$ & $3.1 \mathrm{E}-04$ & $3.1 \mathrm{E}-04$ & $4.9 \mathrm{E}-04$ & $5.6 \mathrm{E}-04$ \\
\hline Texas & TX & $1.2 \mathrm{E}-04$ & $1.0 \mathrm{E}-04$ & $2.2 \mathrm{E}-04$ & $3.3 \mathrm{E}-04$ & $3.7 \mathrm{E}-04$ & $6.5 \mathrm{E}-04$ \\
\hline Oklahoma & OK & $9.9 \mathrm{E}-05$ & $9.5 \mathrm{E}-05$ & $2.4 \mathrm{E}-04$ & $3.2 \mathrm{E}-04$ & $5.3 \mathrm{E}-04$ & 8.3E-04 \\
\hline Arkansas & AR & $1.5 \mathrm{E}-04$ & $9.0 \mathrm{E}-05$ & $2.8 \mathrm{E}-04$ & $5.1 \mathrm{E}-04$ & $6.2 \mathrm{E}-04$ & $1.4 \mathrm{E}-03$ \\
\hline Louisiana & LA & $2.1 \mathrm{E}-04$ & $2.9 \mathrm{E}-04$ & $6.7 \mathrm{E}-04$ & $5.7 \mathrm{E}-04$ & $1.0 \mathrm{E}-03$ & $1.2 \mathrm{E}-03$ \\
\hline Mississippi & MS & $2.4 \mathrm{E}-04$ & $1.8 \mathrm{E}-04$ & $4.4 \mathrm{E}-04$ & $7.1 \mathrm{E}-04$ & 7.3E-04 & $1.5 \mathrm{E}-03$ \\
\hline Alabama & $\mathrm{AL}$ & $2.1 \mathrm{E}-04$ & $2.0 \mathrm{E}-04$ & $4.0 \mathrm{E}-04$ & 7.7E-04 & $7.0 \mathrm{E}-04$ & $1.7 \mathrm{E}-03$ \\
\hline Kentucky & $\mathrm{KY}$ & $4.2 \mathrm{E}-05$ & $7.0 \mathrm{E}-05$ & $1.6 \mathrm{E}-04$ & $2.3 \mathrm{E}-04$ & $5.6 \mathrm{E}-04$ & $8.9 \mathrm{E}-04$ \\
\hline Tennessee & $\mathrm{TN}$ & 7.7E-05 & $6.5 \mathrm{E}-05$ & $1.4 \mathrm{E}-04$ & $4.0 \mathrm{E}-04$ & $4.2 \mathrm{E}-04$ & $1.2 \mathrm{E}-03$ \\
\hline Florida & FL & $4.1 \mathrm{E}-04$ & $3.4 \mathrm{E}-04$ & $5.3 \mathrm{E}-04$ & $1.2 \mathrm{E}-03$ & $7.4 \mathrm{E}-04$ & 2.3E-03 \\
\hline Georgia & GA & $1.7 \mathrm{E}-04$ & $2.2 \mathrm{E}-04$ & $3.1 \mathrm{E}-04$ & $7.8 \mathrm{E}-04$ & $6.3 \mathrm{E}-04$ & $1.7 \mathrm{E}-03$ \\
\hline North Carolina & $\mathrm{NC}$ & $1.1 \mathrm{E}-04$ & $1.4 \mathrm{E}-04$ & $1.4 \mathrm{E}-04$ & $9.0 \mathrm{E}-04$ & $5.2 \mathrm{E}-04$ & $2.2 \mathrm{E}-03$ \\
\hline South Carolina & SC & $1.7 \mathrm{E}-04$ & $4.2 \mathrm{E}-04$ & $4.1 \mathrm{E}-04$ & $1.4 \mathrm{E}-03$ & $1.1 \mathrm{E}-03$ & $3.2 \mathrm{E}-03$ \\
\hline Virginia & $\mathrm{VA}$ & $6.8 \mathrm{E}-05$ & $7.3 \mathrm{E}-05$ & $1.3 \mathrm{E}-04$ & $6.6 \mathrm{E}-04$ & $5.2 \mathrm{E}-04$ & $1.9 \mathrm{E}-03$ \\
\hline West Virginia & $\mathrm{WV}$ & $4.3 \mathrm{E}-05$ & $1.2 \mathrm{E}-04$ & $2.4 \mathrm{E}-04$ & $2.7 \mathrm{E}-04$ & $7.8 \mathrm{E}-04$ & $1.0 \mathrm{E}-03$ \\
\hline Delaware & $\mathrm{DE}$ & $6.5 \mathrm{E}-05$ & $7.4 \mathrm{E}-05$ & $1.2 \mathrm{E}-04$ & $1.1 \mathrm{E}-03$ & $8.5 \mathrm{E}-04$ & $4.2 \mathrm{E}-03$ \\
\hline Washington DC & $\mathrm{DC}$ & $5.4 \mathrm{E}-05$ & $3.2 \mathrm{E}-05$ & $6.7 \mathrm{E}-05$ & $2.7 \mathrm{E}-04$ & $2.3 \mathrm{E}-04$ & $1.3 \mathrm{E}-03$ \\
\hline Maryland & MD & $5.0 \mathrm{E}-05$ & $6.2 \mathrm{E}-05$ & $1.1 \mathrm{E}-04$ & $4.0 \mathrm{E}-04$ & $5.7 \mathrm{E}-04$ & $1.9 \mathrm{E}-03$ \\
\hline Connecticut & CT & $3.5 \mathrm{E}-05$ & $4.5 \mathrm{E}-05$ & $5.4 \mathrm{E}-05$ & $3.1 \mathrm{E}-04$ & $2.8 \mathrm{E}-04$ & 2.3E-03 \\
\hline New Jersey & $\mathrm{NJ}$ & $3.4 \mathrm{E}-05$ & $4.1 \mathrm{E}-05$ & $5.0 \mathrm{E}-05$ & $2.4 \mathrm{E}-04$ & $2.5 \mathrm{E}-04$ & $1.6 \mathrm{E}-03$ \\
\hline New York & $\mathrm{NY}$ & $2.4 \mathrm{E}-05$ & $2.2 \mathrm{E}-05$ & $4.6 \mathrm{E}-05$ & $2.4 \mathrm{E}-04$ & $2.6 \mathrm{E}-04$ & $1.4 \mathrm{E}-03$ \\
\hline Pennsylvania & PA & $2.7 \mathrm{E}-05$ & $3.6 \mathrm{E}-05$ & $6.2 \mathrm{E}-05$ & $2.1 \mathrm{E}-04$ & $2.9 \mathrm{E}-04$ & $1.2 \mathrm{E}-03$ \\
\hline Rhode Island & RI & $4.4 \mathrm{E}-05$ & $4.7 \mathrm{E}-05$ & $7.2 \mathrm{E}-05$ & $4.0 \mathrm{E}-04$ & $2.9 \mathrm{E}-04$ & $3.2 \mathrm{E}-03$ \\
\hline Massachusetts & MA & $4.3 \mathrm{E}-05$ & $4.0 \mathrm{E}-05$ & $8.0 \mathrm{E}-05$ & $3.5 \mathrm{E}-04$ & $3.2 \mathrm{E}-04$ & $3.0 \mathrm{E}-03$ \\
\hline New Hampshire & $\mathrm{NH}$ & $5.4 \mathrm{E}-05$ & $3.2 \mathrm{E}-05$ & $1.1 \mathrm{E}-04$ & $5.1 \mathrm{E}-04$ & $3.9 \mathrm{E}-04$ & $4.1 \mathrm{E}-03$ \\
\hline Vermont & VT & $5.8 \mathrm{E}-05$ & $3.1 \mathrm{E}-05$ & $1.4 \mathrm{E}-04$ & $6.4 \mathrm{E}-04$ & $4.6 \mathrm{E}-04$ & $3.4 \mathrm{E}-03$ \\
\hline Maine & $\mathrm{ME}$ & $1.4 \mathrm{E}-04$ & $6.1 \mathrm{E}-05$ & $3.2 \mathrm{E}-04$ & $1.3 \mathrm{E}-03$ & $6.0 \mathrm{E}-04$ & $5.4 \mathrm{E}-03$ \\
\hline Ohio & $\mathrm{OH}$ & $2.8 \mathrm{E}-05$ & $3.3 \mathrm{E}-05$ & $6.6 \mathrm{E}-05$ & $1.5 \mathrm{E}-04$ & $2.8 \mathrm{E}-04$ & 7.9E-04 \\
\hline Indiana & IN & $2.8 \mathrm{E}-05$ & $2.6 \mathrm{E}-05$ & $5.3 \mathrm{E}-05$ & $1.4 \mathrm{E}-04$ & $2.3 \mathrm{E}-04$ & $6.8 \mathrm{E}-04$ \\
\hline Illinois & IL & $3.0 \mathrm{E}-05$ & $3.6 \mathrm{E}-05$ & $7.4 \mathrm{E}-05$ & $1.7 \mathrm{E}-04$ & $3.1 \mathrm{E}-04$ & $6.7 \mathrm{E}-04$ \\
\hline Michigan & MI & $3.0 \mathrm{E}-05$ & $1.8 \mathrm{E}-05$ & $5.9 \mathrm{E}-05$ & $1.9 \mathrm{E}-04$ & $2.6 \mathrm{E}-04$ & $8.7 \mathrm{E}-04$ \\
\hline Wisconsin & WI & $3.2 \mathrm{E}-05$ & $1.9 \mathrm{E}-05$ & $6.2 \mathrm{E}-05$ & $2.1 \mathrm{E}-04$ & $3.0 \mathrm{E}-04$ & 9.3E-04 \\
\hline Minnesota & $\mathrm{MN}$ & $4.8 \mathrm{E}-05$ & $3.1 \mathrm{E}-05$ & $1.1 \mathrm{E}-04$ & $2.9 \mathrm{E}-04$ & $3.4 \mathrm{E}-04$ & $8.0 \mathrm{E}-04$ \\
\hline Iowa & IA & 4.7E-05 & $3.5 \mathrm{E}-05$ & $8.6 \mathrm{E}-05$ & $3.0 \mathrm{E}-04$ & $3.3 \mathrm{E}-04$ & $9.0 \mathrm{E}-04$ \\
\hline Missouri & $\mathrm{MO}$ & $6.0 \mathrm{E}-05$ & $5.0 \mathrm{E}-05$ & $1.4 \mathrm{E}-04$ & $2.8 \mathrm{E}-04$ & $3.5 \mathrm{E}-04$ & $8.0 \mathrm{E}-04$ \\
\hline Kansas & KS & $1.0 \mathrm{E}-04$ & $1.2 \mathrm{E}-04$ & $2.6 \mathrm{E}-04$ & $3.3 \mathrm{E}-04$ & $5.7 \mathrm{E}-04$ & 7.7E-04 \\
\hline Nebraska & $\mathrm{NE}$ & $9.8 \mathrm{E}-05$ & $1.2 \mathrm{E}-04$ & $2.2 \mathrm{E}-04$ & $3.3 \mathrm{E}-04$ & $5.1 \mathrm{E}-04$ & $7.2 \mathrm{E}-04$ \\
\hline North Dakota & ND & $8.4 \mathrm{E}-05$ & $1.1 \mathrm{E}-04$ & $2.7 \mathrm{E}-04$ & $4.7 \mathrm{E}-04$ & $5.0 \mathrm{E}-04$ & 7.3E-04 \\
\hline South Dakota & SD & $1.1 \mathrm{E}-04$ & $6.5 \mathrm{E}-05$ & $2.2 \mathrm{E}-04$ & $4.4 \mathrm{E}-04$ & $3.9 \mathrm{E}-04$ & $9.4 \mathrm{E}-04$ \\
\hline
\end{tabular}


Table A8 Fate level characterization factors for $\mathrm{NO}_{\mathrm{x}}$ (July-December)

\begin{tabular}{|c|c|c|c|c|c|c|c|}
\hline State & & July & August & September & October & November & December \\
\hline Oregon & OR & $1.6 \mathrm{E}-03$ & $4.2 \mathrm{E}-04$ & $5.5 \mathrm{E}-04$ & $1.4 \mathrm{E}-04$ & 5.3E-05 & $2.1 \mathrm{E}-05$ \\
\hline Washington & WA & $6.4 \mathrm{E}-04$ & $4.0 \mathrm{E}-04$ & $3.2 \mathrm{E}-04$ & $9.0 \mathrm{E}-05$ & $3.6 \mathrm{E}-05$ & $1.5 \mathrm{E}-05$ \\
\hline Wyoming & $\mathrm{WY}$ & $6.7 \mathrm{E}-04$ & $1.0 \mathrm{E}-03$ & $8.4 \mathrm{E}-04$ & $4.4 \mathrm{E}-04$ & $4.5 \mathrm{E}-04$ & $1.6 \mathrm{E}-04$ \\
\hline Idaho & ID & $9.5 \mathrm{E}-04$ & $2.6 \mathrm{E}-04$ & $1.7 \mathrm{E}-04$ & $6.3 \mathrm{E}-05$ & $5.4 \mathrm{E}-05$ & $2.7 \mathrm{E}-05$ \\
\hline Montana & MT & $8.2 \mathrm{E}-04$ & $5.5 \mathrm{E}-04$ & $4.6 \mathrm{E}-04$ & $2.9 \mathrm{E}-04$ & $1.9 \mathrm{E}-04$ & $7.4 \mathrm{E}-05$ \\
\hline California & CA & $7.4 \mathrm{E}-04$ & $5.4 \mathrm{E}-04$ & $4.0 \mathrm{E}-04$ & $4.9 \mathrm{E}-04$ & $1.7 \mathrm{E}-04$ & $6.5 \mathrm{E}-05$ \\
\hline Nevada & NV & $9.2 \mathrm{E}-04$ & $4.6 \mathrm{E}-04$ & $4.5 \mathrm{E}-04$ & $4.2 \mathrm{E}-04$ & $2.0 \mathrm{E}-04$ & $1.7 \mathrm{E}-04$ \\
\hline Utah & UT & $8.2 \mathrm{E}-04$ & $6.7 \mathrm{E}-04$ & $5.1 \mathrm{E}-04$ & $3.1 \mathrm{E}-04$ & $2.4 \mathrm{E}-04$ & $1.4 \mathrm{E}-04$ \\
\hline Colorado & $\mathrm{CO}$ & $8.2 \mathrm{E}-04$ & $4.3 \mathrm{E}-04$ & $2.9 \mathrm{E}-04$ & $1.4 \mathrm{E}-04$ & $1.6 \mathrm{E}-04$ & $7.5 \mathrm{E}-05$ \\
\hline Arizona & $\mathrm{AZ}$ & $1.1 \mathrm{E}-03$ & $1.0 \mathrm{E}-03$ & 5.9E-04 & $6.2 \mathrm{E}-04$ & $4.2 \mathrm{E}-04$ & $3.9 \mathrm{E}-04$ \\
\hline New Mexico & NM & $9.9 \mathrm{E}-04$ & $1.1 \mathrm{E}-03$ & $6.3 \mathrm{E}-04$ & $3.3 \mathrm{E}-04$ & $4.0 \mathrm{E}-04$ & $2.8 \mathrm{E}-04$ \\
\hline Texas & $\mathrm{TX}$ & $6.8 \mathrm{E}-04$ & $4.1 \mathrm{E}-04$ & $4.1 \mathrm{E}-04$ & $2.6 \mathrm{E}-04$ & $1.9 \mathrm{E}-04$ & $9.5 \mathrm{E}-05$ \\
\hline Oklahoma & $\mathrm{OK}$ & 7.5E-04 & $5.9 \mathrm{E}-04$ & $4.4 \mathrm{E}-04$ & $2.7 \mathrm{E}-04$ & $2.1 \mathrm{E}-04$ & $8.2 \mathrm{E}-05$ \\
\hline Arkansas & AR & $1.3 \mathrm{E}-03$ & $8.9 \mathrm{E}-04$ & $6.2 \mathrm{E}-04$ & $3.3 \mathrm{E}-04$ & $3.3 \mathrm{E}-04$ & $9.9 \mathrm{E}-05$ \\
\hline Louisiana & $\overline{\mathrm{LA}}$ & $1.3 \mathrm{E}-03$ & $1.9 \mathrm{E}-03$ & $1.2 \mathrm{E}-03$ & $6.1 \mathrm{E}-04$ & $6.3 \mathrm{E}-04$ & $2.7 \mathrm{E}-04$ \\
\hline Mississippi & MS & $1.8 \mathrm{E}-03$ & $1.2 \mathrm{E}-03$ & $8.1 \mathrm{E}-04$ & $3.9 \mathrm{E}-04$ & 4.5E-04 & $1.6 \mathrm{E}-04$ \\
\hline Alabama & AL & $1.7 \mathrm{E}-03$ & $1.2 \mathrm{E}-03$ & $8.2 \mathrm{E}-04$ & $4.1 \mathrm{E}-04$ & $3.9 \mathrm{E}-04$ & $1.7 \mathrm{E}-04$ \\
\hline Kentucky & KY & $8.5 \mathrm{E}-04$ & $8.3 \mathrm{E}-04$ & $6.0 \mathrm{E}-04$ & $2.3 \mathrm{E}-04$ & $1.6 \mathrm{E}-04$ & $5.3 \mathrm{E}-05$ \\
\hline Tennessee & $\overline{\mathrm{TN}}$ & $1.2 \mathrm{E}-03$ & $7.1 \mathrm{E}-04$ & $4.3 \mathrm{E}-04$ & $2.3 \mathrm{E}-04$ & $1.6 \mathrm{E}-04$ & $4.4 \mathrm{E}-05$ \\
\hline Florida & FL & $2.1 \mathrm{E}-03$ & $1.3 \mathrm{E}-03$ & $1.1 \mathrm{E}-03$ & $5.5 \mathrm{E}-04$ & $4.1 \mathrm{E}-04$ & $2.3 \mathrm{E}-04$ \\
\hline Georgia & GA & $1.4 \mathrm{E}-03$ & $1.5 \mathrm{E}-03$ & $6.7 \mathrm{E}-04$ & $3.8 \mathrm{E}-04$ & $3.1 \mathrm{E}-04$ & $1.4 \mathrm{E}-04$ \\
\hline North Carolina & $\mathrm{NC}$ & $2.1 \mathrm{E}-03$ & $1.0 \mathrm{E}-03$ & $4.1 \mathrm{E}-04$ & $3.3 \mathrm{E}-04$ & $1.3 \mathrm{E}-04$ & $6.8 \mathrm{E}-05$ \\
\hline South Carolina & SC & $2.5 \mathrm{E}-03$ & $1.9 \mathrm{E}-03$ & $8.1 \mathrm{E}-04$ & $5.9 \mathrm{E}-04$ & $2.8 \mathrm{E}-04$ & $1.7 \mathrm{E}-04$ \\
\hline Virginia & $\overline{\mathrm{VA}}$ & $1.8 \mathrm{E}-03$ & $9.8 \mathrm{E}-04$ & $4.6 \mathrm{E}-04$ & $3.3 \mathrm{E}-04$ & $1.1 \mathrm{E}-04$ & $4.2 \mathrm{E}-05$ \\
\hline West Virginia & WV & $9.9 \mathrm{E}-04$ & $1.5 \mathrm{E}-03$ & $9.8 \mathrm{E}-04$ & $4.1 \mathrm{E}-04$ & $2.0 \mathrm{E}-04$ & $9.4 \mathrm{E}-05$ \\
\hline Delaware & $\overline{\mathrm{DE}}$ & $3.8 \mathrm{E}-03$ & $1.2 \mathrm{E}-03$ & $5.8 \mathrm{E}-04$ & $4.6 \mathrm{E}-04$ & $9.1 \mathrm{E}-05$ & $3.6 \mathrm{E}-05$ \\
\hline Washington DC & $\mathrm{DC}$ & $1.2 \mathrm{E}-03$ & $4.6 \mathrm{E}-04$ & $2.0 \mathrm{E}-04$ & $9.6 \mathrm{E}-05$ & $5.1 \mathrm{E}-05$ & $2.0 \mathrm{E}-05$ \\
\hline Maryland & MD & $1.8 \mathrm{E}-03$ & $1.0 \mathrm{E}-03$ & $4.6 \mathrm{E}-04$ & $2.6 \mathrm{E}-04$ & $9.2 \mathrm{E}-05$ & $3.4 \mathrm{E}-05$ \\
\hline Connecticut & CT & $1.5 \mathrm{E}-03$ & $6.6 \mathrm{E}-04$ & $3.5 \mathrm{E}-04$ & $1.2 \mathrm{E}-04$ & $4.6 \mathrm{E}-05$ & $2.9 \mathrm{E}-05$ \\
\hline New Jersey & $\mathrm{NJ}$ & $1.2 \mathrm{E}-03$ & 5.1E-04 & $2.4 \mathrm{E}-04$ & $1.0 \mathrm{E}-04$ & $4.4 \mathrm{E}-05$ & $2.0 \mathrm{E}-05$ \\
\hline New York & NY & $1.0 \mathrm{E}-03$ & $5.1 \mathrm{E}-04$ & $2.8 \mathrm{E}-04$ & $8.7 \mathrm{E}-05$ & $3.5 \mathrm{E}-05$ & $1.3 \mathrm{E}-05$ \\
\hline Pennsylvania & PA & $1.1 \mathrm{E}-03$ & $5.9 \mathrm{E}-04$ & $3.1 \mathrm{E}-04$ & $9.8 \mathrm{E}-05$ & $4.9 \mathrm{E}-05$ & $2.1 \mathrm{E}-05$ \\
\hline Rhode Island & RI & $2.0 \mathrm{E}-03$ & $9.5 \mathrm{E}-04$ & $4.4 \mathrm{E}-04$ & $1.5 \mathrm{E}-04$ & 5.5E-05 & $3.3 \mathrm{E}-05$ \\
\hline Massachusetts & MA & $2.2 \mathrm{E}-03$ & $9.9 \mathrm{E}-04$ & $4.7 \mathrm{E}-04$ & $1.5 \mathrm{E}-04$ & $5.7 \mathrm{E}-05$ & $2.9 \mathrm{E}-05$ \\
\hline New Hampshire & $\mathrm{NH}$ & $2.8 \mathrm{E}-03$ & $1.1 \mathrm{E}-03$ & $6.1 \mathrm{E}-04$ & $2.0 \mathrm{E}-04$ & $7.2 \mathrm{E}-05$ & $2.6 \mathrm{E}-05$ \\
\hline Vermont & VT & $2.4 \mathrm{E}-03$ & $1.0 \mathrm{E}-03$ & $5.6 \mathrm{E}-04$ & $2.1 \mathrm{E}-04$ & $7.1 \mathrm{E}-05$ & $2.7 \mathrm{E}-05$ \\
\hline Maine & $\mathrm{ME}$ & $3.6 \mathrm{E}-03$ & $1.9 \mathrm{E}-03$ & $8.3 \mathrm{E}-04$ & $3.3 \mathrm{E}-04$ & $1.6 \mathrm{E}-04$ & $5.1 \mathrm{E}-05$ \\
\hline Ohio & $\mathrm{OH}$ & $7.4 \mathrm{E}-04$ & $4.2 \mathrm{E}-04$ & $3.1 \mathrm{E}-04$ & $8.9 \mathrm{E}-05$ & $4.5 \mathrm{E}-05$ & $2.8 \mathrm{E}-05$ \\
\hline Indiana & IN & $7.0 \mathrm{E}-04$ & $3.8 \mathrm{E}-04$ & $2.4 \mathrm{E}-04$ & 7.5E-05 & $4.1 \mathrm{E}-05$ & $1.9 \mathrm{E}-05$ \\
\hline Illinois & $\mathrm{IL}$ & 7.5E-04 & $4.8 \mathrm{E}-04$ & $3.0 \mathrm{E}-04$ & $1.1 \mathrm{E}-04$ & $6.2 \mathrm{E}-05$ & $2.3 \mathrm{E}-05$ \\
\hline Michigan & MI & $7.9 \mathrm{E}-04$ & $4.3 \mathrm{E}-04$ & $2.3 \mathrm{E}-04$ & $7.5 \mathrm{E}-05$ & $2.8 \mathrm{E}-05$ & $1.7 \mathrm{E}-05$ \\
\hline Wisconsin & WI & $8.6 \mathrm{E}-04$ & $4.9 \mathrm{E}-04$ & $2.7 \mathrm{E}-04$ & $1.1 \mathrm{E}-04$ & $4.4 \mathrm{E}-05$ & $1.4 \mathrm{E}-05$ \\
\hline Minnesota & $\mathrm{MN}$ & $9.1 \mathrm{E}-04$ & $5.1 \mathrm{E}-04$ & $3.5 \mathrm{E}-04$ & $1.5 \mathrm{E}-04$ & $7.6 \mathrm{E}-05$ & $1.7 \mathrm{E}-05$ \\
\hline Iowa & IA & $1.1 \mathrm{E}-03$ & $6.2 \mathrm{E}-04$ & $3.4 \mathrm{E}-04$ & $1.4 \mathrm{E}-04$ & $7.1 \mathrm{E}-05$ & $2.1 \mathrm{E}-05$ \\
\hline Missouri & $\mathrm{MO}$ & $9.1 \mathrm{E}-04$ & $5.8 \mathrm{E}-04$ & $3.5 \mathrm{E}-04$ & $1.7 \mathrm{E}-04$ & $1.2 \mathrm{E}-04$ & $4.2 \mathrm{E}-05$ \\
\hline Kansas & KS & $9.3 \mathrm{E}-04$ & $9.3 \mathrm{E}-04$ & $5.5 \mathrm{E}-04$ & $3.1 \mathrm{E}-04$ & $2.4 \mathrm{E}-04$ & $7.9 \mathrm{E}-05$ \\
\hline Nebraska & $\mathrm{NE}$ & $1.0 \mathrm{E}-03$ & $8.3 \mathrm{E}-04$ & $4.9 \mathrm{E}-04$ & $2.6 \mathrm{E}-04$ & $1.7 \mathrm{E}-04$ & $6.9 \mathrm{E}-05$ \\
\hline North Dakota & ND & $1.1 \mathrm{E}-03$ & $7.7 \mathrm{E}-04$ & $4.8 \mathrm{E}-04$ & $2.2 \mathrm{E}-04$ & $1.4 \mathrm{E}-04$ & $5.4 \mathrm{E}-05$ \\
\hline South Dakota & SD & $1.3 \mathrm{E}-03$ & $8.1 \mathrm{E}-04$ & $3.9 \mathrm{E}-04$ & $1.9 \mathrm{E}-04$ & $1.5 \mathrm{E}-04$ & $3.3 \mathrm{E}-05$ \\
\hline
\end{tabular}


Table A9 Fate level characterization factors for VOC (January - June)

\begin{tabular}{|c|c|c|c|c|c|c|c|}
\hline State & & January & February & March & April & May & June \\
\hline Oregon & OR & $8.1 \mathrm{E}-06$ & $1.3 \mathrm{E}-05$ & $1.3 \mathrm{E}-05$ & $3.4 \mathrm{E}-05$ & $1.2 \mathrm{E}-05$ & $2.7 \mathrm{E}-05$ \\
\hline Washington & WA & $7.8 \mathrm{E}-06$ & $1.3 \mathrm{E}-05$ & $1.7 \mathrm{E}-05$ & $4.0 \mathrm{E}-05$ & $1.8 \mathrm{E}-05$ & 4.3E-05 \\
\hline Wyoming & $\overline{W Y}$ & $3.8 \mathrm{E}-05$ & $4.5 \mathrm{E}-05$ & $3.6 \mathrm{E}-05$ & $7.5 \mathrm{E}-06$ & 5.3E-06 & $1.7 \mathrm{E}-06$ \\
\hline Idaho & ID & $2.0 \mathrm{E}-05$ & $2.3 \mathrm{E}-05$ & $1.9 \mathrm{E}-05$ & $4.0 \mathrm{E}-05$ & $3.5 \mathrm{E}-06$ & $1.3 \mathrm{E}-05$ \\
\hline Montana & MT & $2.4 \mathrm{E}-05$ & $3.4 \mathrm{E}-05$ & $2.3 \mathrm{E}-05$ & $1.3 \mathrm{E}-05$ & $3.7 \mathrm{E}-06$ & $2.2 \mathrm{E}-06$ \\
\hline California & CA & $2.2 \mathrm{E}-05$ & $2.7 \mathrm{E}-05$ & $4.4 \mathrm{E}-05$ & $7.2 \mathrm{E}-05$ & $4.2 \mathrm{E}-05$ & $6.7 \mathrm{E}-05$ \\
\hline Nevada & NV & $2.4 \mathrm{E}-05$ & $2.5 \mathrm{E}-05$ & $1.6 \mathrm{E}-05$ & $1.7 \mathrm{E}-05$ & $5.5 \mathrm{E}-06$ & $8.9 \mathrm{E}-06$ \\
\hline Utah & UT & $3.8 \mathrm{E}-05$ & $3.6 \mathrm{E}-05$ & $3.2 \mathrm{E}-05$ & $1.8 \mathrm{E}-05$ & $1.5 \mathrm{E}-05$ & $7.3 \mathrm{E}-06$ \\
\hline Colorado & $\mathrm{CO}$ & $4.2 \mathrm{E}-05$ & $3.2 \mathrm{E}-05$ & $3.2 \mathrm{E}-05$ & $2.1 \mathrm{E}-05$ & $7.9 \mathrm{E}-06$ & $8.3 \mathrm{E}-06$ \\
\hline \begin{tabular}{|l|} 
Arizona \\
\end{tabular} & $\overline{A Z}$ & $3.9 \mathrm{E}-05$ & $3.4 \mathrm{E}-05$ & $2.0 \mathrm{E}-05$ & $2.2 \mathrm{E}-05$ & $1.3 \mathrm{E}-05$ & $1.4 \mathrm{E}-05$ \\
\hline New Mexico & NM & $4.3 \mathrm{E}-05$ & $2.7 \mathrm{E}-05$ & $2.0 \mathrm{E}-05$ & $8.3 \mathrm{E}-06$ & $4.0 \mathrm{E}-06$ & $3.3 \mathrm{E}-06$ \\
\hline Texas & TX & $3.9 \mathrm{E}-05$ & $2.8 \mathrm{E}-05$ & $3.6 \mathrm{E}-05$ & $2.9 \mathrm{E}-05$ & $1.8 \mathrm{E}-05$ & $2.7 \mathrm{E}-05$ \\
\hline Oklahoma & OK & $4.8 \mathrm{E}-05$ & $4.2 \mathrm{E}-05$ & $5.6 \mathrm{E}-05$ & $2.5 \mathrm{E}-05$ & $9.1 \mathrm{E}-06$ & $6.1 \mathrm{E}-06$ \\
\hline Arkansas & AR & $6.4 \mathrm{E}-05$ & $4.7 \mathrm{E}-05$ & $5.7 \mathrm{E}-05$ & $3.0 \mathrm{E}-05$ & $8.3 \mathrm{E}-06$ & $7.8 \mathrm{E}-06$ \\
\hline Louisiana & $\mathrm{LA}$ & $5.7 \mathrm{E}-05$ & $5.1 \mathrm{E}-05$ & $6.5 \mathrm{E}-05$ & $3.4 \mathrm{E}-05$ & $4.0 \mathrm{E}-05$ & $3.1 \mathrm{E}-05$ \\
\hline Mississippi & $\overline{\mathrm{MS}}$ & $6.7 \mathrm{E}-05$ & $5.3 \mathrm{E}-05$ & $6.0 \mathrm{E}-05$ & $3.9 \mathrm{E}-05$ & $1.2 \mathrm{E}-05$ & $1.8 \mathrm{E}-05$ \\
\hline Alabama & $\overline{\mathrm{AL}}$ & $6.6 \mathrm{E}-05$ & $4.6 \mathrm{E}-05$ & $5.8 \mathrm{E}-05$ & $4.4 \mathrm{E}-05$ & $1.0 \mathrm{E}-05$ & $1.2 \mathrm{E}-05$ \\
\hline Kentucky & $\mathrm{KY}$ & $5.0 \mathrm{E}-05$ & $5.0 \mathrm{E}-05$ & $7.9 \mathrm{E}-05$ & $3.3 \mathrm{E}-05$ & $3.9 \mathrm{E}-05$ & $2.0 \mathrm{E}-05$ \\
\hline Tennessee & $\mathrm{TN}$ & $5.9 \mathrm{E}-05$ & $4.8 \mathrm{E}-05$ & $6.7 \mathrm{E}-05$ & $4.5 \mathrm{E}-05$ & $1.5 \mathrm{E}-05$ & $1.0 \mathrm{E}-05$ \\
\hline Florida & FL & $5.3 \mathrm{E}-05$ & $5.7 \mathrm{E}-05$ & $5.7 \mathrm{E}-05$ & $1.1 \mathrm{E}-04$ & $4.1 \mathrm{E}-05$ & $1.3 \mathrm{E}-04$ \\
\hline Georgia & $\mathrm{GA}$ & $6.6 \mathrm{E}-05$ & $4.6 \mathrm{E}-05$ & $6.1 \mathrm{E}-05$ & $5.6 \mathrm{E}-05$ & $1.8 \mathrm{E}-05$ & $1.5 \mathrm{E}-05$ \\
\hline North Carolina & $\mathrm{NC}$ & $4.7 \mathrm{E}-05$ & $4.8 \mathrm{E}-05$ & $5.8 \mathrm{E}-05$ & $7.0 \mathrm{E}-05$ & $1.7 \mathrm{E}-05$ & $1.8 \mathrm{E}-05$ \\
\hline South Carolina & $\mathrm{SC}$ & $6.0 \mathrm{E}-05$ & $6.1 \mathrm{E}-05$ & $6.0 \mathrm{E}-05$ & $5.0 \mathrm{E}-05$ & $1.2 \mathrm{E}-05$ & $1.0 \mathrm{E}-05$ \\
\hline Virginia & $\mathrm{VA}$ & $4.1 \mathrm{E}-05$ & $4.6 \mathrm{E}-05$ & $6.4 \mathrm{E}-05$ & $7.1 \mathrm{E}-05$ & $3.5 \mathrm{E}-05$ & $5.1 \mathrm{E}-05$ \\
\hline West Virginia & $\mathrm{WV}$ & $4.8 \mathrm{E}-05$ & $4.9 \mathrm{E}-05$ & $7.8 \mathrm{E}-05$ & $2.3 \mathrm{E}-05$ & $3.6 \mathrm{E}-05$ & $1.4 \mathrm{E}-05$ \\
\hline Delaware & $\mathrm{DE}$ & $4.4 \mathrm{E}-05$ & $5.4 \mathrm{E}-05$ & $7.2 \mathrm{E}-05$ & $1.4 \mathrm{E}-04$ & $5.6 \mathrm{E}-05$ & $1.4 \mathrm{E}-04$ \\
\hline Washington DC & $\overline{\mathrm{DC}}$ & $3.9 \mathrm{E}-05$ & $4.3 \mathrm{E}-05$ & $6.6 \mathrm{E}-05$ & $1.3 \mathrm{E}-04$ & $6.9 \mathrm{E}-05$ & $2.0 \mathrm{E}-04$ \\
\hline Maryland & $\mathrm{MD}$ & $3.9 \mathrm{E}-05$ & $4.7 \mathrm{E}-05$ & $6.7 \mathrm{E}-05$ & $8.6 \mathrm{E}-05$ & $6.5 \mathrm{E}-05$ & $9.9 \mathrm{E}-05$ \\
\hline Connecticut & CT & $3.9 \mathrm{E}-05$ & $3.7 \mathrm{E}-05$ & $5.2 \mathrm{E}-05$ & $1.7 \mathrm{E}-04$ & $8.3 \mathrm{E}-05$ & $3.3 \mathrm{E}-04$ \\
\hline New Jersey & NJ & $3.4 \mathrm{E}-05$ & $3.5 \mathrm{E}-05$ & $4.9 \mathrm{E}-05$ & $1.3 \mathrm{E}-04$ & $7.2 \mathrm{E}-05$ & $2.9 \mathrm{E}-04$ \\
\hline New York & $\mathrm{NY}$ & $3.0 \mathrm{E}-05$ & $3.5 \mathrm{E}-05$ & $5.5 \mathrm{E}-05$ & $1.1 \mathrm{E}-04$ & $4.8 \mathrm{E}-05$ & $1.4 \mathrm{E}-04$ \\
\hline Pennsylvania & $\mathrm{PA}$ & $3.2 \mathrm{E}-05$ & $3.9 \mathrm{E}-05$ & $5.8 \mathrm{E}-05$ & $7.2 \mathrm{E}-05$ & $4.9 \mathrm{E}-05$ & 8.7E-05 \\
\hline Rhode Island & RI & $3.8 \mathrm{E}-05$ & $3.5 \mathrm{E}-05$ & $5.2 \mathrm{E}-05$ & $1.6 \mathrm{E}-04$ & $9.1 \mathrm{E}-05$ & $3.9 \mathrm{E}-04$ \\
\hline Massachusetts & MA & $3.6 \mathrm{E}-05$ & $3.5 \mathrm{E}-05$ & $5.3 \mathrm{E}-05$ & $1.3 \mathrm{E}-04$ & $8.6 \mathrm{E}-05$ & $4.5 \mathrm{E}-04$ \\
\hline New Hampshire & $\mathrm{NH}$ & $4.3 \mathrm{E}-05$ & $4.8 \mathrm{E}-05$ & $7.2 \mathrm{E}-05$ & $1.5 \mathrm{E}-04$ & $5.2 \mathrm{E}-05$ & $1.9 \mathrm{E}-04$ \\
\hline Vermont & VT & $4.6 \mathrm{E}-05$ & $5.1 \mathrm{E}-05$ & $8.6 \mathrm{E}-05$ & $1.7 \mathrm{E}-04$ & $2.1 \mathrm{E}-05$ & $4.0 \mathrm{E}-05$ \\
\hline Maine & $\mathrm{ME}$ & $5.8 \mathrm{E}-05$ & $6.3 \mathrm{E}-05$ & $8.1 \mathrm{E}-05$ & $1.6 \mathrm{E}-04$ & $2.4 \mathrm{E}-05$ & $2.0 \mathrm{E}-05$ \\
\hline Ohio & $\overline{\mathrm{OH}}$ & $4.0 \mathrm{E}-05$ & $4.4 \mathrm{E}-05$ & $7.0 \mathrm{E}-05$ & 5.6E-05 & $5.0 \mathrm{E}-05$ & $7.7 \mathrm{E}-05$ \\
\hline Indiana & IN & $4.7 \mathrm{E}-05$ & $5.4 \mathrm{E}-05$ & 7.3E-05 & $6.4 \mathrm{E}-05$ & $4.2 \mathrm{E}-05$ & $6.1 \mathrm{E}-05$ \\
\hline Illinois & IL & $4.2 \mathrm{E}-05$ & $5.5 \mathrm{E}-05$ & $7.1 \mathrm{E}-05$ & $5.1 \mathrm{E}-05$ & $3.9 \mathrm{E}-05$ & $5.6 \mathrm{E}-05$ \\
\hline Michigan & MI & $3.5 \mathrm{E}-05$ & $4.1 \mathrm{E}-05$ & $5.8 \mathrm{E}-05$ & $7.7 \mathrm{E}-05$ & $4.2 \mathrm{E}-05$ & $9.3 \mathrm{E}-05$ \\
\hline Wisconsin & WI & $3.7 \mathrm{E}-05$ & 5.3E-05 & $6.9 \mathrm{E}-05$ & $7.2 \mathrm{E}-05$ & $3.2 \mathrm{E}-05$ & $4.4 \mathrm{E}-05$ \\
\hline Minnesota & $\mathrm{MN}$ & $3.4 \mathrm{E}-05$ & 5.7E-05 & $7.4 \mathrm{E}-05$ & $6.8 \mathrm{E}-05$ & $2.4 \mathrm{E}-05$ & $2.0 \mathrm{E}-05$ \\
\hline Iowa & IA & $4.4 \mathrm{E}-05$ & $6.0 \mathrm{E}-05$ & $8.0 \mathrm{E}-05$ & 5.3E-05 & $2.1 \mathrm{E}-05$ & $1.4 \mathrm{E}-05$ \\
\hline Missouri & $\mathrm{MO}$ & $5.3 \mathrm{E}-05$ & $5.5 \mathrm{E}-05$ & $7.9 \mathrm{E}-05$ & $4.6 \mathrm{E}-05$ & $2.2 \mathrm{E}-05$ & $1.8 \mathrm{E}-05$ \\
\hline Kansas & KS & 5.3E-05 & $6.3 \mathrm{E}-05$ & $6.7 \mathrm{E}-05$ & $2.4 \mathrm{E}-05$ & $1.2 \mathrm{E}-05$ & $5.6 \mathrm{E}-06$ \\
\hline Nebraska & $\mathrm{NE}$ & $4.4 \mathrm{E}-05$ & $6.0 \mathrm{E}-05$ & $6.4 \mathrm{E}-05$ & $2.8 \mathrm{E}-05$ & $1.5 \mathrm{E}-05$ & 9.3E-06 \\
\hline North Dakota & ND & $2.8 \mathrm{E}-05$ & $6.0 \mathrm{E}-05$ & 5.7E-05 & $3.9 \mathrm{E}-05$ & $9.5 \mathrm{E}-06$ & $4.5 \mathrm{E}-06$ \\
\hline South Dakota & $\mathrm{SD}$ & $3.9 \mathrm{E}-05$ & $6.2 \mathrm{E}-05$ & $6.8 \mathrm{E}-05$ & $4.1 \mathrm{E}-05$ & $1.0 \mathrm{E}-05$ & 6.1E-06 \\
\hline
\end{tabular}


Table A10 Fate level characterization factors for VOC (July-December)

\begin{tabular}{|c|c|c|c|c|c|c|c|}
\hline State & & July & August & September & October & November & December \\
\hline Oregon & OR & 3.7E-05 & $1.0 \mathrm{E}-05$ & $1.4 \mathrm{E}-05$ & $1.2 \mathrm{E}-05$ & 7.5E-06 & $5.6 \mathrm{E}-06$ \\
\hline Washington & WA & $5.6 \mathrm{E}-05$ & 2.3E-05 & $2.1 \mathrm{E}-05$ & $1.4 \mathrm{E}-05$ & 7.2E-06 & $6.2 \mathrm{E}-06$ \\
\hline Wyoming & WY & $2.0 \mathrm{E}-06$ & 4.3E-06 & 8.7E-06 & $1.7 \mathrm{E}-05$ & $2.8 \mathrm{E}-05$ & $3.7 \mathrm{E}-05$ \\
\hline Idaho & ID & $6.6 \mathrm{E}-06$ & $2.1 \mathrm{E}-06$ & $4.9 \mathrm{E}-06$ & $1.1 \mathrm{E}-05$ & $1.6 \mathrm{E}-05$ & $1.9 \mathrm{E}-05$ \\
\hline Montana & MT & $1.3 \mathrm{E}-06$ & $2.7 \mathrm{E}-06$ & $6.0 \mathrm{E}-06$ & $1.6 \mathrm{E}-05$ & $1.8 \mathrm{E}-05$ & $2.3 \mathrm{E}-05$ \\
\hline California & CA & $6.6 \mathrm{E}-05$ & $4.5 \mathrm{E}-05$ & $4.4 \mathrm{E}-05$ & $6.3 \mathrm{E}-05$ & $3.2 \mathrm{E}-05$ & $2.9 \mathrm{E}-05$ \\
\hline Nevada & $\mathrm{NV}$ & $8.2 \mathrm{E}-06$ & $3.8 \mathrm{E}-06$ & $5.9 \mathrm{E}-06$ & $2.1 \mathrm{E}-05$ & $1.6 \mathrm{E}-05$ & $3.4 \mathrm{E}-05$ \\
\hline Utah & UT & $1.1 \mathrm{E}-05$ & $8.6 \mathrm{E}-06$ & $1.4 \mathrm{E}-05$ & $2.0 \mathrm{E}-05$ & $2.4 \mathrm{E}-05$ & $3.8 \mathrm{E}-05$ \\
\hline Colorado & $\mathrm{CO}$ & $1.4 \mathrm{E}-05$ & $1.0 \mathrm{E}-05$ & $1.3 \mathrm{E}-05$ & $1.6 \mathrm{E}-05$ & $3.2 \mathrm{E}-05$ & 4.2E-05 \\
\hline Arizona & $\overline{\mathrm{AZ}}$ & $1.7 \mathrm{E}-05$ & $1.6 \mathrm{E}-05$ & $1.1 \mathrm{E}-05$ & $2.2 \mathrm{E}-05$ & $2.3 \mathrm{E}-05$ & $4.6 \mathrm{E}-05$ \\
\hline New Mexico & $\mathrm{NM}$ & $4.1 \mathrm{E}-06$ & 7.7E-06 & 7.9E-06 & $1.3 \mathrm{E}-05$ & $2.1 \mathrm{E}-05$ & 4.7E-05 \\
\hline Texas & TX & $3.2 \mathrm{E}-05$ & $2.3 \mathrm{E}-05$ & $1.8 \mathrm{E}-05$ & 3.3E-05 & $2.5 \mathrm{E}-05$ & $3.4 \mathrm{E}-05$ \\
\hline Oklahoma & $\mathrm{OK}$ & $6.0 \mathrm{E}-06$ & $1.0 \mathrm{E}-05$ & $1.8 \mathrm{E}-05$ & $3.5 \mathrm{E}-05$ & $3.8 \mathrm{E}-05$ & $4.9 \mathrm{E}-05$ \\
\hline Arkansas & AR & $7.0 \mathrm{E}-06$ & $1.1 \mathrm{E}-05$ & $2.0 \mathrm{E}-05$ & $3.4 \mathrm{E}-05$ & $4.8 \mathrm{E}-05$ & 5.1E-05 \\
\hline Louisiana & LA & $5.8 \mathrm{E}-05$ & $8.4 \mathrm{E}-05$ & $3.2 \mathrm{E}-05$ & $5.5 \mathrm{E}-05$ & $5.2 \mathrm{E}-05$ & 4.3E-05 \\
\hline Mississippi & MS & $1.7 \mathrm{E}-05$ & $1.6 \mathrm{E}-05$ & $2.1 \mathrm{E}-05$ & $3.6 \mathrm{E}-05$ & 4.4E-05 & $4.8 \mathrm{E}-05$ \\
\hline Alabama & $\mathrm{AL}$ & $1.2 \mathrm{E}-05$ & $1.1 \mathrm{E}-05$ & $2.1 \mathrm{E}-05$ & $4.3 \mathrm{E}-05$ & $4.8 \mathrm{E}-05$ & $4.2 \mathrm{E}-05$ \\
\hline Kentucky & KY & 2.3E-05 & $5.0 \mathrm{E}-05$ & $6.6 \mathrm{E}-05$ & 5.3E-05 & $5.8 \mathrm{E}-05$ & 3.7E-05 \\
\hline Tennessee & $\overline{\mathrm{TN}}$ & $1.2 \mathrm{E}-05$ & $1.8 \mathrm{E}-05$ & $3.2 \mathrm{E}-05$ & $5.0 \mathrm{E}-05$ & $6.8 \mathrm{E}-05$ & $3.8 \mathrm{E}-05$ \\
\hline Florida & FL & 1.7E-04 & 8.7E-05 & 8.8E-05 & $6.1 \mathrm{E}-05$ & $3.4 \mathrm{E}-05$ & 4.7E-05 \\
\hline Georgia & GA & $1.8 \mathrm{E}-05$ & $2.0 \mathrm{E}-05$ & $2.9 \mathrm{E}-05$ & $5.8 \mathrm{E}-05$ & $5.2 \mathrm{E}-05$ & $4.4 \mathrm{E}-05$ \\
\hline North Carolina & $\mathrm{NC}$ & $2.7 \mathrm{E}-05$ & $2.4 \mathrm{E}-05$ & $3.2 \mathrm{E}-05$ & 7.3E-05 & $5.2 \mathrm{E}-05$ & $3.8 \mathrm{E}-05$ \\
\hline South Carolina & SC & $1.5 \mathrm{E}-05$ & $1.7 \mathrm{E}-05$ & $2.8 \mathrm{E}-05$ & $6.7 \mathrm{E}-05$ & 5.1E-05 & $4.8 \mathrm{E}-05$ \\
\hline Virginia & VA & $4.9 \mathrm{E}-05$ & $5.2 \mathrm{E}-05$ & $5.1 \mathrm{E}-05$ & 7.4E-05 & $5.0 \mathrm{E}-05$ & $3.1 \mathrm{E}-05$ \\
\hline West Virginia & WV & $1.6 \mathrm{E}-05$ & $6.2 \mathrm{E}-05$ & $6.8 \mathrm{E}-05$ & $6.1 \mathrm{E}-05$ & $4.9 \mathrm{E}-05$ & $3.5 \mathrm{E}-05$ \\
\hline Delaware & $\mathrm{DE}$ & $9.1 \mathrm{E}-05$ & $8.4 \mathrm{E}-05$ & $7.5 \mathrm{E}-05$ & $1.0 \mathrm{E}-04$ & $4.3 \mathrm{E}-05$ & $3.3 \mathrm{E}-05$ \\
\hline Washington DC & $\mathrm{DC}$ & $1.4 \mathrm{E}-04$ & $1.1 \mathrm{E}-04$ & 7.8E-05 & 7.0E-05 & $3.9 \mathrm{E}-05$ & $2.8 \mathrm{E}-05$ \\
\hline Maryland & MD & $7.2 \mathrm{E}-05$ & $9.6 \mathrm{E}-05$ & $7.6 \mathrm{E}-05$ & $7.8 \mathrm{E}-05$ & $3.9 \mathrm{E}-05$ & $2.9 \mathrm{E}-05$ \\
\hline Connecticut & CT & $2.0 \mathrm{E}-04$ & $1.2 \mathrm{E}-04$ & $1.0 \mathrm{E}-04$ & $6.4 \mathrm{E}-05$ & $2.5 \mathrm{E}-05$ & $2.2 \mathrm{E}-05$ \\
\hline New Jersey & $\mathrm{NJ}$ & $2.0 \mathrm{E}-04$ & $1.2 \mathrm{E}-04$ & $9.0 \mathrm{E}-05$ & $5.8 \mathrm{E}-05$ & $2.6 \mathrm{E}-05$ & $2.2 \mathrm{E}-05$ \\
\hline New York & $\mathrm{NY}$ & $1.1 \mathrm{E}-04$ & $7.4 \mathrm{E}-05$ & $6.1 \mathrm{E}-05$ & $3.9 \mathrm{E}-05$ & $2.5 \mathrm{E}-05$ & $2.1 \mathrm{E}-05$ \\
\hline Pennsylvania & $\mathrm{PA}$ & $6.8 \mathrm{E}-05$ & $8.2 \mathrm{E}-05$ & $6.5 \mathrm{E}-05$ & $4.7 \mathrm{E}-05$ & $3.2 \mathrm{E}-05$ & $2.2 \mathrm{E}-05$ \\
\hline Rhode Island & RI & $2.7 \mathrm{E}-04$ & $1.3 \mathrm{E}-04$ & $1.1 \mathrm{E}-04$ & $5.4 \mathrm{E}-05$ & $2.3 \mathrm{E}-05$ & $1.9 \mathrm{E}-05$ \\
\hline Massachusetts & MA & $3.5 \mathrm{E}-04$ & $1.4 \mathrm{E}-04$ & $9.8 \mathrm{E}-05$ & $5.1 \mathrm{E}-05$ & $2.2 \mathrm{E}-05$ & $1.8 \mathrm{E}-05$ \\
\hline New Hampshire & $\mathrm{NH}$ & $1.9 \mathrm{E}-04$ & $7.8 \mathrm{E}-05$ & $6.0 \mathrm{E}-05$ & 4.6E-05 & $2.6 \mathrm{E}-05$ & $2.7 \mathrm{E}-05$ \\
\hline Vermont & VT & 7.7E-05 & $3.2 \mathrm{E}-05$ & 3.3E-05 & 2.7E-05 & 2.6E-05 & $3.3 \mathrm{E}-05$ \\
\hline Maine & $\mathrm{ME}$ & $3.2 \mathrm{E}-05$ & $2.7 \mathrm{E}-05$ & $1.9 \mathrm{E}-05$ & $2.4 \mathrm{E}-05$ & $2.4 \mathrm{E}-05$ & $2.9 \mathrm{E}-05$ \\
\hline Ohio & $\mathrm{OH}$ & $6.5 \mathrm{E}-05$ & 9.6E-05 & 8.4E-05 & 4.5E-05 & $3.5 \mathrm{E}-05$ & $3.1 \mathrm{E}-05$ \\
\hline Indiana & IN & $5.7 \mathrm{E}-05$ & $6.9 \mathrm{E}-05$ & $6.9 \mathrm{E}-05$ & 4.9E-05 & $3.8 \mathrm{E}-05$ & $3.6 \mathrm{E}-05$ \\
\hline Illinois & $\mathrm{IL}$ & $5.2 \mathrm{E}-05$ & $6.2 \mathrm{E}-05$ & $5.8 \mathrm{E}-05$ & $4.8 \mathrm{E}-05$ & $3.4 \mathrm{E}-05$ & $3.2 \mathrm{E}-05$ \\
\hline Michigan & MI & $8.2 \mathrm{E}-05$ & 7.5E-05 & $6.4 \mathrm{E}-05$ & $3.6 \mathrm{E}-05$ & $2.6 \mathrm{E}-05$ & $3.0 \mathrm{E}-05$ \\
\hline Wisconsin & WI & $3.7 \mathrm{E}-05$ & $4.1 \mathrm{E}-05$ & $4.9 \mathrm{E}-05$ & $4.3 \mathrm{E}-05$ & $2.8 \mathrm{E}-05$ & $3.2 \mathrm{E}-05$ \\
\hline Minnesota & $\mathrm{MN}$ & $1.9 \mathrm{E}-05$ & $2.7 \mathrm{E}-05$ & $3.6 \mathrm{E}-05$ & $3.4 \mathrm{E}-05$ & $3.0 \mathrm{E}-05$ & $2.8 \mathrm{E}-05$ \\
\hline Iowa & IA & $1.7 \mathrm{E}-05$ & 2.7E-05 & $3.5 \mathrm{E}-05$ & 4.2E-05 & $3.2 \mathrm{E}-05$ & $3.6 \mathrm{E}-05$ \\
\hline Missouri & $\mathrm{MO}$ & $1.8 \mathrm{E}-05$ & 2.7E-05 & 3.7E-05 & $4.7 \mathrm{E}-05$ & $4.4 \mathrm{E}-05$ & 4.7E-05 \\
\hline Kansas & KS & $3.8 \mathrm{E}-06$ & $1.4 \mathrm{E}-05$ & $2.5 \mathrm{E}-05$ & $3.9 \mathrm{E}-05$ & 4.2E-05 & $5.2 \mathrm{E}-05$ \\
\hline Nebraska & $\mathrm{NE}$ & $8.8 \mathrm{E}-06$ & $1.9 \mathrm{E}-05$ & $2.7 \mathrm{E}-05$ & $3.7 \mathrm{E}-05$ & $3.4 \mathrm{E}-05$ & 4.0E-05 \\
\hline North Dakota & ND & $4.3 \mathrm{E}-06$ & $1.0 \mathrm{E}-05$ & $1.4 \mathrm{E}-05$ & $2.8 \mathrm{E}-05$ & $2.6 \mathrm{E}-05$ & $2.7 \mathrm{E}-05$ \\
\hline South Dakota & SD & $3.9 \mathrm{E}-06$ & $1.0 \mathrm{E}-05$ & $1.5 \mathrm{E}-05$ & $2.9 \mathrm{E}-05$ & $3.2 \mathrm{E}-05$ & $3.6 \mathrm{E}-05$ \\
\hline
\end{tabular}


Table A11 Human exposure level characterization factors for $\mathrm{NO}_{\mathrm{x}}$ (January-June)

\begin{tabular}{|c|c|c|c|c|c|c|c|}
\hline State & & January & February & March & April & May & June \\
\hline Oregon & $\overline{O R}$ & $6.4 \mathrm{E}+00$ & $1.2 \mathrm{E}+01$ & $2.3 \mathrm{E}+01$ & $3.4 \mathrm{E}+01$ & $5.7 \mathrm{E}+01$ & $6.3 \mathrm{E}+01$ \\
\hline Washington & WA & $3.5 \mathrm{E}+00$ & $6.4 \mathrm{E}+00$ & $1.4 \mathrm{E}+01$ & $2.6 \mathrm{E}+01$ & $3.9 \mathrm{E}+01$ & $4.1 \mathrm{E}+01$ \\
\hline Wyoming & $\mathrm{WY}$ & $1.3 \mathrm{E}+01$ & $1.4 \mathrm{E}+01$ & $1.9 \mathrm{E}+01$ & $2.3 \mathrm{E}+01$ & $2.3 \mathrm{E}+01$ & $3.4 \mathrm{E}+01$ \\
\hline Idaho & ID & $1.2 \mathrm{E}+01$ & $1.4 \mathrm{E}+01$ & $2.0 \mathrm{E}+01$ & $2.5 \mathrm{E}+01$ & $2.6 \mathrm{E}+01$ & $3.5 \mathrm{E}+01$ \\
\hline Montana & MT & $1.2 \mathrm{E}+01$ & $1.4 \mathrm{E}+01$ & $2.1 \mathrm{E}+01$ & $2.8 \mathrm{E}+01$ & $2.4 \mathrm{E}+01$ & $3.2 \mathrm{E}+01$ \\
\hline California & CA & $1.0 \mathrm{E}+01$ & $1.4 \mathrm{E}+01$ & $3.4 \mathrm{E}+01$ & $4.4 \mathrm{E}+01$ & $1.0 \mathrm{E}+02$ & $1.0 \mathrm{E}+02$ \\
\hline Nevada & $\mathrm{NV}$ & $1.9 \mathrm{E}+01$ & $1.7 \mathrm{E}+01$ & $3.0 \mathrm{E}+01$ & $3.3 \mathrm{E}+01$ & $4.4 \mathrm{E}+01$ & $5.3 \mathrm{E}+01$ \\
\hline Utah & UT & $1.3 \mathrm{E}+01$ & $1.3 \mathrm{E}+01$ & $1.9 \mathrm{E}+01$ & $2.2 \mathrm{E}+01$ & $2.6 \mathrm{E}+01$ & $3.5 \mathrm{E}+01$ \\
\hline Colorado & $\mathrm{CO}$ & $1.2 \mathrm{E}+01$ & $1.2 \mathrm{E}+01$ & $1.8 \mathrm{E}+01$ & $2.3 \mathrm{E}+01$ & $2.9 \mathrm{E}+01$ & $4.3 \mathrm{E}+01$ \\
\hline Arizona & $\overline{A Z}$ & $1.6 \mathrm{E}+01$ & $1.7 \mathrm{E}+01$ & $2.5 \mathrm{E}+01$ & $3.0 \mathrm{E}+01$ & $5.0 \mathrm{E}+01$ & $5.4 \mathrm{E}+01$ \\
\hline New Mexico & NM & $1.4 \mathrm{E}+01$ & $1.3 \mathrm{E}+01$ & $1.9 \mathrm{E}+01$ & $2.0 \mathrm{E}+01$ & $2.7 \mathrm{E}+01$ & $3.0 \mathrm{E}+01$ \\
\hline Texas & TX & $9.5 \mathrm{E}+00$ & $1.1 \mathrm{E}+01$ & $2.0 \mathrm{E}+01$ & $3.4 \mathrm{E}+01$ & $5.8 \mathrm{E}+01$ & $7.5 \mathrm{E}+01$ \\
\hline Oklahoma & OK & $1.0 \mathrm{E}+01$ & $1.0 \mathrm{E}+01$ & $2.4 \mathrm{E}+01$ & $3.6 \mathrm{E}+01$ & $6.0 \mathrm{E}+01$ & $7.5 \mathrm{E}+01$ \\
\hline Arkansas & $\mathrm{AR}$ & $1.0 \mathrm{E}+01$ & $1.1 \mathrm{E}+01$ & $2.9 \mathrm{E}+01$ & $5.3 \mathrm{E}+01$ & $8.0 \mathrm{E}+01$ & $1.1 \mathrm{E}+02$ \\
\hline Louisiana & $\overline{L A}$ & $6.3 \mathrm{E}+00$ & $1.1 \mathrm{E}+01$ & $1.9 \mathrm{E}+01$ & $3.9 \mathrm{E}+01$ & $5.9 \mathrm{E}+01$ & $8.1 \mathrm{E}+01$ \\
\hline Mississippi & MS & $8.7 \mathrm{E}+00$ & $1.5 \mathrm{E}+01$ & $2.6 \mathrm{E}+01$ & $5.4 \mathrm{E}+01$ & $8.2 \mathrm{E}+01$ & $1.1 \mathrm{E}+02$ \\
\hline Alabama & $\mathrm{AL}$ & $7.2 \mathrm{E}+00$ & $1.6 \mathrm{E}+01$ & $2.2 \mathrm{E}+01$ & $5.9 \mathrm{E}+01$ & $8.8 \mathrm{E}+01$ & $1.1 \mathrm{E}+02$ \\
\hline Kentucky & KY & $3.3 \mathrm{E}+00$ & $4.8 \mathrm{E}+00$ & $9.6 \mathrm{E}+00$ & $3.4 \mathrm{E}+01$ & $6.3 \mathrm{E}+01$ & $1.0 \mathrm{E}+02$ \\
\hline Tennessee & $\mathrm{TN}$ & $5.4 \mathrm{E}+00$ & $8.2 \mathrm{E}+00$ & $1.5 \mathrm{E}+01$ & $4.8 \mathrm{E}+01$ & $7.8 \mathrm{E}+01$ & $1.2 \mathrm{E}+02$ \\
\hline Florida & FL & $1.1 \mathrm{E}+01$ & $1.9 \mathrm{E}+01$ & $2.6 \mathrm{E}+01$ & $4.7 \mathrm{E}+01$ & $6.9 \mathrm{E}+01$ & $9.2 \mathrm{E}+01$ \\
\hline Georgia & GA & $5.5 \mathrm{E}+00$ & $1.6 \mathrm{E}+01$ & $1.7 \mathrm{E}+01$ & $5.0 \mathrm{E}+01$ & $8.6 \mathrm{E}+01$ & $1.1 \mathrm{E}+02$ \\
\hline North Carolina & $\mathrm{NC}$ & $2.9 \mathrm{E}+00$ & $9.9 \mathrm{E}+00$ & $9.6 \mathrm{E}+00$ & $4.5 \mathrm{E}+01$ & $8.7 \mathrm{E}+01$ & $1.5 \mathrm{E}+02$ \\
\hline South Carolina & $\mathrm{SC}$ & $4.8 \mathrm{E}+00$ & $1.8 \mathrm{E}+01$ & $1.5 \mathrm{E}+01$ & $4.9 \mathrm{E}+01$ & $9.3 \mathrm{E}+01$ & $1.3 \mathrm{E}+02$ \\
\hline Virginia & VA & $1.4 \mathrm{E}+00$ & $4.9 \mathrm{E}+00$ & $7.1 \mathrm{E}+00$ & $4.1 \mathrm{E}+01$ & $7.7 \mathrm{E}+01$ & $1.5 \mathrm{E}+02$ \\
\hline West Virginia & WV & $1.5 \mathrm{E}+00$ & $3.1 \mathrm{E}+00$ & $7.1 \mathrm{E}+00$ & $2.7 \mathrm{E}+01$ & $5.2 \mathrm{E}+01$ & $8.7 \mathrm{E}+01$ \\
\hline Delaware & $\mathrm{DE}$ & $4.8 \mathrm{E}-01$ & $3.0 \mathrm{E}+00$ & $6.8 \mathrm{E}+00$ & $5.1 \mathrm{E}+01$ & $1.1 \mathrm{E}+02$ & $2.5 \mathrm{E}+02$ \\
\hline Washington DC & $\overline{\mathrm{DC}}$ & $6.6 \mathrm{E}-01$ & $2.0 \mathrm{E}+00$ & $4.1 \mathrm{E}+00$ & $2.3 \mathrm{E}+01$ & $5.2 \mathrm{E}+01$ & $1.6 \mathrm{E}+02$ \\
\hline Maryland & $\mathrm{MD}$ & $6.0 \mathrm{E}-01$ & $2.3 \mathrm{E}+00$ & $4.8 \mathrm{E}+00$ & $2.8 \mathrm{E}+01$ & $6.2 \mathrm{E}+01$ & $1.6 \mathrm{E}+02$ \\
\hline Connecticut & CT & $3.1 \mathrm{E}-01$ & $7.1 \mathrm{E}-01$ & $4.8 \mathrm{E}+00$ & $1.7 \mathrm{E}+01$ & $3.3 \mathrm{E}+01$ & $1.4 \mathrm{E}+02$ \\
\hline New Jersey & NJ & $3.8 \mathrm{E}-01$ & $9.9 \mathrm{E}-01$ & $3.8 \mathrm{E}+00$ & $1.8 \mathrm{E}+01$ & $3.8 \mathrm{E}+01$ & $1.3 \mathrm{E}+02$ \\
\hline New York & NY & $7.7 \mathrm{E}-01$ & $1.6 \mathrm{E}+00$ & $6.8 \mathrm{E}+00$ & $2.2 \mathrm{E}+01$ & $4.4 \mathrm{E}+01$ & $1.2 \mathrm{E}+02$ \\
\hline Pennsylvania & $\mathrm{PA}$ & 7.3E-01 & $1.9 \mathrm{E}+00$ & $5.1 \mathrm{E}+00$ & $2.3 \mathrm{E}+01$ & $4.8 \mathrm{E}+01$ & $1.3 \mathrm{E}+02$ \\
\hline Rhode Island & $\mathrm{RI}$ & $1.9 \mathrm{E}-01$ & $5.8 \mathrm{E}-01$ & $6.2 \mathrm{E}+00$ & $1.5 \mathrm{E}+01$ & $3.5 \mathrm{E}+01$ & $1.1 \mathrm{E}+02$ \\
\hline Massachusetts & MA & $2.0 \mathrm{E}-01$ & $5.4 \mathrm{E}-01$ & $6.0 \mathrm{E}+00$ & $1.4 \mathrm{E}+01$ & $3.4 \mathrm{E}+01$ & $8.0 \mathrm{E}+01$ \\
\hline New Hampshire & $\mathrm{NH}$ & $3.4 \mathrm{E}-01$ & $8.4 \mathrm{E}-01$ & $9.1 \mathrm{E}+00$ & $2.1 \mathrm{E}+01$ & $3.8 \mathrm{E}+01$ & $7.9 \mathrm{E}+01$ \\
\hline Vermont & VT & $7.8 \mathrm{E}-01$ & $2.0 \mathrm{E}+00$ & $1.3 \mathrm{E}+01$ & $3.2 \mathrm{E}+01$ & $4.9 \mathrm{E}+01$ & $6.8 \mathrm{E}+01$ \\
\hline Maine & $\mathrm{ME}$ & $4.9 \mathrm{E}-01$ & 5.2E-01 & $1.3 \mathrm{E}+01$ & $2.7 \mathrm{E}+01$ & $3.5 \mathrm{E}+01$ & $3.5 \mathrm{E}+01$ \\
\hline Ohio & $\mathrm{OH}$ & $1.5 \mathrm{E}+00$ & $2.7 \mathrm{E}+00$ & $6.0 \mathrm{E}+00$ & $2.2 \mathrm{E}+01$ & $5.1 \mathrm{E}+01$ & $9.8 \mathrm{E}+01$ \\
\hline Indiana & IN & $2.6 \mathrm{E}+00$ & $3.9 \mathrm{E}+00$ & $7.7 \mathrm{E}+00$ & $2.5 \mathrm{E}+01$ & $5.6 \mathrm{E}+01$ & $9.0 \mathrm{E}+01$ \\
\hline Illinois & $\mathrm{IL}$ & $3.7 \mathrm{E}+00$ & $5.0 \mathrm{E}+00$ & $1.0 \mathrm{E}+01$ & $2.9 \mathrm{E}+01$ & $6.0 \mathrm{E}+01$ & $9.2 \mathrm{E}+01$ \\
\hline Michigan & MI & $3.1 \mathrm{E}+00$ & $4.4 \mathrm{E}+00$ & $1.2 \mathrm{E}+01$ & $2.7 \mathrm{E}+01$ & $4.9 \mathrm{E}+01$ & $9.3 \mathrm{E}+01$ \\
\hline Wisconsin & WI & $4.6 \mathrm{E}+00$ & $6.3 \mathrm{E}+00$ & $1.4 \mathrm{E}+01$ & $3.1 \mathrm{E}+01$ & $5.6 \mathrm{E}+01$ & $8.8 \mathrm{E}+01$ \\
\hline Minnesota & MN & $7.8 \mathrm{E}+00$ & $9.6 \mathrm{E}+00$ & $1.9 \mathrm{E}+01$ & $3.6 \mathrm{E}+01$ & $4.8 \mathrm{E}+01$ & $6.3 \mathrm{E}+01$ \\
\hline Iowa & IA & $7.3 \mathrm{E}+00$ & $8.5 \mathrm{E}+00$ & $1.6 \mathrm{E}+01$ & $4.1 \mathrm{E}+01$ & $6.7 \mathrm{E}+01$ & $9.0 \mathrm{E}+01$ \\
\hline Missouri & $\mathrm{MO}$ & $6.7 \mathrm{E}+00$ & $7.8 \mathrm{E}+00$ & $1.9 \mathrm{E}+01$ & $4.0 \mathrm{E}+01$ & $6.7 \mathrm{E}+01$ & $8.6 \mathrm{E}+01$ \\
\hline Kansas & KS & $1.1 \mathrm{E}+01$ & $1.1 \mathrm{E}+01$ & $2.2 \mathrm{E}+01$ & $3.4 \mathrm{E}+01$ & $5.1 \mathrm{E}+01$ & $5.8 \mathrm{E}+01$ \\
\hline Nebraska & $\mathrm{NE}$ & $1.3 \mathrm{E}+01$ & $1.3 \mathrm{E}+01$ & $2.0 \mathrm{E}+01$ & $3.5 \mathrm{E}+01$ & $4.8 \mathrm{E}+01$ & $5.3 \mathrm{E}+01$ \\
\hline North Dakota & ND & $1.2 \mathrm{E}+01$ & $1.3 \mathrm{E}+01$ & $2.1 \mathrm{E}+01$ & $3.5 \mathrm{E}+01$ & $3.6 \mathrm{E}+01$ & $3.7 \mathrm{E}+01$ \\
\hline South Dakota & SD & $1.4 \mathrm{E}+01$ & $1.5 \mathrm{E}+01$ & $2.6 \mathrm{E}+01$ & $4.0 \mathrm{E}+01$ & $5.2 \mathrm{E}+01$ & $6.1 \mathrm{E}+01$ \\
\hline
\end{tabular}


Table A12 Human exposure level characterization factors for $\mathrm{NO}_{\mathrm{x}}$ (July-December)

\begin{tabular}{|c|c|c|c|c|c|c|c|}
\hline State & & July & August & September & October & November & December \\
\hline Oregon & OR & $8.0 \mathrm{E}+01$ & $6.6 \mathrm{E}+01$ & $6.6 \mathrm{E}+01$ & $2.5 \mathrm{E}+01$ & $1.1 \mathrm{E}+01$ & $5.0 \mathrm{E}+00$ \\
\hline Washington & WA & $4.8 \mathrm{E}+01$ & $5.7 \mathrm{E}+01$ & $5.3 \mathrm{E}+01$ & $1.6 \mathrm{E}+01$ & $5.3 \mathrm{E}+00$ & $3.0 \mathrm{E}+00$ \\
\hline Wyoming & WY & $1.9 \mathrm{E}+01$ & $2.5 \mathrm{E}+01$ & $2.8 \mathrm{E}+01$ & $1.8 \mathrm{E}+01$ & $1.6 \mathrm{E}+01$ & $9.7 \mathrm{E}+00$ \\
\hline Idaho & ID & $2.6 \mathrm{E}+01$ & $3.1 \mathrm{E}+01$ & $3.0 \mathrm{E}+01$ & $1.7 \mathrm{E}+01$ & $1.3 \mathrm{E}+01$ & $8.6 \mathrm{E}+00$ \\
\hline Montana & MT & $2.1 \mathrm{E}+01$ & $2.4 \mathrm{E}+01$ & $2.6 \mathrm{E}+01$ & $2.3 \mathrm{E}+01$ & $1.2 \mathrm{E}+01$ & $8.8 \mathrm{E}+00$ \\
\hline California & CA & $9.4 \mathrm{E}+01$ & $1.2 \mathrm{E}+02$ & $8.7 \mathrm{E}+01$ & $5.7 \mathrm{E}+01$ & $2.4 \mathrm{E}+01$ & $7.3 \mathrm{E}+00$ \\
\hline Nevada & NV & $3.5 \mathrm{E}+01$ & $4.0 \mathrm{E}+01$ & $3.9 \mathrm{E}+01$ & $4.5 \mathrm{E}+01$ & $2.8 \mathrm{E}+01$ & $1.3 \mathrm{E}+01$ \\
\hline Utah & UT & $2.9 \mathrm{E}+01$ & $3.4 \mathrm{E}+01$ & $3.0 \mathrm{E}+01$ & $2.3 \mathrm{E}+01$ & $1.8 \mathrm{E}+01$ & $1.1 \mathrm{E}+01$ \\
\hline Colorado & $\mathrm{CO}$ & $5.4 \mathrm{E}+01$ & $4.3 \mathrm{E}+01$ & $3.9 \mathrm{E}+01$ & $1.8 \mathrm{E}+01$ & $1.7 \mathrm{E}+01$ & $8.6 \mathrm{E}+00$ \\
\hline Arizona & $\mathrm{AZ}$ & $5.7 \mathrm{E}+01$ & $6.7 \mathrm{E}+01$ & $5.0 \mathrm{E}+01$ & $4.9 \mathrm{E}+01$ & $3.8 \mathrm{E}+01$ & $1.1 \mathrm{E}+01$ \\
\hline New Mexico & $\overline{\mathrm{NM}}$ & $3.7 \mathrm{E}+01$ & $3.4 \mathrm{E}+01$ & $3.1 \mathrm{E}+01$ & $2.3 \mathrm{E}+01$ & $2.2 \mathrm{E}+01$ & $1.2 \mathrm{E}+01$ \\
\hline Texas & TX & $7.5 \mathrm{E}+01$ & $7.0 \mathrm{E}+01$ & $5.0 \mathrm{E}+01$ & $3.3 \mathrm{E}+01$ & $2.5 \mathrm{E}+01$ & $8.1 \mathrm{E}+00$ \\
\hline Oklahoma & OK & $6.8 \mathrm{E}+01$ & $7.0 \mathrm{E}+01$ & $5.1 \mathrm{E}+01$ & $3.2 \mathrm{E}+01$ & $2.9 \mathrm{E}+01$ & $8.1 \mathrm{E}+00$ \\
\hline Arkansas & AR & $1.1 \mathrm{E}+02$ & $9.8 \mathrm{E}+01$ & $6.6 \mathrm{E}+01$ & $4.1 \mathrm{E}+01$ & $4.2 \mathrm{E}+01$ & $7.4 \mathrm{E}+00$ \\
\hline Louisiana & LA & $7.3 \mathrm{E}+01$ & $8.0 \mathrm{E}+01$ & $4.1 \mathrm{E}+01$ & $3.2 \mathrm{E}+01$ & $2.4 \mathrm{E}+01$ & $5.2 \mathrm{E}+00$ \\
\hline Mississippi & MS & $1.1 \mathrm{E}+02$ & $1.1 \mathrm{E}+02$ & $6.2 \mathrm{E}+01$ & $4.5 \mathrm{E}+01$ & $3.9 \mathrm{E}+01$ & $7.4 \mathrm{E}+00$ \\
\hline Alabama & $\mathrm{AL}$ & $1.1 \mathrm{E}+02$ & $1.1 \mathrm{E}+02$ & $6.8 \mathrm{E}+01$ & $4.4 \mathrm{E}+01$ & $3.2 \mathrm{E}+01$ & $6.6 \mathrm{E}+00$ \\
\hline Kentucky & $\mathrm{KY}$ & $9.7 \mathrm{E}+01$ & $7.1 \mathrm{E}+01$ & $4.8 \mathrm{E}+01$ & $2.3 \mathrm{E}+01$ & $1.6 \mathrm{E}+01$ & $3.2 \mathrm{E}+00$ \\
\hline Tennessee & TN & $1.1 \mathrm{E}+02$ & $9.9 \mathrm{E}+01$ & $6.5 \mathrm{E}+01$ & $3.6 \mathrm{E}+01$ & $2.8 \mathrm{E}+01$ & $4.3 \mathrm{E}+00$ \\
\hline Florida & FL & $7.4 \mathrm{E}+01$ & $1.1 \mathrm{E}+02$ & $6.9 \mathrm{E}+01$ & $3.3 \mathrm{E}+01$ & $2.1 \mathrm{E}+01$ & $9.6 \mathrm{E}+00$ \\
\hline Georgia & GA & $1.1 \mathrm{E}+02$ & $1.2 \mathrm{E}+02$ & $7.3 \mathrm{E}+01$ & $4.3 \mathrm{E}+01$ & $2.5 \mathrm{E}+01$ & $6.8 \mathrm{E}+00$ \\
\hline North Carolina & NC & $1.5 \mathrm{E}+02$ & $1.1 \mathrm{E}+02$ & $8.3 \mathrm{E}+01$ & $5.6 \mathrm{E}+01$ & $1.9 \mathrm{E}+01$ & $5.3 \mathrm{E}+00$ \\
\hline South Carolina & SC & $1.3 \mathrm{E}+02$ & $1.2 \mathrm{E}+02$ & $8.9 \mathrm{E}+01$ & $5.8 \mathrm{E}+01$ & $2.6 \mathrm{E}+01$ & $8.5 \mathrm{E}+00$ \\
\hline Virginia & VA & $1.6 \mathrm{E}+02$ & $1.0 \mathrm{E}+02$ & $7.7 \mathrm{E}+01$ & $4.4 \mathrm{E}+01$ & $1.2 \mathrm{E}+01$ & $2.5 \mathrm{E}+00$ \\
\hline West Virginia & WV & $8.5 \mathrm{E}+01$ & $7.3 \mathrm{E}+01$ & $5.2 \mathrm{E}+01$ & $2.2 \mathrm{E}+01$ & $9.7 \mathrm{E}+00$ & $2.0 \mathrm{E}+00$ \\
\hline Delaware & $\overline{\mathrm{DE}}$ & $2.7 \mathrm{E}+02$ & $1.5 \mathrm{E}+02$ & $1.0 \mathrm{E}+02$ & $6.0 \mathrm{E}+01$ & $8.5 \mathrm{E}+00$ & $1.3 \mathrm{E}+00$ \\
\hline Washington DC & $\mathrm{DC}$ & $1.4 \mathrm{E}+02$ & $7.8 \mathrm{E}+01$ & $4.3 \mathrm{E}+01$ & $1.6 \mathrm{E}+01$ & $5.4 \mathrm{E}+00$ & $9.8 \mathrm{E}-01$ \\
\hline Maryland & MD & $1.6 \mathrm{E}+02$ & $9.5 \mathrm{E}+01$ & $5.8 \mathrm{E}+01$ & $2.7 \mathrm{E}+01$ & $6.4 \mathrm{E}+00$ & $1.1 \mathrm{E}+00$ \\
\hline Connecticut & $\mathrm{CT}$ & $8.0 \mathrm{E}+01$ & $9.2 \mathrm{E}+01$ & $4.6 \mathrm{E}+01$ & $1.1 \mathrm{E}+01$ & $3.6 \mathrm{E}+00$ & $6.6 \mathrm{E}-01$ \\
\hline New Jersey & NJ & $1.2 \mathrm{E}+02$ & $7.2 \mathrm{E}+01$ & $4.2 \mathrm{E}+01$ & $1.4 \mathrm{E}+01$ & $4.2 \mathrm{E}+00$ & 7.4E-01 \\
\hline New York & $\mathrm{NY}$ & $9.5 \mathrm{E}+01$ & $8.9 \mathrm{E}+01$ & $6.1 \mathrm{E}+01$ & $1.4 \mathrm{E}+01$ & $5.3 \mathrm{E}+00$ & $1.3 \mathrm{E}+00$ \\
\hline Pennsylvania & PA & $1.2 \mathrm{E}+02$ & $8.5 \mathrm{E}+01$ & $5.5 \mathrm{E}+01$ & $1.4 \mathrm{E}+01$ & $5.6 \mathrm{E}+00$ & $1.1 \mathrm{E}+00$ \\
\hline Rhode Island & RI & $6.1 \mathrm{E}+01$ & $9.0 \mathrm{E}+01$ & $4.5 \mathrm{E}+01$ & $1.1 \mathrm{E}+01$ & $3.7 \mathrm{E}+00$ & $5.9 \mathrm{E}-01$ \\
\hline Massachusetts & MA & $5.0 \mathrm{E}+01$ & $7.6 \mathrm{E}+01$ & $4.3 \mathrm{E}+01$ & $9.9 \mathrm{E}+00$ & $3.5 \mathrm{E}+00$ & $6.7 \mathrm{E}-01$ \\
\hline New Hampshire & $\mathrm{NH}$ & $6.8 \mathrm{E}+01$ & $8.4 \mathrm{E}+01$ & $5.3 \mathrm{E}+01$ & $1.2 \mathrm{E}+01$ & $3.8 \mathrm{E}+00$ & $1.0 \mathrm{E}+00$ \\
\hline Vermont & VT & $8.5 \mathrm{E}+01$ & $8.7 \mathrm{E}+01$ & $6.5 \mathrm{E}+01$ & $1.5 \mathrm{E}+01$ & $4.8 \mathrm{E}+00$ & $1.6 \mathrm{E}+00$ \\
\hline Maine & $\mathrm{ME}$ & $5.1 \mathrm{E}+01$ & $7.4 \mathrm{E}+01$ & $4.8 \mathrm{E}+01$ & $1.1 \mathrm{E}+01$ & $4.5 \mathrm{E}+00$ & $1.3 \mathrm{E}+00$ \\
\hline Ohio & $\mathrm{OH}$ & $9.8 \mathrm{E}+01$ & $7.2 \mathrm{E}+01$ & $5.1 \mathrm{E}+01$ & $1.3 \mathrm{E}+01$ & $6.8 \mathrm{E}+00$ & $1.8 \mathrm{E}+00$ \\
\hline Indiana & IN & $1.0 \mathrm{E}+02$ & $7.2 \mathrm{E}+01$ & $4.8 \mathrm{E}+01$ & $1.5 \mathrm{E}+01$ & $8.8 \mathrm{E}+00$ & $2.4 \mathrm{E}+00$ \\
\hline Illinois & IL & $1.1 \mathrm{E}+02$ & $7.3 \mathrm{E}+01$ & $5.0 \mathrm{E}+01$ & $1.8 \mathrm{E}+01$ & $1.1 \mathrm{E}+01$ & $3.1 \mathrm{E}+00$ \\
\hline Michigan & MI & $1.2 \mathrm{E}+02$ & $9.0 \mathrm{E}+01$ & $6.5 \mathrm{E}+01$ & $1.1 \mathrm{E}+01$ & $6.7 \mathrm{E}+00$ & $2.4 \mathrm{E}+00$ \\
\hline Wisconsin & WI & $1.1 \mathrm{E}+02$ & $7.6 \mathrm{E}+01$ & $5.5 \mathrm{E}+01$ & $1.3 \mathrm{E}+01$ & $8.9 \mathrm{E}+00$ & $3.2 \mathrm{E}+00$ \\
\hline Minnesota & $\mathrm{MN}$ & $6.9 \mathrm{E}+01$ & $5.9 \mathrm{E}+01$ & $3.6 \mathrm{E}+01$ & $1.5 \mathrm{E}+01$ & $1.3 \mathrm{E}+01$ & $5.1 \mathrm{E}+00$ \\
\hline Iowa & IA & $9.2 \mathrm{E}+01$ & $8.2 \mathrm{E}+01$ & $5.8 \mathrm{E}+01$ & $2.5 \mathrm{E}+01$ & $1.5 \mathrm{E}+01$ & $5.1 \mathrm{E}+00$ \\
\hline Missouri & $\mathrm{MO}$ & $9.1 \mathrm{E}+01$ & $8.1 \mathrm{E}+01$ & $5.6 \mathrm{E}+01$ & $2.7 \mathrm{E}+01$ & $2.2 \mathrm{E}+01$ & $5.4 \mathrm{E}+00$ \\
\hline Kansas & KS & $4.8 \mathrm{E}+01$ & $5.7 \mathrm{E}+01$ & $4.6 \mathrm{E}+01$ & $2.8 \mathrm{E}+01$ & $2.5 \mathrm{E}+01$ & $8.4 \mathrm{E}+00$ \\
\hline Nebraska & $\mathrm{NE}$ & $3.7 \mathrm{E}+01$ & $4.9 \mathrm{E}+01$ & $4.0 \mathrm{E}+01$ & $2.6 \mathrm{E}+01$ & $1.9 \mathrm{E}+01$ & $8.3 \mathrm{E}+00$ \\
\hline North Dakota & ND & $3.1 \mathrm{E}+01$ & $3.6 \mathrm{E}+01$ & $2.7 \mathrm{E}+01$ & $2.2 \mathrm{E}+01$ & $1.3 \mathrm{E}+01$ & $8.9 \mathrm{E}+00$ \\
\hline South Dakota & SD & $3.9 \mathrm{E}+01$ & $5.3 \mathrm{E}+01$ & $3.9 \mathrm{E}+01$ & $3.2 \mathrm{E}+01$ & $2.1 \mathrm{E}+01$ & $9.1 \mathrm{E}+00$ \\
\hline
\end{tabular}


Table A13 Human exposure level characterization factors for VOC (January-June)

\begin{tabular}{|c|c|c|c|c|c|c|c|}
\hline State & & January & February & March & April & May & June \\
\hline Oregon & $\overline{\mathrm{OR}}$ & $1.0 \mathrm{E}+00$ & $1.4 \mathrm{E}+00$ & $2.0 \mathrm{E}+00$ & $2.3 E+00$ & $1.9 \mathrm{E}+00$ & $1.9 \mathrm{E}+0 \mathrm{c}$ \\
\hline Washington & WA & $1.1 \mathrm{E}+00$ & $1.4 \mathrm{E}+00$ & $2.3 \mathrm{E}+00$ & $3.5 \mathrm{E}+00$ & $4.2 \mathrm{E}+00$ & $4.8 \mathrm{E}+0 \mathrm{C}$ \\
\hline Wyoming & WY & $4.7 \mathrm{E}+00$ & $4.0 \mathrm{E}+00$ & $3.5 \mathrm{E}+00$ & $1.8 \mathrm{E}+00$ & $8.6 \mathrm{E}-01$ & $6.6 \mathrm{E}-01$ \\
\hline \begin{tabular}{|l} 
Idaho \\
\end{tabular} & ID & $2.8 \mathrm{E}+00$ & $2.7 \mathrm{E}+00$ & $2.2 \mathrm{E}+00$ & $1.6 \mathrm{E}+00$ & $6.5 \mathrm{E}-01$ & $6.6 \mathrm{E}-01$ \\
\hline Montana & MT & $3.9 \mathrm{E}+00$ & $3.5 \mathrm{E}+00$ & $3.1 \mathrm{E}+00$ & $1.9 \mathrm{E}+00$ & $8.1 \mathrm{E}-01$ & $6.0 \mathrm{E}-01$ \\
\hline California & CA & $2.9 \mathrm{E}+00$ & $5.2 \mathrm{E}+00$ & $7.4 \mathrm{E}+00$ & $8.7 \mathrm{E}+00$ & $1.3 \mathrm{E}+01$ & $1.2 \mathrm{E}+01$ \\
\hline Nevada & NV & $3.0 \mathrm{E}+00$ & $2.9 \mathrm{E}+00$ & $2.9 \mathrm{E}+00$ & $2.5 \mathrm{E}+00$ & $1.8 \mathrm{E}+00$ & $1.5 \mathrm{E}+0 \mathrm{C}$ \\
\hline Utah & UT & $3.7 \mathrm{E}+00$ & $3.3 \mathrm{E}+00$ & $3.1 \mathrm{E}+00$ & $2.2 \mathrm{E}+00$ & $1.9 \mathrm{E}+00$ & $1.2 \mathrm{E}+0 \mathrm{C}$ \\
\hline Colorado & $\mathrm{CO}$ & $4.5 \mathrm{E}+00$ & $3.2 \mathrm{E}+00$ & $3.3 \mathrm{E}+00$ & $2.2 \mathrm{E}+00$ & $1.8 \mathrm{E}+00$ & $1.7 \mathrm{E}+00$ \\
\hline \begin{tabular}{|l|} 
Arizona \\
\end{tabular} & $\overline{A Z}$ & $2.9 \mathrm{E}+00$ & $3.2 \mathrm{E}+00$ & $3.0 \mathrm{E}+00$ & $2.8 \mathrm{E}+00$ & $2.7 \mathrm{E}+00$ & $2.5 \mathrm{E}+0 \mathrm{c}$ \\
\hline New Mexico & $\mathrm{NM}$ & $3.6 \mathrm{E}+00$ & $2.3 \mathrm{E}+00$ & $1.8 \mathrm{E}+00$ & $1.1 \mathrm{E}+00$ & 5.9E-01 & $5.6 \mathrm{E}-01$ \\
\hline \begin{tabular}{|l|} 
Texas \\
\end{tabular} & TX & $3.8 \mathrm{E}+00$ & $2.8 \mathrm{E}+00$ & $3.5 \mathrm{E}+00$ & $3.5 \mathrm{E}+00$ & $3.8 \mathrm{E}+00$ & $4.7 \mathrm{E}+0 \mathrm{C}$ \\
\hline Oklahoma & OK & $5.7 \mathrm{E}+00$ & $3.9 \mathrm{E}+00$ & $5.1 \mathrm{E}+00$ & $2.7 \mathrm{E}+00$ & $1.4 \mathrm{E}+00$ & $9.6 \mathrm{E}-01$ \\
\hline Arkansas & $\mathrm{AR}$ & $5.7 \mathrm{E}+00$ & $4.2 \mathrm{E}+00$ & $5.1 \mathrm{E}+00$ & $3.4 \mathrm{E}+00$ & $1.7 \mathrm{E}+00$ & $1.3 \mathrm{E}+0 \mathrm{C}$ \\
\hline Louisiana & $\overline{\text { LA }}$ & $2.4 \mathrm{E}+00$ & $2.9 \mathrm{E}+00$ & $3.6 \mathrm{E}+00$ & $4.4 \mathrm{E}+00$ & $3.9 \mathrm{E}+00$ & $3.7 \mathrm{E}+0 \mathrm{C}$ \\
\hline Mississippi & MS & $3.5 \mathrm{E}+00$ & $3.1 \mathrm{E}+00$ & $3.6 \mathrm{E}+00$ & $3.3 \mathrm{E}+00$ & $1.6 \mathrm{E}+00$ & $1.6 \mathrm{E}+0 \mathrm{C}$ \\
\hline Alabama & $\mathrm{AL}$ & $3.0 \mathrm{E}+00$ & $3.1 \mathrm{E}+00$ & $3.3 \mathrm{E}+00$ & $3.6 \mathrm{E}+00$ & $1.7 \mathrm{E}+00$ & $1.2 \mathrm{E}+0 \mathrm{C}$ \\
\hline Kentucky & KY & $5.1 \mathrm{E}+00$ & $5.2 \mathrm{E}+00$ & $7.7 \mathrm{E}+00$ & $7.1 \mathrm{E}+00$ & $6.9 \mathrm{E}+00$ & $4.3 \mathrm{E}+0 \mathrm{C}$ \\
\hline Tennessee & TN & $4.9 \mathrm{E}+00$ & $4.3 \mathrm{E}+00$ & $5.7 \mathrm{E}+00$ & $4.5 \mathrm{E}+00$ & $2.8 \mathrm{E}+00$ & $1.4 \mathrm{E}+0 \mathrm{C}$ \\
\hline Florida & FL & $2.3 \mathrm{E}+00$ & $2.7 \mathrm{E}+00$ & $3.3 \mathrm{E}+00$ & $4.8 \mathrm{E}+00$ & $4.5 \mathrm{E}+00$ & $6.4 \mathrm{E}+0 \mathrm{C}$ \\
\hline Georgia & $\overline{\mathrm{GA}}$ & $2.8 \mathrm{E}+00$ & $3.5 \mathrm{E}+00$ & $3.8 \mathrm{E}+00$ & $4.1 \mathrm{E}+00$ & $3.1 \mathrm{E}+00$ & $2.1 \mathrm{E}+00$ \\
\hline \begin{tabular}{|l|} 
North Carolina \\
\end{tabular} & $\mathrm{NC}$ & $2.1 \mathrm{E}+00$ & $3.4 \mathrm{E}+00$ & $3.1 \mathrm{E}+00$ & $3.0 \mathrm{E}+00$ & $2.5 \mathrm{E}+00$ & $1.5 \mathrm{E}+0 \mathrm{c}$ \\
\hline \begin{tabular}{|l|} 
South Carolina \\
\end{tabular} & SC & $2.5 \mathrm{E}+00$ & $3.8 \mathrm{E}+00$ & $2.9 \mathrm{E}+00$ & $2.5 \mathrm{E}+00$ & $1.7 \mathrm{E}+00$ & $8.2 \mathrm{E}-01$ \\
\hline Virginia & VA & $1.9 \mathrm{E}+00$ & $3.1 \mathrm{E}+00$ & $4.2 \mathrm{E}+00$ & $4.8 \mathrm{E}+00$ & $5.2 \mathrm{E}+00$ & $5.7 \mathrm{E}+0 \mathrm{C}$ \\
\hline West Virginia & WV & $3.2 \mathrm{E}+00$ & $3.8 \mathrm{E}+00$ & $6.7 \mathrm{E}+00$ & $5.3 \mathrm{E}+00$ & $5.6 \mathrm{E}+00$ & $3.3 \mathrm{E}+0 \mathrm{C}$ \\
\hline Delaware & $\mathrm{DE}$ & $8.7 \mathrm{E}-01$ & $2.4 \mathrm{E}+00$ & $3.6 \mathrm{E}+00$ & $5.2 \mathrm{E}+00$ & $5.4 \mathrm{E}+00$ & $6.4 \mathrm{E}+00$ \\
\hline Washington DC & $\overline{\mathrm{DC}}$ & $1.7 \mathrm{E}+00$ & $2.4 \mathrm{E}+00$ & $4.3 \mathrm{E}+00$ & $7.0 \mathrm{E}+00$ & $1.1 \mathrm{E}+01$ & $1.6 \mathrm{E}+01$ \\
\hline Maryland & $\mathrm{MD}$ & $1.6 \mathrm{E}+00$ & $2.6 \mathrm{E}+00$ & $4.3 \mathrm{E}+00$ & $6.6 \mathrm{E}+00$ & $8.1 \mathrm{E}+00$ & $1.0 \mathrm{E}+01$ \\
\hline Connecticut & CT & $7.2 \mathrm{E}-01$ & $7.2 \mathrm{E}-01$ & $3.5 \mathrm{E}+00$ & $6.7 \mathrm{E}+00$ & $6.8 \mathrm{E}+00$ & $1.1 \mathrm{E}+01$ \\
\hline New Jersey & NJ & $1.0 \mathrm{E}+00$ & $1.1 \mathrm{E}+00$ & $3.4 \mathrm{E}+00$ & $6.7 \mathrm{E}+00$ & $7.8 \mathrm{E}+00$ & $1.6 \mathrm{E}+01$ \\
\hline New York & NY & $1.6 \mathrm{E}+00$ & $1.9 \mathrm{E}+00$ & $5.2 \mathrm{E}+00$ & $6.4 \mathrm{E}+00$ & $5.3 \mathrm{E}+00$ & $9.1 \mathrm{E}+0 \mathrm{C}$ \\
\hline Pennsylvania & $\mathrm{PA}$ & $2.1 \mathrm{E}+00$ & $2.7 \mathrm{E}+00$ & $5.5 \mathrm{E}+00$ & $6.6 \mathrm{E}+00$ & $7.0 \mathrm{E}+00$ & $8.5 \mathrm{E}+0 \mathrm{C}$ \\
\hline Rhode Island & RI & $3.3 \mathrm{E}-01$ & $4.1 \mathrm{E}-01$ & $2.9 \mathrm{E}+00$ & $4.5 \mathrm{E}+00$ & $7.0 \mathrm{E}+00$ & $6.1 \mathrm{E}+0 \mathrm{c}$ \\
\hline Massachusetts & MA & $3.5 \mathrm{E}-01$ & $4.6 \mathrm{E}-01$ & $3.0 \mathrm{E}+00$ & $4.3 \mathrm{E}+00$ & $6.3 \mathrm{E}+00$ & $5.1 \mathrm{E}+0 \mathrm{c}$ \\
\hline New Hampshire & $\mathrm{NH}$ & $4.4 \mathrm{E}-01$ & $5.5 \mathrm{E}-01$ & $3.8 \mathrm{E}+00$ & $4.6 \mathrm{E}+00$ & $3.5 \mathrm{E}+00$ & $1.8 \mathrm{E}+0 \mathrm{C}$ \\
\hline \begin{tabular}{|l|} 
Vermont \\
\end{tabular} & VT & $7.6 \mathrm{E}-01$ & $8.7 \mathrm{E}-01$ & $4.5 \mathrm{E}+00$ & $5.2 \mathrm{E}+00$ & $1.5 \mathrm{E}+00$ & $7.9 \mathrm{E}-01$ \\
\hline Maine & ME & $2.3 \mathrm{E}-01$ & $1.9 \mathrm{E}-01$ & $2.0 \mathrm{E}+00$ & $2.6 \mathrm{E}+00$ & $1.8 \mathrm{E}+00$ & $2.1 \mathrm{E}-01$ \\
\hline Ohio & $\mathrm{OH}$ & $4.1 \mathrm{E}+00$ & $4.6 \mathrm{E}+00$ & $8.1 \mathrm{E}+00$ & $7.1 \mathrm{E}+00$ & $8.0 \mathrm{E}+00$ & $7.7 \mathrm{E}+00$ \\
\hline Indiana & IN & $6.0 \mathrm{E}+00$ & $6.1 \mathrm{E}+00$ & $9.6 \mathrm{E}+00$ & $7.7 \mathrm{E}+00$ & $7.6 \mathrm{E}+00$ & $6.8 \mathrm{E}+0 \mathrm{C}$ \\
\hline Illinois & $\mathrm{IL}$ & $6.3 \mathrm{E}+00$ & $6.4 \mathrm{E}+00$ & $1.0 \mathrm{E}+01$ & $7.6 \mathrm{E}+00$ & $7.5 \mathrm{E}+00$ & $8.1 \mathrm{E}+0 \mathrm{C}$ \\
\hline \begin{tabular}{|l} 
Michigan \\
\end{tabular} & MI & $4.4 \mathrm{E}+00$ & $5.5 \mathrm{E}+00$ & $8.5 \mathrm{E}+00$ & $7.7 \mathrm{E}+00$ & $6.3 \mathrm{E}+00$ & $7.9 \mathrm{E}+0 \mathrm{C}$ \\
\hline Wisconsin & WI & $5.6 \mathrm{E}+00$ & $7.4 \mathrm{E}+00$ & $1.1 \mathrm{E}+01$ & $7.7 \mathrm{E}+00$ & $5.7 \mathrm{E}+00$ & $4.6 \mathrm{E}+00$ \\
\hline Minnesota & MN & $5.9 \mathrm{E}+00$ & $7.9 \mathrm{E}+00$ & $1.2 \mathrm{E}+01$ & $7.1 \mathrm{E}+00$ & $4.2 \mathrm{E}+00$ & $2.7 \mathrm{E}+00$ \\
\hline Iowa & IA & $7.1 \mathrm{E}+00$ & $7.2 \mathrm{E}+00$ & $1.1 \mathrm{E}+01$ & $5.7 \mathrm{E}+00$ & $3.9 \mathrm{E}+00$ & $1.9 \mathrm{E}+0 \mathrm{C}$ \\
\hline Missouri & $\mathrm{MO}$ & $6.9 \mathrm{E}+00$ & $5.9 \mathrm{E}+00$ & $9.0 \mathrm{E}+00$ & $5.3 \mathrm{E}+00$ & $4.4 \mathrm{E}+00$ & $2.6 \mathrm{E}+0 \mathrm{C}$ \\
\hline Kansas & KS & $7.1 \mathrm{E}+00$ & $6.0 \mathrm{E}+00$ & $6.5 \mathrm{E}+00$ & $3.3 \mathrm{E}+00$ & $1.8 \mathrm{E}+00$ & $1.1 \mathrm{E}+0 \mathrm{c}$ \\
\hline Nebraska & $\mathrm{NE}$ & $6.8 \mathrm{E}+00$ & $6.3 \mathrm{E}+00$ & $7.1 \mathrm{E}+00$ & $4.0 \mathrm{E}+00$ & $2.6 \mathrm{E}+00$ & $1.6 \mathrm{E}+0 \mathrm{c}$ \\
\hline North Dakota & $\mathrm{ND}$ & $4.6 \mathrm{E}+00$ & $6.6 \mathrm{E}+00$ & $6.3 \mathrm{E}+00$ & $4.2 \mathrm{E}+00$ & $1.6 \mathrm{E}+00$ & $7.8 \mathrm{E}-01$ \\
\hline South Dakota & SD & $6.4 \mathrm{E}+00$ & $7.0 \mathrm{E}+00$ & $7.8 \mathrm{E}+00$ & $4.2 \mathrm{E}+00$ & $2.0 \mathrm{E}+00$ & $9.8 \mathrm{E}-01$ \\
\hline
\end{tabular}


Table A14 Human exposure level characterization factors for VOC (July-December)

\begin{tabular}{|c|c|c|c|c|c|c|c|}
\hline State & & July & August & September & October & November & December \\
\hline Oregon & $\overline{\mathrm{OR}}$ & $2.1 \mathrm{E}+00$ & $1.9 \mathrm{E}+00$ & $2.1 \mathrm{E}+00$ & $1.9 \mathrm{E}+00$ & $1.1 \mathrm{E}+00$ & $7.5 \mathrm{E}-01$ \\
\hline Washington & WA & $4.4 \mathrm{E}+00$ & $4.3 \mathrm{E}+00$ & $4.7 \mathrm{E}+00$ & $2.3 \mathrm{E}+00$ & $9.6 \mathrm{E}-01$ & $7.5 \mathrm{E}-01$ \\
\hline Wyoming & $\overline{W Y}$ & $2.9 \mathrm{E}-01$ & $6.2 \mathrm{E}-01$ & $1.2 \mathrm{E}+00$ & $2.2 \mathrm{E}+00$ & $3.2 \mathrm{E}+00$ & $3.7 \mathrm{E}+00$ \\
\hline Idaho & ID & $3.0 \mathrm{E}-01$ & $4.1 \mathrm{E}-01$ & $8.6 \mathrm{E}-01$ & $1.7 \mathrm{E}+00$ & $2.1 \mathrm{E}+00$ & $2.0 \mathrm{E}+00$ \\
\hline Montana & MT & $1.9 \mathrm{E}-01$ & $4.6 \mathrm{E}-01$ & $9.8 \mathrm{E}-01$ & $2.5 \mathrm{E}+00$ & $2.4 \mathrm{E}+00$ & $2.8 \mathrm{E}+00$ \\
\hline California & CA & $1.1 \mathrm{E}+01$ & $1.2 \mathrm{E}+01$ & $1.4 \mathrm{E}+01$ & $9.4 \mathrm{E}+00$ & $4.9 \mathrm{E}+00$ & $2.5 \mathrm{E}+00$ \\
\hline Nevada & NV & $1.1 \mathrm{E}+00$ & $1.1 \mathrm{E}+00$ & $1.6 \mathrm{E}+00$ & $3.1 \mathrm{E}+00$ & $3.1 \mathrm{E}+00$ & $2.7 \mathrm{E}+00$ \\
\hline Utah & UT & $8.8 \mathrm{E}-01$ & $1.4 \mathrm{E}+00$ & $1.8 \mathrm{E}+00$ & $2.3 \mathrm{E}+00$ & $2.8 \mathrm{E}+00$ & $3.3 \mathrm{E}+00$ \\
\hline Colorado & $\mathrm{CO}$ & $2.3 \mathrm{E}+00$ & $2.1 \mathrm{E}+00$ & $2.7 \mathrm{E}+00$ & $2.0 \mathrm{E}+00$ & $3.7 \mathrm{E}+00$ & $3.2 \mathrm{E}+00$ \\
\hline Arizona & AZ & $3.0 \mathrm{E}+00$ & $3.8 \mathrm{E}+00$ & $2.7 \mathrm{E}+00$ & $2.8 \mathrm{E}+00$ & $3.3 \mathrm{E}+00$ & $1.9 \mathrm{E}+00$ \\
\hline New Mexico & NM & $7.4 \mathrm{E}-01$ & $9.4 \mathrm{E}-01$ & $1.1 \mathrm{E}+00$ & $1.3 \mathrm{E}+00$ & $2.5 \mathrm{E}+00$ & $2.8 \mathrm{E}+00$ \\
\hline Texas & $\mathrm{TX}$ & $5.0 \mathrm{E}+00$ & $5.3 \mathrm{E}+00$ & $3.2 \mathrm{E}+00$ & $4.3 \mathrm{E}+00$ & $3.5 \mathrm{E}+00$ & $2.7 \mathrm{E}+00$ \\
\hline Oklahoma & $\overline{\mathrm{OK}}$ & 9.5E-01 & $1.8 \mathrm{E}+00$ & $2.3 \mathrm{E}+00$ & $3.9 \mathrm{E}+00$ & $5.1 \mathrm{E}+00$ & $4.2 \mathrm{E}+00$ \\
\hline Arkansas & AR & $1.3 \mathrm{E}+00$ & $2.1 \mathrm{E}+00$ & $2.5 \mathrm{E}+00$ & $4.1 \mathrm{E}+00$ & $5.7 \mathrm{E}+00$ & $3.4 \mathrm{E}+00$ \\
\hline Louisiana & LA & $3.8 \mathrm{E}+00$ & $5.5 \mathrm{E}+00$ & $2.7 \mathrm{E}+00$ & $4.9 \mathrm{E}+00$ & $3.5 \mathrm{E}+00$ & $1.6 \mathrm{E}+00$ \\
\hline Mississippi & MS & $1.1 \mathrm{E}+00$ & $1.8 \mathrm{E}+00$ & $1.7 \mathrm{E}+00$ & $3.8 \mathrm{E}+00$ & $4.1 \mathrm{E}+00$ & $2.3 \mathrm{E}+00$ \\
\hline Alabama & $\mathrm{AL}$ & $1.0 \mathrm{E}+00$ & $1.5 \mathrm{E}+00$ & $1.9 \mathrm{E}+00$ & $4.6 \mathrm{E}+00$ & $4.9 \mathrm{E}+00$ & $2.1 \mathrm{E}+00$ \\
\hline Kentucky & $\mathrm{KY}$ & $4.7 \mathrm{E}+00$ & $7.4 \mathrm{E}+00$ & $8.2 \mathrm{E}+00$ & $7.3 \mathrm{E}+00$ & $8.8 \mathrm{E}+00$ & $3.8 \mathrm{E}+00$ \\
\hline Tennessee & TN & $1.3 \mathrm{E}+00$ & $2.7 \mathrm{E}+00$ & $3.9 \mathrm{E}+00$ & $6.4 \mathrm{E}+00$ & $8.9 \mathrm{E}+00$ & $3.1 \mathrm{E}+00$ \\
\hline Florida & FL & $5.0 \mathrm{E}+00$ & $7.7 \mathrm{E}+00$ & $6.5 \mathrm{E}+00$ & $3.4 \mathrm{E}+00$ & $1.8 \mathrm{E}+00$ & $1.6 \mathrm{E}+00$ \\
\hline Georgia & GA & $2.4 \mathrm{E}+00$ & $3.4 \mathrm{E}+00$ & $3.9 \mathrm{E}+00$ & $6.8 \mathrm{E}+00$ & $5.7 \mathrm{E}+00$ & $2.2 \mathrm{E}+00$ \\
\hline North Carolina & NC & $2.3 \mathrm{E}+00$ & $2.6 \mathrm{E}+00$ & $4.6 \mathrm{E}+00$ & $8.8 \mathrm{E}+00$ & $5.8 \mathrm{E}+00$ & $2.3 \mathrm{E}+00$ \\
\hline South Carolina & $\mathrm{SC}$ & $1.4 \mathrm{E}+00$ & $1.8 \mathrm{E}+00$ & $3.2 \mathrm{E}+00$ & $7.0 \mathrm{E}+00$ & $5.3 \mathrm{E}+00$ & $2.5 \mathrm{E}+00$ \\
\hline Virginia & VA & $5.4 \mathrm{E}+00$ & $6.3 \mathrm{E}+00$ & $8.2 \mathrm{E}+00$ & $9.6 \mathrm{E}+00$ & $6.1 \mathrm{E}+00$ & $1.9 \mathrm{E}+00$ \\
\hline West Virginia & WV & $4.1 \mathrm{E}+00$ & $7.9 \mathrm{E}+00$ & $1.0 \mathrm{E}+01$ & $7.7 \mathrm{E}+00$ & $6.5 \mathrm{E}+00$ & $2.4 \mathrm{E}+00$ \\
\hline Delaware & $\mathrm{DE}$ & $5.0 \mathrm{E}+00$ & $6.0 \mathrm{E}+00$ & $9.0 \mathrm{E}+00$ & $9.0 \mathrm{E}+00$ & $4.2 \mathrm{E}+00$ & $1.3 \mathrm{E}+00$ \\
\hline Washington DC & $\overline{D C}$ & $1.2 \mathrm{E}+01$ & $1.4 \mathrm{E}+01$ & $1.3 \mathrm{E}+01$ & $7.9 \mathrm{E}+00$ & $4.6 \mathrm{E}+00$ & $1.5 \mathrm{E}+00$ \\
\hline Maryland & $\mathrm{MD}$ & $7.8 \mathrm{E}+00$ & $1.0 \mathrm{E}+01$ & $1.2 \mathrm{E}+01$ & $8.3 \mathrm{E}+00$ & $4.6 \mathrm{E}+00$ & $1.6 \mathrm{E}+00$ \\
\hline Connecticut & CT & $7.4 \mathrm{E}+00$ & $1.0 \mathrm{E}+01$ & $1.0 \mathrm{E}+01$ & $3.6 \mathrm{E}+00$ & $1.9 \mathrm{E}+00$ & $6.9 \mathrm{E}-01$ \\
\hline New Jersey & $\mathrm{NJ}$ & $1.3 \mathrm{E}+01$ & $1.3 \mathrm{E}+01$ & $1.2 \mathrm{E}+01$ & $4.6 \mathrm{E}+00$ & $2.7 \mathrm{E}+00$ & $1.0 \mathrm{E}+00$ \\
\hline New York & NY & $7.7 \mathrm{E}+00$ & $9.8 \mathrm{E}+00$ & $1.1 \mathrm{E}+01$ & $3.6 \mathrm{E}+00$ & $3.1 \mathrm{E}+00$ & $1.3 \mathrm{E}+00$ \\
\hline Pennsylvania & PA & $8.1 \mathrm{E}+00$ & $1.2 \mathrm{E}+01$ & $1.3 \mathrm{E}+01$ & $5.8 \mathrm{E}+00$ & $4.4 \mathrm{E}+00$ & $1.7 \mathrm{E}+00$ \\
\hline Rhode Island & $\mathrm{RI}$ & $4.4 \mathrm{E}+00$ & $8.2 \mathrm{E}+00$ & $7.5 \mathrm{E}+00$ & $2.5 \mathrm{E}+00$ & $1.4 \mathrm{E}+00$ & $3.9 \mathrm{E}-01$ \\
\hline Massachusetts & MA & $4.3 \mathrm{E}+00$ & $7.4 \mathrm{E}+00$ & $7.2 \mathrm{E}+00$ & $2.1 \mathrm{E}+00$ & $1.3 \mathrm{E}+00$ & $4.6 \mathrm{E}-01$ \\
\hline New Hampshire & $\mathrm{NH}$ & $2.9 \mathrm{E}+00$ & $4.5 \mathrm{E}+00$ & $5.2 \mathrm{E}+00$ & $1.7 \mathrm{E}+00$ & $1.3 \mathrm{E}+00$ & $6.3 \mathrm{E}-01$ \\
\hline Vermont & VT & $2.0 \mathrm{E}+00$ & $2.9 \mathrm{E}+00$ & $4.3 \mathrm{E}+00$ & $1.2 \mathrm{E}+00$ & $1.4 \mathrm{E}+00$ & $9.0 \mathrm{E}-01$ \\
\hline Maine & $\mathrm{ME}$ & $1.1 \mathrm{E}+00$ & $1.8 \mathrm{E}+00$ & $1.9 \mathrm{E}+00$ & $6.5 \mathrm{E}-01$ & $6.2 \mathrm{E}-01$ & $2.9 \mathrm{E}-01$ \\
\hline Ohio & $\mathrm{OH}$ & $8.2 \mathrm{E}+00$ & $1.4 \mathrm{E}+01$ & $1.5 \mathrm{E}+01$ & $6.6 \mathrm{E}+00$ & $5.4 \mathrm{E}+00$ & $2.7 \mathrm{E}+00$ \\
\hline Indiana & IN & $7.3 \mathrm{E}+00$ & $1.1 \mathrm{E}+01$ & $1.1 \mathrm{E}+01$ & $7.7 \mathrm{E}+00$ & $5.8 \mathrm{E}+00$ & $3.9 \mathrm{E}+00$ \\
\hline Illinois & $\mathrm{IL}$ & $9.2 \mathrm{E}+00$ & $9.5 \mathrm{E}+00$ & $1.0 \mathrm{E}+01$ & $7.6 \mathrm{E}+00$ & $5.2 \mathrm{E}+00$ & $4.0 \mathrm{E}+00$ \\
\hline Michigan & MI & $8.6 \mathrm{E}+00$ & $1.1 \mathrm{E}+01$ & $1.4 \mathrm{E}+01$ & $4.7 \mathrm{E}+00$ & $3.9 \mathrm{E}+00$ & $2.2 \mathrm{E}+00$ \\
\hline Wisconsin & WI & $4.6 \mathrm{E}+00$ & $6.0 \mathrm{E}+00$ & $8.3 \mathrm{E}+00$ & $5.5 \mathrm{E}+00$ & $4.1 \mathrm{E}+00$ & $3.2 \mathrm{E}+00$ \\
\hline Minnesota & MN & $2.7 \mathrm{E}+00$ & $4.2 \mathrm{E}+00$ & $4.6 \mathrm{E}+00$ & $4.4 \mathrm{E}+00$ & $4.6 \mathrm{E}+00$ & $4.1 \mathrm{E}+00$ \\
\hline Iowa & IA & $1.7 \mathrm{E}+00$ & $3.9 \mathrm{E}+00$ & $4.6 \mathrm{E}+00$ & $6.4 \mathrm{E}+00$ & $5.1 \mathrm{E}+00$ & $4.8 \mathrm{E}+00$ \\
\hline Missouri & $\mathrm{MO}$ & $2.7 \mathrm{E}+00$ & $4.6 \mathrm{E}+00$ & $5.5 \mathrm{E}+00$ & $7.0 \mathrm{E}+00$ & $6.8 \mathrm{E}+00$ & $5.0 \mathrm{E}+00$ \\
\hline Kansas & $\mathrm{KS}$ & $6.3 \mathrm{E}-01$ & $1.9 \mathrm{E}+00$ & $3.0 \mathrm{E}+00$ & $5.3 E+00$ & $6.3 \mathrm{E}+00$ & $5.2 \mathrm{E}+00$ \\
\hline Nebraska & $\mathrm{NE}$ & $9.7 \mathrm{E}-01$ & $2.4 \mathrm{E}+00$ & $3.1 \mathrm{E}+00$ & $5.3 \mathrm{E}+00$ & $5.2 \mathrm{E}+00$ & $4.9 \mathrm{E}+00$ \\
\hline North Dakota & ND & $4.3 \mathrm{E}-01$ & $1.3 \mathrm{E}+00$ & $1.7 \mathrm{E}+00$ & $4.0 \mathrm{E}+00$ & $3.5 \mathrm{E}+00$ & $3.9 \mathrm{E}+00$ \\
\hline South Dakota & SD & $3.7 \mathrm{E}-01$ & $1.5 \mathrm{E}+00$ & $1.8 \mathrm{E}+00$ & $4.9 \mathrm{E}+00$ & $4.7 \mathrm{E}+00$ & $4.9 \mathrm{E}+00$ \\
\hline
\end{tabular}


Table A15 Ecosystem exposure level characterization factors for NOx (January-June)

\begin{tabular}{|c|c|c|c|c|c|c|c|}
\hline State & & January & February & March & April & May & June \\
\hline Oregon & OR & $2.2 \mathrm{E}-01$ & $2.0 \mathrm{E}-01$ & $2.4 \mathrm{E}-01$ & $2.1 \mathrm{E}-01$ & $2.2 \mathrm{E}-01$ & $2.7 \mathrm{E}-01$ \\
\hline Washington & WA & $1.9 \mathrm{E}-01$ & $1.6 \mathrm{E}-01$ & $2.8 \mathrm{E}-01$ & $3.4 \mathrm{E}-01$ & 4.4E-01 & 5.0E-01 \\
\hline Wyoming & WY & 8.0E-01 & $8.4 \mathrm{E}-01$ & $9.1 \mathrm{E}-01$ & 4.0E-01 & $4.4 \mathrm{E}-01$ & $5.2 \mathrm{E}-01$ \\
\hline Idaho & ID & 3.5E-01 & 5.9E-01 & $3.9 \mathrm{E}-01$ & $3.1 \mathrm{E}-01$ & 3.3E-01 & 4.7E-01 \\
\hline Montana & $\mathrm{MT}$ & 6.6E-01 & $6.6 \mathrm{E}-01$ & 6.8E-01 & 5.9E-01 & $7.1 \mathrm{E}-01$ & 7.0E-01 \\
\hline California & CA & 8.5E-02 & $1.4 \mathrm{E}-01$ & $1.5 \mathrm{E}-01$ & 5.2E-02 & $9.0 \mathrm{E}-02$ & 8.3E-02 \\
\hline Nevada & $\mathrm{NV}$ & $1.7 \mathrm{E}-01$ & $3.1 \mathrm{E}-01$ & $3.8 \mathrm{E}-01$ & 1.3E-01 & $1.6 \mathrm{E}-01$ & $1.8 \mathrm{E}-01$ \\
\hline Utah & UT & $2.1 \mathrm{E}-01$ & $4.4 \mathrm{E}-01$ & 5.0E-01 & $1.8 \mathrm{E}-01$ & $2.3 \mathrm{E}-01$ & $2.1 \mathrm{E}-01$ \\
\hline Colorado & $\mathrm{CO}$ & 8.3E-01 & 5.8E-01 & 9.1E-01 & 3.1E-01 & $4.1 \mathrm{E}-01$ & $4.1 \mathrm{E}-01$ \\
\hline Arizona & $\mathrm{AZ}$ & $1.8 \mathrm{E}-01$ & 3.1E-01 & 3.8E-01 & $1.2 \mathrm{E}-01$ & 2.3E-01 & $1.7 \mathrm{E}-01$ \\
\hline New Mexico & NM & 7.7E-01 & 4.3E-01 & 8.0E-01 & $2.0 \mathrm{E}-01$ & 4.2E-01 & $2.2 \mathrm{E}-01$ \\
\hline Texas & $\mathrm{TX}$ & $3.5 \mathrm{E}+00$ & $1.4 \mathrm{E}+00$ & $4.9 \mathrm{E}+00$ & 8.5E-01 & $2.3 \mathrm{E}+00$ & $4.0 \mathrm{E}+00$ \\
\hline Oklahoma & $\mathrm{OK}$ & $1.7 \mathrm{E}+00$ & 1.9E-01 & $4.2 \mathrm{E}+00$ & 4.2E-01 & $6.4 \mathrm{E}-01$ & $4.6 \mathrm{E}-01$ \\
\hline Arkansas & AR & $2.9 \mathrm{E}+00$ & $1.3 \mathrm{E}+00$ & $2.6 \mathrm{E}+00$ & $1.6 \mathrm{E}+00$ & $2.3 \mathrm{E}+00$ & $2.0 \mathrm{E}+00$ \\
\hline Louisiana & LA & $7.6 \mathrm{E}+00$ & $3.2 \mathrm{E}+00$ & $6.3 \mathrm{E}+00$ & $4.8 \mathrm{E}+00$ & $7.3 \mathrm{E}+00$ & $1.9 \mathrm{E}+01$ \\
\hline Mississippi & MS & $7.3 \mathrm{E}+00$ & $2.7 \mathrm{E}+00$ & $2.6 \mathrm{E}+00$ & $5.8 \mathrm{E}+00$ & $1.3 \mathrm{E}+01$ & $8.5 \mathrm{E}+00$ \\
\hline Alabama & $\mathrm{AL}$ & $3.5 \mathrm{E}+00$ & $2.9 \mathrm{E}+00$ & $1.1 \mathrm{E}+00$ & $3.6 \mathrm{E}+00$ & $1.8 \mathrm{E}+00$ & $2.8 \mathrm{E}+00$ \\
\hline Kentucky & KY & $3.1 \mathrm{E}-01$ & $3.6 \mathrm{E}-01$ & $5.1 \mathrm{E}-01$ & $2.8 \mathrm{E}-01$ & $2.2 \mathrm{E}-01$ & $4.6 \mathrm{E}-01$ \\
\hline Tennessee & $\mathrm{TN}$ & 7.9E-01 & 6.0E-01 & 4.5E-01 & $5.2 \mathrm{E}-01$ & 3.7E-01 & $8.8 \mathrm{E}-01$ \\
\hline Florida & FL & $2.5 \mathrm{E}+00$ & $2.1 \mathrm{E}+00$ & $5.4 \mathrm{E}-01$ & $3.9 \mathrm{E}+00$ & $4.9 \mathrm{E}+00$ & $1.2 \mathrm{E}+00$ \\
\hline Georgia & GA & $1.4 \mathrm{E}+00$ & $8.4 \mathrm{E}-01$ & $1.5 \mathrm{E}-01$ & $6.2 \mathrm{E}-01$ & $4.5 \mathrm{E}-01$ & 8.7E-01 \\
\hline North Carolina & $\mathrm{NC}$ & 1.3E-01 & 3.7E-01 & 4.4E-02 & 2.2E-01 & $9.2 \mathrm{E}-01$ & 2.8E-01 \\
\hline South Carolina & $\mathrm{SC}$ & $3.0 \mathrm{E}-01$ & $5.2 \mathrm{E}-01$ & $4.8 \mathrm{E}-02$ & 3.7E-01 & 7.9E-01 & 3.7E-01 \\
\hline Virginia & VA & $3.6 \mathrm{E}-02$ & $1.2 \mathrm{E}-01$ & 4.3E-02 & $9.6 \mathrm{E}-02$ & $7.0 \mathrm{E}-01$ & $1.7 \mathrm{E}-01$ \\
\hline West Virginia & WV & $3.6 \mathrm{E}-02$ & $1.6 \mathrm{E}-01$ & 9.5E-02 & $1.0 \mathrm{E}-01$ & $2.6 \mathrm{E}-01$ & $2.0 \mathrm{E}-01$ \\
\hline Delaware & $\mathrm{DE}$ & 1.5E-02 & $1.5 \mathrm{E}-02$ & $2.1 \mathrm{E}-02$ & 4.7E-02 & $1.1 \mathrm{E}+00$ & $1.0 \mathrm{E}-01$ \\
\hline Washington DC & $\mathrm{DC}$ & $1.8 \mathrm{E}-02$ & $3.4 \mathrm{E}-02$ & $2.8 \mathrm{E}-02$ & $3.9 \mathrm{E}-02$ & $3.5 \mathrm{E}-01$ & $7.4 \mathrm{E}-02$ \\
\hline Maryland & $\mathrm{MD}$ & $1.7 \mathrm{E}-02$ & $2.4 \mathrm{E}-02$ & 2.7E-02 & $4.1 \mathrm{E}-02$ & 5.1E-01 & 7.8E-02 \\
\hline Connecticut & $\mathrm{CT}$ & 9.9E-03 & 2.9E-03 & 7.9E-03 & $2.3 \mathrm{E}-02$ & $2.4 \mathrm{E}-01$ & 3.6E-02 \\
\hline New Jersey & $\mathrm{NJ}$ & $1.0 \mathrm{E}-02$ & $5.7 \mathrm{E}-03$ & $1.4 \mathrm{E}-02$ & $3.1 \mathrm{E}-02$ & $2.9 \mathrm{E}-01$ & $3.6 \mathrm{E}-02$ \\
\hline New York & NY & 9.5E-03 & 4.3E-03 & $2.0 \mathrm{E}-02$ & $3.7 \mathrm{E}-02$ & $2.2 \mathrm{E}-01$ & 5.9E-02 \\
\hline Pennsylvania & PA & $1.5 \mathrm{E}-02$ & 2.0E-02 & 2.9E-02 & 4.0E-02 & 2.5E-01 & 7.7E-02 \\
\hline Rhode Island & RI & 9.6E-03 & $1.4 \mathrm{E}-03$ & 5.9E-03 & $2.0 \mathrm{E}-02$ & $2.2 \mathrm{E}-01$ & $4.5 \mathrm{E}-02$ \\
\hline Massachusetts & MA & $7.6 \mathrm{E}-03$ & $1.4 \mathrm{E}-03$ & $6.0 \mathrm{E}-03$ & $2.2 \mathrm{E}-02$ & $2.1 \mathrm{E}-01$ & $4.3 \mathrm{E}-02$ \\
\hline New Hampshire & $\mathrm{NH}$ & $5.4 \mathrm{E}-03$ & $1.7 \mathrm{E}-03$ & $1.1 \mathrm{E}-02$ & 3.5E-02 & 3.0E-01 & $6.2 \mathrm{E}-02$ \\
\hline Vermont & $\mathrm{VT}$ & 4.0E-03 & $3.0 \mathrm{E}-03$ & $2.2 \mathrm{E}-02$ & 5.5E-02 & $4.2 \mathrm{E}-01$ & $9.5 \mathrm{E}-02$ \\
\hline Maine & $\mathrm{ME}$ & $3.2 \mathrm{E}-03$ & $3.6 \mathrm{E}-03$ & $2.5 \mathrm{E}-02$ & $6.7 \mathrm{E}-02$ & $4.6 \mathrm{E}-01$ & $1.5 \mathrm{E}-01$ \\
\hline Ohio & $\mathrm{OH}$ & 4.3E-02 & 1.3E-01 & 9.3E-02 & $6.3 \mathrm{E}-02$ & $1.0 \mathrm{E}-01$ & $1.6 \mathrm{E}-01$ \\
\hline Indiana & IN & $1.7 \mathrm{E}-01$ & $2.8 \mathrm{E}-01$ & $3.0 \mathrm{E}-01$ & $1.4 \mathrm{E}-01$ & $1.6 \mathrm{E}-01$ & $2.1 \mathrm{E}-01$ \\
\hline Illinois & $\mathrm{IL}$ & 2.8E-01 & 3.8E-01 & 5.3E-01 & $2.4 \mathrm{E}-01$ & 6.3E-01 & 3.7E-01 \\
\hline Michigan & MI & $9.5 \mathrm{E}-02$ & $6.5 \mathrm{E}-02$ & $1.2 \mathrm{E}-01$ & 7.7E-02 & 1.6E-01 & 2.3E-01 \\
\hline Wisconsin & WI & $1.7 \mathrm{E}-01$ & $2.2 \mathrm{E}-01$ & 2.0E-01 & 1.3E-01 & 2.0E-01 & $3.2 \mathrm{E}-01$ \\
\hline Minnesota & $\mathrm{MN}$ & $2.4 \mathrm{E}-01$ & $2.8 \mathrm{E}-01$ & $2.2 \mathrm{E}-01$ & $2.8 \mathrm{E}-01$ & $3.8 \mathrm{E}-01$ & $6.0 \mathrm{E}-01$ \\
\hline Iowa & IA & 3.2E-01 & 7.2E-01 & $3.2 \mathrm{E}-01$ & 3.3E-01 & $1.7 \mathrm{E}+00$ & 4.7E-01 \\
\hline Missouri & $\mathrm{MO}$ & 7.8E-01 & 7.9E-01 & $2.3 \mathrm{E}+00$ & $6.8 \mathrm{E}-01$ & $1.2 \mathrm{E}+00$ & 7.2E-01 \\
\hline Kansas & $\mathrm{KS}$ & $1.0 \mathrm{E}+00$ & $2.6 \mathrm{E}-01$ & $2.5 \mathrm{E}+00$ & 5.3E-01 & 5.5E-01 & $4.8 \mathrm{E}-01$ \\
\hline Nebraska & $\mathrm{NE}$ & $7.1 \mathrm{E}-01$ & 6.7E-01 & 6.7E-01 & $5.4 \mathrm{E}-01$ & $1.0 \mathrm{E}+00$ & $6.5 \mathrm{E}-01$ \\
\hline North Dakota & $\mathrm{ND}$ & $5.6 \mathrm{E}-01$ & $4.5 \mathrm{E}-01$ & 3.7E-01 & $6.5 \mathrm{E}-01$ & 9.3E-01 & $9.4 \mathrm{E}-01$ \\
\hline South Dakota & SD & 6.6E-01 & 7.0E-01 & $5.4 \mathrm{E}-01$ & $4.6 \mathrm{E}-01$ & 9.2E-01 & $6.2 \mathrm{E}-01$ \\
\hline
\end{tabular}


Table A16 Ecosystem exposure level characterization factors for NOx (July-December)

\begin{tabular}{|c|c|c|c|c|c|c|c|}
\hline State & & July & August & September & October & November & December \\
\hline Oregon & OR & $1.8 \mathrm{E}-01$ & $2.6 \mathrm{E}-01$ & $3.1 \mathrm{E}-01$ & $1.3 \mathrm{E}-01$ & $2.7 \mathrm{E}-01$ & 2.3E-01 \\
\hline Washington & WA & $4.3 \mathrm{E}-01$ & $5.1 \mathrm{E}-01$ & $4.5 \mathrm{E}-01$ & $2.0 \mathrm{E}-01$ & $1.2 \mathrm{E}-01$ & 2.2E-01 \\
\hline Wyoming & WY & $2.8 \mathrm{E}-01$ & $3.9 \mathrm{E}-01$ & $8.4 \mathrm{E}-01$ & $3.5 \mathrm{E}-01$ & $2.0 \mathrm{E}+00$ & $1.3 \mathrm{E}+00$ \\
\hline Idaho & ID & $3.1 \mathrm{E}-01$ & 3.6E-01 & $6.4 \mathrm{E}-01$ & $2.2 \mathrm{E}-01$ & $9.2 \mathrm{E}-01$ & 3.6E-01 \\
\hline Montana & MT & $6.9 \mathrm{E}-01$ & $7.2 \mathrm{E}-01$ & $1.1 \mathrm{E}+00$ & $3.9 \mathrm{E}-01$ & $1.2 \mathrm{E}+00$ & $1.2 \mathrm{E}+00$ \\
\hline California & CA & $1.0 \mathrm{E}-01$ & $1.1 \mathrm{E}-01$ & $1.6 \mathrm{E}-01$ & $7.3 \mathrm{E}-02$ & $2.9 \mathrm{E}-01$ & $9.0 \mathrm{E}-02$ \\
\hline Nevada & NV & $2.2 \mathrm{E}-01$ & $2.9 \mathrm{E}-01$ & $4.2 \mathrm{E}-01$ & $1.6 \mathrm{E}-01$ & $7.7 \mathrm{E}-01$ & $1.6 \mathrm{E}-01$ \\
\hline Utah & UT & $1.7 \mathrm{E}-01$ & $2.0 \mathrm{E}-01$ & $4.2 \mathrm{E}-01$ & $1.7 \mathrm{E}-01$ & $1.1 \mathrm{E}+00$ & $2.5 \mathrm{E}-01$ \\
\hline Colorado & $\mathrm{CO}$ & $2.1 \mathrm{E}-01$ & $3.1 \mathrm{E}-01$ & $6.2 \mathrm{E}-01$ & $3.4 \mathrm{E}-01$ & $2.0 \mathrm{E}+00$ & $1.3 \mathrm{E}+00$ \\
\hline Arizona & $\mathrm{AZ}$ & $1.8 \mathrm{E}-01$ & $1.7 \mathrm{E}-01$ & $3.7 \mathrm{E}-01$ & $1.8 \mathrm{E}-01$ & $8.1 \mathrm{E}-01$ & $1.3 \mathrm{E}-01$ \\
\hline New Mexico & NM & $2.3 \mathrm{E}-01$ & $2.6 \mathrm{E}-01$ & $4.2 \mathrm{E}-01$ & $3.6 \mathrm{E}-01$ & $1.7 \mathrm{E}+00$ & $6.2 \mathrm{E}-01$ \\
\hline Texas & TX & $2.4 \mathrm{E}+00$ & $1.4 \mathrm{E}+01$ & $4.1 \mathrm{E}+00$ & $2.1 \mathrm{E}+00$ & $3.8 \mathrm{E}+00$ & $1.8 \mathrm{E}+00$ \\
\hline Oklahoma & $\mathrm{OK}$ & $4.1 \mathrm{E}-01$ & $1.0 \mathrm{E}+00$ & $6.7 \mathrm{E}-01$ & $7.0 \mathrm{E}-01$ & $2.3 \mathrm{E}+00$ & $1.5 \mathrm{E}+00$ \\
\hline Arkansas & AR & $8.9 \mathrm{E}-01$ & $2.5 \mathrm{E}+00$ & $1.6 \mathrm{E}+00$ & $1.5 \mathrm{E}+00$ & $4.6 \mathrm{E}+00$ & $1.8 \mathrm{E}+00$ \\
\hline Louisiana & $\overline{L A}$ & $2.1 \mathrm{E}+01$ & $5.8 \mathrm{E}+01$ & $1.7 \mathrm{E}+01$ & $1.1 \mathrm{E}+01$ & $1.1 \mathrm{E}+01$ & $2.8 \mathrm{E}+00$ \\
\hline Mississippi & MS & $1.2 \mathrm{E}+01$ & $1.3 \mathrm{E}+01$ & $1.3 \mathrm{E}+01$ & $\overline{5.7 \mathrm{E}+00}$ & $2.8 \mathrm{E}+01$ & $2.8 \mathrm{E}+00$ \\
\hline Alabama & $\mathrm{AL}$ & $3.5 \mathrm{E}+00$ & $2.4 \mathrm{E}+00$ & $1.3 \mathrm{E}+01$ & $4.0 \mathrm{E}+00$ & $2.1 \mathrm{E}+01$ & 7.5E-01 \\
\hline Kentucky & $\mathrm{KY}$ & $4.0 \mathrm{E}-01$ & $3.0 \mathrm{E}-01$ & $8.2 \mathrm{E}-01$ & $1.8 \mathrm{E}-01$ & $1.9 \mathrm{E}+00$ & $2.9 \mathrm{E}-01$ \\
\hline Tennessee & $\mathrm{TN}$ & 7.7E-01 & $4.4 \mathrm{E}-01$ & $2.8 \mathrm{E}+00$ & 3.3E-01 & $5.0 \mathrm{E}+00$ & $4.1 \mathrm{E}-01$ \\
\hline Florida & FL & $6.3 \mathrm{E}+00$ & $9.1 \mathrm{E}+00$ & $5.9 \mathrm{E}+00$ & $9.7 \mathrm{E}+00$ & $1.7 \mathrm{E}+00$ & $1.5 \mathrm{E}+00$ \\
\hline Georgia & GA & $1.5 \mathrm{E}+00$ & $2.8 \mathrm{E}-01$ & $8.1 \mathrm{E}+00$ & $1.7 \mathrm{E}+00$ & $5.5 \mathrm{E}+00$ & $7.8 \mathrm{E}-01$ \\
\hline North Carolina & $\mathrm{NC}$ & $9.7 \mathrm{E}-01$ & $3.8 \mathrm{E}-02$ & $4.3 \mathrm{E}+00$ & $5.9 \mathrm{E}-01$ & $1.4 \mathrm{E}+00$ & $3.4 \mathrm{E}-01$ \\
\hline South Carolina & $\mathrm{SC}$ & $8.3 \mathrm{E}-01$ & $1.2 \mathrm{E}-01$ & $5.3 \mathrm{E}+00$ & $1.9 \mathrm{E}+00$ & $1.8 \mathrm{E}+00$ & $7.8 \mathrm{E}-01$ \\
\hline Virginia & VA & $8.4 \mathrm{E}-01$ & $2.0 \mathrm{E}-02$ & $2.7 \mathrm{E}+00$ & $1.9 \mathrm{E}-01$ & 4.4E-01 & $1.0 \mathrm{E}-01$ \\
\hline West Virginia & WV & 3.3E-01 & $4.2 \mathrm{E}-02$ & $8.7 \mathrm{E}-01$ & $1.5 \mathrm{E}-01$ & $2.6 \mathrm{E}-01$ & $1.2 \mathrm{E}-01$ \\
\hline Delaware & $\mathrm{DE}$ & $1.3 \mathrm{E}+00$ & $1.6 \mathrm{E}-02$ & $2.6 \mathrm{E}+00$ & $2.0 \mathrm{E}-01$ & $3.9 \mathrm{E}-01$ & $3.0 \mathrm{E}-03$ \\
\hline Washington DC & $\mathrm{DC}$ & 5.6E-01 & $1.1 \mathrm{E}-02$ & $1.5 \mathrm{E}+00$ & $1.1 \mathrm{E}-01$ & $2.0 \mathrm{E}-01$ & $7.8 \mathrm{E}-03$ \\
\hline Maryland & $\mathrm{MD}$ & $7.4 \mathrm{E}-01$ & $1.4 \mathrm{E}-02$ & $1.8 \mathrm{E}+00$ & $1.4 \mathrm{E}-01$ & $2.5 \mathrm{E}-01$ & $6.0 \mathrm{E}-03$ \\
\hline Connecticut & CT & $1.2 \mathrm{E}-01$ & $2.7 \mathrm{E}-02$ & $1.2 \mathrm{E}+00$ & $9.7 \mathrm{E}-02$ & $1.4 \mathrm{E}-01$ & $8.4 \mathrm{E}-04$ \\
\hline New Jersey & NJ & $3.1 \mathrm{E}-01$ & $1.5 \mathrm{E}-02$ & $1.2 \mathrm{E}+00$ & $1.4 \mathrm{E}-01$ & $2.8 \mathrm{E}-01$ & $1.0 \mathrm{E}-03$ \\
\hline New York & NY & $1.5 \mathrm{E}-01$ & $6.2 \mathrm{E}-02$ & $9.8 \mathrm{E}-01$ & $8.6 \mathrm{E}-02$ & $2.0 \mathrm{E}-01$ & $2.2 \mathrm{E}-03$ \\
\hline Pennsylvania & PA & $3.0 \mathrm{E}-01$ & $2.8 \mathrm{E}-02$ & $1.2 \mathrm{E}+00$ & $1.2 \mathrm{E}-01$ & $2.3 \mathrm{E}-01$ & $7.5 \mathrm{E}-03$ \\
\hline Rhode Island & RI & $6.7 \mathrm{E}-02$ & $3.2 \mathrm{E}-02$ & $1.1 \mathrm{E}+00$ & $7.3 \mathrm{E}-02$ & $4.9 \mathrm{E}-02$ & $8.6 \mathrm{E}-04$ \\
\hline Massachusetts & MA & $7.5 \mathrm{E}-02$ & $3.6 \mathrm{E}-02$ & $1.0 \mathrm{E}+00$ & $6.2 \mathrm{E}-02$ & $4.4 \mathrm{E}-02$ & $8.8 \mathrm{E}-04$ \\
\hline New Hampshire & $\mathrm{NH}$ & $1.2 \mathrm{E}-01$ & $7.2 \mathrm{E}-02$ & $9.3 \mathrm{E}-01$ & $6.5 \mathrm{E}-02$ & $4.4 \mathrm{E}-02$ & $1.1 \mathrm{E}-03$ \\
\hline Vermont & VT & $1.6 \mathrm{E}-01$ & $1.4 \mathrm{E}-01$ & $7.4 \mathrm{E}-01$ & $7.5 \mathrm{E}-02$ & $8.8 \mathrm{E}-02$ & $2.0 \mathrm{E}-03$ \\
\hline Maine & $\mathrm{ME}$ & $4.2 \mathrm{E}-01$ & $1.8 \mathrm{E}-01$ & $7.0 \mathrm{E}-01$ & $5.1 \mathrm{E}-02$ & $1.9 \mathrm{E}-02$ & $2.5 \mathrm{E}-03$ \\
\hline Ohio & $\mathrm{OH}$ & $2.2 \mathrm{E}-01$ & $7.1 \mathrm{E}-02$ & $4.2 \mathrm{E}-01$ & $1.3 \mathrm{E}-01$ & $1.3 \mathrm{E}-01$ & 6.7E-02 \\
\hline Indiana & IN & $2.4 \mathrm{E}-01$ & $2.5 \mathrm{E}-01$ & $4.1 \mathrm{E}-01$ & $1.6 \mathrm{E}-01$ & $4.2 \mathrm{E}-01$ & 1.7E-01 \\
\hline Illinois & IL & $2.7 \mathrm{E}-01$ & $3.5 \mathrm{E}-01$ & $4.1 \mathrm{E}-01$ & $1.5 \mathrm{E}-01$ & $7.7 \mathrm{E}-01$ & $2.7 \mathrm{E}-01$ \\
\hline Michigan & MI & $3.1 \mathrm{E}-01$ & $2.1 \mathrm{E}-01$ & $5.2 \mathrm{E}-01$ & $1.9 \mathrm{E}-01$ & $9.2 \mathrm{E}-02$ & $4.7 \mathrm{E}-02$ \\
\hline Wisconsin & $\overline{\mathrm{WI}}$ & $4.6 \mathrm{E}-01$ & $3.4 \mathrm{E}-01$ & $4.2 \mathrm{E}-01$ & $2.6 \mathrm{E}-01$ & $2.3 \mathrm{E}-01$ & $8.3 \mathrm{E}-02$ \\
\hline Minnesota & MN & 7.2E-01 & $5.6 \mathrm{E}-01$ & $6.5 \mathrm{E}-01$ & $2.6 \mathrm{E}-01$ & $3.8 \mathrm{E}-01$ & $1.1 \mathrm{E}-01$ \\
\hline Iowa & IA & $4.1 \mathrm{E}-01$ & $4.6 \mathrm{E}-01$ & $6.3 \mathrm{E}-01$ & $1.7 \mathrm{E}-01$ & $7.1 \mathrm{E}-01$ & $4.3 \mathrm{E}-01$ \\
\hline Missouri & $\mathrm{MO}$ & $2.7 \mathrm{E}-01$ & $7.7 \mathrm{E}-01$ & $5.2 \mathrm{E}-01$ & $1.7 \mathrm{E}-01$ & $2.1 \mathrm{E}+00$ & $8.1 \mathrm{E}-01$ \\
\hline Kansas & KS & $3.0 \mathrm{E}-01$ & $6.9 \mathrm{E}-01$ & $7.4 \mathrm{E}-01$ & $5.1 \mathrm{E}-01$ & $1.8 \mathrm{E}+00$ & $1.4 \mathrm{E}+00$ \\
\hline Nebraska & $\mathrm{NE}$ & $3.5 \mathrm{E}-01$ & $5.4 \mathrm{E}-01$ & $9.6 \mathrm{E}-01$ & $2.8 \mathrm{E}-01$ & $1.2 \mathrm{E}+00$ & $1.1 \mathrm{E}+00$ \\
\hline North Dakota & ND & $1.2 \mathrm{E}+00$ & $1.0 \mathrm{E}+00$ & $1.3 \mathrm{E}+00$ & $2.9 \mathrm{E}-01$ & $6.1 \mathrm{E}-01$ & 9.1E-01 \\
\hline South Dakota & SD & $7.2 \mathrm{E}-01$ & 7.6E-01 & $1.2 \mathrm{E}+00$ & $2.7 \mathrm{E}-01$ & $1.4 \mathrm{E}+00$ & $8.7 \mathrm{E}-01$ \\
\hline
\end{tabular}


Table A17 Ecosystem exposure level characterization factors for VOC (January-June)

\begin{tabular}{|c|c|c|c|c|c|c|c|}
\hline State & & January & February & March & April & May & June \\
\hline Oregon & OR & 9.5E-02 & $3.4 \mathrm{E}-02$ & $3.1 \mathrm{E}-02$ & $1.3 \mathrm{E}-02$ & 4.3E-03 & $5.6 \mathrm{E}-03$ \\
\hline Washington & WA & $1.2 \mathrm{E}-01$ & $4.1 \mathrm{E}-02$ & $4.4 \mathrm{E}-02$ & $3.1 \mathrm{E}-02$ & $2.5 \mathrm{E}-02$ & 3.0E-02 \\
\hline Wyoming & WY & 4.1E-01 & $2.4 \mathrm{E}-01$ & $1.5 \mathrm{E}-01$ & $2.5 \mathrm{E}-02$ & $1.0 \mathrm{E}-02$ & 7.7E-03 \\
\hline Idaho & ID & $1.9 \mathrm{E}-01$ & $1.3 \mathrm{E}-01$ & $6.0 \mathrm{E}-02$ & $1.8 \mathrm{E}-02$ & $5.4 \mathrm{E}-03$ & $6.2 \mathrm{E}-03$ \\
\hline Montana & MT & 3.2E-01 & 2.1E-01 & 9.1E-02 & $3.0 \mathrm{E}-02$ & $1.0 \mathrm{E}-02$ & $6.4 \mathrm{E}-03$ \\
\hline California & CA & $2.5 \mathrm{E}-02$ & $4.1 \mathrm{E}-02$ & $3.2 \mathrm{E}-02$ & $7.2 \mathrm{E}-03$ & 5.5E-03 & $4.0 \mathrm{E}-03$ \\
\hline Nevada & $\mathrm{NV}$ & 3.5E-02 & $5.7 \mathrm{E}-02$ & 4.0E-02 & $7.0 \mathrm{E}-03$ & $3.0 \mathrm{E}-03$ & $1.8 \mathrm{E}-03$ \\
\hline Utah & $\mathrm{UT}$ & $1.1 \mathrm{E}-01$ & $1.3 \mathrm{E}-01$ & 8.5E-02 & $1.6 \mathrm{E}-02$ & 9.8E-03 & $6.2 \mathrm{E}-03$ \\
\hline Colorado & $\mathrm{CO}$ & $4.4 \mathrm{E}-01$ & $1.6 \mathrm{E}-01$ & $1.5 \mathrm{E}-01$ & $2.1 \mathrm{E}-02$ & $1.3 \mathrm{E}-02$ & $7.4 \mathrm{E}-03$ \\
\hline Arizona & $\mathrm{AZ}$ & $2.7 \mathrm{E}-02$ & $7.0 \mathrm{E}-02$ & $4.8 \mathrm{E}-02$ & $6.7 \mathrm{E}-03$ & $5.5 \mathrm{E}-03$ & $2.8 \mathrm{E}-03$ \\
\hline New Mexico & NM & 3.6E-01 & $8.4 \mathrm{E}-02$ & $9.4 \mathrm{E}-02$ & $8.1 \mathrm{E}-03$ & $6.2 \mathrm{E}-03$ & $3.2 \mathrm{E}-03$ \\
\hline Texas & $\mathrm{TX}$ & $1.3 \mathrm{E}+00$ & $2.1 \mathrm{E}-01$ & $6.4 \mathrm{E}-01$ & $7.6 \mathrm{E}-02$ & $1.8 \mathrm{E}-01$ & $3.1 \mathrm{E}-01$ \\
\hline Oklahoma & $\mathrm{OK}$ & $1.1 \mathrm{E}+00$ & $7.4 \mathrm{E}-02$ & 7.2E-01 & $2.4 \mathrm{E}-02$ & $1.3 \mathrm{E}-02$ & $6.0 \mathrm{E}-03$ \\
\hline Arkansas & AR & $1.2 \mathrm{E}+00$ & $3.8 \mathrm{E}-01$ & 4.7E-01 & 5.3E-02 & $4.7 \mathrm{E}-02$ & $1.6 \mathrm{E}-02$ \\
\hline Louisiana & LA & $2.1 \mathrm{E}+00$ & 7.0E-01 & $1.4 \mathrm{E}+00$ & 3.9E-01 & $2.8 \mathrm{E}-01$ & $1.2 \mathrm{E}+00$ \\
\hline Mississippi & MS & $1.8 \mathrm{E}+00$ & $5.2 \mathrm{E}-01$ & $4.3 \mathrm{E}-01$ & $1.1 \mathrm{E}-01$ & $8.0 \mathrm{E}-02$ & $7.3 \mathrm{E}-02$ \\
\hline Alabama & $\mathrm{AL}$ & $1.1 \mathrm{E}+00$ & $5.4 \mathrm{E}-01$ & $1.3 \mathrm{E}-01$ & 6.8E-02 & $2.0 \mathrm{E}-02$ & $2.3 E-02$ \\
\hline Kentucky & KY & $2.7 \mathrm{E}-01$ & $2.7 E-01$ & 4.0E-01 & 4.6E-02 & $1.1 \mathrm{E}-02$ & $1.3 \mathrm{E}-02$ \\
\hline Tennessee & $\mathrm{TN}$ & 7.4E-01 & 3.5E-01 & $1.8 \mathrm{E}-01$ & 4.1E-02 & $1.1 \mathrm{E}-02$ & $1.3 \mathrm{E}-02$ \\
\hline Florida & FL & 2.9E-01 & $2.1 \mathrm{E}-01$ & $4.8 \mathrm{E}-02$ & $1.7 \mathrm{E}-01$ & $1.4 \mathrm{E}-01$ & $3.2 \mathrm{E}-02$ \\
\hline Georgia & GA & $6.6 \mathrm{E}-01$ & $1.8 \mathrm{E}-01$ & 4.0E-02 & $2.6 \mathrm{E}-02$ & 7.5E-03 & $7.6 \mathrm{E}-03$ \\
\hline North Carolina & $\mathrm{NC}$ & 7.7E-02 & $1.1 \mathrm{E}-01$ & $3.5 \mathrm{E}-02$ & $1.7 \mathrm{E}-02$ & $2.2 \mathrm{E}-02$ & $3.7 \mathrm{E}-03$ \\
\hline South Carolina & SC & $1.6 \mathrm{E}-01$ & $1.2 \mathrm{E}-01$ & $2.1 \mathrm{E}-02$ & $1.6 \mathrm{E}-02$ & $1.2 \mathrm{E}-02$ & $3.8 \mathrm{E}-03$ \\
\hline Virginia & VA & 2.5E-02 & 5.9E-02 & 5.7E-02 & $1.2 \mathrm{E}-02$ & $3.6 \mathrm{E}-02$ & $4.5 \mathrm{E}-03$ \\
\hline West Virginia & WV & 2.7E-02 & 9.3E-02 & $1.6 \mathrm{E}-01$ & 2.1E-02 & 1.3E-02 & 5.3E-03 \\
\hline Delaware & $\mathrm{DE}$ & 5.5E-03 & $6.7 \mathrm{E}-03$ & $2.8 \mathrm{E}-02$ & $8.4 \mathrm{E}-03$ & $3.1 \mathrm{E}-02$ & $3.1 \mathrm{E}-03$ \\
\hline Washington DC & $\mathrm{DC}$ & $1.1 \mathrm{E}-02$ & $1.5 \mathrm{E}-02$ & $5.0 \mathrm{E}-02$ & $8.5 \mathrm{E}-03$ & $4.5 \mathrm{E}-02$ & $5.6 \mathrm{E}-03$ \\
\hline Maryland & $\mathrm{MD}$ & $9.1 \mathrm{E}-03$ & $1.2 \mathrm{E}-02$ & $4.4 \mathrm{E}-02$ & $8.9 \mathrm{E}-03$ & $3.9 \mathrm{E}-02$ & $4.2 \mathrm{E}-03$ \\
\hline Connecticut & $\mathrm{CT}$ & $2.0 \mathrm{E}-03$ & $1.4 \mathrm{E}-03$ & 8.8E-03 & $6.7 \mathrm{E}-03$ & $2.4 \mathrm{E}-02$ & $1.7 \mathrm{E}-03$ \\
\hline New Jersey & $\mathrm{NJ}$ & $2.6 \mathrm{E}-03$ & $2.6 \mathrm{E}-03$ & $2.1 \mathrm{E}-02$ & $7.3 \mathrm{E}-03$ & $2.7 \mathrm{E}-02$ & $2.6 \mathrm{E}-03$ \\
\hline New York & NY & 3.7E-03 & $3.1 \mathrm{E}-03$ & 2.3E-02 & 9.6E-03 & $1.5 \mathrm{E}-02$ & 1.7E-03 \\
\hline Pennsylvania & PA & 7.6E-03 & $1.2 \mathrm{E}-02$ & $5.2 \mathrm{E}-02$ & $1.0 \mathrm{E}-02$ & $2.2 \mathrm{E}-02$ & $2.8 \mathrm{E}-03$ \\
\hline Rhode Island & RI & $1.5 \mathrm{E}-03$ & $6.5 \mathrm{E}-04$ & $3.7 \mathrm{E}-03$ & $5.4 \mathrm{E}-03$ & $2.4 \mathrm{E}-02$ & $1.6 \mathrm{E}-03$ \\
\hline Massachusetts & MA & $1.3 \mathrm{E}-03$ & $7.2 \mathrm{E}-04$ & $3.7 \mathrm{E}-03$ & $6.3 \mathrm{E}-03$ & $2.2 \mathrm{E}-02$ & $1.7 \mathrm{E}-03$ \\
\hline New Hampshire & $\mathrm{NH}$ & $1.1 \mathrm{E}-03$ & $1.1 \mathrm{E}-03$ & $4.6 \mathrm{E}-03$ & $8.4 \mathrm{E}-03$ & $1.5 \mathrm{E}-02$ & $6.7 \mathrm{E}-04$ \\
\hline Vermont & $\mathrm{VT}$ & $1.4 \mathrm{E}-03$ & $1.9 \mathrm{E}-03$ & 8.7E-03 & $1.1 \mathrm{E}-02$ & $6.4 \mathrm{E}-03$ & 3.7E-04 \\
\hline Maine & $\mathrm{ME}$ & 8.7E-04 & $1.2 \mathrm{E}-03$ & 3.6E-03 & $6.2 \mathrm{E}-03$ & 7.3E-03 & 1.7E-04 \\
\hline Ohio & $\mathrm{OH}$ & 4.7E-02 & $1.3 \mathrm{E}-01$ & $1.9 \mathrm{E}-01$ & $2.0 \mathrm{E}-02$ & 9.4E-03 & $6.8 \mathrm{E}-03$ \\
\hline Indiana & IN & $2.2 \mathrm{E}-01$ & 3.3E-01 & $4.1 \mathrm{E}-01$ & 3.7E-02 & 1.5E-02 & $1.4 \mathrm{E}-02$ \\
\hline Illinois & $\mathrm{IL}$ & 3.3E-01 & 3.6E-01 & 4.2E-01 & 4.7E-02 & 2.7E-02 & $1.5 \mathrm{E}-02$ \\
\hline Michigan & MI & $1.6 \mathrm{E}-01$ & $1.0 \mathrm{E}-01$ & $1.5 \mathrm{E}-01$ & $1.8 \mathrm{E}-02$ & 9.2E-03 & $6.1 \mathrm{E}-03$ \\
\hline Wisconsin & WI & $3.1 \mathrm{E}-01$ & 3.0E-01 & $2.2 \mathrm{E}-01$ & $3.2 \mathrm{E}-02$ & 9.9E-03 & $5.8 \mathrm{E}-03$ \\
\hline Minnesota & $\mathrm{MN}$ & 3.1E-01 & 3.5E-01 & 1.5E-01 & 5.8E-02 & 1.3E-02 & 5.7E-03 \\
\hline Iowa & IA & 4.3E-01 & 6.3E-01 & $2.1 \mathrm{E}-01$ & 4.3E-02 & 4.6E-02 & $6.8 \mathrm{E}-03$ \\
\hline Missouri & $\mathrm{MO}$ & 7.3E-01 & $4.5 \mathrm{E}-01$ & $8.6 \mathrm{E}-01$ & 7.2E-02 & 4.3E-02 & $1.5 \mathrm{E}-02$ \\
\hline Kansas & $\mathrm{KS}$ & 7.5E-01 & $1.2 \mathrm{E}-01$ & $6.1 \mathrm{E}-01$ & $3.9 \mathrm{E}-02$ & $1.5 \mathrm{E}-02$ & $7.9 \mathrm{E}-03$ \\
\hline Nebraska & $\mathrm{NE}$ & $4.8 \mathrm{E}-01$ & $4.7 \mathrm{E}-01$ & $1.7 \mathrm{E}-01$ & 5.0E-02 & 3.3E-02 & $1.1 \mathrm{E}-02$ \\
\hline North Dakota & $\mathrm{ND}$ & $2.6 \mathrm{E}-01$ & 3.6E-01 & $1.1 \mathrm{E}-01$ & 7.1E-02 & 1.5E-02 & $9.1 \mathrm{E}-03$ \\
\hline South Dakota & $\mathrm{SD}$ & 4.0E-01 & 5.0E-01 & $1.5 \mathrm{E}-01$ & 5.5E-02 & 2.5E-02 & 6.3E-03 \\
\hline
\end{tabular}


Table A18 Ecosystem exposure level characterization factors for VOC (July-December)

\begin{tabular}{|c|c|c|c|c|c|c|c|}
\hline State & & July & August & September & October & November & December \\
\hline Oregon & OR & $2.0 \mathrm{E}-03$ & $6.4 \mathrm{E}-03$ & $6.3 \mathrm{E}-03$ & $1.2 \mathrm{E}-02$ & 4.3E-02 & 8.9E-02 \\
\hline Washington & WA & $2.0 \mathrm{E}-02$ & $2.1 \mathrm{E}-02$ & $2.3 \mathrm{E}-02$ & $3.0 \mathrm{E}-02$ & $3.3 \mathrm{E}-02$ & $1.1 \mathrm{E}-01$ \\
\hline Wyoming & WY & $1.8 \mathrm{E}-03$ & $8.8 \mathrm{E}-03$ & $2.6 \mathrm{E}-02$ & $4.6 \mathrm{E}-02$ & $4.7 \mathrm{E}-01$ & $6.1 \mathrm{E}-01$ \\
\hline Idaho & ID & $1.5 \mathrm{E}-03$ & $4.2 \mathrm{E}-03$ & $1.5 \mathrm{E}-02$ & $2.2 \mathrm{E}-02$ & $2.1 \mathrm{E}-01$ & $1.7 \mathrm{E}-01$ \\
\hline Montana & MT & $2.3 \mathrm{E}-03$ & $7.2 \mathrm{E}-03$ & $2.5 \mathrm{E}-02$ & $4.4 \mathrm{E}-02$ & $1.9 \mathrm{E}-01$ & $6.2 \mathrm{E}-01$ \\
\hline California & CA & $4.3 \mathrm{E}-03$ & 5.7E-03 & $1.1 \mathrm{E}-02$ & $6.5 \mathrm{E}-03$ & $6.8 \mathrm{E}-02$ & $4.2 \mathrm{E}-02$ \\
\hline Nevada & NV & $1.7 \mathrm{E}-03$ & $5.4 \mathrm{E}-03$ & $8.4 \mathrm{E}-03$ & 5.7E-03 & $1.4 \mathrm{E}-01$ & $5.5 \mathrm{E}-02$ \\
\hline Utah & UT & $2.3 \mathrm{E}-03$ & $6.9 \mathrm{E}-03$ & $2.0 \mathrm{E}-02$ & $1.6 \mathrm{E}-02$ & $3.2 \mathrm{E}-01$ & $1.2 \mathrm{E}-01$ \\
\hline Colorado & $\mathrm{CO}$ & $3.0 \mathrm{E}-03$ & $1.0 \mathrm{E}-02$ & $2.7 \mathrm{E}-02$ & $3.6 \mathrm{E}-02$ & $5.0 \mathrm{E}-01$ & $5.8 \mathrm{E}-01$ \\
\hline Arizona & $\mathrm{AZ}$ & $2.5 \mathrm{E}-03$ & $4.2 \mathrm{E}-03$ & $9.2 \mathrm{E}-03$ & 7.6E-03 & $8.7 \mathrm{E}-02$ & $3.2 \mathrm{E}-02$ \\
\hline New Mexico & NM & $1.9 \mathrm{E}-03$ & $7.2 \mathrm{E}-03$ & $1.1 \mathrm{E}-02$ & $1.5 \mathrm{E}-02$ & $2.5 \mathrm{E}-01$ & $2.0 \mathrm{E}-01$ \\
\hline Texas & TX & $1.3 \mathrm{E}-01$ & $1.1 \mathrm{E}+00$ & 3.3E-01 & $2.5 \mathrm{E}-01$ & 5.7E-01 & $8.1 \mathrm{E}-01$ \\
\hline Oklahoma & $\mathrm{OK}$ & $3.2 \mathrm{E}-03$ & $3.3 \mathrm{E}-02$ & $2.3 \mathrm{E}-02$ & $8.0 \mathrm{E}-02$ & $3.9 \mathrm{E}-01$ & 7.7E-01 \\
\hline Arkansas & AR & $4.4 \mathrm{E}-03$ & $8.0 \mathrm{E}-02$ & $5.2 \mathrm{E}-02$ & $1.5 \mathrm{E}-01$ & 7.6E-01 & $1.2 \mathrm{E}+00$ \\
\hline Louisiana & LA & $1.6 \mathrm{E}+00$ & $3.6 \mathrm{E}+00$ & $1.3 \mathrm{E}+00$ & $1.8 \mathrm{E}+00$ & $1.7 \mathrm{E}+00$ & $1.1 \mathrm{E}+00$ \\
\hline Mississippi & MS & $1.1 \mathrm{E}-01$ & $2.6 \mathrm{E}-01$ & $2.0 \mathrm{E}-01$ & 4.6E-01 & $2.0 \mathrm{E}+00$ & $1.0 \mathrm{E}+00$ \\
\hline Alabama & $\mathrm{AL}$ & $1.9 \mathrm{E}-02$ & $6.0 \mathrm{E}-02$ & $1.9 \mathrm{E}-01$ & $3.0 \mathrm{E}-01$ & $1.9 \mathrm{E}+00$ & $2.0 \mathrm{E}-01$ \\
\hline Kentucky & KY & $1.1 \mathrm{E}-02$ & $2.7 \mathrm{E}-02$ & $1.1 \mathrm{E}-01$ & $9.3 \mathrm{E}-02$ & $5.2 \mathrm{E}-01$ & $2.7 \mathrm{E}-01$ \\
\hline Tennessee & $\mathrm{TN}$ & $5.5 \mathrm{E}-03$ & $2.1 \mathrm{E}-02$ & $1.3 \mathrm{E}-01$ & $1.1 \mathrm{E}-01$ & $1.3 \mathrm{E}+00$ & $2.7 \mathrm{E}-01$ \\
\hline Florida & FL & $2.0 \mathrm{E}-01$ & 4.6E-01 & $1.0 \mathrm{E}-01$ & 6.3E-01 & $1.1 \mathrm{E}-01$ & $9.2 \mathrm{E}-02$ \\
\hline Georgia & GA & $9.6 \mathrm{E}-03$ & $1.1 \mathrm{E}-02$ & $2.3 \mathrm{E}-01$ & $2.3 \mathrm{E}-01$ & $1.1 \mathrm{E}+00$ & $2.2 \mathrm{E}-01$ \\
\hline North Carolina & $\mathrm{NC}$ & $5.8 \mathrm{E}-03$ & $1.1 \mathrm{E}-03$ & $1.6 \mathrm{E}-01$ & $1.1 \mathrm{E}-01$ & 5.9E-01 & $1.5 \mathrm{E}-01$ \\
\hline South Carolina & SC & $4.8 \mathrm{E}-03$ & $2.0 \mathrm{E}-03$ & $1.4 \mathrm{E}-01$ & $2.1 \mathrm{E}-01$ & $5.5 \mathrm{E}-01$ & $2.4 \mathrm{E}-01$ \\
\hline Virginia & $\mathrm{VA}$ & $1.3 \mathrm{E}-02$ & $1.1 \mathrm{E}-03$ & $1.9 \mathrm{E}-01$ & $4.6 \mathrm{E}-02$ & $2.7 \mathrm{E}-01$ & $6.1 \mathrm{E}-02$ \\
\hline West Virginia & WV & $1.4 \mathrm{E}-02$ & $6.5 \mathrm{E}-03$ & $1.1 \mathrm{E}-01$ & $5.6 \mathrm{E}-02$ & $1.3 \mathrm{E}-01$ & $1.1 \mathrm{E}-01$ \\
\hline Delaware & $\mathrm{DE}$ & $8.9 \mathrm{E}-03$ & $6.7 \mathrm{E}-04$ & $1.7 \mathrm{E}-01$ & $3.3 \mathrm{E}-02$ & $2.8 \mathrm{E}-01$ & $1.6 \mathrm{E}-03$ \\
\hline Washington DC & DC & $2.3 \mathrm{E}-02$ & $1.2 \mathrm{E}-03$ & $2.8 \mathrm{E}-01$ & $2.4 \mathrm{E}-02$ & $2.1 \mathrm{E}-01$ & $5.3 \mathrm{E}-03$ \\
\hline Maryland & MD & $1.6 \mathrm{E}-02$ & $1.1 \mathrm{E}-03$ & $2.4 \mathrm{E}-01$ & $2.7 \mathrm{E}-02$ & $2.4 \mathrm{E}-01$ & $3.9 \mathrm{E}-03$ \\
\hline Connecticut & CT & $4.7 \mathrm{E}-03$ & $2.0 \mathrm{E}-03$ & $1.3 \mathrm{E}-01$ & $2.3 \mathrm{E}-02$ & $5.7 \mathrm{E}-02$ & $3.7 \mathrm{E}-04$ \\
\hline New Jersey & NJ & $1.1 \mathrm{E}-02$ & $2.0 \mathrm{E}-03$ & $1.9 \mathrm{E}-01$ & $3.1 \mathrm{E}-02$ & $1.6 \mathrm{E}-01$ & $5.8 \mathrm{E}-04$ \\
\hline New York & NY & $7.5 \mathrm{E}-03$ & $3.2 \mathrm{E}-03$ & $1.4 \mathrm{E}-01$ & $2.3 \mathrm{E}-02$ & $1.4 \mathrm{E}-01$ & $2.1 \mathrm{E}-03$ \\
\hline Pennsylvania & PA & $1.4 \mathrm{E}-02$ & $3.1 \mathrm{E}-03$ & $2.0 \mathrm{E}-01$ & $3.2 \mathrm{E}-02$ & $1.9 \mathrm{E}-01$ & $6.4 \mathrm{E}-03$ \\
\hline Rhode Island & RI & $2.2 \mathrm{E}-03$ & $2.2 \mathrm{E}-03$ & $8.7 \mathrm{E}-02$ & $1.4 \mathrm{E}-02$ & $1.4 \mathrm{E}-02$ & $2.5 \mathrm{E}-04$ \\
\hline Massachusetts & MA & $2.9 \mathrm{E}-03$ & $2.4 \mathrm{E}-03$ & $8.7 \mathrm{E}-02$ & $1.2 \mathrm{E}-02$ & $1.4 \mathrm{E}-02$ & $2.9 \mathrm{E}-04$ \\
\hline New Hampshire & $\mathrm{NH}$ & $1.9 \mathrm{E}-03$ & $1.4 \mathrm{E}-03$ & $6.7 \mathrm{E}-02$ & $9.6 \mathrm{E}-03$ & $1.3 \mathrm{E}-02$ & $4.8 \mathrm{E}-04$ \\
\hline Vermont & VT & $1.5 \mathrm{E}-03$ & $1.0 \mathrm{E}-03$ & $5.4 \mathrm{E}-02$ & $8.8 \mathrm{E}-03$ & $2.5 \mathrm{E}-02$ & $1.0 \mathrm{E}-03$ \\
\hline Maine & ME & $1.0 \mathrm{E}-03$ & 7.1E-04 & 2.3E-02 & $2.8 \mathrm{E}-03$ & $2.4 \mathrm{E}-03$ & $4.9 \mathrm{E}-04$ \\
\hline Ohio & $\mathrm{OH}$ & $1.9 \mathrm{E}-02$ & $1.5 \mathrm{E}-02$ & $1.1 \mathrm{E}-01$ & $5.3 \mathrm{E}-02$ & $8.0 \mathrm{E}-02$ & $6.8 \mathrm{E}-02$ \\
\hline Indiana & IN & $1.5 \mathrm{E}-02$ & $3.4 \mathrm{E}-02$ & $1.1 \mathrm{E}-01$ & $1.1 \mathrm{E}-01$ & $1.8 \mathrm{E}-01$ & $1.9 \mathrm{E}-01$ \\
\hline Illinois & IL & $1.7 \mathrm{E}-02$ & $3.1 \mathrm{E}-02$ & $8.1 \mathrm{E}-02$ & $1.1 \mathrm{E}-01$ & $3.2 \mathrm{E}-01$ & $2.5 \mathrm{E}-01$ \\
\hline Michigan & MI & $2.1 \mathrm{E}-02$ & $1.4 \mathrm{E}-02$ & 1.3E-01 & $7.9 \mathrm{E}-02$ & $7.4 \mathrm{E}-02$ & $5.1 \mathrm{E}-02$ \\
\hline Wisconsin & WI & $1.4 \mathrm{E}-02$ & $1.4 \mathrm{E}-02$ & $5.7 \mathrm{E}-02$ & $1.3 \mathrm{E}-01$ & $1.6 \mathrm{E}-01$ & $1.1 \mathrm{E}-01$ \\
\hline Minnesota & MN & $8.9 \mathrm{E}-03$ & $1.3 \mathrm{E}-02$ & $5.5 \mathrm{E}-02$ & $1.0 \mathrm{E}-01$ & $1.8 \mathrm{E}-01$ & $1.3 \mathrm{E}-01$ \\
\hline Iowa & IA & $5.3 \mathrm{E}-03$ & $2.1 \mathrm{E}-02$ & $5.2 \mathrm{E}-02$ & $6.4 \mathrm{E}-02$ & $2.3 \mathrm{E}-01$ & $3.6 \mathrm{E}-01$ \\
\hline Missouri & $\mathrm{MO}$ & $4.4 \mathrm{E}-03$ & $5.1 \mathrm{E}-02$ & $5.0 \mathrm{E}-02$ & $4.9 \mathrm{E}-02$ & $5.7 \mathrm{E}-01$ & $7.5 \mathrm{E}-01$ \\
\hline Kansas & $\mathrm{KS}$ & $2.1 \mathrm{E}-03$ & $2.6 \mathrm{E}-02$ & $4.4 \mathrm{E}-02$ & $8.7 \mathrm{E}-02$ & $3.8 \mathrm{E}-01$ & $1.0 \mathrm{E}+00$ \\
\hline Nebraska & NE & $3.1 \mathrm{E}-03$ & $2.0 \mathrm{E}-02$ & $5.2 \mathrm{E}-02$ & $4.9 \mathrm{E}-02$ & $2.8 \mathrm{E}-01$ & $7.1 \mathrm{E}-01$ \\
\hline North Dakota & ND & $7.2 \mathrm{E}-03$ & $1.6 \mathrm{E}-02$ & $4.8 \mathrm{E}-02$ & $7.9 \mathrm{E}-02$ & $1.8 \mathrm{E}-01$ & $4.3 \mathrm{E}-01$ \\
\hline South Dakota & SD & $3.4 \mathrm{E}-03$ & $1.1 \mathrm{E}-02$ & $4.1 \mathrm{E}-02$ & $5.2 \mathrm{E}-02$ & $3.0 \mathrm{E}-01$ & $5.8 \mathrm{E}-01$ \\
\hline
\end{tabular}


Table A19 Human effect level characterization factors for NOx (January-June)

\begin{tabular}{|c|c|c|c|c|c|c|c|}
\hline State & & January & February & March & April & May & June \\
\hline Oregon & OR & $4.7 \mathrm{E}-05$ & $9.5 \mathrm{E}-05$ & $1.9 \mathrm{E}-04$ & $2.9 \mathrm{E}-04$ & $5.1 \mathrm{E}-04$ & $5.5 \mathrm{E}-04$ \\
\hline Washington & WA & $2.2 \mathrm{E}-05$ & $4.8 \mathrm{E}-05$ & $1.0 \mathrm{E}-04$ & $1.9 \mathrm{E}-04$ & $3.1 \mathrm{E}-04$ & $3.1 \mathrm{E}-04$ \\
\hline Wyoming & WY & $1.0 \mathrm{E}-04$ & $1.2 \mathrm{E}-04$ & $1.4 \mathrm{E}-04$ & $1.9 \mathrm{E}-04$ & $1.8 \mathrm{E}-04$ & $2.8 \mathrm{E}-04$ \\
\hline Idaho & ID & $8.8 \mathrm{E}-05$ & $1.0 \mathrm{E}-04$ & $1.4 \mathrm{E}-04$ & $1.8 \mathrm{E}-04$ & $1.8 \mathrm{E}-04$ & $2.6 \mathrm{E}-04$ \\
\hline Montana & MT & $9.3 \mathrm{E}-05$ & $1.1 \mathrm{E}-04$ & $1.6 \mathrm{E}-04$ & $2.1 \mathrm{E}-04$ & $1.9 \mathrm{E}-04$ & $2.5 \mathrm{E}-04$ \\
\hline California & CA & $8.2 \mathrm{E}-05$ & $1.2 \mathrm{E}-04$ & $3.0 \mathrm{E}-04$ & $3.8 \mathrm{E}-04$ & $9.3 \mathrm{E}-04$ & $9.0 \mathrm{E}-04$ \\
\hline Nevada & NV & $1.5 \mathrm{E}-04$ & $1.4 \mathrm{E}-04$ & $2.6 \mathrm{E}-04$ & $2.7 \mathrm{E}-04$ & $4.0 \mathrm{E}-04$ & $4.5 \mathrm{E}-04$ \\
\hline Utah & UT & $1.0 \mathrm{E}-04$ & $9.8 \mathrm{E}-05$ & $1.4 \mathrm{E}-04$ & $1.7 \mathrm{E}-04$ & $1.8 \mathrm{E}-04$ & $2.6 \mathrm{E}-04$ \\
\hline Colorado & $\mathrm{CO}$ & $9.0 \mathrm{E}-05$ & $1.0 \mathrm{E}-04$ & $1.4 \mathrm{E}-04$ & $1.9 \mathrm{E}-04$ & $2.3 \mathrm{E}-04$ & $3.5 \mathrm{E}-04$ \\
\hline Arizona & $\overline{A Z}$ & $1.2 \mathrm{E}-04$ & $1.2 \mathrm{E}-04$ & $2.0 \mathrm{E}-04$ & $2.5 \mathrm{E}-04$ & $4.2 \mathrm{E}-04$ & $4.4 \mathrm{E}-04$ \\
\hline New Mexico & NM & $1.0 \mathrm{E}-04$ & $9.5 \mathrm{E}-05$ & $1.3 \mathrm{E}-04$ & $1.5 \mathrm{E}-04$ & $1.8 \mathrm{E}-04$ & $2.0 \mathrm{E}-04$ \\
\hline Texas & $\mathrm{TX}$ & $6.1 \mathrm{E}-05$ & $8.0 \mathrm{E}-05$ & $1.3 \mathrm{E}-04$ & $2.6 \mathrm{E}-04$ & $4.6 \mathrm{E}-04$ & $5.6 \mathrm{E}-04$ \\
\hline Oklahoma & $\mathrm{OK}$ & $8.7 \mathrm{E}-05$ & $7.9 \mathrm{E}-05$ & $1.9 \mathrm{E}-04$ & $3.1 \mathrm{E}-04$ & $5.4 \mathrm{E}-04$ & $6.5 \mathrm{E}-04$ \\
\hline Arkansas & $\mathrm{AR}$ & $9.2 \mathrm{E}-05$ & $9.5 \mathrm{E}-05$ & $2.4 \mathrm{E}-04$ & $4.8 \mathrm{E}-04$ & $7.6 \mathrm{E}-04$ & $9.7 \mathrm{E}-04$ \\
\hline Louisiana & $\mathrm{LA}$ & $4.8 \mathrm{E}-05$ & $8.8 \mathrm{E}-05$ & $1.4 \mathrm{E}-04$ & $3.3 \mathrm{E}-04$ & 5.5E-04 & 7.3E-04 \\
\hline Mississippi & MS & $7.6 \mathrm{E}-05$ & $1.3 \mathrm{E}-04$ & $2.3 \mathrm{E}-04$ & $5.0 \mathrm{E}-04$ & $7.7 \mathrm{E}-04$ & $9.8 \mathrm{E}-04$ \\
\hline Alabama & $\mathrm{AL}$ & $7.0 \mathrm{E}-05$ & $1.6 \mathrm{E}-04$ & $2.2 \mathrm{E}-04$ & $6.0 \mathrm{E}-04$ & $9.0 \mathrm{E}-04$ & $1.2 \mathrm{E}-03$ \\
\hline Kentucky & KY & $3.6 \mathrm{E}-05$ & $4.8 \mathrm{E}-05$ & $9.9 \mathrm{E}-05$ & $3.6 \mathrm{E}-04$ & $6.7 \mathrm{E}-04$ & $1.1 \mathrm{E}-03$ \\
\hline Tennessee & $\mathrm{TN}$ & $5.6 \mathrm{E}-05$ & $8.5 \mathrm{E}-05$ & $1.5 \mathrm{E}-04$ & $5.1 \mathrm{E}-04$ & $8.3 \mathrm{E}-04$ & $1.3 \mathrm{E}-03$ \\
\hline Florida & FL & $1.4 \mathrm{E}-04$ & $2.2 \mathrm{E}-04$ & $3.4 \mathrm{E}-04$ & 5.6E-04 & $8.3 \mathrm{E}-04$ & $1.2 \mathrm{E}-03$ \\
\hline Georgia & GA & 5.9E-05 & $1.7 \mathrm{E}-04$ & $1.9 \mathrm{E}-04$ & $5.2 \mathrm{E}-04$ & $8.7 \mathrm{E}-04$ & $1.1 \mathrm{E}-03$ \\
\hline North Carolina & $\mathrm{NC}$ & $3.4 \mathrm{E}-05$ & $1.1 \mathrm{E}-04$ & $1.0 \mathrm{E}-04$ & $5.2 \mathrm{E}-04$ & $9.6 \mathrm{E}-04$ & $1.6 \mathrm{E}-03$ \\
\hline South Carolina & SC & $5.5 \mathrm{E}-05$ & $1.9 \mathrm{E}-04$ & $1.6 \mathrm{E}-04$ & $5.3 \mathrm{E}-04$ & $9.8 \mathrm{E}-04$ & $1.4 \mathrm{E}-03$ \\
\hline Virginia & VA & $1.6 \mathrm{E}-05$ & $5.5 \mathrm{E}-05$ & 7.7E-05 & $4.8 \mathrm{E}-04$ & $8.3 \mathrm{E}-04$ & $1.7 \mathrm{E}-03$ \\
\hline West Virginia & $\mathrm{WV}$ & $1.7 \mathrm{E}-05$ & $3.4 \mathrm{E}-05$ & $7.8 \mathrm{E}-05$ & $3.2 \mathrm{E}-04$ & $6.0 \mathrm{E}-04$ & $9.8 \mathrm{E}-04$ \\
\hline Delaware & $\mathrm{DE}$ & $5.3 \mathrm{E}-06$ & $3.6 \mathrm{E}-05$ & $7.9 \mathrm{E}-05$ & $6.5 \mathrm{E}-04$ & $1.3 \mathrm{E}-03$ & $3.0 \mathrm{E}-03$ \\
\hline Washington DC & $\overline{\mathrm{DC}}$ & $7.4 \mathrm{E}-06$ & $2.3 \mathrm{E}-05$ & $4.7 \mathrm{E}-05$ & $2.8 \mathrm{E}-04$ & $5.9 \mathrm{E}-04$ & $1.8 \mathrm{E}-03$ \\
\hline Maryland & MD & $6.8 \mathrm{E}-06$ & $2.7 \mathrm{E}-05$ & $5.5 \mathrm{E}-05$ & $3.6 \mathrm{E}-04$ & $7.3 \mathrm{E}-04$ & $1.9 \mathrm{E}-03$ \\
\hline Connecticut & CT & $3.5 \mathrm{E}-06$ & $8.6 \mathrm{E}-06$ & $5.3 \mathrm{E}-05$ & $2.1 \mathrm{E}-04$ & $3.5 \mathrm{E}-04$ & $1.7 \mathrm{E}-03$ \\
\hline New Jersey & NJ & $4.3 \mathrm{E}-06$ & $1.2 \mathrm{E}-05$ & $4.3 \mathrm{E}-05$ & $2.2 \mathrm{E}-04$ & $4.4 \mathrm{E}-04$ & $1.6 \mathrm{E}-03$ \\
\hline New York & NY & $8.4 \mathrm{E}-06$ & $1.7 \mathrm{E}-05$ & 7.3E-05 & $2.3 \mathrm{E}-04$ & $4.2 \mathrm{E}-04$ & $1.2 \mathrm{E}-03$ \\
\hline Pennsylvania & PA & $8.5 \mathrm{E}-06$ & $2.2 \mathrm{E}-05$ & $5.8 \mathrm{E}-05$ & $2.8 \mathrm{E}-04$ & $5.7 \mathrm{E}-04$ & $1.5 \mathrm{E}-03$ \\
\hline Rhode Island & RI & $2.2 \mathrm{E}-06$ & $7.1 \mathrm{E}-06$ & $6.9 \mathrm{E}-05$ & $1.9 \mathrm{E}-04$ & $3.6 \mathrm{E}-04$ & $1.4 \mathrm{E}-03$ \\
\hline Massachusetts & $\overline{\mathrm{MA}}$ & $2.3 \mathrm{E}-06$ & $6.4 \mathrm{E}-06$ & $6.6 \mathrm{E}-05$ & $1.6 \mathrm{E}-04$ & $3.4 \mathrm{E}-04$ & $9.6 \mathrm{E}-04$ \\
\hline New Hampshire & $\mathrm{NH}$ & $3.4 \mathrm{E}-06$ & $9.4 \mathrm{E}-06$ & $9.6 \mathrm{E}-05$ & $2.3 \mathrm{E}-04$ & $3.1 \mathrm{E}-04$ & 8.6E-04 \\
\hline Vermont & VT & $7.5 \mathrm{E}-06$ & $2.2 \mathrm{E}-05$ & $1.3 \mathrm{E}-04$ & $3.2 \mathrm{E}-04$ & $2.9 \mathrm{E}-04$ & $5.2 \mathrm{E}-04$ \\
\hline Maine & $\mathrm{ME}$ & $2.6 \mathrm{E}-06$ & $2.6 \mathrm{E}-06$ & $1.2 \mathrm{E}-04$ & $2.8 \mathrm{E}-04$ & $2.4 \mathrm{E}-04$ & 2.9E-04 \\
\hline Ohio & $\mathrm{OH}$ & $1.7 \mathrm{E}-05$ & $2.9 \mathrm{E}-05$ & $6.6 \mathrm{E}-05$ & $2.4 \mathrm{E}-04$ & 5.6E-04 & $1.0 \mathrm{E}-03$ \\
\hline Indiana & IN & $2.9 \mathrm{E}-05$ & $3.9 \mathrm{E}-05$ & $7.9 \mathrm{E}-05$ & $2.6 \mathrm{E}-04$ & 5.7E-04 & $8.7 \mathrm{E}-04$ \\
\hline Illinois & IL & $3.8 \mathrm{E}-05$ & $4.7 \mathrm{E}-05$ & $1.0 \mathrm{E}-04$ & $2.8 \mathrm{E}-04$ & $5.6 \mathrm{E}-04$ & $8.0 \mathrm{E}-04$ \\
\hline Michigan & MI & $3.1 \mathrm{E}-05$ & $4.4 \mathrm{E}-05$ & $1.1 \mathrm{E}-04$ & $2.5 \mathrm{E}-04$ & $4.3 \mathrm{E}-04$ & 7.9E-04 \\
\hline Wisconsin & WI & $4.5 \mathrm{E}-05$ & $5.9 \mathrm{E}-05$ & $1.3 \mathrm{E}-04$ & $2.8 \mathrm{E}-04$ & $4.9 \mathrm{E}-04$ & 7.6E-04 \\
\hline Minnesota & MN & $7.2 \mathrm{E}-05$ & $8.3 \mathrm{E}-05$ & $1.7 \mathrm{E}-04$ & $3.0 \mathrm{E}-04$ & $4.0 \mathrm{E}-04$ & $4.9 \mathrm{E}-04$ \\
\hline Iowa & IA & $7.0 \mathrm{E}-05$ & $7.5 \mathrm{E}-05$ & $1.4 \mathrm{E}-04$ & $3.6 \mathrm{E}-04$ & $5.9 \mathrm{E}-04$ & $7.4 \mathrm{E}-04$ \\
\hline Missouri & $\mathrm{MO}$ & $6.5 \mathrm{E}-05$ & $6.7 \mathrm{E}-05$ & $1.6 \mathrm{E}-04$ & $3.7 \mathrm{E}-04$ & $6.4 \mathrm{E}-04$ & $8.0 \mathrm{E}-04$ \\
\hline Kansas & $\mathrm{KS}$ & $9.6 \mathrm{E}-05$ & $9.0 \mathrm{E}-05$ & $1.8 \mathrm{E}-04$ & $2.9 \mathrm{E}-04$ & $4.2 \mathrm{E}-04$ & $4.7 \mathrm{E}-04$ \\
\hline Nebraska & $\mathrm{NE}$ & $1.1 \mathrm{E}-04$ & $1.0 \mathrm{E}-04$ & $1.7 \mathrm{E}-04$ & $3.0 \mathrm{E}-04$ & $4.0 \mathrm{E}-04$ & $4.2 \mathrm{E}-04$ \\
\hline North Dakota & ND & $9.6 \mathrm{E}-05$ & $1.0 \mathrm{E}-04$ & $1.7 \mathrm{E}-04$ & $2.8 \mathrm{E}-04$ & $2.9 \mathrm{E}-04$ & $2.7 \mathrm{E}-04$ \\
\hline South Dakota & SD & $1.2 \mathrm{E}-04$ & $1.2 \mathrm{E}-04$ & $2.0 \mathrm{E}-04$ & $3.2 \mathrm{E}-04$ & $4.2 \mathrm{E}-04$ & $4.6 \mathrm{E}-04$ \\
\hline
\end{tabular}


Table A20 Human effect level characterization factors for NOx (July-December)

\begin{tabular}{|c|c|c|c|c|c|c|c|}
\hline State & & July & August & September & October & November & December \\
\hline Oregon & OR & $7.2 \mathrm{E}-04$ & $5.8 \mathrm{E}-04$ & $5.8 \mathrm{E}-04$ & $2.1 \mathrm{E}-04$ & $8.4 \mathrm{E}-05$ & 3.7E-05 \\
\hline Washington & WA & $3.8 \mathrm{E}-04$ & $4.3 \mathrm{E}-04$ & $4.0 \mathrm{E}-04$ & $1.2 \mathrm{E}-04$ & $3.5 \mathrm{E}-05$ & $2.1 \mathrm{E}-05$ \\
\hline Wyoming & WY & $1.4 \mathrm{E}-04$ & $1.9 \mathrm{E}-04$ & $2.1 \mathrm{E}-04$ & $1.5 \mathrm{E}-04$ & $1.2 \mathrm{E}-04$ & $7.4 \mathrm{E}-05$ \\
\hline Idaho & ID & $1.7 \mathrm{E}-04$ & $2.1 \mathrm{E}-04$ & $2.0 \mathrm{E}-04$ & $1.3 \mathrm{E}-04$ & $9.4 \mathrm{E}-05$ & $6.1 \mathrm{E}-05$ \\
\hline Montana & MT & $1.4 \mathrm{E}-04$ & $1.7 \mathrm{E}-04$ & $1.7 \mathrm{E}-04$ & $1.8 \mathrm{E}-04$ & $9.5 \mathrm{E}-05$ & $6.8 \mathrm{E}-05$ \\
\hline California & CA & $8.5 \mathrm{E}-04$ & $1.0 \mathrm{E}-03$ & $7.8 \mathrm{E}-04$ & $5.0 \mathrm{E}-04$ & $2.0 \mathrm{E}-04$ & $5.9 \mathrm{E}-05$ \\
\hline Nevada & NV & $3.0 \mathrm{E}-04$ & $3.5 \mathrm{E}-04$ & $3.4 \mathrm{E}-04$ & $3.9 \mathrm{E}-04$ & $2.4 \mathrm{E}-04$ & $1.1 \mathrm{E}-04$ \\
\hline Utah & UT & $1.9 \mathrm{E}-04$ & $2.3 \mathrm{E}-04$ & $2.1 \mathrm{E}-04$ & $1.7 \mathrm{E}-04$ & $1.3 \mathrm{E}-04$ & $8.7 \mathrm{E}-05$ \\
\hline Colorado & $\mathrm{CO}$ & $4.3 \mathrm{E}-04$ & $3.5 \mathrm{E}-04$ & $3.0 \mathrm{E}-04$ & $1.4 \mathrm{E}-04$ & $1.3 \mathrm{E}-04$ & $6.5 \mathrm{E}-05$ \\
\hline Arizona & $\mathrm{AZ}$ & $4.7 \mathrm{E}-04$ & $6.0 \mathrm{E}-04$ & $4.3 \mathrm{E}-04$ & $3.7 \mathrm{E}-04$ & $2.9 \mathrm{E}-04$ & $6.6 \mathrm{E}-05$ \\
\hline New Mexico & $\overline{\mathrm{NM}}$ & $2.5 \mathrm{E}-04$ & $2.4 \mathrm{E}-04$ & $2.1 \mathrm{E}-04$ & $1.7 \mathrm{E}-04$ & $1.6 \mathrm{E}-04$ & $7.6 \mathrm{E}-05$ \\
\hline Texas & TX & $6.1 \mathrm{E}-04$ & 5.7E-04 & $2.9 \mathrm{E}-04$ & $2.3 \mathrm{E}-04$ & $1.6 \mathrm{E}-04$ & $4.4 \mathrm{E}-05$ \\
\hline Oklahoma & $\mathrm{OK}$ & $6.2 \mathrm{E}-04$ & $6.5 \mathrm{E}-04$ & $4.0 \mathrm{E}-04$ & $2.6 \mathrm{E}-04$ & $2.3 \mathrm{E}-04$ & $6.0 \mathrm{E}-05$ \\
\hline Arkansas & AR & $1.0 \mathrm{E}-03$ & $9.2 \mathrm{E}-04$ & $5.0 \mathrm{E}-04$ & $3.6 \mathrm{E}-04$ & $3.5 \mathrm{E}-04$ & $6.3 \mathrm{E}-05$ \\
\hline Louisiana & $\overline{\mathrm{LA}}$ & $7.1 \mathrm{E}-04$ & 7.7E-04 & $3.2 \mathrm{E}-04$ & $2.4 \mathrm{E}-04$ & $1.7 \mathrm{E}-04$ & $4.2 \mathrm{E}-05$ \\
\hline Mississippi & MS & $9.8 \mathrm{E}-04$ & $1.0 \mathrm{E}-03$ & $\begin{array}{l}5.2 \mathrm{E}-04 \\
\end{array}$ & $3.9 \mathrm{E}-04$ & $3.1 \mathrm{E}-04$ & $6.7 \mathrm{E}-05$ \\
\hline Alabama & $\mathrm{AL}$ & $1.1 \mathrm{E}-03$ & $1.1 \mathrm{E}-03$ & $6.4 \mathrm{E}-04$ & $4.2 \mathrm{E}-04$ & $2.8 \mathrm{E}-04$ & $6.5 \mathrm{E}-05$ \\
\hline Kentucky & $\mathrm{KY}$ & $1.0 \mathrm{E}-03$ & $7.5 \mathrm{E}-04$ & $4.5 \mathrm{E}-04$ & $2.4 \mathrm{E}-04$ & $1.7 \mathrm{E}-04$ & $3.4 \mathrm{E}-05$ \\
\hline Tennessee & $\mathrm{TN}$ & $1.2 \mathrm{E}-03$ & $1.1 \mathrm{E}-03$ & $6.3 \mathrm{E}-04$ & $3.7 \mathrm{E}-04$ & $2.7 \mathrm{E}-04$ & $4.4 \mathrm{E}-05$ \\
\hline Florida & FL & $9.2 \mathrm{E}-04$ & $1.4 \mathrm{E}-03$ & $9.0 \mathrm{E}-04$ & $3.6 \mathrm{E}-04$ & $2.5 \mathrm{E}-04$ & $1.2 \mathrm{E}-04$ \\
\hline Georgia & GA & $1.1 \mathrm{E}-03$ & $1.2 \mathrm{E}-03$ & $7.0 \mathrm{E}-04$ & $4.3 \mathrm{E}-04$ & $2.5 \mathrm{E}-04$ & $7.1 \mathrm{E}-05$ \\
\hline North Carolina & NC & $1.7 \mathrm{E}-03$ & $1.2 \mathrm{E}-03$ & $9.0 \mathrm{E}-04$ & $6.1 \mathrm{E}-04$ & $2.0 \mathrm{E}-04$ & 5.9E-05 \\
\hline South Carolina & $\mathrm{SC}$ & $1.4 \mathrm{E}-03$ & $1.3 \mathrm{E}-03$ & $9.4 \mathrm{E}-04$ & $6.0 \mathrm{E}-04$ & $2.9 \mathrm{E}-04$ & $9.1 \mathrm{E}-05$ \\
\hline Virginia & $\overline{V A}$ & $1.9 \mathrm{E}-03$ & $1.2 \mathrm{E}-03$ & $8.1 \mathrm{E}-04$ & $4.8 \mathrm{E}-04$ & $1.4 \mathrm{E}-04$ & $2.8 \mathrm{E}-05$ \\
\hline West Virginia & $\mathrm{WV}$ & $1.0 \mathrm{E}-03$ & $8.2 \mathrm{E}-04$ & $5.4 \mathrm{E}-04$ & $2.4 \mathrm{E}-04$ & $1.1 \mathrm{E}-04$ & $2.3 \mathrm{E}-05$ \\
\hline Delaware & $\mathrm{DE}$ & $3.4 \mathrm{E}-03$ & $1.8 \mathrm{E}-03$ & $1.2 \mathrm{E}-03$ & $7.5 \mathrm{E}-04$ & $9.5 \mathrm{E}-05$ & $1.5 \mathrm{E}-05$ \\
\hline Washington DC & $\mathrm{DC}$ & $1.7 \mathrm{E}-03$ & $8.9 \mathrm{E}-04$ & $4.5 \mathrm{E}-04$ & $1.9 \mathrm{E}-04$ & $6.0 \mathrm{E}-05$ & $1.1 \mathrm{E}-05$ \\
\hline Maryland & MD & $2.0 \mathrm{E}-03$ & $1.1 \mathrm{E}-03$ & $\begin{array}{l}6.3 \mathrm{E}-04 \\
\end{array}$ & $3.1 \mathrm{E}-04$ & $7.1 \mathrm{E}-05$ & $1.3 \mathrm{E}-05$ \\
\hline Connecticut & CT & $1.0 \mathrm{E}-03$ & $1.1 \mathrm{E}-03$ & $5.2 \mathrm{E}-04$ & $1.2 \mathrm{E}-04$ & $4.1 \mathrm{E}-05$ & $7.7 \mathrm{E}-06$ \\
\hline New Jersey & NJ & $1.6 \mathrm{E}-03$ & $8.7 \mathrm{E}-04$ & $4.8 \mathrm{E}-04$ & $1.6 \mathrm{E}-04$ & $4.7 \mathrm{E}-05$ & $8.6 \mathrm{E}-06$ \\
\hline New York & NY & $1.1 \mathrm{E}-03$ & $9.2 \mathrm{E}-04$ & $6.3 \mathrm{E}-04$ & $1.3 \mathrm{E}-04$ & $5.6 \mathrm{E}-05$ & $1.4 \mathrm{E}-05$ \\
\hline Pennsylvania & PA & $1.6 \mathrm{E}-03$ & $9.9 \mathrm{E}-04$ & $6.0 \mathrm{E}-04$ & $1.6 \mathrm{E}-04$ & $6.2 \mathrm{E}-05$ & $1.3 \mathrm{E}-05$ \\
\hline Rhode Island & RI & $7.5 \mathrm{E}-04$ & $1.1 \mathrm{E}-03$ & $5.0 \mathrm{E}-04$ & $1.2 \mathrm{E}-04$ & $4.1 \mathrm{E}-05$ & $6.9 \mathrm{E}-06$ \\
\hline Massachusetts & MA & $5.6 \mathrm{E}-04$ & $9.0 \mathrm{E}-04$ & $4.7 \mathrm{E}-04$ & $1.1 \mathrm{E}-04$ & $3.8 \mathrm{E}-05$ & $7.8 \mathrm{E}-06$ \\
\hline New Hampshire & $\mathrm{NH}$ & $6.9 \mathrm{E}-04$ & $9.1 \mathrm{E}-04$ & $5.4 \mathrm{E}-04$ & $1.1 \mathrm{E}-04$ & $4.0 \mathrm{E}-05$ & $1.1 \mathrm{E}-05$ \\
\hline Vermont & VT & $6.9 \mathrm{E}-04$ & $7.7 \mathrm{E}-04$ & $5.7 \mathrm{E}-04$ & $1.1 \mathrm{E}-04$ & $4.4 \mathrm{E}-05$ & $1.5 \mathrm{E}-05$ \\
\hline Maine & $\mathrm{ME}$ & $3.7 \mathrm{E}-04$ & 7.5E-04 & $4.3 \mathrm{E}-04$ & $9.0 \mathrm{E}-05$ & $4.2 \mathrm{E}-05$ & $1.2 \mathrm{E}-05$ \\
\hline Ohio & $\mathrm{OH}$ & $1.1 \mathrm{E}-03$ & $7.6 \mathrm{E}-04$ & $5.1 \mathrm{E}-04$ & $1.3 \mathrm{E}-04$ & $7.3 \mathrm{E}-05$ & $2.0 \mathrm{E}-05$ \\
\hline Indiana & IN & $1.0 \mathrm{E}-03$ & $7.0 \mathrm{E}-04$ & $4.3 \mathrm{E}-04$ & $1.5 \mathrm{E}-04$ & $8.7 \mathrm{E}-05$ & $2.6 \mathrm{E}-05$ \\
\hline Illinois & IL & $\begin{array}{l}9.5 \mathrm{E}-04 \\
\end{array}$ & $6.7 \mathrm{E}-04$ & $4.3 \mathrm{E}-04$ & $1.7 \mathrm{E}-04$ & $1.0 \mathrm{E}-04$ & $3.1 \mathrm{E}-05$ \\
\hline Michigan & MI & $1.1 \mathrm{E}-03$ & $8.1 \mathrm{E}-04$ & $5.9 \mathrm{E}-04$ & $8.7 \mathrm{E}-05$ & $6.2 \mathrm{E}-05$ & $2.3 \mathrm{E}-05$ \\
\hline Wisconsin & $\overline{\mathrm{WI}}$ & $9.4 \mathrm{E}-04$ & $6.5 \mathrm{E}-04$ & $4.6 \mathrm{E}-04$ & $1.1 \mathrm{E}-04$ & $8.1 \mathrm{E}-05$ & $3.1 \mathrm{E}-05$ \\
\hline Minnesota & $\mathrm{MN}$ & $5.3 \mathrm{E}-04$ & $4.7 \mathrm{E}-04$ & $2.7 \mathrm{E}-04$ & $1.2 \mathrm{E}-04$ & $1.1 \mathrm{E}-04$ & $4.7 \mathrm{E}-05$ \\
\hline Iowa & IA & $7.3 \mathrm{E}-04$ & $6.9 \mathrm{E}-04$ & $4.7 \mathrm{E}-04$ & $2.1 \mathrm{E}-04$ & $1.3 \mathrm{E}-04$ & $4.9 \mathrm{E}-05$ \\
\hline Missouri & MO & $8.6 \mathrm{E}-04$ & $7.7 \mathrm{E}-04$ & $4.6 \mathrm{E}-04$ & $2.4 \mathrm{E}-04$ & $2.0 \mathrm{E}-04$ & $4.9 \mathrm{E}-05$ \\
\hline Kansas & KS & $3.7 \mathrm{E}-04$ & $4.8 \mathrm{E}-04$ & $3.5 \mathrm{E}-04$ & $2.3 \mathrm{E}-04$ & $2.1 \mathrm{E}-04$ & $6.8 \mathrm{E}-05$ \\
\hline Nebraska & $\mathrm{NE}$ & $2.6 \mathrm{E}-04$ & 3.9E-04 & $3.0 \mathrm{E}-04$ & $2.1 \mathrm{E}-04$ & $1.6 \mathrm{E}-04$ & $7.1 \mathrm{E}-05$ \\
\hline North Dakota & ND & $2.1 \mathrm{E}-04$ & $2.7 \mathrm{E}-04$ & $2.0 \mathrm{E}-04$ & $1.8 \mathrm{E}-04$ & $1.0 \mathrm{E}-04$ & $7.9 \mathrm{E}-05$ \\
\hline South Dakota & SD & $2.6 \mathrm{E}-04$ & $4.1 \mathrm{E}-04$ & $2.8 \mathrm{E}-04$ & $2.6 \mathrm{E}-04$ & $1.7 \mathrm{E}-04$ & 7.9E-05 \\
\hline
\end{tabular}


Table A21 Human effect level characterization factors for VOC (January-June)

\begin{tabular}{|c|c|c|c|c|c|c|c|}
\hline State & & January & February & March & April & May & June \\
\hline Oregon & OR & $8.4 \mathrm{E}-06$ & 1.1E-05 & $1.7 \mathrm{E}-05$ & $2.0 \mathrm{E}-05$ & $1.8 \mathrm{E}-05$ & $1.8 \mathrm{E}-05$ \\
\hline Washington & WA & $8.5 \mathrm{E}-06$ & $1.1 \mathrm{E}-05$ & $1.7 \mathrm{E}-05$ & $2.6 \mathrm{E}-05$ & 3.3E-05 & $3.4 \mathrm{E}-05$ \\
\hline Wyoming & WY & $3.8 \mathrm{E}-05$ & $3.2 \mathrm{E}-05$ & $2.8 \mathrm{E}-05$ & $1.5 \mathrm{E}-05$ & $7.5 \mathrm{E}-06$ & 6.2E-06 \\
\hline Idaho & ID & $2.2 \mathrm{E}-05$ & $2.1 \mathrm{E}-05$ & $1.7 \mathrm{E}-05$ & $1.3 \mathrm{E}-05$ & $5.4 \mathrm{E}-06$ & $5.9 \mathrm{E}-06$ \\
\hline Montana & MT & $3.2 \mathrm{E}-05$ & $2.8 \mathrm{E}-05$ & $2.6 \mathrm{E}-05$ & $1.6 \mathrm{E}-05$ & 7.5E-06 & $5.7 \mathrm{E}-06$ \\
\hline California & CA & $2.5 \mathrm{E}-05$ & $4.6 \mathrm{E}-05$ & $6.6 \mathrm{E}-05$ & $7.8 \mathrm{E}-05$ & $1.2 \mathrm{E}-04$ & $1.1 \mathrm{E}-04$ \\
\hline Nevada & $\mathrm{NV}$ & $2.5 \mathrm{E}-05$ & $2.5 \mathrm{E}-05$ & $2.7 \mathrm{E}-05$ & $2.4 \mathrm{E}-05$ & $2.0 \mathrm{E}-05$ & $1.7 \mathrm{E}-05$ \\
\hline Utah & $\overline{\mathrm{UT}}$ & $2.9 \mathrm{E}-05$ & $2.6 \mathrm{E}-05$ & $2.3 \mathrm{E}-05$ & $1.7 \mathrm{E}-05$ & $1.4 \mathrm{E}-05$ & $9.8 \mathrm{E}-06$ \\
\hline Colorado & $\mathrm{CO}$ & $3.6 \mathrm{E}-05$ & $2.6 \mathrm{E}-05$ & $2.7 \mathrm{E}-05$ & $1.9 \mathrm{E}-05$ & $1.6 \mathrm{E}-05$ & $1.6 \mathrm{E}-05$ \\
\hline Arizona & $\overline{A Z}$ & $2.3 \mathrm{E}-05$ & $2.7 \mathrm{E}-05$ & $2.8 \mathrm{E}-05$ & $2.8 \mathrm{E}-05$ & $2.9 \mathrm{E}-05$ & $2.7 \mathrm{E}-05$ \\
\hline New Mexico & NM & $2.7 \mathrm{E}-05$ & $1.7 \mathrm{E}-05$ & $1.4 \mathrm{E}-05$ & $1.0 \mathrm{E}-05$ & $5.1 \mathrm{E}-06$ & $5.1 \mathrm{E}-06$ \\
\hline Texas & TX & $2.6 \mathrm{E}-05$ & $2.1 \mathrm{E}-05$ & $2.5 \mathrm{E}-05$ & $2.7 \mathrm{E}-05$ & $2.9 \mathrm{E}-05$ & 3.3E-05 \\
\hline Oklahoma & $\mathrm{OK}$ & $4.9 \mathrm{E}-05$ & $3.1 \mathrm{E}-05$ & $4.2 \mathrm{E}-05$ & $2.5 \mathrm{E}-05$ & $1.4 \mathrm{E}-05$ & 9.6E-06 \\
\hline Arkansas & $\overline{\mathrm{AR}}$ & $5.3 \mathrm{E}-05$ & $3.7 \mathrm{E}-05$ & $4.3 \mathrm{E}-05$ & $3.2 \mathrm{E}-05$ & $1.8 \mathrm{E}-05$ & $1.4 \mathrm{E}-05$ \\
\hline Louisiana & $\overline{\mathrm{LA}}$ & $1.8 \mathrm{E}-05$ & $2.5 \mathrm{E}-05$ & $2.8 \mathrm{E}-05$ & $4.0 \mathrm{E}-05$ & $3.9 \mathrm{E}-05$ & $3.6 \mathrm{E}-05$ \\
\hline Mississippi & MS & $3.0 \mathrm{E}-05$ & $2.8 \mathrm{E}-05$ & $3.1 \mathrm{E}-05$ & $3.2 \mathrm{E}-05$ & $1.7 \mathrm{E}-05$ & $1.6 \mathrm{E}-05$ \\
\hline Alabama & $\mathrm{AL}$ & $2.9 \mathrm{E}-05$ & $3.1 \mathrm{E}-05$ & $3.5 \mathrm{E}-05$ & $3.7 \mathrm{E}-05$ & $1.8 \mathrm{E}-05$ & $1.4 \mathrm{E}-05$ \\
\hline Kentucky & $\mathrm{KY}$ & $5.6 \mathrm{E}-05$ & $5.4 \mathrm{E}-05$ & $8.0 \mathrm{E}-05$ & $7.7 \mathrm{E}-05$ & 7.5E-05 & $4.8 \mathrm{E}-05$ \\
\hline Tennessee & TN & $5.1 \mathrm{E}-05$ & $4.4 \mathrm{E}-05$ & $5.8 \mathrm{E}-05$ & $4.7 \mathrm{E}-05$ & $2.9 \mathrm{E}-05$ & $1.6 \mathrm{E}-05$ \\
\hline Florida & FL & $3.0 \mathrm{E}-05$ & $3.4 \mathrm{E}-05$ & $4.1 \mathrm{E}-05$ & $6.0 \mathrm{E}-05$ & 5.9E-05 & $8.8 \mathrm{E}-05$ \\
\hline Georgia & GA & $3.0 \mathrm{E}-05$ & 3.6E-05 & $4.1 \mathrm{E}-05$ & 4.3E-05 & $3.2 \mathrm{E}-05$ & $2.1 \mathrm{E}-05$ \\
\hline North Carolina & $\mathrm{NC}$ & $2.4 \mathrm{E}-05$ & $3.7 \mathrm{E}-05$ & $3.3 \mathrm{E}-05$ & $3.4 \mathrm{E}-05$ & $2.8 \mathrm{E}-05$ & $1.8 \mathrm{E}-05$ \\
\hline South Carolina & SC & $2.8 \mathrm{E}-05$ & $4.1 \mathrm{E}-05$ & $3.1 \mathrm{E}-05$ & $2.8 \mathrm{E}-05$ & $1.8 \mathrm{E}-05$ & $9.6 \mathrm{E}-06$ \\
\hline Virginia & VA & $2.1 \mathrm{E}-05$ & $3.4 \mathrm{E}-05$ & $4.5 \mathrm{E}-05$ & $5.6 \mathrm{E}-05$ & $5.8 \mathrm{E}-05$ & $6.5 \mathrm{E}-05$ \\
\hline West Virginia & $\mathrm{WV}$ & $3.6 \mathrm{E}-05$ & $4.2 \mathrm{E}-05$ & $7.3 \mathrm{E}-05$ & $6.2 \mathrm{E}-05$ & $6.4 \mathrm{E}-05$ & $3.8 \mathrm{E}-05$ \\
\hline Delaware & $\overline{\mathrm{DE}}$ & $1.0 \mathrm{E}-05$ & $2.9 \mathrm{E}-05$ & $4.1 \mathrm{E}-05$ & 6.3E-05 & $6.3 \mathrm{E}-05$ & 7.9E-05 \\
\hline Washington DC & $\overline{\mathrm{DC}}$ & $1.9 \mathrm{E}-05$ & $2.7 \mathrm{E}-05$ & $4.8 \mathrm{E}-05$ & $8.4 \mathrm{E}-05$ & $1.2 \mathrm{E}-04$ & $1.9 \mathrm{E}-04$ \\
\hline Maryland & $\mathrm{MD}$ & $2.0 \mathrm{E}-05$ & $3.0 \mathrm{E}-05$ & $4.8 \mathrm{E}-05$ & $8.2 \mathrm{E}-05$ & $9.6 \mathrm{E}-05$ & $1.3 \mathrm{E}-04$ \\
\hline Connecticut & CT & $8.8 \mathrm{E}-06$ & $8.5 \mathrm{E}-06$ & $3.7 \mathrm{E}-05$ & $8.2 \mathrm{E}-05$ & $7.0 \mathrm{E}-05$ & 1.3E-04 \\
\hline New Jersey & NJ & $1.3 \mathrm{E}-05$ & $1.4 \mathrm{E}-05$ & $3.7 \mathrm{E}-05$ & $8.2 \mathrm{E}-05$ & $8.8 \mathrm{E}-05$ & $1.9 \mathrm{E}-04$ \\
\hline New York & NY & $1.7 \mathrm{E}-05$ & $2.0 \mathrm{E}-05$ & $5.4 \mathrm{E}-05$ & $7.3 \mathrm{E}-05$ & $5.1 \mathrm{E}-05$ & $1.1 \mathrm{E}-04$ \\
\hline Pennsylvania & PA & $2.5 \mathrm{E}-05$ & $3.1 \mathrm{E}-05$ & $6.1 \mathrm{E}-05$ & $8.1 \mathrm{E}-05$ & $8.1 \mathrm{E}-05$ & $1.1 \mathrm{E}-04$ \\
\hline Rhode Island & RI & $4.0 \mathrm{E}-06$ & $4.9 \mathrm{E}-06$ & $3.1 \mathrm{E}-05$ & $5.4 \mathrm{E}-05$ & $7.2 \mathrm{E}-05$ & 7.9E-05 \\
\hline Massachusetts & MA & $4.0 \mathrm{E}-06$ & 5.3E-06 & $3.2 \mathrm{E}-05$ & $5.1 \mathrm{E}-05$ & $6.3 \mathrm{E}-05$ & $6.4 \mathrm{E}-05$ \\
\hline New Hampshire & $\mathrm{NH}$ & $4.5 \mathrm{E}-06$ & $5.8 \mathrm{E}-06$ & $3.9 \mathrm{E}-05$ & 5.2E-05 & $3.2 \mathrm{E}-05$ & $2.1 \mathrm{E}-05$ \\
\hline Vermont & VT & $6.9 \mathrm{E}-06$ & $7.0 \mathrm{E}-06$ & $4.4 \mathrm{E}-05$ & $5.4 \mathrm{E}-05$ & $1.1 \mathrm{E}-05$ & $9.0 \mathrm{E}-06$ \\
\hline Maine & $\mathrm{ME}$ & $1.2 \mathrm{E}-06$ & $1.0 \mathrm{E}-06$ & $2.0 \mathrm{E}-05$ & $2.7 \mathrm{E}-05$ & $1.7 \mathrm{E}-05$ & $2.4 \mathrm{E}-06$ \\
\hline Ohio & $\mathrm{OH}$ & $4.5 \mathrm{E}-05$ & $4.9 \mathrm{E}-05$ & $8.7 \mathrm{E}-05$ & $7.8 \mathrm{E}-05$ & $8.4 \mathrm{E}-05$ & $8.5 \mathrm{E}-05$ \\
\hline Indiana & IN & $6.4 \mathrm{E}-05$ & $6.0 \mathrm{E}-05$ & $9.7 \mathrm{E}-05$ & $8.0 \mathrm{E}-05$ & $7.5 \mathrm{E}-05$ & $6.1 \mathrm{E}-05$ \\
\hline Illinois & IL & $6.4 \mathrm{E}-05$ & 5.9E-05 & $1.0 \mathrm{E}-04$ & $7.4 \mathrm{E}-05$ & 6.7E-05 & 6.7E-05 \\
\hline Michigan & MI & $4.3 \mathrm{E}-05$ & $5.5 \mathrm{E}-05$ & $8.5 \mathrm{E}-05$ & $7.9 \mathrm{E}-05$ & $5.8 \mathrm{E}-05$ & 7.3E-05 \\
\hline Wisconsin & WI & $5.5 \mathrm{E}-05$ & $7.0 \mathrm{E}-05$ & $1.1 \mathrm{E}-04$ & $7.4 \mathrm{E}-05$ & $5.2 \mathrm{E}-05$ & 4.3E-05 \\
\hline Minnesota & MN & $5.5 \mathrm{E}-05$ & $6.7 \mathrm{E}-05$ & $1.0 \mathrm{E}-04$ & $6.5 \mathrm{E}-05$ & $3.8 \mathrm{E}-05$ & $2.4 \mathrm{E}-05$ \\
\hline Iowa & IA & $6.8 \mathrm{E}-05$ & $6.1 \mathrm{E}-05$ & $9.8 \mathrm{E}-05$ & $5.3 \mathrm{E}-05$ & $3.7 \mathrm{E}-05$ & $1.8 \mathrm{E}-05$ \\
\hline Missouri & $\mathrm{MO}$ & $6.7 \mathrm{E}-05$ & $5.0 \mathrm{E}-05$ & $8.1 \mathrm{E}-05$ & $5.1 \mathrm{E}-05$ & $4.4 \mathrm{E}-05$ & $2.8 \mathrm{E}-05$ \\
\hline Kansas & KS & $6.1 \mathrm{E}-05$ & $4.6 \mathrm{E}-05$ & $5.5 \mathrm{E}-05$ & $3.0 \mathrm{E}-05$ & $1.6 \mathrm{E}-05$ & $1.0 \mathrm{E}-05$ \\
\hline Nebraska & $\mathrm{NE}$ & $6.0 \mathrm{E}-05$ & $4.8 \mathrm{E}-05$ & $6.1 \mathrm{E}-05$ & $3.5 \mathrm{E}-05$ & $2.4 \mathrm{E}-05$ & $1.5 \mathrm{E}-05$ \\
\hline North Dakota & ND & $4.0 \mathrm{E}-05$ & $5.2 \mathrm{E}-05$ & $5.3 \mathrm{E}-05$ & $3.7 \mathrm{E}-05$ & $1.4 \mathrm{E}-05$ & 7.1E-06 \\
\hline South Dakota & SD & $5.6 \mathrm{E}-05$ & $5.4 \mathrm{E}-05$ & $6.6 \mathrm{E}-05$ & $3.7 \mathrm{E}-05$ & $1.9 \mathrm{E}-05$ & $9.1 \mathrm{E}-06$ \\
\hline
\end{tabular}


Table A22 Human effect level characterization factors for VOC (July-December)

\begin{tabular}{|c|c|c|c|c|c|c|c|}
\hline State & & July & August & September & October & November & December \\
\hline Oregon & OR & $2.0 \mathrm{E}-05$ & $1.8 \mathrm{E}-05$ & $2.0 \mathrm{E}-05$ & $1.7 \mathrm{E}-05$ & 8.5E-06 & 5.8E-06 \\
\hline Washington & WA & $3.5 \mathrm{E}-05$ & $3.4 \mathrm{E}-05$ & $3.6 \mathrm{E}-05$ & $1.9 \mathrm{E}-05$ & $7.2 \mathrm{E}-06$ & $5.6 \mathrm{E}-06$ \\
\hline Wyoming & WY & $2.5 \mathrm{E}-06$ & $5.6 \mathrm{E}-06$ & $1.0 \mathrm{E}-05$ & $1.9 \mathrm{E}-05$ & $2.6 \mathrm{E}-05$ & $2.8 \mathrm{E}-05$ \\
\hline Idaho & ID & $2.5 \mathrm{E}-06$ & 3.5E-06 & 7.3E-06 & $1.4 \mathrm{E}-05$ & $1.7 \mathrm{E}-05$ & $1.5 \mathrm{E}-05$ \\
\hline Montana & MT & $1.7 \mathrm{E}-06$ & 4.3E-06 & $8.5 \mathrm{E}-06$ & $2.2 \mathrm{E}-05$ & $2.0 \mathrm{E}-05$ & $2.3 \mathrm{E}-05$ \\
\hline California & CA & $1.1 \mathrm{E}-04$ & $1.2 \mathrm{E}-04$ & $1.3 \mathrm{E}-04$ & $8.6 \mathrm{E}-05$ & $4.3 \mathrm{E}-05$ & $2.1 \mathrm{E}-05$ \\
\hline Nevada & NV & $1.2 \mathrm{E}-05$ & $1.3 \mathrm{E}-05$ & $1.8 \mathrm{E}-05$ & $3.0 \mathrm{E}-05$ & $2.7 \mathrm{E}-05$ & $2.2 \mathrm{E}-05$ \\
\hline Utah & UT & $6.8 \mathrm{E}-06$ & $1.1 \mathrm{E}-05$ & $1.3 \mathrm{E}-05$ & $1.8 \mathrm{E}-05$ & $2.2 \mathrm{E}-05$ & $2.5 \mathrm{E}-05$ \\
\hline Colorado & $\mathrm{CO}$ & $2.1 \mathrm{E}-05$ & $1.9 \mathrm{E}-05$ & $2.3 \mathrm{E}-05$ & $1.7 \mathrm{E}-05$ & $3.0 \mathrm{E}-05$ & $2.3 \mathrm{E}-05$ \\
\hline Arizona & $\mathrm{AZ}$ & $3.3 \mathrm{E}-05$ & $4.3 \mathrm{E}-05$ & $2.9 \mathrm{E}-05$ & $2.6 \mathrm{E}-05$ & $2.8 \mathrm{E}-05$ & $1.3 \mathrm{E}-05$ \\
\hline New Mexico & $\overline{\mathrm{NM}}$ & $6.9 \mathrm{E}-06$ & $8.6 \mathrm{E}-06$ & $1.0 \mathrm{E}-05$ & $1.1 \mathrm{E}-05$ & $1.9 \mathrm{E}-05$ & $1.9 \mathrm{E}-05$ \\
\hline Texas & TX & $3.9 \mathrm{E}-05$ & $4.1 \mathrm{E}-05$ & $2.0 \mathrm{E}-05$ & $3.1 \mathrm{E}-05$ & $2.5 \mathrm{E}-05$ & $1.6 \mathrm{E}-05$ \\
\hline Oklahoma & $\mathrm{OK}$ & $1.0 \mathrm{E}-05$ & $1.9 \mathrm{E}-05$ & $2.0 \mathrm{E}-05$ & $3.2 \mathrm{E}-05$ & $4.2 \mathrm{E}-05$ & $3.1 \mathrm{E}-05$ \\
\hline Arkansas & AR & $1.4 \mathrm{E}-05$ & $2.3 \mathrm{E}-05$ & $1.9 \mathrm{E}-05$ & $3.7 \mathrm{E}-05$ & $4.9 \mathrm{E}-05$ & $3.0 \mathrm{E}-05$ \\
\hline Louisiana & $\overline{\mathrm{LA}}$ & $4.2 \mathrm{E}-05$ & $5.9 \mathrm{E}-05$ & $2.3 \mathrm{E}-05$ & $3.8 \mathrm{E}-05$ & $2.5 \mathrm{E}-05$ & $1.4 \mathrm{E}-05$ \\
\hline Mississippi & MS & $1.2 \mathrm{E}-05$ & $1.9 \mathrm{E}-05$ & $1.4 \mathrm{E}-05$ & $3.5 \mathrm{E}-05$ & $3.4 \mathrm{E}-05$ & $2.1 \mathrm{E}-05$ \\
\hline Alabama & $\mathrm{AL}$ & $1.2 \mathrm{E}-05$ & $1.7 \mathrm{E}-05$ & $1.9 \mathrm{E}-05$ & $4.5 \mathrm{E}-05$ & $4.5 \mathrm{E}-05$ & $2.1 \mathrm{E}-05$ \\
\hline Kentucky & $\mathrm{KY}$ & 5.5E-05 & $8.1 \mathrm{E}-05$ & $7.9 \mathrm{E}-05$ & $7.5 \mathrm{E}-05$ & $9.1 \mathrm{E}-05$ & $4.1 \mathrm{E}-05$ \\
\hline Tennessee & $\mathrm{TN}$ & $1.5 \mathrm{E}-05$ & $2.9 \mathrm{E}-05$ & $3.7 \mathrm{E}-05$ & $6.5 \mathrm{E}-05$ & $8.8 \mathrm{E}-05$ & $3.1 \mathrm{E}-05$ \\
\hline Florida & FL & $6.8 \mathrm{E}-05$ & $1.0 \mathrm{E}-04$ & $8.8 \mathrm{E}-05$ & $4.0 \mathrm{E}-05$ & $2.1 \mathrm{E}-05$ & $2.2 \mathrm{E}-05$ \\
\hline Georgia & GA & $2.5 \mathrm{E}-05$ & $3.4 \mathrm{E}-05$ & $3.8 \mathrm{E}-05$ & $7.0 \mathrm{E}-05$ & $5.6 \mathrm{E}-05$ & $2.3 \mathrm{E}-05$ \\
\hline North Carolina & NC & $2.8 \mathrm{E}-05$ & $3.1 \mathrm{E}-05$ & $4.9 \mathrm{E}-05$ & $9.5 \mathrm{E}-05$ & $6.2 \mathrm{E}-05$ & $2.5 \mathrm{E}-05$ \\
\hline South Carolina & $\mathrm{SC}$ & $1.6 \mathrm{E}-05$ & $2.0 \mathrm{E}-05$ & $3.4 \mathrm{E}-05$ & $7.3 \mathrm{E}-05$ & 5.5E-05 & $2.6 \mathrm{E}-05$ \\
\hline Virginia & $\mathrm{VA}$ & $6.5 \mathrm{E}-05$ & $7.2 \mathrm{E}-05$ & $8.7 \mathrm{E}-05$ & $1.0 \mathrm{E}-04$ & $6.6 \mathrm{E}-05$ & $2.1 \mathrm{E}-05$ \\
\hline West Virginia & $\mathrm{WV}$ & $5.1 \mathrm{E}-05$ & $8.9 \mathrm{E}-05$ & $1.1 \mathrm{E}-04$ & $8.1 \mathrm{E}-05$ & $7.1 \mathrm{E}-05$ & $2.7 \mathrm{E}-05$ \\
\hline Delaware & $\mathrm{DE}$ & $6.4 \mathrm{E}-05$ & 7.3E-05 & $1.0 \mathrm{E}-04$ & $1.1 \mathrm{E}-04$ & $4.7 \mathrm{E}-05$ & $1.5 \mathrm{E}-05$ \\
\hline Washington DC & $\mathrm{DC}$ & $1.5 \mathrm{E}-04$ & $1.6 \mathrm{E}-04$ & $1.4 \mathrm{E}-04$ & $8.9 \mathrm{E}-05$ & $5.0 \mathrm{E}-05$ & $1.7 \mathrm{E}-05$ \\
\hline Maryland & MD & $1.0 \mathrm{E}-04$ & $1.2 \mathrm{E}-04$ & $1.3 \mathrm{E}-04$ & $9.6 \mathrm{E}-05$ & $5.0 \mathrm{E}-05$ & $1.9 \mathrm{E}-05$ \\
\hline Connecticut & CT & $9.4 \mathrm{E}-05$ & $1.3 \mathrm{E}-04$ & $1.2 \mathrm{E}-04$ & $4.2 \mathrm{E}-05$ & $2.2 \mathrm{E}-05$ & $8.5 \mathrm{E}-06$ \\
\hline New Jersey & NJ & $1.7 \mathrm{E}-04$ & $1.6 \mathrm{E}-04$ & $1.4 \mathrm{E}-04$ & 5.5E-05 & $3.1 \mathrm{E}-05$ & $1.2 \mathrm{E}-05$ \\
\hline New York & NY & $9.0 \mathrm{E}-05$ & $1.1 \mathrm{E}-04$ & $1.2 \mathrm{E}-04$ & $3.7 \mathrm{E}-05$ & $3.2 \mathrm{E}-05$ & $1.4 \mathrm{E}-05$ \\
\hline Pennsylvania & PA & $1.1 \mathrm{E}-04$ & $1.4 \mathrm{E}-04$ & $1.5 \mathrm{E}-04$ & $6.4 \mathrm{E}-05$ & $4.7 \mathrm{E}-05$ & $2.0 \mathrm{E}-05$ \\
\hline Rhode Island & RI & $5.6 \mathrm{E}-05$ & $1.0 \mathrm{E}-04$ & $8.6 \mathrm{E}-05$ & $2.9 \mathrm{E}-05$ & $1.6 \mathrm{E}-05$ & $4.9 \mathrm{E}-06$ \\
\hline Massachusetts & MA & $5.3 \mathrm{E}-05$ & $8.9 \mathrm{E}-05$ & $8.0 \mathrm{E}-05$ & $2.3 \mathrm{E}-05$ & $1.5 \mathrm{E}-05$ & $5.6 \mathrm{E}-06$ \\
\hline New Hampshire & $\mathrm{NH}$ & $3.3 \mathrm{E}-05$ & 5.3E-05 & 5.5E-05 & $1.7 \mathrm{E}-05$ & $1.3 \mathrm{E}-05$ & $6.9 \mathrm{E}-06$ \\
\hline Vermont & VT & $2.1 \mathrm{E}-05$ & $3.3 \mathrm{E}-05$ & $4.4 \mathrm{E}-05$ & $9.7 \mathrm{E}-06$ & $1.3 \mathrm{E}-05$ & $7.6 \mathrm{E}-06$ \\
\hline Maine & $\mathrm{ME}$ & $1.2 \mathrm{E}-05$ & $2.0 \mathrm{E}-05$ & $1.9 \mathrm{E}-05$ & $6.0 \mathrm{E}-06$ & $6.0 \mathrm{E}-06$ & $2.6 \mathrm{E}-06$ \\
\hline Ohio & $\mathrm{OH}$ & $9.8 \mathrm{E}-05$ & $1.5 \mathrm{E}-04$ & $1.6 \mathrm{E}-04$ & $6.5 \mathrm{E}-05$ & $5.5 \mathrm{E}-05$ & $2.9 \mathrm{E}-05$ \\
\hline Indiana & IN & $6.8 \mathrm{E}-05$ & $9.9 \mathrm{E}-05$ & $1.1 \mathrm{E}-04$ & $7.6 \mathrm{E}-05$ & $5.7 \mathrm{E}-05$ & $4.1 \mathrm{E}-05$ \\
\hline Illinois & IL & $7.3 \mathrm{E}-05$ & $8.3 \mathrm{E}-05$ & $8.8 \mathrm{E}-05$ & $7.1 \mathrm{E}-05$ & $4.9 \mathrm{E}-05$ & $4.0 \mathrm{E}-05$ \\
\hline Michigan & MI & $8.3 \mathrm{E}-05$ & $1.1 \mathrm{E}-04$ & $1.4 \mathrm{E}-04$ & $4.2 \mathrm{E}-05$ & $3.7 \mathrm{E}-05$ & $2.1 \mathrm{E}-05$ \\
\hline Wisconsin & $\overline{\mathrm{WI}}$ & $4.3 \mathrm{E}-05$ & 5.7E-05 & $7.3 \mathrm{E}-05$ & $5.0 \mathrm{E}-05$ & $3.9 \mathrm{E}-05$ & $3.0 \mathrm{E}-05$ \\
\hline Minnesota & $\mathrm{MN}$ & $2.4 \mathrm{E}-05$ & $4.0 \mathrm{E}-05$ & $3.7 \mathrm{E}-05$ & $3.8 \mathrm{E}-05$ & $4.2 \mathrm{E}-05$ & $3.8 \mathrm{E}-05$ \\
\hline Iowa & IA & $1.6 \mathrm{E}-05$ & $3.6 \mathrm{E}-05$ & $3.9 \mathrm{E}-05$ & $5.8 \mathrm{E}-05$ & $4.6 \mathrm{E}-05$ & $4.6 \mathrm{E}-05$ \\
\hline Missouri & $\mathrm{MO}$ & $3.1 \mathrm{E}-05$ & $4.8 \mathrm{E}-05$ & $4.8 \mathrm{E}-05$ & $6.7 \mathrm{E}-05$ & $6.1 \mathrm{E}-05$ & $4.8 \mathrm{E}-05$ \\
\hline Kansas & KS & $6.1 \mathrm{E}-06$ & $1.8 \mathrm{E}-05$ & $2.4 \mathrm{E}-05$ & $4.5 \mathrm{E}-05$ & $5.3 \mathrm{E}-05$ & $4.3 \mathrm{E}-05$ \\
\hline Nebraska & $\mathrm{NE}$ & $9.1 \mathrm{E}-06$ & $2.2 \mathrm{E}-05$ & $2.5 \mathrm{E}-05$ & $4.6 \mathrm{E}-05$ & $4.5 \mathrm{E}-05$ & $4.3 \mathrm{E}-05$ \\
\hline North Dakota & ND & $3.7 \mathrm{E}-06$ & $1.2 \mathrm{E}-05$ & $1.4 \mathrm{E}-05$ & $3.5 \mathrm{E}-05$ & $3.1 \mathrm{E}-05$ & $3.5 \mathrm{E}-05$ \\
\hline South Dakota & SD & $3.3 \mathrm{E}-06$ & $1.4 \mathrm{E}-05$ & $1.5 \mathrm{E}-05$ & $4.3 \mathrm{E}-05$ & $4.0 \mathrm{E}-05$ & $4.4 \mathrm{E}-05$ \\
\hline
\end{tabular}


Table A23 Characterization factors and emissions for the sensitivity scenarios

\begin{tabular}{|c|c|c|c|c|c|c|c|}
\hline \multirow[b]{2}{*}{ State } & & \multicolumn{2}{|c|}{ NOx-Fate } & \multicolumn{2}{|c|}{ VOC-Fate } & \multicolumn{2}{|c|}{ NOx-Hum-Exp } \\
\hline & & Scenario1 & Scenario2 & Scenario1 & Scenario2 & Scenario1 & Scenario2 \\
\hline Oregon & OR & $1.7 \mathrm{E}-03$ & $2.4 \mathrm{E}-03$ & $1.6 \mathrm{E}-05$ & $4.5 \mathrm{E}-06$ & $8.4 \mathrm{E}+01$ & $1.1 \mathrm{E}+02$ \\
\hline Washington & WA & $7.1 \mathrm{E}-04$ & $9.6 \mathrm{E}-04$ & $2.9 \mathrm{E}-05$ & $1.6 \mathrm{E}-05$ & $5.4 \mathrm{E}+01$ & $8.5 \mathrm{E}+01$ \\
\hline Wyoming & WY & $9.0 \mathrm{E}-04$ & $1.0 \mathrm{E}-03$ & $3.4 \mathrm{E}-06$ & $1.7 \mathrm{E}-06$ & $2.6 \mathrm{E}+01$ & $3.0 \mathrm{E}+01$ \\
\hline Idaho & ID & $1.3 \mathrm{E}-03$ & $1.4 \mathrm{E}-03$ & $2.7 \mathrm{E}-06$ & $6.5 \mathrm{E}-07$ & $3.5 \mathrm{E}+01$ & $3.8 \mathrm{E}+01$ \\
\hline Montana & MT & $7.8 \mathrm{E}-04$ & $7.5 \mathrm{E}-04$ & $1.8 \mathrm{E}-06$ & $6.9 \mathrm{E}-07$ & $2.6 \mathrm{E}+01$ & $2.5 \mathrm{E}+01$ \\
\hline California & CA & $1.1 \mathrm{E}-03$ & $1.7 \mathrm{E}-03$ & $5.8 \mathrm{E}-05$ & $2.1 \mathrm{E}-05$ & $1.3 \mathrm{E}+02$ & $2.6 \mathrm{E}+02$ \\
\hline Nevada & NV & $1.6 \mathrm{E}-03$ & $2.0 \mathrm{E}-03$ & $8.7 \mathrm{E}-06$ & $2.1 \mathrm{E}-06$ & $5.8 \mathrm{E}+01$ & $7.7 \mathrm{E}+01$ \\
\hline Utah & UT & $1.3 \mathrm{E}-03$ & $1.5 \mathrm{E}-03$ & $9.4 \mathrm{E}-06$ & $2.5 \mathrm{E}-06$ & $3.9 \mathrm{E}+01$ & $4.8 \mathrm{E}+01$ \\
\hline Colorado & $\mathrm{CO}$ & $8.7 \mathrm{E}-04$ & $1.0 \mathrm{E}-03$ & $9.5 \mathrm{E}-06$ & $6.6 \mathrm{E}-06$ & $4.7 \mathrm{E}+01$ & $6.1 \mathrm{E}+01$ \\
\hline Arizona & $\mathrm{AZ}$ & $1.1 \mathrm{E}-03$ & $1.4 \mathrm{E}-03$ & $1.6 \mathrm{E}-05$ & $8.4 \mathrm{E}-06$ & $6.8 \mathrm{E}+01$ & $9.8 \mathrm{E}+01$ \\
\hline New Mexico & NM & $9.6 \mathrm{E}-04$ & $1.0 \mathrm{E}-03$ & $4.6 \mathrm{E}-06$ & $3.3 \mathrm{E}-06$ & $3.5 \mathrm{E}+01$ & $3.7 \mathrm{E}+01$ \\
\hline Texas & TX & $6.9 \mathrm{E}-04$ & $8.2 \mathrm{E}-04$ & $2.7 \mathrm{E}-05$ & $1.9 \mathrm{E}-05$ & $8.1 \mathrm{E}+01$ & $1.0 \mathrm{E}+02$ \\
\hline Oklahoma & OK & $9.1 \mathrm{E}-04$ & $9.9 \mathrm{E}-04$ & $6.0 \mathrm{E}-06$ & $2.8 \mathrm{E}-06$ & $8.3 E+01$ & $9.4 \mathrm{E}+01$ \\
\hline Arkansas & $\mathrm{AR}$ & $1.5 \mathrm{E}-03$ & $1.7 \mathrm{E}-03$ & $7.5 \mathrm{E}-06$ & $2.2 \mathrm{E}-06$ & $1.3 \mathrm{E}+02$ & $1.6 \mathrm{E}+02$ \\
\hline Louisiana & LA & $1.1 \mathrm{E}-03$ & $1.2 \mathrm{E}-03$ & $7.0 \mathrm{E}-05$ & $6.8 \mathrm{E}-05$ & $8.7 \mathrm{E}+01$ & $1.0 \mathrm{E}+02$ \\
\hline Mississippi & MS & $1.7 \mathrm{E}-03$ & $2.0 \mathrm{E}-03$ & $1.6 \mathrm{E}-05$ & $6.3 \mathrm{E}-06$ & $1.3 \mathrm{E}+02$ & $1.6 \mathrm{E}+02$ \\
\hline Alabama & $\mathrm{AL}$ & $1.8 \mathrm{E}-03$ & $2.2 \mathrm{E}-03$ & $1.0 \mathrm{E}-05$ & $3.5 \mathrm{E}-06$ & $1.5 \mathrm{E}+02$ & $1.9 \mathrm{E}+02$ \\
\hline Kentucky & KY & $1.2 \mathrm{E}-03$ & $1.7 \mathrm{E}-03$ & $3.8 \mathrm{E}-05$ & $9.3 \mathrm{E}-06$ & $1.2 \mathrm{E}+02$ & $1.9 \mathrm{E}+02$ \\
\hline Tennessee & $\mathrm{TN}$ & $1.5 \mathrm{E}-03$ & $2.1 \mathrm{E}-03$ & $1.1 \mathrm{E}-05$ & $2.1 \mathrm{E}-06$ & $1.4 \mathrm{E}+02$ & $1.9 \mathrm{E}+02$ \\
\hline Florida & FL & $1.8 \mathrm{E}-03$ & $2.7 \mathrm{E}-03$ & $8.8 \mathrm{E}-05$ & $3.2 \mathrm{E}-05$ & $1.1 \mathrm{E}+02$ & $1.8 \mathrm{E}+02$ \\
\hline Georgia & GA & $1.9 \mathrm{E}-03$ & $2.5 \mathrm{E}-03$ & $1.4 \mathrm{E}-05$ & $3.1 \mathrm{E}-06$ & $1.5 \mathrm{E}+02$ & $2.2 \mathrm{E}+02$ \\
\hline North Carolina & NC & $2.5 \mathrm{E}-03$ & $3.7 \mathrm{E}-03$ & $1.5 \mathrm{E}-05$ & $4.1 \mathrm{E}-06$ & $1.8 \mathrm{E}+02$ & $2.6 \mathrm{E}+02$ \\
\hline South Carolina & $\overline{\mathrm{SC}}$ & $3.0 \mathrm{E}-03$ & $4.1 \mathrm{E}-03$ & $1.1 \mathrm{E}-05$ & $3.0 \mathrm{E}-06$ & $1.6 \mathrm{E}+02$ & $2.2 \mathrm{E}+02$ \\
\hline Virginia & $\overline{\mathrm{VA}}$ & $2.2 \mathrm{E}-03$ & $2.9 \mathrm{E}-03$ & $4.1 \mathrm{E}-05$ & $2.0 \mathrm{E}-05$ & $1.8 \mathrm{E}+02$ & $2.6 \mathrm{E}+02$ \\
\hline West Virginia & WV & $1.4 \mathrm{E}-03$ & $2.2 \mathrm{E}-03$ & $3.5 \mathrm{E}-05$ & $9.6 \mathrm{E}-06$ & $1.0 \mathrm{E}+02$ & $1.7 \mathrm{E}+02$ \\
\hline Delaware & $\mathrm{DE}$ & $5.1 \mathrm{E}-03$ & $6.5 \mathrm{E}-03$ & $5.5 \mathrm{E}-05$ & $2.9 \mathrm{E}-05$ & $3.2 \mathrm{E}+02$ & $4.3 \mathrm{E}+02$ \\
\hline District of Colum & DC & $1.3 \mathrm{E}-03$ & $2.1 \mathrm{E}-03$ & $8.6 \mathrm{E}-05$ & $3.4 \mathrm{E}-05$ & $1.5 \mathrm{E}+02$ & $2.8 \mathrm{E}+02$ \\
\hline Maryland & MD & $2.1 \mathrm{E}-03$ & $3.3 \mathrm{E}-03$ & $6.9 \mathrm{E}-05$ & $3.3 \mathrm{E}-05$ & $1.8 \mathrm{E}+02$ & $2.9 \mathrm{E}+02$ \\
\hline Connecticut & CT & $2.1 \mathrm{E}-03$ & $3.3 \mathrm{E}-03$ & $1.2 \mathrm{E}-04$ & $5.8 \mathrm{E}-05$ & $9.4 \mathrm{E}+01$ & $1.7 \mathrm{E}+02$ \\
\hline New Jersey & NJ & $1.5 \mathrm{E}-03$ & $2.5 \mathrm{E}-03$ & $1.3 \mathrm{E}-04$ & $8.6 \mathrm{E}-05$ & $1.3 \mathrm{E}+02$ & $2.6 \mathrm{E}+02$ \\
\hline New York & NY & $1.4 \mathrm{E}-03$ & $2.0 \mathrm{E}-03$ & $6.1 \mathrm{E}-05$ & $3.4 \mathrm{E}-05$ & $1.1 \mathrm{E}+02$ & $1.7 \mathrm{E}+02$ \\
\hline Pennsylvania & PA & $1.3 \mathrm{E}-03$ & $1.8 \mathrm{E}-03$ & $5.4 \mathrm{E}-05$ & $2.5 \mathrm{E}-05$ & $1.3 \mathrm{E}+02$ & $2.0 \mathrm{E}+02$ \\
\hline Rhode Island & RI & $2.9 \mathrm{E}-03$ & $4.4 \mathrm{E}-03$ & $1.4 \mathrm{E}-04$ & $6.7 \mathrm{E}-05$ & $7.5 \mathrm{E}+01$ & $1.3 \mathrm{E}+02$ \\
\hline Massachusetts & MA & $2.7 \mathrm{E}-03$ & $4.3 \mathrm{E}-03$ & $1.6 \mathrm{E}-04$ & $9.2 \mathrm{E}-05$ & $5.0 \mathrm{E}+01$ & $9.5 \mathrm{E}+01$ \\
\hline New Hampshire & $\mathrm{NH}$ & $3.2 \mathrm{E}-03$ & $4.6 \mathrm{E}-03$ & $6.9 \mathrm{E}-05$ & $3.0 \mathrm{E}-05$ & $6.2 \mathrm{E}+01$ & $9.3 \mathrm{E}+01$ \\
\hline Vermont & $\begin{array}{l}\text { VT } \\
\end{array}$ & $3.4 \mathrm{E}-03$ & $4.4 \mathrm{E}-03$ & $2.1 \mathrm{E}-05$ & $9.0 \mathrm{E}-06$ & $8.1 \mathrm{E}+01$ & $1.1 \mathrm{E}+02$ \\
\hline Maine & $\mathrm{ME}$ & $4.8 \mathrm{E}-03$ & $5.9 \mathrm{E}-03$ & $1.8 \mathrm{E}-05$ & $5.5 \mathrm{E}-06$ & $3.6 \mathrm{E}+01$ & $4.4 \mathrm{E}+01$ \\
\hline Ohio & $\mathrm{OH}$ & $1.0 \mathrm{E}-03$ & $1.7 \mathrm{E}-03$ & $5.5 \mathrm{E}-05$ & $1.8 \mathrm{E}-05$ & $1.2 \mathrm{E}+02$ & $2.0 \mathrm{E}+02$ \\
\hline Indiana & IN & $8.8 \mathrm{E}-04$ & $1.3 \mathrm{E}-03$ & $4.0 \mathrm{E}-05$ & $1.7 \mathrm{E}-05$ & $1.2 \mathrm{E}+02$ & $1.8 \mathrm{E}+02$ \\
\hline Illinois & IL & $8.9 \mathrm{E}-04$ & $1.3 \mathrm{E}-03$ & $4.3 \mathrm{E}-05$ & $1.9 \mathrm{E}-05$ & $1.2 \mathrm{E}+02$ & $1.9 \mathrm{E}+02$ \\
\hline Michigan & MI & $1.0 \mathrm{E}-03$ & $1.4 \mathrm{E}-03$ & $3.9 \mathrm{E}-05$ & $1.5 \mathrm{E}-05$ & $1.1 \mathrm{E}+02$ & $1.6 \mathrm{E}+02$ \\
\hline Wisconsin & WI & $1.0 \mathrm{E}-03$ & $1.4 \mathrm{E}-03$ & $2.1 \mathrm{E}-05$ & $6.9 \mathrm{E}-06$ & $1.0 \mathrm{E}+02$ & $1.4 \mathrm{E}+02$ \\
\hline Minnesota & MN & $9.3 \mathrm{E}-04$ & $1.2 \mathrm{E}-03$ & $1.3 \mathrm{E}-05$ & $3.3 \mathrm{E}-06$ & $7.0 \mathrm{E}+01$ & $9.2 \mathrm{E}+01$ \\
\hline Iowa & IA & $1.1 \mathrm{E}-03$ & $1.3 \mathrm{E}-03$ & $9.5 \mathrm{E}-06$ & $3.1 \mathrm{E}-06$ & $9.7 \mathrm{E}+01$ & $1.2 \mathrm{E}+02$ \\
\hline Missouri & $\mathrm{MO}$ & $1.0 \mathrm{E}-03$ & $1.3 \mathrm{E}-03$ & $1.7 \mathrm{E}-05$ & $5.1 \mathrm{E}-06$ & $1.2 \mathrm{E}+02$ & $1.6 \mathrm{E}+02$ \\
\hline Kansas & KS & $9.1 \mathrm{E}-04$ & $1.0 \mathrm{E}-03$ & $7.6 \mathrm{E}-06$ & $3.5 \mathrm{E}-06$ & $6.6 \mathrm{E}+01$ & $7.9 \mathrm{E}+01$ \\
\hline Nebraska & $\mathrm{NE}$ & $9.1 \mathrm{E}-04$ & $1.0 \mathrm{E}-03$ & $9.7 \mathrm{E}-06$ & $3.9 \mathrm{E}-06$ & $5.0 \mathrm{E}+01$ & $6.4 \mathrm{E}+01$ \\
\hline North Dakota & ND & $8.5 \mathrm{E}-04$ & $9.5 \mathrm{E}-04$ & $5.1 \mathrm{E}-06$ & $1.5 \mathrm{E}-06$ & $3.7 \mathrm{E}+01$ & $4.2 \mathrm{E}+01$ \\
\hline South Dakota & SD & $1.1 \mathrm{E}-03$ & $1.3 \mathrm{E}-03$ & $3.6 \mathrm{E}-06$ & $1.2 \mathrm{E}-06$ & $5.0 \mathrm{E}+01$ & $5.6 \mathrm{E}+01$ \\
\hline
\end{tabular}


Table A23 continued

\begin{tabular}{|c|c|c|c|c|c|c|c|}
\hline \multirow[b]{2}{*}{ State } & & \multicolumn{2}{|c|}{ VOC-Hum-Exp } & \multicolumn{2}{|c|}{ NOx-Eco-Exp } & \multicolumn{2}{|c|}{ VOC-Eco-Exp } \\
\hline & & Scenario1 & Scenario2 & Scenario1 & Scenario2 & Scenario1 & Scenario2 \\
\hline Oregon & OR & $2.1 \mathrm{E}+00$ & $9.4 \mathrm{E}-01$ & $1.4 \mathrm{E}-01$ & $1.6 \mathrm{E}-01$ & $1.8 \mathrm{E}-03$ & $1.4 \mathrm{E}-03$ \\
\hline Washington & WA & $4.9 \mathrm{E}+00$ & $3.3 \mathrm{E}+00$ & $4.8 \mathrm{E}-01$ & $5.7 \mathrm{E}-01$ & $2.1 \mathrm{E}-02$ & $1.6 \mathrm{E}-02$ \\
\hline Wyoming & WY & $3.7 \mathrm{E}-01$ & $1.6 \mathrm{E}-01$ & $1.2 \mathrm{E}-01$ & $1.4 \mathrm{E}-01$ & $1.1 \mathrm{E}-03$ & $1.3 \mathrm{E}-03$ \\
\hline Idaho & ID & $4.1 \mathrm{E}-01$ & $1.3 \mathrm{E}-01$ & $1.9 \mathrm{E}-01$ & $2.0 \mathrm{E}-01$ & $8.1 \mathrm{E}-04$ & $8.3 \mathrm{E}-04$ \\
\hline Montana & MT & $2.9 \mathrm{E}-01$ & $1.1 \mathrm{E}-01$ & 5.5E-01 & $4.9 \mathrm{E}-01$ & $2.0 \mathrm{E}-03$ & $1.5 \mathrm{E}-03$ \\
\hline California & $\mathrm{CA}$ & $1.6 \mathrm{E}+01$ & $1.0 \mathrm{E}+01$ & $3.5 \mathrm{E}-02$ & $5.2 \mathrm{E}-02$ & $1.3 \mathrm{E}-03$ & $1.1 \mathrm{E}-03$ \\
\hline Nevada & NV & $2.1 \mathrm{E}+00$ & $8.3 \mathrm{E}-01$ & $6.1 \mathrm{E}-02$ & $8.8 \mathrm{E}-02$ & $6.8 \mathrm{E}-04$ & 8.6E-04 \\
\hline Utah & UT & $1.4 \mathrm{E}+00$ & $4.0 \mathrm{E}-01$ & $4.7 \mathrm{E}-02$ & $6.8 \mathrm{E}-02$ & $1.1 \mathrm{E}-03$ & $1.5 \mathrm{E}-03$ \\
\hline Colorado & $\mathrm{CO}$ & $1.8 \mathrm{E}+00$ & $9.5 \mathrm{E}-01$ & $6.5 \mathrm{E}-02$ & $9.3 \mathrm{E}-02$ & $1.3 \mathrm{E}-03$ & $1.6 \mathrm{E}-03$ \\
\hline Arizona & AZ & $3.9 \mathrm{E}+00$ & $2.6 \mathrm{E}+00$ & $6.3 \mathrm{E}-02$ & $8.4 \mathrm{E}-02$ & $1.1 \mathrm{E}-03$ & $8.1 \mathrm{E}-04$ \\
\hline New Mexico & NM & $7.4 \mathrm{E}-01$ & $3.7 \mathrm{E}-01$ & $5.2 \mathrm{E}-02$ & $7.0 \mathrm{E}-02$ & $9.8 \mathrm{E}-04$ & $1.3 \mathrm{E}-03$ \\
\hline Texas & TX & $5.6 \mathrm{E}+00$ & $3.8 \mathrm{E}+00$ & $2.3 \mathrm{E}+00$ & $5.2 \mathrm{E}+00$ & $2.3 \mathrm{E}-01$ & $2.5 \mathrm{E}-01$ \\
\hline Oklahoma & $\mathrm{OK}$ & $1.1 \mathrm{E}+00$ & $4.5 \mathrm{E}-01$ & $9.9 \mathrm{E}-02$ & $1.4 \mathrm{E}-01$ & $1.2 \mathrm{E}-03$ & $1.5 \mathrm{E}-03$ \\
\hline Arkansas & $\mathrm{AR}$ & $1.7 \mathrm{E}+00$ & $6.6 \mathrm{E}-01$ & $1.2 \mathrm{E}-01$ & $2.5 \mathrm{E}-01$ & $1.6 \mathrm{E}-03$ & $2.8 \mathrm{E}-03$ \\
\hline Louisiana & $\overline{L A}$ & $5.8 \mathrm{E}+00$ & $5.1 \mathrm{E}+00$ & $1.3 \mathrm{E}+01$ & $3.9 \mathrm{E}+01$ & $1.3 \mathrm{E}+00$ & $2.9 \mathrm{E}+00$ \\
\hline Mississippi & MS & $2.1 \mathrm{E}+00$ & $8.6 \mathrm{E}-01$ & $1.3 \mathrm{E}+00$ & $3.3 \mathrm{E}+00$ & $7.2 \mathrm{E}-02$ & $1.2 \mathrm{E}-01$ \\
\hline Alabama & $\mathrm{AL}$ & $1.7 \mathrm{E}+00$ & 6.4E-01 & $4.8 \mathrm{E}-01$ & $1.1 \mathrm{E}+00$ & $1.1 \mathrm{E}-02$ & $1.7 \mathrm{E}-02$ \\
\hline Kentucky & $\mathrm{KY}$ & $5.4 \mathrm{E}+00$ & $1.7 \mathrm{E}+00$ & $5.8 \mathrm{E}-02$ & $1.1 \mathrm{E}-01$ & $2.5 \mathrm{E}-03$ & $1.8 \mathrm{E}-03$ \\
\hline Tennessee & $\mathrm{TN}$ & $1.9 \mathrm{E}+00$ & $5.4 \mathrm{E}-01$ & $1.0 \mathrm{E}-01$ & $1.9 \mathrm{E}-01$ & $2.0 \mathrm{E}-03$ & $2.5 \mathrm{E}-03$ \\
\hline Florida & FL & $7.5 \mathrm{E}+00$ & $4.5 \mathrm{E}+00$ & $1.8 \mathrm{E}+01$ & $3.7 \mathrm{E}+01$ & $8.8 \mathrm{E}-01$ & $7.0 \mathrm{E}-01$ \\
\hline Georgia & GA & $3.3 \mathrm{E}+00$ & $1.1 \mathrm{E}+00$ & $3.5 \mathrm{E}-01$ & $7.0 \mathrm{E}-01$ & $5.4 \mathrm{E}-03$ & $6.7 \mathrm{E}-03$ \\
\hline North Carolina & NC & $2.3 \mathrm{E}+00$ & $9.1 \mathrm{E}-01$ & $1.8 \mathrm{E}-01$ & $2.7 \mathrm{E}-01$ & $4.3 \mathrm{E}-03$ & $3.7 \mathrm{E}-03$ \\
\hline South Carolina & SC & $1.5 \mathrm{E}+00$ & $5.8 \mathrm{E}-01$ & $4.3 \mathrm{E}-01$ & $5.2 \mathrm{E}-01$ & $6.9 \mathrm{E}-03$ & $6.5 \mathrm{E}-03$ \\
\hline Virginia & $\mathrm{VA}$ & $6.0 \mathrm{E}+00$ & $3.1 \mathrm{E}+00$ & $9.3 \mathrm{E}-02$ & $1.2 \mathrm{E}-01$ & $4.6 \mathrm{E}-03$ & $3.3 \mathrm{E}-03$ \\
\hline West Virginia & WV & $4.1 \mathrm{E}+00$ & $1.4 \mathrm{E}+00$ & $4.6 \mathrm{E}-02$ & $7.6 \mathrm{E}-02$ & $2.9 \mathrm{E}-03$ & $2.1 \mathrm{E}-03$ \\
\hline Delaware & $\mathrm{DE}$ & $5.4 \mathrm{E}+00$ & $3.4 \mathrm{E}+00$ & $1.1 \mathrm{E}-01$ & $1.2 \mathrm{E}-01$ & $4.0 \mathrm{E}-03$ & $2.7 \mathrm{E}-03$ \\
\hline District of Colun & $\mathrm{DC}$ & $1.3 \mathrm{E}+01$ & $5.5 \mathrm{E}+00$ & $6.0 \mathrm{E}-02$ & 7.4E-02 & $7.5 \mathrm{E}-03$ & $4.5 \mathrm{E}-03$ \\
\hline Maryland & $\mathrm{MD}$ & $8.4 \mathrm{E}+00$ & $4.3 \mathrm{E}+00$ & $7.3 \mathrm{E}-02$ & $9.0 \mathrm{E}-02$ & $5.8 \mathrm{E}-03$ & $3.6 \mathrm{E}-03$ \\
\hline Connecticut & CT & $7.3 \mathrm{E}+00$ & $4.2 \mathrm{E}+00$ & $3.1 \mathrm{E}-02$ & $4.9 \mathrm{E}-02$ & $1.7 \mathrm{E}-03$ & $9.1 \mathrm{E}-04$ \\
\hline New Jersey & $\mathrm{NJ}$ & $1.5 \mathrm{E}+01$ & $1.3 \mathrm{E}+01$ & $4.2 \mathrm{E}-02$ & $6.0 \mathrm{E}-02$ & $3.8 \mathrm{E}-03$ & $2.6 \mathrm{E}-03$ \\
\hline New York & NY & $7.4 \mathrm{E}+00$ & $6.0 \mathrm{E}+00$ & $6.4 \mathrm{E}-02$ & $9.2 \mathrm{E}-02$ & $3.2 \mathrm{E}-03$ & $1.8 \mathrm{E}-03$ \\
\hline Pennsylvania & PA & $8.1 \mathrm{E}+00$ & $4.5 \mathrm{E}+00$ & $6.1 \mathrm{E}-02$ & $7.9 \mathrm{E}-02$ & $4.7 \mathrm{E}-03$ & $3.0 \mathrm{E}-03$ \\
\hline Rhode Island & RI & $4.6 \mathrm{E}+00$ & $2.4 \mathrm{E}+00$ & $3.3 \mathrm{E}-02$ & $5.3 \mathrm{E}-02$ & $1.2 \mathrm{E}-03$ & $4.4 \mathrm{E}-04$ \\
\hline Massachusetts & MA & $3.8 \mathrm{E}+00$ & $2.8 \mathrm{E}+00$ & $3.7 \mathrm{E}-02$ & $5.9 \mathrm{E}-02$ & $1.5 \mathrm{E}-03$ & $6.3 \mathrm{E}-04$ \\
\hline New Hampshire & $\mathrm{NH}$ & $1.7 \mathrm{E}+00$ & $8.5 \mathrm{E}-01$ & $5.5 \mathrm{E}-02$ & $7.9 \mathrm{E}-02$ & $5.9 \mathrm{E}-04$ & 2.3E-04 \\
\hline Vermont & VT & $9.2 \mathrm{E}-01$ & $5.0 \mathrm{E}-01$ & $1.1 \mathrm{E}-01$ & $1.4 \mathrm{E}-01$ & $3.3 \mathrm{E}-04$ & $1.8 \mathrm{E}-04$ \\
\hline Maine & $\mathrm{ME}$ & $3.8 \mathrm{E}-01$ & $1.7 \mathrm{E}-01$ & $1.8 \mathrm{E}-01$ & $2.0 \mathrm{E}-01$ & $2.0 \mathrm{E}-04$ & $8.2 \mathrm{E}-05$ \\
\hline Ohio & $\mathrm{OH}$ & $7.8 \mathrm{E}+00$ & $2.8 \mathrm{E}+00$ & $4.9 \mathrm{E}-02$ & $8.5 \mathrm{E}-02$ & $3.6 \mathrm{E}-03$ & $2.2 \mathrm{E}-03$ \\
\hline Indiana & IN & $6.8 \mathrm{E}+00$ & $4.1 \mathrm{E}+00$ & $5.8 \mathrm{E}-02$ & $1.0 \mathrm{E}-01$ & $3.4 \mathrm{E}-03$ & $2.3 \mathrm{E}-03$ \\
\hline Illinois & IL & $8.1 \mathrm{E}+00$ & $4.7 \mathrm{E}+00$ & $7.8 \mathrm{E}-02$ & $1.3 \mathrm{E}-01$ & $4.1 \mathrm{E}-03$ & $2.3 \mathrm{E}-03$ \\
\hline Michigan & MI & $6.4 \mathrm{E}+00$ & $3.3 \mathrm{E}+00$ & $1.8 \mathrm{E}-01$ & $2.6 \mathrm{E}-01$ & $4.3 \mathrm{E}-03$ & $2.3 \mathrm{E}-03$ \\
\hline Wisconsin & $\overline{\mathrm{WI}}$ & $3.8 \mathrm{E}+00$ & $1.5 \mathrm{E}+00$ & $2.5 \mathrm{E}-01$ & $3.3 \mathrm{E}-01$ & $3.2 \mathrm{E}-03$ & $1.5 \mathrm{E}-03$ \\
\hline Minnesota & MN & $2.8 \mathrm{E}+00$ & $9.6 \mathrm{E}-01$ & $5.1 \mathrm{E}-01$ & $6.4 \mathrm{E}-01$ & $3.8 \mathrm{E}-03$ & $1.4 \mathrm{E}-03$ \\
\hline Iowa & IA & $1.7 \mathrm{E}+00$ & $6.4 \mathrm{E}-01$ & $1.7 \mathrm{E}-01$ & $2.1 \mathrm{E}-01$ & $1.5 \mathrm{E}-03$ & $8.6 \mathrm{E}-04$ \\
\hline Missouri & $\mathrm{MO}$ & $3.5 \mathrm{E}+00$ & $1.3 \mathrm{E}+00$ & 8.7E-02 & $1.3 \mathrm{E}-01$ & $1.7 \mathrm{E}-03$ & $1.3 \mathrm{E}-03$ \\
\hline Kansas & KS & $1.1 \mathrm{E}+00$ & $4.6 \mathrm{E}-01$ & $1.1 \mathrm{E}-01$ & $1.4 \mathrm{E}-01$ & $1.0 \mathrm{E}-03$ & $9.8 \mathrm{E}-04$ \\
\hline Nebraska & $\mathrm{NE}$ & $1.3 \mathrm{E}+00$ & $5.0 \mathrm{E}-01$ & $1.8 \mathrm{E}-01$ & $2.0 \mathrm{E}-01$ & $1.6 \mathrm{E}-03$ & $1.0 \mathrm{E}-03$ \\
\hline North Dakota & ND & 5.9E-01 & $2.0 \mathrm{E}-01$ & $9.0 \mathrm{E}-01$ & $1.0 \mathrm{E}+00$ & $5.9 \mathrm{E}-03$ & $2.6 \mathrm{E}-03$ \\
\hline South Dakota & SD & 5.3E-01 & $1.9 \mathrm{E}-01$ & $4.4 \mathrm{E}-01$ & $4.8 \mathrm{E}-01$ & $1.5 \mathrm{E}-03$ & $1.0 \mathrm{E}-03$ \\
\hline
\end{tabular}


Table A23 continued

\begin{tabular}{|c|c|c|c|c|c|c|c|}
\hline \multirow[b]{2}{*}{ State } & & \multicolumn{2}{|c|}{ NOx-Hum-Eff } & \multicolumn{2}{|c|}{ VOC-Hum-Eff } & \multicolumn{2}{|c|}{ NOx-Emis } \\
\hline & & Scenario1 & Scenario2 & Scenario1 & Scenario2 & Scenario1 & Scenario2 \\
\hline Oregon & OR & $7.5 \mathrm{E}-04$ & $1.0 \mathrm{E}-03$ & $2.1 \mathrm{E}-05$ & $9.4 \mathrm{E}-06$ & $5.7 \mathrm{E}+02$ & $3.3 \mathrm{E}+02$ \\
\hline Washington & WA & $4.2 \mathrm{E}-04$ & $6.9 \mathrm{E}-04$ & $3.9 \mathrm{E}-05$ & $2.5 \mathrm{E}-05$ & $1.2 \mathrm{E}+03$ & $7.6 \mathrm{E}+02$ \\
\hline Wyoming & $\overline{\mathrm{WY}}$ & $1.8 \mathrm{E}-04$ & $2.1 \mathrm{E}-04$ & $3.2 \mathrm{E}-06$ & $1.3 \mathrm{E}-06$ & $7.5 \mathrm{E}+02$ & $5.4 \mathrm{E}+02$ \\
\hline Idaho & ID & $2.2 \mathrm{E}-04$ & $2.5 \mathrm{E}-04$ & $3.4 \mathrm{E}-06$ & $1.1 \mathrm{E}-06$ & $4.8 \mathrm{E}+02$ & $3.4 \mathrm{E}+02$ \\
\hline Montana & MT & $1.7 \mathrm{E}-04$ & $1.7 \mathrm{E}-04$ & $2.6 \mathrm{E}-06$ & $9.3 \mathrm{E}-07$ & $5.9 \mathrm{E}+02$ & $4.1 \mathrm{E}+02$ \\
\hline California & CA & $1.2 \mathrm{E}-03$ & $2.4 \mathrm{E}-03$ & $1.5 \mathrm{E}-04$ & $1.0 \mathrm{E}-04$ & $3.5 \mathrm{E}+03$ & $2.0 \mathrm{E}+03$ \\
\hline Nevada & $\mathrm{NV}$ & $5.4 \mathrm{E}-04$ & $7.6 \mathrm{E}-04$ & $2.6 \mathrm{E}-05$ & $1.0 \mathrm{E}-05$ & $3.3 \mathrm{E}+02$ & $2.0 \mathrm{E}+02$ \\
\hline Utah & UT & $2.7 \mathrm{E}-04$ & $3.3 \mathrm{E}-04$ & $1.1 \mathrm{E}-05$ & $3.2 \mathrm{E}-06$ & $8.8 \mathrm{E}+02$ & $6.2 \mathrm{E}+02$ \\
\hline Colorado & $\mathrm{CO}$ & $3.7 \mathrm{E}-04$ & $4.9 \mathrm{E}-04$ & $1.6 \mathrm{E}-05$ & $8.5 \mathrm{E}-06$ & $9.2 \mathrm{E}+02$ & $5.6 \mathrm{E}+02$ \\
\hline \begin{tabular}{|l|} 
Arizona \\
\end{tabular} & $\mathrm{AZ}$ & $6.1 \mathrm{E}-04$ & $9.4 \mathrm{E}-04$ & $4.4 \mathrm{E}-05$ & $3.0 \mathrm{E}-05$ & $1.1 \mathrm{E}+03$ & $5.7 \mathrm{E}+02$ \\
\hline New Mexico & NM & $2.5 \mathrm{E}-04$ & $2.6 \mathrm{E}-04$ & $7.0 \mathrm{E}-06$ & $3.4 \mathrm{E}-06$ & $8.3 \mathrm{E}+02$ & $6.3 \mathrm{E}+02$ \\
\hline Texas & TX & $6.5 \mathrm{E}-04$ & $8.1 \mathrm{E}-04$ & $4.1 \mathrm{E}-05$ & $2.4 \mathrm{E}-05$ & $5.2 \mathrm{E}+03$ & $3.2 \mathrm{E}+03$ \\
\hline$\overline{\text { Oklahoma }}$ & OK & $7.5 \mathrm{E}-04$ & $8.5 \mathrm{E}-04$ & $1.2 \mathrm{E}-05$ & $4.4 \mathrm{E}-06$ & $1.1 \mathrm{E}+03$ & $7.4 \mathrm{E}+02$ \\
\hline Arkansas & AR & $1.2 \mathrm{E}-03$ & $1.5 \mathrm{E}-03$ & $1.9 \mathrm{E}-05$ & 7.1E-06 & $1.0 \mathrm{E}+03$ & $5.7 \mathrm{E}+02$ \\
\hline$\overline{\text { Louisiana }}$ & LA & $8.0 \mathrm{E}-04$ & $9.3 \mathrm{E}-04$ & $5.9 \mathrm{E}-05$ & $5.2 \mathrm{E}-05$ & $2.3 \mathrm{E}+03$ & $1.7 \mathrm{E}+03$ \\
\hline Mississippi & MS & $1.3 \mathrm{E}-03$ & $1.5 \mathrm{E}-03$ & $2.2 \mathrm{E}-05$ & $8.8 \mathrm{E}-06$ & $1.0 \mathrm{E}+03$ & $5.6 \mathrm{E}+02$ \\
\hline Alabama & $\mathrm{AL}$ & $1.6 \mathrm{E}-03$ & $2.0 \mathrm{E}-03$ & $1.9 \mathrm{E}-05$ & $7.1 \mathrm{E}-06$ & $1.3 \mathrm{E}+03$ & $6.3 \mathrm{E}+02$ \\
\hline Kentucky & KY & $1.3 \mathrm{E}-03$ & $2.0 \mathrm{E}-03$ & $6.1 \mathrm{E}-05$ & $2.0 \mathrm{E}-05$ & $2.5 \mathrm{E}+03$ & $1.0 \mathrm{E}+03$ \\
\hline Tennessee & $\mathrm{TN}$ & $1.5 \mathrm{E}-03$ & $2.1 \mathrm{E}-03$ & $2.1 \mathrm{E}-05$ & $5.9 \mathrm{E}-06$ & $1.2 \mathrm{E}+03$ & $5.0 \mathrm{E}+02$ \\
\hline Florida & FL & $1.3 \mathrm{E}-03$ & $2.2 \mathrm{E}-03$ & $9.8 \mathrm{E}-05$ & $6.1 \mathrm{E}-05$ & $2.8 \mathrm{E}+03$ & $1.1 \mathrm{E}+03$ \\
\hline Georgia & GA & $1.6 \mathrm{E}-03$ & $2.2 \mathrm{E}-03$ & $3.4 \mathrm{E}-05$ & $1.1 \mathrm{E}-05$ & $1.7 \mathrm{E}+03$ & $7.7 \mathrm{E}+02$ \\
\hline North Carolina & $\mathrm{NC}$ & $2.1 \mathrm{E}-03$ & $3.1 \mathrm{E}-03$ & $2.9 \mathrm{E}-05$ & $1.1 \mathrm{E}-05$ & $1.7 \mathrm{E}+03$ & $6.7 \mathrm{E}+02$ \\
\hline \begin{tabular}{|l|} 
South Carolina \\
\end{tabular} & SC & $1.8 \mathrm{E}-03$ & $2.4 \mathrm{E}-03$ & $1.8 \mathrm{E}-05$ & $6.9 \mathrm{E}-06$ & $1.0 \mathrm{E}+03$ & $4.8 \mathrm{E}+02$ \\
\hline Virginia & $\mathrm{VA}$ & $2.1 \mathrm{E}-03$ & $3.0 \mathrm{E}-03$ & $7.2 \mathrm{E}-05$ & $3.8 \mathrm{E}-05$ & $1.5 \mathrm{E}+03$ & $9.2 \mathrm{E}+02$ \\
\hline West Virginia & $\overline{\mathrm{WV}}$ & $1.2 \mathrm{E}-03$ & $2.0 \mathrm{E}-03$ & $5.0 \mathrm{E}-05$ & $1.8 \mathrm{E}-05$ & $1.2 \mathrm{E}+03$ & $4.0 \mathrm{E}+02$ \\
\hline Delaware & $\mathrm{DE}$ & $4.1 \mathrm{E}-03$ & $5.6 \mathrm{E}-03$ & $6.9 \mathrm{E}-05$ & $4.4 \mathrm{E}-05$ & $4.2 \mathrm{E}+01$ & $2.6 \mathrm{E}+01$ \\
\hline District of Colum & $\mathrm{DC}$ & $1.8 \mathrm{E}-03$ & $3.4 \mathrm{E}-03$ & $1.6 \mathrm{E}-04$ & $6.7 \mathrm{E}-05$ & $9.3 \mathrm{E}+01$ & $4.9 \mathrm{E}+01$ \\
\hline Maryland & $\mathrm{MD}$ & $2.3 \mathrm{E}-03$ & $3.8 \mathrm{E}-03$ & $1.1 \mathrm{E}-04$ & $5.8 \mathrm{E}-05$ & $6.0 \mathrm{E}+02$ & $3.1 \mathrm{E}+02$ \\
\hline Connecticut & CT & $1.2 \mathrm{E}-03$ & $2.2 \mathrm{E}-03$ & $9.4 \mathrm{E}-05$ & $5.4 \mathrm{E}-05$ & $3.9 \mathrm{E}+02$ & $1.9 \mathrm{E}+02$ \\
\hline New Jersey & NJ & $1.7 \mathrm{E}-03$ & $3.3 \mathrm{E}-03$ & $1.9 \mathrm{E}-04$ & $1.6 \mathrm{E}-04$ & $1.3 \mathrm{E}+03$ & $6.9 \mathrm{E}+02$ \\
\hline New York & NY & $1.1 \mathrm{E}-03$ & $1.8 \mathrm{E}-03$ & $8.7 \mathrm{E}-05$ & $7.2 \mathrm{E}-05$ & $1.8 \mathrm{E}+03$ & $8.9 \mathrm{E}+02$ \\
\hline Pennsylvania & $\mathrm{PA}$ & $1.6 \mathrm{E}-03$ & $2.6 \mathrm{E}-03$ & $1.1 \mathrm{E}-04$ & $6.0 \mathrm{E}-05$ & $2.1 \mathrm{E}+03$ & $1.1 \mathrm{E}+03$ \\
\hline Rhode Island & $\mathrm{RI}$ & $9.4 \mathrm{E}-04$ & $1.7 \mathrm{E}-03$ & $6.0 \mathrm{E}-05$ & $3.2 \mathrm{E}-05$ & $2.4 \mathrm{E}+02$ & $1.4 \mathrm{E}+02$ \\
\hline Massachusetts & MA & $5.8 \mathrm{E}-04$ & $1.1 \mathrm{E}-03$ & $4.6 \mathrm{E}-05$ & $3.5 \mathrm{E}-05$ & $7.1 \mathrm{E}+02$ & $3.6 \mathrm{E}+02$ \\
\hline New Hampshire & $\mathrm{NH}$ & $6.4 \mathrm{E}-04$ & $9.7 \mathrm{E}-04$ & $2.0 \mathrm{E}-05$ & $9.8 \mathrm{E}-06$ & $1.9 \mathrm{E}+02$ & $9.9 \mathrm{E}+01$ \\
\hline Vermont & VT & $6.1 \mathrm{E}-04$ & $8.3 \mathrm{E}-04$ & $9.8 \mathrm{E}-06$ & $5.1 \mathrm{E}-06$ & $1.1 \mathrm{E}+02$ & $5.4 \mathrm{E}+01$ \\
\hline Maine & $\mathrm{ME}$ & $2.6 \mathrm{E}-04$ & $3.2 \mathrm{E}-04$ & $4.2 \mathrm{E}-06$ & $1.8 \mathrm{E}-06$ & $2.3 \mathrm{E}+02$ & $1.4 \mathrm{E}+02$ \\
\hline Ohio & $\mathrm{OH}$ & $1.3 \mathrm{E}-03$ & $2.2 \mathrm{E}-03$ & $8.7 \mathrm{E}-05$ & $3.2 \mathrm{E}-05$ & $2.6 \mathrm{E}+03$ & $1.1 \mathrm{E}+03$ \\
\hline Indiana & IN & $1.1 \mathrm{E}-03$ & $1.7 \mathrm{E}-03$ & $6.0 \mathrm{E}-05$ & $2.7 \mathrm{E}-05$ & $2.1 \mathrm{E}+03$ & $9.8 \mathrm{E}+02$ \\
\hline Illinois & $\mathrm{IL}$ & $1.1 \mathrm{E}-03$ & $1.6 \mathrm{E}-03$ & $6.2 \mathrm{E}-05$ & $2.8 \mathrm{E}-05$ & $2.4 \mathrm{E}+03$ & $1.0 \mathrm{E}+03$ \\
\hline Michigan & MI & $9.4 \mathrm{E}-04$ & $1.3 \mathrm{E}-03$ & $5.7 \mathrm{E}-05$ & $2.6 \mathrm{E}-05$ & $2.0 \mathrm{E}+03$ & $1.1 \mathrm{E}+03$ \\
\hline Wisconsin & WI & $8.5 \mathrm{E}-04$ & $1.2 \mathrm{E}-03$ & $3.5 \mathrm{E}-05$ & $1.3 \mathrm{E}-05$ & $1.1 \mathrm{E}+03$ & $5.4 \mathrm{E}+02$ \\
\hline Minnesota & MN & $5.2 \mathrm{E}-04$ & $7.1 \mathrm{E}-04$ & $2.5 \mathrm{E}-05$ & $8.8 \mathrm{E}-06$ & $1.3 \mathrm{E}+03$ & $7.4 \mathrm{E}+02$ \\
\hline Iowa & IA & $7.6 \mathrm{E}-04$ & $9.8 \mathrm{E}-04$ & $1.5 \mathrm{E}-05$ & $5.2 \mathrm{E}-06$ & $8.4 \mathrm{E}+02$ & $4.3 \mathrm{E}+02$ \\
\hline Missouri & $\mathrm{MO}$ & $1.1 \mathrm{E}-03$ & $1.6 \mathrm{E}-03$ & $3.7 \mathrm{E}-05$ & $1.3 \mathrm{E}-05$ & $1.7 \mathrm{E}+03$ & $8.6 \mathrm{E}+02$ \\
\hline Kansas & $\mathrm{KS}$ & $5.3 \mathrm{E}-04$ & $6.3 \mathrm{E}-04$ & $9.9 \mathrm{E}-06$ & $4.1 \mathrm{E}-06$ & $1.0 \mathrm{E}+03$ & $6.0 \mathrm{E}+02$ \\
\hline Nebraska & $\mathrm{NE}$ & $3.7 \mathrm{E}-04$ & $4.8 \mathrm{E}-04$ & $1.2 \mathrm{E}-05$ & $4.4 \mathrm{E}-06$ & $8.1 \mathrm{E}+02$ & $4.8 \mathrm{E}+02$ \\
\hline North Dakota & $\mathrm{ND}$ & $2.5 \mathrm{E}-04$ & $2.8 \mathrm{E}-04$ & $5.0 \mathrm{E}-06$ & $1.6 \mathrm{E}-06$ & $6.5 \mathrm{E}+02$ & $3.7 \mathrm{E}+02$ \\
\hline South Dakota & SD & $3.3 \mathrm{E}-04$ & $3.8 \mathrm{E}-04$ & $4.5 \mathrm{E}-06$ & $1.6 \mathrm{E}-06$ & $3.4 \mathrm{E}+02$ & $1.5 \mathrm{E}+02$ \\
\hline
\end{tabular}




\section{APPENDIX C}

\section{DETAILS OF THE CASE STUDY}

The detailed LCI data, emission factors for NOx and VOC, and the results of the LCIA are listed in this section.

Table A24 Monthly energy consumption by type for the studied cases

\begin{tabular}{|l|c|c|c|c|c|c|c|c|c|c|}
\hline & \multicolumn{3}{|c}{ PIT } & \multicolumn{2}{c}{ PIT_HP } & \multicolumn{2}{c}{ MIN AUS } & \multicolumn{3}{c|}{ POR } \\
\cline { 2 - 14 } & $\begin{array}{l}\text { Heating } \\
\text { KWh th }\end{array}$ & $\begin{array}{c}\text { Cooling } \\
\text { KWh el }\end{array}$ & $\begin{array}{c}\text { Heating } \\
\text { KWh el }\end{array}$ & $\begin{array}{c}\text { Cooling } \\
\text { KWh el }\end{array}$ & $\begin{array}{c}\text { Heating } \\
\text { KWh th }\end{array}$ & $\begin{array}{c}\text { Cooling } \\
\text { KWh el }\end{array}$ & $\begin{array}{c}\text { Heating } \\
\text { KWh th }\end{array}$ & $\begin{array}{c}\text { Cooling } \\
\text { KWh el }\end{array}$ & $\begin{array}{c}\text { Heating } \\
\text { KWh th }\end{array}$ & $\begin{array}{c}\text { Cooling } \\
\text { KWh el }\end{array}$ \\
\hline January & 2843 & 0 & 2200 & 0 & 3664 & 0 & 1260 & 10 & 227 & 0 \\
\hline February & 2315 & 0 & 1890 & 0 & 2696 & 0 & 1084 & 30 & 1583 & 0 \\
\hline March & 1260 & 12 & 890 & 10 & 1846 & 0 & 615 & 80 & 1231 & 10 \\
\hline April & 791 & 22 & 530 & 30 & 674 & 26 & 0 & 140 & 791 & 19 \\
\hline May & 176 & 99 & 70 & 130 & 147 & 117 & 0 & 230 & 381 & 68 \\
\hline June & 0 & 181 & 0 & 250 & 0 & 189 & 0 & 320 & 0 & 99 \\
\hline July & 0 & 196 & 0 & 270 & 0 & 231 & 0 & 400 & 0 & 154 \\
\hline August & 0 & 202 & 0 & 270 & 0 & 205 & 0 & 390 & 0 & 162 \\
\hline September & 0 & 103 & 0 & 140 & 88 & 93 & 0 & 260 & 176 & 104 \\
\hline October & 674 & 36 & 380 & 50 & 791 & 24 & 0 & 170 & 762 & 25 \\
\hline November & 1524 & 3 & 1010 & 0 & 1964 & 0 & 469 & 70 & 1377 & 0 \\
\hline December & 2345 & 0 & 1780 & 0 & 3283 & 0 & 1231 & 20 & 2052 & 0 \\
\hline
\end{tabular}


Table A25 Emission factors for energy consumption

\begin{tabular}{|c|c|c|c|c|c|c|c|}
\hline & \multicolumn{5}{|c|}{ Electricity (per KWh elec) } & \multicolumn{2}{|c|}{ NG (per KWh thermal) } \\
\hline & \multicolumn{2}{|c|}{$\begin{array}{l}\text { Extraction \& } \\
\text { processing }\end{array}$} & \multirow{2}{*}{$\begin{array}{c}\text { Transportation } \\
\text { Coal }\end{array}$} & \multicolumn{2}{|c|}{ Combustion } & \multirow{2}{*}{$\begin{array}{c}\text { Extraction \& } \\
\text { processing }\end{array}$} & \multirow[t]{2}{*}{ Combustion } \\
\hline & Coal & NG & & Coal & NG & & \\
\hline $\mathrm{NOx}(\mathrm{MT})$ & $3.7 \mathrm{E}-07$ & $7.4 \mathrm{E}-08$ & 3.9E-08 & $3.2 \mathrm{E}-06$ & 4.4E-07 & $2.6 \mathrm{E}-08$ & $1.1 \mathrm{E}-07$ \\
\hline VOC (MT-MIR) & $1.3 \mathrm{E}-08$ & $4.0 \mathrm{E}-07$ & 3.9E-09 & $2.6 \mathrm{E}-08$ & $2.5 \mathrm{E}-08$ & $1.4 \mathrm{E}-07$ & 8.6E-09 \\
\hline
\end{tabular}

Table A26 LCI and LCIA results for furnace and AC at Pittsburgh

\begin{tabular}{|c|c|c|c|c|c|c|}
\hline & \multicolumn{6}{|c|}{ Pittsburgh (furnace and AC) } \\
\hline & \multicolumn{4}{|c|}{ LCI } & \multicolumn{2}{|c|}{ LCIA } \\
\hline & \multicolumn{2}{|c|}{$\mathrm{NOx}(\mathrm{MT})$} & \multicolumn{2}{|c|}{ VOC (MT-MIR) } & \multirow{2}{*}{$\begin{array}{c}\text { Human } \\
\text { exposure }\end{array}$} & \multirow{2}{*}{$\begin{array}{c}\text { Ecosystem } \\
\text { exposure }\end{array}$} \\
\hline & PA & LA & PA & LA & & \\
\hline January & $3.0 \mathrm{E}-04$ & 7.3E-05 & $2.4 \mathrm{E}-05$ & 3.9E-04 & $1.7 \mathrm{E}-03$ & $1.4 \mathrm{E}-03$ \\
\hline February & $2.4 \mathrm{E}-04$ & 5.9E-05 & $2.0 \mathrm{E}-05$ & $3.2 \mathrm{E}-04$ & $2.1 \mathrm{E}-03$ & $4.2 \mathrm{E}-04$ \\
\hline March & $1.6 \mathrm{E}-04$ & 3.2E-05 & $1.1 \mathrm{E}-05$ & 1.7E-04 & 2.1E-03 & 4.6E-04 \\
\hline April & $1.3 \mathrm{E}-04$ & 2.0E-05 & $7.4 \mathrm{E}-06$ & $1.1 \mathrm{E}-04$ & 4.2E-03 & $1.5 \mathrm{E}-04$ \\
\hline May & $2.2 \mathrm{E}-04$ & 4.9E-06 & 4.0E-06 & $2.6 \mathrm{E}-05$ & $1.1 \mathrm{E}-02$ & 9.9E-05 \\
\hline June & 3.7E-04 & $6.7 \mathrm{E}-07$ & 4.5E-06 & $3.6 \mathrm{E}-06$ & $4.6 \mathrm{E}-02$ & $4.5 \mathrm{E}-05$ \\
\hline July & $4.0 \mathrm{E}-04$ & 7.3E-07 & 4.9E-06 & $3.9 \mathrm{E}-06$ & $4.8 \mathrm{E}-02$ & $1.4 \mathrm{E}-04$ \\
\hline August & 4.1E-04 & 7.5E-07 & 5.0E-06 & $4.0 \mathrm{E}-06$ & 3.5E-02 & 7.0E-05 \\
\hline September & $2.1 \mathrm{E}-04$ & $3.8 \mathrm{E}-07$ & $2.6 \mathrm{E}-06$ & $2.1 \mathrm{E}-06$ & $1.2 \mathrm{E}-02$ & 2.7E-04 \\
\hline October & $1.4 \mathrm{E}-04$ & 1.7E-05 & 6.7E-06 & 9.3E-05 & 3.1E-03 & 3.7E-04 \\
\hline November & $1.7 \mathrm{E}-04$ & 3.9E-05 & 1.3E-05 & $2.1 \mathrm{E}-04$ & 2.7E-03 & 8.0E-04 \\
\hline December & $2.5 \mathrm{E}-04$ & 6.0E-05 & $2.0 \mathrm{E}-05$ & $3.2 \mathrm{E}-04$ & $1.2 \mathrm{E}-03$ & 5.3E-04 \\
\hline
\end{tabular}

Table A27 LCI and LCIA results for heat pump at Pittsburgh

\begin{tabular}{|c|c|c|c|c|c|c|}
\hline & \multicolumn{6}{|c|}{ Pittsburgh (heat pump) } \\
\hline & \multicolumn{4}{|c|}{ LCI } & \multicolumn{2}{|c|}{ LCIA } \\
\hline & \multicolumn{2}{|c|}{$\mathrm{NOx}(\mathrm{MT})$} & \multicolumn{2}{|c|}{ VOC (MT-MIR) } & \multirow{2}{*}{$\begin{array}{l}\text { Human } \\
\text { exposure }\end{array}$} & \multirow{2}{*}{$\begin{array}{c}\text { Ecosystem } \\
\text { exposure }\end{array}$} \\
\hline & $\mathrm{PA}$ & LA & $\mathrm{PA}$ & LA & & \\
\hline January & 4.5E-03 & 8.2E-06 & $5.5 \mathrm{E}-05$ & $4.4 \mathrm{E}-05$ & $3.5 \mathrm{E}-03$ & $2.2 \mathrm{E}-04$ \\
\hline February & 3.8E-03 & 7.0E-06 & 4.7E-05 & $3.8 \mathrm{E}-05$ & $7.5 \mathrm{E}-03$ & $1.3 \mathrm{E}-04$ \\
\hline March & $1.8 \mathrm{E}-03$ & $3.3 \mathrm{E}-06$ & $2.2 \mathrm{E}-05$ & $1.8 \mathrm{E}-05$ & $9.6 \mathrm{E}-03$ & $1.0 \mathrm{E}-04$ \\
\hline April & $1.1 \mathrm{E}-03$ & $2.1 \mathrm{E}-06$ & $1.4 \mathrm{E}-05$ & $1.1 \mathrm{E}-05$ & $2.6 \mathrm{E}-02$ & $6.0 \mathrm{E}-05$ \\
\hline May & $4.1 \mathrm{E}-04$ & $7.4 \mathrm{E}-07$ & $5.0 \mathrm{E}-06$ & $4.0 \mathrm{E}-06$ & $2.0 \mathrm{E}-02$ & $1.1 \mathrm{E}-04$ \\
\hline June & $5.1 \mathrm{E}-04$ & 9.3E-07 & $6.2 \mathrm{E}-06$ & $5.0 \mathrm{E}-06$ & $6.4 \mathrm{E}-02$ & $6.3 \mathrm{E}-05$ \\
\hline July & $5.5 \mathrm{E}-04$ & $1.0 \mathrm{E}-06$ & $6.7 \mathrm{E}-06$ & $5.4 \mathrm{E}-06$ & $6.7 \mathrm{E}-02$ & $1.9 \mathrm{E}-04$ \\
\hline August & $5.5 \mathrm{E}-04$ & $1.0 \mathrm{E}-06$ & 6.7E-06 & $5.4 \mathrm{E}-06$ & 4.7E-02 & 9.3E-05 \\
\hline September & $2.8 \mathrm{E}-04$ & 5.2E-07 & $3.5 \mathrm{E}-06$ & $2.8 \mathrm{E}-06$ & $1.6 \mathrm{E}-02$ & $3.6 \mathrm{E}-04$ \\
\hline October & 8.7E-04 & $1.6 \mathrm{E}-06$ & $1.1 \mathrm{E}-05$ & 8.6E-06 & $1.3 \mathrm{E}-02$ & $1.4 \mathrm{E}-04$ \\
\hline November & $2.0 \mathrm{E}-03$ & $3.8 \mathrm{E}-06$ & $2.5 \mathrm{E}-05$ & 2.0E-05 & $1.2 \mathrm{E}-02$ & 5.5E-04 \\
\hline December & $3.6 \mathrm{E}-03$ & 6.6E-06 & $4.4 \mathrm{E}-05$ & 3.6E-05 & $4.3 \mathrm{E}-03$ & $8.6 \mathrm{E}-05$ \\
\hline
\end{tabular}


Table A28 LCI and LCIA results for furnace and AC at Minneapolis

\begin{tabular}{|c|c|c|c|c|c|c|c|c|}
\hline & \multicolumn{8}{|c|}{ Minneapolis } \\
\hline & \multicolumn{5}{|c|}{ LCI } & \multirow[b]{3}{*}{ SD } & \multicolumn{2}{|c|}{ LCIA } \\
\hline & \multicolumn{2}{|c|}{ NOx (MT) } & & \multicolumn{2}{|c|}{ VOC (MT-MIR) } & & \multirow{2}{*}{$\begin{array}{c}\text { Human } \\
\text { exposure }\end{array}$} & \multirow{2}{*}{$\begin{array}{c}\text { Ecosystem } \\
\text { exposure }\end{array}$} \\
\hline & $\mathrm{MN}$ & WY & SD & MN & WY & & & \\
\hline January & $3.9 \mathrm{E}-04$ & 9.4E-05 & $0.0 \mathrm{E}+00$ & $3.2 \mathrm{E}-05$ & $5.0 \mathrm{E}-04$ & $0.0 \mathrm{E}+00$ & $6.8 \mathrm{E}-03$ & 3.9E-04 \\
\hline February & $2.8 \mathrm{E}-04$ & 6.9E-05 & $0.0 \mathrm{E}+00$ & $2.3 \mathrm{E}-05$ & $3.7 \mathrm{E}-04$ & $0.0 \mathrm{E}+00$ & $5.4 \mathrm{E}-03$ & $2.3 \mathrm{E}-04$ \\
\hline March & $1.9 \mathrm{E}-04$ & 4.7E-05 & $0.0 \mathrm{E}+00$ & $1.6 \mathrm{E}-05$ & $2.5 \mathrm{E}-04$ & $0.0 \mathrm{E}+00$ & $5.7 \mathrm{E}-03$ & $1.3 \mathrm{E}-04$ \\
\hline April & $1.3 \mathrm{E}-04$ & $1.9 \mathrm{E}-05$ & $2.6 \mathrm{E}-06$ & $6.9 \mathrm{E}-06$ & $9.3 \mathrm{E}-05$ & $2.6 \mathrm{E}-07$ & $5.6 \mathrm{E}-03$ & $4.9 \mathrm{E}-05$ \\
\hline May & $3.0 \mathrm{E}-04$ & $1.1 \mathrm{E}-05$ & $1.2 \mathrm{E}-05$ & 6.4E-06 & $2.2 \mathrm{E}-05$ & $1.2 \mathrm{E}-06$ & $1.5 \mathrm{E}-02$ & $1.3 \mathrm{E}-04$ \\
\hline June & $4.6 \mathrm{E}-04$ & $1.2 \mathrm{E}-05$ & $1.9 \mathrm{E}-05$ & $8.3 \mathrm{E}-06$ & $3.8 \mathrm{E}-06$ & $1.9 \mathrm{E}-06$ & $3.1 \mathrm{E}-02$ & $3.0 \mathrm{E}-04$ \\
\hline July & $5.7 \mathrm{E}-04$ & $1.5 \mathrm{E}-05$ & $2.3 \mathrm{E}-05$ & $1.0 \mathrm{E}-05$ & $4.6 \mathrm{E}-06$ & $2.3 \mathrm{E}-06$ & $4.0 \mathrm{E}-02$ & $4.3 \mathrm{E}-04$ \\
\hline August & $5.0 \mathrm{E}-04$ & $1.3 \mathrm{E}-05$ & $2.1 \mathrm{E}-05$ & $9.0 \mathrm{E}-06$ & $4.1 \mathrm{E}-06$ & $2.1 \mathrm{E}-06$ & $3.1 \mathrm{E}-02$ & $3.0 \mathrm{E}-04$ \\
\hline September & $2.4 \mathrm{E}-04$ & $8.3 \mathrm{E}-06$ & $9.4 \mathrm{E}-06$ & $4.8 \mathrm{E}-06$ & $1.4 \mathrm{E}-05$ & $9.3 \mathrm{E}-07$ & $9.3 \mathrm{E}-03$ & $1.7 \mathrm{E}-04$ \\
\hline October & $1.4 \mathrm{E}-04$ & $2.2 \mathrm{E}-05$ & $2.4 \mathrm{E}-06$ & $7.9 \mathrm{E}-06$ & $1.1 \mathrm{E}-04$ & $2.4 \mathrm{E}-07$ & $2.8 \mathrm{E}-03$ & 5.1E-05 \\
\hline November & $2.1 \mathrm{E}-04$ & $5.0 \mathrm{E}-05$ & $0.0 \mathrm{E}+00$ & $1.7 \mathrm{E}-05$ & $2.7 \mathrm{E}-04$ & $0.0 \mathrm{E}+00$ & $4.3 \mathrm{E}-03$ & $3.1 \mathrm{E}-04$ \\
\hline December & $3.5 \mathrm{E}-04$ & $8.4 \mathrm{E}-05$ & $0.0 \mathrm{E}+00$ & $2.8 \mathrm{E}-05$ & $4.5 \mathrm{E}-04$ & $0.0 \mathrm{E}+00$ & $4.3 \mathrm{E}-03$ & $4.3 \mathrm{E}-04$ \\
\hline
\end{tabular}

Table A29 LCI and LCIA results for furnace and AC at Austin

\begin{tabular}{|c|c|c|c|c|}
\hline & \multicolumn{4}{|c|}{ Austin } \\
\hline & \multicolumn{2}{|c|}{ LCI } & \multicolumn{2}{|c|}{$\overline{\text { LCIA }}$} \\
\hline & NOx (MT) & VOC (MT-MIR) & Human & Ecosystem \\
\hline & TX & TX & exposure & exposure \\
\hline January & $1.8 \mathrm{E}-04$ & $1.9 \mathrm{E}-04$ & $2.5 \mathrm{E}-03$ & $9.0 \mathrm{E}-04$ \\
\hline February & $2.0 \mathrm{E}-04$ & $1.6 \mathrm{E}-04$ & $2.7 \mathrm{E}-03$ & $3.2 \mathrm{E}-04$ \\
\hline March & $2.4 \mathrm{E}-04$ & $1.0 \mathrm{E}-04$ & $5.2 \mathrm{E}-03$ & $1.2 \mathrm{E}-03$ \\
\hline April & $2.7 \mathrm{E}-04$ & $2.5 \mathrm{E}-05$ & $9.4 \mathrm{E}-03$ & $2.3 \mathrm{E}-04$ \\
\hline May & $4.5 \mathrm{E}-04$ & $4.2 \mathrm{E}-05$ & $2.6 \mathrm{E}-02$ & $1.0 \mathrm{E}-03$ \\
\hline June & $6.2 \mathrm{E}-04$ & $5.8 \mathrm{E}-05$ & $4.7 \mathrm{E}-02$ & $2.5 \mathrm{E}-03$ \\
\hline July & $7.8 \mathrm{E}-04$ & $7.3 \mathrm{E}-05$ & $5.9 \mathrm{E}-02$ & $1.9 \mathrm{E}-03$ \\
\hline August & $7.6 \mathrm{E}-04$ & $7.1 \mathrm{E}-05$ & $5.4 \mathrm{E}-02$ & $1.0 \mathrm{E}-02$ \\
\hline$\overline{\text { September }}$ & $5.1 \mathrm{E}-04$ & $4.7 \mathrm{E}-05$ & $2.6 \mathrm{E}-02$ & $2.1 \mathrm{E}-03$ \\
\hline October & $3.3 \mathrm{E}-04$ & $3.1 \mathrm{E}-05$ & $1.1 \mathrm{E}-02$ & $7.0 \mathrm{E}-04$ \\
\hline November & $2.0 \mathrm{E}-04$ & $8.1 \mathrm{E}-05$ & $5.1 \mathrm{E}-03$ & $8.0 \mathrm{E}-04$ \\
\hline December & $2.0 \mathrm{E}-04$ & $1.8 \mathrm{E}-04$ & $2.1 \mathrm{E}-03$ & $5.0 \mathrm{E}-04$ \\
\hline
\end{tabular}


Table A30 LCI and LCIA results for furnace and AC at Portland

\begin{tabular}{|c|c|c|c|c|c|c|c|c|}
\hline & \multicolumn{8}{|c|}{ Portland } \\
\hline & \multicolumn{5}{|c|}{ LCI } & & \multicolumn{2}{|c|}{ LCIA } \\
\hline & \multicolumn{2}{|c|}{ NOx (MT) } & & \multicolumn{2}{|c|}{ VOC (MT-MIR) } & \multirow[b]{2}{*}{ ID } & \multirow{2}{*}{$\begin{array}{c}\text { Human } \\
\text { exposure }\end{array}$} & \multirow{2}{*}{$\begin{array}{r}\text { Ecosystem } \\
\text { exposure }\end{array}$} \\
\hline & OR & WY & ID & OR & WY & & & \\
\hline January & $2.3 \mathrm{E}-04$ & 5.7E-05 & $0.0 \mathrm{E}+00$ & $1.9 \mathrm{E}-05$ & 3.1E-04 & $0.0 \mathrm{E}+00$ & 3.7E-03 & $2.3 \mathrm{E}-04$ \\
\hline February & 1.7E-04 & $4.0 \mathrm{E}-05$ & $0.0 \mathrm{E}+00$ & 1.4E-05 & $2.2 \mathrm{E}-04$ & $0.0 \mathrm{E}+00$ & $3.4 \mathrm{E}-03$ & $1.2 \mathrm{E}-04$ \\
\hline March & $1.3 \mathrm{E}-04$ & $3.2 \mathrm{E}-05$ & $9.0 \mathrm{E}-08$ & $1.1 \mathrm{E}-05$ & $1.7 \mathrm{E}-04$ & 8.9E-09 & $4.2 \mathrm{E}-03$ & $8.8 \mathrm{E}-05$ \\
\hline April & $9.0 \mathrm{E}-05$ & $2.1 \mathrm{E}-05$ & $1.7 \mathrm{E}-07$ & $7.6 \mathrm{E}-06$ & $1.1 \mathrm{E}-04$ & $1.7 \mathrm{E}-08$ & $3.8 \mathrm{E}-03$ & $3.0 \mathrm{E}-05$ \\
\hline May & $6.5 \mathrm{E}-05$ & $1.2 \mathrm{E}-05$ & $6.1 \mathrm{E}-07$ & 6.3E-06 & $5.4 \mathrm{E}-05$ & 6.1E-08 & 4.1E-03 & $2.0 \mathrm{E}-05$ \\
\hline June & 3.7E-05 & $2.7 \mathrm{E}-06$ & $8.9 \mathrm{E}-07$ & 4.4E-06 & $2.0 \mathrm{E}-06$ & $8.8 \mathrm{E}-08$ & $2.4 \mathrm{E}-03$ & $1.2 \mathrm{E}-05$ \\
\hline July & $5.7 \mathrm{E}-05$ & $4.2 \mathrm{E}-06$ & $1.4 \mathrm{E}-06$ & $6.8 \mathrm{E}-06$ & $3.1 \mathrm{E}-06$ & $1.4 \mathrm{E}-07$ & $4.7 \mathrm{E}-03$ & $1.2 \mathrm{E}-05$ \\
\hline August & 6.0E-05 & $4.5 \mathrm{E}-06$ & $1.5 \mathrm{E}-06$ & 7.1E-06 & $3.2 \mathrm{E}-06$ & $1.4 \mathrm{E}-07$ & $4.1 \mathrm{E}-03$ & $1.8 \mathrm{E}-05$ \\
\hline September & 5.7E-05 & $7.4 \mathrm{E}-06$ & 9.3E-07 & 6.1E-06 & $2.6 \mathrm{E}-05$ & 9.3E-08 & 4.0E-03 & $2.5 \mathrm{E}-05$ \\
\hline October & 8.9E-05 & $2.0 \mathrm{E}-05$ & $2.2 \mathrm{E}-07$ & 7.7E-06 & 1.1E-04 & $2.2 \mathrm{E}-08$ & $2.8 \mathrm{E}-03$ & $2.4 \mathrm{E}-05$ \\
\hline November & $1.5 \mathrm{E}-04$ & $3.5 \mathrm{E}-05$ & $0.0 \mathrm{E}+00$ & $1.2 \mathrm{E}-05$ & $1.9 \mathrm{E}-04$ & $0.0 \mathrm{E}+00$ & $2.8 \mathrm{E}-03$ & $2.0 \mathrm{E}-04$ \\
\hline December & $2.2 \mathrm{E}-04$ & 5.2E-05 & $0.0 \mathrm{E}+00$ & $1.8 \mathrm{E}-05$ & $2.8 \mathrm{E}-04$ & $0.0 \mathrm{E}+00$ & $2.6 \mathrm{E}-03$ & $2.9 \mathrm{E}-04$ \\
\hline
\end{tabular}




\section{REFERENCES}

Andersson-Sköld, Y., Grennfelt, P., Pliejel, K. (1992). Photochemical ozone creation potentials: a study of different concepts. Journal of the Air and Waste Management Association, 42, 1152-1158.

Arya, S.P. (1999). Air pollution meteorology and dispersion. New York, NY, USA: Oxford University Press.

Bare, J.C., Hofstetter, P., Pennington, D.W., Udo de Haes, H.A. (2000). Midpoints versus endpoints: the sacrifices and benefits. International Journal of Life Cycle Assessment, 5 (6), 319-326.

Bare, J.C., Norris, G.A, Pennington, D.W., McKone, T. (2003). TRACI: The Tool for the Reduction and Assessment of Chemical and other environmental Impacts. Journal of Industrial Ecology, 6 (3), 49-78.

Bare, J. C. (2008). Personal communication. January 15.

Bell, M. I., McDermott, A., Zeger, S. L., Samet, J. M., Dominici, F. (2004). Ozone and shortterm mortality in 95 US urban communities, 1987-2000. Journal of the American Medical Association, 292 (19), 2372-2378.

Bell, M. L., Peng, R. D., Dominici, F. (2006). The exposure-response curve for ozone and risk of mortality and the adequacy of the current ozone regulations. Enviromental Health Perspectives, 114 (4), 532-536.

Bellekom, S., Potting, J., Benders, R. (2006). Feasibility of applying site-dependent impact assessment of acidification in LCA. International Journal of Life Cycle Assessment, 11 (6), 417-424.

Byun, D. W., Moon, N., In, H., Song, C. K., Jacob, D., Park, R. (2005). Linking GEOS-Chem with CMAQ: consistency in meteorology and chemistry. Paper presented at the $2^{\text {nd }}$ GEOS-Chem User’s Meeting, April 4-6, Cambridge MA, USA.

Carter, W. P. L. (1994). Development of ozone reactivity scales for volatile organic compounds. Journal of the Air and Waste Management Association, 44, 881-899. 
Carter, W. P. L. (1998). Updated maximum incremental reactivity scale for regulatory applications. Preliminary report to California Air Resource Board. August 6. Retrieved on June 21, 2007 from http://pah.cert.ucr.edu/ carter/bycarter.htm.

Carter, W. P. L., Crosley, D. R., Golden, D. M., Iraci, L. T., Johnston, J. C., Makar, P. A. (1999). $V O C$ reactivity science assessment: atmospheric chemistry and chemical mechanisms. Retrieved on July 5, 2007 from http://www.narsto.org/section.src?SID=10.

Carter, W. P. L. (2000). Documentation of the SAPRC99 mechanism for VOC reactivity assessment. Preliminary report to California Air Resource Board. May 8. Retrieved on June 21, 2007 from http://pah.cert.ucr.edu/ carter/bycarter.htm.

Carter, W. P. L., Tonnesen, G., Yarwood, G. (2003). Investigation of VOC reactivity effects using existing regional air quality models. Report to American Chemical Council. April 17.

Carter, W. P. L. (2007). Development of the SAPRC-07 chemical mechanism and updated ozone reactivity scales. Final report to California Air Resource Board. August 31. Retrieved on September 15, 2007 from http://pah.cert.ucr.edu/ carter/SAPRC/

Cohen, D. S. (2004). Photochemical formation and cost-efficient abatement of ozone: high-order sensitivity analysis. PhD Dissertation. Georgia, USA: Georgia Institute of Technology.

Derwent, R. G., Jenkin, M. E. (1991). Hydrocarbons and the long range transport of ozone and PAN across Europe. Atmospheric Environment, $25 A$ (8), 1661-1678.

Derwent, R. G., Jenkin, M. E., Saunders, S. M. (1996). Photochemical ozone creation potential for a large number of reactive hydrocarbons under European conditions. Atmospheric Environment, 30 (2), 181-199.

Derwent, R. G., Jenkin, M. E., Saunders, S. M., Pilling, M. J. (2001). Characterization of the reactivities of volatile organic compounds using a master chemical mechanism. Journal of the Air and Waste Management Association, 51, 699-707.

Environ International Corporation, Alpine Geophysics LLC, UC Riverside. (2003). VISTAS emissions and air quality modeling — phase I task 2 report: recommended model configurations and evaluation methodology for Phase I modeling. Retrieved on July 15, 2007 from http://pah.cert.ucr.edu/vistas/docs.shtml.

Environ International Corporation. (2006). CAMx user's guide v4.40. Retrieved on March 4, 2007 from http://www.camx.com/publ/index.php.

European Union. (2002). Directive 2002/3/EC of the European Parliament and of the Council. Official Journal of the European Communities. L 67, 9 March 2002, 14-30.

Fava, J., Denison, R., Jones, B., Curran, M.A., Vigon, B. Selke, B., et al. (1991). A technical framework for life-cycle assessment. Pensacola FL, USA: SETAC. 
Fava, J., Consoli, F., Denison, R., Dickson, K., Mohin, T., Vigon, B. (1993). A conceptual framework for life cycle impact assessment. Pensacola FL.: SETAC.

Fiore, A.M., Jacob, D., Bey, I., Yantosca, R. M., Field, B. D., Fusco, A., Wilkinson, J. (2002). Background ozone over the United States in summer: origin, trend, and contribution to pollution episodes. Journal of Geophysical Research, 107 (D15), 4275, doi:10.1029/2001JD000982.

Fiore, A., Jacob, D. J., Liu, H., Yantosca, R. M., Fairlie, T. D., Li, Q. (2003). Variability in surface ozone background over the United States: implications for air-quality policy. Journal of Geophysical Research, 108 (D24), 4787, doi:10.1029/2003JD003855.

Forster, P., Ramaswamy, V., Artaxo, P., Berntsen, T., Betts, R., Fahey, D.W., et al. (2007). Changes in atmospheric constituents and in radiative forcing. In Solomon, S., D. Qin, M. Manning, Z. Chen, M. Marquis, K.B. Averyt, M. Tignor and H.L. Miller (eds.) Climate Change 2007: The Physical Science Basis. Contribution of Working Group I to the Fourth Assessment Report of the Intergovernmental Panel on Climate Change. Cambridge, United Kingdom and New York, NY, USA: Cambridge University Press.

Goedkoop, M., Spriensma, R. (2001). The eco-indicator 99 A damage oriented method for life cycle impact assessment. Retrieved on May 12, 2007 from http://www.pre.nl.

Grey, M. W., Whitten, G. Z., Killus, J. P., Dodge, M.C. (1989). A photochemical kinetics mechanism for urban and regional scale computer modeling. Journal of Geophysical Research, 94, 12925-12956.

Guinée, J.B., Gorrée, M., Heijungs, R., Huppes, G., Kleijn, R., de Koning, A., et al. (2002). Handbook on Life Cycle Assessment: Operational Guide to the ISO Standards. Dordrecht, Netherlands: Kluwer. Retrieved on June 23, 2007 from http://www.leidenuniv.nl/cml/ssp/projects/lca2/lca2.html.

Hanna, S. R., Lu, Z., Frey, H. C., Wheeler, N., Vukovich, J., Arunachalam, S., et al. (2001). Uncertainties in predicted ozone concentrations due to input uncertainties for the UAM-V photochemical grid model applied to the July 1995 OTAG domain. Atmospheric Environment, 35, 891-903.

Haushild, M., Wenzel, H. (1998). Environmental assessment of products Vol. 2 - Scientific background. Boston MA, USA: Kluwer.

Hauschild, M., Potting, J. (2005). Spatial differentiation in life cycle impact assessment - the EDIP2003 methodology. Environmental News No. 80. Copenhagen, Denmark: Danish Environmental Protection Agency.

Hellweg, S., Hofstetter, T. B., Hungerbuhler, K. (2003). Discounting and the environment. International Journal of Life Cycle Assessment, 8 (1), 8-18. 
Heyes, C., Schopp, W., Amann, M., Unger, S. (1996). A reduced form model to predict long term ozone concentrations in Europe. Interim report WP-96-12/Decemeber. Laxenburg, Austria: International Institute for Applied Systems Analysis.

Heyes, C., Schopp, W., Amann, M, Bertok, I., Cofala, J., Gyarfas, F., et al. (1997). A model for optimizing strategies for controlling ground level ozone in Europe. Interim report IR-97002/ January 1997. . Laxenburg, Austria: International Institute for Applied Systems Analysis.

Hofstetter, P. (1998). Perspectives in life cycle impact assessment: A structured approach to combine models of the technosphere, ecosphere, and valuesphere. Boston MA, USA: Kluwer.

International Code Council (ICC). (2003). International Energy Conservation Code. Washington DC, USA: Author.

ISO. (1997). Environmental management-life cycle assessment-principles and framework. ISO 14040:1997. Geneva, Switzerland: ISO.

ISO. (2000). Environmental management-life cycle assessment-life cycle impact assessment. ISO 14042:2000. Geneva, Switzerland: ISO.

Kasibhatla, P., Chameides, W. L., Saylor, R. D., Olerud, D. (1998). Relationships between regional ozone pollution and emissions of nitrogen oxides in the eastern United States. Journal of Geophysical Research, 103 (D17), 22663-22669.

Kemball-Cook, S., Jia, Y., Emery, C., Morris, R., Wang, Z., Tonnesen, G. (2004). 2002 annual mm5 simulation to support WRAP CMAQ visibility modeling for the section 308 sip/tip: MM5 sensitivity simulations to identify a more optimal MM5 configuration for simulating meteorology in the Western United States. Retrieved on July 12, 2007 from http://pah.cert.ucr.edu/aqm/308/reports/2002MM5_SensRpt_091004.pdf

Lee, S. M., Fernando, H. J. S., Clarke-Grossman, S. (2007). MM5-SMOKE-CMAQ as a modeling tool for the 8-h ozone regulatory enforcement: application to the state of Arizona. Environmental Modeling and Assessment, 12, 63-74.

Mao, Q., Gautney, L. L., Cook, T. M., Jacobs, M. E., Smith, S. N., Kelose, J. J. (2006). Numerical experiments on MM5-CMAQ sensitivity to various PBL schemes. Atmospheric Environment, 40, 3092-3110.

McNally, D. (2003). Annual application of MM5 for calendar year 2001. Retrieved on June 14, 2007 from http://www.epa.gov/scram001/reportsindex.htm.

Morris, R., Koo, B., Tonnesen, G., Chien, C. J. (2004). VISTAS phase II task 7 results CB4/SAPRC99 comparisons for urban sites. Presentation at the VISTAS Modeling Conference Call. Retrieved on July 25, 2007 from http://pah.cert.ucr.edu/vistas/vistas2/docs.shtml. 
Murray, C. J. L., Lopez, A. D. (1996). The global burden of disease. Executive Summary. Retrieved on October 20, 2007 from http://hsph.harvard.edu/organizations/bdu.pdf.

National Ambient Air Quality Standards for Ozone; Proposed Rule, 72 Federal Register 37818 (2007).

National Research Council. (1991). Rethinking the ozone problem in urban and regional air pollution. Washington D.C., USA: National Academy Press.

Norris, G. A. (2003). Impact characterization in the Tool for the Reduction and Assessment of Chemical and other environmental Impacts. Journal of Industrial Ecology, 6 (3-4), 79101.

Pennington, D.W., Potting, J., Finnveden, G., Lindeijer, E., Jolliet, O., Rydberg, T., et al. (2004). Life cycle assessment part 2: Current impact assessment practice. Environment International, 30, 721-739.

Potting, J., Haushild, M. (2005). Background for spatial differentiation in life cycle impact assessment - the EDIP2003 methodology. Environmental Project No. 996. Copenhagen, Denmark: Danish Environmental Protection Agency. Retrieved March 10, 2007 from http://www2.mst.dk/Udgiv/publications/2005/87-7614-581-6/html/indhold_eng.htm.

Potting, J., Hauschild, M. (2006). Spatial differentiation in life cycle impact assessment. International Journal of Life Cycle Assessment, 11 (Sp. Iss. 1), 11-13.

Russell, A. G. (1997). Regional photochemical air quality modeling: model formulations, history, and state of science. Annual Review of Energy and the Environment, 22, 537-588.

Russell, A.G., Dennis, R. (2000). NARSTO critical review of photochemical models and modeling. Atmospheric Environment, 34, 2283-2324.

Reynolds, S. D., Roth, P. M., Seinfeld, J. H. (1973). Mathematical model of photochemical air pollution, I. Formulation of the model. Atmospheric Environment, 7, 1033-1061.

Seppala, J. (2003). Life cycle impact assessment based on decision analysis. Doctoral dissertation, Helsinki University of Technology. Retrieved on December 7, 2007 from http://www.sal.hut.fi/Publications/r-index.html.

Shah, V. P., Col Debella, D., Ries, R. J. (2008). Life cycle assessment of residential heating and cooling systems in four regions in the United States. Energy and Buildings, 40 (4), 503513.

Shannon, J. D. (1991). A model of regional long-term average sulfur atmospheric pollution, surface removal, and net horizontal flux. Atmospheric Environment, 15 (5), 689-701.

Simpson, D. (1993). Photochemical model calculations over Europe for two extended summer periods: 1985 and 1989. Model results and comparison with observations. Atmospheric Environment, 27A (6), 921-943. 
Tonnesen, G., Wang, Z., Omary, M., Chien, C. J., Morris, R., Mansell, G. (2005). Final report for the Western Regional Air Partnership (WRAP) Regional Modeling Center (RMC) for the project period March 1, 2004 through February 28, 2005. Retrieved on September 18, 2007 from http://pah.cert.ucr.edu/aqm/308/reports/final.

Udo de Haes, H.A., Jolliet, O., Finnveden, G., Hauschild, M., Krewitt, W., Muller-Wank, R. (1999). Best available practice regarding impact categories and category indicators in life cycle impact assessment. International Journal of Life Cycle Assessment, 4 (2), 66-74.

United Nations Environment Program. (2003). Evaluation of environmental impacts in life cycle assessment. Retrieved on December 15, 2007 from http://cinitiative.unep.fr/default.asp?site=lcinit\&page_id=F511DC47-8407-41E9-AB5D6493413088FB\%20\%20.

United States Environmental Protection Agency (USEPA). (2002). 2002 national emissions inventory booklet. Washington DC, USA: USEPA. Retrieved on November 15, 2007 from http://www.epa.gov/ttn/chief/net/2002neibooklet.pdf.

UESPA. (2005a). Technical support document for the final Clean Air Interstate Rule. Air quality modeling. Retrieved on September 8, 2007 from http://www.epa.gov/scram001/reportsindex.htm.

USEPA. (2005b). Clean Air Interstate Rule emissions inventory technical support document. Retrieved on May 25, 2007 from http://www.epa.gov/scram001/reportsindex.htm.

USEPA. (2005c). Regulatory impact analysis for the final Clean Air Interstate Rule. EPA452/R-05-002. Retrieved on January 18, 2008 from http://www.epa.gov/cair/pdfs/finaltech08.pdf.

USEPA. (2006a). Air quality criteria for ozone and related photochemical oxidants Volume I. EPA 600/R-05/004aF, 2006. Retrieved on November 1, 2007, from http://cfpub.epa.gov/ncea/cfm/recordisplay.cfm?deid=149923.

USEPA. (2006b). Air quality criteria for ozone and related photochemical oxidants Volume II. EPA 600/R-05/004bF. Retrieved on November 1, 2007, from http://cfpub.epa.gov/ncea/cfm/recordisplay.cfm?deid=149923.

USEPA. (2007a). Review of the National Ambient Air Quality Standards for ozone: Policy assessment of scientific and technical information. EPA 452/R-07-007. Retrieved on November 1, 2007, from http://www.epa.gov/ttn/naaqs/standards/ozone/s_o3_cr_sp.html.

USEPA. (2007b). Guidance on the use of models and other analyses for demonstrating attainment of air quality goals for ozone, PM2.5, and regional haze. EPA-454/B-07-002. Retrieved on May 16, 2007 from http://www.epa.gov/scram001/guidance_sip.htm.

World Health Organization. (2005). WHO air quality guidelines for particulate matter, ozone, nitrogen dioxide and sulfur dioxide. Global Update 2005. Summary of risk assessment. WHO/SDE/PHE/OEH/06.02. Geneva, Switzerland: WHO. 
Yarwood, G., Morris, R., Lau, S., Ganesh, U., Tonnesen, G. (2003). Guidance on the application of CAMx probing tools. CRC project A-37-2. Retrieved on September 16, 2007 from http://www.crcao.org/publications/atmosphereImpacts/index.html.

Yarwood, G., Rao, S., Yocke, M., Whitten, G. Z. (2005a). Updates to the carbon bond mechanism: CB05. Retrieved on May 21, 2007 from http://www.camx.com/publ/index.php.

Yarwood, G., Morris, R., Rao., S., Lau, S., Fujita, E., Cambell, D., et al. (2005b) Evaluating VOC receptor models using grid-model simulations. CRC project A-34. Retrieved on September 16, 2007 from http://www.crcao.org/publications/atmosphereImpacts/index.html. 